\title{
Harnessing iNKT cells to improve in situ vaccination for cancer therapy
}

By

Kef Kawin Prasit

\begin{abstract}
A thesis
submitted to the Victoria University of Wellington

in fulfilment of the requirements for the degree of

Doctor of Philosophy
\end{abstract}

Victoria University of Wellington

2020 


\section{Acknowledgements}

Thank you to Professor Ian Hermans and Professor Gavin Painter for the supervision and support they have provided over the years. Additional thanks to the VUW Doctoral Scholarship and the Maurice Wilkins Center for providing funding during my studies.

I would also like to thank the following people:

- Laura Ferrer-Font and Phoebe Harmos for their help with the immune profiling flow cytometry experiments

- Alfonso Schmidt for helping with the preliminary confocal experiments

- Jennifer Chen and Rod Dunbar for providing the analysis and staining for the larger confocal experiment

- Johannes Mayer for helping with the draining lymph node removal surgeries

- Gribbles Veterinary Service for assaying liver ALT levels in multiple samples

- Ching Wen Tang and Anna Mooney for informing me about their own results related to the conjugate vaccines and mouse models that helped inform the discussion in this thesis.

- Christophe Pellefigues for providing information on the IFN $\alpha \mathrm{R}$ antibody dosing 


\begin{abstract}
Toll-like receptor (TLR) agonism in combination with the activation of type I NKT (iNKT) cells through systemic administration of their respective agonists has been shown to have a cooperative effect on activating antigen-presenting cells, stimulating cytokine production, and inducing adaptive immune responses to co-administered antigens. Here, it was hypothesised that it might be possible to harness these activities to treat solid tumours locally via intratumoural treatment to combat tumour growth while reducing toxicity to other organs.

An intratumoural treatment model combining the stimulatory activity of unmethylated DNA oligonucleotides consisting of synthetic cytosine-guanine motifs (CpG), a TLR9 agonist, with activation of iNKT cells through administration of the CD1d-binding iNKT agonist $\alpha$ galactosylceramide ( $\alpha$-GalCer) intratumourally was shown to have significant anti-tumour activity. The treatment regimen showed superior efficacy to that achieved with either agent alone in several in vivo models representing different types of cancer. In some models, the combination of $\alpha$-GalCer and $\mathrm{CpG}$ was effective at inducing the complete rejection of both treated and untreated tumours through the induction of a systemic adaptive immune response. Post tumour rejection, a memory response protected against rechallenge with the same, or similar, tumours. Intratumoural administration of the agents was associated with increases in IFN- $\alpha$ in the tumour (rather than the serum), and blockade or removal of the IFN- $\alpha$ receptor abrogated the antitumour response.
\end{abstract}

The importance of the draining lymph node and spleen in anti-tumour activity (as shown by the excision of these organs), and liver enzyme responses, suggested that some of the agonists/antigens may have dispersed into the lymphoid organs and liver to support the response. 
Nonetheless, the anti-tumour effect was dependent on local effects of the intratumoural administration on the tumour microenvironment, as subcutaneous and peritumoural routes of administration only minimally affected tumour growth despite the reagents potentially having greater exposure to lymphoid organs.

Through the use of various techniques including knockout mice, neutralising monoclonal antibodies, confocal microscopy and flow cytometry, it was shown that the combination of $\alpha$ GalCer and $\mathrm{CpG}$ was dependent on the effector activity of $\mathrm{CD}^{+}$cells. However, optimal activity was associated with changes in other immune cell types, notably recruitment of iNKT cells into the tumour bed, and was also associated with induction of serum antibodies that could transfer some protection to naïve hosts. Induction of a successful response was dependent on conventional dendritic cells (DCs) of the "cDC1" phenotype, which are known to be effective at antigen cross-presentation to $\mathrm{CD} 8^{+} \mathrm{T}$ cells, while full tumour rejection also required the activity of plasmacytoid DCs, which are significant producers of IFN- $\alpha$. In less immunogenic tumour models, the addition of relevant tumour associated antigens (TAAs) improved the anti-tumour response. The TAAs could be added as part of an admix, but improved responses were obtained when TAAs were chemically conjugated to $\alpha$-GalCer via an enzymatically cleavable linker. Alternatively, intratumoural administration of $\alpha-\mathrm{GalCer}$ and $\mathrm{CpG}$ as free agents could be combined effectively with low dose systemic chemotherapy to induce curative responses, potentially through a mechanism involving immunogenic cell death to improve the immunogenicity of TAAs in situ. 
Harnessing iNKT cells to improve in situ vaccination for cancer therapy ..................1

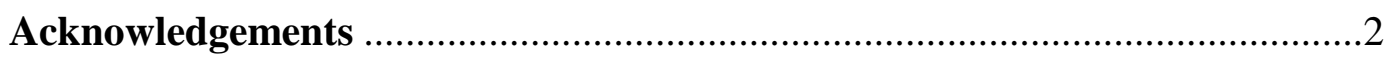

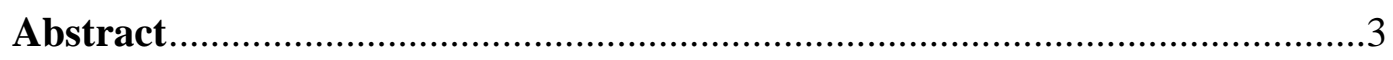

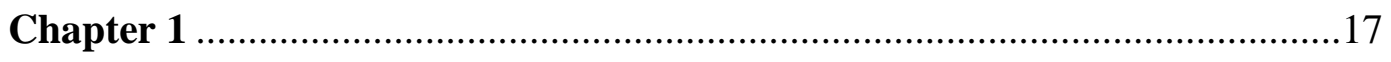

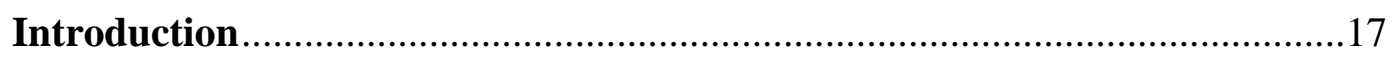

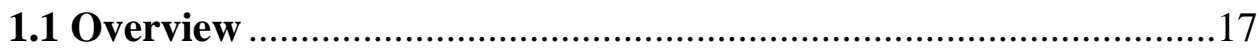

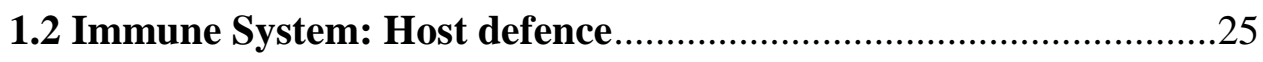

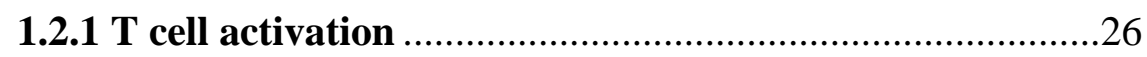

1.2.2 Major histocompatibility complex (MHC) ....................29

1.3 The role of antigen-presenting cells in adaptive immune responses 31

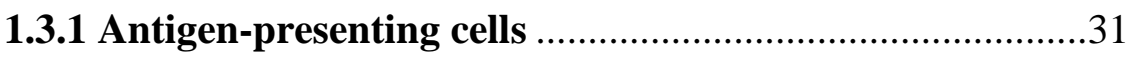

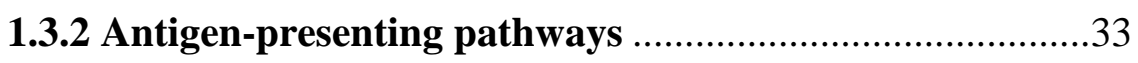

1.3.3 CD8 ${ }^{+}$T cell responses ............................................44

1.4 Toll-Like Receptors (TLRs) ..............................................46

1.4.1 MyD88 Pathway ...................................................

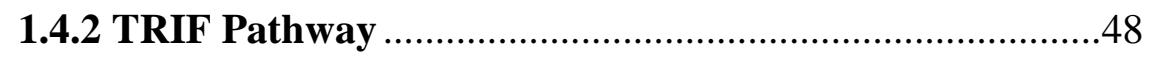

1.5 Properties of Invariant Natural Killer T (iNKT) cells and their role in the

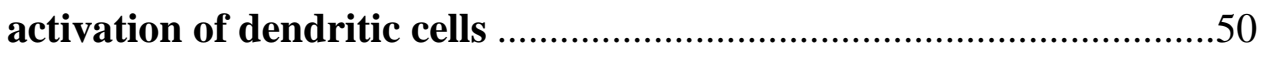

1.5.1 iNKT Cell Biology ..................................................50

1.6 Challenges posed by immune evasion .....................................58

1.7 Use of TLR ligands clinically ...............................................62

1.8 Use of unmethylated CpG ODN to stimulate TLR9 ....................64 
1.9 Intratumoural Delivery of TLR and iNKT cell agonists as a therapy?

Aims

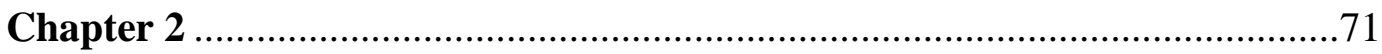

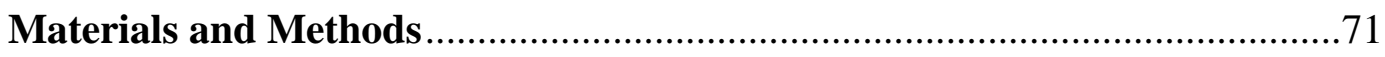

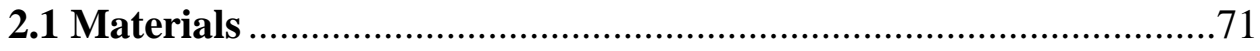

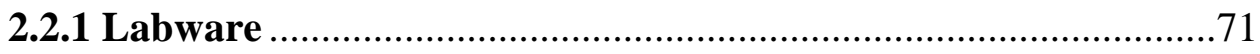

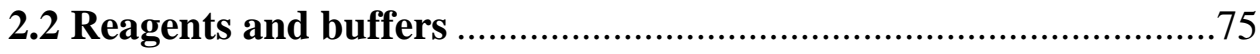

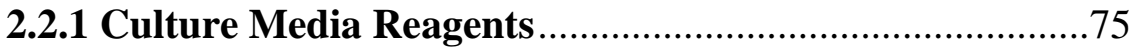

2.2.2 Enzyme and tissue processing …………….......................77

2.2.3 Flow cytometry reagents …………………........................79

2.2.4 Monoclonal antibodies used for in vivo studies .................80

2.2.5 Fluorescently conjugated antibodies .................................80

2.2.6 Immunohistochemistry (IHC) antibodies .........................82

2.2.7 Enzyme-Linked Immunosorbent Assay (ELISA) .............82

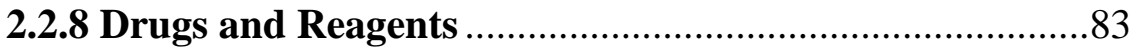

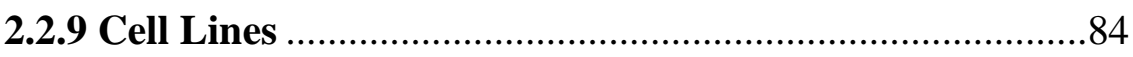

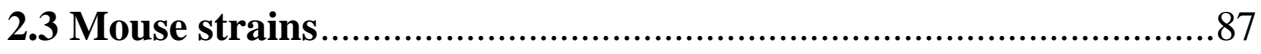

2.3.1 Dendritic cell specific knockouts ..........................................

2.4 Methods

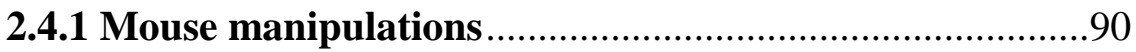

2.4.2 Intratumoural Administration Models .............................91

2.4.3 Tissue preparation ........................................................94 


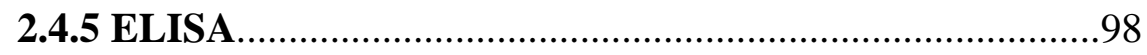

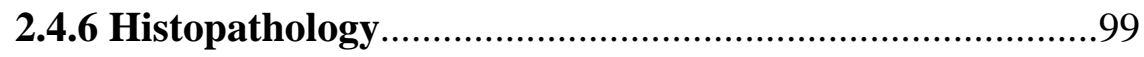

2.4.7 Histology Staining, Analysis \& Microscopy ...................99

Chapter 3

Antitumour effect of combining iNKT cell activation with intratumoural CpG administration in murine tumour models................................................ 101

3.1 Introduction...................................................................... 101

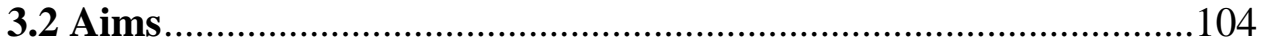

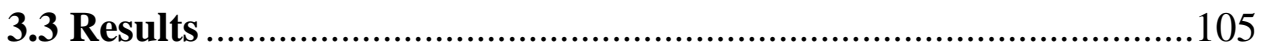

3.3.1 Effect of combining iNKT cell activation with intratumoural CpG administration in a murine lymphoma model. 105

3.3.2 In situ vaccination with a CpG and $\alpha$-GalCer administration in established EL4 Tumours.

3.3.3 Inducing abscopal effect in EL4 tumours

3.3.4 Inducing abscopal effect in EG7.OVA tumours. 115

3.3.5 Comparing the efficacy of different routes of administration

3.3.6 In situ vaccination with a CpG and $\alpha$-GalCer administration in established B16-F1 tumours 120

3.3.7 In situ vaccination with a CpG and $\alpha$-GalCer administration in established TC1 Tumours 
3.3.8 Evaluation of liver toxicity of intratumoural delivery $\alpha$-GalCer +

$$
\text { CpG }
$$

3.4 Discussion 126

Chapter 4

Delineating the role of different immune cell subsets in $\alpha$-GalCer + CpG treatment strategy

4.1 Introduction

4.2 Aims 134

4.3 Results 135

4.3.1 Determining the impact of $\alpha$-GalCer and $\mathrm{CpG}$ on peptidespecific $\mathbf{T}$ cell responses. 135 4.3.2 $\alpha$-GalCer and CpG induce changes in CD8 and iNKT cell populations in the tumours, spleen and draining lymph node139 4.3.3 Influx of immune cells into the tumour microenvironment 180 4.3.4 Exploring the role of $\mathrm{CD8}^{+} \mathrm{T}$ cells 185

4.3.5 Exploring the role of iNKT cells 189

4.3.6 Exploring the role of DC subsets 192

4.3.7 Role of B-cells 197

4.4 Discussion 199

Chapter 5 205

The role of secondary lymphoid organs in the efficacy of intratumoural CpG and $\alpha$ GalCer. 205

5.1 Introduction 205 


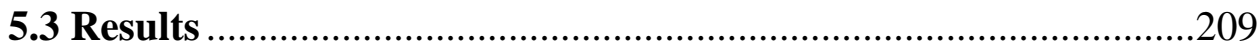

5.3.1 Importance of tumour draining lymph node...............209

5.3.2 Requirement of the spleen for treatment efficacy .........215

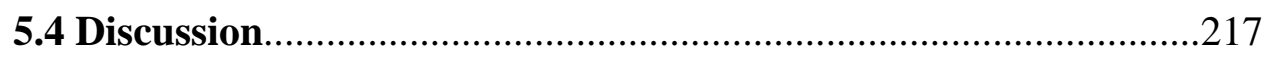

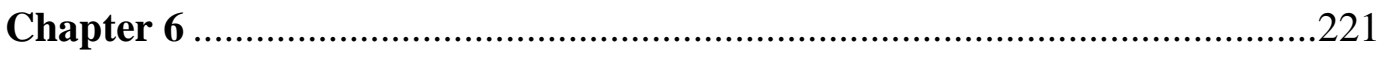

Role of type I IFN in $\boldsymbol{\alpha}$-GalCer + CpG combination therapy ....................221

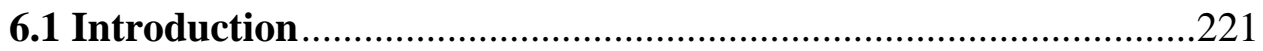

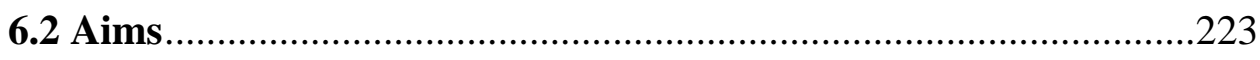

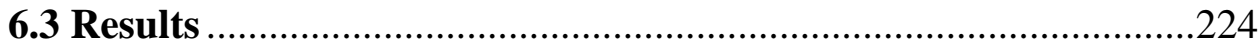

6.3.1 Determining levels of IFN- $\alpha$ in response to therapy ......224

6.3.2 Investigating impact of the blockade of type I IFN signalling 226

6.3.3 Requirement for type I IFN signalling by DCs ............228

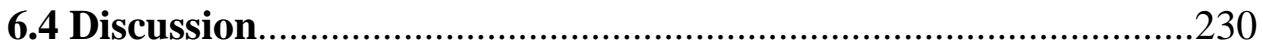

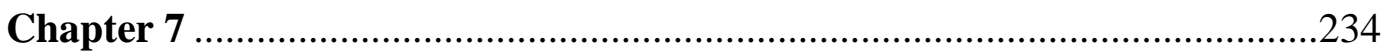

Overcoming non-responsive tumours - developing rational combination strategies

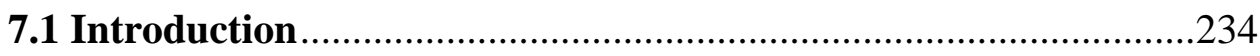

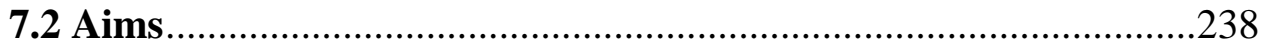

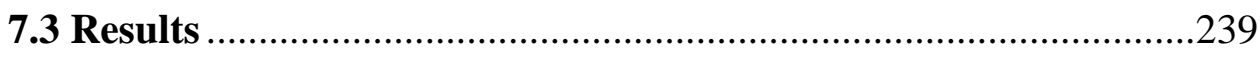


7.3.1 Impact of the addition of tumour associated antigens to $\alpha$ GalCer/CpG: Addition of OVA peptide in EG7.OVA tumour model

7.3.2 Impact of the addition of tumour associated antigens to $\alpha$ GalCer/CpG: Addition of HPV E7 epitope in the TC1 tumour model

7.3.3 Inducing abscopal effect of $\alpha$-GalCer-HPV conjugate + CpG in TC1 and comparison of the conjugate with admix of individual components 246

7.3.4 Impact of $\alpha$-GalCer-Neoepitope conjugate + CpG in B16.OVA tumours 250

7.3.5 Taking the brake off: Impact of $\alpha-$ GalCer + CpG + checkpoint blockade (PD-1) in CT26 tumours. 252 7.3.6 Increasing immunogenicity by inducing immunogenic cell death .256

7.4 Discussion. 260

Chapter 8 .266

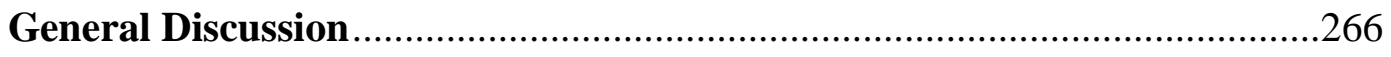

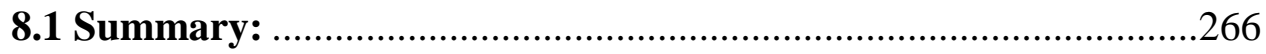

8.2 The abscopal effect: .........................................................269

8.3 Tumour antigen-specific $\mathbf{T}$ cell responses with memory:...........272

8.4 Quality of T cells and other effector immune cells: ..................274

8.5 Treating non-immunogenic tumours: .....................................227 
8.6 Overall mechanism:

8.7 Comparison with closely related approaches:.........................282

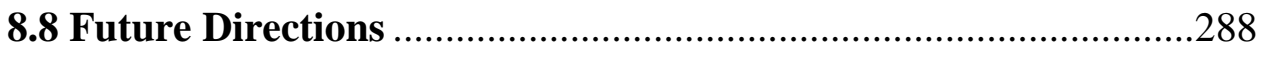

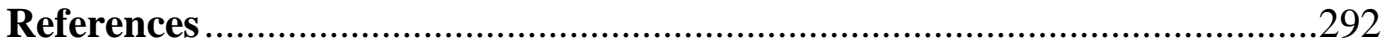

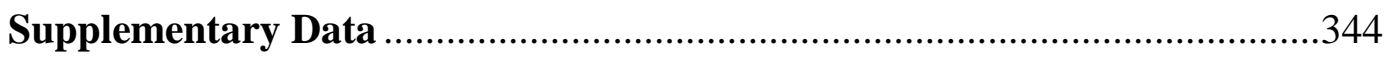




\section{Table of Figures}

Figure 1: The basic MHC class II antigen presentation pathway ....................................... 35

Figure 2: The basic MHC class I antigen presentation pathway. ............................................ 38

Figure 3: Models of phagosomal antigen cross-presentation by MHC-I................................ 40

Figure 4: TLRs and their signalling pathways ..................................................................49

Figure 5: Invariant NKT cells and their role in immunity ....................................................... 54

Figure 6: The chemical structure of a CD1d ligand: $\alpha$-galactosylceramide ( $\alpha$-GalCer).......... 57

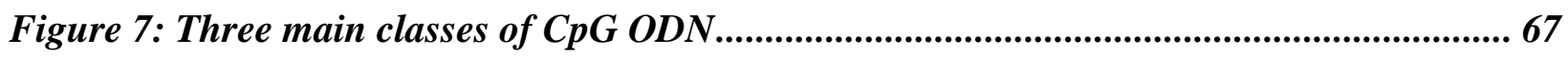

Figure 8 Intratumoural treatment of EG7.OVA tumours with $\alpha$-GalCer and CpG lead to

tumour regression .................................................................................................................. 109

Figure 9 EG7.OVA cured mice can successfully reject rechallenge with EL4.LA tumour cells

Figure 10 Intratumoural treatment of EL4.LA tumours with $\alpha$-GalCer and CpG leads to tumour regression

Figure 11 Intratumoural treatment of EL4.LA tumours with a-GalCer and CpG leads to tumour regression and abscopal effect

Figure 12 Intratumoural treatment of EG7.OVA tumours with $\alpha$-GalCer and CpG leads to tumour regression and abscopal effect

Figure 13 Intratumoural therapy is the most effective route of administration for $\alpha$-GalCer and $C p G$

Figure 14 In situ vaccination with $\alpha$-GalCer and CpG in the B16-F1 tumour model. Dosing schedules were as in earlier experiments. 
Figure 16 Intratumoural delivery of $2 \mu \mathrm{g}$ of $\alpha$-GalCer and $25 \mu \mathrm{g}$ of CpG results in lower alanine transaminase (ALT) levels than intravenous delivery of $\alpha$-GalCer in the serum of mice collected via cheek bleeding 18 hours after the first treatment or 6 hours after CpG treatment in the case of $\alpha-G a l C e r+C p G(6 h)$ 125

Figure 17 Gating strategy for CD8+ T cells with $\mathrm{OVA}^{-p e n t a m e r}{ }^{+}, \mathrm{CD}^{4} 4^{+}, \mathrm{CD}^{2} 2 \mathrm{~L}^{+}$, and KLRGI+ subsets.

Figure 18 The combination of $\alpha$-GalCer and CpG does not increase OVA-specific CD8 ${ }^{+} \mathrm{T}$ cells in the blood collected 7 days after the start of treatment.. 138

Figure 19 Gating strategies for $\mathrm{B}$ cells, Tregs, $\mathrm{CD}^{+}, \mathrm{CD}^{+}$and iNKT cells 141

Figure 20 Intratumoural combination therapy of $\alpha-G a l C e r$ and $C p G$ does not increase the frequency of OVA-specific $\mathrm{CDB}^{+} \mathrm{T}$ cells in tumours 143

Figure 21 Intratumoural combination therapy of $\alpha$-GalCer and CpG decreases the proportion of regulatory $T$ cells (Tregs) in treated tumours relative to $\alpha$-GalCer and vehicle. 144 Figure 22 Intratumoural combination therapy of $\alpha$-GalCer and CpG does not induce significant changes in the $\mathrm{CD4}^{+} \mathrm{T}$ cell populations in tumours 147

Figure 23 Intratumoural combination therapy of $\alpha-G a l C e r$ and $C p G$ does not induce significant changes in the $\mathrm{CD}^{+} \mathrm{T}$ cell populations in tumours 150

Figure 24 Intratumoural combination therapy of $\alpha$-GalCer and CpG induces significant changes to the iNKT cell populations in tumours 154

Figure 25 Intratumoural combination therapy of $\alpha-G a l C e r$ and $C p G$ does not increase the frequency of B cells in tumours 155

Figure 26 Intratumoural combination therapy of $\alpha-G a l C e r$ and $C p G$ does not increase the frequency of OVA-specific CD8 ${ }^{+}$T cells in the draining lymph node (dLN). 
Figure 27 Intratumoural combination therapy of $\alpha$-GalCer and $C p G$ decreases the proportion of regulatory $T$ cells (Tregs) in dLN near treated tumours relative to $\alpha$-GalCer and vehicle 157 Figure 28 Intratumoural combination therapy of $\alpha$-GalCer and $C p G$ does not induce significant changes in the $\mathrm{CD}^{+} \mathrm{T}$ cell populations in $\mathrm{dLN}$. 162

Figure 29 Intratumoural combination therapy of $\alpha$-GalCer and CpG does not induce significant changes in the $C D 8^{+} T$ cell populations in $d L N$ 167

Figure 30 Intratumoural combination therapy of $\alpha$-GalCer and CpG induces significant changes to the iNKT cell populations in the draining lymph node

Figure 31 Intratumoural combination therapy of $\alpha$-GalCer and CpG increases the frequency of $B$ cells in the dLN of treated tumours

Figure 32 Intratumoural combination therapy of $\alpha$-GalCer and CpG does not increase the frequency of $O V A$-specific $C D 8^{+} T$ cells in the spleen

Figure 33 Intratumoural combination therapy of $\alpha$-GalCer and CpG does not induce changes in the proportion of regulatory $T$ cells (Tregs) in the spleen 174

Figure 34 Intratumoural combination therapy of $\alpha$-GalCer and $C p G$ induces a reduction in the $\mathrm{CD4}^{+} \mathrm{T}$ cells in the spleen. 175

Figure 35 Intratumoural combination therapy of $\alpha$-GalCer and CpG does not induce significant changes in the $\mathrm{CDB}^{+} \mathrm{T}$ cell subpopulations in the spleen. 176 Figure 36 Intratumoural combination therapy of $\alpha$-GalCer and CpG induces significant changes to the iNKT cell populations in the spleen. 178 Figure 37 Intratumoural combination therapy of $\alpha$-GalCer and CpG does not increase the frequency of $B$ cells in the spleen 179 
Figure 38 Intratumoural combination of a-GalCer and $\mathrm{CpG}$ induces an influx of $\mathrm{CD4}^{+}, \mathrm{CD}^{+}$, $\mathrm{CD64}^{+}$and $\mathrm{CD4}^{+} \mathrm{CD64}^{+}$cells

Figure 39 Treatment with intratumourally administered a-GalCer and CpG induces infiltration by NK, T cells and macrophages in treated tumours and to a lesser extent the untreated contralateral tumour

Figure 40:Memory against EL4.LA is CD8 mediated as mice depleted of CD8 ${ }^{+}$cells lose memory against rechalleng. 187

Figure $41 \alpha$-GalCer/CpG combination therapy requires $\mathrm{CD}^{+}$cells 188

Figure $42 \alpha$-GalCer/CpG combination therapy requires iNKT cells...... 191

Figure $43 \alpha$-GalCer and CpG mediated anti-tumour response requires $C l e c 9 a^{+} D C s$. 194

Figure $44 \alpha$-GalCer and CpG mediated anti-tumour response requires Siglec-H $\mathrm{H}^{+} \mathrm{DCs}$ 196

Figure 45 Transfer of serum from mice cured of EG7.OVA into naïve mice protects against EG7.OVA challenge. 198

Figure 46 Intratumoural injection of 40uL calligraphy dye drains that the inguinal lymph nodes within 1 hour of injection, indicating the inguinal lymph node is the primary draining lymph node. 210

Figure 47 Removal of draining lymph node partially abolishes anti-tumour immunity in an EG7.OVA tumour model.. 213

Figure 48 Removal of the tumour draining lymph node diminishes the anti-tumour effect of intratumoural $\alpha$-GalCer and CpG in the ELA.LA tumour model. 214

Figure 49 Intratumoural $\alpha$-GalCer and CpG is ineffective after the removal of the spleen compared to sham surgery 216 
Figure 50 Type 1 IFN detected by ELISA is increased in the tumour but not the serum 6 hours

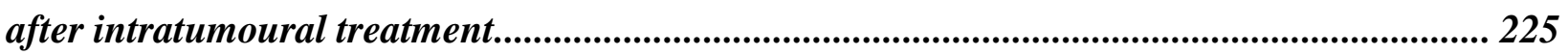

Figure 51 IFN $\alpha$ R blockade leads to complete abrogation of $\alpha$-GalCer and CpG mediated anti-

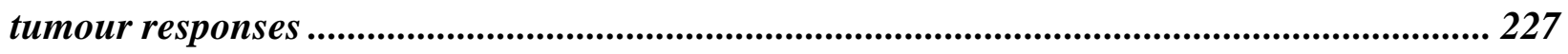

Figure 52 IFNaR knockout on CD11 ${ }^{+}$cells leads to loss of $\alpha$-GalCer and CpG mediated anti-

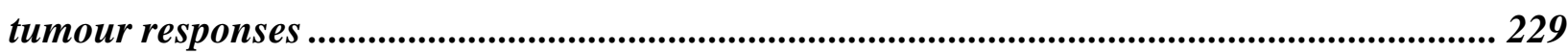

Figure 53 Intratumoural administration of $\alpha$-GalCer-OVA conjugate and CpG combine to induce regression in EG7.OVA model

Figure 54 Intatumoural combination of $\alpha$-GalCer-E6 conjugate and CpG induces regression

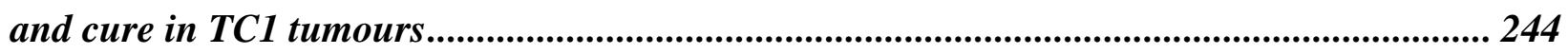

Figure 55 Mice cured of TC1 tumours are immune to rechallenge ..................................... 245

Figure 56 Intratumoural combination of $\alpha$-GalCer-E7 conjugate and CpG induces regression and cure in bilaterally implanted TC1 tumours............................................................. 248

Figure 57 TC1 anti-tumour memory is CD8 mediated.................................................... 249

Figure 58 Intatumoural combination of $\alpha$-GalCer-neoepitope conjugate and CpG induces

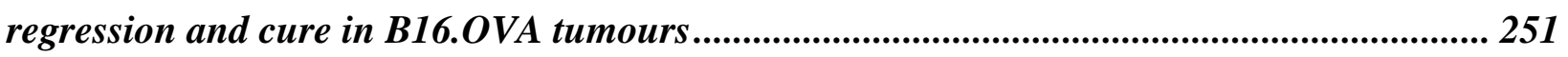

Figure 59 In situ vaccination with $\alpha$-GalCer and CpG in a CT26 tumour model .................. 254

Figure 60 in situ vaccination induced anti-tumour memory is CD8 mediated ..................... 255

Figure 61 Intatumoural combination of $\alpha$-GalCer and $C p G$ with low dose doxorubicin induces regression and cure in B16.F10 tumours............................................................... 259

Figure 62 Simplified Mechanistic Model........................................................................ 282 


\section{Chapter 1}

\section{Introduction}

\subsection{Overview}

Vaccination has revolutionised modern medicine by harnessing the immune system to prevent and eradicate infectious diseases, saving millions of lives in the process. For centuries, people lived under the constant threat of deadly infectious diseases, like smallpox, polio, and hepatitis. Edward Jenner developed the world's first standardised vaccine in 1796 by experimentally validating a well-known countryside folklore, where milkmaids who suffered the mild disease of cowpox never contracted smallpox, which was as prevalent as it was deadly during that period, especially among children. He discovered that he could protect people from the devastating disease by inoculating people with matter from a fresh lesion from a milkmaid with the related, though relatively benign, cowpox virus. This ushered the beginning of modern vaccinology: the use of attenuated, killed pathogens or a component of the whole pathogen to induce adaptive immunity and protect against illness and death following a future exposure to that pathogen. For many centuries before this experiment, the much less safe technique of variolation was used to protect against death from smallpox infection, which involved exposing healthy individuals to pus from a smallpox-infected individual to induce a mild infection ${ }^{1}$. Vaccination developed further during the nineteenth century when Pasteur created the first attenuated bacterial vaccine against cholera in chickens, and further developed vaccines against rabies, cholera, and typhoid plague that would eventually be used in humans ${ }^{2}$.

There are several FDA-approved vaccines for cancer prevention such as the hepatitis B vaccine and the human papillomavirus (HPV) vaccines, which work by preventing infection 
from cancer-causing viruses. By preventing the viral infection, these vaccines block processes that may eventually result in runaway cancer cell growth; however, most cancers are not caused by viruses ${ }^{3}$. On the other hand, therapeutic vaccines are used as a method of treatment. Similar to regular vaccines, therapeutic vaccines work by stimulating the immune system in order to target an infection or cancer cells. However, a therapeutic cancer vaccine would be used to treat existing cancers by alerting the immune system to recognise and destroy cancer cells, or by boosting weak responses that may have been induced naturally ${ }^{4}$.

Just like any other effective immunisation approaches, therapeutic cancer vaccines need to focus responses against antigens, substances that cause an immune response, expressed explicitly in the target tissue. These antigens need to be injected and then acquired in vivo by antigen-presenting cells (APCs) to provoke an adaptive immune response. It is now clear that responses mediated by antigen-specific T cells can induce tumour regression; hence, APCs need to be programmed with the capacity to stimulate $\mathrm{T}$ cell activation. $\mathrm{T}$ cells are a type of white blood cell that develop in the thymus gland and play a central role in the immune response. This can be achieved by injecting vaccines with adjuvants, which are immunostimulatory compounds that directly, or indirectly, modulate APC function. The tumour-associated antigens targeted by $\mathrm{T}$ cells arise as a consequence of the mutational load associated with disease progression (which could include virus-derived antigens), resulting in a dysregulated or mutated protein profile. Since this profile is generally patient-specific and even shows diversity between cancer lesions, there is a paucity of universal tumour-associated antigens for a vaccine design of general utility. Also, defining and preparing individualised vaccines remains technically challenging (although progress is being made), so the development of cancer vaccines has been slow ${ }^{5}$. In addition, the immunosuppressive nature of the tumour microenvironment generally renders otherwise 
promising vaccines ineffective. Thus, the first and only therapeutic vaccine approved for any cancer remains an autologous cell-based vaccine sipuleucel-T. Sipuleucel-T consists of activated autologous APCs loaded with prostatic acid phosphatase, an enzyme produced by the prostate whose level proportionally increases with prostate cancer progression. In a pivotal phase III trial, sipuleucel-T gave a median overall survival of 25.8 months compared with 21.7 months for placebo-treated patients. In addition, it also resulted in a $22 \%$ relative reduction in the risk of death ${ }^{6}$. However, due to the high cost and the small efficacy advantage, sipuleucel-T was not widely used.

Although cancer vaccine design has been progressing, notably with recent advances in developing personalised vaccines based on bioinformatic analysis of the mutational load in a patient's tumour ${ }^{7,8}$, more straightforward approaches are needed. The major hurdles in the development of cancer vaccines are: 1) the identification of universal antigens that can be used in 'off-the-shelf' vaccines for common cancers, 2) the bioinformatic and manufacturing bottleneck associated with the use of personalised synthetic vaccines, 3) the manufacturing and logistical hurdles associated with alternative ways to make individualised vaccines based on resected tumour or biopsy material, 4) tumour-induced immunosuppression limiting efficacy, and 5) the production of an immune response of sufficient quality and magnitude. "In situ vaccination", the topic of this thesis, represents an alternative approach in which the cancer tissue is modified through treatment in vivo so that it acts like a vaccine to promote tumour-associated antigen (TAA)-specific adaptive immune responses. The treatment may also overturn some of the immunosuppressive networks that limit on-going immunity. Importantly, this can be done without the need to identify and isolate the TAAs previously. The strategy relies on improving the immunogenicity of TAA released by stressed, dying, or dead tumour cells that are processed 
and presented by APCs. This kind of approach allows for the development of "vaccines" in patients themselves, without the requirement for many of the ex vivo processing steps or synthetic pathways required to prepare typical parenterally injected vaccines. In addition, this strategy can take advantage of the complete antigenic repertoire of a tumour and not be limited to pre-selected TAA's that may exclude undetectable or uncharacterized antigens. The efficiency of in situ vaccination can potentially be enhanced by boosting specific steps, such as using conventional therapies to stimulate tumour cell death to release TAAs in an immunogenic manner (“immunogenic cell death"), involving enhanced antigen uptake and presentation by APCs ${ }^{9,10}$. Ideally, an in situ vaccine should be able to induce anti-tumour $\mathrm{T}$ cell responses that will result in systemic anti-tumour immunity.

The induction of $\mathrm{T}$ cell responses requires specialised APCs. The most important are dendritic cells (DCs), which have a high capacity for acquiring, processing, and presenting antigens via major histocompatibility complex (MHC) molecules to antigen-specific T cells. The main function of MHC molecules is to present peptide fragments from foreign antigens on the cell surface for recognition by the appropriate $\mathrm{T}$ cells. The function of DCs, and hence their ability to stimulate $\mathrm{T}$ cells, can be modulated through multiple signals. These signals include recognition of pathogen-associated molecular patterns (PAMPs) via innate pattern recognition receptors, of which the Toll-like receptors (TLRs) are prominent examples (to be discussed in more detail below). Another way to stimulate DCs is via signals provided by rapidly activated leukocytes in the local environment, including $\mathrm{T}$ cells themselves. In this context, within the lymphoid tissues are subpopulations of "innate-like" $\mathrm{T}$ cells that respond to a limited range of defined antigens that can be rapidly activated to perform this function. For example, invariant natural killer T (iNKT) cells, otherwise known as type I NKT cells, are specific for a small range 
of glycolipids, and administration of these glycolipids in vivo can induce rapid iNKT cell activation, which subsequently leads to activation of DCs (more detail on this series of events will follow). At the outset of this $\mathrm{PhD}$ study, our group had already shown that activation of iNKT cells could condition multiple different DC subsets to respond more effectively to TLR ligation. Depending on the TLR agonist used, among the effects observed were the activation of plasmacytoid DCs (pDCs), a cell type associated with the release of the immunostimulatory cytokine interferon $\alpha(\mathrm{IFN} \alpha)$, and enhanced activation of conventional DCs (cDCs). Activated cDCs increase expression of molecules required for T cell stimulation (including MHC molecules), and increase production of cytokines, notably the $\mathrm{T}$ cell differentiation cytokine IL12. Ultimately, the combination of signals from iNKT cells and TLR ligation "conditioned" the cDCs to induce stronger $\mathrm{T}$ cell responses to injected antigens ${ }^{11}$. Given that some TLR ligands have been approved for clinical use (e.g. the TLR7 agonist imiquimod) and newer ones are being tested as potential cancer treatments, we speculated that the addition of an iNKT cell-activating glycolipid to a TLR agonist could enhance therapeutic effects in a cancer setting.

The main focus of this work, therefore, was to test the hypothesis that "harnessing the immune-stimulating activities generated by the activation of iNKT cells, together with TLR ligation in situ, will drive superior anti-tumour immune responses". To achieve this, compounds that drive these two activities were administered intratumourally (directly into the tumours). The local administration was also anticipated to reduce the systemic release of cytokines, which can be dose-limiting by systemic administration routes. Intratumoural delivery of immunostimulatory compounds should induce a local effect, including increased pro-inflammatory cytokines that may enhance immune infiltration and antitumour activity. The intratumoural approach should, therefore, provide a superior therapeutic index (the ratio between the dose that gives efficacy 
versus the dose that causes side effects) compared to other routes, particularly intravenous administration where numerous tissues are unnecessarily exposed to damaging pro-inflammatory cytokines.

If a tumour can be accessed to inject, why not simply remove it? If the primary tumour is easily accessible, or is not dangerously near vital organs and has not metastasised, resectioning of the tumour would be the first line of treatment of choice. However, in cases where tumours are near a vital organ, or they have metastasised making resection impractical, intratumoural injection offers an alternative route. Intratumoural injections can be considered for any tumour where the primary lesion or its metastatic lesions are accessible either percutaneously via direct injection or specific procedures such as colonoscopy, cystoscopy, bronchoscopy, thoracoscopy, coelioscopy, or even surgery ${ }^{12}$. Injection at some tumour sites might require the support of the use of imaging such as ultrasound, computed tomography (CT) and magnetic resonance for guidance. In addition, direct injection into the tumour might not only reduce systemic exposure, off-target toxicities, and the amounts of drugs required, but may also induce systemic antitumour activities that have a clinical impact on distant non-injected tumour lesions. This is the so-called "abscopal effect” ('ab' — away from, 'scopus' — target) and obviates the need to inject into every tumour.

In order to provide the background necessary to understand the experiments conducted in this thesis concerning in situ vaccination, the sections that follow will cover important immunological concepts and the cell-types involved. A primary focus is on the concept that TLR agonists can enhance adaptive immunity, specifically mediated by $\mathrm{T}$ cells. The other arm of adaptive immunity involves antibody-producing B cells, which do not feature significantly in this study and are thus only described briefly. In both mice (the principal tool used here) and 
humans, there are two major $\mathrm{T}$ cell populations defined by expression of CD4 or CD8. Activated $\mathrm{CD} 4^{+} \mathrm{T}$ cells function as "helper" cells $(\mathrm{Th})$ that release cytokines when an antigen is recognised and can be sub-grouped further based on the cytokines they produce. In certain situations, some may even be suppressive, and play a role in supporting tumour growth; these cells, known as regulatory $\mathrm{T}$ cells (Treg), are defined by expression of the transcription marker FOXP $3{ }^{13}$. On the other hand, activated $\mathrm{CD} 8^{+} \mathrm{T}$ cells can differentiate into cytotoxic $\mathrm{T}$ lymphocytes (CTL) with the capacity to kill cellular targets that express a target antigen. These $\mathrm{T}$ cells have been commonly regarded as the most critical anti-tumour effectors, although it is becoming increasingly apparent that $\mathrm{CD} 4^{+} \mathrm{T}$ cells can also play a significant role ${ }^{14-16} . \mathrm{CD} 4^{+} \mathrm{T}$-cells are considered "helper" cells because they provide signals to support adaptive immunity. This "help" is required to activate B cells to secrete antibodies, to stimulate macrophages to destroy ingested microbes, and to help stimulate CD8 T cells to differentiate into CTLs capable of killing target cells ${ }^{17}$. Importantly, activated $\mathrm{T}$ cell populations clonally proliferate in order to exert their activities, but then reduce upon resolution of disease, leaving behind a population of "memory" cells that are highly active when the same antigen is encountered at a later date; this is the basis of prophylactic vaccination. Most $\mathrm{T}$ cells recognise antigens in the form of peptide fragments presented on the target cell's surface by MHC molecules, so the capacity of a target cell to present such peptides is critical to clinical impact.

As already noted, this thesis will bring significant focus to a less known population of $\mathrm{T}$ cells, known as iNKT cells ${ }^{18}$, which recognise glycolipids presented via the MHC-like molecule CD1d. iNKT cells are distinct from natural killer (NK) cells; although these two cell-types share many features, such as rapid production of cytokines and expression of certain cell-surface markers, iNKT cells (like all $\mathrm{T}$ cells) express a $\mathrm{T}$ cell receptor (TCR) to recognise antigen, while 
NK cells do not (although they can recognise and kill cells that show features of stress).

However, the activities of iNKT cells and NK cells are linked, as will be described later.

Several concepts and cell populations are a significant feature of in situ vaccination, and these concepts will be discussed in more detail below. Briefly, an essential feature of in situ vaccination is the acquisition of antigen and its presentation to $\mathrm{T}$ cells. The quality of immune responses is often defined by the APCs involved in antigen-presentation to T cells. A description of the critical APCs, with a focus on different DC populations, is therefore provided below. Finally, the tumour microenvironment is often highly resistant to adaptive immunity. This concept, and cell populations involved, will be explored in detail below, as well as how the microenvironment needs to be altered for in situ vaccination to work.

Ultimately, this introduction will give background on cancer therapy with TLR agonists, providing the reasons why treatment with the TLR9 agonist $\mathrm{CpG}$ (defined below) is a significant feature of this thesis, and why it was important to combine this approach with the activation of iNKT cells. 


\subsection{Immune System: Host defence}

The immune system is a complex network of immune cells and organs designed to defend the host from pathogens and for regulating the host's cells to prevent dysregulation. By understanding the interplay between immune cells, we can design strategies that harness the immune response to treat various diseases.

In adult mammals, immune cells develop in the bone marrow and thymus, and then migrate and reside within peripheral tissues and secondary lymphoid tissues (lymph nodes and spleen $)^{19,20}$. Innate immune cells can defend against a wide variety of pathogens via patternrecognition receptors (PRRs), which can recognise pathogen-associated molecular patterns (PAMPs) such as bacterial cell wall components or viral capsids. Upon stimulation, innate immune cells respond quickly by phagocytosing the pathogen or by releasing pro-inflammatory cytokines to induce inflammatory responses and kill the pathogen ${ }^{21,22}$. Adaptive immune cells on the other hand, such as B lymphocytes and $\mathrm{CD} 4^{+}$and $\mathrm{CD} 8^{+} \mathrm{T}$ lymphocytes, have randomly generated receptors, and undergo a process of clonal selection after antigen exposure that involves amplifying clones that recognise epitopes within specific antigen structures. Thus, upon recognition of antigen through the B cell receptor (BCR) on B cells, or via presentation by APCs via $\mathrm{MHC}$ molecules to the $\mathrm{T}$ cell receptor (TCR) on $\mathrm{T}$ cells, the adaptive immune cells are activated against that particular antigen, proliferate extensively, and elicit effector functions to fight the disease. After clearing the disease, memory cells are left behind so that an effector response can be mounted at an increased rate when re-encountering an antigen ${ }^{23}$. The adaptive immune response is vital for providing a significant, highly specific response that directly targets and eliminates pathogens while providing long-term protection against reinfection. 
Activated B cells produce antibodies that recognise and bind to specific pathogenassociated structures expressed on the surface of pathogen-derived molecules, which prevents the pathogen from infecting host cells ${ }^{24}$. Antibodies can also bind to pathogens to cause opsonisation, targeting the pathogen for elimination by phagocytic cells that recognise the constant region of the antibody structure $(\mathrm{Fc})^{25}$. Similarly, Fc region recognition-mediated antibody binding to surface-expressed pathogen-associated target antigens on a host cell can also trigger direct cytotoxicity by natural killer (NK) cells, macrophages, neutrophils or eosinophils in a process called antibody-dependent cell-mediated cytotoxicity (ADCC). However, antibodies cannot bind to intracellular antigens associated with infection, precipitating the need for T cells

\subsection{1 $\mathrm{T}$ cell activation}

$\mathrm{T}$ progenitor cells are formed in the bone marrow and migrate to the thymus (hence the name "T cell") to mature. In the thymus, the developing T cells start to express randomly generated TCRs and CD4 and CD8 receptors. All T cells express TCRs, and CD4 or CD8 or a combination thereof. Since there are fewer than 30,000 genes in the human body, it is impossible to have specific genes encoding receptors for all possible antigens. Instead, the DNA of the developing $\mathrm{T}$ cells residing in the bone marrow is shuffled to create cells with unique receptors

23. The TCR genes undergo rearrangement, giving rise to almost limitless potential combinations which allow for binding diversity. While this binding diversity shapes the immune response against pathogens, it could potentially lead to accidental binding against self-molecules, which could cause autoimmunity. Under optimal conditions, mature T cells should only recognise foreign antigens combined with self-MHC molecules in order to mount an appropriate immune response. In order to achieve this, $\mathrm{T}$ cells undergo positive and negative selection. In positive selection, T cells in the thymus with TCRs that have moderate binding avidity to MHC 
complexes receive survival signals. In negative selection, $\mathrm{T}$ cells with TCRs that bind too strongly to MHC complexes, and will likely be self-reactive, undergo arrest and entry into the death programme known as apoptosis. Likewise, T cells with TCRs that do not bind to MHC complexes at all also undergo apoptosis ${ }^{23}$. These two selection processes are designed to protect host cells and tissues from being a target of an immune response, and without these selection processes, autoimmune diseases would likely kill the host. Cells that survive the selection process then differentiate into either naïve $\mathrm{CD} 4^{+}$or $\mathrm{CD} 8^{+} \mathrm{T}$ cells depending on whether their TCR recognises an MHC class I-presented antigen $\left(\mathrm{CD}^{+} \mathrm{T}\right.$ cell) or an MHC class II-presented antigen $\left(\mathrm{CD} 4^{+} \mathrm{T}\right.$ cell). From here, they move into the blood and populate the lymphoid organs, with constant recirculation. At this stage, they are referred to as mature, having completed the $\mathrm{T}$ cell differentiation process. Some of the $\mathrm{CD} 8^{+} \mathrm{T}$ cells will go on to become cytotoxic $\mathrm{T}$ cells (CTL) following their activation with an MHC class I-restricted antigen ${ }^{23} . \mathrm{CD}^{+} \mathrm{T}$ cells become activated by a specific peptide antigen that is presented by MHC class II molecules expressed on the surface of APCs. Once activated, they divide rapidly and secrete cytokines that regulate or assist the active immune response, including maturation of B cells into plasma cells and memory B cells ${ }^{26}$. At least four distinct CD4 T-cell subsets have been shown to exist, Th1, Th2, Th17, and Treg cells. Th1 cells, driven by transcription factors STAT4 and T-bet, mainly secrete IL-12 and IFN- $\gamma$; whereas Th2 cells, which are modulated by transcription factors STAT6 and GATA3, secrete IL-4, IL-5, IL-9, IL-10, IL-13, and IL-25 ${ }^{27}$. Functionally, Th1 cells predominantly promote cell-mediated immunity and help in clearance of intracellular pathogens, while Th2 cells are responsible for humoural immunity protecting against extracellular invaders, notably helminths. The balance between exposure to the T cell polarising cytokine IL-12, favouring Th1 responses, or IL-4, favouring Th2 responses, determines the bias expressed by the immune 
response $^{27}$. The formation of Th17 cells is controlled by the transcription factors STAT3, ROR $\gamma$ and ROR $\alpha$ in combination with a milieu of cytokines including TGF- $\beta$, IL6, IL-21 and IL-23. Th17 cells are pro-inflammatory and play an important role in host defense against infection by recruiting neutrophils and macrophages to infected tissues. In contrast, Tregs, defined by expression of the transcription factor FOXP3, suppress immune responses, thereby maintaining homeostasis and self-tolerance.

T cells require at least two receptor-mediated signals and soluble factors to become activated. The first signal is provided by antigen presentation where a peptide is presented to the TCR via MHC molecules. The second involves stimulation from co-stimulatory molecules expressed by T cells such as CD28, which interacts with CD80 (B7.1) and CD86 (B7.2) on the membrane of APCs ${ }^{28}$. This T cell co-stimulation is necessary for T cell proliferation, differentiation, and survival. Activation of T cells without co-stimulation may lead to T cell anergy (a period of exhaustion where cells are not responsive to further stimulation), T cell deletion, or the development of immune tolerance ${ }^{29}$. Once activated, the $\mathrm{CD}^{+}$and $\mathrm{CD} 4^{+} \mathrm{T}$ cells undergo clonal expansion with the help of the cytokine IL-2, a growth and differentiation factor for T cells, and other pro-inflammatory cytokines, such as IL-12 and type I IFN. These factors increase the number of antigen-specific cells that can then travel throughout the body in search of somatic cells that express the targeted antigen ${ }^{30,31}$. When exposed to infected or dysfunctional cells such as cancer (the focus of this thesis), $\mathrm{CD} 8^{+} \mathrm{T}$ cells release the cytotoxins such as perforin and granzymes. Perforin creates pores in the cell membrane of a target cell, allowing granzymes to enter the cytoplasm and use their serine protease function to trigger a caspase cascade, a series of cysteine proteases that eventually result in apoptosis (programmed cell death) ${ }^{32}$. 


\subsubsection{Major histocompatibility complex (MHC)}

The MHC is a set of genes that code for cell-surface proteins that are essential for T cells to recognise foreign molecules in vertebrates. Because the genes are highly polymorphic, there is considerable diversity in the structure of MHC molecules between individuals. In a transplant setting, a recipient's $\mathrm{T}$ cells will become activated via foreign $\mathrm{MHC}$ molecules on the donor tissue, triggering a rejection reaction; hence $\mathrm{MHC}$ determines histocompatibility (tissue compatibility). The primary function of MHC molecules is to present processed peptides from antigens derived from pathogens and display them on the cell surface for recognition by the appropriate T-cells ${ }^{33}$. Of the three MHC classes identified, attention commonly focuses on classes I and II, with class III molecules being poorly defined structurally and functionally. All nucleated cells express MHC class I molecules, which present peptide fragments derived from proteins that are endogenous to the cell, which in the case of infected or malignant host cells, includes pathogen-derived antigens or tumour-associated antigens. Only $\mathrm{CD}^{+} \mathrm{T}$ cells recognise antigenic peptides in the context of MHC class I molecules. If $\mathrm{CD}^{+} \mathrm{T}$ cells have been activated in the context of infection or any other form of "danger" to the host, and have differentiated into antigen-specific CTL, these cells can kill infected or neoplastic cells presenting relevant MHC/peptide complexes on their cell surface. Activated $\mathrm{CD}^{+} \mathrm{T}$ cells also release a variety of cytokines that serve to limit infection or malignancy ${ }^{34}$ On the other hand, MHC class II molecules are only expressed by APCs (with the expression of these molecules being a defining feature of this cell type). All APCs can acquire antigens from the local environment for presentation on MHC class II - again in the form of processed peptide - which can be recognised only by antigen-specific $\mathrm{CD}^{+}{ }^{+}$cells ${ }^{35}$. If activated in the context of danger to the host, $\mathrm{CD}^{+}{ }^{+} \mathrm{T}$ cells differentiate into effectors cells that limit infection, in this case by either producing 
cytokines in the affected tissues, or by causing activation of other cells types including phagocytes that have acquired foreign antigens. Another vital function of $\mathrm{CD} 4^{+} \mathrm{T}$ cells is to provide "help" in the form of CD40 signalling to antigen-specific B cells, thereby signalling antibody isotype switching and differentiation into antibody-producing plasma cells. A similar CD40-mediated helper function can be provided by $\mathrm{CD} 4^{+} \mathrm{T}$ cells to antigen-bearing DCs, which effectively license these potent APCs to stimulate priming of antigen-specific $\mathrm{CD} 8^{+} \mathrm{T}$ cells to become $\mathrm{CTL}^{34}$. Adaptive T cell responses therefore commonly involve the coordinated activity of $\mathrm{CD}^{+}$and $\mathrm{CD} 8^{+} \mathrm{T}$ cells, restricted by their respective MHC molecules, although there are situations in which one or the other cell-type dominates. 


\subsection{The role of antigen-presenting cells in adaptive immune responses}

\subsubsection{Antigen-presenting cells}

Antigen-presenting cells (APCs) are a heterogeneous group of immune cells that mediate the cellular immune response by processing and presenting antigens for recognition by lymphocytes such as T cells. Classical APCs include DCs, macrophages, and B cells. APCs are vital to the production of adaptive immunity, as they can acquire and process exogenous antigens found in the extracellular environment ${ }^{36,37}$. Before loading MHC class I and II molecules with antigens, large-molecular weight proteins must be degraded to a length between 8 to 30 amino acids ${ }^{36}$. Once processed, these antigens are presented on MHC class II molecules to $\mathrm{CD}^{+}{ }^{\mathrm{T}}$ cells, or MHC class I molecules to $\mathrm{CD}^{+} \mathrm{T}^{\mathrm{T}}$ cells ${ }^{37}$.

APCs are also are capable of providing surface-bound and soluble (cytokines) costimulatory molecules to prime antigen-specific T cells. These molecules and MHC itself are upregulated upon activation via interactions with pathogenic molecules by pattern-recognition receptors (PRRs) ${ }^{38}$, or through helper interactions with other lymphocytes. In the absence of this co-stimulation, $\mathrm{T}$ cells that are specific against the antigen presented on MHC molecules become unresponsive to further stimulation (anergic) or are deleted through apoptosis ${ }^{29}$. Another costimulatory molecule present on APCs besides CD80 and CD86 is CD40, which binds to the CD40L (CD154) on T cells. The binding of CD40L to CD40 enhances the activation potential of APCs via increased CD80/86 expression and IL-12 production, further augmenting T cell responses ${ }^{39,40}$. Interleukin 12 (termed IL-12p70 in its active form and commonly designated IL12) is an important immune-regulatory cytokine that is produced mainly by APCs ${ }^{41}$. The expression of IL-12 during infection regulates innate responses and determines the type of adaptive immune response that occurs. IL-12 induces IFN- $\gamma$ production and triggers $\mathrm{CD} 4^{+} \mathrm{T}$ 
cells to differentiate into Th1 cells. Studies have suggested that IL-12 plays a vital role in responses to many diseases, such as viral and bacterial infections and cancers ${ }^{41}$. IL-12 produced by iNKT cell-matured DCs stimulates natural killer (NK), iNKT, and MHC-restricted T cells to produce more IFN- $\gamma$ which can secondarily activate other anti-tumour-promoting effector lymphocytes ${ }^{42}$. As IL-12 has multiple biological activities, it is important in the treatment of many diseases, including cancer, where a Th1 response is desirable.

DCs, macrophages and B cells are regarded as professional APCs that specialise in presenting antigens to $\mathrm{T}$ cells. Of these, DCs are specialised in priming naive $\mathrm{T}$ cells. They are deployed in all tissues of the body and lymphoid organs, acting as part of an immune surveillance system ${ }^{43}$. Most DCs are lymphoid residents where they survey circulating lymphatics and blood for pathogens. DCs are phagocytes that can detect, rapidly engulf, and digest pathogens and foreign cells to form many different fragments of the antigen. These fragments will then be transported to the surface of the APC, where they can be presented to cells of the adaptive immune system ${ }^{44,45}$. These cells then migrate into the $\mathrm{T}$ cell zones of the lymphoid tissues (primarily draining lymph nodes and spleen) where they report to T cells what antigens are present in the tissues. This is the obligatory pathway for the immune surveillance of antigens that are not endogenously expressed in professional APCs. In addition to releasing their antigens, dying cells also release factors, such as heat-shock proteins, uric acid, the high mobility group box 1 protein (HMGB1), double-stranded genomic DNA (dsDNA), into their cytoplasm that selectively stimulate this surveillance process and enhance the generation of CD4 and CD8 T cell responses ${ }^{46}$. DCs are critical orchestrators of immune responses that are adept at presenting exogenous and endogenous antigens to $\mathrm{T}$ cells and regulating $\mathrm{T}$ cell proliferation, survival, and effector functions. Macrophages, on the other hand, are highly proficient at 
phagocytosis ${ }^{47}$, though they are also capable of presenting antigen to $\mathrm{T}$ cells. The presentation of antigen by macrophages is thought to be critical for the effector phase of immune responses (i.e., T-cell dependent activation of macrophages) rather than in the afferent phase of responses (stimulating naive T cells) ${ }^{37,48}$. B cells can also function as APCs before activation and differentiation. However, B cells have a limited capacity to present some antigens due to their inherent difficulties in internalising large particulate materials; in such instances, B cells may interact with the solubilised proteins released by the macrophages ${ }^{49}$.

\subsubsection{Antigen-presenting pathways}

Most nucleated cells are capable of presenting antigen; however, it is a crucial characteristic of APCs and can occur via different pathways. There are two classical pathways of presentation to T cells, to be described in detail below, as well as antigen cross-presentation pathways, that are relevant for the stimulation of $\mathrm{CD} 8^{+} \mathrm{T}$ cell responses. 


\subsubsection{Presentation of acquired antigens via MHC class II to $\mathrm{CD4}^{+} \mathrm{T}$ cells}

APCs require multiple cellular machinery processes in order to present acquired antigen via MHC class II to stimulate $\mathrm{CD} 4^{+} \mathrm{T}$ cells ${ }^{50}$. The processes of phagocytosis, pinocytosis, or receptor-mediated endocytosis are required to bring proteins into the APC. Internalised proteins are then degraded by resident proteases in the lysosome, generating peptides between approximately 15 to 24 amino acids long as potential antigenic peptide MHC class II ligands, with the peptides of the appropriate physical characteristics then binding to the MHC class II binding pocket ${ }^{37,50-52}$. The MHC class II molecules themselves are initially generated as $\alpha$ - and $\beta$-chains in the ER, which form a heterodimer that is stabilised by an invariant chain peptide inserted into the binding groove formed. The MHC class II:invariant chain peptide complexes are then transported from the ER through the Golgi into the MHC class II compartment (MIIC); they then merge with lysosomes where they are digested by proteases, leaving class II-associated invariant chain peptide (CLIP) in the peptide-binding groove of the MHC class II heterodimer. The CLIP is then exchanged for antigenic peptide by the dedicated chaperone HLA-DM (H-2-M in mice). At this point, the peptide-loaded MHC II molecules are transported to the plasma membrane ${ }^{53,54}$, where they are available to stimulate antigen-specific $\mathrm{CD} 4^{+} \mathrm{T}$ cells with cognate receptors ${ }^{55}$. 


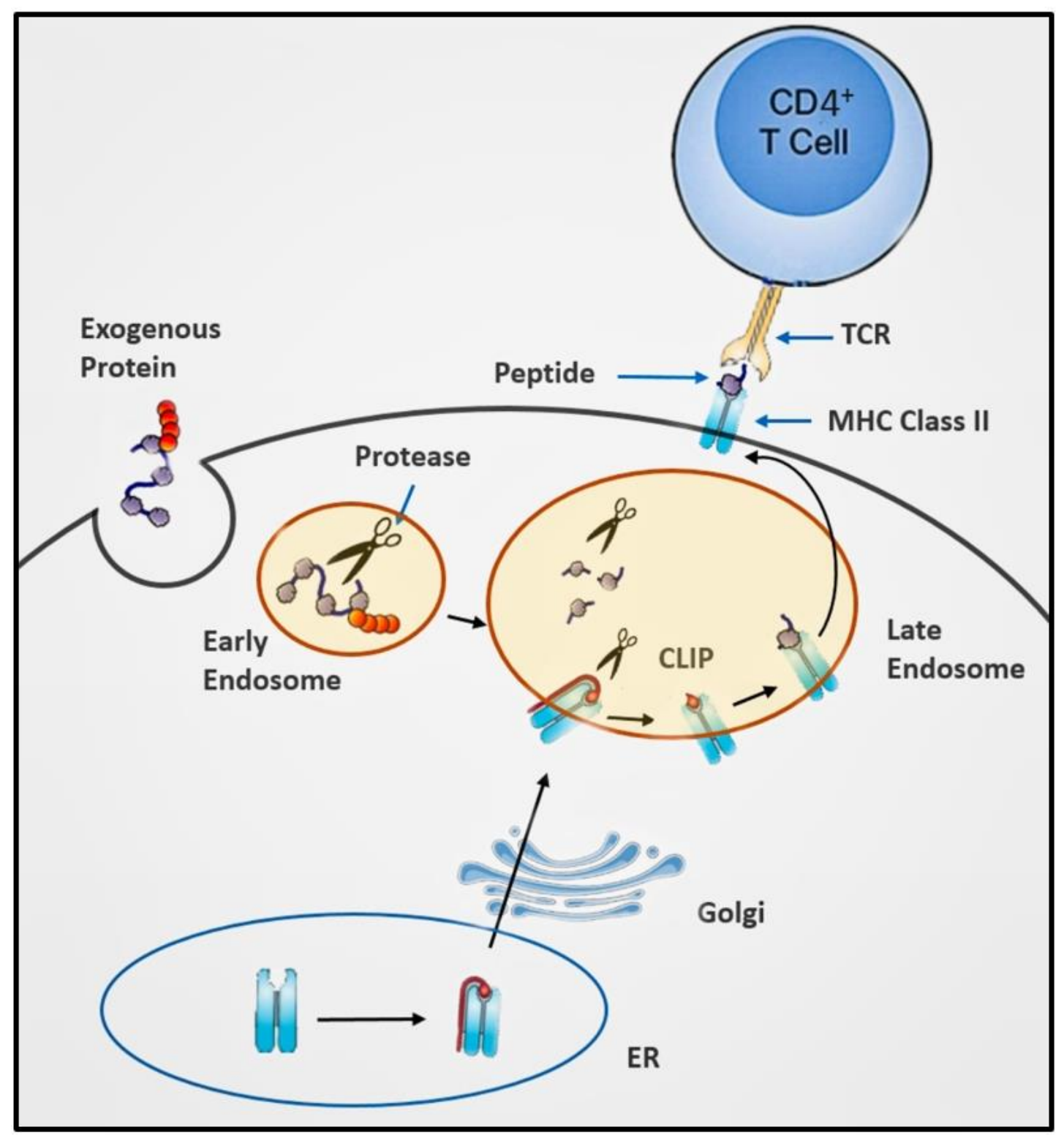

Figure 1: The basic MHC class II antigen presentation pathway. MHC class II proteins are assembled in ER and form a complex with the invariant chain. The MHC:peptide heterotrimer is transported through the Golgi to the MHC class II compartment (MIIC), either directly and/or via the plasma membrane. Endocytosed proteins are degraded by resident proteases in the MIIC. The class II-associated invariant chain peptide (CLIP) fragment remains in the peptide-binding groove of the MHC class II dimer and is exchanged for an antigenic peptide by the dedicated chaperone HLA-DM (known as H2-M in mice). MHC class II molecules are then transported to the plasma membrane to present antigenic peptides to $C D 4^{+} T$ cells.

(Adapted from Towards a systems understanding of MHC class I and MHC class II antigen presentation, Nature Reviews Immunology volume 11, pages $823-836(2011)^{56}$ 


\subsubsection{Presentation of endogenous antigens via $\mathrm{MHC}$ class $\mathrm{I}$ to $\mathrm{CDB}^{+} \mathrm{T}$ cells by the classical pathway}

As noted above, MHC class I molecules are expressed by all nucleated cells and present peptides that are typically derived from protein antigens in the cytosol that arise from conventional as well as cryptic translational reading frames ${ }^{56-58}$. These endogenous proteins include proteins derived from viruses and bacteria that may have infected the cells ${ }^{59,60}$. Cytosolic and nuclear proteasomes degrade these proteins into short peptides ( $\sim 8$ to 10 amino acids long) and these peptides are translocated into the endoplasmic reticulum (ER) by the transporter associated with antigen presentation (TAP) to access MHC class I molecules. TAP consists of two parts, TAP1 and TAP2, which are members of the ABC transporter superfamily. The characteristic feature of $\mathrm{ABC}$ transporters is their organisation into two transmembrane domains (TMDs) and two nucleotide-binding domains (NBDs). These two intramolecular domains are coupled to each other, and ATP binding induces conformational changes in the TMDs, allowing products that have been degraded by proteasomes to move across the membrane. TAP recognises and transports the peptides produced in the cytosol straight into the ER where they are chaperoned by the calnexin-calreticulin system, while tapasin recognises peptides that can form stable complexes with MHC class I in a process known as peptide proofreading or editing. Peptides selected through proofreading ${ }^{61}$ improve MHC class I stability; tapasin also contributes to the editing of immunogenic peptide epitopes. The catalytic mechanism of peptide proofreading is performed by tapasin and TAPBPR (TAP-binding proteinrelated, a tapasin homologue) ${ }^{62}$.

The vesicles containing the peptide-MHC class I complexes separate from the ER and travel through the Golgi to the cell surface, where they can be presented to $\mathrm{CD} 8^{+} \mathrm{T}$ cells with the 
appropriate antigen-specific TCR ${ }^{55,63}$. Peptides and MHC class I molecules that fail to associate in the ER are returned to the cytosol for degradation ${ }^{64,65}$. 


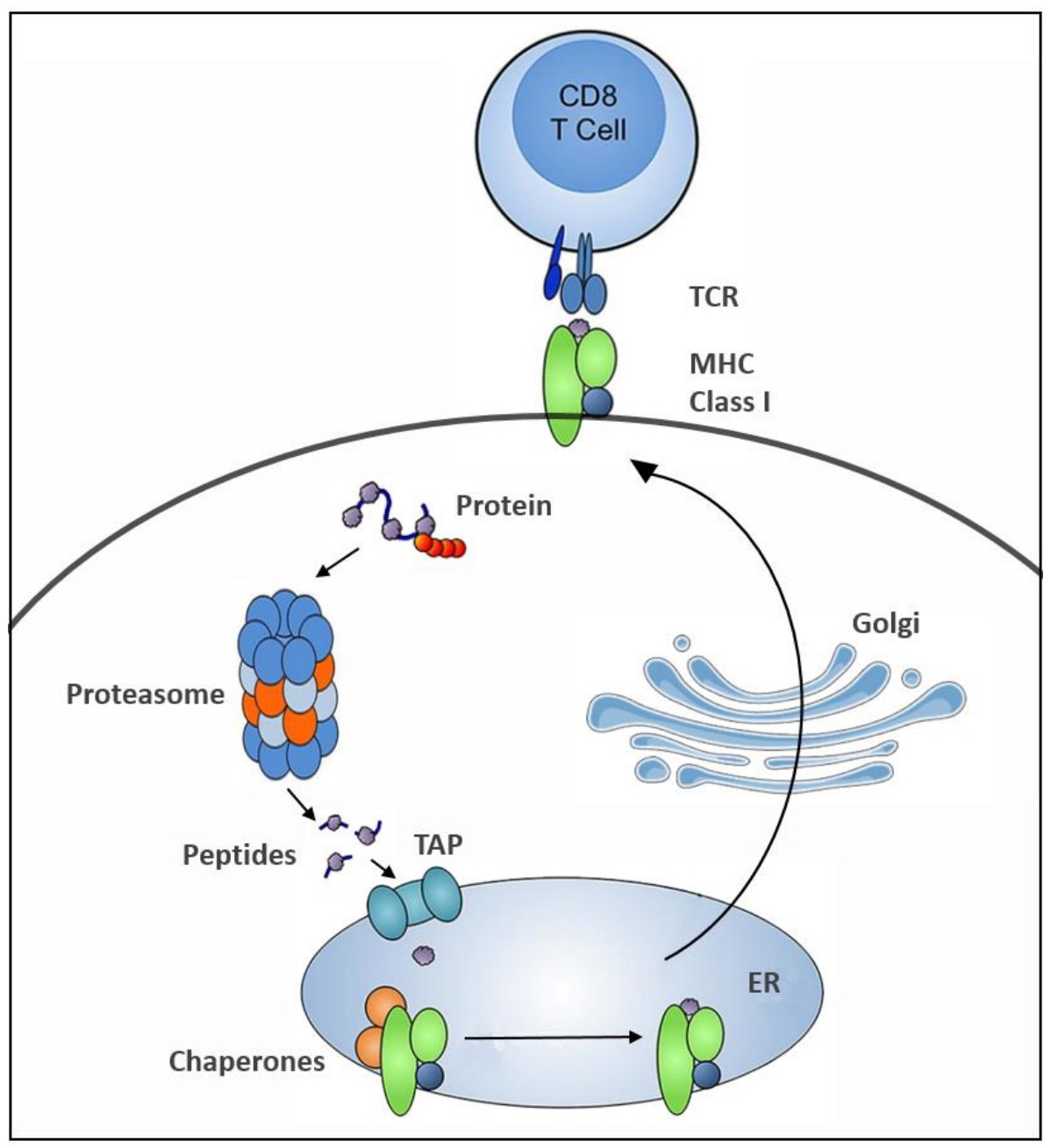

Figure 2: The basic MHC class I antigen presentation pathway.

The presentation of intracellular antigenic peptides by MHC class I molecules starts with antigens being degraded by the proteasome. Then, the resulting peptides are translocated via transporter associated with antigen presentation (TAP) into the ER lumen and loaded onto MHC class I molecules. The peptide:MHC class I complexes are then released from the ER and transported via the Golgi to the plasma membrane for antigen presentation to $C D 8^{+} T$ cells.

(Adapted from Towards a systems understanding of MHC class I and MHC class II antigen presentation, Nature Reviews Immunology volume 11, pages $823-836(2011)^{56}$ 


\subsubsection{Presentation of exogenous antigens via $\mathrm{MHC}$ class $\mathrm{I}$ to $\mathrm{CD8}^{+} \mathrm{T}$ cells by cross- presentation pathways}

In 1976, Bevan had noted that a cytotoxic $\mathrm{CD}^{+} \mathrm{T}$ response could be induced against antigens introduced exogenously from adoptively transferred allogeneic cells ${ }^{66,67}$. This process was not accounted for in the classical pathway described above where only endogenous antigens could be presented on MHC class I molecules to $\mathrm{CD} 8^{+} \mathrm{T}$ cells. It was hypothesised that antigens could be presented in a process that involved uptake of exogenous antigens into APCs followed by crossover into the MHC class I presentation pathway. This process, termed "crosspresentation" allowed for exogenous antigens to be presented on MHC class I molecules to CD8 ${ }^{+}$ T cells. The process of activating $\mathrm{CD}^{+} \mathrm{T}$ cells via this pathway is called "cross-priming". Crosspriming of CTL is useful for addressing intracellular infections that do not specifically infect APCs. Furthermore, this process is a prerequisite for vaccination strategies using exogenous antigens to induce cytotoxic $\mathrm{CD}^{+} \mathrm{T}$ cell responses to be possible, including against cancer ${ }^{28}$. 


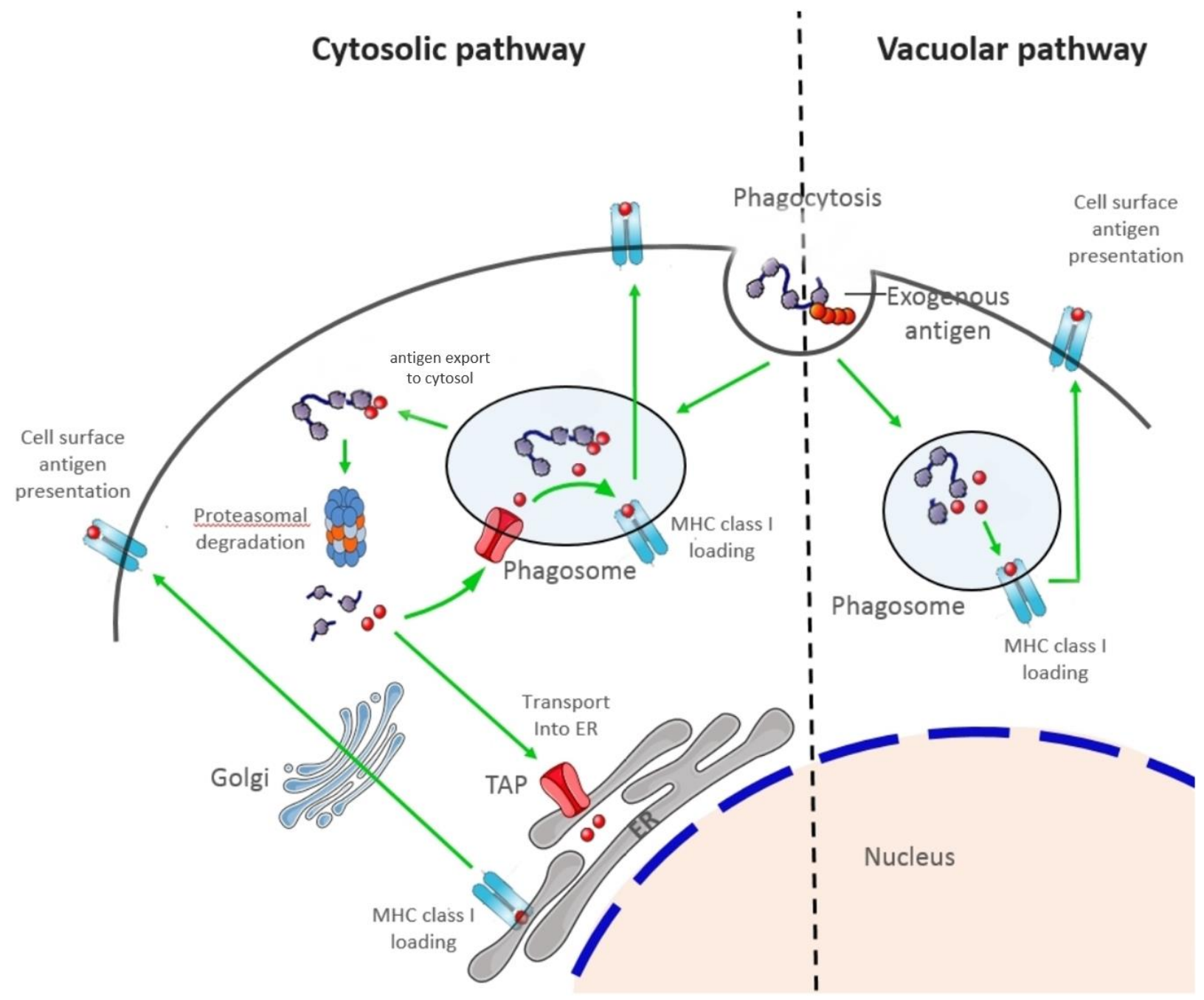

Figure 3: Models of phagosomal antigen cross-presentation by MHC-I. There are two main models of intracellular pathways leading to antigen cross-presentation in DCs.

Left: TAP-and proteasome-dependent cytosolic pathway. Antigens on phagocytosed particles are transported to the cytosol by an ERAD-associated retrotranslocon, where they are ubiquitylated and sent to the proteasome for degradation. The generated peptides are then either translocated back into the phagosome by TAP and loaded onto MHC-I or sent into the TAP-dependent MHC-I antigen presentation pathway in the ER.

Right: TAP-and proteasome-independent vacuolar pathway. Peptides are generated from antigens on phagocytosed particles within phagosomes by cathepsins. These peptides are then loaded onto MHC-I molecules within the phagosome. ${ }^{68}$ (Adapted from Presentation of phagocytosed antigens by MHC class I and II, Mantegazza et al., Traffic. 2013 Feb; 14(2): 135$152)^{68}$ 
Shown in Fig 3 above are the two main proposed models for intracellular pathways leading to cross-presentation of exogenous antigens in DCs. Illustrated on the left is the TAPand proteasome-dependent cytosolic pathway. In this scenario, antigens present on phagocytosed material are transferred to the cytosol by an ERAD-associated retrotranslocon, ubiquitylated, and directed to the proteasome for degradation ${ }^{68}$. The generated peptides are then either translocated back into the phagosome by TAP and loaded onto MHC-I or fed into the classical TAPdependent MHC-I antigen presentation pathway in the ER.

On the right is the TAP-and proteasome-independent vacuolar pathway. In this pathway, peptides are generated from antigens on phagocytosed particles within phagosomes by proteolysis mediated by cathepsins. These peptides are then loaded onto MHC-I molecules within the phagosome ${ }^{68}$. The composition of the phagocytosed material determines the pathway that is used for antigen cross-presentation. For example, iron oxide beads containing chicken ovalbumin protein (OVA) are heavily dependent on TAP, whereas poly-lactide poly-glycolide microspheres containing OVA are cross-presented in a TAP-deficient model ${ }^{69}$. This difference led to the development of TAP-independent vacuolar pathway theory described above.

\section{Dendritic cells}

As mentioned above, DCs are superior APCs due to their ability to efficiently present antigen and their strong co-stimulatory capacities. However, there is a high level of heterogeneity (Fig 3) within the DC population, with different subsets possessing a higher capacity for different functions. The DC populations have been widely studied and characterised in mice. DCs originate from monocyte and dendritic precursors in the bone marrow, which then convert into either the common DC precursor or monocytes ${ }^{70,71}$. The monocytes can then be 
differentiated into monocyte-derived inflammatory DCs in response to inflammation, including infections and auto-immune disease ${ }^{72-76}$. At the same time, the common dendritic precursor can be differentiated into either the plasmacytoid DC (pDC) or conventional DC (cDC) ${ }^{70,71}$. pDCs are identified as SiglecH $\mathrm{H}^{+} \mathrm{B} 22 \mathrm{O}^{+} \mathrm{CD} 11 \mathrm{c}^{\text {low }}$ cells and have some antigen cross-presentation ability in humans and mice, but this is still up for debate ${ }^{77,78}$. However, while pDCs are somewhat capable of these other functions, such as cross-presentation, the most identifiable function of this subset is their high responsiveness to nucleic acid molecules (like unmethylated 5 ' cytosinephosphate-guanine 3' - "CpG" - motifs from prokaryotic DNA), resulting in significant type I IFN production by these cells ${ }^{79,80}$.

cDCs can be further divided into two major subsets recently renamed cDC1s and cDC2s ${ }^{81}$. cDCs express the integrin CD11c, and MHC class II and each subset can be distinguished by additional markers ${ }^{82,83}$. While various markers have historically been used to identify cDC subsets, a recent analysis suggests that a more consistent and straightforward identification of these cells across most tissues is possible by gating $\mathrm{cDCs}$ as $\mathrm{CD} 11 \mathrm{c}^{+} \mathrm{MHC}$ class $\mathrm{II}^{+} \mathrm{CD} 26^{+} \mathrm{CD} 64^{-} \mathrm{F} 4 / 80^{-}$, and within this population are cDC1s as XCR $1^{+}$and $\mathrm{cDC} 2 \mathrm{~s}$ as $\operatorname{Sirp} \alpha+{ }^{84}$. pDCs (defined by the transcription factors E2-2, ZEB2, and IRF8) also express CD11c and MHC class II but can be segregated by their additional expression of B220, Siglec-H, and BST2 ${ }^{78,85}$. cDC1s (defined by the transcription factors, IRF4, ID2, IRF8, and BATF3) excel in the activation of CTLs, through a heightened propensity for cross-priming, and also activate NK cells, and iNKT cells, which are all critical effector cell types in anti-tumour immunity. cDC2s on the other hand (defined by the transcription factors ID2, ZEB2, IRF4, Notch2 and KLF4), have a lower capacity for cross-priming but are efficient stimulators of $\mathrm{CD} 4^{+} \mathrm{T}$ cells, including 
Th2 cells and Th17 cells ${ }^{86-88}$. In this way, each cDC subset responds to a specific type of threat and activates both the innate and adaptive defences best suited for overcoming it. 


\subsection{3 $\mathrm{CDB}^{+} \mathrm{T}$ cell responses}

\subsubsection{Induction of $\mathrm{CD8}^{+} \mathrm{T}$ cell responses}

The induction of $\mathrm{CD} 8^{+} \mathrm{T}$ cell responses is vital for addressing intracellular infections and presents an opportunity to be utilised by vaccines for the treatment of diseases, including cancer immunotherapy. To this end, vaccines would harness the cytotoxic killing function of activated $\mathrm{CD} 8^{+} \mathrm{T}$ cells to eliminate infected cells or neoplastic tissue ${ }^{60}$. There are several factors involved in the activation of $\mathrm{CD} 8^{+} \mathrm{T}$ cells including DCs presenting MHC class I-peptide complexes to the TCR, co-stimulatory molecule interactions such as CD40L/CD40 which in turn regulate the costimulatory activity of APCs, with upregulated expression of CD80 (B7.1) and CD86 (B7.2), integrins such as CD54, and a "third signal" mediated by IL-12 binding to the IL-12R ${ }^{89-91}$. All these processes together result in the activation of $\mathrm{CD}^{+} \mathrm{T}$ cells with increased capacity for IFN$\gamma$ production ${ }^{92}$. These cells can, in turn, activate and recruit other immune cells; for example, macrophages can be recruited for protection against bacterial infection ${ }^{93}$. The $\mathrm{CD} 8^{+} \mathrm{T}$ cells are converted into CTLs with increased expression of perforin and granzymes that degrade cellular components within targeted tissues ${ }^{32}$. For example, perforin targets the lamins that maintain the integrity of the nuclear membrane, while granzymes cause DNA fragmentation, and induce the caspase pathways for apoptosis ${ }^{94-98}$. CTLs are also able to induce apoptosis in target cells via the Fas/FasL pathway of caspase induction ${ }^{99,100}$, which is compounded by IFN- $\gamma$ mediated upregulation of Fas on target cells, thus enhancing Fas-mediated killing by CTLs ${ }^{101}$.

The understanding of how the host immune system mounts an immunological response to bacterial infections and viral infections will contribute to therapies for diseases such as cancer 59,102,103. Since DCs are the most efficient APC, ex vivo loaded and stimulated DCs were initially used to present antigens to effector cells in order to eradicate primary and metastasised cancer 
cells. However, the ex vivo generation and modification of DCs turned out to be a labourintensive, time- and money-consuming procedure. Constructing an effective, reliable, tolerable, and safe cancer vaccine is the core principle of cancer immunotherapy. Novel methods are being investigated for specifically targeting DCs by vaccination in order to induce strong antigenspecific CTL responses ${ }^{104}$. 


\subsection{Toll-Like Receptors (TLRs)}

Perhaps the most encouraging preclinical data on anti-tumour vaccines have been generated with agonists that engage TLRs. These receptors are type 1 transmembrane glycoproteins that are a critical component in the immune response to microbial agents. They are classified as either pathogen-associated molecular patterns (PAMPs) if derived from microbes and danger-associated molecular patterns (DAMPs) if derived from damaged tissues ${ }^{105}$. Ligation of these agonists to TLRs causes DCs to produce cytokines as well as increasing the expression of MHC and co-stimulatory molecules. In humans, ten genes encode for different TLRs.

Downstream of TLR signalling molecules are nuclear factor-kB (NF- $\kappa \mathrm{B})$, mitogen-activated protein (MAP) kinases and interferon-regulatory factors (IRFs) involved in the expression of cytokine genes relevant to inflammation ${ }^{106}$ such as tumour necrosis factor-alpha (TNF $\alpha$ ), IL-1 $\beta$, and IL-12 they are expressed on the cell surface (TLR1, 2, 4, 5, 6) and type 1 IFN if they are endosomal (TLR3, 7, 8, 9) ${ }^{11}$. TLRs regulate essential processes for initiating T-cell immunity, including antigen uptake, processing and presentation of antigen by APCs, as well as inducing expression of molecules required for T-cell activation ${ }^{107}$. As such, TLR agonists demonstrate therapeutic promise as immunological adjuvants for anticancer immunotherapy. To date, two TLR agonists have been approved by the FDA for use in cancer patients ${ }^{108}$ : monophosphoryl lipid A (MPL) derived from the lipopolysaccharide of Salmonella Minnesota is included in the formulation of Cervarix, a vaccine against human papillomavirus-16 and -18, and Imiquimod (a synthetic imidazoquinoline) that is routinely employed for actinic keratosis, superficial basal cell carcinoma, and external genital warts (condylomata acuminata) ${ }^{108}$. Different TLR agonists are derived from different foreign materials (or synthetic mimetics thereof): TLR1 and 2 are stimulated by the lipidated bacterial components of gram-positive bacteria, TLR3 is stimulated 
by viral double-stranded RNA, TLR4 by lipopolysaccharide (LPS) from bacterial cell wall components, TLR5 by the flagella of bacteria, TLR7 and 8 by purine-rich viral single-stranded RNA, and TLR9 by unmethylated CpG motifs from prokaryotic DNA ${ }^{109,110}$.

There are two primary TLR signalling pathways, the myeloid differentiation primary response 88 (MyD88) pathway, and the Toll/interferon response factor (TRIF) pathway. The majority of TLRs utilise the MyD88 pathway, while TLR3 utilises TRIF, and TLR4 can utilise both ${ }^{109,110}$.

\subsubsection{MyD88 Pathway}

The TLR signalling pathway to the downstream nuclear factor $\kappa$-light-chain-enhancer of activated B cells (NF- $\mathrm{KB})$ transcription factor, which controls cytokine production and cell survival, is complex. The adaptor protein MyD88 is composed of two parts, a Toll/interleukin-1 receptor (TIR) domain and a death domain. Upon stimulation with ligands, MyD88 recruits IL-1 receptor-associated kinase-4 (IRAK-4) to TLRs through the interaction of the death domains of both molecules. IRAK4 is initially activated, which in turn phosphorylates and activates IRAK1. After IRAK4 and IRAK1 have been sequentially phosphorylated, they dissociate from MyD88 and interact with tumour necrosis factor receptor (TNFR)-associated factor 6 (TRAF6). TRAF6 is an E3 ubiquitin ligase. Ubiquitinated TRAF6 subsequently recruits a protein kinase complex involving transforming growth factor- $\beta$-activated kinase-1 (TAK1) which then activates the ІкB kinase (IKK) complex, leading to activation of mitogen-activated protein kinase (MAP) kinases c-Jun N-terminal kinases (JNK), p38 and MAP kinase (MAPK) and eventually NF-кB. This IKK complex is composed of two kinase subunits (IKK $\alpha$ and IKK $\beta$ ) and IKK, a regulatory subunit which is also known as NFkB essential modulator (NEMO) (Fig 4A). The activation of 
$\mathrm{NF}-\mathrm{\kappa B}$ is important for eliciting innate immune responses as well as for the subsequent development of adaptive immune responses ${ }^{110,111}$.

\subsubsection{TRIF Pathway}

As for the TRIF pathway: upon TLR3 or TLR4 (if endocytosed) ligand binding, TRIF is recruited to their TIR domains. However, in the case of TLR4, TRIF-adaptor molecule (TRAM) is also recruited to its TIR domain. Subsequently, inducible IKK (IKKi; IKKe), TANK-binding kinase 1 (TBK1) and TRAF3 are recruited to the TRIF-TIR or TRAM-TRIF-TIR complexes. TBK1 phosphorylates interferon regulatory factors (IRF) 3 and 7, which, in conjunction with p300 and CREB binding protein (CBP) upregulate the expression of interferon-inducible genes IP-10 and RANTES. TRAF6 binding to TRIF also induces the release of pro-inflammatory cytokines via NFKB again eliciting innate immune responses ${ }^{105}$ (Fig 4B). 


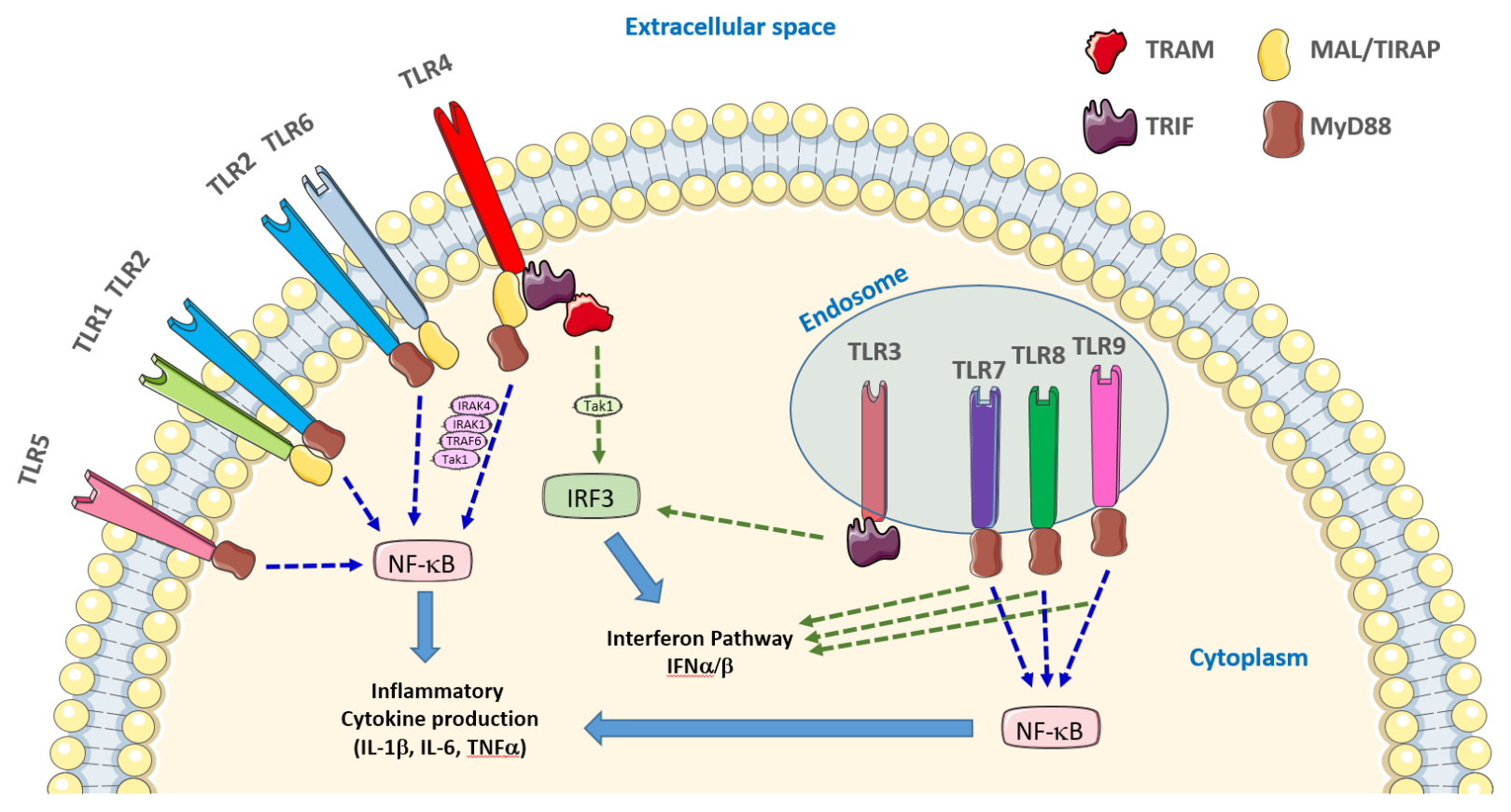

Figure 4: TLRs and their signalling pathways

A) MyD88 signalling pathway. After ligand binding (except TLR3), TLRs recruit the adaptor molecule MyD88 (brown) to their TIR domains. Upon stimulation, IRAK-4, IRAK-1, and TRAF6 are recruited to the receptor, which induces the association of IRAK-1 and MyD88 via the death domains. IRAK-4 then phosphorylates IRAK-1. Phosphorylated IRAK-1, together with TRAF6, dissociates from the receptor and then TRAF6 interacts with TAK1, TAB1, and TAB2 to form a larger complex, which induces the activation of TAK1. Activated TAK1 induces the activation of the transcription factors $N F-k B$.

B) TRIF signalling pathway. After activation of TLR4 and TLR3, TRIF is recruited to their TIR domains. TRAM (TRIF-adaptor molecule) is another adaptor recruited to the TIR domain of TLR4. Through a series of phosphorylations, IRF3 is activated. Phosphorylated IRF3 forms homodimers and heterodimers with IRF7 and translocates to the nucleus. The dimer complex of IRFs binds to DNA target sequences to transcribe IFN and IFN-inducible genes

(Adapted from Toll-like receptors and breast cancer, Sun et al., Integr Cancer Sci Therap, 2016, Volume 3(2): 432-436; doi: 10.15761/ICST.1000183) ${ }^{110}$ 


\subsection{Properties of Invariant Natural Killer T (iNKT) cells and their role in the activation of dendritic cells}

As already noted in this introduction, a significant focus of this thesis is on utilising the capacity of activated iNKT cells to activate DCs and enhance T cell immunity. An overview of this cell-type is provided in the following sections.

\subsection{1 iNKT Cell Biology}

iNKT cells are a subpopulation of T lymphocytes with phenotypic properties of both $\mathrm{T}$ and NK cells and display a wide range of immune effector properties against pathogens, tumours allergens, and self-antigens ${ }^{112,113}$. A subset of these cells, known as type I NKT cells, or “invariant” NKT cells (iNKT cells,) express a TCR structure that is largely invariant and can, therefore, recognise only a limited range of antigens. A clear preferred structural pattern of the recognised antigens has emerged which consists of a glycolipid with six-membered ring sugar, linked to a lipid backbone that can either be based on a ceramide, a diacylglycerol, or potentially a cholesterol moiety ${ }^{114}$. These innate-like $\mathrm{T}$ cells are $\mathrm{CD}^{+} \mathrm{T}$ cells, and they exhibit many features of NK cells, including an innate cytotoxic function. These innate and innate-like cells can augment $\mathrm{CD}^{+} \mathrm{T}$ cell responses against a variety of infections. For example, NK cells increase $\mathrm{CD}^{+} \mathrm{T}$ cell responses to herpes simplex virus ${ }^{115}$ and iNKT cells aid in the $\mathrm{CD}^{+} \mathrm{T}$ cell responses to West Nile virus ${ }^{116}$. The role of iNKT cells in providing help to $\mathrm{CD}^{+} \mathrm{T}$ cells ${ }^{117}$ will be described below in some detail.

iNKT cells are defined by their expression of a unique invariant TCR $\alpha$ chain $(\mathrm{V} \alpha 14 \mathrm{~J} \alpha 18$ in mice and $\mathrm{V} \alpha 24 \mathrm{~J} \alpha 18$ in humans) paired with a limited repertoire of TCR $\beta$ chains (highly enriched for $\mathrm{V} \beta 8, \mathrm{~V} \beta 7, \mathrm{~V} \beta 2$ in mice and $\mathrm{V} \beta 11$ in humans) ${ }^{118}$. The TCRs of iNKT cells are selected for recognition of a variety of lipid and glycolipid antigens bound to the non- 
polymorphic MHC class I-like molecule CD1d ${ }^{118,119}$, which controls the thymic selection of iNKT cells, as well as their activation in many situations during immune responses ${ }^{120}$. They reside in several locations, including the spleen, liver, lymph nodes, and thymus ${ }^{121}$. Upon activation, iNKT cells rapidly secrete a wide array of cytokines that enhance immune responses ${ }^{122}$, including IFN- $\gamma$ and IL-4, which support the proliferation and activation of different T cells 119,123 and are capable of cytotoxic activity ${ }^{119}$ against pathogens and tumour cells ${ }^{124}$. This suggests a potential immunotherapeutic use for iNKT cell activation for the treatment of cancer. The Va14-expressing iNKT cells constitute a major population of all NKT cells in mice. In the adult mouse, iNKT cell frequency is highest in the liver (12-30\% of liver lymphocytes), with lower frequencies in the spleen (1-3\%), lungs (5-10\%), thymus $(0.5-1 \%)$, bone marrow $(0.4-8 \%)$, lymph nodes $(0.2-1 \%)$, intestines $(0.05-0.6 \%)$ and blood $(0.2 \%)^{125,126}$. The frequency of iNKT cells in humans is approximately ten-times lower in studied tissues, and the frequency in peripheral blood shows substantial variation between different individuals with a mean value of approximately $0.1 \%{ }^{127}$. Although human and mouse iNKT cells display conserved phenotypic and functional features, this significant difference in frequency has challenged the extrapolation of iNKT cell-based immunotherapy from mice to humans. However, a study in pigs found that the frequency of iNKT cells in peripheral blood prior to treatment was a poor predictor of how an animal would respond to iNKT cell therapy; despite significant variation in iNKT cell frequency between individual pigs it was still possible to harness iNKT cells to protect against infectious diseases ${ }^{128}$.

The glycolipid $\alpha$-Galactosylceramide ( $\alpha-$ GalCer), derived from extracts of the marine sponge Agelas Mauritianus, was found to activate virtually all iNKT cells potently. The subsequent development of a synthetic version of $\alpha$-GalCer designated KRN7000 (hereafter the 
version referred to as $\alpha-$ GalCer in this thesis) allowed for the ability to induce a robust activation of iNKT cells both in vitro and in vivo ${ }^{129}$. Since $\alpha-$ GalCer binds to CD1d to form a complex with very high affinity for the TCRs of mouse and human iNKT cells, this compound also enabled an accurate detection and quantification of iNKT cells by using fluorescentlylabelled $\alpha$-GalCer-loaded CD1d tetramers for flow cytometry ${ }^{130}$. Interestingly, the CD1d receptor is highly conserved among humans, non-human primates, and mice as well as a few other species, making it a highly translatable therapeutic target ${ }^{131-133}$. The crystal structure of human CD1d in complex with $\alpha$-GalCer has been elucidated ${ }^{134}$, which facilitated the understanding of the binding mode between the ligand and the receptor which involves the lipid tails of $\alpha$-GalCer binding to the $A^{\prime}$ and F' pockets of CD1d and the galactose ring being presented to the TCR of iNKT cells as a result (see Fig 6). This understanding further helped in the design of more potent ligands.

Several studies have shown that $\alpha$-GalCer can serve as an adjuvant for various vaccines, including viral, parasite, DNA, RNA, and protein vaccines ${ }^{132}$. However, the efficacy of the vaccine can be improved when $\alpha$-GalCer is used as an adjuvant with peptide antigens, including TAAs ${ }^{135-138}$. Similarly, the addition of $\alpha$-GalCer enhanced the anti-tumour activity of irradiated tumour cells ${ }^{135-138}$. Furthermore, the use of $\alpha-$ GalCer as a single agent has been shown to be protective against multiple tumour types in preclinical models of liver metastasis, including in Colon26 adenocarcinoma and B16 melanoma ${ }^{139}$. Mice that had been challenged with an intrasplenic dose of Colon26 adenocarcinoma were protected against liver metastases when a large dose of $\alpha-$ GalCer, $100 \mu \mathrm{g} / \mathrm{kg}$, was injected intravenously ${ }^{140}$. Similarly, for mice with B16 melanoma liver metastases, $\alpha-$ GalCer significantly inhibited tumour growth in the liver with similar potency as IL-2 ${ }^{139}$. Mice that had been given $\alpha$-GalCer were significantly more likely to 
be cured and acquired tumour-specific immunity. This suggests that $\alpha-$ GalCer and by extension iNKT cell activation may be useful for controlling liver metastases ${ }^{139}$. Following the injection of $\alpha$-GalCer in vivo, DCs upregulate costimulatory molecules such as CD40, CD80, and CD86, and secrete proinflammatory cytokines such as IL-12 and TNF- $\alpha$. These changes constitute an adjuvant cascade that increases the activation of peptide antigen-specific $\mathrm{CD} 4^{+}$and $\mathrm{CD} 8^{+} \mathrm{T}$ cells 141-143. The adjuvant effects of $\alpha$-GalCer are dependent on iNKT cells and require the interaction between CD40 on DCs with CD40L on the surface of iNKT cells ${ }^{141,142,144}$ (Fig 5). This amplification of $\mathrm{T}$ cell responses is particularly pronounced in the cross-priming of $\mathrm{CD} 8^{+} \mathrm{T}$ cell responses in both mouse and non-human primate models ${ }^{140}$.

In addition, $\alpha-G a l C e r$ administration results in NK cells being transactivated by IL-12p70 released from activated APCs leading to a significant release of IFN- $\gamma$ and increased capacity for cytotoxicity (Fig 5), which can lead directly to tumour cell killing or through blocking angiogenesis ${ }^{145}$. The observed anti-tumour effects seen in early studies with $\alpha$-GalCer could, therefore, be due to iNKT cells releasing a significant volume of IFN- $\gamma$ and increased cytotoxicity ${ }^{119,146-148}$.

In addition to the effects on T cells, the activation of iNKT cells also enhances antibody production by B cells (Fig 5). This phenomenon is not restricted to mouse models, as the stimulation of human peripheral blood in culture with $\alpha$-GalCer induces antibody production ${ }^{149}$. This is consistent with the observation that the expression of CD1d by B cells is required for iNKT cell-induced antibody enhancement ${ }^{150}$, which suggests the potential for iNKT mediated antibodies contributing to anti-tumour activity. 


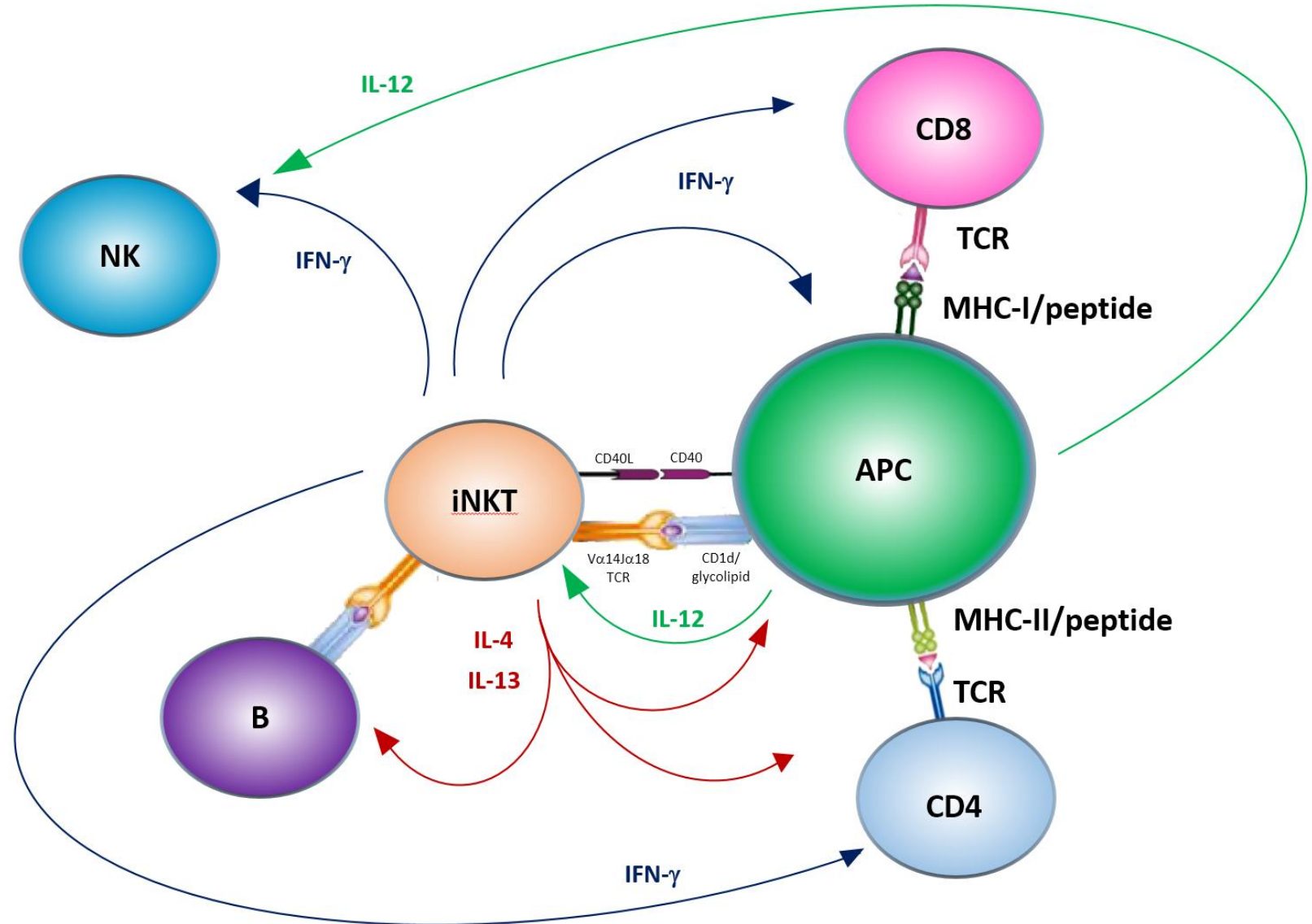

Figure 5: Invariant NKT cells and their role in immunity. iNKT cells express a characteristic antigen receptor that includes an invariant TCR $\alpha$ chain. These TCRs recognize specific glycolipid ligands bound to CD1d molecules, which are expressed mainly on APCs such as dendritic cells and B lymphocytes. After TCR ligation, iNKT cells rapidly secrete multiple Th1 and Th2 type cytokines, such as IFNy and IL-4. These cytokines, along with surface molecules expressed on activated iNKT cells, influence the activity of many other cells in the immune system, and contribute to transactivation of NK cells, maturation of DCs and enhancement of specific $T$-cell and B-cell responses and memory ${ }^{151}$.

(Adapted from Synthetic glycolipid activators of natural killer T cells as immunotherapeutic agents, Carreno et al., Clinical \& Translational Immunology, 2016, Volume 5: e69; doi:10.1038/cti.2016.14) ${ }^{152}$ 
In most clinical trials, $\alpha$-GalCer as a single agent did not show robust clinical efficacy either as an anti-tumour or an antiviral agent ${ }^{153}$. However, one report of responses to $\alpha$-GalCer loaded DC in cancer patients did show enhanced CTL responses to both a chronic viral infection and also to an influenza vaccine received by one subject during the trial period ${ }^{154}$, suggesting that activated iNKT cells enhanced the function of resident DCs in these patients (in this case harbouring viral antigens). A phase I study found that DCs loaded with $\alpha$-GalCer and the cancertestis antigen NY-ESO-1 was a safe and immunologically effective therapy. The therapy led to increases in NY-ESO-1 specific T cells in circulation in the majority of patients. Also, iNKT cell proliferation and associated cytokine secretion were detected in most patients, while in vitro restimulation showed that the therapy increased the number of $\mathrm{CD} 4^{+}$and $\mathrm{CD} 8^{+} \mathrm{T}$ cells that could produce multiple cytokines simultaneously ${ }^{137}$. For the most part, $\alpha$-GalCer was well tolerated in clinical studies, with only mild adverse events, including fatigue, myalgia, back pain, headache, rhinitis, fever, chills, and dizziness ${ }^{155,156}$. In a Phase1 study, $\alpha$-GalCer has been safely administered intravenously to patients given weekly for three consecutive weeks to patients with refractory solid tumours over a wide dose range up to $4,800 \mathrm{ug} / \mathrm{M}^{2}$ or $\sim 130 \mu \mathrm{g} / \mathrm{Kg}$ without serious adverse events ${ }^{155}$. In a trial using ABX196, an $\alpha$-GalCer analogue with stronger agonist activity, there was a strong adjuvant effect when combined with HBV antigens, with a single dose being sufficient to generate immunity. However, administration of this ligand did result in a number of mild to moderate adverse events (headache and asthenia), that became more severe in a handful of subjects when combined with antigen (increased systemic IFN- $\gamma$ and a transitory increased ALT/AST) ${ }^{157}$. Overall, it appears that $\alpha$-GalCer is safe and efficacious in both preclinical and clinical models. 
It is well established that DCs are the primary cell population responsible for deciding between immunity or immune tolerance, depending on the stimuli that they receive ${ }^{158,159}$. For example, the development of tolerogenic DCs will lead to antigen tolerance through the expansion of regulatory T cells (Tregs). On the other hand, TLR agonists such as CpG can significantly promote the maturation of pro-inflammatory DCs, which favours type $1 \mathrm{~T}$ cell responses while restricting the expansion of Tregs ${ }^{160}$. Recently, several studies have demonstrated that combining TLR ligation with iNKT cell activation has a cooperative effect on DC maturation, resulting in highly pro-inflammatory DCs, as seen by enhanced expression of activation markers ${ }^{161,162}$. However, similar to the systemic administration of chemotherapy drugs, the administration of the combination of TLR agonist and iNKT cell activator will result in significant off-target toxicities as a result of significant systemic cytokine release ${ }^{11}$. To address this, in this thesis intratumoural delivery was adopted as the method of choice, with the aim of lowering the amount of drug in circulation to minimise the amount of cytokines in the blood, while maximising the amount of cytokines at the site of injection, the tumour. 

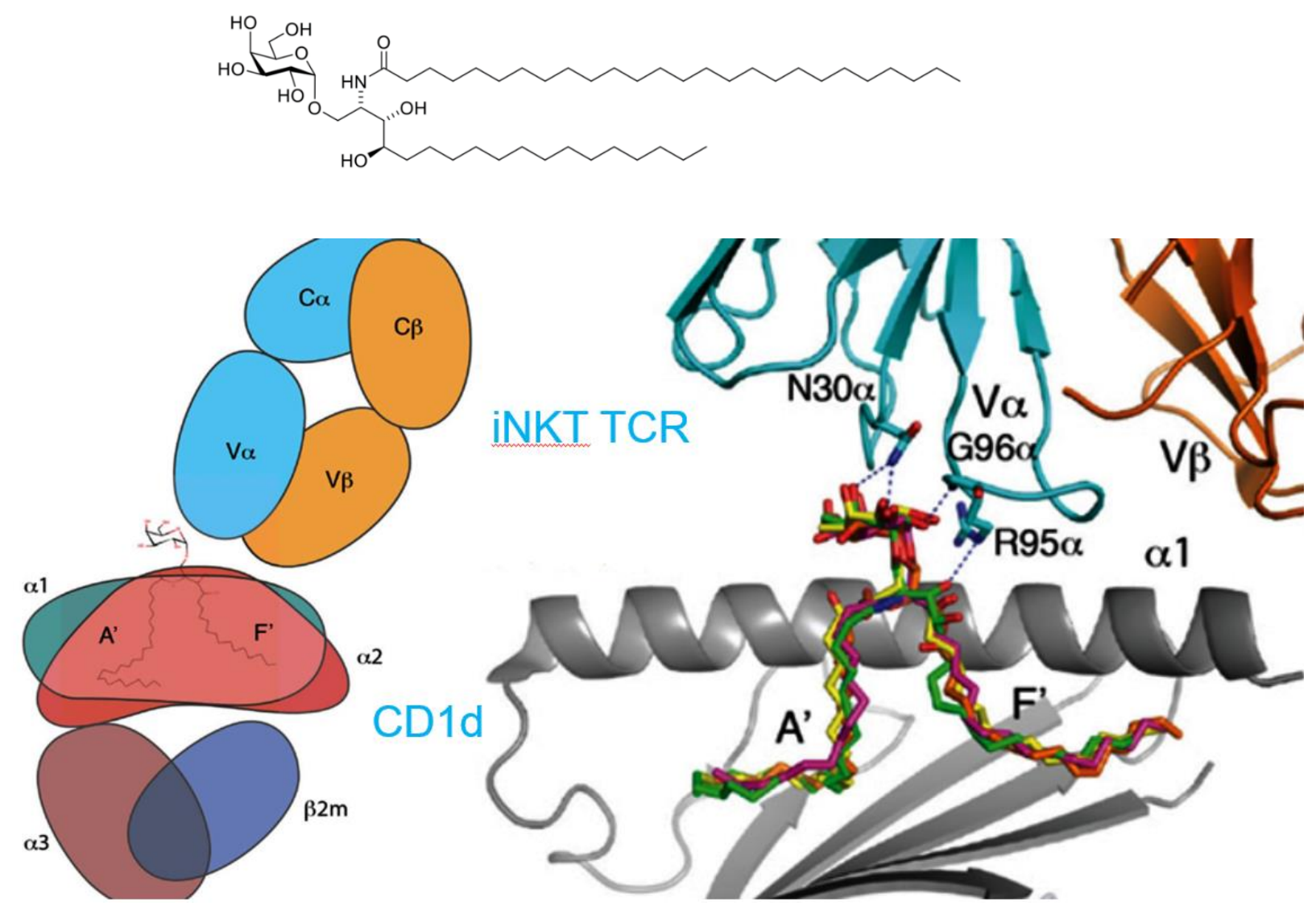

Figure 6: Top, The chemical structure of a CDId ligand: $\alpha$-galactosylceramide ( $\alpha$-GalCer); Lower left, a pictorial representation of how the lipophilic tails of $\alpha$-GalCer are anchored in the CDId receptor (through pocket $A^{\prime}$ and $F^{\prime}$ ) presenting the galactose moiety to the TCR of iNKT cell. Lower right, actual X-ray structure showing the binding of $\alpha$-GalCer (and other analogues) to CDId and TCR of iNKT cells ${ }^{114}$ (Adapted from Recognition of microbial glycolipids by natural killer T cells DM Zajonc and E Girardi, Front Immunol. 2015 Aug 4;6:400. doi: 10.3389/fimmu.2015.00400. eCollection 2015) ${ }^{114}$ 


\subsection{Challenges posed by immune evasion}

The role of therapeutic vaccines is to enlarge the pool of tumour-specific $\mathrm{T}$ cells from both the naive pool as well as reactivating existing tumour-specific $\mathrm{T}$ cells that may be in a dormant state. In addition, these $\mathrm{T}$ cells have to home to tumours to exert their effector functions within the tumour microenvironment. However, these tumour-specific T cells face an immuneregulatory environment that has physiologically evolved to dampen acute inflammatory

responses or to stimulate wound healing in otherwise healthy individuals ${ }^{36,163}$. Tumour cells can exploit these immune-suppressive mechanisms to avoid immune recognition and elimination. Although much remains to be done to elucidate the mechanisms of immune evasion fully, research is beginning to unravel the mechanism of resistance. There are tumour cell-intrinsic mechanisms, such as avoidance of immune recognition, and tumour cell-extrinsic mechanisms, such as the creation of an immune-suppressive microenvironment. Some of the major mechanisms are outlined below.

- Defects in major histocompatibility complex (MHC) class I proteins: MHC class I proteins are essential for immune detection by $\mathrm{CD} 8^{+} \mathrm{T}$ cells. Short TAA bind in the grooves of MHC class I molecules and are displayed at the cell surface for surveillance by T cells. A defect in, a decrease in, or loss of MHC class I expression will allow tumour cells to avoid detection ${ }^{164,165}$.

- Loss of expression of antigen-processing machinery components: is observed in a wide variety of human tumour types. Silencing of the antigen processing machinery (for example, transporter associated with antigen processing (TAP) and endoplasmic 
reticulum aminopeptidases (ERAPs) is strongly correlated with disease progression and metastasis ${ }^{58,166}$

- Loss of TAA through de-differentiation: for example, de-differentiation of melanoma cells results in antigen-deficient tumour cell variants and the induction of T-cell tolerance ${ }^{167}$.

- Increasing resistance to cell death by immune cells: Cellular FLICE (FADD-like IL$1 \beta$-converting enzyme)-inhibitory protein (c-FLIP; also known as CFLAR or inhibitor of apoptosis proteins (IAPs)) is a major anti-apoptotic protein. Upregulation of c-FLIP has been correlated with a poor clinical outcome through the prevention of apoptosis ${ }^{168}$.

- Expression of ligands for inhibitory receptors: Programmed cell death protein 1 ligand 1 (PD-L1), the ligand for PD-1, is highly expressed in several cancers. PD-L1 expression is an immune evasion mechanism exploited by various malignancies and is generally associated with poorer prognosis ${ }^{169}$.

- Infiltration of immune suppressive cells: Cells such as regulatory T cells, macrophages and myeloid-derived suppressor cells (MDSCs) play an important role in normal physiology by moderating immune destruction and preventing autoimmune disease, but in solid tumours, they promote immunosuppression by several mechanisms including secretion of immunosuppressive cytokines such as transforming growth factor$\beta$ (TGF $\beta$ ), interleukin-10 (IL-10) and vascular endothelial growth factor (VEGF) ${ }^{170}$.

- Deprivation of nutrients and oxygen: Rapidly growing tumour cells outgrow their blood supply resulting in a microenvironment with reduced oxygen and nutrients. Tumour cells can adapt to this stressful environment by inducing angiogenesis and altering metabolic strategies, thus ensuring survival and proliferation through the 
upregulation of various enzymes and factors such as indoleamine 2,3-dioxygenase (IDO), and Hypoxia-Inducible Factor (HIF-1). The generated hypoxic stress has a substantial impact on tumour cell biology. For example, it may contribute to increased tumour heterogeneity by selecting for certain cell subpopulations, thereby facilitating the emergence of therapeutic resistant cancer clones ${ }^{171}$.

Cancer's ability to induce tumour tolerance is incredibly complex as these immune escape mechanisms do not act in isolation. Together, the overlap between various signalling pathways and the interactions between several immunosuppressive molecules leads to resistance.

Combinations of different therapies will likely be more effective than single-agent therapies for the treatment of a given patient. However, determining which of these combinations is most effective remains a challenge ${ }^{172}$.

Combining TLR ligation with iNKT cells activation may provide a potential remedy for overcoming immune evasion. Treatment with $\mathrm{CpG}$, or iNKT cell activators such as $\alpha$-GalCer, have been the subject of a large number of clinical trials, and while safe, the magnitude of the immunity induced appears to be inadequate to control the spread of established cancers ${ }^{153}$. However, preclinical studies have shown additive or even synergistic effects upon combining iNKT activators and TLR agonists ${ }^{173}$. The enhanced innate and adaptive immune responses result in stronger tumour inhibition in animal models that correlates with increased numbers of antigen-specific CTLs at the tumour site. Thus a combination of iNKT cell activation and TLR ligation may overcome tumour immune evasion by resetting the immunosuppressive tumour microenvironment. This could occur via the activation local APCs, shifting the cytokine milieu away from a pro-tumourigenic towards an anti-tumour focus. Secondly, stimuli through TLR would promote the egress of these APCs to lymphoid organs to stimulate T cells as well as 
enhancing chemokine and adhesion expression to attract these T cells towards the tumour. This effect would be compounded by iNKT cells, also releasing factors that further promote APC activation, as well as providing direct anti-tumour cytotoxic activity. 


\subsection{Use of TLR ligands clinically}

Mycobacterium Bovis Bacillus Calmette-Guérin (BCG) was initially used as a vaccine against tuberculosis but is now FDA-approved for the treatment of non-invasive transitional cell

carcinoma of the bladder ${ }^{174}$. It is an attenuated form of the bacteria Mycobacterium Bovis, which is immunologically related to M. tuberculosis. Although the mechanism of action of BCG is not entirely understood, signalling via TLR2, TLR4, and TLR9 have all been implicated in the host response to this agent leading to an anti-tumour response ${ }^{175}$. Guanosine or uridine-rich ssRNA is a ligand for TLR7, and this suggests that cells of the innate immune system can detect RNA virus infection by sensing viral ssRNA in endosomes ${ }^{176}$. The successful use of BCG clinically has provided much of the impetus to investigate specific TLR agonists in the cancer setting.

The first clinically approved specific TLR agonist was Imiquimod, also called Aldara® (imiquimod 5\% cream is commercially available) or R-837, which is a TLR7 agonist used for the treatment of actinic keratosis, external genital/perianal warts (condylomata acuminata), and superficial basal cell skin cancers (BCCs) ${ }^{174}$. It works by releasing cytokines such as IFNs. Another approved TLR agonist, MPL, is a detoxified derivative of Salmonella Minnesota lipopolysaccharide (LPS) that is employed as an adjuvant in Cervarix, a human papillomavirus (HPV) bivalent (Types 16 and 18) vaccine that is used to induce adaptive responses as prophylaxis against infection ${ }^{177}$. MPL is thought to signal through TLR4 binding on dendritic cells leading to increased T-helper cells. MPL forms part of the so-called "Adjuvant System 04" (AS04) together with aluminium salt used for treating or protecting against HPV infection and associated pre-cancerous lesions ${ }^{177,178}$.

Additionally, several experimental TLR ligands have been investigated over the past few years for their ability to mediate clinically beneficial immunostimulatory effects ${ }^{179}$. For example, 
it was shown that TLR7/TLR8 agonism, compared to TLR3 and TLR9 agonism, drives an NK cell-dependent immune response that can eradicate tumours in animal models that have escaped immunosurveillance following MHC class I downregulation ${ }^{15}$, which is in line with data from other groups demonstrating the ability of TLR7 ligands to trigger NK cell-dependent tumour control $^{180,181}$. A small-molecule agonist of TLRs 7/8 (NKTR-262) in combination with pegylated IL-2 (NKTR-214) is in a phase 1 trial for the treatment of solid tumours. NKTR-262 is given intratumourally and is designed to be retained in the tumour microenvironment (being lipophilic) in order to activate APCs, such as dendritic cells, to create new antigen-specific cytotoxic T cells (trial NCT03435640).

The stabilized double-stranded RNA polyinosinic-polycytidylic acid stabilized with polylysine and carboxymethylcellulose (Poly-ICLC) is a viral mimic that serves as a TLR3 agonist. It was evaluated in a phase 1 trial for the treatment of acute myeloid leukaemia as an adjuvant for the fusion protein DEC-205/NY-ESO-1 (trial NCT01834248), which is a fully human monoclonal antibody directed against the endocytic DC receptor, DEC-205, linked to NY-ESO-1. The tumour antigen NY-ESO-1 is cancer-testis antigen ${ }^{182}$, meaning it is expressed in human tumours of different histological origin, but not in normal tissues except for testis and placenta. The trial found that the treatment-induced immunity to NY-ESO-1 in patients with tumours expressing NY-ESO-1. This immune response lead to the stabilisation of disease in 13 patients and tumour regression in 2 patients. This suggests that protein vaccines targeting DCs are potentially viable and efficacious. 


\subsection{Use of unmethylated CpG ODN to stimulate TLR9}

Perhaps the most promising preclinical data going into the clinic involves TLR9 agonists. SD-101, a proprietary unmethylated $\mathrm{CpG}$ oligodinucleotide, is being evaluated in multiple clinical trials in a variety of cancer indications intratumourally. Since the unmethylated $\mathrm{CpG}$ sequence is characteristic of bacterial DNA, it can be detected by TLR9 while methylated CpG is not a ligand for TLR9. SD-101 activates the two principal TLR9 signalling pathways; one pathway leads to rapid interferon- $\alpha$ (IFN- $\alpha$ ) production, which in turn stimulates many critical activities including activating NK cells, blocking tumour-mediated immune suppression, and promoting $\mathrm{T}$ cell homing into the tumour. The second pathway complements TCR-induced signals to enhance effector T cell proliferation, survival and cytokine production ${ }^{183}$. SD-101 in combination with anti-OX40 antibody (BMS 986178), an agonistic antibody for a co-stimulatory molecule that helps drive $\mathrm{T}$ cell responses, is being evaluated for the treatment of lymphoma and solid malignancies (NCT03831295; NCT03410901). Agonistic anti-OX40 monoclonal antibody selectively binds to and activates the OX40 receptor, which induces proliferation of memory and effector T lymphocytes. SD-101 is also being explored in combination with Ibrutinib, a Bruton's tyrosine kinase (BTK) inhibitor, and radiation therapy for patients with relapsed or refractory follicular lymphoma (NCT02927964). The rationale for this combination is because Bruton's tyrosine kinase (BTK) is crucial in B-cell development and survival, and as a result is a critical factor in the pathogenesis of B-cell malignancies where it is over-expressed.

TLR9 agonists are generally well tolerated in cancer patients with the most common adverse events observed with the administration of TLR9 agonists being local injection-site reactions (for example, erythema, oedema, inflammation, and pain). However, unlike other therapeutic immunotherapies, TLR9 agonist therapy has not been associated with clinically 
significant autoimmune diseases ${ }^{184}$. A distinguishing characteristic of TLR9 agonists, such as CpG-ODNs, is their ability to induce strong $\mathrm{CD}^{+}$and $\mathrm{CD}^{+} \mathrm{T}$-cell responses and as well as rapidly produce antigen-specific antibodies when used as a vaccine adjuvant ${ }^{185}$.

At present, the use of TLR9 as adjuvants in tumour vaccination has been assessed clinically. The most advanced cancer vaccine program was a phase III, randomised, controlled, clinical trial of the tumour antigen MAGE-A3 combined with clinical-grade CpG (CpG7909) in resected stage III melanoma ${ }^{186}$. Unfortunately, the treated arm did not fare any better than the placebo arm in terms of the time for disease-free survival. However, this may not be the fault of $\mathrm{CpG}$ as used, as MAGE-A3 may not be have been a good antigen.

Treatment with the $\mathrm{CpG}$ has also been combined with chemotherapy. A phase 2 clinical trial, where treatment with the CpG7909 was used in combination with taxane/platinum chemotherapy, for advanced-stage non-small-cell initially showed promising results. The primary endpoint was the objective response rate, which was 38\% in the CPG7909 and chemotherapy arm $(n=74)$ and $19 \%$ in the chemotherapy-alone arm $(n=37)$. The median overall survival (OS) times were 12.3 months in the CPG7909 and chemotherapy arm and 6.8 months in the chemotherapy-alone arm, and the 1-year survival rates were $50 \%$ and $33 \%$, respectively ${ }^{187}$. Unfortunately, the phase 3 trial was stopped after the interim analysis indicated the lack of benefit from the addition of CPG7909. Grade 3-4 neutropenia, thrombocytopenia, and anaemia were all reported more commonly for patients in the CPG7909 arm, which was administered subcutaneously. Therefore, the true therapeutic potential of TLR9 agonists as a cancer treatment remains to be determined, although there are close to 80 entries registering $\mathrm{CpG}$ for the treatment of cancer at clinicaltrials.gov website. Most of these, however, are completed or terminated some time ago without publication of the results. However, there was an encouraging 
study where CpG7909 was administered intratumourally in combination with local low-dose radiotherapy. Fifteen patients with relapsed, low-grade lymphoma had low-dose radiotherapy applied to one of the tumour sites with the combined injection of CpG7909 at the same site. Tumour regression was observed in several patients with an objective response rate of $27 \%{ }^{188}$. There are three major classes of stimulatory CpG ODNs (Fig 7) that have been defined based on molecular structure and activity in human peripheral blood mononuclear cells (PBMCs), in particular, B cells and pDCs ${ }^{189-191}$. Class A CpG ODNs (CpG-A ODN) induce high IFN- $\alpha$ production from pDCs but are weak stimulators of TLR9-dependent NF- $\mathrm{B}$ signalling and pro-inflammatory cytokine (e.g. IL-6) production. Class B CpG-B ODNs (CpG-B ODN) are potent stimulators of B cells and TLR9-dependent NF- $\kappa \mathrm{B}$ signalling but weakly stimulate IFN- $\alpha$ secretion. Class C ODNs (CpG-C ODN) combines features of both classes A and $\mathrm{B}$, inducing strong IFN- $\alpha$ production from $\mathrm{pDCs}$ as well as B cell stimulation. The clinical grade CPG7909 is a human-specific CpG-B ODN. For the preclinical work in this thesis, the agonist CpG-ODN 1826 was used. While CpG-ODN 1826 is mouse-specific, it is in the same class as CPG7909. This should minimise any unforeseen complications or potential lack of translation when going from mice to humans. At the outset of this thesis, it was known that CpGB ODN induces rapid IFN- $\alpha$ production, which in turn, activates NK cells, blocks tumourmediated immune suppression, and leads to efficient generation and activation of tumourspecific cytotoxic CD8 ${ }^{+}$T cells ${ }^{189-191}$. 


\section{CpG ODN Classes}

\section{A-Class 5'-(9)(9)(G)(G) (A) C.:-(A) \\ 3 -(9)(9-(9)-(9) (T) - (T)}

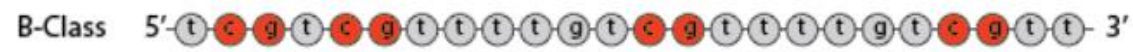

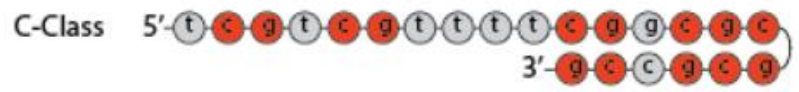

(I) Phosphorothioate link, (N) Phosphodiester link, CO CG dinucleotide

Figure 7: Three main classes of $C p G$ ODN. Top. CpG-A ODNs induce high IFN- $\alpha$ production from pDCs but are weak stimulators of TLR9-dependent $N F-\kappa B$ signalling and pro-inflammatory cytokine (e.g. IL-6) production. Middle. Cp G-B ODNs are potent stimulators of $B$ cells and TLR9-dependent $N F-\kappa B$ signalling but weakly stimulate IFN- $\alpha$ secretion. Bottom. $C p G-C$ ODNs combine features of both classes $A$ and B. They induce strong IFN- $\alpha$ production from $p D C s$ as well as $B$ cell stimulation. Adapted from invivogen.com.

Overall, from multiple clinical trials ranging from hepatitis B virus ${ }^{192}$ to cancer ${ }^{193}$, the clinical data indicates that $\mathrm{CpG}$ ODNs are safe. Also, in mice, CpG-ODNs improve humoral responses in addition to cellular immune responses, accelerating the production of protective antibodies at higher and more persistent titres when combined with protein-based vaccines. Based on data gathered from clinicaltrials.gov discussed above, when used as a single agent or in combination with known antigens, CpG-ODNs show some potential for clinical efficacy in cancer. However, the magnitude of the induced immunity does not appear sufficient to control the spread of established cancers ${ }^{194,195}$. 


\subsection{Intratumoural Delivery of TLR and iNKT cell agonists as a therapy?}

Our group observed enhanced activation of APCs when a TLR agonist was coadministered intravenously with $\alpha$-GalCer to stimulate iNKT cells, which when also combined with co-delivery of antigen, resulted in enhanced priming of $\mathrm{CD} 8^{+} \mathrm{T}$ cell responses ${ }^{11}$. This was seen for a range of TLR agonists targeting different TLRs, except for agonists targeting TLR7. The latter may be explained because, in mice, TLR7 is not expressed on DCs of the conventional "cDC1" phenotype that have a high propensity for stimulating $\mathrm{CD} 8^{+} \mathrm{T}$ cells. Interestingly, for all TLR agonists, when administered up to $24 \mathrm{~h}$ after $\alpha$-GalCer, significant increases in cytokine release were observed, including TNF $\alpha$ and IL-12p70. Depending on agonist used, and the timing between the different agents, in some cases a cytokine "storm" was induced that severely incapacitated the animals. These results indicate that APCs that have been exposed to activated iNKT cells are particularly sensitive to further stimulation via TLR agonists ${ }^{11}$. While the production of these cytokines could ultimately have anti-tumour activity, it is likely that this dosing arrangement would not be well tolerated by the subjects in a systemic treatment setting.

We, therefore, hypothesised that an intratumourally delivered combination of iNKT cells agonist ( $\alpha$-GalCer) and a TLR agonist such as $\mathrm{CpG}$ (up to $24 \mathrm{~h}$ apart) might minimise systemic cytokine release, while still create changes to the local tissue and draining lymphoid tissues that help drive an antigen-specific response. An additional advantage of delivering the drugs intratumourally is that it could permit high local intratumoural concentrations to be achieved, with a corresponding low systemic concentration, thus minimising potential side effects associated with high doses ${ }^{196}$.

Furthermore, there are multiple examples where intratumoural treatment can induce antitumour responses at distal sites, the abscopal effect. For example, in patients with metastatic 
melanoma, intratumoural injections of BCG induced regressions in about $90 \%$ of the injected tumour sites and approximately $20 \%$ of distant, uninjected tumour sites ${ }^{197}$. In another study, local delivery of the TLR7 agonist imiquimod coupled with intralesional BCG was able to induce complete remission in 5 out of 9 cutaneous melanoma patients ${ }^{198}$. Finally, a recent report of intratumoural administration of $\mathrm{CpG}$ and an anti-OX40 mAb produced an impressive immune response against established and distal tumours in mice ${ }^{189}$. At the time of this writing, according to ClinicalTrials.Gov, there are/have been over 500 clinical trials that use intratumoural delivery for the treatment of cancers which implies that this is a clinically acceptable delivery technique. While iNKT cells can mediate lysis of tumour cells, their anti-tumour effects likely depend in large part on their ability to activate other immune cells, such as NK and DCs, and initiate adaptive immunity. The ability of $\mathrm{CpG}$ to prime DCs via TLR9 leading to DC maturation and activation makes $\mathrm{CpG}$ an ideal combination partner to $\alpha$-GalCer, an iNKT cell activator, bridging innate and adaptive immunity.

To address the hypothesis that an intratumourally administered combination of iNKT cell agonist and TLR9 agonist will induce powerful antitumour responses, this thesis started from baseline knowledge that intratumoural $\mathrm{CpG}$ had been shown to have some anti-tumour activity on its own in animal models of cancer ${ }^{199-201}$. The intention here was to investigate whether introducing $\alpha$-GalCer to activate iNKT cells could improve on this activity, to uncover the molecular and cellular activities involved, and to explore further approaches to improving the therapy. The thesis, therefore, had the following aims: 


\begin{abstract}
Aims
1. To assess whether the outcome to intratumoural TLR9 ligation with $\mathrm{CpG}$ can be improved by harnessing the activity of iNKT cells.
\end{abstract}

2. Determine if the intratumoral administration of combination therapy provided a larger therapeutic window i.e. efficacy with concomitant reduction in safety signals such as liver toxicity and systemic cytokine release as compared to systemic administration.

3. To determine the cellular mechanisms involved in the anti-tumour response to the combination of TLR9 ligation and activation of iNKT cells.

4. To determine whether the efficacy of combination therapy can be enhanced by intratumoral administration of peptide from tumour-associated antigens

5. Determine if the efficacy of combination therapy can be enhanced by combination with other cancer therapies. 


\section{Chapter 2}

\section{Materials and Methods}

2.1 Materials

2.2.1 Labware

\begin{tabular}{|l|l|}
\hline Supplier/Manufacturer & Product \\
\hline & \\
\hline Axygen Scientific, & $\quad 0.5-20 \mu \mathrm{L}, 200 \mu \mathrm{L}, 1000 \mu \mathrm{L}$ Tips \\
Inc., Union City CA USA. & Racked and Pre- sterilised \\
& $\bullet \quad 0.6,1.7$ and 2.0 mL MAXYmum \\
& Recovery ${ }^{\mathrm{TM}}$ Microtubes \\
\hline
\end{tabular}




\begin{tabular}{|c|c|}
\hline $\begin{array}{l}\text { Becton Dickson Pty, Ltd } \\
\text { Mountain View, CA, USA. }\end{array}$ & 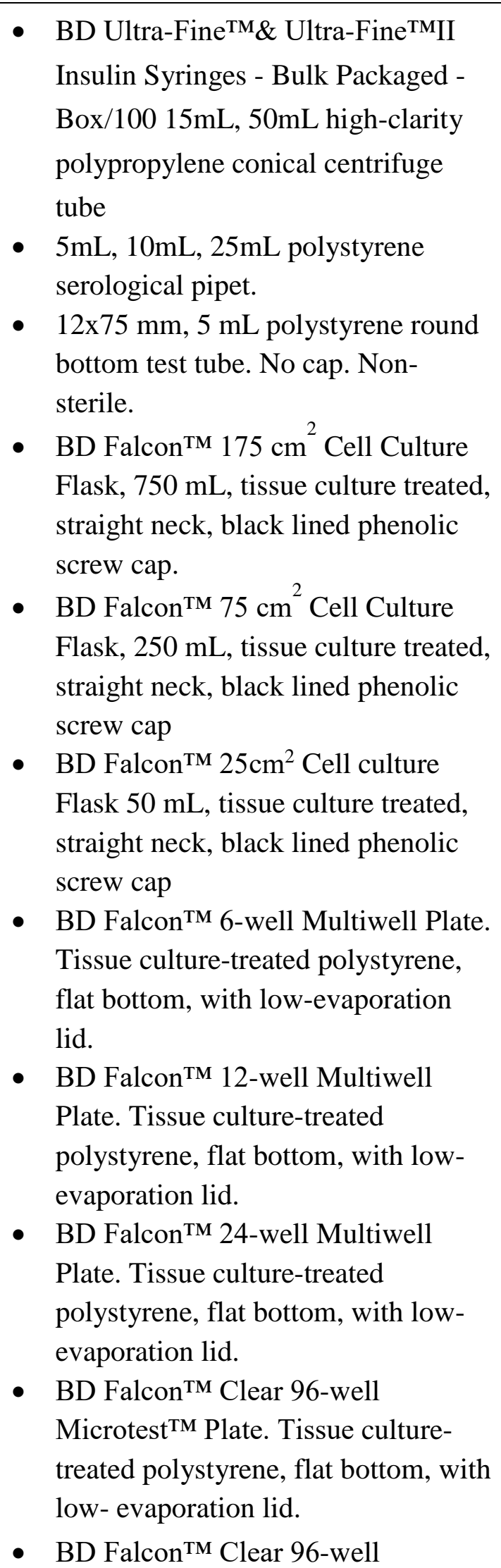 \\
\hline
\end{tabular}




\begin{tabular}{|c|c|}
\hline & $\begin{array}{l}\text { Microtest }{ }^{\mathrm{TM}} \text { Plate. Tissue- culture- } \\
\text { treated polystyrene, U-bottom, with } \\
\text { low- evaporation lid. } \\
\text { - } \text { BD Plastipak }{ }^{\mathrm{TM}} \text { Syringes } 30 \mathrm{~mL}, \\
\text { Luer- Lok } \\
\text { - } \quad \text { BD Syringe } 1 \mathrm{~mL}, 2 \mathrm{~mL}, 3 \mathrm{~mL}, 5 \mathrm{~mL} \text {, } \\
\text { 10mL Luer-Slip Tuberculin } \\
\text { - Cell Strainer with } 70 \mu \mathrm{m} \text { nylon } \\
\text { mesh, white frame colour. Sterile. } \\
\text { - } \text { BD Veo }{ }^{\mathrm{TM}} \text { insulin syringes with BD } \\
\text { Ultra-Fine }\end{array}$ \\
\hline Millipore Sigma & $\begin{array}{l}\text { - Millipore Pure Express }{ }^{\mathrm{TM}} \text { Plus } \\
\text { Stericup and Steritopp. } 0.22 \mu \mathrm{m} \text { non- } \\
\text { pyrogenic pre-sterilized. Vacuum } \\
\text { driven. }\end{array}$ \\
\hline Sefar Filter Specialist, Nelson, New Zealand & $\begin{array}{l}\text { - } \quad 70 \mu \mathrm{m} \text { nylon gauze } \\
\text { - } \quad 80 \mu \mathrm{m} \text { stainless steel mesh }\end{array}$ \\
\hline Sigma-Aldrich & - Isofluorane \\
\hline $\begin{array}{l}\text { Thermo Fisher Scientific, Waltham, MA, } \\
\text { USA. }\end{array}$ & $\begin{array}{l}\text { - Nunc CryoTube } 1.0 \mathrm{~mL} 1.8 \mathrm{~mL} \text { PP } \\
\text { sterile Nunc-Immuno }{ }^{\mathrm{TM}} \text { Plates } \\
\text { Polystyrene, without lids. } 96 \text { wells } \\
\text { per plate. MaxiSorp®. } \\
\text { - } \quad \text { Transfer pipette graduated } 1 \mathrm{~mL} \\
\text { large bulb } \cdot \text { SecureSeal } \\
\text { AdM Thermal } \\
\text { Adhe Sealing Film for PCR }\end{array}$ \\
\hline
\end{tabular}




\begin{tabular}{|c|c|}
\hline & $\begin{array}{l}\text { application } \\
\text { - Slide-A-Lyzer Dialysis Cassettes, } \\
10000 \text { MWCO } \\
\text { - } \text { Glass Pasteur pipette } 150 \mathrm{~mm} \\
(\mathrm{p} / 1,000) \\
\text { - }\end{array}$ \\
\hline TESA® Micromaster $®$ & $\begin{array}{l}\text { - } \text { Micromaster® electronic } \\
\text { micrometer with digital display } \\
\text { - } 0-30 \mathrm{~mm}\end{array}$ \\
\hline $\begin{array}{l}\text { Cortex Technology Smedevaenget } \\
\text { Hadsund, Denmark }\end{array}$ & $\begin{array}{l}\text { - DermaLab USB Single Parameter } \\
\text { TEWL probe, SKINLABSoftware }\end{array}$ \\
\hline Sarstedt & $\begin{array}{l}\text { Microvette }{ }^{\circledR} 200 \mathrm{Z}-\mathrm{Gel} \text { tubes. } \\
200 \mu \mathrm{L} \text { polypropylene tubes with } \\
\text { clotting activator for serum. }\end{array}$ \\
\hline $\begin{array}{l}\text { Thermo Scientific }{ }^{\mathrm{TM}} \text {, } \\
\text { New Zealand Ltd. }\end{array}$ & $\begin{array}{l}\text { - Tissue Loc }{ }^{\mathrm{TM}} \text { histological tissue } \\
\text { embedding cassettes }\end{array}$ \\
\hline Briscoes, Wellington, New Zealand & - Breville Fast slow cooker \\
\hline Thermo Scientific $^{\mathrm{TM}}$ & $\begin{array}{l}\text { - } \text { Citadal } 2000 \text { Carousel Tissue } \\
\text { processor } \\
\text { - HistoSTar Embedding Center } \\
\text { - HM } 325 \text { Rotary Microtome }\end{array}$ \\
\hline Olympus,Wellington, New Zealand & $\begin{array}{ll}\text { - } & \text { Brightfield BX51 Microscope } \\
\text { - } & \text { Laser Scanning Confocal } \\
& \text { Microscope FV1200® }\end{array}$ \\
\hline
\end{tabular}




\subsection{Reagents and buffers}

\subsubsection{Culture Media Reagents}

Fetal bovine serum (FBS)

FBS was purchased from Invitrogen (Gibco, Auckland, New Zealand, 12203C) and stored in $25 \mathrm{~mL}$ aliquots at $-20^{\circ} \mathrm{C}$

Complete Iscove's modified Dulbecco's medium (cIMDM5)

IMDM was purchased from Invitrogen (Auckland, New Zealand, 31980-097) and supplemented with 1\% Penicillin-Streptomycin from Invitrogen (Gibco, Auckland New Zealand, 15140-122), and 5\% FBS. Media was filter sterilised and stored at $4^{0} \mathrm{C}$ for a maximum of 14 days.

\section{Complete RPMI (cRPMI10)}

RPMI was purchased from Invitrogen (Auckland, New Zealand, 42401-018) and supplemented with 1\% Penicillin-Streptomycin from Invitrogen (Gibco, Auckland New Zealand, 15140-122), $10 \%$ FBS, $1 \%$ Glutamax, and $1 \%$ sodium pyruvate. Media was filter sterilised and stored at $4^{0} \mathrm{C}$ for a maximum of 14 days.

Complete Dulbecco's Modified Eagle's Medium (cDMEM10)

DMEM was purchased from Invitrogen (Auckland, New Zealand, 42401-018) and supplemented with $1 \%$ penicillin-streptomycin, $2 \mathrm{mM}$ Glutamax, $10 \%$ fetal bovine serum, and $100 \mu \mathrm{g} / \mathrm{mL}$ normocin. 
Ethylene-diamine-tetra-acetic acid (EDTA)

Diluted EDTA (0.5M) was purchased from Invitrogen (Auckland, New Zealand, 15575-020) and stored at room temperature.

\section{$\underline{\text { Trypsin-EDTA }}$}

Trypsin containing $0.05 \%$ Ethylenediaminetetraacetic acid (EDTA) and phenol red was purchased from Invitrogen (Gibco, Auckland, New Zealand, 25300062) and stored in 25mL aliquots at $-20^{\circ} \mathrm{C}$.

$\underline{\text { Blasticidin }}$

Blasticidin selective antibiotic was purchased from Invivogen (San Diego, California, USA, antbl-1) and stored at $-20^{\circ} \mathrm{C}$.

\section{$\underline{\text { Geneticin }}$}

Geneticin was purchased from Invitrogen (Gibco, Auckland, New Zealand, 10131027) and stored in $5 \mathrm{~mL}$ aliquots at $-20^{\circ} \mathrm{C}$.

\section{$\underline{\text { Normocin }}$}

Normocin was purchased from Invivogen (San Diego, California, USA, ant-nr-1) and stored at $20^{0} \mathrm{C}$. 
$\underline{\text { Zeocin }}$

Zeocin selective antibiotics were purchased from Invivogen (San Diego, California, USA, ant$\mathrm{zn}-1)$ and stored at $-20^{\circ} \mathrm{C}$.

\section{$\underline{\text { HEK-Blue Detection medium }}$}

HEK-Blue Detection medium was purchased from Invivogen (San Diego, California, USA, hb$\operatorname{det} 2$ ) and stored at $4^{0} \mathrm{C}$ until used. For usage, the medium was reconstituted in $50 \mathrm{~mL}$ of endotoxin-free water and frozen at $-20^{\circ} \mathrm{C}$. The assay was performed. HEK-Blue ${ }^{\mathrm{TM}}$ Detection detects SEAP (Secreted embryonic alkaline phosphatase) reporter proteins secreted by the cells. The hydrolysis of the substrate by SEAP produces a blue colour that can be seen with the naked eye or measured with a spectrophotometer.

\subsubsection{Enzyme and tissue processing}

\section{$\underline{\text { Bicinchoninic acid }(\mathrm{BCA})^{\mathrm{TM}} \text { protein assay kit }}$}

$\mathrm{BCA}^{\mathrm{TM}}$ protein assay kit was purchased from Thermo Scientific Ltd (Auckland, New Zealand) and stored at room temperature.

\section{Cell Lysis Buffer}

Cell Signalling ${ }^{\mathrm{TM}}$ Cell Lysis Buffer (10X) was purchased from Life Technologies Ltd (Auckland, New Zealand, 78430) and stored at $-20^{\circ} \mathrm{C}$. 


\section{$\underline{\text { Dulbecco's phosphate-buffered saline (dPBS) }}$}

$\mathrm{dPBS}$ is a powder containing no calcium or magnesium was purchased from Invitrogen (Gibco, Auckland, New Zealand, 21600-069) and dissolved in deionised water (dH20).

\section{$\underline{\text { Liberase TL }}$}

Liberase TL was purchased from Roche Diagnostics New Zealand Ltd (Auckland, New Zealand, 5401020001). The lyophilised powder was reconstituted in IMDM to a concentration of $1 \mathrm{mg} / \mathrm{mL}$ and stored in aliquots at $-20^{\circ} \mathrm{C}$ for up to three months.

\section{$\underline{\text { DNase } 1}$}

DNase 1 was purchased from Roche Diagnostics New Zealand Ltd (Auckland, New Zealand, 10104159001) as a lyophilised powder and dissolved in cIMDM to a concentration of $10 \mathrm{mg} / \mathrm{mL}$ and stored at $20{ }^{\circ} \mathrm{C}$.

\section{$\underline{\text { Tissue digestion buffer }}$}

Liberase TL at a final concentration of $2.5 \mathrm{mg} / \mathrm{mL}$ is made from a stock of $50 \mathrm{mg} / \mathrm{mL}$ and DNAse 1 at $120 \mu \mathrm{g} / \mathrm{mL}$ from a stock of $10 \mathrm{mg} / \mathrm{mL}$ were made up in IMDM to the required volume.

\section{$\underline{\text { Protease inhibitor }}$}

Halt ${ }^{\mathrm{TM}}$ Protease Inhibitor Cocktail (100X) was purchased from Life Technologies Ltd (Auckland, New Zealand, 78430) and stored at $4^{0} \mathrm{C}$.

$\underline{\text { Red blood cell lysis buffer }}$

Red blood cell lysis buffer was purchased from Sigma-Aldrich, New Zealand Ltd (Auckland, New Zealand, R7757) and stored at room temperature. 


\subsubsection{Flow cytometry reagents}

\section{4,6-Diamidino-2-Phenlinodole dihydrochloride (DAPI)}

DAPI was purchased as a lyophilised powder from Invitrogen (Auckland, New Zealand D1306) and dissolved in sterile $\mathrm{dH}_{2} \mathrm{O}$ to a concentration of $5 \mathrm{mg} / \mathrm{mL}$ and stored at $-70^{\circ} \mathrm{C}$. The stock was further diluted in flow cytometry buffer to a concentration of $200 \mathrm{mg} / \mathrm{mL}$ in FC buffer and stored at $4^{0} \mathrm{C}$.

$\underline{\text { Live/Dead }^{\circledR}}{ }^{\circledR}$ fixable dead cell staining kit

Live/Dead kit was purchased from Life Technologies New Zealand Ltd, Molecular Probes (Auckland, New Zealand L23105) and stored at $-20^{\circ} \mathrm{C}$. The lyophilised powder was dissolved in $50 \mu \mathrm{L}$ anhydrous DMSO (components of the kit) per vial before use and stored at $-20^{0} \mathrm{C}$ for a maximum of 6 weeks.

\section{Compensation beads}

OneComp beads were purchased from eBioscience (San Diego, CA, USA, 0111142) and were stored at $4^{0} \mathrm{C}$. These beads react with antibodies of mouse, rat and hamster origin.

Flow cytometry (FC) buffer

NaN3, FBS and EDTA were added to IL dPBS to give final concentration of $0.01 \% \mathrm{NaN} 3,1 \%$ FBS and $1 \mathrm{mM}$ of EDTA. FC buffer was stored at $4^{0} \mathrm{C}$. 


\subsubsection{Monoclonal antibodies used for in vivo studies}

$\underline{\text { Rat anti-mouse CD8 (Clone 2.43) }}$

Anti-mouse CD8 was purchased from Bio X cell (West Lebanon, New Hampshire USA,

BE0061). It was stored at shipping concentration at $4^{0} \mathrm{C}$.

$\underline{\text { Rat anti-mouse IFNaR1 (Clone MAR1-5A3) }}$

Anti-mouse IFNaR1 was purchased from Bio X cell (West Lebanon, New Hampshire USA, BE00241). It was stored at shipping concentration at $4^{0} \mathrm{C}$.

$\underline{\text { Rat anti-mouse CD1d (Clone 19G11) }}$

Anti-mouse CD1d was purchased from Bio X cell (West Lebanon, New Hampshire USA, BE0000). It was stored at shipping concentration at $4^{0} \mathrm{C}$.

$\underline{\text { Rat anti-mouse IgG1 Isotype Control, unknown specificity (MOPC-21) }}$

Anti-mouse IgG1 Isotype Control, unknown specificity was purchased from Bio X cell (West

Lebanon, New Hampshire USA, BE0083). It was stored at shipping concentration at $4^{0} \mathrm{C}$.

\subsubsection{Fluorescently conjugated antibodies}

Table 2.1 - Flow cytometry antibodies

\begin{tabular}{|l|l|l|l|}
\hline Specificity & Fluorophore & Clone & Company \\
\hline B220 & PerCP & RA3-6B2 & BioLegend \\
\hline CD1d Tetramer & BV421 & & NIH \\
\hline CD3 & BV510 & 17 A2 & BD Pharmingen \\
\hline CD4 & APCH7 & GK1.5 & BioLegend \\
\hline
\end{tabular}




\begin{tabular}{|c|c|c|c|}
\hline CD8a & FITC/BV421/AF700 & $53-6.7$ & BioLegend \\
\hline CD11b & APC-Сy7/BUV737 & M1/70 & BioLegend \\
\hline CD11c & PE-Cy7/APC/BV786 & N418 & BioLegend \\
\hline CD44 & BV711 & IM7 & BioLegend \\
\hline CD45 & BV786 & 30-F11 & BD Pharmingen \\
\hline CD62L & FITC & MEL-14 & BioLegend \\
\hline CD69 & PerCP-Сy5.5 & $\mathrm{H} 1.2 \mathrm{~F} 3$ & Biolegend \\
\hline CD80 & APC & 16-10A1 & Biolegend \\
\hline CD86 & $\mathrm{PE}$ & GL-1 & BD Pharmingen \\
\hline CD103 & PE/FITC & 2E7 & BioLegend \\
\hline CD127 & PE Cy7 & A7R34 & Biolegend \\
\hline CD317 (BST2) & APC & 927 & BioLegend \\
\hline DAPI & DAPI & & Biolegend \\
\hline $\mathrm{F} 4 / 80$ & PerCP & BM8 & BioLegend \\
\hline KLRG1 & APC & KLRG1 & BioLegend \\
\hline Ly6C & PE-Cy7 & HK1.4 & BioLegend \\
\hline MHC II (I-A/I-E) & A488/BV421 & M5/114.15.2 & BioLegend \\
\hline NK1.1 & BV650 & PK136 & BioLegend \\
\hline PD-1 (CD279) & AF647 & RMP1-30 & BioLegend \\
\hline PD-L1 (CD274) & BV711 & 10F.9G2 & BioLegend \\
\hline PD-L2 (CD273) & BV421 & TY25 & BioLegend \\
\hline $\begin{array}{l}\mathrm{H}-2 \mathrm{~Kb} / \mathrm{SIINFEKL} \\
\text { Pentamer }\end{array}$ & $\mathrm{PE}$ & 25.D1.16 & eBioscience \\
\hline
\end{tabular}




\begin{tabular}{|l|l|l|l|}
\hline Siglec-H & PE & 551 & BioLegend \\
\hline XCR1 & FITC & ZET & BioLegend \\
\hline
\end{tabular}

\subsubsection{Immunohistochemistry (IHC) antibodies}

$\underline{\text { Rat anti-mouse CD4 (Clone GK1.5) }}$

anti-mouse CD4 was purchased from BioLegend, (Auckland, New Zealand, 100437) and stored at $4^{0} \mathrm{C}$

$\underline{\text { Rat anti-mouse CD8a (IL9R) (Clone 53-6.7) }}$

CD8a monoclonal antibody was purchased from BD Biosciences. (San Jose CA, USA, 564459) and stored at $4^{0} \mathrm{C}$.

Mouse anti-mouse CD64 (FcgRI) (Clone X54-5/7.1)

anti-mouse CD64 was purchased from the BioLegend (Auckland, New Zealand) and stored at $4^{0} \mathrm{C}$.

\subsubsection{Enzyme-Linked Immunosorbent Assay (ELISA)}

ELISAs were all performed to detect cytokine production in either serum samples and or in tissue lysate samples. ELISA kits were purchased from either BD Pharmingen (Auckland New Zealand), R \& D Systems (Minneapolis Minnesota, USA) or eBioscience (San Diego, CA, USA). Reagents were all stored as per manufacturers' specifications. 
Table 2.2 ELISA Kits for cytokine detection

\begin{tabular}{|l|l|l|l|}
\hline Species & ELISA kit & Cat NO & Company \\
\hline Mouse & $\begin{array}{l}\text { Invitrogen eBioscience } \\
\text { Mouse IFN alpha }\end{array}$ & BMS6027 & Invitrogen \\
& Platinum ELISA kit & & \\
\hline
\end{tabular}

\subsubsection{Drugs and Reagents}

$\underline{\text { Anaesthetic }}$

10x stock solution was diluted in sterile dPBS to $8.6 \mathrm{mg} / \mathrm{mL}$ ketamine and $0.26 \mathrm{mg} / \mathrm{mL}$ xylazine working solution.

\section{$\underline{\text { Diptheria Toxin (DT) }}$}

DT was purchased as a lyophilised powder from Sigma-Aldrich, New Zealand Ltd, (Auckland, New Zealand, D2918). DT was reconstituted in $\mathrm{dH}_{2} \mathrm{O}$ at a stock concentration of $2 \mathrm{mg} / \mathrm{mL}$. Stock aliquots of DT were further diluted in $1 \mathrm{ML}$ of sterile dPBS to obtain a $20 \mathrm{ng} / \mathrm{g}$ working solution.

$\underline{\text { Ethanol }(\mathrm{EtOH})}$

Absolute ethanol was purchased from Pure Science (Porirua, New Zealand, N1034705).

$\underline{\alpha-G a l a c t o s y l c e r a m i d e ~(a-G a l C e r) ~ a n d ~ C o n j u g a t e s ~}$

$\alpha$-GalCer, peptides and $\alpha$-GalCer-peptide conjugates were all synthesised and provided by the Ferrier Research Institute Victoria University of Wellington (Gracefield, Wellington, NZ). 
Doxorubicin Hydrochloride

Doxorubicin hydrochloride was originally purchased from Sapphire Bioscience (Redfern, Australia, 15007).

\section{$\underline{\text { CpG Oligonucleotides }}$}

Class B CpG 1826 (5' TCC ATG ACG TTC CTG ACG TT 3') was custom synthesised by Trilink Biotechnologies (San Diego, California, USA, O-4100).

\subsubsection{Cell Lines}

\section{B16-F1 (CRL-6323)}

The B16-F1 cell line was originally acquired from ATCC and maintained in house using cIMDM5.

\section{$\underline{\text { B16-F10 (CRL-6475) }}$}

The B16.F10 was originally acquired from ATCC and maintained in house using cIMD5.

\section{$\underline{\text { B16.OVA (CRL-6322) }}$}

The B16.OVA cell line was originally acquired from ATCC and maintained in house using cIMDM5. B16.OVA cells were generated from B16.F10 cells that were transfected with the plasmid pAc-neo-OVA, which carries a complete copy of chicken ovalbumin (OVA) mRNA and the neomycin (G418) resistance gene via electroporation. 
$\underline{\mathrm{CT} 26}$

The CT26 cell line was originally purchased from the American Type Culture Collection (ATCC; Manassas, Virginia, USA) and maintained in house using cIMD5 (5\% FBS, 1\% P/S). CT26 is an N-nitroso-N-methylurethane-(NNMU) induced, undifferentiated colon carcinoma cell line. It was cloned to generate the cell line designated CT26.WT

\section{$\underline{\text { EL4.LA (TIB-39) }}$}

The original EL4 murine lymphoma was purchased from the American Type Culture Collection (ATCC; Manassas, Virginia, USA) and maintained in house using cIMDM5. The EL4 cell line was established from a lymphoma induced in a C57BL mouse by 9,10-dimethyl-1,2benzanthracene. Extended passage resulted in genetic drift and increased immunogenicity as determined via sequencing and tumour experiments.

\section{EG7.OVA (CRL-2113)}

EG7.OVA lymphoma was originally purchased from ATCC. EG7.OVA and maintained using cIMDM5. EG7.OVA cells were derived from EL4 cells that were transfected with the plasmid pAc-neo-OVA, which carries a complete copy of chicken ovalbumin (OVA) mRNA and the neomycin (G418) resistance gene via electroporation.

\section{$\underline{\text { HEK-Blue mTLR9 }}$}

HEK-Blue mTLR9 cells were originally purchased from Invivogen (San Diego, California, USA, hkb-mtlr9) and maintained using cDMEM10. After 2 passages, selective antibiotics were added (30ug/mL of blasticidin and $100 \mathrm{ug} / \mathrm{mL}$ of zeocin). Cells were maintained and SEAP activity 
determined in the manner described in the HEK-Blue mTLR9 technical data sheet with parental cell line HEK-Blue Null1 cells being used as controls.

\section{$\underline{\text { HEK-Blue Null1 }}$}

HEK-Blue Null1 cells were originally purchased from Invivogen (San Diego, California, USA, hkb-null1) and maintained using cDMEM10. After 2 passages, selective antibiotics were added (30 $\mu \mathrm{g} / \mathrm{mL}$ of blasticidin and $100 \mu \mathrm{g} / \mathrm{mL}$ of zeocin). Cells were maintained and SEAP activity determined in the manner described in the HEK-Blue Null1 technical datasheet.

\section{$\underline{\mathrm{TC} 1}$}

The TC1 murine lung epithelial cancer cell line was kindly provided by T-C. Wu (Inje University, Seoul, Republic of Korea). The cell line was generated by transfecting lung epithelial cancer cells with the HPV E6 and E7 proteins via electroporation. The TC1 cell line was maintained in cRPMI10 (10\% FBS, 1\% Penicillin/streptomycin, $1 \%$ glutamax and 1\% sodium pyruvate). 


\subsection{Mouse strains}

Mice were bred and housed within the Biomedical Research Unit of the Malaghan Institute of Medical Research, under specific pathogen-free conditions. Majority of the experiments involved mice between 6 - 8 weeks of age and mice were sex-matched as best as possible. Experimental procedures were approved by the Animal Ethics Committee at Victoria University and carried out in accordance with guidelines of Victoria University of Wellington, New Zealand, The mouse experiments in this thesis were under the following ethics protocols:

23784: Synthetic Vaccines That Exploit the Innate Immune Response 26384: Preventing Metastasis by Vaccination

26774: Maurice Wilkins Centre: Reducing immunosuppression and banishing hypoxia from the tumour microenvironment to improve immunotherapy

\section{$\underline{\mathrm{C} 57 \mathrm{BL} / 6 \mathrm{~J}}$}

C57BL/6J mice were obtained from The Jackson Laboratory (Bar Harbor, MA, USA) and maintained as an in house strain.

\section{$\underline{B a l B / B y J}$}

Balbc/ByJ mice were obtained from The Jackson Laboratory (Bar Harbor, MA, USA) and maintained as an in house strain.

\section{$\underline{\text { Balb/c x C57 mouse strain }}$}

Balb/c and C57BL/6 breeding pairs obtained from Jackson Laboratory (Bar Harbour, Maine, USA) and were crossed in house. 


\section{$\underline{\text { CD1d Knockout }}$}

CD1d knockout breeding pairs were obtained from Jackson Laboratory (Bar Harbour, Maine, USA) and maintained as an inbred strain. CD1 deficient mice were knocked out of both copies of the CD1 glycoprotein. CD1 is a glycoprotein expressed on the surface of various antigenpresenting cells and is involved in activating iNKT cells which in turn produce cytokines such as IFN $\gamma$ and IL-4. Therefore mice deficient in both copies do not have an iNKT cell subset. The Th2 response is normal however, in knockouts.

\section{$\underline{\text { Traj18 Knockout }}$}

Traj18 (T cell receptor joining 18) knockout breeding pairs were obtained from Jackson Laboratory (Bar Harbour, Maine, USA), backcrossed onto Malaghan C57BL/6J mice and maintained as an inbred strain. Traj18 knockout mice cannot form the iNKT cell receptor $\alpha$-chain and consequently lack iNKT cells. Rearrangements of T cell receptor J $\alpha$ regions upstream or downstream of Traj18 are not affected.

\subsubsection{Dendritic cell specific knockouts}

\section{Siglec-H Diphtheria Toxin Receptor (DTR) Mice}

Siglec-H-DTR mice, by Christiane Ruedl (Nanyang Technological University, Singapore) and maintained as an in house strain. F1 crosses of Siglec-H DTR and C57BL/6J mice were used for experiments. 


\section{Clec9a Diphtheria Toxin Receptor (DTR) Mice}

Clec9a-DTR mice were kindly provided by Christiane Ruedl (Nanyang Technological University, Singapore) and maintained as an in-house strain. F1 crosses of Clec9a-DTR and C57BL/6J mice were used for experiments.

$\underline{\text { Itgax }^{\text {cre }} \text { mice }}$

$\operatorname{Itgax}^{\mathrm{cre}}$ mice express Cre recombinase under the control of the Itgax (CD11c) promotor within a BAC transgene. CD11c cre mice can be crossed with mice containing a loxP flanked ("floxed") gene of interest. This results in the deletion of that gene sequence in CD11c/Cre expressing cells by Cre-mediated recombination. Breeding pairs were obtained from QIMR Berghofer Medical Research Institute (Brisbane, QLD, Australia).

\section{$\underline{\mathrm{IFNaR}^{\mathrm{fl} / \mathrm{fl}} \text { mice }}$}

Ifnar ${ }^{\mathrm{fl} / \mathrm{fl}}$ mice have loxP sequences flanking exon 3 of the gene encoding for the type I interferon- $\alpha \beta$ receptor (IFNaR). Crossing $\mathrm{IFNaR}^{\mathrm{fl} / \mathrm{fl}}$ mice to $\mathrm{CD} 11 \mathrm{c}_{\text {cre }}$ mice deletes IFN $\alpha$ R from all CD11c expressing cells, rendering them unresponsive to IFN-I signalling. Itgax $^{\text {cre-neg }}$.Ifnar ${ }^{\text {fl/fl }}\left(\right.$ IFNARwt) littermates were used as controls for Itgax ${ }^{\text {cre-pos }}$.Ifnar ${ }^{\text {fl/fl }}$ (IFNAR $\triangle$ CD11c) mice. Breeding pairs were obtained from QIMR Berghofer Medical Research Institute (Brisbane, QLD, Australia). 


\subsection{Methods}

\subsubsection{Mouse manipulations}

\section{$\underline{\text { Tumour Size Measurement }}$}

Tumour size is measured (with a digital calliper) every 1-2 days.

$\underline{\text { Intraperitoneal (i.p.) administration }}$

Solutions were administered to mice in volume of $100-250 \mu \mathrm{L}$ via using a $1 \mathrm{ml}$ insulin syringe.

Intraperitoneal administration of anti-CD1d (19G11)

Treatment of $250 \mu \mathrm{g}$ of anti - CD1d and the isotype control antibody was administered by i.p. every alternate day from the day 8 to day 19 .

Intraperitoneal administration of anti-CD8 (2.43)

Treatment of $250 \mu \mathrm{g}$ of anti-CD8 antibody was administered by i.p. on day -2 , day -1 before tumour rechallenge. For depletion during treatment, $250 \mu \mathrm{g}$ of anti-CD8 antibody was administered on days 8,9 , and 12 post tumour inoculation.

$\underline{\text { Intraperitoneal administration of anti }- \text { PK136 }}$

Treatment of $250 \mu \mathrm{g}$ of anti-PK136 antibody was administered by i.p. on day 4 and 5 post tumour inoculation

Intraperitoneal administration of anti - IFN $\alpha$ R (MAR1-5A3)

Treatment of $250 \mu \mathrm{g}$ of anti - IFNaR and the isotype control antibody was administered by i.p. on days 4,5 and 8 


\section{Intraperitoneal administration of Diphtheria Toxin}

Treatment of $15 \mathrm{ng} / \mathrm{g}$ of DT was diluted to a working solution in dPBS and administered by i.p. on days 4,5 , and 8 post tumour inoculation

\section{$\underline{\text { Subcutaneous injection of tumour cells }}$}

For tumour challenge, EL4 and EG7.OVA were washed $3 \times$ in medium, and $10^{6}$ tumour cells were injected s.c. into the flank of mice. For B16-F1/F10/OVA, $3 \times 10^{5}$ cells were injected subcutaneously into the right flank. TC1 tumours were initiated by injecting $10^{5}$ cells subcutaneously into the right flank. Finally for CT26, $2 \times 10^{5}$ cells were injected into the flank(s). Tumour size was measured using callipers and mice were culled when tumour sizes reached 200 $\mathrm{mm}^{2}$.

\subsubsection{Intratumoural Administration Models}

$\underline{\text { Intratumoural application of } \alpha \text {-GalCer }}$

All mice were between 6 - 8 weeks of age, and were sex matched. Once the tumour size reached $36 \mathrm{~mm}^{2}-42 \mathrm{~mm}^{2}$ mice are anaesthetized using isofluorane. Once under sedation $40 \mu \mathrm{L}$ of the 58.25 $\mathrm{uM} \alpha$-GalCer working solution was injected intratumourally using a $300 \mu \mathrm{L}$ insulin needle. The control group was treated with PBS. Treatment with GalCer was conducted once on the first day of treatment only.

\section{$\underline{\text { Intratumoural } \mathrm{CpG} \text { administration }}$}

All mice were between 6 - 8 weeks of age, and were sex matched. Once the tumour size reached $36 \mathrm{~mm}^{2}-42 \mathrm{~mm}^{2}$ mice are anaesthetized using isofluorane. Once under sedation $40 \mu \mathrm{L}$ of the 98.2071uM CpG working solution was injected intratumourally using a $300 \mu \mathrm{L}$ insulin needle. 
The control group was treated with PBS. CpG was given for 6 doses in the following example schedule: days $6,7,10,11,14$, and 15 .

\section{$\underline{\text { Admixed intratumoural } \alpha-\mathrm{GalCer} \text { and } \mathrm{CpG} \text { administration }}$}

Full treatment involved 1 injection of $\alpha$-GalCer and $\mathrm{CpG}$ delivered intratumourally starting when tumour size reached $36 \mathrm{~mm}^{2}-42 \mathrm{~mm}^{2}$ (Day 5-9) followed by an intratumoural injection of CpG. Mice are rested for 2 days and then receive intratumoural CpG 2 times over the course of 2 days. Mice are again rested for 2 days and the $\mathrm{CpG}$ regimen is repeated. For example, mice could receive treatment on (assuming treatment starts on day 6): days $6,7,10,11,14$, and 15 .

\section{Splenectomy}

Mice were anaesthetised with $100 \mathrm{mg} / \mathrm{kg}$ Ketamine and $10 \mathrm{mg} / \mathrm{kg}$ Xylazine i.p., followed by s.c. administration of $0.1 \mathrm{mg} / \mathrm{kg}$ Buprenorphine for perioperative analgesia. Lacrilube (Allergan New Zealand Ltd, NZ) was applied to corneas to prevent desiccation. The surgical area was sterilised with ethanol, followed by an incision on the left flank to access the spleen. The primary artery and vein were tied to sever blood flow to the spleen, which was followed quickly by cutting off the blood vessels to release the spleen. In the sham controls, an incision was made into the peritoneum, but no other manipulation was made. The incisions were then sutured and mice recovered on a heating pad. The mice were monitored every hour post-op until they woke up from the anaesthetic, in addition, daily monitoring health monitoring was also done for an additional 5 days. Subcutaneous administration of $5 \mathrm{mg} / \mathrm{kg}$ Carprofen was used for the two days following surgery for postoperative analgesia. 


\section{Endpoint procedures}

Experimental endpoint for flow cytometry was typically day 8 and/or day 11. Typical endpoint for a tumour experiment was once the tumour reached $200 \mathrm{~mm}^{2}$.

\section{Euthanasia}

Mice were euthanized by $\mathrm{CO}_{2}$ asphyxiation or cervical dislocation.

Tumour and lymph node collection for histology

At experimental endpoint, tumour and lymph node was carefully removed with a forceps and scissors, for histological analysis, and placed in a 6 well plate. The sample was then transferred into moulds and covered in OCT and frozen using the GentleJane Freezing system.

\section{$\underline{\text { Tumour collection for ELISA }}$}

At experimental endpoint, the tumour was carefully removed with a forceps and scissors, for ELISA analysis. The tissue was then placed in ice cold PBS and homogenised as stated below. 


\subsubsection{Tissue preparation}

$\underline{\text { Blood }}$

Mice were restrained by hand, and a puncture into the cheek was made, blood was collected (4-6 drops) into $1.5 \mathrm{ml}$ Eppendorf tubes containing $200 \mu \mathrm{PBS}$ (+ $10 \mathrm{mM}$ EDTA). For larger blood volumes the mice were euthanized by asphyxiation with $\mathrm{CO}_{2}$ and a 27 gauge needle inserted into the left ventricle, the blood was then extracted into placed in Eppendorf tubes as above. One ml of red blood cell lysis solution (Qiagen, CA, USA) was added to each tube and incubated at $37^{\circ} \mathrm{C}$ for $30 \mathrm{~min}$. The white blood cells were pelleted by centrifugation at $706 \mathrm{xg}$ for $5 \mathrm{~min}$ in a Biofuge Fresco (Heraeus, ThermoFisher Scientific, New Zealand), the supernatant removed, and the cells resuspended in $200 \mu \mathrm{FACS}$ buffer for flow cytometric analysis.

\section{$\underline{\text { Serum }}$}

Mice were restrained by hand, and a puncture into the cheek was made, Blood was collected (810 drops) into $1.5 \mathrm{ml}$ Eppendorf tubes, stored at $4^{\circ} \mathrm{C}$ overnight, and centrifuged at 13,250 x $\mathrm{g}$ for $10 \mathrm{~min}$. The top serum layer within the tube after centrifugation was transferred into new tubes and stored at $-20^{\circ} \mathrm{C}$ until analysis.

\section{Spleen}

Mice were culled by asphyxiation, followed by cervical dislocation. Spleens were collected and placed into IMDM resting on ice. Spleens were injected with $0.5 \mathrm{ml}$ Liberase/DNase digestion buffer and incubated at $37^{\circ} \mathrm{C}$ for $30 \mathrm{~min}$. Spleens were then dissociated by mulching through 70 $\mu \mathrm{m}$ filters (Becton Dickinson), washed with IMDM, then the cells were pelleted by centrifugation at $645 \mathrm{x}$ g for $4 \mathrm{~min}$ in a Megafuge $4 \mathrm{KR}$ (Heraeus, ThermoFisher Scientific). The 
supernatant was discarded, and red blood cells were lysed by brief vortexing with $3 \mathrm{ml}$ red blood cell lysis solution. Cells were pelleted again by centrifugation, the supernatant was discarded, and cells were washed with DPBS. For extraction of T or B cells from spleens, the tissue was dissociated immediately after extraction, without incubating with Liberase/DNase digestion buffer.

\section{Lymph nodes}

Mice were culled by asphyxiation, followed by cervical dislocation. The outer skin layer was cut and peeled away for collection of the Inguinal, Auxiliary and Brachial lymph nodes with tweezers. The muscle wall was cut and spread for access to the mesenteric lymph node. All lymph nodes were collected in IMDM on ice, mulched through a $70 \mu \mathrm{m}$ filter and then washed with IMDM. Cells were pelleted by centrifugation at $645 \AA \sim \mathrm{g}$ for $4 \mathrm{~min}$, the supernatant discarded, and the cells were washed with DPBS.

\section{$\underline{\text { Viable cell counts }}$}

An aliquot of $10 \mu \mathrm{L}$ of cells was added to $190 \mu \mathrm{L}$ 0.4\% trypan blue (Gibco, Life Technologies) at a known dilution. A small volume of the mix was placed onto a haemocytometer. Under the microscope, the viable cells were identified as white cells, whereas the dead cells were blue. The cells were counted and the total cell count calculated using the following formula:

Total cell number $=($ Average cell count per ninth area $) \times($ dilution factor $) \times\left(10^{4}\right)=$ (total volume) 


\subsubsection{Flow cytometry staining}

\section{Cell surface staining}

Cells were resuspended, into $5 \mathrm{~mL}$ polystyrene round-bottom tubes, at a volume of $2 \mathrm{~mL}$ and filtered through a $70 \mu \mathrm{m}$ gauze. Thereafter, cells are pelleted at $419 \mathrm{X}$ g for 4 minutes. Following this, cells were resuspended in $50 \mu \mathrm{L}$ of mouse Fc Block TM (Clone 2.4G2) and incubated for 10 minutes at $4^{0} \mathrm{C}$. At this stage, $50 \mu \mathrm{L}$ of fluorophore bound antibody cocktail was added to the tubes and incubated for another 30 minutes at $4^{0} \mathrm{C}$. Staining was halted by the addition of $4 \mathrm{~mL}$ of flow cytometry buffer and spun at $419 \mathrm{X}$ g for 4 minutes. Finally, samples were resuspended in $125 \mu \mathrm{L}$ of flow cytometry buffer, and $125 \mu \mathrm{L}$ of DAPI solution was added. DAPI was added to the final cell suspension just prior to acquisition on the cytometer.

\section{$\underline{\text { Fluorescence-activated cell sorting }}$}

Cells were stained for cell surface markers, and single stain controls were prepared as described in 2.2.5.2. After staining the cells were resuspended in sterile flow buffer with viability dye (DAPI) at a volume of $4 \times 10^{6}$ cell $/ \mathrm{mL}$. The voltages of each channel were set up, compensation adjusted using unstained cells and single stains. 


\section{Acquisition of flow cytometry data}

All FACS data was acquired on LSRFortessa SORPTM or LSRII SORPTM flow cytometer with FACS DIVA software (Becton Dickinson, San Jose, CA, USA). The staff of the HGCC at MIMR performed daily cytometer setup and recorded the cytometer performance to ensure calibration of the cytometers was maintained. Photomultiplier tube (PMT) voltages and compensation was set for each experiment. To do this, both unstained cells and OneComp eBeads (eBioscience, CA, USA) were stained with each fluorophore. Compensation was verified manually using fluorescence minus one (FMO) controls prior to sample acquisition.

\section{Analysis of flow cytometry data}

FACS data was analysed using FlowJo software (version 9.9; Treestar, Inc, CA, USA). Doublets were excluded based on forward, and side scatter properties and with the use of viability dye; dead cells were excluded. Cells were gated based on fluorescence minus one (FMO) controls and or isotype controls where appropriate.

\section{$\underline{\text { FACS data visualization and display }}$}

FACS data were visualized by utilizing plots generated from FlowJo software (version 9.9; Treestar Inc, CA, USA). All graphical representation was done using Graph Pad Prism software (version 5 for Mac OS X; Graph Pad Software, CA, USA). 


\subsubsection{ELISA}

$\underline{\text { Serum alanine transferase }}$

Measurement of serum ALT levels was performed with a modular analyzer (Roche/Hitachi Modular P800, Roche Diagnostics, Indianapolis, IN) by Gribbles Veterinary Clinic (Hamilton, New Zealand) according to a standard operating procedure approved by International Accreditation New Zealand.

\section{Tumour Lysate for ELISA}

Homogenization buffer was made up of Halt ${ }^{\mathrm{TM}}$ protease inhibitor in 1x Cell Lysis Buffer and kept at on ice. Several $50 \mathrm{ml}$ Falcon tubes are filled with $20 \mathrm{ml}$ cold dPBS. The tubes of dPBS were used to wash the homogenizer between samples and groups. Tumour tissue is placed in $1.5 \mathrm{~mL}$ of homogenizing buffer and homogenized. Homogenate samples were transferred to 1.5mL Eppendorf tubes using a pipette. Samples are then centrifuged at $15000 \mathrm{~g}$ for $10 \mathrm{minutes}$ at $4^{0} \mathrm{C}$. The aqueous phase was pipetted into a new Eppendorf tube and spun as above. After the second spin, the aqueous phase was aliquoted into $0.6 \mathrm{ml}$ tubes and stored at $-70^{\circ} \mathrm{C}$ until ELISAs were performed.

\section{$\underline{\text { Total protein quantification }}$}

Total protein concentrations were quantified using Bicinchoninic acid $\mathrm{BCA}^{\mathrm{TM}}$ protein assay kits according to manufactures"' specifications.

\section{$\underline{\text { ELISA Kits }}$}

ELISAs were all performed to detect cytokine production in either serum samples and or in tissue lysate samples. Manufactures' protocols were followed for IFN- $\alpha$. Samples were either serially 
diluted to get optimal readings or $100 \mu \mathrm{L}$ of neat sample was loaded. The absorbance was read on a VersaMax $^{\mathrm{TM}}$ plate reader according to the protocol, and concentration was determined using the standard curve and dilution factors.

\subsubsection{Histopathology}

$\underline{\text { Histology processing }}$

Tissue was immersed in 4\% PFA for fixation, immediately after removal from the mouse. After 24 hours in PFA, it was then placed in histological cassettes in 70\% EtOH for a maximum of 14 days. Following this, samples were processed in a tissue processor overnight and then embedded in paraffin wax. The tissue processor involves 10 dehydration steps from $70 \%$ EtOH to Xylene, and the final two steps ensure the tissue is fixed and infiltrated with paraffin wax. Tissue sections were serially sectioned on a microtome at 3-4 $\mu \mathrm{m}$ onto adhesive microscope slides. Slides were dewaxed in xylene and then rehydrated using an ethanol concentration gradient from $100 \%$ to $70 \%$ prior to staining.

\subsubsection{Histology Staining, Analysis \& Microscopy}

\section{Confocal microscopy staining}

Whole-mount staining was utilized to stain ear tissue with fluorescent antibodies that were detected on the Olympus Laser Scanning Confocal microscope. Briefly, whole tumour tissue was excised from the mouse and snap frozen using the GentleJane freezing system in OCT. The tissue was then sectioned and placed on slides. Tumour tissue was then blocked with $10 \%$ of the appropriate serum (i.e. rat, goat and or rabbit) in dPBS for one hour at room temperature. Block was flicked off the tissue and tissue stained with $100 \mu \mathrm{L}$ of antibody, overnight at $4^{0} \mathrm{C}$. The following day ear tissue was rinsed in $\mathrm{dPBS}$ three times and mounted onto a slide using Faramount aqueous mounting media. 
Table 2.3 Antibodies for confocal microscopy

\begin{tabular}{|l|l|l|l|}
\hline Specificity & Flurophore & Clone & Company \\
\hline CD4 & BV421 & GK1.5 & BioLegend \\
\hline CD8 & BB515 & $53-6.7$ & BD Biosciences \\
\hline CD64 & AF647 & X54-5/7.1 & BioLegend \\
\hline
\end{tabular}

$\underline{\text { Imaging and analysis }}$

All data collected from confocal microscopy were analysed using Image $\mathrm{J}$ software. 3D reconstructions were recorded of the co-stained whole ear tissue, along the Z-axis between 17$50 \mu \mathrm{m}$. Z- stacks were recorded from bottom-top and assessed. Images in the results section (chapter 6 ) were a depiction of both the first $4 \mu \mathrm{m}$ and the last $4 \mu \mathrm{m}$ that was recorded. 6 slides of tissue were imaged per group in each experiment.

\section{$\underline{\text { Data visualization and statistical analysis }}$}

All graphical representation of data was done using Graph Pad Prism software (version 5 for Mac OS X; Graph Pad Software, CA, USA). Statistical analyses used were stated in each figure legend, and $\mathrm{p}<0.05$ was considered significant. For comparison between two similarly-sized groups, Student's t-test was used. A log-rank Mantel-Cox test was used to compare survival distributions of two samples. Analysis examining a single parameter of three or more groups were compared with one-way ANOVA with Tukey's post-tests. For experiments examining two or more groups, Two-way ANOVA with Holm-Bonferroni's post-test was used. Repeated measures tests were used in the experiments that compared the same biological sample. Unless specifically stated, symbols represent individual samples, and errors bars represent mean \pm the standard error of the mean (S.E.M.). 


\section{Chapter 3}

\section{Antitumour effect of combining iNKT cell activation with intratumoural CpG administration in murine tumour models}

\subsection{Introduction}

The tumour microenvironment can limit the impact of immunotherapy through multiple mechanisms, generally driven by hypoxia, that exclude or suppress the activity of immune effector cells. The majority of immune suppression is due to the presence of normal immune regulatory cells and molecules within the tumour that inhibit $\mathrm{T}$ cell priming or suppress cytotoxic T cell function. One way to overcome the immunosuppressive tumour microenvironment is to deliver immunostimulatory compounds intratumourally, thereby stimulating cells of the infiltrate and encouraging appropriate cross-talk with draining lymphoid tissues. Studies have shown that intratumoural targeting of pattern recognition receptors, most notably TLRs, not only enhance endogenous adaptive anti-tumour responses that have been constrained locally but can unleash responses that act on tumours at distant locations in the so-called "abscopal effect". Ideally, for intratumoural delivery, the treatment needs to be conveniently administered into a single tumour to treat existing cancer and eradicate cancer cells at both the treatment site, as well as distal tumours. As already discussed in the Introduction chapter, the most clinically advanced intratumoural treatment involving TLR ligation is stimulation via TLR9 with synthetic oligodeoxynucleotides containing unmethylated cytosine-guanine motifs (CpG). Multiple studies have looked into the intratumoural therapeutic potential of $\mathrm{CpG}$ in murine models often in combination with other agents such as an anti-4-1BB (CD137) agonistic antibody, OX40L, and checkpoint blockade (anti-PD1) ${ }^{189,202,203}$. The results from some of these murine models are 
currently being validated in patients in the clinic ${ }^{204-206}$. However, there is a dearth of published data regarding the combination of iNKT cell agonism and TLR9 mediated immune stimulation. One study showed that the combined iNKT cell agonism and CpG-based immunisation, given systemically, led to the expansion of NK and antigen-specific CTLs, likely resulting from the maturation of highly pro-inflammatory DCs as seen by a cooperative increase in serum IL-12 ${ }^{173}$. The enhanced innate and adaptive immune responses resulted in higher tumour inhibition that correlated with increased numbers of antigen-specific CTLs at the tumour site. Antibodymediated depletion experiments further demonstrated that CTLs rather than NK cells were essential for the enhanced tumour inhibition ${ }^{173}$. While the cooperative action between TLR stimulation and iNKT cell activation on DC function is exhibited by different TLR ligands, in both mouse and human systems ${ }^{162}, \mathrm{CpG}$ was shown to be more suitable in inducing specific $\mathrm{CD}^{+} \mathrm{T}$ cell proliferation and pro-inflammatory cytokines when the antigen was targeted to $\mathrm{CD} 8 \alpha^{+}$DCs (cDC1s). TLR9 signalling was essential for this response, whereas TLR5 signalling, for example, did not seem to play a significant role ${ }^{207}$.

In other published work, the combination of $\alpha$-GalCer with $\mathrm{CpG}$ gave the most robust $\mathrm{CD}^{+} \mathrm{T}$ cell proliferation over combinations with other TLRs such as flagellin (TLR5) or poly I:C (TLR3) ${ }^{162}$. Human DCs exposed to TLR ligands and activated iNKT cells in vitro had enhanced expression of maturation markers, suggesting that a cooperative action of TLR ligands and iNKT cells on DC function is a generalisable phenomenon across species. Interestingly, depletion of pDCs or myeloid DCs revealed that both DC subsets were necessary for the activation of iNKT cells by $\alpha$-GalCer and CpG. While pDCs were responsible for the stimulation of iNKT cells with CpG, myeloid DCs presented $\alpha$-GalCer via CD1d ${ }^{208}$. Overall, these studies 
highlight the potential for manipulating the interactions between TLR ligands and iNKT cell activation in the design of effective vaccine adjuvants ${ }^{162}$.

It is known that when TLR agonists and an iNKT cell agonist are administered intravenously into mice, they can cooperate to increase the number of antigen-specific $\mathrm{CD} 8^{+} \mathrm{T}$ cells and the release of cytokines such as IFN- $\gamma$ and TNF- $\alpha$ that may help in driving anti-tumour responses ${ }^{209}$. The activated iNKT cells appeared to provide a potent immune adjuvant effect. Furthermore, it is known that this cooperative activity can be increased by delaying the administration of the TLR agonist after activating the iNKT cells with $\alpha$-GalCer, however, this could be counterproductive in that it produces a significant cytokine release syndrome (CRS, "cytokine storm") which can result in the death of the mice ${ }^{11}$. In order to circumvent this issue, in this thesis, intratumoural delivery was therefore explored as a potentially safer route of administration. It was hypothesised that the localised nature of the injection would sequester the initial innate immune response primarily to the injected tumour, thereby limiting the toxicity of the compounds, while still maintaining high drug concentrations in the tumour microenvironment which in principle should manifest in superior efficacy as compared to systemic i.v. administration due to the higher therapeutic index. This, of course, is predicated on the hypothesis that the reagents have minimal leakage as free agents into the systemic circulation and that their drainage to the lymphoid tissues, perhaps in the context of cellular debris, reduces the risk of toxicity. Importantly, leakage into the systemic circulation could be monitored by measuring the production of cytokines and other biomarkers such as liver enzymes in the blood, which was conducted as part of the overall exercise. 


\subsection{Aims}

The hypothesis addressed in this chapter is that "iNKT cell activation via intratumoural administration of the iNKT cell agonist $\alpha$-GalCer together with intratumoural administration of the TLR9 agonist CpG will have a cooperative effect, eliciting stronger anti-tumour responses than either agent on it own". If applicable across multiple tumour types, this could form the foundation for a general strategy of in situ vaccination.

To address this hypothesis, the following Aims were pursued:

1. To identify an intratumoural dosing regimen of $\alpha$-GalCer to facilitate iNKT cellmediated improvement to an established $\mathrm{CpG}$ treatment schedule

2. To determine if intratumoural route of administration of the two agents is superior to systemic delivery and displays a larger therapeutic index

3. To determine if the combined treatment can improve the abscopal effect of intratumoural $\mathrm{CpG}$

4. To determine whether the induced response is associated with immunological memory

5. To establish whether the combined treatment is effective in multiple tumour types 


\subsection{Results}

\subsubsection{Effect of combining iNKT cell activation with intratumoural $\mathrm{CpG}$ administration in a murine lymphoma model}

To evaluate whether the anti-tumour activity of TLR agonism with intratumoural CpG combined effectively with contemporaneous iNKT cell activation with $\alpha$-GalCer, a tumour experiment was designed whereby $\alpha-G a l C e r$ and $\mathrm{CpG}$ as single agents were compared to an admix combination of $\alpha-\mathrm{GalCer}$ and $\mathrm{CpG}$. To this end, C57BL/6J mice were inoculated with $1 \times 10^{6}$ EG7.OVA lymphoma cells into the flank and treatment started when the tumours reached approximately $36 \mathrm{~mm}^{2}$ (day 6). The EG7.OVA cell line was used because it is transfected with ovalbumin protein (OVA) as a model of a tumour-associated neoantigen, and tools are available for evaluating OVA-specific T cell responses via flow cytometry. Anti-tumour effects of intratumoural $\mathrm{CpG}$ have been reported in mice using a dosing regimen consisting of three treatments four days apart, where each treatment consisted of $50 \mu \mathrm{g}$ was injected directly into established tumours on two consecutive days (S.R. Mattarollo and S. Chisholm, personal communication, 2016). Others have used $30 \mu \mathrm{g}$ of $\mathrm{CpG}$ per tumour and demonstrated a tumour growth delay suggesting a lowered amount is adequate ${ }^{210}$. A lower dose of $25 \mu \mathrm{g}$ was ultimately chosen. This was still assumed to be well in excess of the dose required for agonism, based on the initial analysis of $\mathrm{CpG}$ activity in vitro using a HEK cell-line transfected with a TLR9 reporter. In this assay, the concentration of $\mathrm{CpG}$ that gave half-maximal response $\left(\mathrm{EC}_{50}\right)$ was $0.4 \mu \mathrm{M}$, (the response plateaued at $\sim 2 \mu \mathrm{M}$ ) (Supplementary fig 1 ). With a starting tumour size of $\sim 100 \mathrm{~mm}^{3}$ (bisecting diameters of $\sim 6 \mathrm{~mm}$ each), $25 \mu \mathrm{g}$ of $\mathrm{CpG}$ could reach $\sim 40 \mu \mathrm{M}$, which is $\sim 100$ times greater than the $\mathrm{EC}_{50}$ and $\sim 20$ times greater than maximal activity in the in vitro 
assay. Using this modified regimen, the impact of including an additional single intratumoural injection of $\alpha$-GalCer in the repeated CpG dosing schedule was examined. A dose of $2 \mu \mathrm{g}$ of $\alpha$ GalCer was used, which was known to induce activation of DCs when injected intravenously ${ }^{11}$. Previous experiments from our group had shown that the order in which TLR ligation and iNKT cell activation take place significantly increased the production of Th1 cytokines; however, this had not been specifically investigated in combination with $\mathrm{CpG}$, and nor had it been investigated in the context of potentially enhancing an anti-tumour response ${ }^{11}$. The possibility that the combination of $\mathrm{CpG}$ and $\alpha$-GalCer would display a similar temporal effect that could affect the anti-tumour response against EG7.OVA lymphoma cells was therefore assessed by having an additional treatment group where the first $\mathrm{CpG}$ treatment was delayed until after $\alpha$-GalCer. Tumours were allowed to reach an average of $\sim 6 \mathrm{~mm} \times 6 \mathrm{~mm}$ in size (hereafter referred to as 36 $\mathrm{mm}^{2}$ as a product of bisecting diameters) before intratumoural treatment was initiated (day 6). The different treatment groups were: $\mathrm{CpG}$ alone, where three treatments $(25 \mu \mathrm{g} \mathrm{CpG}$ on two consecutive days) were given four days apart; $\alpha$-GalCer alone, where a single $2 \mu \mathrm{g}$ dose was followed by vehicle (PBS) to the same schedule as $\mathrm{CpG}$; an admix of $\alpha$-GalCer and $\mathrm{CpG}$ where the $\alpha$-GalCer and first $\mathrm{CpG}$ dose were delivered together and then subsequent $\mathrm{CpG}$ doses delivered alone; a similar admix where the first $\mathrm{CpG}$ treatment was delayed until 6 hours after $\alpha$ GalCer; and a control group injected with PBS according to the same regimen as CpG to control for injection effects (Fig 8a).

Intratumourally administered $\mathrm{CpG}$ alone trended towards a modest anti-tumour response in terms of reduced tumour growth (Fig 8b) and increased survival (Fig 8c) which was improved when combined with $\alpha$-GalCer, and there was a slight further improvement when $\mathrm{CpG}$ administration was delayed by 6 hours after $\alpha$-GalCer administration (Fig 8b). While the 
combination therapies were significantly improved over single treatments (which was seen in repeated experiments), the impact of delaying $\mathrm{CpG}$ was small but consistent. In aggregating data from four separate experiments together and comparing survival, delaying CpG by 6 hours led to significantly improved survival over simultaneously delivered admix (Fig 8d, p =0.0258, logrank Mantel-Cox test). Treated mice appeared normal in terms of weight gain as well as their behaviour, unlike previous experiences in our laboratory of using TLR agonists after iNKT cell activation via intravenous administration ${ }^{11,209}$.

Following up on this experiment, there were two important questions to answer: 1) is the anti-tumour effect observed durable and 2) if it is durable, whether the anti-tumour memory is OVA-dependent, and hence the antitumour response could be evaluated by focusing on the OVA-specific response. To this end, mice whose tumours had been eradicated by the combined treatment were re-challenged in the opposite flank with $1 \times 10^{6}$ lab-adapted EL4 lymphoma cells (EL4.LA). This cell line is derived from the parental EL4 cell line used to generate EG7.OVA, but without the OVA model antigen. Genetic analysis with microsatellite markers (IDEXX BioResearch, Columbia MO, USA) had shown that this version had undergone some genetic drift from the original EL4 cell line, and our laboratory had shown it also has increased immunogenicity. However, the cell line otherwise maintains many of the same features as classical EL4 cells, including morphology, growth rate and phenotype by flow cytometry (e.g. CD45.2 expression). The EL4.LA tumours initially grafted to the mice and began to grow, but in the end, tumours in the vast majority of the re-challenged mice ended up regressing and were cleared without the need for intervention (Fig 9). This result showed that the mice developed immunity to antigens other than OVA (which we had assumed may be a dominant antigen), presumably targeted at shared epitopes between EL4.LA and EG7.OVA derived from TAAs 
expressed in common. It remained to be established whether OVA was a rejection antigen at all in EG7.OVA; this work is described in a later chapter. 


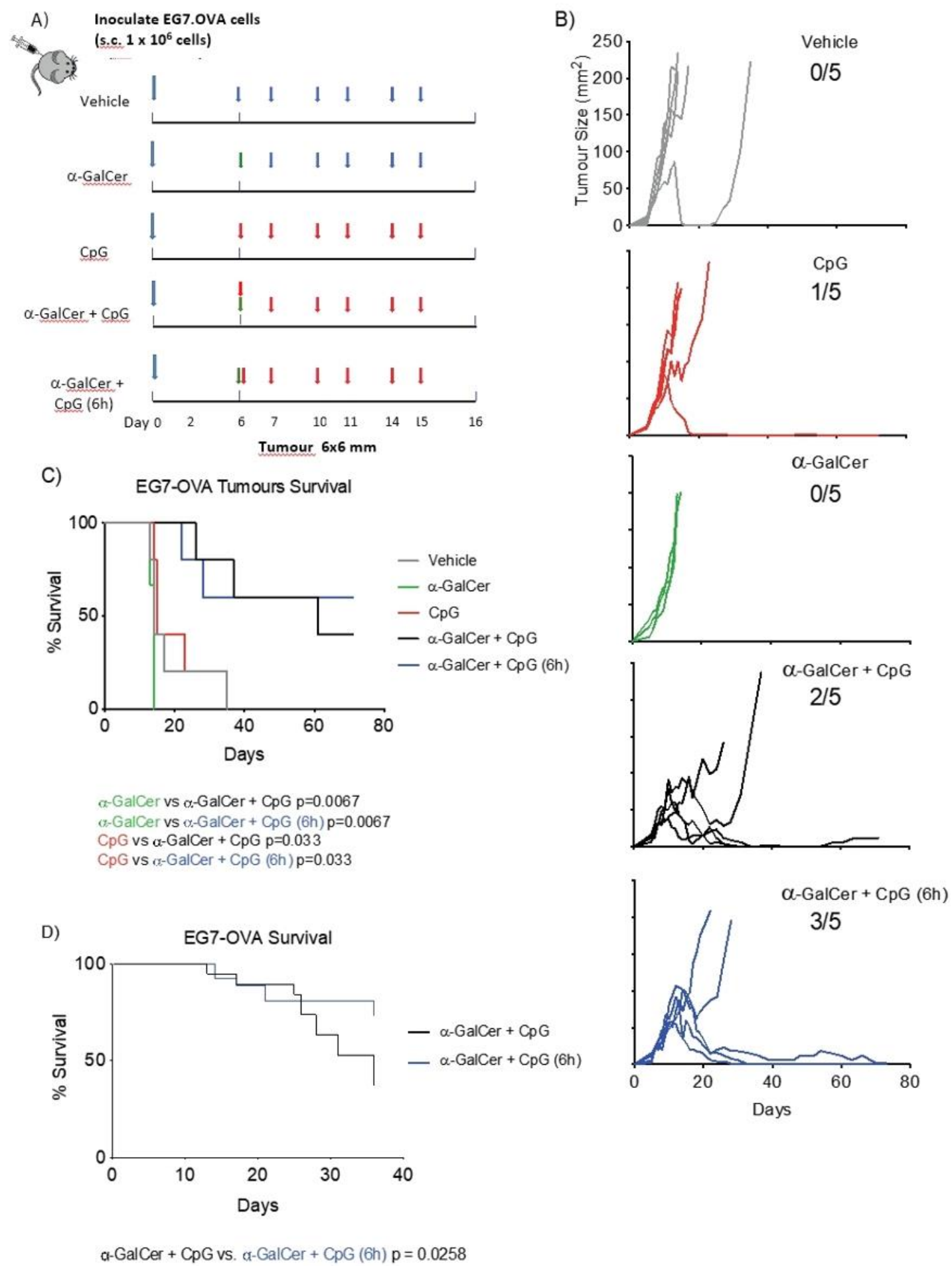

Figure 8 Intratumoural treatment of EG7.OVA tumours with $\alpha$-GalCer and CpG lead to tumour regression. A) Treatment protocol. Treatment was with either with a single dose of $2 \mu \mathrm{g} \alpha$-GalCer (shown as arrows in green), repeated doses of $25 \mu \mathrm{g}$ $C p G$ (red arrows), or a combination of both where the single dose of $\alpha$-Galcer was either given at the time of the first dose of $C p G$, or 6 h prior to the first dose of CpG. PBS was injected as a vehicle control for all injections (blue arrows). B) Tumour growth curves plotted for each mouse. Numbers of animals that rejected tumour per group are indicated. The experiment is representative of 5 similar experiments $C$ ) Survival curves for the different treatment groups (scored as tumour free), with statistical differences indicated (log-rank Mantel-Cox test). D) Survival of aggregated EG7.OVA experiments comparing admix to delayed CpG administration with statistics indicated (log-rank Mantel-Cox test). 
A)

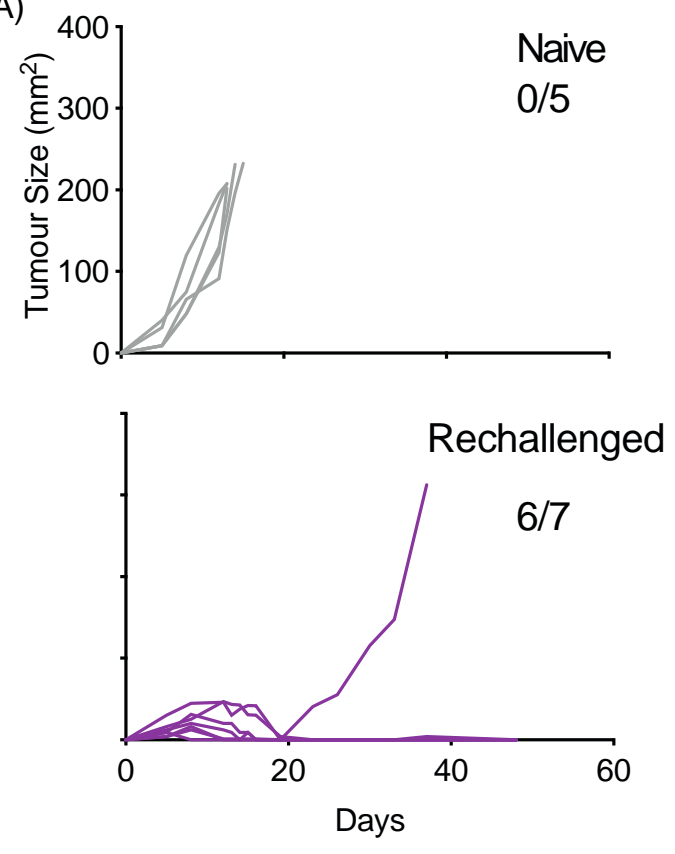

B)

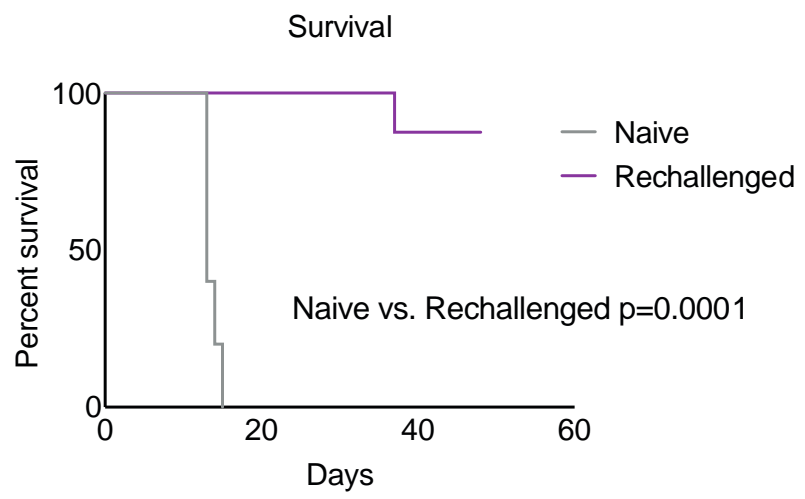

Figure 9 EG7.OVA cured mice can successfully reject rechallenge with EL4.LA tumour cells. Treatment was as in Fig 8. All mice that rejected EG7.OVA were rechallenged with 1x106 ELA.LA cells in the opposite flank A) Tumour growth curves for EL4.LA plotted for each mouse. Numbers of animals that rejected tumour per group are indicted B) Survival curve with statistics indicated (log-rank Mantel-Cox test). 


\subsubsection{In situ vaccination with a CpG and $\alpha$-GalCer administration in established EL4}

\section{Tumours}

Next, it was investigated whether the intratumoural combination of $\alpha-$ GalCer and $\mathrm{CpG}$ could induce regression in mice bearing a tumour not expressing an immunodominant epitope such as potentially provided by OVA. To this end, we utilised a similar experimental design as above and treated EL4.LA tumours.

Treatment with repeated $\mathrm{CpG}$, or a single dose of $\alpha-\mathrm{GalCer}$, as single agents did not cause tumours to regress completely. Intratumoural $\mathrm{CpG}$ as a single agent has been shown by others to, at best, delay tumour growth with the occasional, rare tumour regression ${ }^{211}$. However, the combination treatment with admix $\alpha-G a l C e r$ and $\mathrm{CpG}$ resulted in the majority of mice showing complete regression, while all mice ultimately rejected tumours when $\mathrm{CpG}$ administration was delayed by 6 hours (Fig 10). Overall these data suggest that combining TLR ligation and iNKT cell activation through the intratumoural combination of $\mathrm{CpG}$ and $\alpha$-GalCer is an effective therapy that involves targeting of antigens shared by the two $\mathrm{T}$ cell lymphoma lines, and that while it remains possible that OVA may be targeted (to be explored later), expression of this antigen is not necessary for rejection to occur. In general, this combination therapy is further improved by delaying the first administration of CpG by 6 hours after $\alpha$-GalCer. 
A)

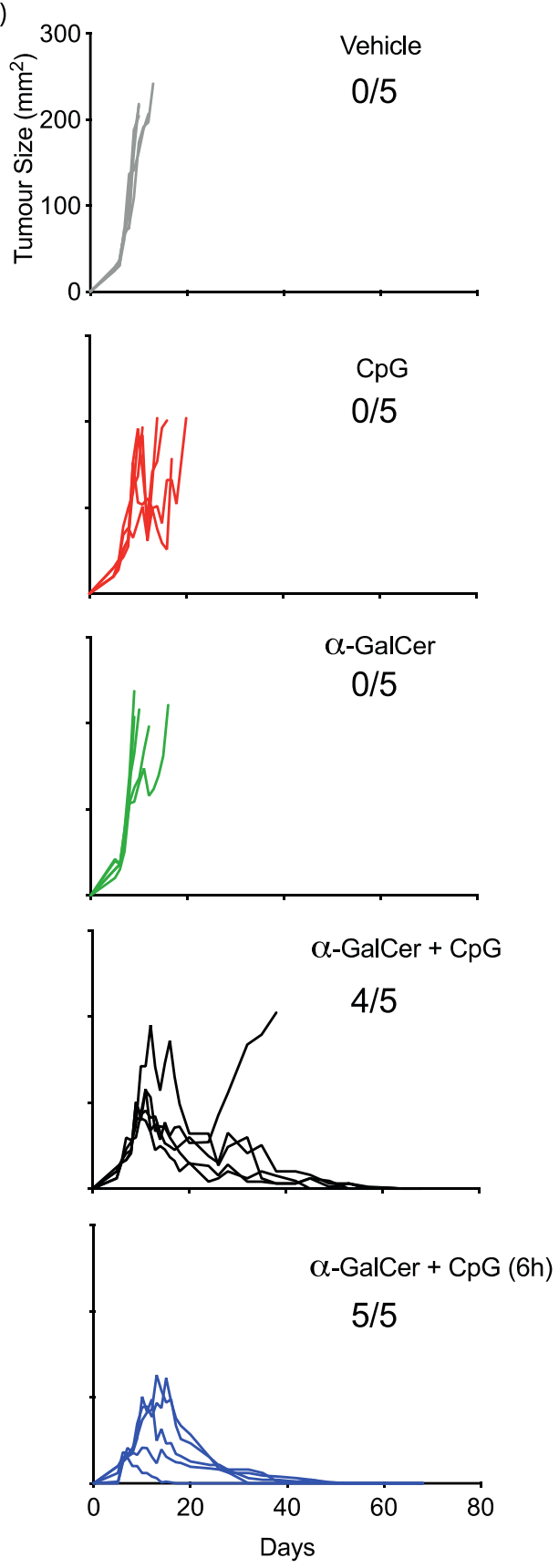

B)

Survival

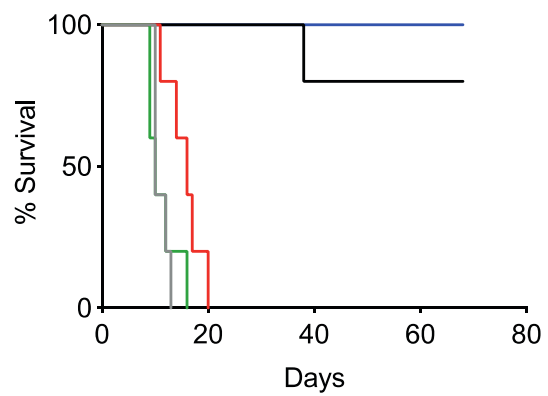

$\alpha-$ GalCer vs $\alpha$-GalCer + CpG $p=0.0017$

$\alpha$-GalCer vs $\alpha$-GalCer + CpG (6h) $p=0.0017$

CpG vs $\alpha-\mathrm{GalCer}+\mathrm{CpG} p=0.0018$

CpG vs $\alpha-$ GalCer + CpG (6h) p $=0.0018$

$$
\begin{aligned}
& - \text { Vehicle } \\
& -\quad \alpha \text {-GalCer } \\
& -\quad \text { CpG } \\
& -\quad \alpha \text {-GalCer + CpG } \\
& -\quad \alpha-\text {-GalCer + CpG (6h) }
\end{aligned}
$$




\subsubsection{Inducing abscopal effect in EL4 tumours}

Next, it was investigated whether the treatment of a single tumour could result in the regression of a distal untreated tumour. This is an essential question since intratumoural delivery for cancer will not be a viable approach if every single tumour has to be injected as this is not practical. To ensure that any weak abscopal effect was not missed, in initial experiments, the distal tumour was engrafted later so that these tumours were smaller at the time of treatment. In subsequent experiments, tumours were generated at the same time, and the same conclusions were drawn. Thus, in the initial experiments, C57BL/6J mice were injected with $1 \times 10^{6}$ EL4 tumour cells into the right flank on day 0 , and then two days later, $1 \times 10^{6}$ EL4 cells were injected into the opposite flank. Treatment was initiated when the "treated" tumour reached approximately $36 \mathrm{~mm}^{2}$ (day 5), and the distal tumour reached approximately $12 \mathrm{~mm}^{2}$. Mice were injected intratumourally with the same dosing schedules used in earlier experiments.

Single-agent treatment was mostly ineffective; however, $\mathrm{CpG}$ alone treatment resulted in regression in both tumours in one mouse. Throughout this thesis, on occasions, $\mathrm{CpG}$ as a single agent occasionally eradicated tumours in a low percentage of mice. On the other hand, both the admix of $\alpha-\mathrm{GalCer}$ and $\mathrm{CpG}$ and $\alpha$-GalCer with 6 hours delayed $\mathrm{CpG}$ resulted in a significantly superior response, with the complete rejection of both treated and distal tumours, highlighting an effective abscopal effect (Fig 11). 
A

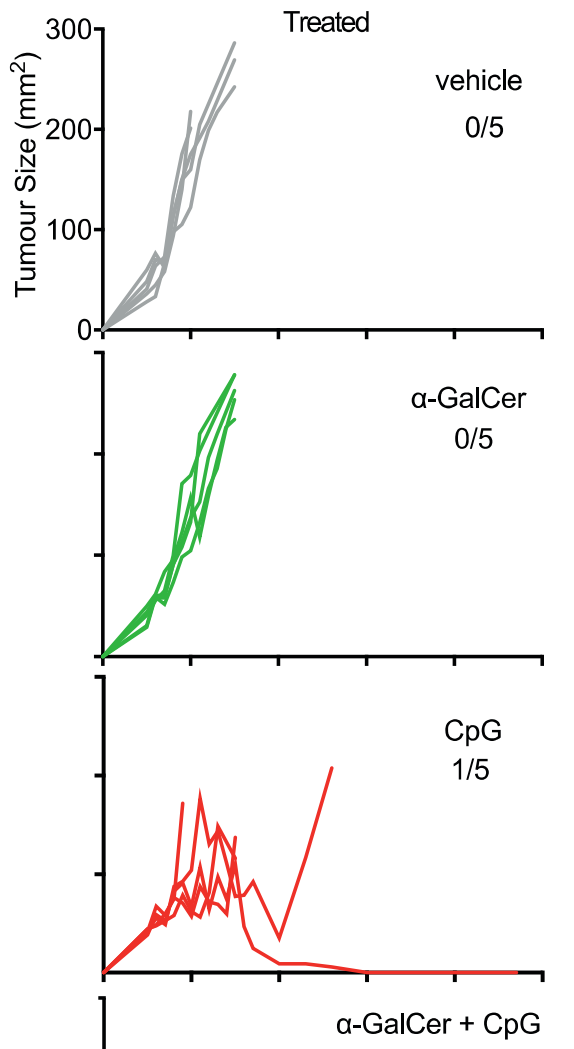

$8 / 8$
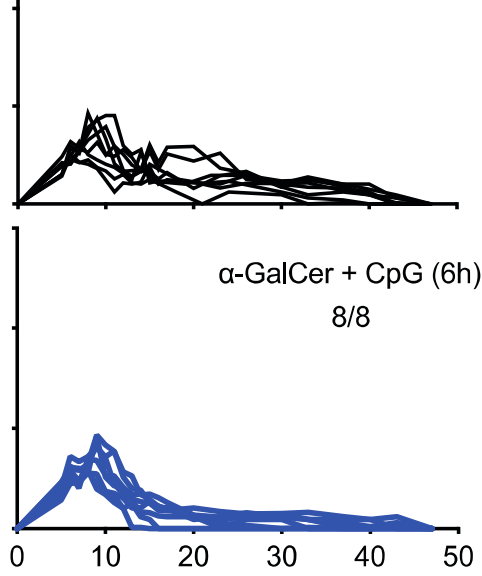

B
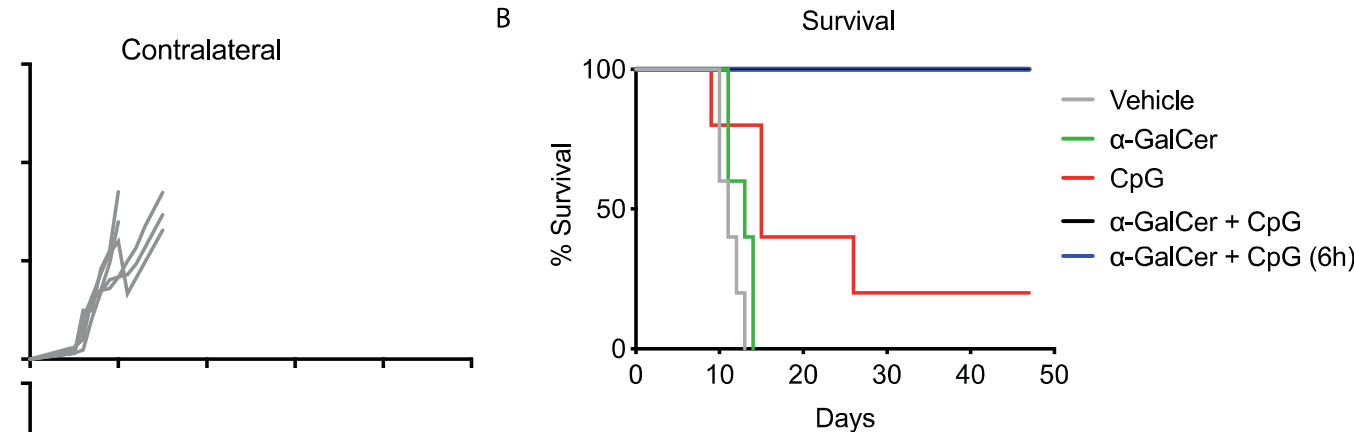

$\alpha$-GalCer vs $\alpha$-GalCer + CpG $p=0.0002$

$\alpha$-GalCer vs $\alpha$-GalCer + CpG (6h) $p=0.0002$

$\mathrm{CpG}$ vs $\alpha$-GalCer $+\mathrm{CpG} p=0.0021$

CpG vs $\alpha$-GalCer + CpG (6h) p=0.0021

Figure 11 Intratumoural treatment of EL4.LA tumours with a-GalCer and CpG leads to tumour regression and abscopal effect A) Tumour growth curves plotted for each mouse, with graphs for injected tumours on the left, and untreated contralateral

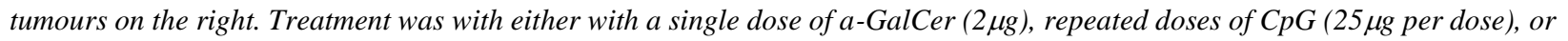
a combination of both where a single dose of $\alpha$-GalCer $(2 \mu \mathrm{g})$ was either given at the time of the first dose of CpG (25 $\mu \mathrm{g})$, or $6 \mathrm{~h}$ prior to the first dose of CpG. Scheduling was as shown in the schema in Figure 8A. B) Survival curves for the different treatment groups, with statistical differences indicated (log-rank Mantel-Cox test). The experiment shown is representative of 2 repeated experiments 


\subsubsection{Inducing abscopal effect in EG7.OVA tumours}

Following from the previous experiment, it was investigated whether regression could be induced in bilaterally implanted EG7.OVA tumours. To do this, C57BL/6J mice were inoculated with $1 \times 10^{6}$ EG7.OVA subcutaneously into both flanks at the same time on day 0 . Unlike the previous experiment, both tumours were roughly the same size. When tumours reached approximately $36 \mathrm{~mm}^{2}$, treatment of the tumour on the right flank was initiated as described previously in Fig 8a.

Again, single agents offered little protection against tumour challenge, while admixes of $\alpha$-GalCer and CpG induced complete regression in some of the mice. Consistent with previous observations, $\alpha$-GalCer with 6 hours delayed $\mathrm{CpG}$ induced complete regression in the majority of mice of both their treated and untreated tumours (Fig 12). The regression rate in EG7.OVA tumours was slightly lower than EL4.LA tumours, suggesting that the latter cell line may be more immunogenic than the former (in line with earlier observations in the laboratory). 
A

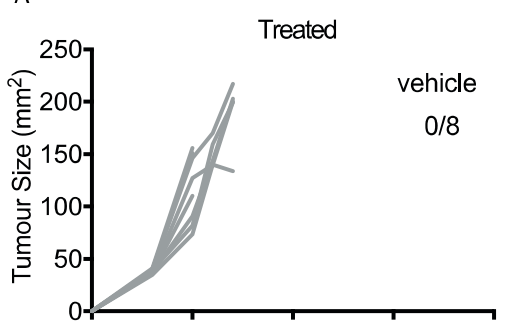

a-GalCer

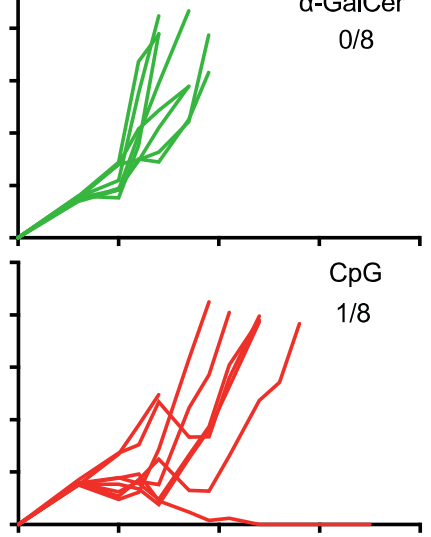

$\alpha-G a l C e r+C p G$
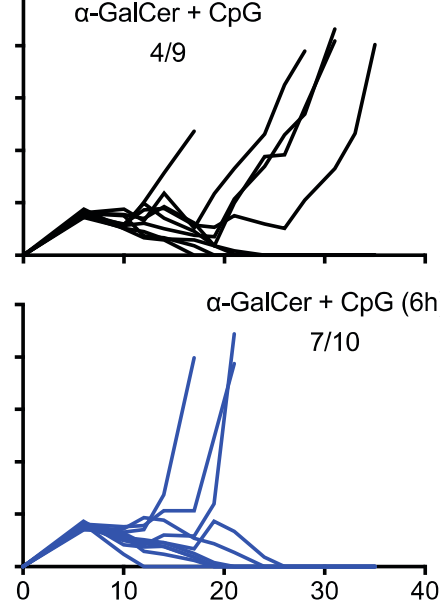

Contralateral
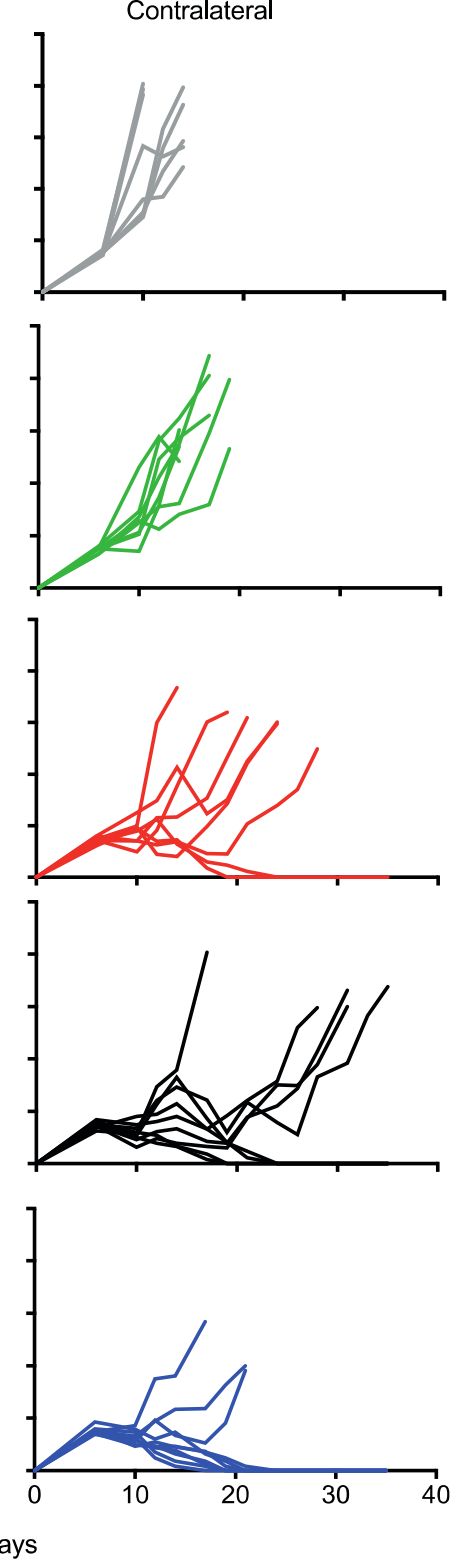

B

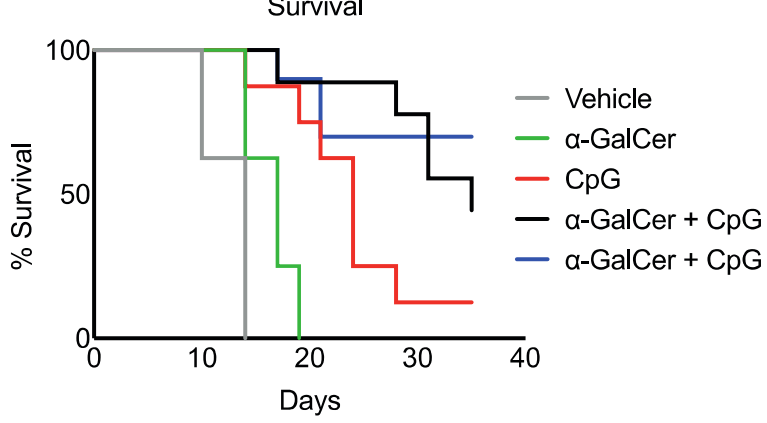

$\alpha$-GalCer vs $\alpha$-GalCer + CpG $p=0.0002$ $\alpha$-GalCer vs $\alpha$-GalCer + CpG (6h) $p<0.0001$ CpG vs $\alpha$-GalCer + CpG p $=0.0293$ CpG vs $\alpha$-GalCer + CpG (6h) p=0.037

Figure 12 Intratumoural treatment of EG7.OVA tumours with $\alpha$-GalCer and CpG leads to tumour regression and abscopal effect. A) Tumour growth curves plotted for each mouse, with graphs for injected tumours on the left, and for untreated

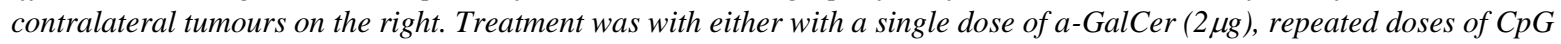

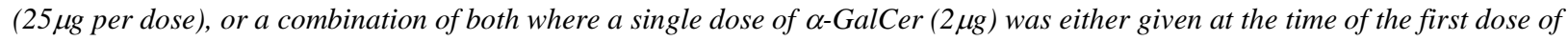
$C p G(25 \mu \mathrm{g})$, or $6 \mathrm{~h}$ prior to the first dose of CpG. Scheduling was as shown in the schema in Figure 8A. B) Survival curves for the different treatment groups, with statistical differences indicated (log-rank Mantel-Cox test). The experiment shown is representative of 2 repeated experiments 


\subsubsection{Comparing the efficacy of different routes of administration}

A pertinent question was whether the combination of $\alpha-\mathrm{GalCer}$ and $\mathrm{CpG}$ had to be administered intratumourally to achieve a high cure rate. Would other routes of administration, such as subcutaneous or peritumoural, be similarly effective at the same dose? To assess subcutaneous treatment, therapy was injected into the forelimb of the mouse that was most distal from the tumour (i.e. the left forelimb). The aim was to determine whether having the compounds drain to a non-tumour draining lymph node (and potentially some systemic circulation) would affect how the treatment performs. Peritumoural, on the other hand, involved injecting the compounds around the tumour but not puncturing the tumour itself; this was to address whether administering therapy near the tumour draining lymph node is sufficient, or was the therapy required to be injected directly, potentially bypassing the fibrotic tissues surrounding the tumours, to alter the tumour microenvironment.

For this experiment, C57BL/6J mice were inoculated with $1 \times 10^{6}$ EG7.OVA tumour cells and treatment started when the tumours reached approximately $6 \times 6 \mathrm{~mm}$. The groups were as follows: intratumourally administered PBS, intratumourally administered $\alpha$-GalCer with 6 hours delayed $\mathrm{CpG}$, subcutaneous $\alpha$-GalCer with 6 hours delayed $\mathrm{CpG}$, and peritumoural $\alpha$-GalCer with $6 \mathrm{~h}$ delayed $\mathrm{CpG}$. The number of administrations was the same for all treatments and followed the regimen as described in Fig 8a.

Of the three routes of administration, intratumourally administering $\alpha$-GalCer with 6 hours delayed $\mathrm{CpG}$ was the most effective at inducing tumour regression, with the majority of the mice completely rejecting the tumours. On the other hand, peritumoural was dramatically less effective with significantly reduced survival, and most mice just experiencing tumour growth delay, while subcutaneous administration into the distal forelimb only induced tumour 
growth delay, and all animals ultimately succumbed to unchecked tumour growth (Fig 13). This suggests that in order for the therapy to be maximally effective, the combination has to be administered intratumourally. This result also lends credence to the fact that the systemic circulation of the reagents via leakage from the injection site is not the primary cause of the antitumour effect. If this were true, the subcutaneous administration would have been efficacious. The reagents have to be in the tumour microenvironment to affect the immune changes associated with the improved activity as evidenced by the low regression rate with the peritumoural route. 

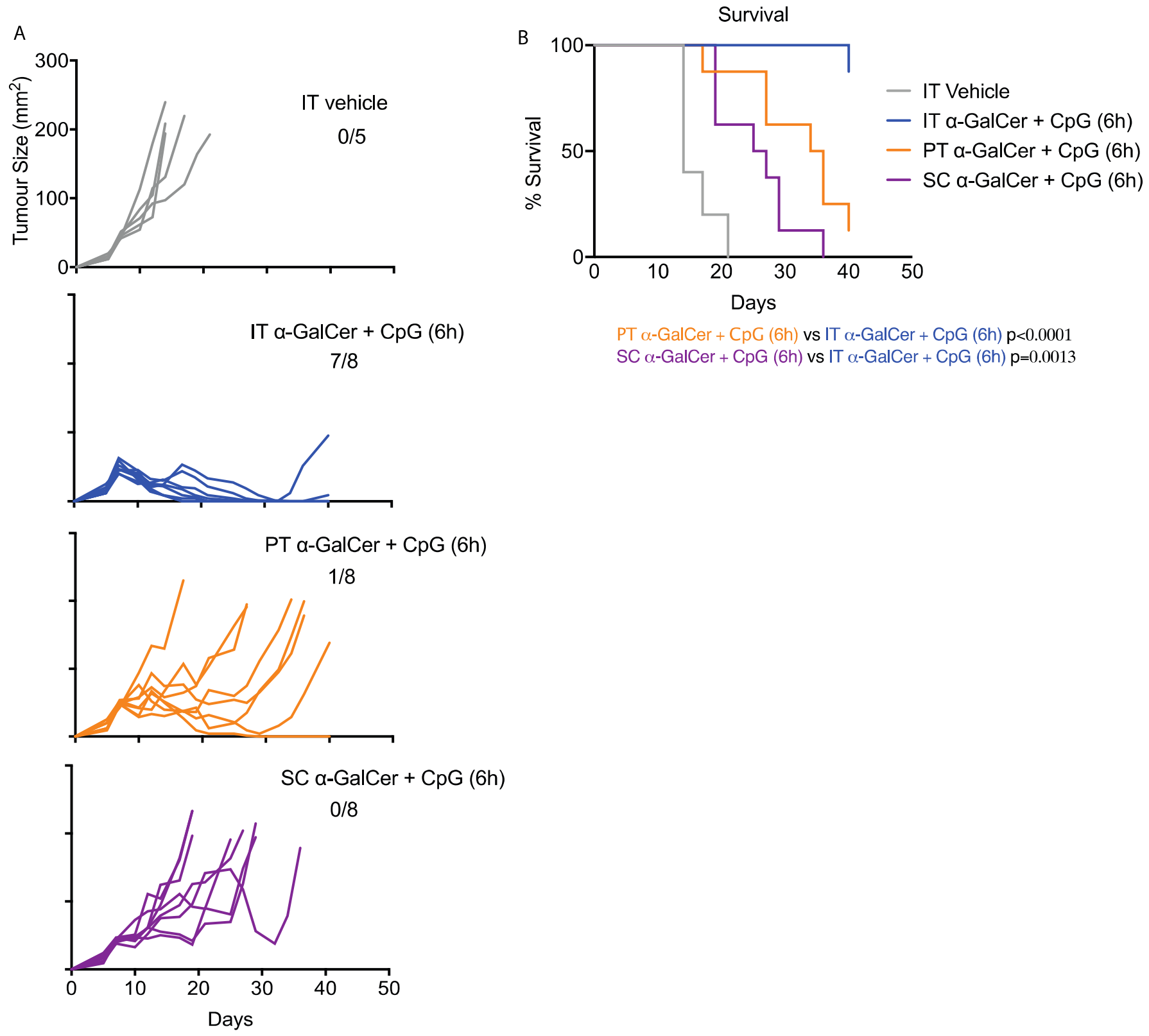

PT $\alpha$-GalCer + CpG (6h) vs IT $\alpha$-GalCer + CpG (6h) p $<0.0001$ $\mathrm{SC} \alpha$-GalCer + CpG (6h) vs IT $\alpha-$ GalCer + CpG (6h) p=0.0013

Figure 13 Intratumoural therapy is the most effective route of administration for $\alpha$-GalCer and CpG. Dosing schedule for the combined was as in earlier experiments, with the first $C p G$ delayed by $6 \mathrm{~h}$, except that reagents were either administered intratumourally (IT), subcutaneously (SC) or peritumorally (PT). A) Tumour growth curves plotted for each mouse (Scored as tumour free). B) Survival curves for the different treatment groups, with statistical differences indicated (log-rank Mantel-Cox test). 


\subsubsection{In situ vaccination with a CpG and $\alpha$-GalCer administration in established B16-F1 tumours}

Next, it was determined whether the therapeutic efficacy observed in the lymphoma models translated over to different tumour types, particularly in less immunogenic solid tumours. Mice engrafted with the murine melanoma cell line B16-F1 do not respond to immunotherapy with immune checkpoint blockade (anti-PD-1 or anti-CTLA-4) in our hands (C.W. Tang, personal communication, 2017), so this was considered to be a tumour of limited immunogenicity. Others have also reported that the B16 tumour lines (B16-F1 and the more metastatic version B16-F10) are poorly immunogenic ${ }^{212,213}$. However, the antigenicity is not totally compromised, as the tumours could respond to strategies that enhance immunogenicity ${ }^{214}$. To assess intratumoural $\mathrm{CpG}$ and $\alpha$-GalCer administration in B16-F1, C57BL/6J mice were inoculated with $3.5 \times 10^{5}$ cells into the flank on day 0 , and then once the tumours reached approximately $36 \mathrm{~mm}^{2}$ (day 9), mice were treated with the single agents or combined therapy in groups as described in Fig 8A. Intratumourally administered $\alpha$-GalCer with 6 hours delayed $\mathrm{CpG}$ led to complete regression in some mice while admixing $\alpha-\mathrm{GalCer}$ and $\mathrm{CpG}$, as well as single agents, only led to a growth delay relative to PBS (Fig 14). The relatively low level of tumour eradication with B16-F1 tumour is consistent with it being less immunogenic than the lymphoma cell lines. 

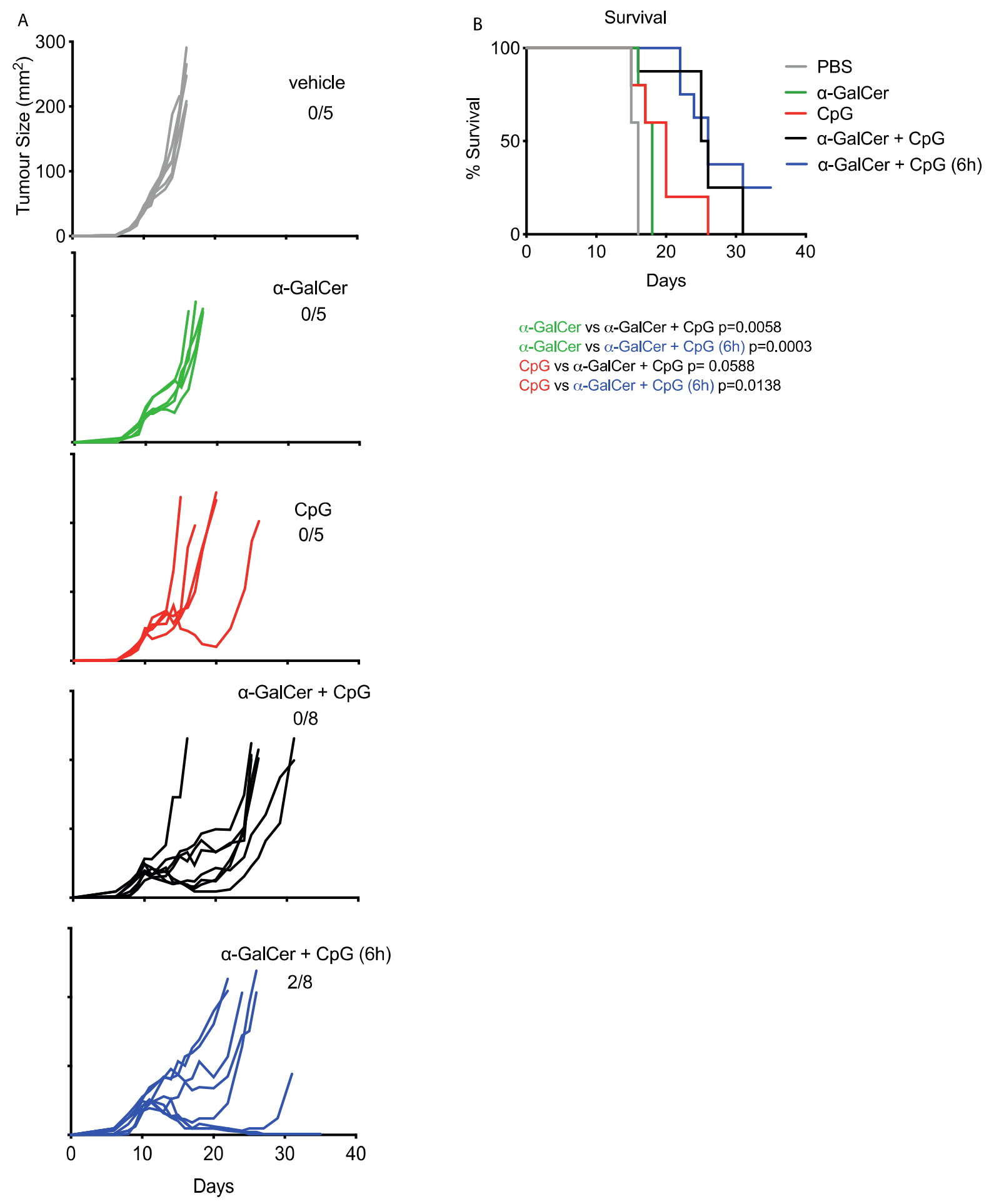

$\alpha-$ GalCer vs $\alpha$-GalCer + CpG $p=0.0058$ $\alpha$-GalCer vs $\alpha$-GalCer + CpG (6h) $p=0.0003$

$\mathrm{CpG}$ vs $\alpha$-GalCer $+\mathrm{CpG} p=0.0588$

CpG vs $\alpha-$ GalCer + CpG (6h) p=0.0138

Figure 14 In situ vaccination with $\alpha$-GalCer and CpG in the B16-F1 tumour model. Dosing schedules were as in earlier experiments A) Tumour growth curves plotted for each mouse. B) Survival curves for the different treatment groups, with statistical differences indicated (log-rank Mantel-Cox test). The experiment shown is representative of 2 repeated experiments. 


\subsubsection{In situ vaccination with a CpG and $\alpha$-GalCer administration in established TC1}

\section{Tumours}

Another poorly immunogenic tumour is $\mathrm{TC} 1$. The TC1 tumour cell line is a transformed murine lung epithelial cancer cell line co-transfected with human papillomavirus (HPV)-16 E6 and E7 genes and the activated human Ha-ras (G12V) oncogene ${ }^{215}$. Established TC1 derived tumours are refractory to treatment by anti-4-1BB mAb and anti-PDL-1 mAb ${ }^{216,217}$. To test responses to the combined treatment $\mathrm{C} 57 \mathrm{BL} / 6 \mathrm{~J}$ mice were inoculated with $1 \times 10^{5} \mathrm{TC} 1$ tumour cells into the flank on day 0 , and when the tumours reached approximately $36 \mathrm{~mm}^{2}$ (day 9) mice were treated intratumourally as before.

Unlike previously used tumour types, the intratumoural combination of $\alpha$-GalCer and $\mathrm{CpG}$, both as an admix and with delayed $\mathrm{CpG}$, only resulted in a tumour growth delay relative to PBS with no full regressions achieved (Fig 15). The combination of $\alpha$-GalCer and $\mathrm{CpG}$ is therefore ineffective at eradicating a tumour of such low immunogenicity. 
A

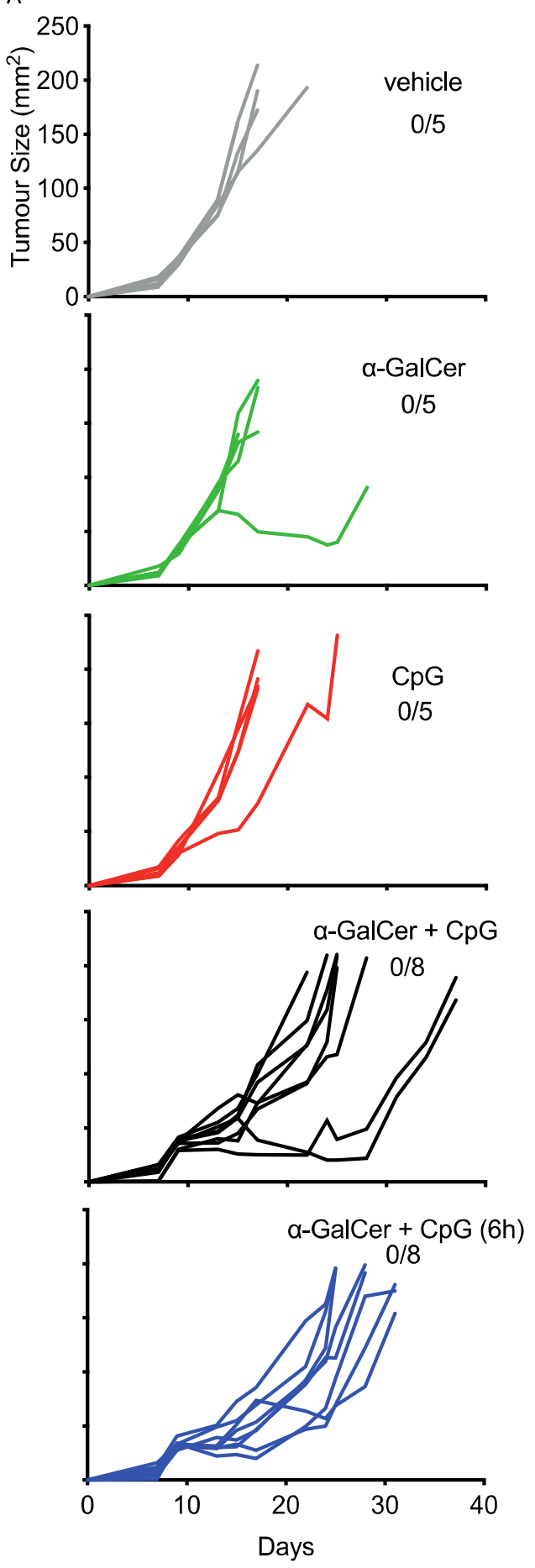

B

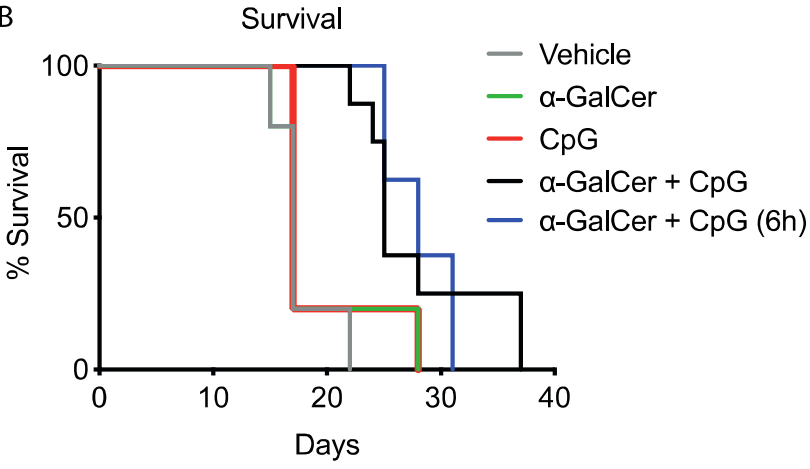

$\alpha$-GalCer vs $\alpha$-GalCer $+\mathrm{CpG} p=0.0443$

$\alpha$-GalCer vs $\alpha$-GalCer + CpG (6h) $p=0.0130$

CpG vs $\alpha-G a l C e r+C p G p=0.0533$

CpG vs $\alpha-$ GalCer + CpG (6h) p $=0.0156$

Figure 15 In situ vaccination with $\alpha$-GalCer and CpG in TC1 tumour model. Dosing schedules were as in earlier experiments A) Tumour growth curves plotted for each mouse. B) Survival curves for the different treatment groups, with statistical differences indicated (log-rank Mantel-Cox test). Experiment shown is representative of 2 repeated experiments. 


\subsubsection{Evaluation of liver toxicity of intratumoural delivery $\alpha$-GalCer + CpG}

The systemic administration of $\alpha$-GalCer to induce activation of iNKT cells is known to cause a transient elevation of liver function enzymes such as alanine transferase (ALT) when given systemically in rodents ${ }^{218}$, reflecting a phase of hepatotoxicity caused by activation of liver-resident iNKT cells that peaks by 18 hours and resolves by 72 hours ${ }^{219}$. It was anticipated that intratumoural delivery of $\alpha$-GalCer should cause less of an increase in ALT since it is primarily localised at the tumour and is not expected to be significantly exposed to the liver. In order to test this, serum was collected 18 hours after mice were treated intratumourally with the one dose of either PBS, CpG (25 $\mu \mathrm{g}), \alpha$-GalCer $(2 \mu \mathrm{g})$, or an admix $\alpha$-GalCer and CpG. In addition, a group was treated with $\alpha$-GalCer followed 6 hours later with a single dose of $\mathrm{CpG}$; in this case, serum was collected 12 hours after $\mathrm{CpG}$ treatment. Serum was also collected from mice that had been treated 18 hours prior with intravenous (i.v.) $\alpha$-GalCer $(2 \mu \mathrm{g})$, which is known to induce an ALT rise, as a point of comparison. The serum was sent to Gribbles Veterinary Services to analyse for ALT levels using an approved standard operating procedure. It was found that while intratumourally delivered $\alpha-\mathrm{GalCer}, \mathrm{CpG}$ or the combinations did increase serum ALT levels slightly beyond the normal range seen in PBS (but not in a statistically significant manner), the ALT levels were significantly lower than those mice that were administered $2 \mu \mathrm{g}$ of $\alpha$-GalCer intravenously as a single agent (Fig 16) where one expects the liver to be fully exposed to the reagents. This suggests that the intratumoural route of delivery is resulting in less liver toxicity, and this is consistent with lower systemic exposure compared to intravenous delivery. Although the differences in mean ALTs after the intratumoural treatments were not significant compared to the mean of the vehicle-treated group, some of the individual increases exceeded a 3-4 fold increase from normal range (range:14-56 IU/L representing 2.5th 
and 97.5 th percentiles ${ }^{220}$. Some limited leakage of reagents into circulation (and to the liver) may, therefore, have occurred in these animals.

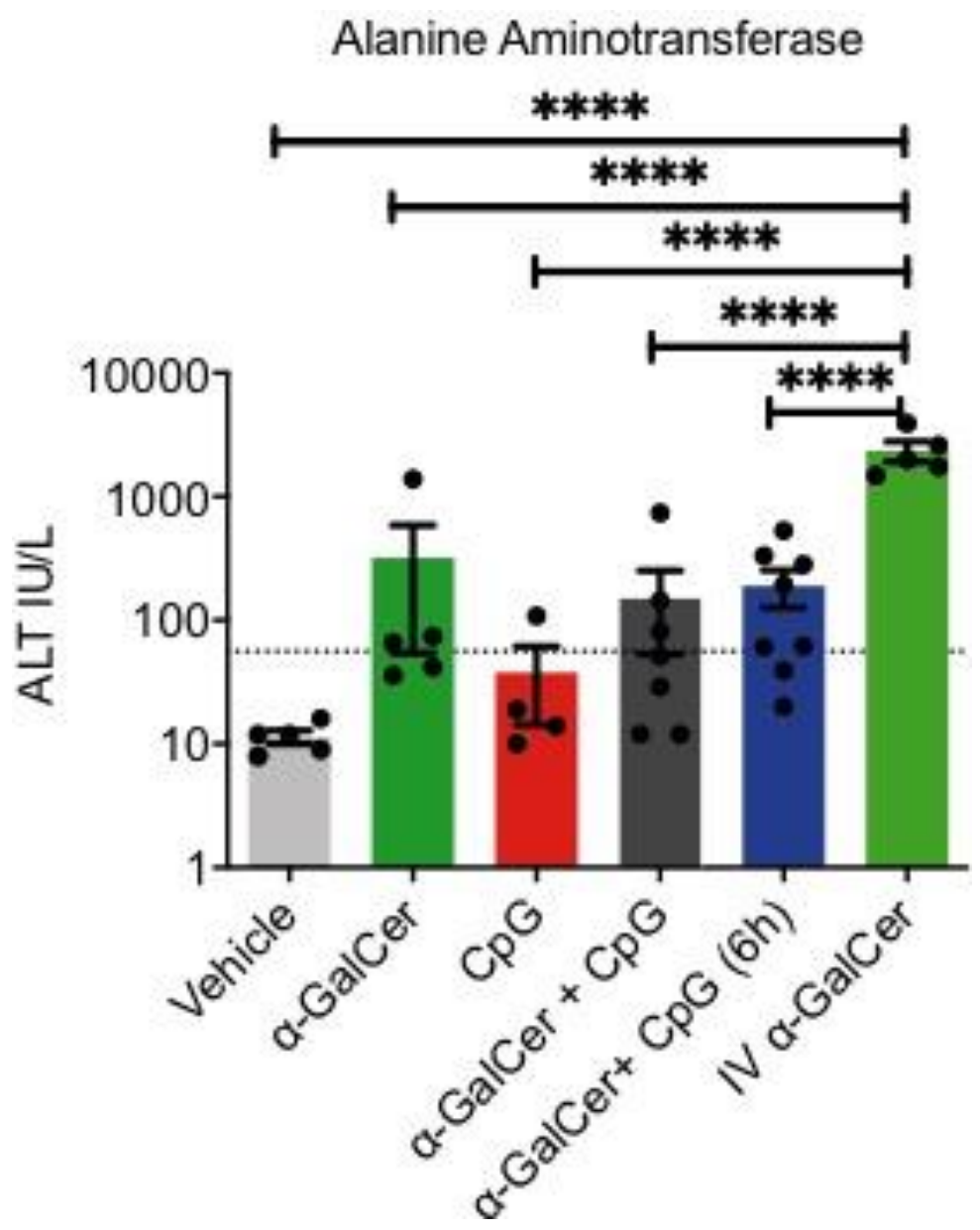

Figure 16 Intratumoural delivery of $2 \mu \mathrm{g}$ of $\alpha$-GalCer and $25 \mu \mathrm{g}$ of CpG results in lower alanine transaminase (ALT) levels than intravenous delivery of $\alpha$-GalCer in the serum of mice collected via cheek bleeding 18 hours after the first treatment or 6 hours after $C p G$ treatment in the case of $\alpha$-GalCer $+C p G(6 h)$. Statistically significant differences are indicated, $\pm S E M$, $* P<0.05$, **P<0.01, ***P<0.001, ****P<0.0001 (One-Way ANOVA with Tukey's Post-Hoc Test, mean \pm SEM). The dotted line represents the upper normal limit of ALT (reference range $14-56$ IU/L for the 2.5 to 97.5 interval ${ }^{220}$ ). 


\subsection{Discussion}

This chapter describes the development and optimisation of an intratumoural combination of an iNKT cell agonist ( $\alpha$-GalCer) and a TLR9 agonist $(\mathrm{CpG})$ treatment strategy for the treatment of various cancers. It has been shown that the local intratumoural administration of CpG together with $\alpha$-GalCer was very effective in inducing the rejection of the tumours at both the treated and distant sites in syngeneic lymphoma models. The success of this combination has prompted the generation of multiple hypotheses regarding the mechanism of action which will be explored further in later chapters using neutralising antibodies and knockout mice.

The intratumoural administration of the combination of $\alpha-\mathrm{GalCer}$ and $\mathrm{CpG}$ leads to an effective, systemic and durable anti-tumour response. This anti-tumour response can eradicate large established tumours in a subcutaneous lymphoma model, and induce memory. It was notable that mice that were previously cured of genetically modified tumours expressing OVA did not develop new tumours upon re-challenge with either the same tumour or the parental, unmodified, cell line. This suggests that the immune system had been trained to recognise antigen(s) endogenously expressed within the tumour, and not just through the artificial introduction of a model antigen.

An interesting finding was that the anti-tumour response was improved when the administration of $\mathrm{CpG}$ was delayed by 6 hours, highlighting the importance of time and order of administration. It has previously been demonstrated that CD80 and CD86 were expressed at higher levels when $\mathrm{CpG}$ was intravenously combined with $\alpha$-GalCer and are likely to contribute to the stronger proliferative responses and increased cytokine induction of the responder T cells ${ }^{209}$. Cell surface interactions are not the only factors however, as $\mathrm{CpG}$ was able to induce significant levels of IL-12p70 in the absence of iNKT cell stimulation, and this was enhanced 
when combined with $\alpha$-GalCer ${ }^{162}$. The variability in IL-12 responses supports the hypothesis that plasticity in DC cytokine responses is modulated by pattern recognition ${ }^{221,222}$. It has been reported that IL-12p70 release requires a CD40 signal in the context of combined TLR and $\alpha$ GalCer agonism ${ }^{223}$, as CD40L-deficient animals were unable to demonstrate increases in IL12 p70 after $\alpha$-GalCer administration ${ }^{162}$. Thus, one of the critical signals provided by activated iNKT cells to DCs is likely to be CD40L. Therefore, in the context of the delayed CpG treatment, it is possible that iNKT cells needed to be activated first to license DCs to prime a T cell response and induce CD40 ligation ${ }^{224}$. Subsequent TLR9 activation of the "sensitised" DCs then releases an abundance of IL-12 while pDCs concomitantly release type 1 IFN, as has been reported with systemic administration of other TLR agonists after $\alpha$-GalCer. This would be supplemented with iNKT cells producing cytokines such as IFN- $\gamma$ to help in the cytotoxic response ${ }^{11}$.

Previous data has shown that iNKT cell-dependent DC maturation is enhanced by the cooperative action of TLRs on DCs in both murine and human systems ${ }^{162}$. The increased costimulatory capacity correlated with the induction of more potent $\mathrm{T}$ cell responses both in vitro and in vivo. The antigen-specific T cell populations detected in the blood had a more substantial proportion of cells with an effector phenotype (TE) when induced in the presence of both iNKT cell and TLR stimulation ${ }^{225,226}$. Furthermore, the provision of IL-12 at priming is closely associated with improved intrinsic survival properties of memory CD8 + T cells ${ }^{30,227}$. Interestingly, combining TLR ligation with iNKT cell stimulation with co-administered OVA antigen also results in high OVA-specific IgG responses ${ }^{162}$. The data presented in this chapter show that the combined treatment was associated with immunological memory, implying involvement of adaptive immune cells. It remained to be established whether T cells, antibodies 
or perhaps both, contribute to the anti-tumour responses seen here. Applying the above mechanistic rationale to the phenomena of delaying TLR agonism after iNKT cell stimulation, it would seem that iNKT cell agonism is sensitising APCs to TLR stimulation leading to an increased release of IL-12p70 as well as increasing CD80/86 expression. This could lead to greater antigen cross-priming potential and thus better initiate a $\mathrm{CD} 8^{+} \mathrm{T}$ cell response, which may be a key effector mechanism in the anti-tumour activity observed. This will be covered in a later chapter.

Delineating the presence of a systemic immune response, mice that had bilateral tumours, but only received intratumoural treatment into one of their tumours were able to successfully reject both tumours in the form of the abscopal effect. The eradication of the contralateral tumour may have been affected in multiple possible ways. First, the abscopal effect relies on the priming of adaptive immune responses that then recirculate to the distal tumour. As noted, the fact that memory responses are generated suggests that adaptive responses are indeed initiated. As the priming of adaptive responses occurs in the lymphoid tissues, this process would rely on antigen being transferred to the lymphoid tissues, either as free material, or loaded onto local APCs that migrate to these tissues. It also possible that it is incorporated into dead or dying cells that access lymphoid tissues. While $\mathrm{CpG}$ injected into the treated tumour could possibly stimulate tumourresident APCs to make the journey to the lymphoid tissues, the adjuvant effect of $\alpha$-GalCer relies on iNKT cells, which reside primarily in lymphoid tissues. Therefore, the $\alpha$-GalCer also has to be transferred to the lymphoid tissues, either as free material or loaded onto trafficking APCs. If the abscopal effect involves priming of $\mathrm{T}$ cells, then the antigen and $\alpha$-GalCer have to ultimately be presented on the same DCs in the lymphoid tissues to receive the licensing signals from iNKT cells that form the basis of the adjuvant effect of this compound. Second, it could rely on priming 
of adaptive immune responses locally in the tumour that then recirculate to the distal tumour. This goes against the dogma that adaptive responses are initiated in lymphoid tissues, and would potentially have to rely on there being established tertiary lymphoid tissues (lymph node-like structures that form near the sites of chronic inflammation) in the tumour. iNKT cells would also have to be in the tumour to invoke their cellular adjuvant properties, which is unlikely based on other data from our lab, with few iNKT cells seen at baseline in any of our tumour models. Third, it could rely on boosting of existing adaptive immune responses that have already made it to the tumour. There was only limited time for an endogenous response to have developed, but it is possible that this was enhanced in both the tumour and lymphoid tissues. Finally, it is possible that the whole process is mediated by an innate response, perhaps involving iNKT cells and NK cells, although this would require the formation of "innate-memory" cells, which has been described for NK cells ${ }^{228}$ and iNKT cells ${ }^{229}$. Similar to the previous points above, in order to involve iNKT cells, the $\alpha$-GalCer would ultimately have to be presented on DCs in the lymphoid tissues, so leakage from the tumour, or transfer on the APCs would need to be involved. It may also be a mixture of these hypotheses with adaptive responses initiated within the lymphoid tissues and sustained (and potentially boosted) through repeated $\mathrm{CpG}$ administration, which may also favour the infiltration and activity of iNKT cells and NK cells.

As several of these concepts are reliant on $\alpha$-GalCer reaching lymphoid-resident iNKT cells, it was notable that there was not a statistically significant increase in average ALT rise typical of activation of resident iNKT cells; this was interpreted to mean $\alpha$-GalCer did not leach from the tumour to access the liver via the blood. However, in some individual animals, ALT increases were observed with intratumoural treatment that were well above the normal range, suggesting that liver iNKT cells can be accessed with the intratumoural treatment. Given that the 
kinetics of any $\alpha$-GalCer release from the tumour are unknown, it is possible that a measurable peak in the process was missed, or that only very low quantities are involved that don't always cause hepatotoxicity. Although the transient hepatotoxicity associated with $\alpha$-GalCer is heightened in rodents because of the high number of iNKT cells in the liver, this was not seen with intravenous $\alpha$-GalCer in a human safety study ${ }^{155}$. Nonetheless, a reduced toxicity profile with intratumoural treatment would be an advantage to this approach.

In immunogenic tumours such as EL4.LA and EG7.OVA lymphoma models, the combination is highly effective as an in situ vaccine leading to complete eradication of tumours in the majority of mice. On the other hand, with less immunogenic tumours such as the TC1 or B16 tumour models, the use of the combination of iNKT cells agonist and TLR9 agonist was not as effective. Only growth delay was observed with TC1, and only a small fraction of B16 mice achieved full regression. Chapter 7 will outline approaches to successfully overcoming this limitation. 


\section{Chapter 4}

\section{Delineating the role of different immune cell subsets in $\alpha$-GalCer $+\mathrm{CpG}$ treatment strategy}

\subsection{Introduction}

It is well known that both activation of iNKT cells with $\alpha$-GalCer, and stimulation of TLR9 with $\mathrm{CpG}$, can recruit a plethora of innate and adaptive immune cells including $\mathrm{CD} 8^{+} \mathrm{T}$ cells, pDCs, iNKT cells, cDC1s and stimulate B cells to produce antibodies. Having established that the combination of $\alpha$-GalCer and CpG administered intratumourally is an effective antitumour therapy in the mouse, it was next determined what cell types were implicated in the efficacy of the combination therapy. Given that memory responses were observed, the differentiation of $\mathrm{CD}^{+} \mathrm{T}$ cells into CTLs could be critical. Several studies have suggested that direct lysis of tumour cells by CTLs through contact ${ }^{230,231}$ as well as indirect effects of cytokine signalling by activated $\mathrm{T}$ cells, in particular, IFN $\gamma$, are important in tumour control ${ }^{232,233}$. Mechanistically, to stimulate T cell responses including CTL, it is thought that DCs within the tissue take-up antigen and migrate to a draining $\mathrm{LN}$ via afferent lymphatic vessels or that antigens that are released into the lymph or circulation are acquired by lymphoid-resident DCs. In general, DCs can be subdivided into conventional (cDC), plasmacytoid (pDC) or monocytederived (MoDC) subsets ${ }^{234}$. Of relevance here, pDCs (identified in mice by expression of Siglec H) are major producers of type I IFN, while cDC are regarded as the most efficient activators of naïve T cells. The latter can be delineated into two major subsets; cDC1 (which express Clec9a), which are known to have a strong capacity for cross-presentation to $\mathrm{CD} 8^{+} \mathrm{T}$ cells and produce IL-12 to aid in the differentiation of naïve T cells ${ }^{84,234-236}$, and cDC2, which have a strong 
capacity to promote the $\mathrm{CD}^{+} \mathrm{T}$ cell effectors required for a variety of anti-viral, anti-fungal or anti-helminth responses, as well as the production of IL- 23 which mobilises Thelper 17 cells responsible for immunity against pathogens ${ }^{84,236,237}$. Here, T cells were hypothesised to be involved in the response to intratumoural $\mathrm{CpG}$ and $\alpha$-GalCer, with $\mathrm{CD} 8^{+} \mathrm{T}$ cells in particular previously shown to be effectively stimulated via iNKT cell-mediated adjuvancy ${ }^{142,143,238}$ leading to peptide-peptide specific memory generation. If true, specific removal of $\mathrm{CD} 8^{+} \mathrm{T}$ cells would abrogate the anti-tumour response and anti-tumour memory. As $\mathrm{CD} 8^{+} \mathrm{T}$ cells require antigen to be cross-presented to them in order to differentiate and mount an immune response, it stands to reason that $\mathrm{cDC} 1 \mathrm{~s}$ may also be involved; thus their removal could also strongly hamper the anti-tumour response.

Administration of $\mathrm{CpG}$ is known to activate $\mathrm{pDCs}$, which through the production of type I IFN can potentially induce activation of $\mathrm{cDC}^{239}$. The $\mathrm{CpG}$ treatment could also activate other key effectors such as neutrophils ${ }^{210}$, macrophages ${ }^{240}, \mathrm{~B}$ cells ${ }^{241}$ and NK cells ${ }^{242}$. Therefore, it was hypothesized that removal of pDCs would negatively impact the anti-tumour response via a reduction in the production of type I IFN. As the effective intratumoural treatment used here required $\alpha$-GalCer in addition to $\mathrm{CpG}$, the effector properties of iNKT cells must have been involved, either as anti-tumour effectors in their own right ${ }^{243}$ or indirectly by facilitating licensing of APCs to prime T cells ${ }^{243}$. Interestingly, activated iNKT cells also provoke systemic responses that mediate increased DC migration to draining lymph nodes ${ }^{244}$. Again, a way to test for the involvement of iNKT cells is to examine responses in animals where these cells are ablated or severely reduced.

As $\mathrm{CpG}$ is known to stimulate B cells and the combination therapy has been demonstrated to generate an immunological memory against cancers in remission, it was 
possible that antibodies produced by plasma B cells might play a role in the anti-tumour response. If true, one way to test this is to establish whether antibodies found in the serum of mice that had undergone remission could protect naïve animals from tumour challenge.

The role of various cell types could also be elucidated by depletion through various means. The first two methods involve conditional ablation of specific cell types and to study the effects of removing these cells in otherwise healthy mice. First, mice can be treated with antibodies against a defined marker of a target cell (CD8, CD1d, etc.), which would tag cells expressing that marker for removal from circulation via induction of antibody-dependent cell cytotoxicity (ADCC), phagocytosis, or complement-dependent cytotoxicity (CDC) ${ }^{245}$. The second method of depletion is to use diphtheria toxin receptor (DTR)-guided ablation of specific cell types in transgenic mice. Mice are known to be resistant to diphtheria toxin, so transgenic mice have been generated that express the human DTR on specific cell types, making the cells sensitive to the toxin. In this way, upon administration of diphtheria toxin, the cells expressing the DTR will be depleted, while the mice will remain relatively healthy and normal ${ }^{246}$. The final method involves genetically knocking-out specific cell types, making the animals deficient from birth. While this method guarantees complete removal of a cell type, results do have to be considered carefully as there are examples where the immune system compensates for the absence of this cell type, altering the immune environment and its cell populations from what they would normally be 247,248 . 


\subsection{Aims}

The goal of the studies in this chapter was to identify what cells were involved in the anti-tumour response of the combination of $\alpha$-GalCer and CpG. To do this a number of different approaches to depletion of specific cell-types were used. Specifically, the aims were to:

- Determine the role of $\mathrm{CD}^{+}$Cells

- Determine the role of $\mathrm{Clec} 9 \mathrm{a}^{+}$cells (cDC1 cells)

- Determine the role of Siglec- $\mathrm{H}^{+}$cells (pDC)

- Determine the role of iNKT cells

- Determine the protective effect of serum produced as a result of treatment 


\subsection{Results}

\subsubsection{Determining the impact of $\alpha$-GalCer and $\mathrm{CpG}$ on peptide-specific $\mathrm{T}$ cell responses.}

The syngeneic EG7.OVA model is convenient as it enables the analysis of immune responses a tumour-associated antigen, albeit an artificially introduced one. As noted earlier, EG7.OVA cells are mouse thymoma EL4 cells stably transfected with the complementary DNA encoding full OVA protein and thus express OVA epitopes as unique antigens modelling a tumour-associated neoantigen. This cell line is a model system for studying antigen-specific immune responses of cytotoxic $\mathrm{T}$ lymphocytes in mice, as the dominant $\mathrm{CD} 8^{+} \mathrm{T}$ cell epitope for OVA in C57BL/6 mice (an H-2 $\mathrm{K}^{\mathrm{b}}$-binding peptide, $\mathrm{OVA}_{257-264}$ ) is known, and fluorescent MHC/peptide pentamers ("OVA pentamers") are available for analysis by flow cytometry. It was therefore determined whether intratumoural $\alpha-\mathrm{GalCer}$ and $\mathrm{CpG}$ could induce an OVA-specific immune response in this model. Cohorts of tumour-bearing mice were separately treated with vehicle (PBS), $\alpha$-GalCer, $\mathrm{CpG}$, a combination of $\alpha-\mathrm{GalCer}$ and $\mathrm{CpG}$, or a combination where the first $\mathrm{CpG}$ dose was delayed by 6 hours. The dosing regimen was as in previously presented experiments.

Blood was collected for analysis of immune cells by flow cytometry 7 days after the beginning of treatment, when all tumours are typically still progressing (but before the start of regression which usually begins around day 11). To detect OVA-specific T cells, fluorescent MHC/peptide pentamers were used in combination with antibodies for CD8, and the activation markers CD44 (which is upregulated on activated cells), KLRG1 is upregulated on effector T cells, and CD62L is upregulated on central memory T cells. The gating strategy, with fluorophores used for each antibody, is provided in Fig 17. For each antibody, titrations were 
performed to optimise staining. An example titration is provided in Supplementary Fig 2. From the flow cytometry analysis, the percentage of OVA-specific T cells, defined as $\mathrm{CD}^{+} \mathrm{OVA}$ pentamer $^{+}$cells, was not significantly different between groups and there were too few cells detected to reliably infer activation status (Fig 18a). When looking at the overall percentages of $\mathrm{CD}^{+} \mathrm{T}$ cells, irrespective of TCR-specificity, there was some variation, with no consistent significant trends associated with treatment (Fig 18b). When focusing on activated CD8 ${ }^{+} \mathrm{T}$ cells, again irrespective of specificity, the percentage of $\mathrm{CD} 44^{+} \mathrm{KLRG} 1^{+} \mathrm{T}$ cells had an increased trend with all treatment groups as compared to the untreated arm, indicating a potential switch to an effector phenotype (Fig 18c), although this did not reach statistical significance. This suggests that OVA may not be involved in the immune response to EG7.OVA in this treatment model. However, it remained possible that OVA-specific responses were sequestered to the tumour or lymph node. Analysis of the tumour infiltrating lymphocytes, as well as cells in the tumour draining lymph nodes and other lymphoid tissues was therefore required to shed better light on the cells involved, rather than simply evaluating the percentage of these cells in the blood. 

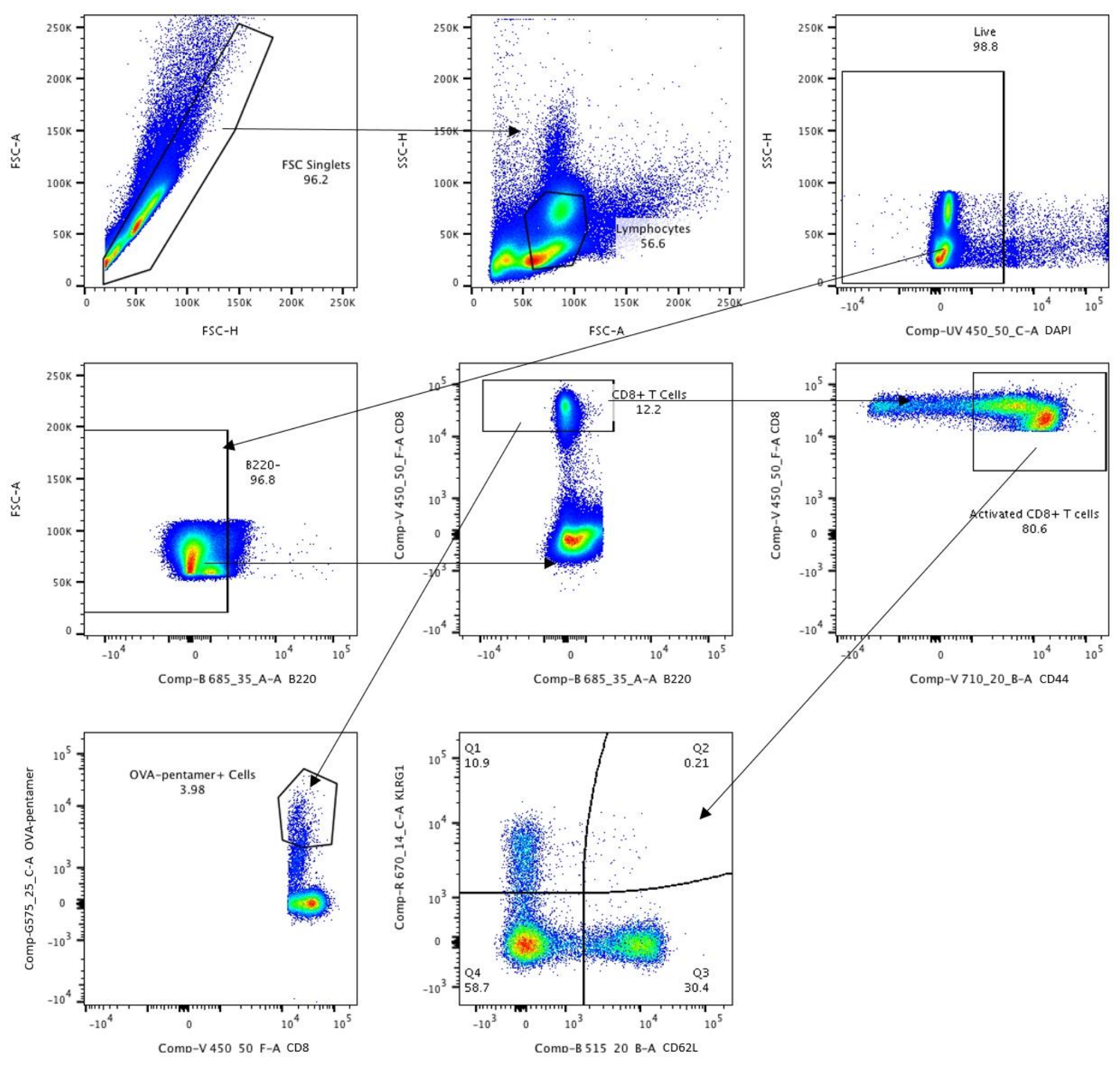

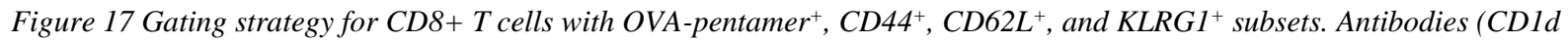
tetramer-BV421, LY6c-eF450, Ki67-BV480, CD3-BV510, CD11b-BV570, NK1.1-BV650, CD127-BV711, CD44-BV750, CD45BV786, CD19-FITC, FoxP3-AF532, OVA pentamer-PE, SCA-1-PE-CF594, CD69-PerCP-Cy5.5, PD-1-PE-Cy7, CD62L-AF647, KLRG1-APC, CD4-APC-H7, CD8-AF700) 


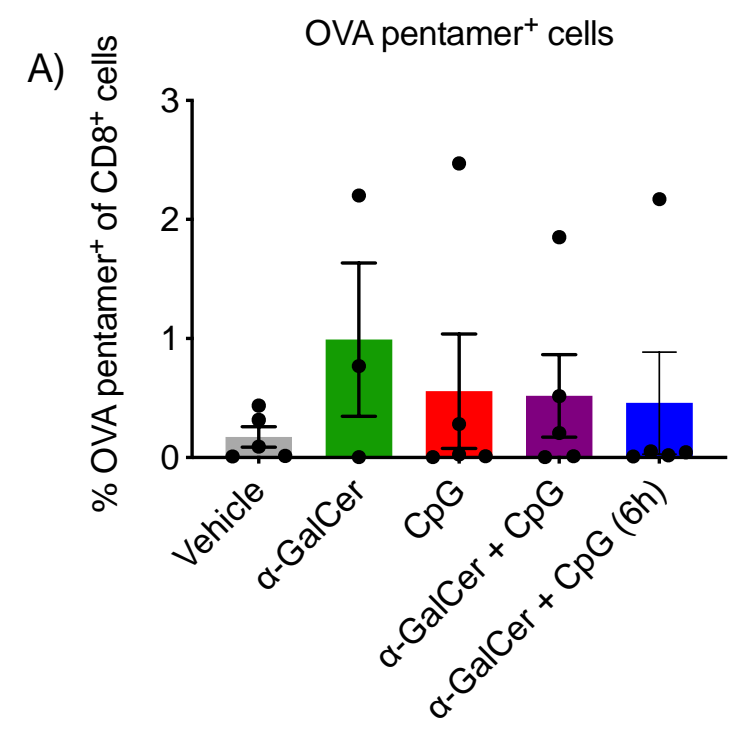

B)

$\mathrm{CD}^{+} \mathrm{T}$ cells
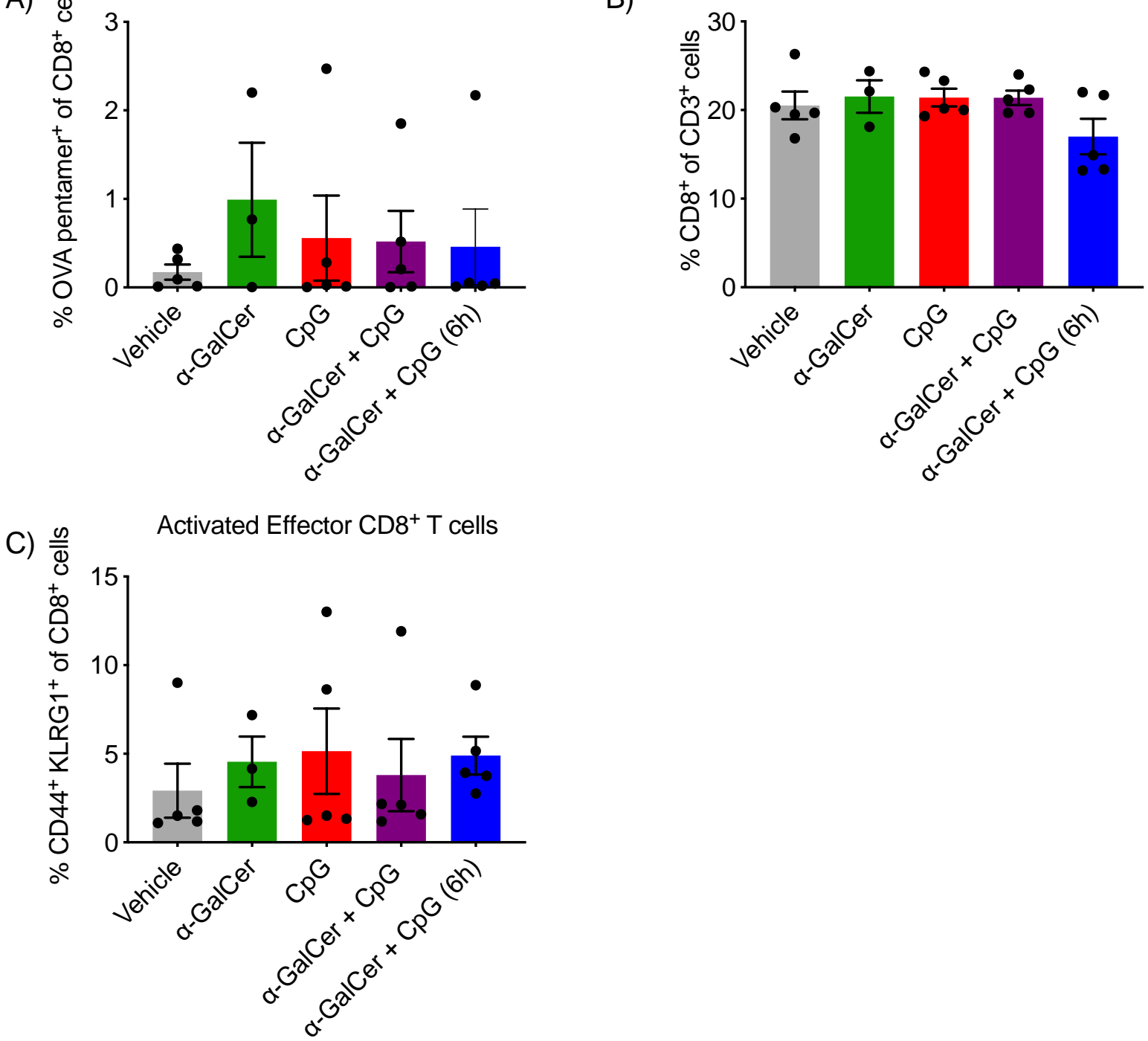

Figure 18 The combination of $\alpha$-GalCer and CpG does not increase OVA-specific CD8 $8^{+} T$ cells in the blood collected 7 days after the start of treatment. A) Analysis of OVA-pentamer ${ }^{+} \mathrm{CD}^{+} \mathrm{T}$ cells showed that treatment did not induce an increase in OVA-specific CD $8^{+} T$ cells in the blood compared to vehicle. B) Treatment did not induce changes in the overall CD $8^{+} T$ cell population. $C$ ) The administration of a-GalCer (as a single agent or in combination with $C p G$ ) had a trend towards increasing the frequency of $C D 44^{+} K L R G 1^{+} C D 8^{+} T$ cells in the blood, but not in a significant manner. Statistically significant differences are indicated $* P<0.05$, **P<0.01, ***P<0.001, ****P<0.0001 (One-Way ANOVA with Tukey's Post-Hoc Test). Gating strategies and antibodies in figure 17. 


\subsection{2 $\alpha$-GalCer and CpG induce changes in CD8 and iNKT cell populations in the tumours, spleen and draining lymph node}

To follow up on the previous experiment, it was next determined whether increases in OVA-specific T cells could be detected in the tumour, spleen or draining lymph node (dLN) in animals bearing EG7.OVA tumours subjected to treatment. In addition, to further elucidate what other lymphocytes were being modulated by treatment, particularly $\mathrm{CD} 4^{+} \mathrm{T}$ cells, regulatory $\mathrm{T}$ cells, iNKT cells and B cells, a broader panel of antibodies was used to examine the anti-tumour response to intratumoural $\alpha-G a l C e r$ and $\mathrm{CpG}$ in the tumours and lymphoid tissues harvested 7 days post-treatment. This time point was chosen when tumours were still progressing immediately before the start of regression with effective treatment, which was when phenotypic changes in keys effectors, such as the T-cell population, might be expected to peak. The tumours, spleen, and dLN were processed into a single cell suspension and analysed via flow cytometry. The experiment was conducted in hosts on a C57BL/6 background with congenic expression of the CD45.2 isoform (whereas C57BL/6 express CD45.1) so that the immune infiltrate could be distinguished from the EG7.OVA tumour cells themselves, which are a $\mathrm{T}$ cell lymphoma derived from C57BL/6 animals and therefore express CD45.1. Also, importantly, each animal was challenged with two tumours, with only one treated, so that analysis of both tumours might define the lymphocytes associated with the induced abscopal effect. A series of antibody panels were used, with the expression of CD45.2 used to define immune cells. Of T cells, it was possible to delineate OVA-specific $\mathrm{T}$ cells $\left(\mathrm{CD} 45.2^{+} \mathrm{CD} 8^{+} \mathrm{OVA}\right.$ pentamer $\left.{ }^{+}\right)$, all $\mathrm{T}$ cells $\left(\mathrm{CD} 45.2^{+} \mathrm{CD}^{+}\right), \mathrm{CD}^{+} \mathrm{T}$ cells $\left(\mathrm{CD} 45.2^{+} \mathrm{CD}^{+} \mathrm{CD}^{+}\right)$, and $\mathrm{CD} 8 \mathrm{~T}$ cells $\left(\mathrm{CD} 45.2^{+} \mathrm{CD}^{+} \mathrm{CD}^{+}\right)$ and regulatory $\mathrm{T}$ cells $\left(\mathrm{CD} 45.2^{+} \mathrm{CD}^{+} \mathrm{CD}^{+} \mathrm{FoxP}^{+}\right)$. The use of antibodies to activation markers enabled the $\mathrm{T}$ cell populations to be further broken down into $\mathrm{CD} 4^{+}$naïve $\mathrm{T}$ cells 
$\left(\mathrm{CD} 45.2^{+} \mathrm{CD}^{+} \mathrm{CD}^{+} \mathrm{CD} 62 \mathrm{~L}^{+}\right), \mathrm{CD} 4^{+}$central memory T cells $\left(\mathrm{CD} 45.2^{+} \mathrm{CD}^{+}\right.$

$\left.\mathrm{CD} 4^{+} \mathrm{CD} 44^{+} \mathrm{CD} 62 \mathrm{~L}^{+}\right), \mathrm{CD} 4^{+}$effector memory $\mathrm{T}$ cells $\left(\mathrm{CD} 45.2^{+} \mathrm{CD} 3^{+} \mathrm{CD} 4^{+} \mathrm{CD} 44^{+} \mathrm{KLRG} 1^{-}\right)$, $\mathrm{CD} 4^{+}$short lived effector $\mathrm{T}$ cells $\left(\mathrm{CD} 45.2^{+} \mathrm{CD}^{+}{ }^{+} \mathrm{CD} 4^{+} \mathrm{CD} 44^{+} \mathrm{KLRG} 1^{+}\right)$, and $\mathrm{CD} 8^{+}$naïve $\mathrm{T}$ cells $\left(\mathrm{CD} 45.2^{+} \mathrm{CD}^{+} \mathrm{CD}^{+} \mathrm{CD} 62 \mathrm{~L}^{+}\right), \mathrm{CD} 8$ central memory $\mathrm{T}$ cells $\left(\mathrm{CD} 45.2^{+} \mathrm{CD}^{+}\right.$ $\left.\mathrm{CD} 4{ }^{+} \mathrm{CD} 44^{+} \mathrm{CD} 62 \mathrm{~L}^{+}\right), \mathrm{CD} 8^{+}$effector memory $\mathrm{T}$ cells $\left(\mathrm{CD} 45.2^{+} \mathrm{CD}^{+}{ }^{+} \mathrm{CD} 8^{+} \mathrm{CD} 44^{+} \mathrm{KLRG1}^{-}\right)$, $\mathrm{CD} 8^{+}$short-lived effector $\mathrm{T}$ cells $\left(\mathrm{CD} 45.2^{+} \mathrm{CD} 3^{+} \mathrm{CD} 8^{+} \mathrm{CD} 44^{+} \mathrm{KLRG} 1^{+}\right)$. For analysis of iNKT cells, fluorescent $\alpha$-GalCer-loaded CD1d tetramers (“CD1d tetramers”) were used to define iNKT cells among the $\mathrm{T}$ cell population $\left(\mathrm{CD} 45.2^{+} \mathrm{CD} 1 \mathrm{~d}\right.$ tetramer $\left.{ }^{+} \mathrm{CD}^{+}\right)$. The iNKT cell population could be broken down further into the recognised phenotypes CD4- NK1.1- ${ }^{-}$NKT cells $\left(\mathrm{CD} 45.2^{+} \mathrm{CD} 1 \mathrm{~d}\right.$ tetramer $\left.{ }^{+} \mathrm{CD}^{+}{ }^{+} \mathrm{CD} 4-\mathrm{NK} 1.1^{-}\right), \mathrm{CD}^{+}{ }^{+} \mathrm{NKT}$ cells $\left(\mathrm{CD} 45.2^{+} \mathrm{CD} 1 \mathrm{~d}\right.$ tetramer ${ }^{+}$ $\left.\mathrm{CD}^{+} \mathrm{CD} 4^{+}\right), \mathrm{NK} 1.1^{+}$iNKT cells $\left(\mathrm{CD} 45.2^{+} \mathrm{CD} 1 \mathrm{~d}\right.$ tetramer $\left.{ }^{+} \mathrm{CD} 3^{+} \mathrm{NK} 1.1^{+}\right) \mathrm{CD} 4^{+} \mathrm{NK} 1.1^{+}$iNKT cells $\left(\mathrm{CD} 45.2^{+} \mathrm{CD} 1 \mathrm{~d}\right.$ tetramer $\left.{ }^{+} \mathrm{CD} 3^{+} \mathrm{CD} 4^{+} \mathrm{NK} 1.1^{+}\right)$and $\mathrm{B}$ cells $\left(\mathrm{CD} 45^{+} \mathrm{CD} 19^{+}\right)$. The gating strategy for each cell-type, with each fluorophore used, is presented in fig 19. 

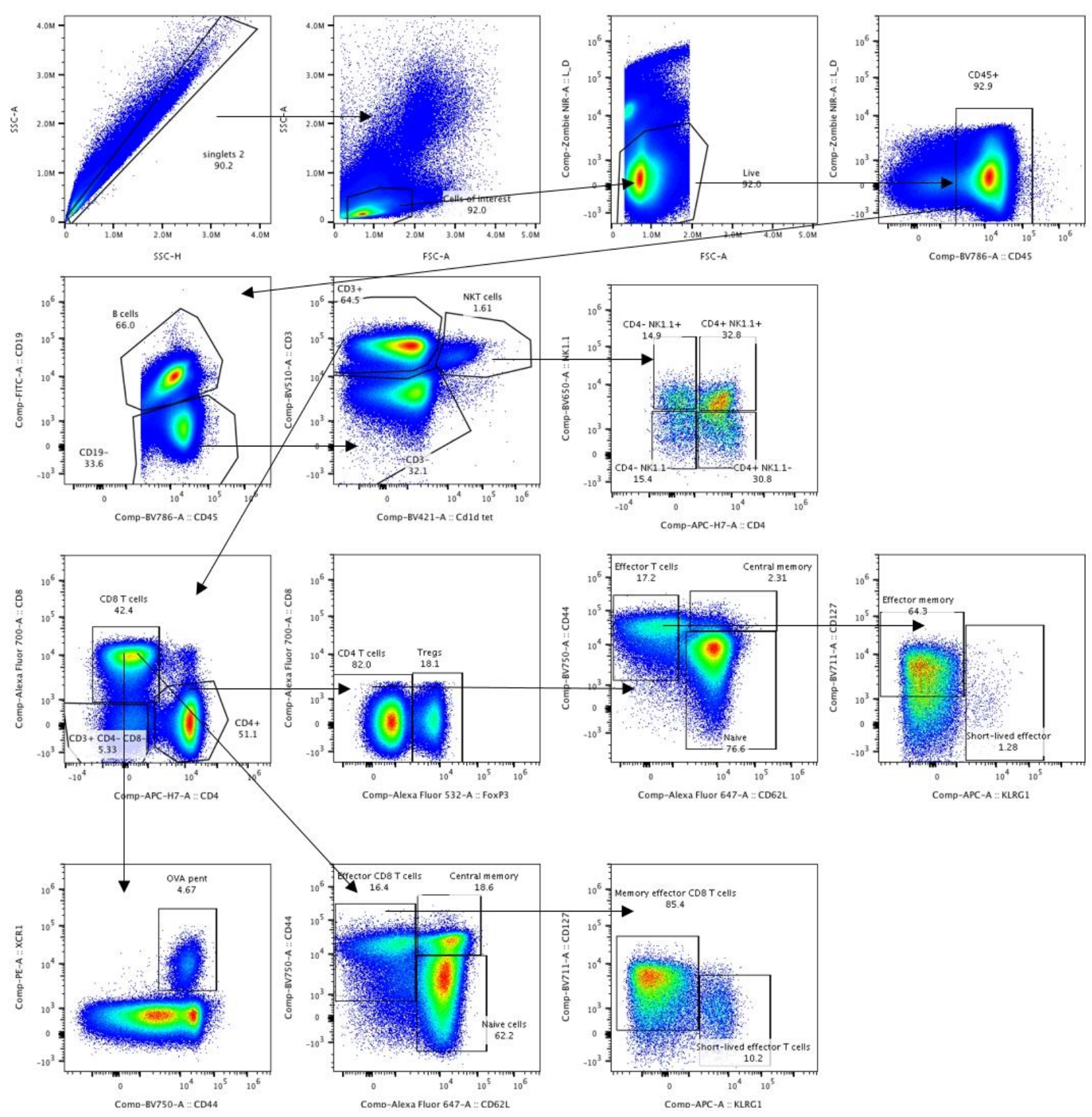

Figure 19 Gating strategies for B cells, Tregs, $C D 4^{+}, C D 8^{+}$and iNKT cells. Antibodies (CDId tetramer-BV421, LY6c-eF450, Ki67-BV480, CD3-BV510, CCD11b-BV570, NK1.1-BV650, CD127-BV711, CD44-BV750, CD45-BV786, CD19-FITC, FoxP3AF532, OVA pentamer-PE, SCA-1-PE-CF594, CD69-PerCP-Cy5.5, PD-1-PE-Cy7, CD62L-AF647, KLRG1-APC, CD4-APCH7, CD8-AF700). Panel design and flow cytometry analysis was conducted in collaboration with Laura Ferrer-Front and Phoebe Harmos. 


\subsubsection{Immune profile of the tumour}

In the tumour, there were no significant changes detected in the frequency of OVAspecific $\mathrm{T}$ cells (Fig 20). Perhaps surprisingly, there were very few changes in all conventional $\mathrm{T}$

cell populations. Tregs were reduced in tumours treated with either $\mathrm{CpG}$ or the combination of CpG and $\alpha-$ GalCer (Fig 21A), though only $\mathrm{CpG}$ treatment as a single agent induced a reduction in the frequency of Tregs in treated tumours compared to untreated (contralateral) tumours (Fig 21B). There were no notable changes in the $\mathrm{CD} 4^{+} \mathrm{T}$ cell populations (Fig 22). As for the $\mathrm{CD} 8^{+} \mathrm{T}$ cell population, there was no significant difference in the overall population (Fig 23A), but when broken down into different phenotypes, there was a trend towards the increased frequency of short-lived effector $\mathrm{CD}^{+} \mathrm{T}$ cells with treatment, but this was only significant in the treated tumours of $\mathrm{CpG}$ treated mice (Fig 23G). 


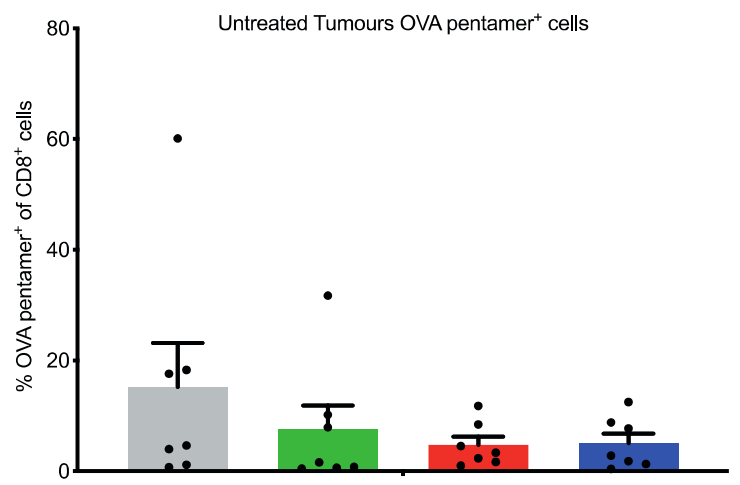

B

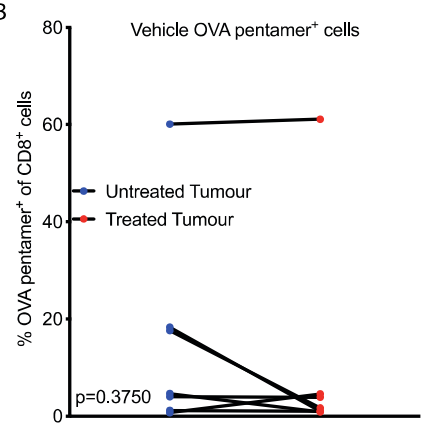

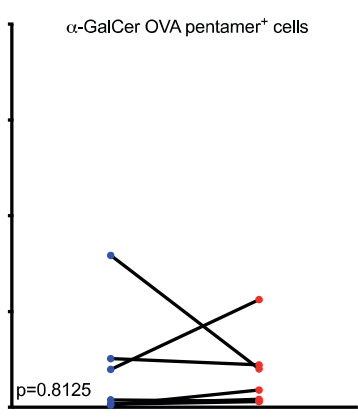
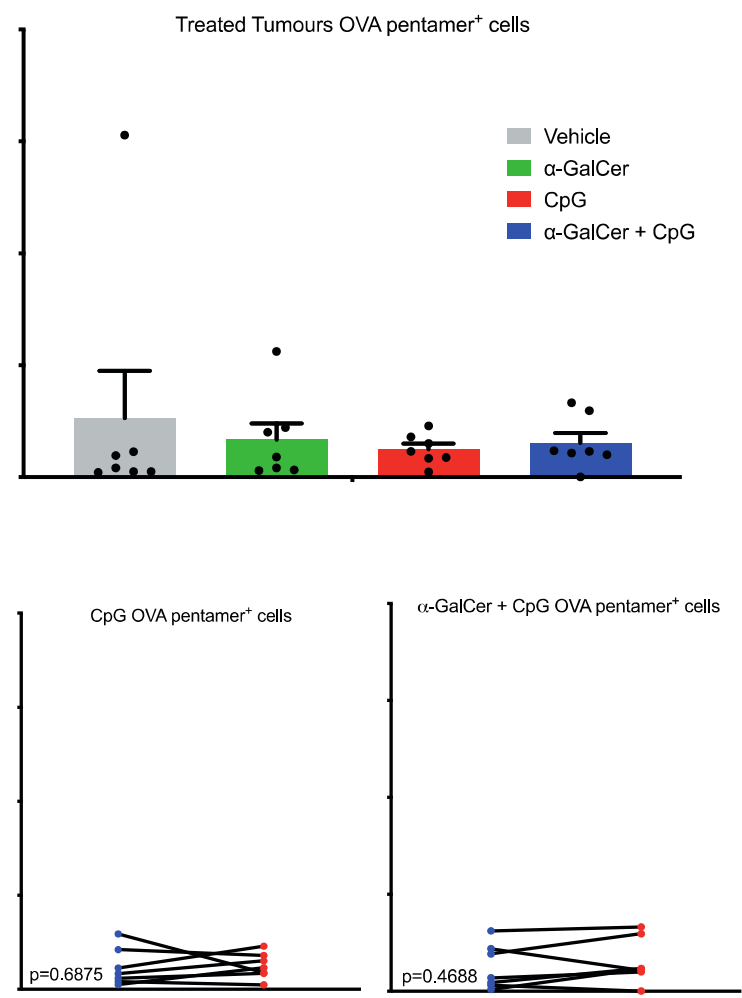

Figure 20 Intratumoural combination therapy of $\alpha$-GalCer and $C p G$ does not increase the frequency of $O V A-s p e c i f i c C D 8^{+} T$ cells in tumours. A) Both treated and untreated (contralateral) tumours exhibited no change in OVA-specific T cells as a result of treatment. Statistically significant differences are indicated $* P<0.05, * * P<0.01$, $* * * P<0.001$, ****P<0.0001 (One-Way ANOVA with Tukey's Post-Hoc Test). B) $\alpha$-GalCer and CpG combination therapy does not induce differences between treated and untreated tumours. Statistically significant differences are indicated (Wilcoxon Signed-Rank Test). 
A
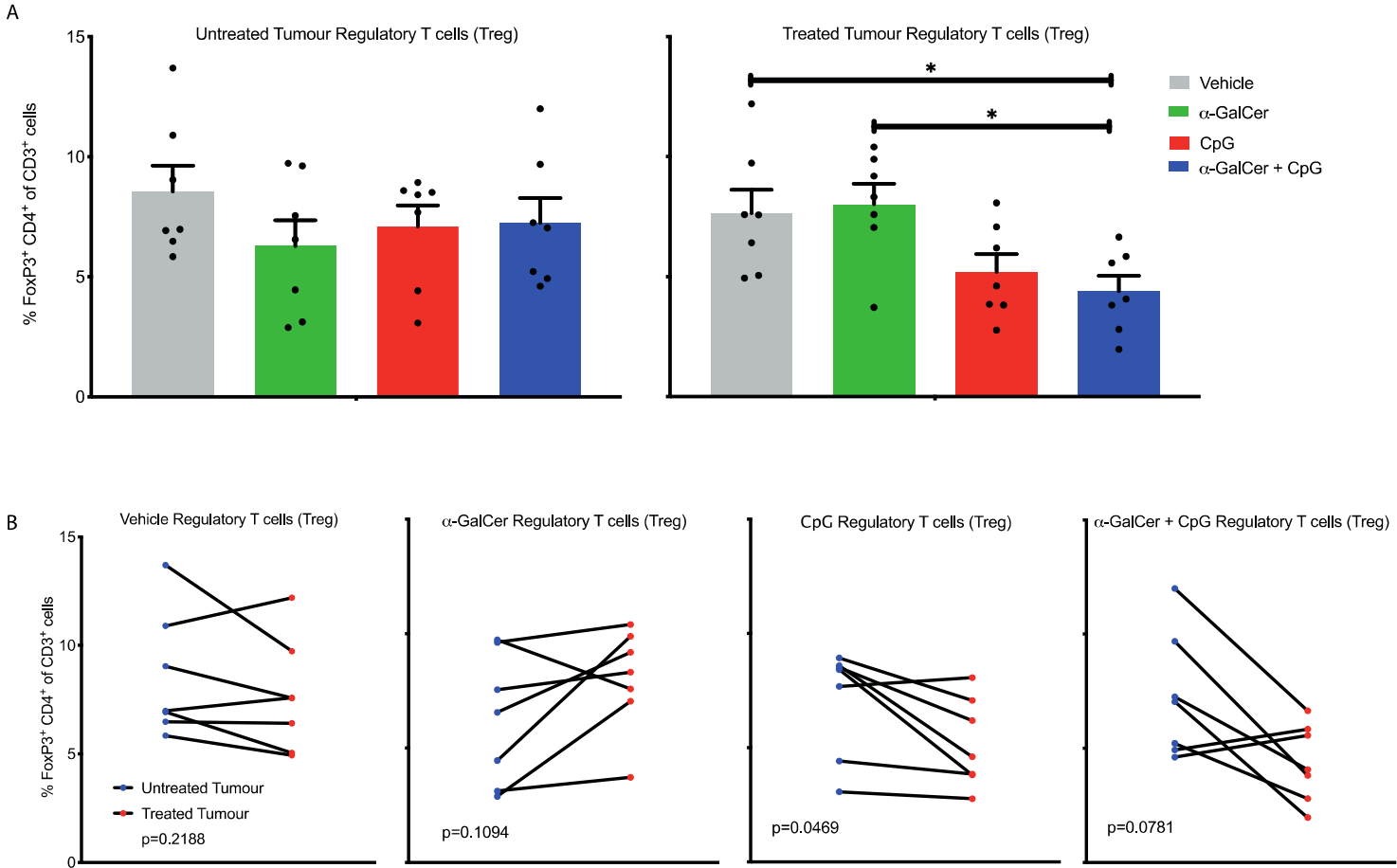

Figure 21 Intratumoural combination therapy of $\alpha$-GalCer and $C p G$ decreases the proportion of regulatory $T$ cells (Tregs) in treated tumours relative to $\alpha$-GalCer and vehicle. A) Tumours treated with the combination saw a significant reduction in Tregs relative to either vehicle or $\alpha$-GalCer as a single agent. Untreated tumours saw no statistically significant differences between groups. Statistically significant differences are indicated $* P<0.05$, $* * P<0.01$, $* * * P<0.001, * * * * P<0.0001$ (One-Way ANOVA with Tukey's Post-Hoc Test). B) $\alpha$-GalCer and CpG combination therapy does not induce differences between treated and untreated tumours, however, $C p G$ treated tumours saw a significant reduction in Tregs compared to untreated tumours. Statistically significant differences are indicated (Wilcoxon Signed-Rank Test). 
A
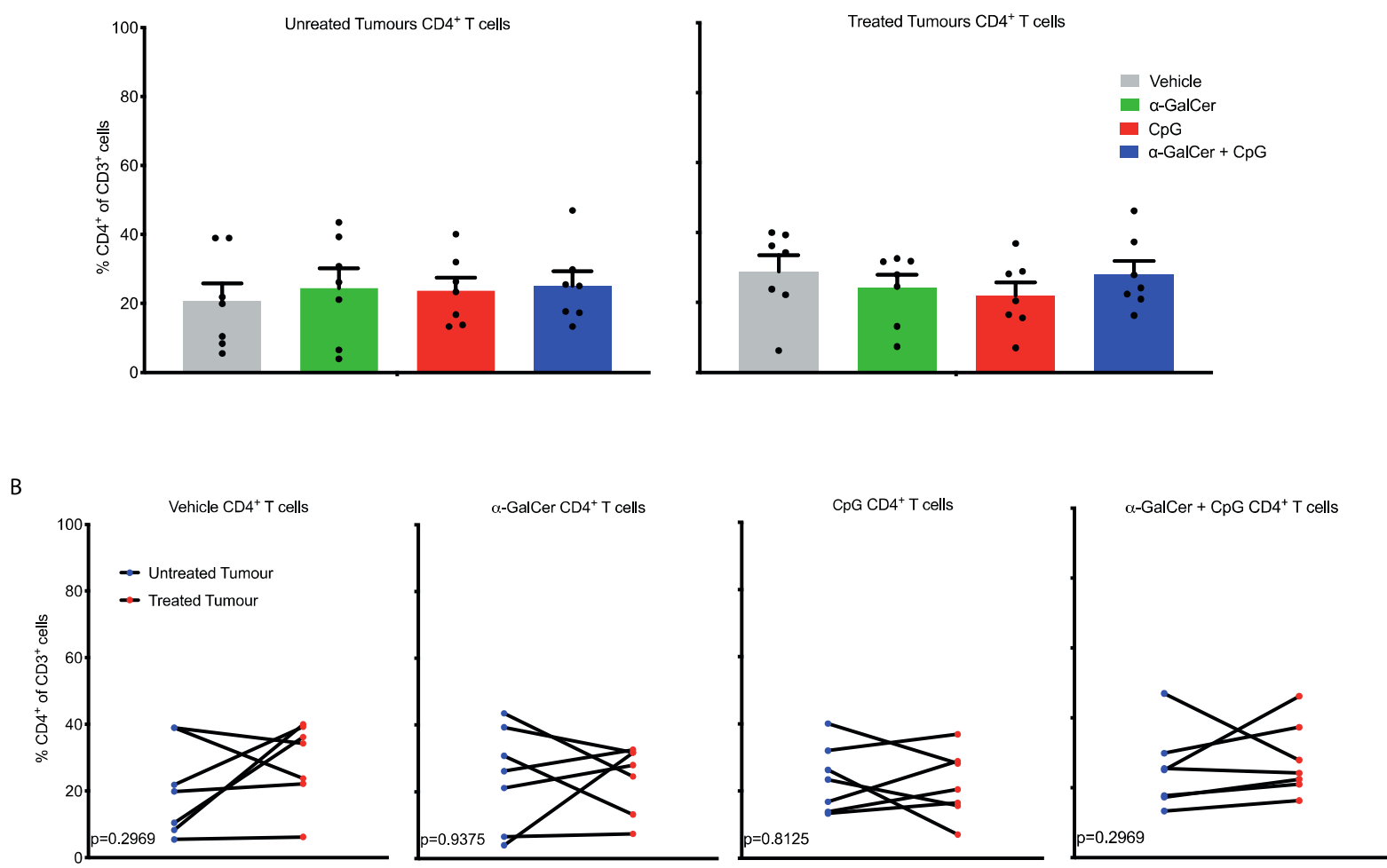

C
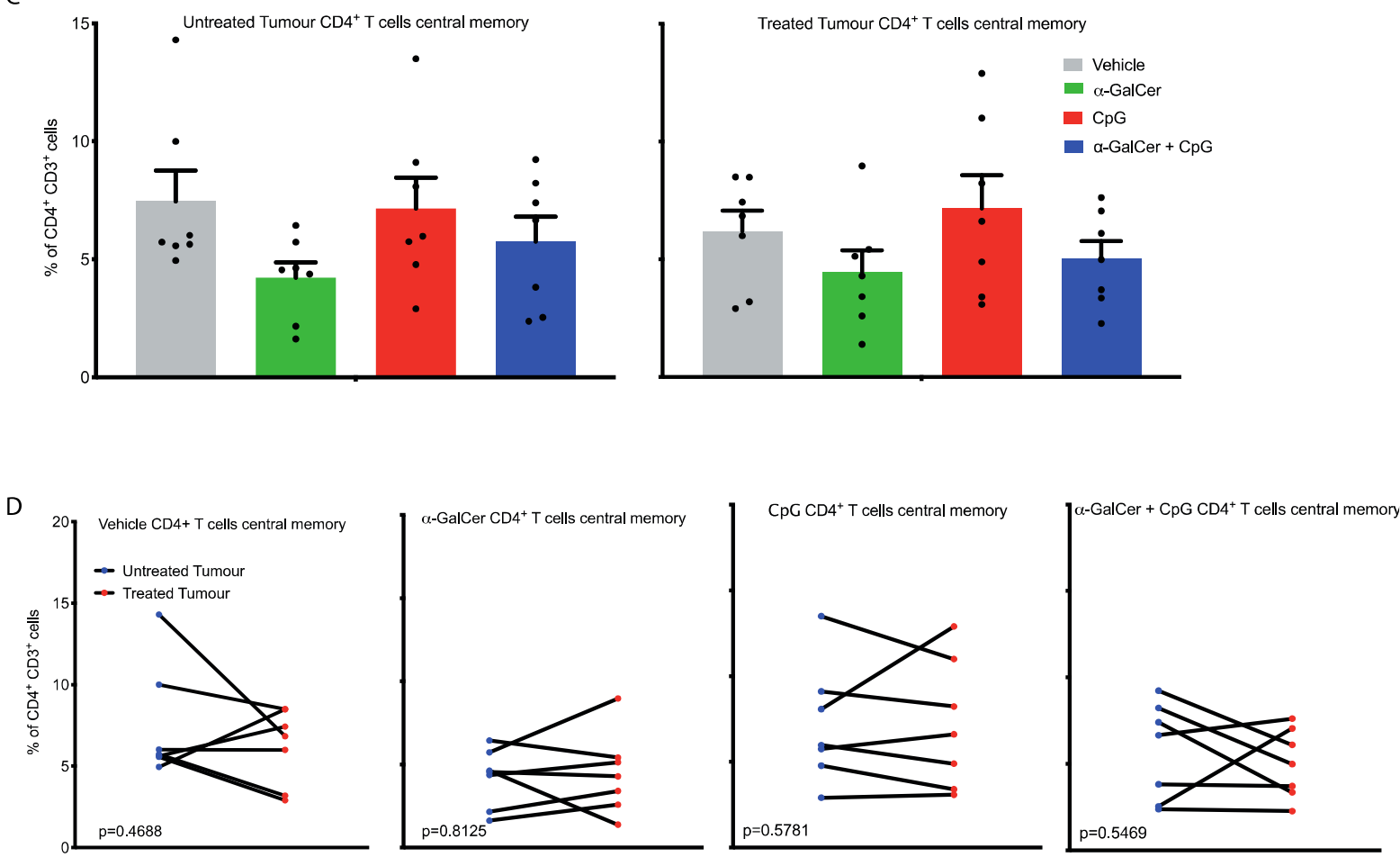

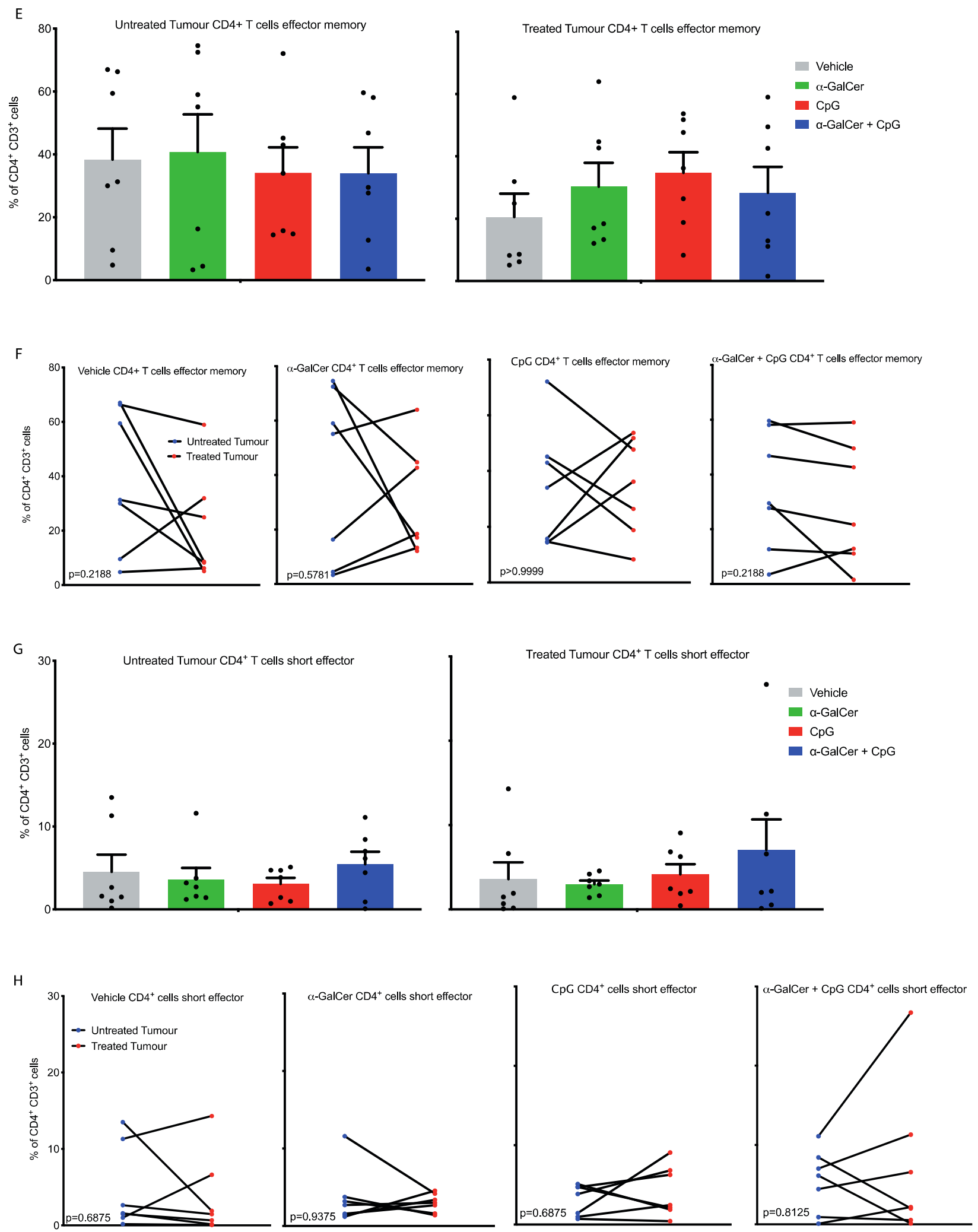

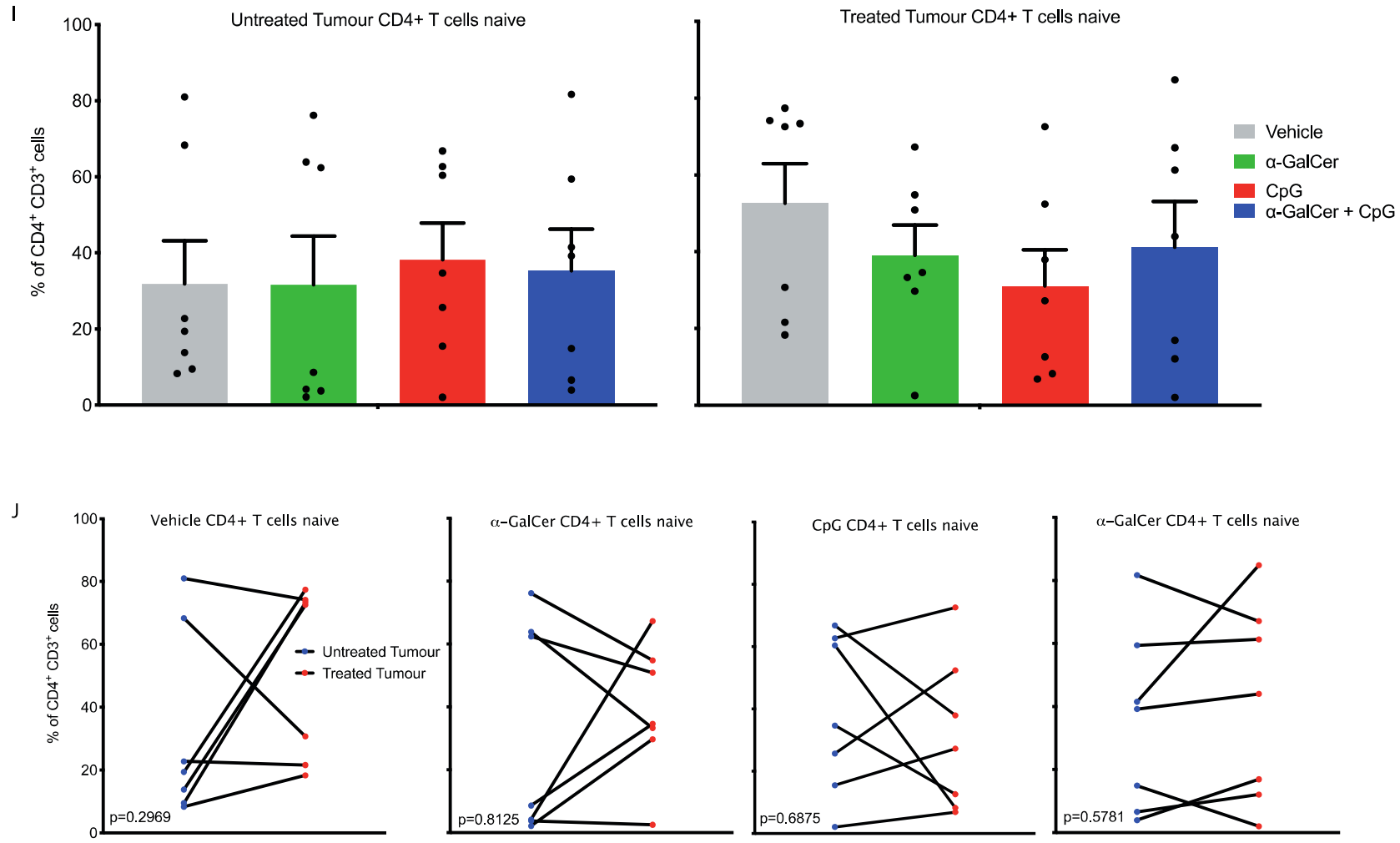

Figure 22 Intratumoural combination therapy of $\alpha$-GalCer and $C p G$ does not induce significant changes in the CD4 ${ }^{+} T$ cell populations in tumours. A) Treated and untreated tumours saw no changes in the overall $C D 4^{+} T$ cell population. B) Treated tumours showed no significant differences to untreated (contralateral tumours) in terms of the overall CD4+ $T$ cell population. C) Treated and untreated tumours saw no changes in the $\mathrm{CD}^{+} \mathrm{T}$ cell central memory population. D) Treated tumours showed no significant differences to untreated (contralateral tumours) in the $\mathrm{CD}^{+} \mathrm{T}$ cell central memory population. E) Combination therapy did not induce changes in the effector memory population. F) Treated tumours did not show significant differences to untreated tumours in the effector memory population. G) Treatment did not induce significant changes in the short lived effector population. H) Treated tumours did not show significant differences to untreated (contralateral tumours) for the short lived effector population. I) Combination therapy did not induce changes in the naïve $\mathrm{CD}^{+} \mathrm{T}$ cell population in both treated and untreated tumours. J) Treated tumours showed no differences in the nä̈ve $C D 4^{+} T$ cell population compared to untreated, contralateral tumours. Statistically significant differences are indicated $* P<0.05, * * P<0.01$, $* * * P<0.001$, $* * * * P<0.0001$ (OneWay ANOVA with Tukey's Post-Hoc Test). Statistically significant differences are indicated (Wilcoxon Signed-Rank Test). 

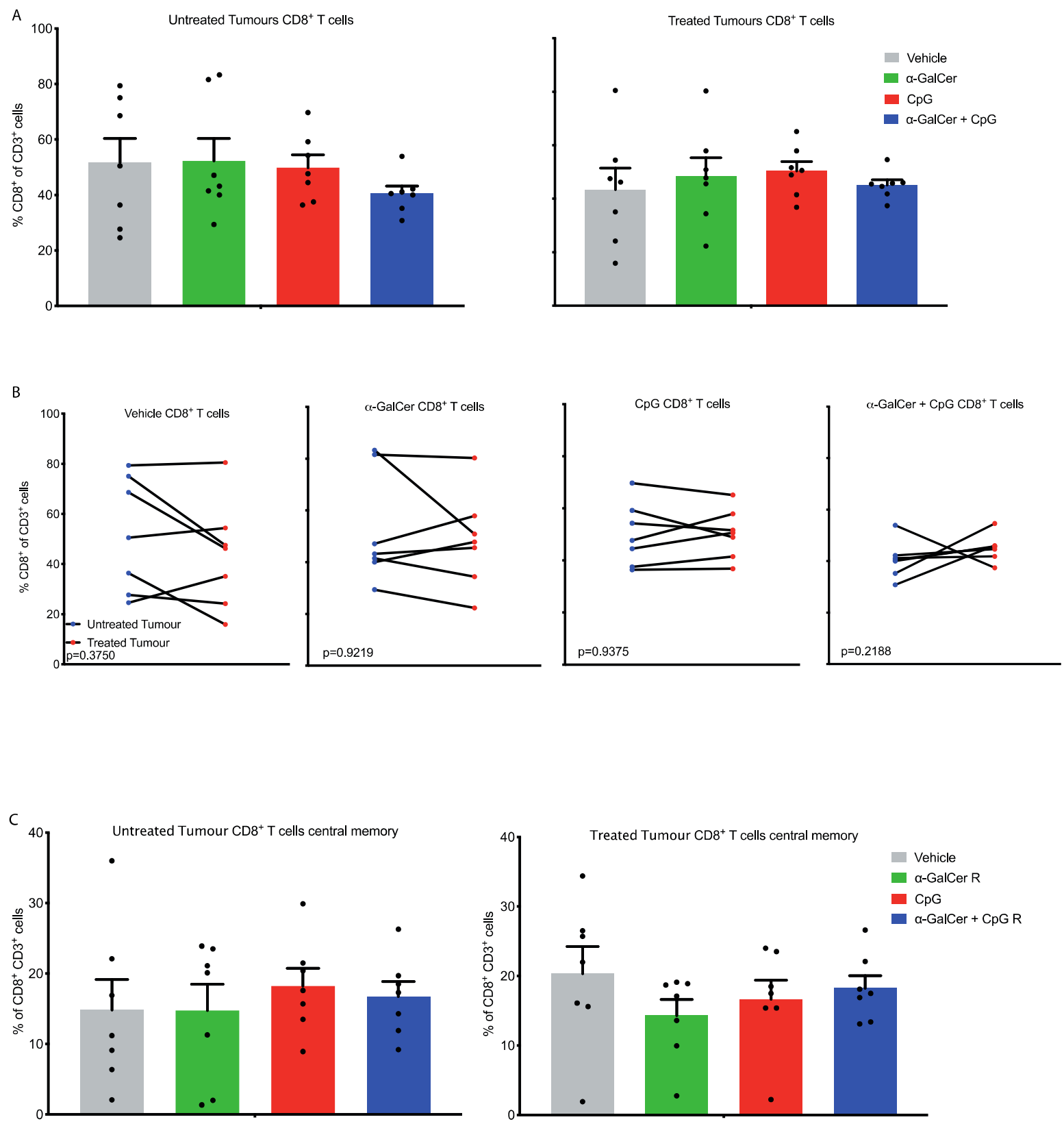

D

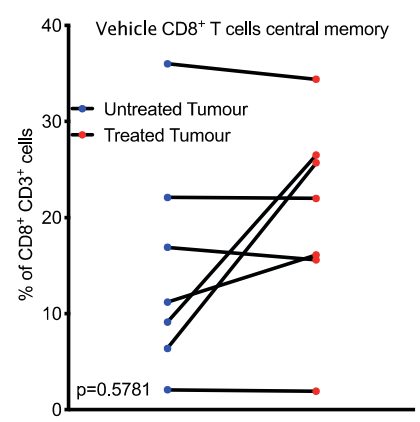

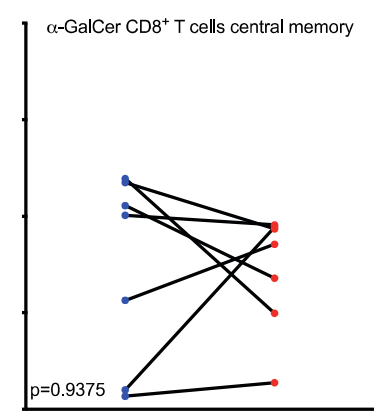

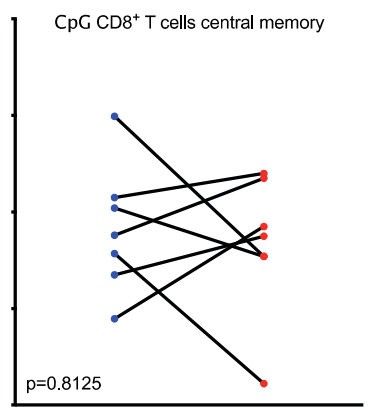

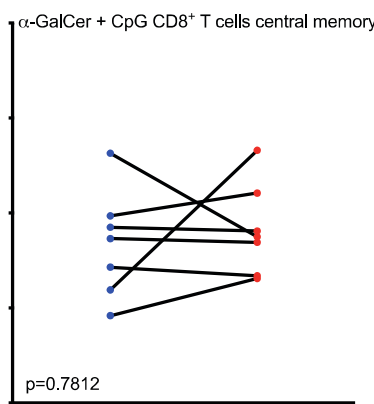


E

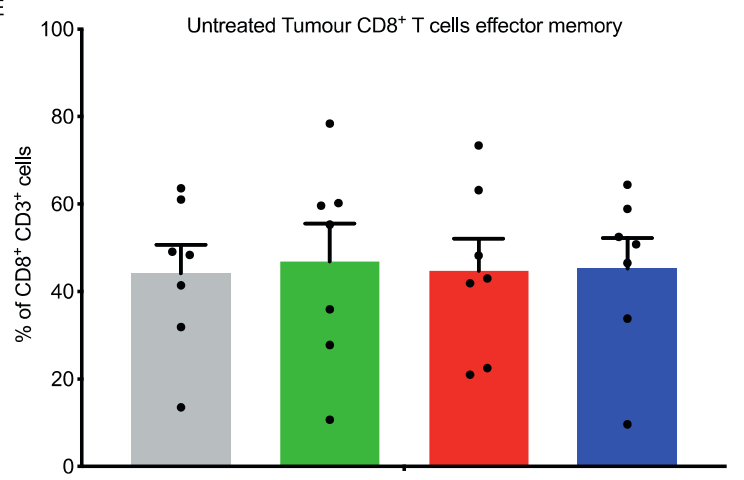

Treated Tumour $\mathrm{CD}^{+} \mathrm{T}$ cells effector memory

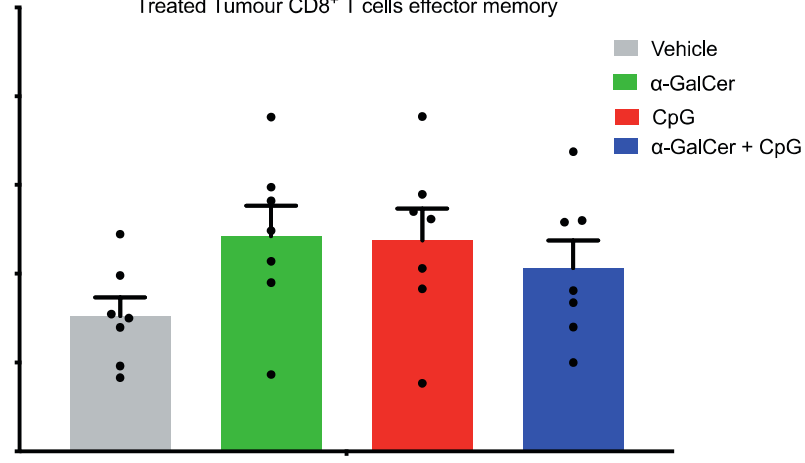

$\mathrm{F}$
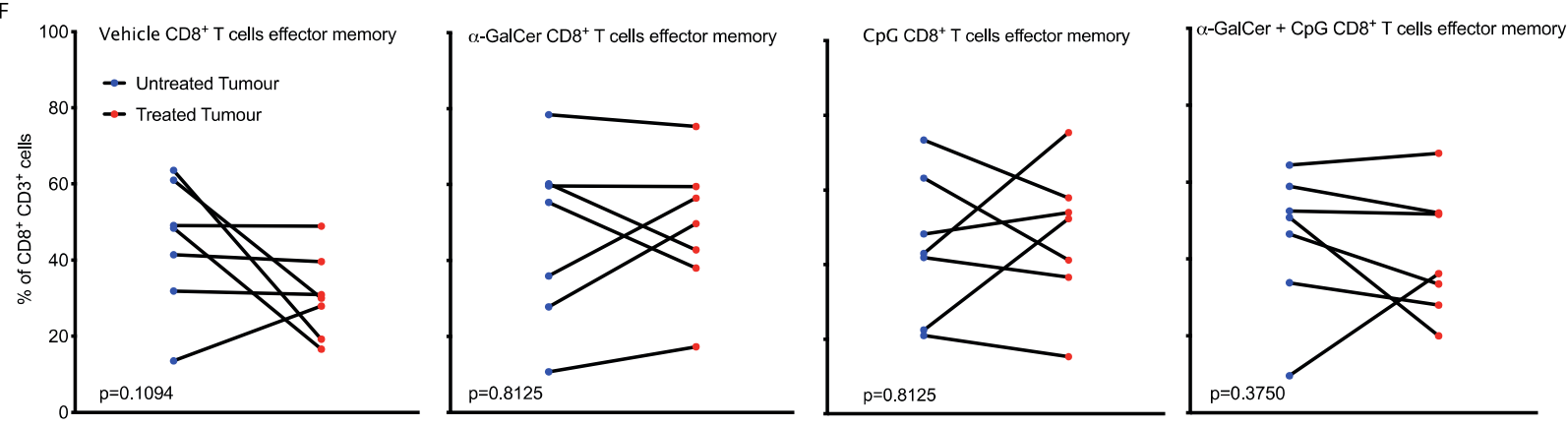
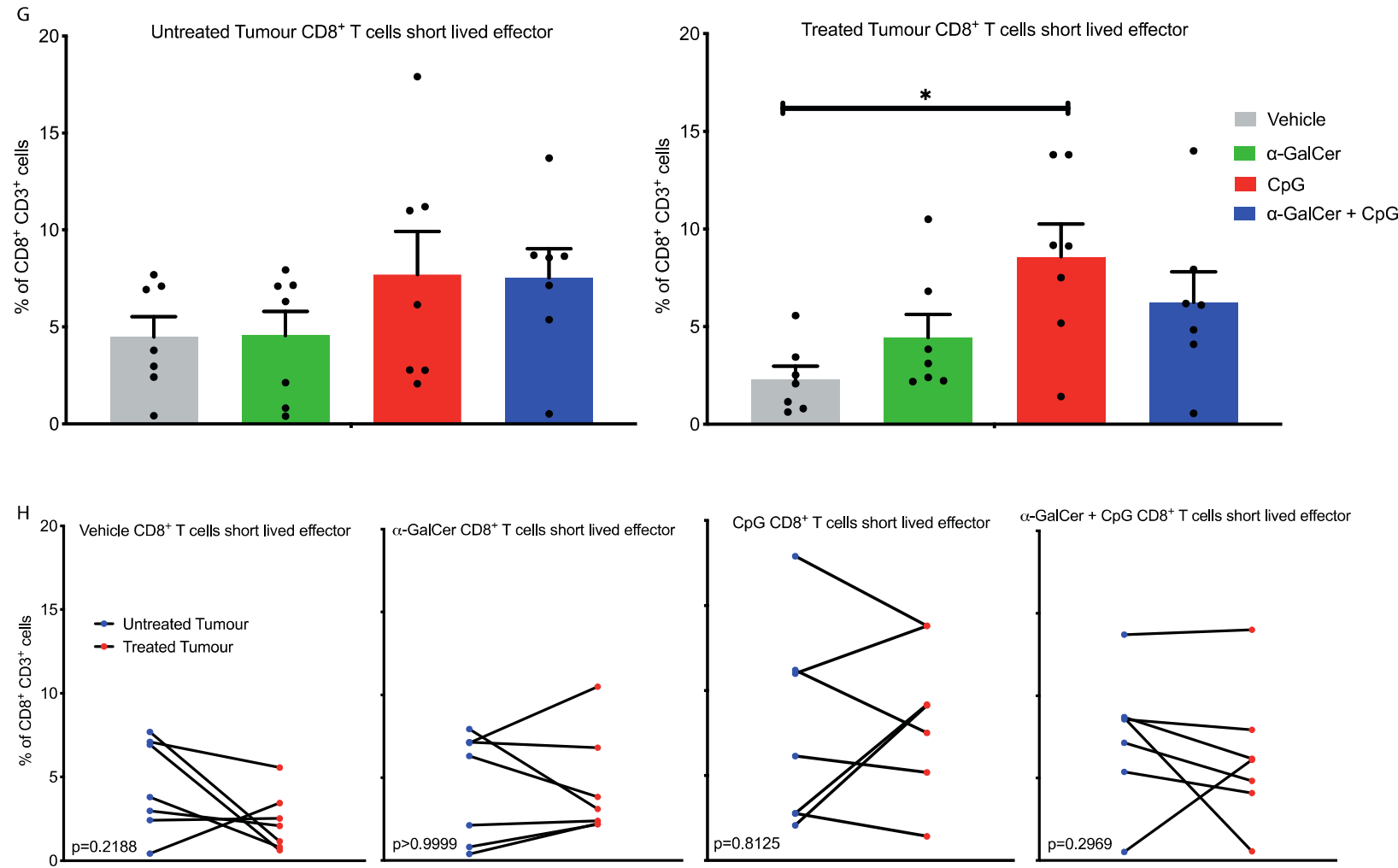

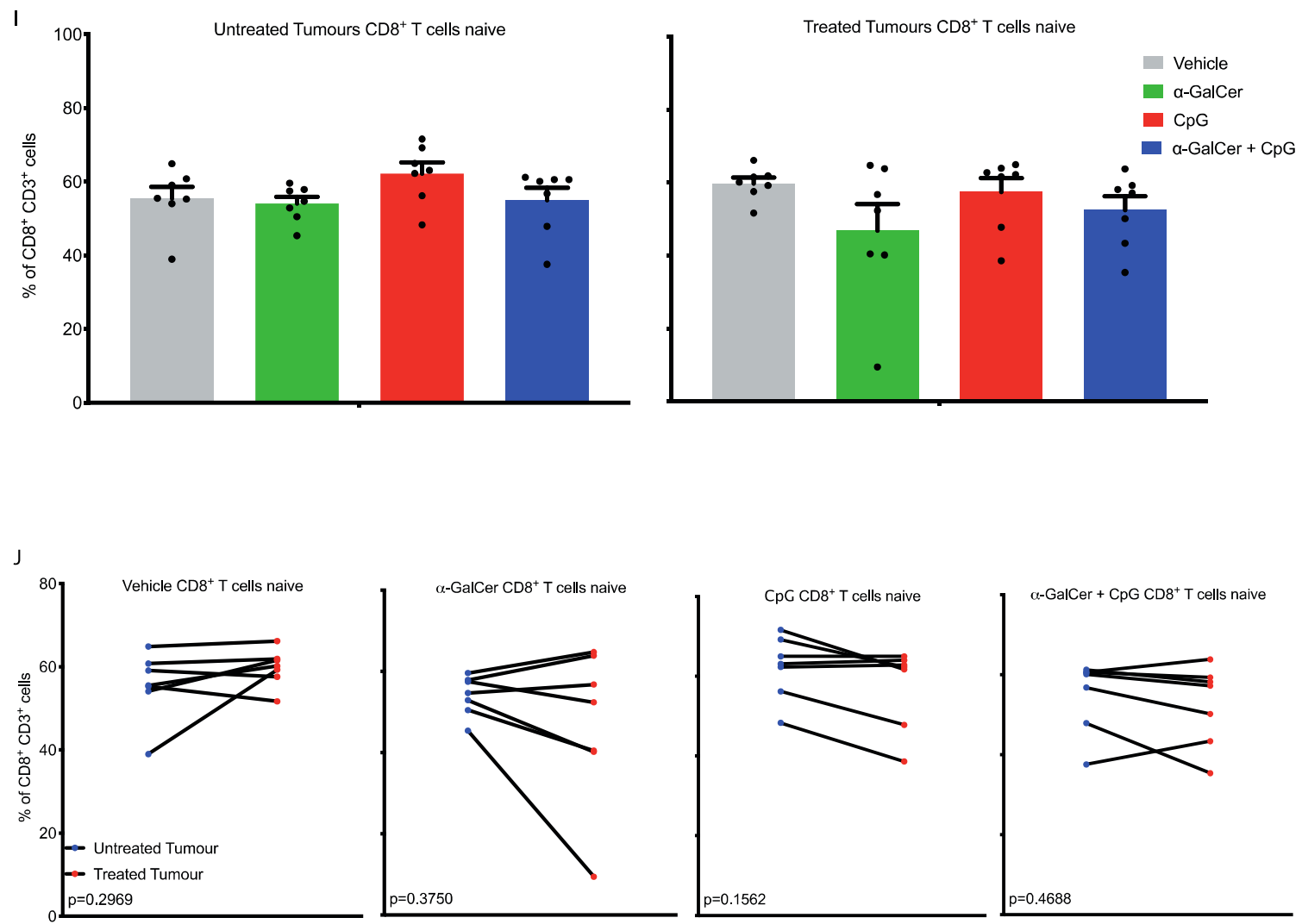

Figure 23 Intratumoural combination therapy of $\alpha$-GalCer and $C p G$ does not induce significant changes in the CD $8^{+} T$ cell populations in tumours. A) Treated and untreated tumours saw no changes in the overall $C D 8^{+} T$ cell population. B) Treated tumours showed no significant differences to untreated (contralateral tumours) in terms of the overall CD $8^{+} T$ cell population. C) Treated and untreated tumours saw no changes in the CD8+ $T$ cell central memory population. D) Treated tumours showed no significant differences to untreated (contralateral tumours) in the CD8 $8^{+} T$ cell central memory population. E) Combination therapy did not induce changes in the effector memory population. F) Treated tumours did not show significant differences to untreated tumours in the effector memory population. G) CpG induced a significant increase in short lived effectors over vehicle. H) Treated tumours did not show significant differences to untreated (contralateral tumours) for the short lived effector population. I) Combination therapy did not induce changes in the naïve $C D 8^{+} T$ cell population in both treated and untreated tumours. J) Treated tumours showed no differences in the naïve $C D 8^{+}$T cell population compared to untreated, contralateral tumours. Statistically significant differences are indicated $* P<0.05, * * P<0.01$, *** $P<0.001$, ****P<0.0001 (One-Way ANOVA with Tukey's Post-Hoc Test). Statistically significant differences are indicated (Wilcoxon Signed-Rank Test).

Of particular note, there was an increase in the frequency of iNKT cells with the combined treatment over controls that was not seen with $\alpha$-GalCer alone, suggesting the additional $\mathrm{CpG}$ treatment facilitated this accumulation. This was seen in both the treated and untreated tumours (Fig 24A). When broken down into the subpopulations, no strong conclusions 
could be drawn, although there was an increase in $\mathrm{CD}^{+}{ }^{+}$iNKT cells with the combined treatment, which was significant in the untreated tumours, (Fig 24E), while there was a reduction in NK1.1 ${ }^{+}$iNKT cells (Fig 24G). This reduction is possibly due to the downregulation of NK1.1 when iNKT cells are activated. When directly comparing untreated tumours to treated tumours the only significant difference was when $\mathrm{CpG}$ alone was used, with an increase in the frequency of NK1.1 ${ }^{+}$iNKT cells observed in treated tumours relative to untreated (Fig 24H). As for B cells, there was no significant change to the population in the tumours associated with treatment (Fig 25).
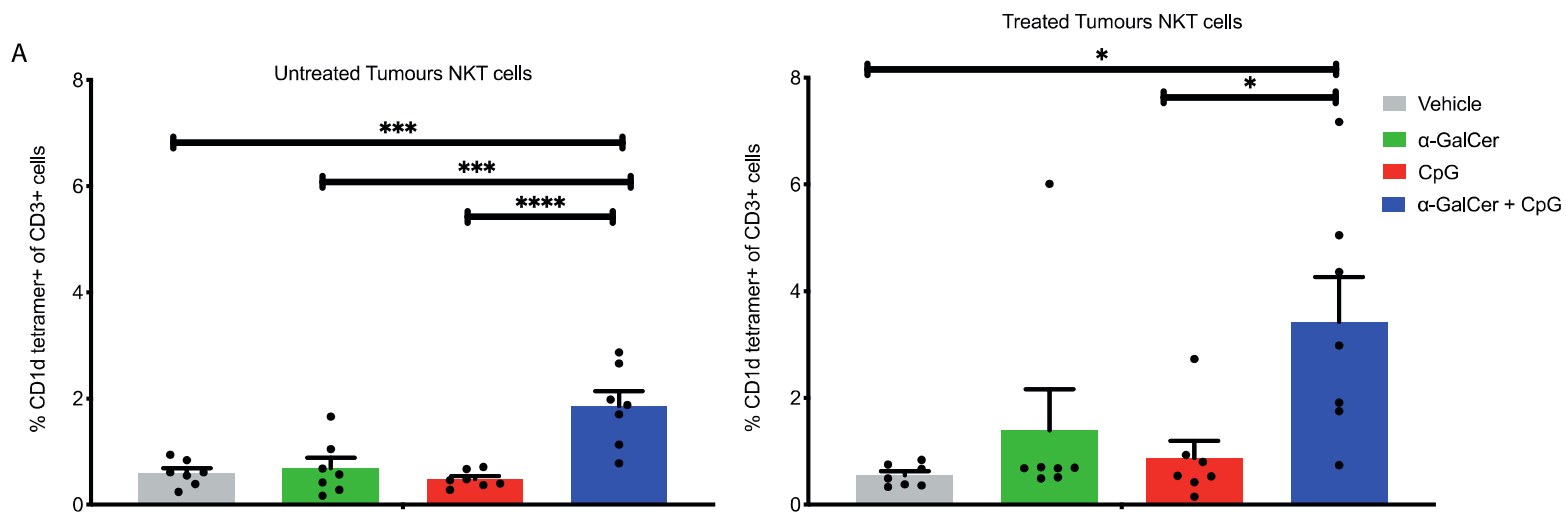

B

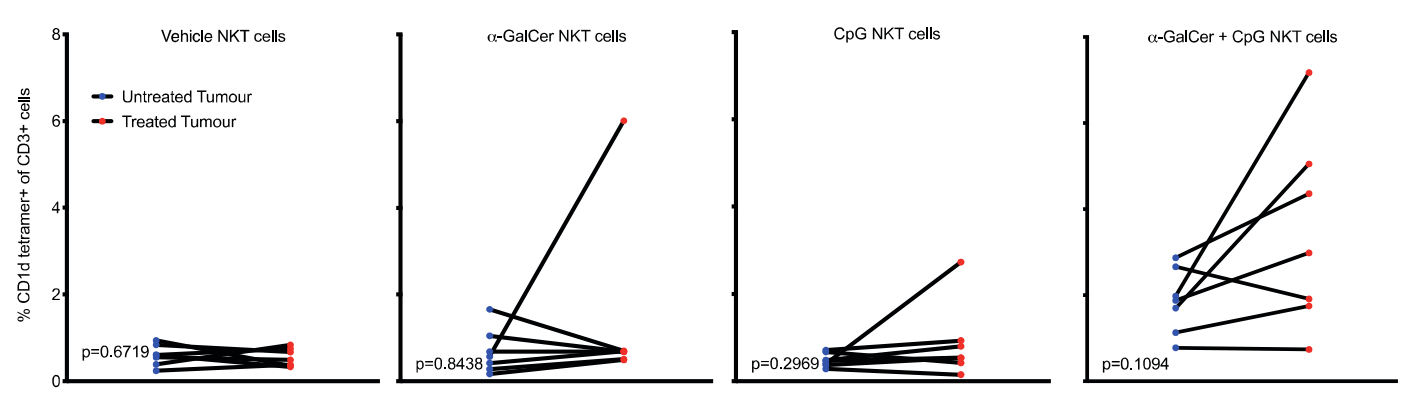


C

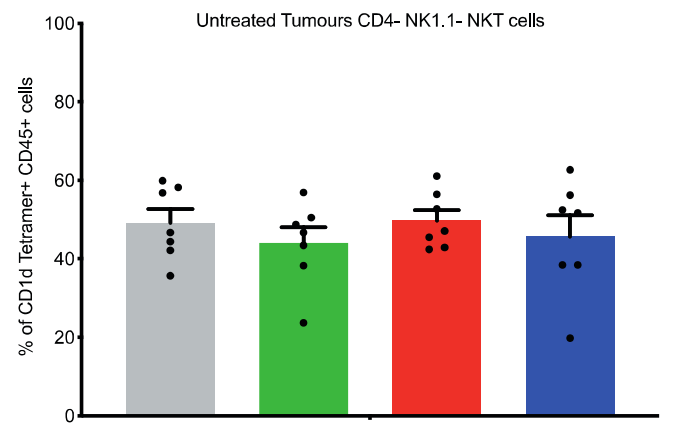

D
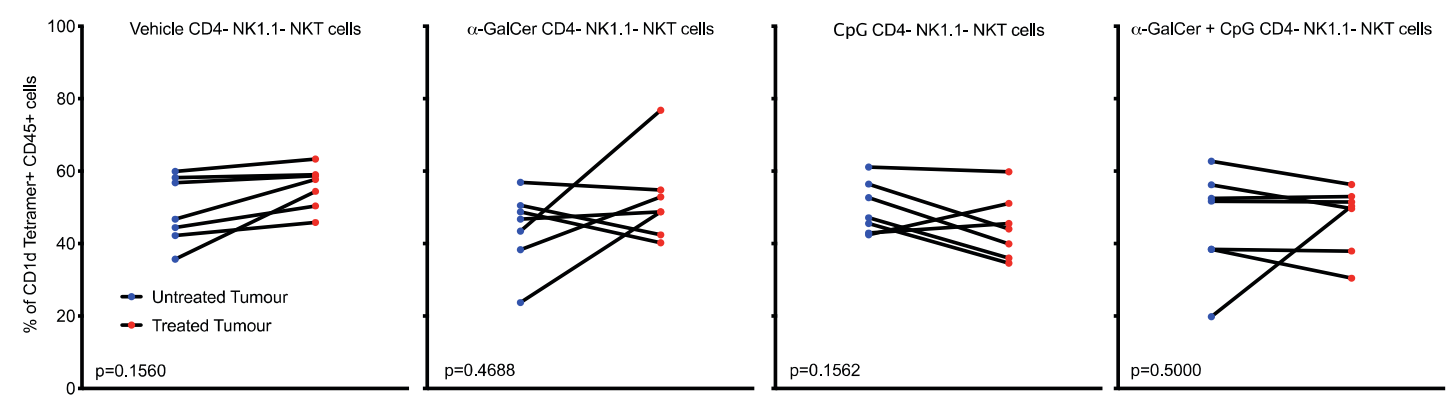

E
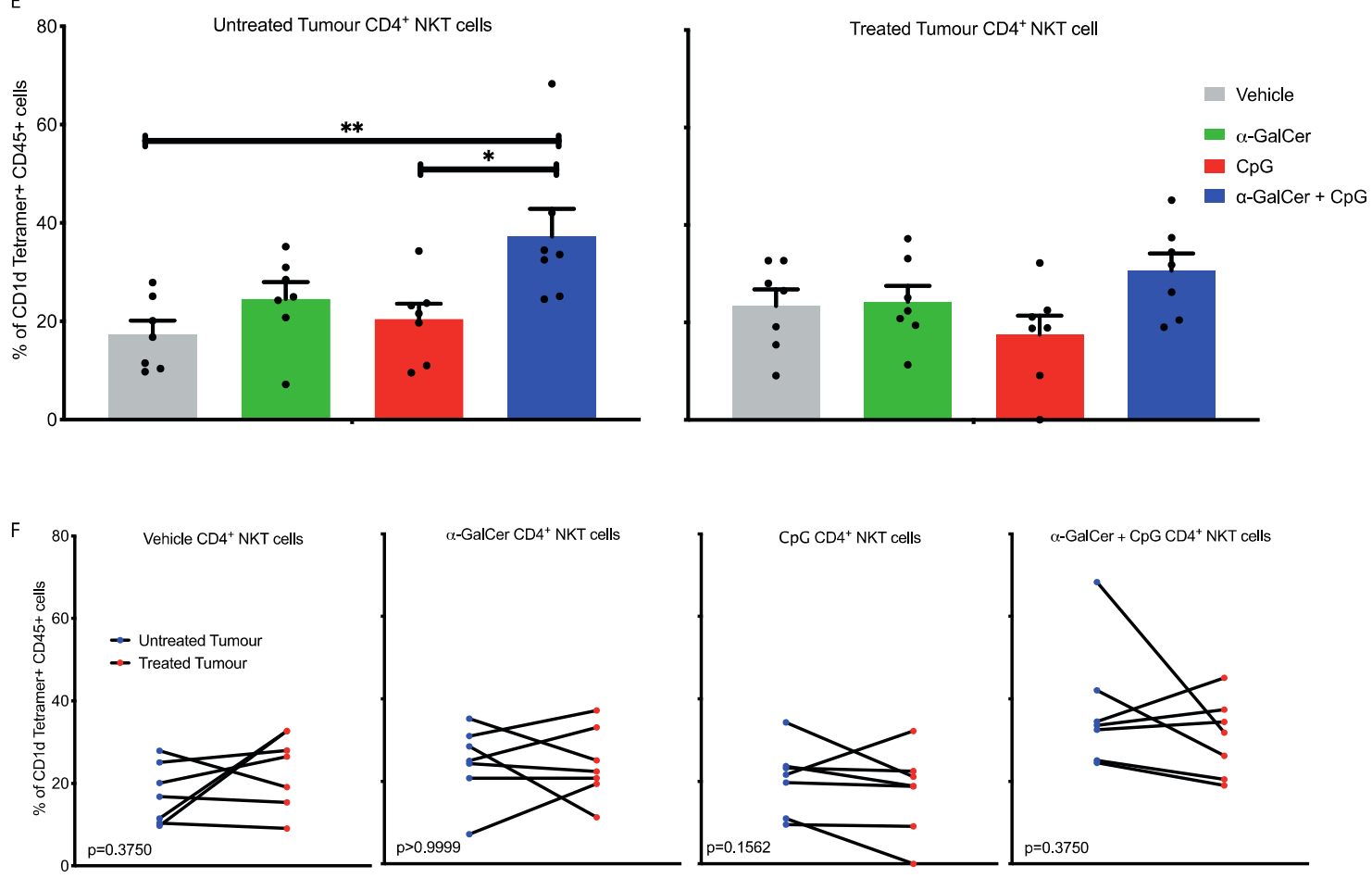

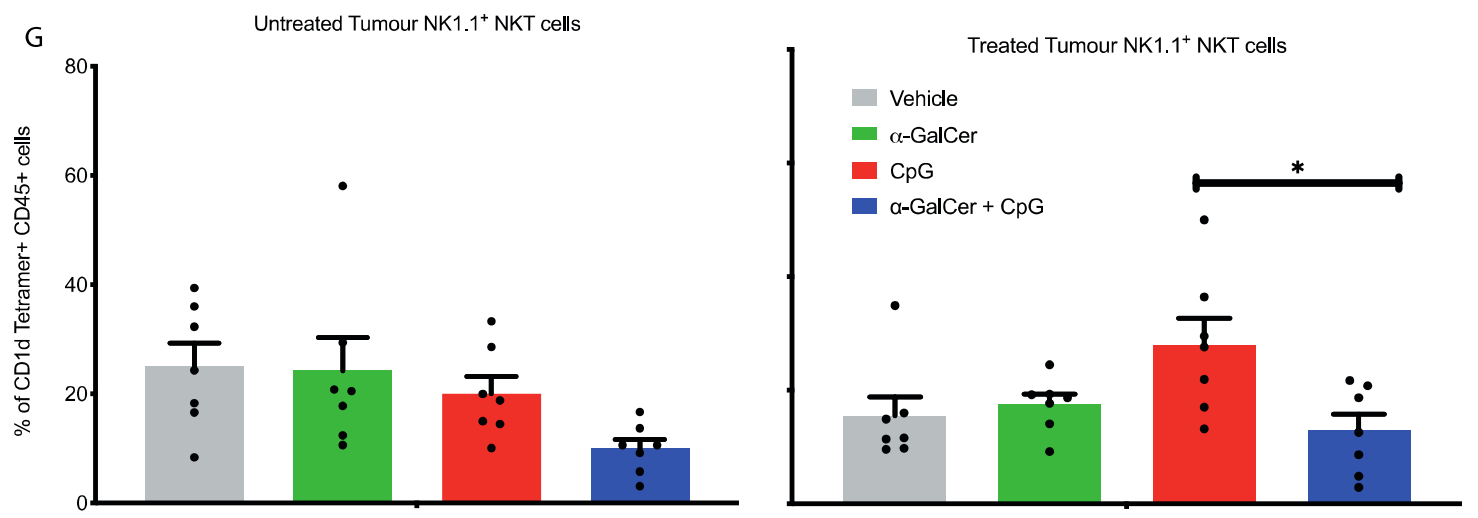

H
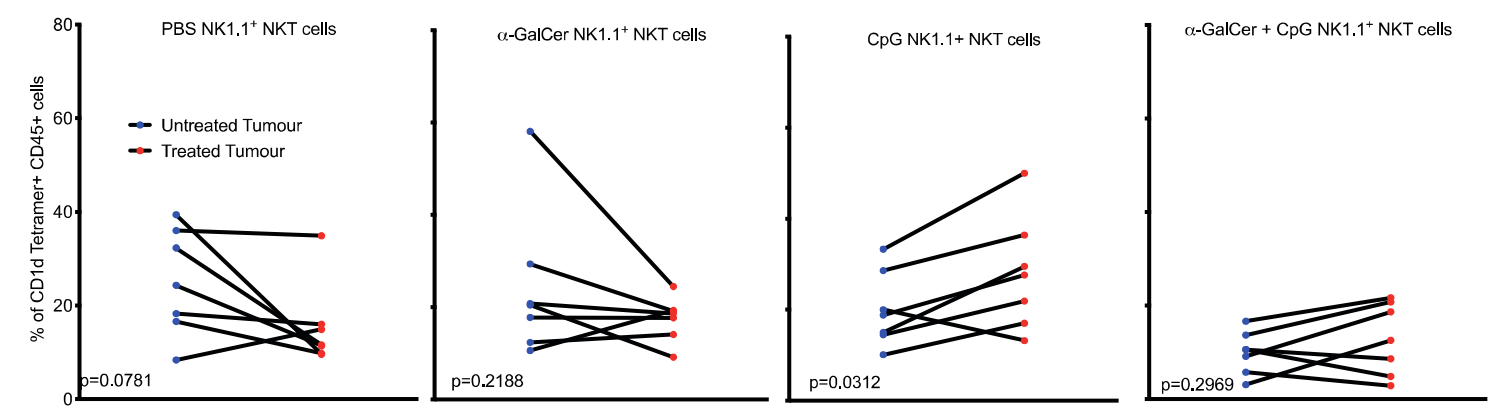

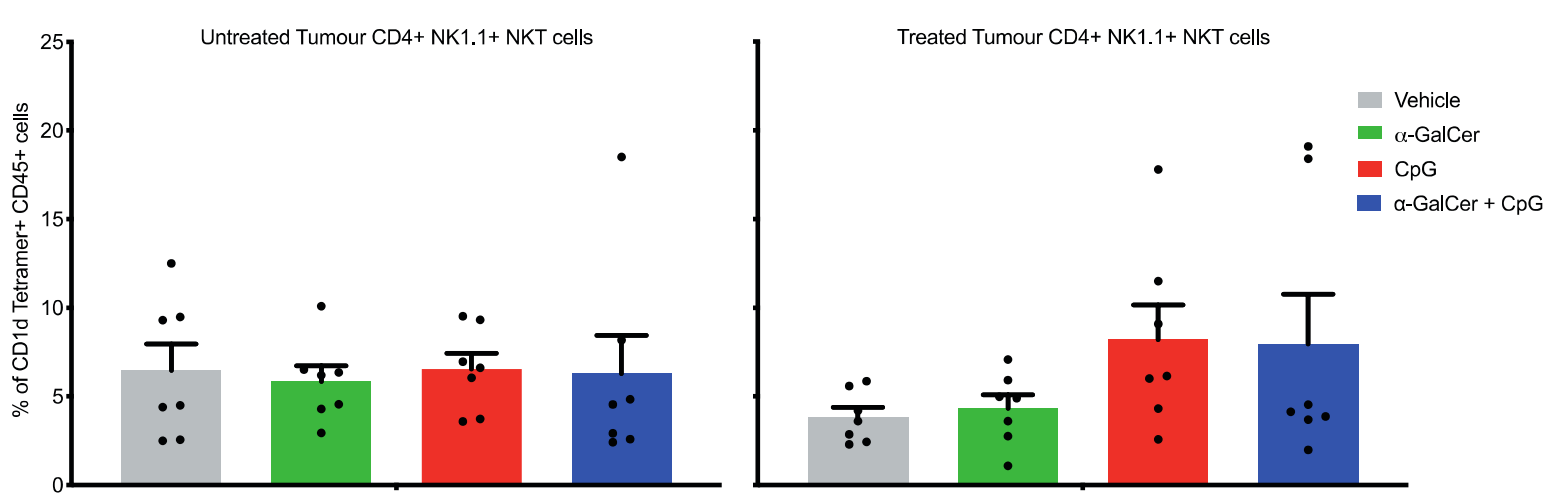

J
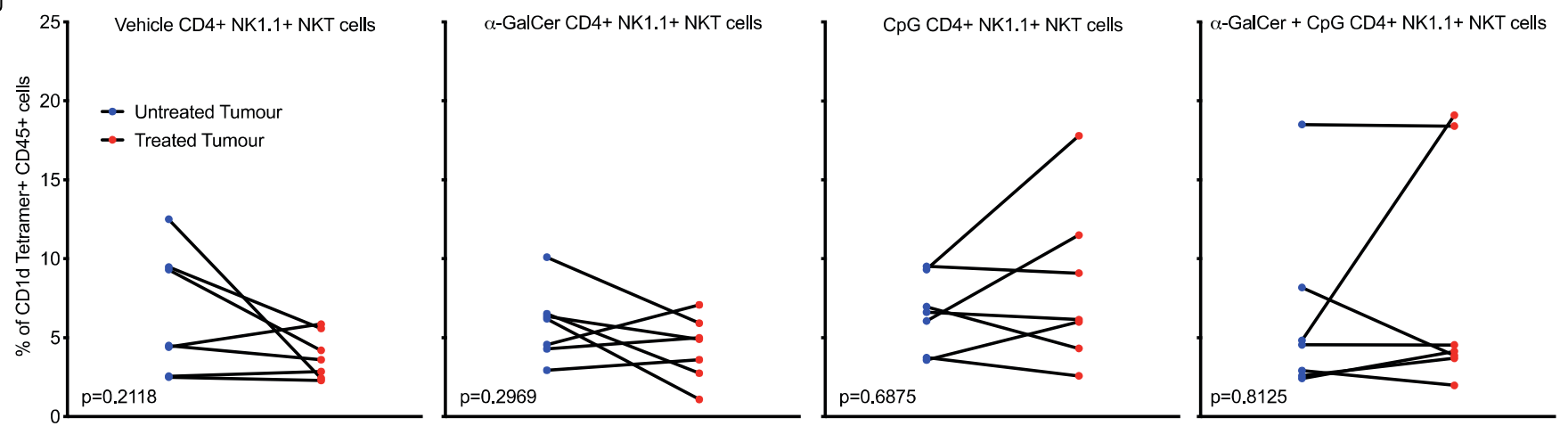

Figure 24 Intratumoural combination therapy of $\alpha$-GalCer and $C p G$ induces significant changes to the iNKT cell populations in tumours. A) Combination therapy treated tumours saw a significant increase in the frequency of iNKT cells compared to vehicle and $C p G$ treated tumours. In the untreated (contralateral) tumours, combination therapy also induced significant increases in iNKT cell frequency compared to vehicle or CpG treated animals. B) Treated tumours showed no significant differences to untreated (contralateral tumours) in terms of the overall iNKT cell population. $C$ ) Treated and untreated tumours saw no changes in the CD4- NK1.1- iNKT cell population. D) Treated tumours showed no significant differences to untreated (contralateral) tumours for the CD4- NK1.1- iNKT cell population. E) Combination therapy induced an increase in CD4+iNKT cell frequency in untreated tumours compared to untreated tumours on vehicle and CpG treated animals. F) Treated tumours did not show significant differences to untreated tumours in the $C D 4^{+} i N K T$ cell population. G) CpG treatment induced a significant increase in NK1.1 $1^{+}$iNKT cells compared to combination therapy in treated tumours. $H$ ) CpG treated tumours saw a significant increase in NK1.1 ${ }^{+}$iNKT cells compared to untreated (contralateral tumours). I) Combination therapy did not induce significant

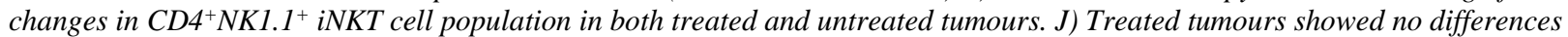
in the $C D 4^{+} N K 1.1^{+} i N K T$ cell population compared to untreated, contralateral tumours. Statistically significant differences are indicated $* P<0.05$, **P<0.01, ***P<0.001, ****P<0.0001 (One-Way ANOVA with Tukey's Post-Hoc Test). Statistically significant differences are indicated (Wilcoxon Signed-Rank Test). 
A

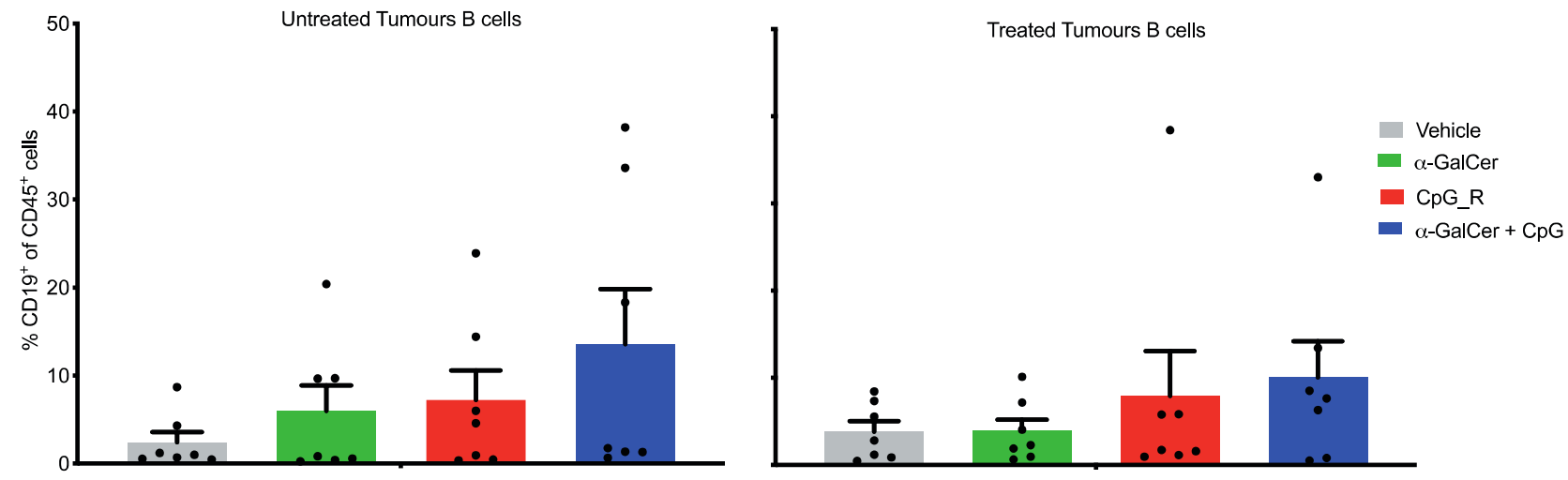

B
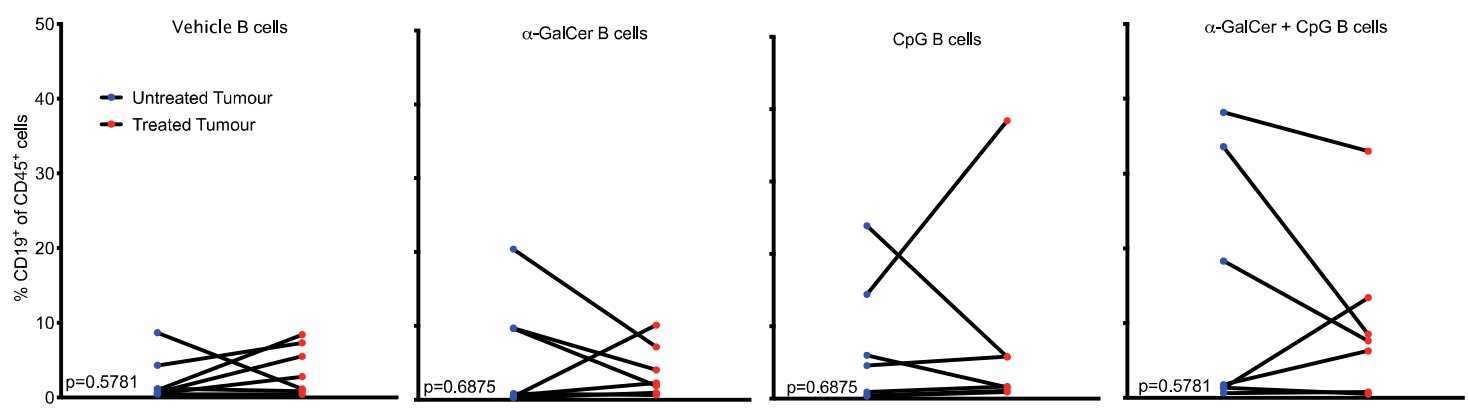

Figure 25 Intratumoural combination therapy of $\alpha$-GalCer and $C p G$ does not increase the frequency of $B$ cells in tumours. A) Both treated and untreated (contralateral) tumours exhibited no change in $B$ cell frequency as a result of treatment. Statistically significant differences are indicated $* P<0.05, * * P<0.01, * * * P<0.001, * * * * P<0.0001$ (One-Way ANOVA with Tukey's Post-Hoc Test). B) $\alpha$-GalCer and CpG combination therapy does not induce differences between treated and untreated tumours. Statistically significant differences are indicated (Wilcoxon Signed-Rank Test).

\subsubsection{Immune profile of the tumour draining lymph node}

In the tumour draining lymph node, flow cytometric analysis demonstrated that, as observed in the blood and tumour, there was no significant change in the frequency of OVAspecific T cells between treatment groups (Fig 26A). Accordingly, there was also no significant change in the frequency of OVA-specific T cells between lymph nodes draining from the treated or untreated tumours for each of the treatments (Fig 26B). 
A

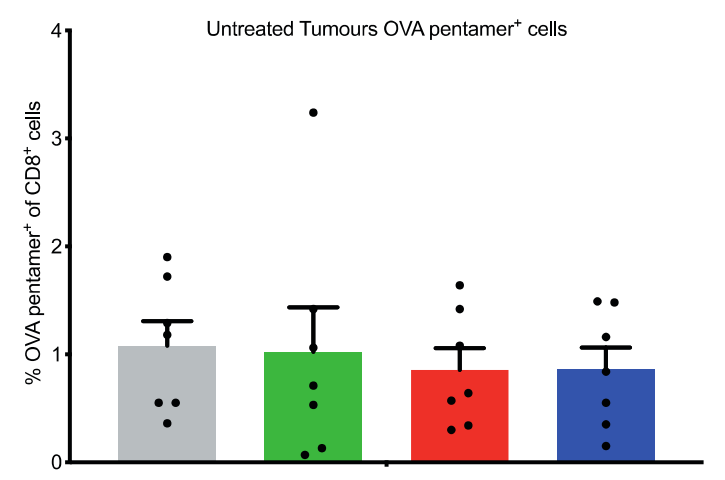

B

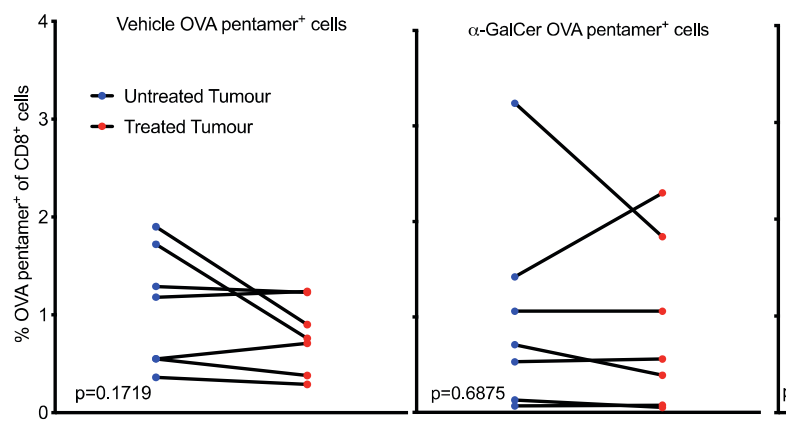

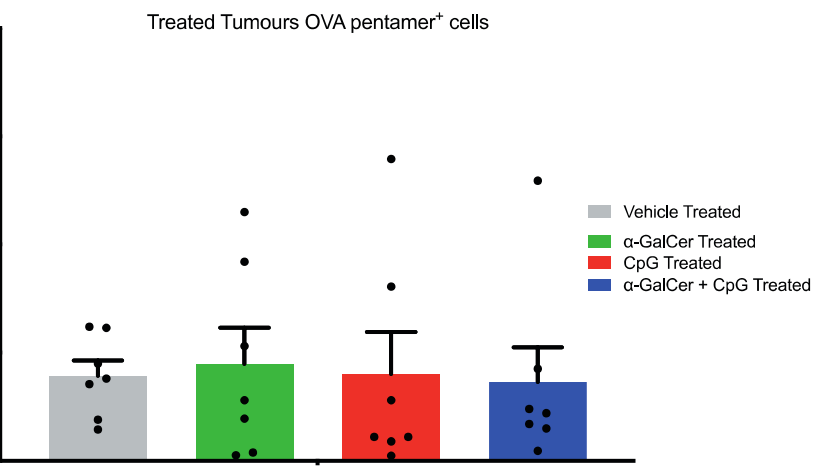
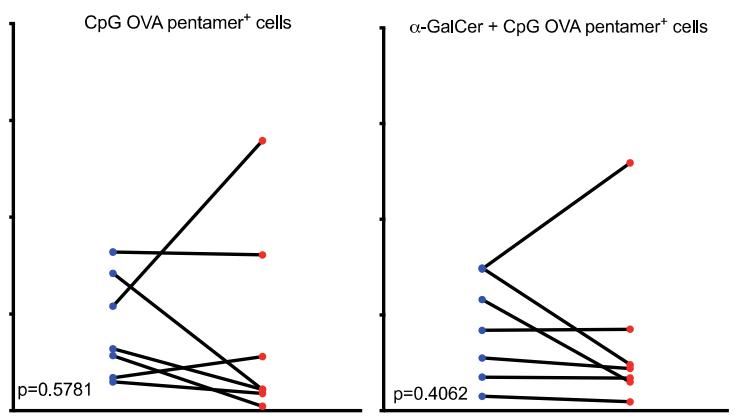

Figure 26 Intratumoural combination therapy of $\alpha$-GalCer and CpG does not increase the frequency of $O V A-s p e c i f i c$ CD8 ${ }^{+} T$ cells in the draining lymph node $(d L N)$. A) Both dLNs near treated and untreated (contralateral) tumours exhibited no change in $O V A$-specific $T$ cells as a result of treatment. Statistically significant differences are indicated $* P<0.05$, $* * P<0.01, * * * P<0.001$, ****P<0.0001 (One-Way ANOVA with Tukey's Post-Hoc Test). B) $\alpha$-GalCer and CpG combination therapy does not induce differences between dLNs near treated and untreated tumours. Statistically significant differences are indicated (Wilcoxon Signed-Rank Test).

Interestingly, the combination induced a significant reduction in the frequency of regulatory T cells in the treated tumour dLN compared to vehicle or $\alpha$-GalCer-treatment alone; however, this was not observed in the untreated tumours, suggesting it was a local effect related to the treatment (Fig 27A and B). There was no significant difference in the $\mathrm{CD} 4^{+} \mathrm{T}$ cell populations (Fig 28). For the $\mathrm{CD} 8^{+} \mathrm{T}$ cell population, the combination treatment induced an increase in $\mathrm{CD} 8^{+} \mathrm{T}$ cell frequency in dLNs draining the treated tumours compared untreated tumours in the group, perhaps reflecting a site of priming (Fig 29B). The dLN of treated tumours of combination therapy treated mice saw an increase in the frequency of CD8+ central memory cells compared to the dLN of the untreated tumours (Fig 29D). Similarly, there was an increase 
in the frequency of short-lived effector cells as a result of combination therapy in the dLN of treated tumours compared to untreated tumours (Fig 29H).
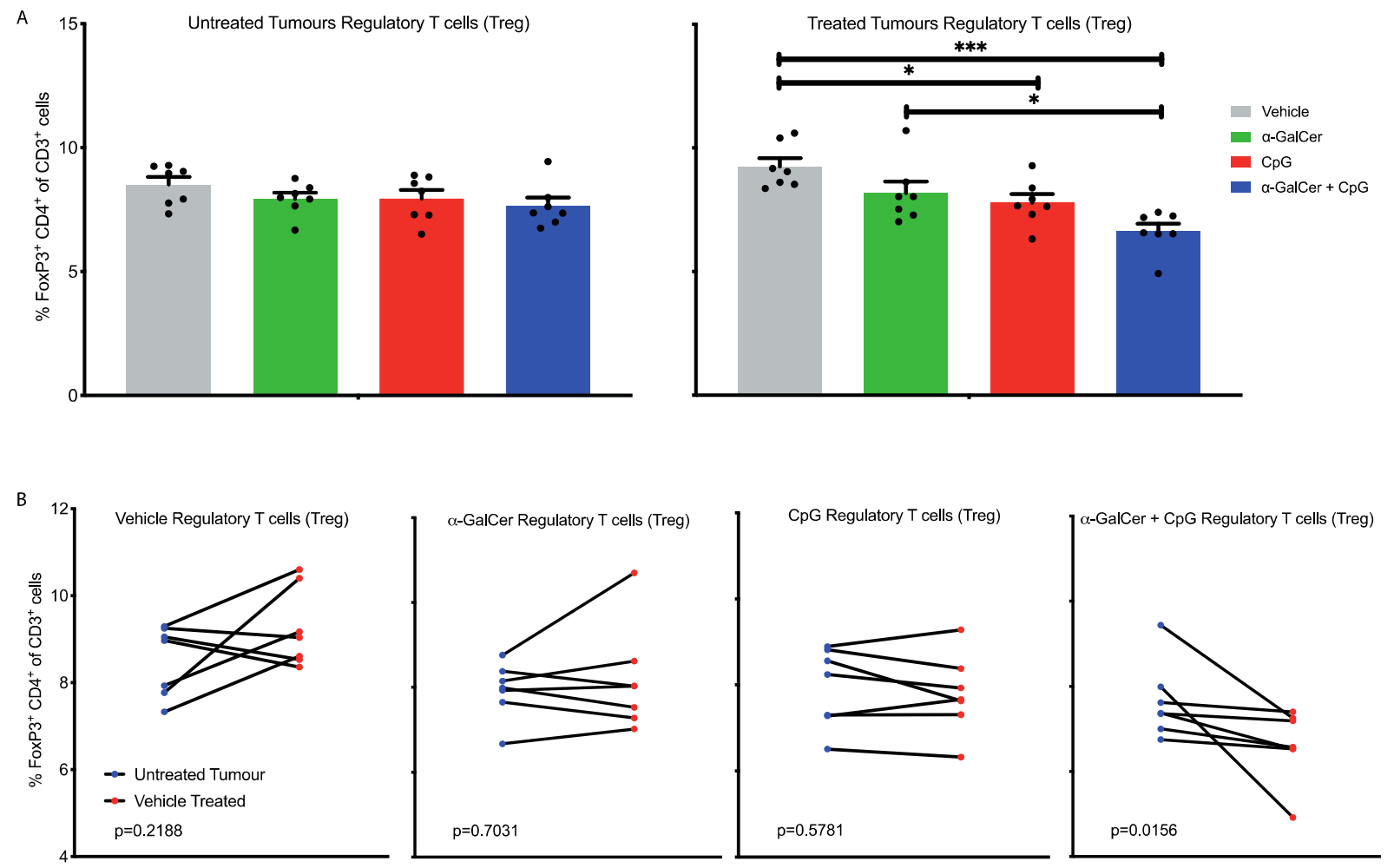

Figure 27 Intratumoural combination therapy of $\alpha$-GalCer and $C p G$ decreases the proportion of regulatory $T$ cells (Tregs) in $d L N$ near treated tumours relative to $\alpha$-GalCer and vehicle. A) dLN near tumours treated with the combination saw a significant reduction in Tregs relative to either vehicle or $\alpha$-GalCer as a single agent, CpG also induced a significant reduction in Tregs compared to vehicle. Untreated tumours saw no statistically significant differences between groups. Statistically significant differences are indicated $* P<0.05, * * P<0.01, * * * P<0.001$, ****P<0.0001 (One-Way ANOVA with Tukey's Post-Hoc Test). B) $\alpha$-GalCer and CpG combination therapy induced a reduction in Tregs in dLN near treated tumours compared to dLN near untreated tumours. Statistically significant differences are indicated (Wilcoxon Signed-Rank Test). 
A

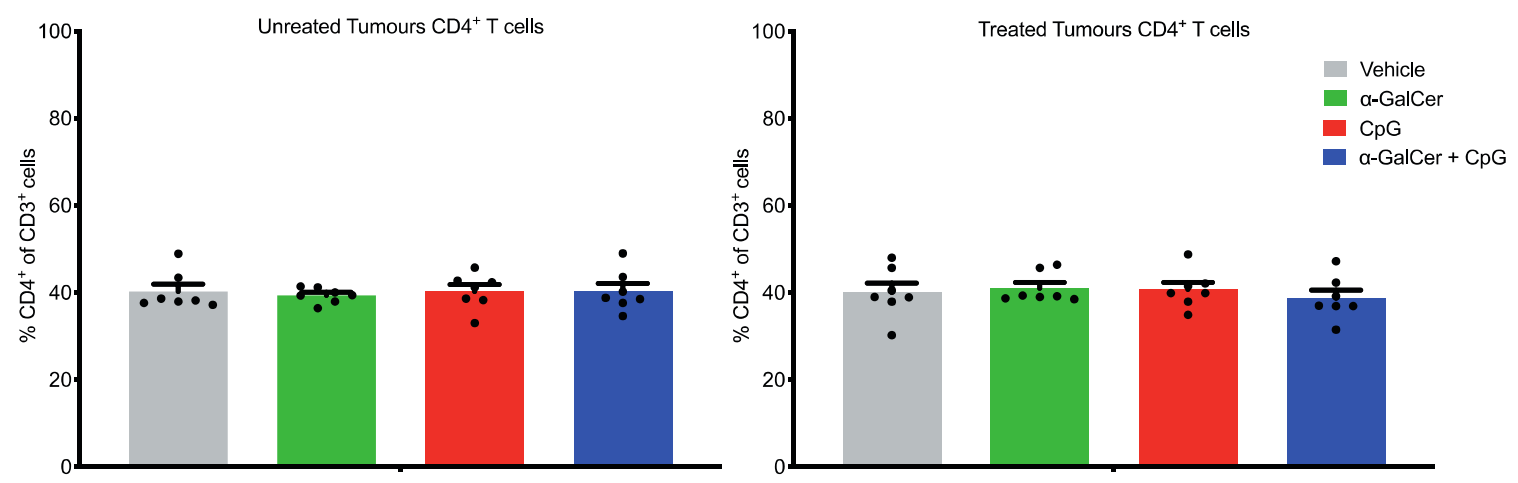

B

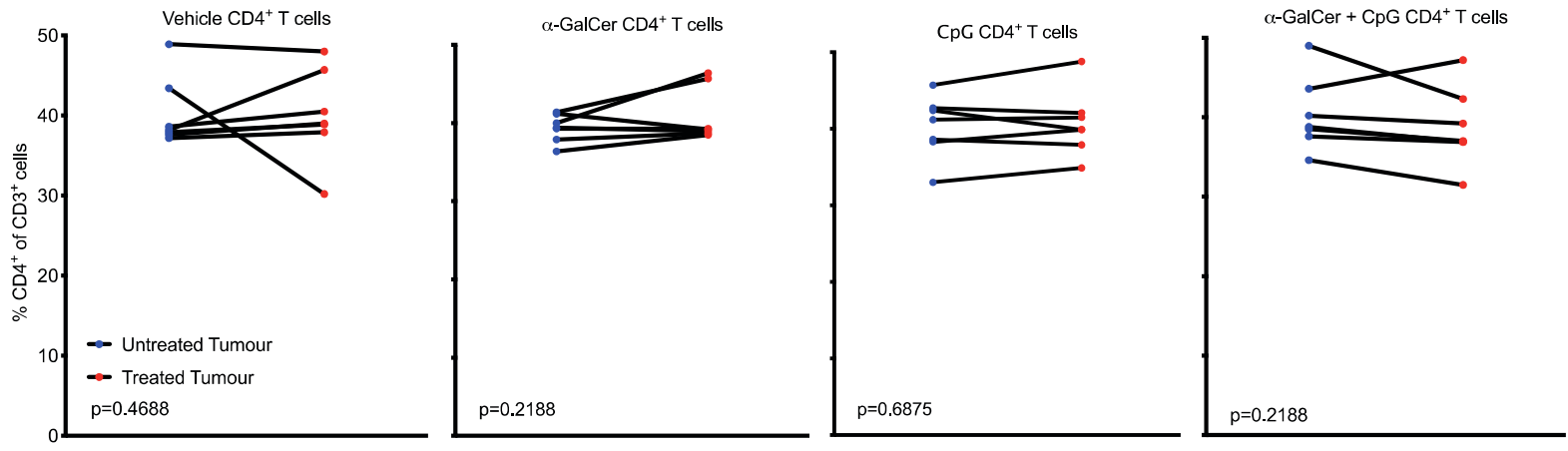


C
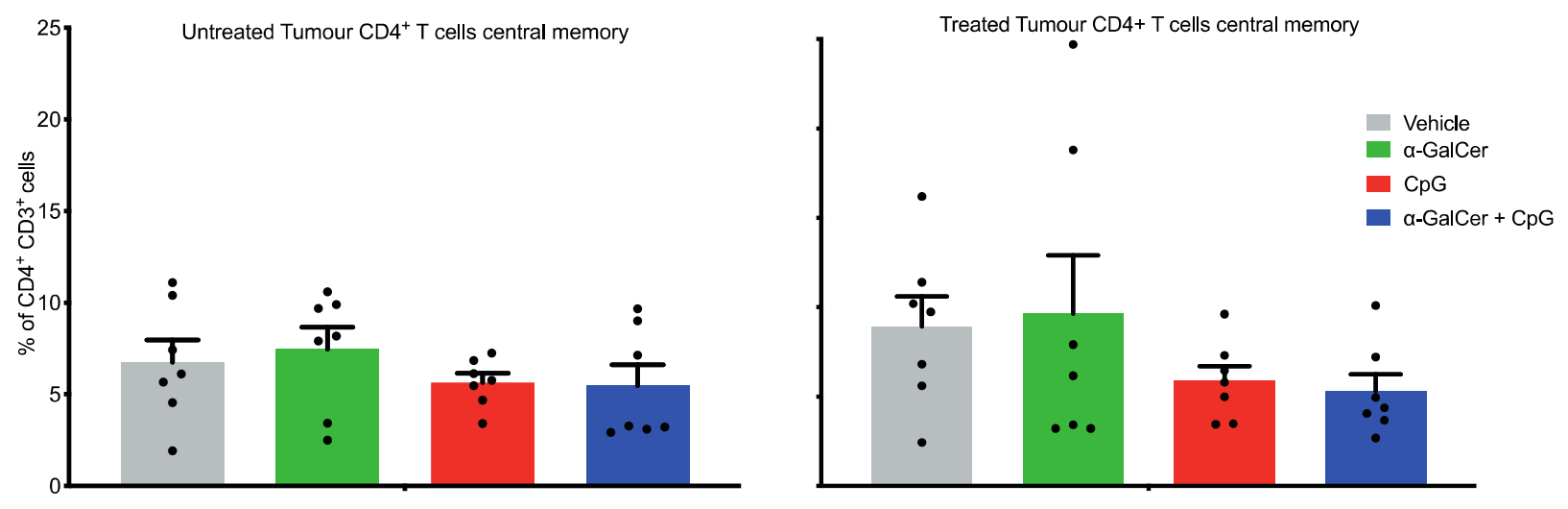

D

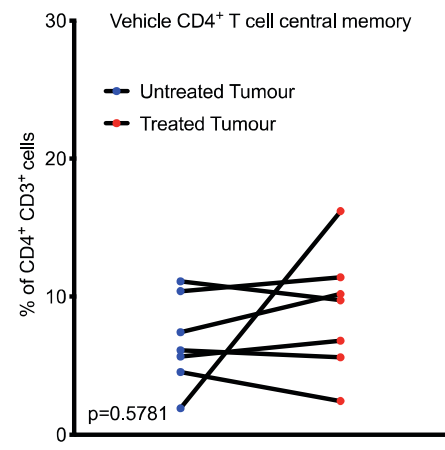

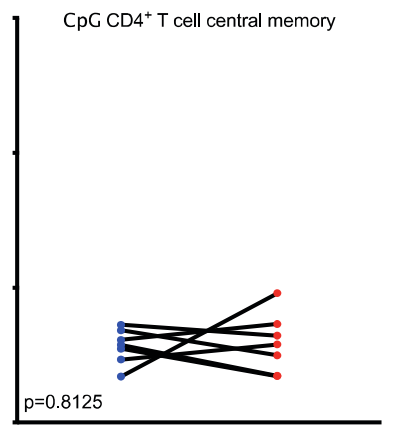

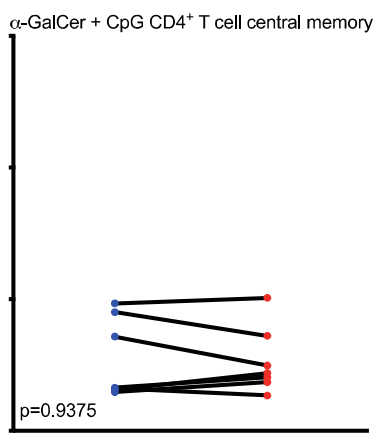



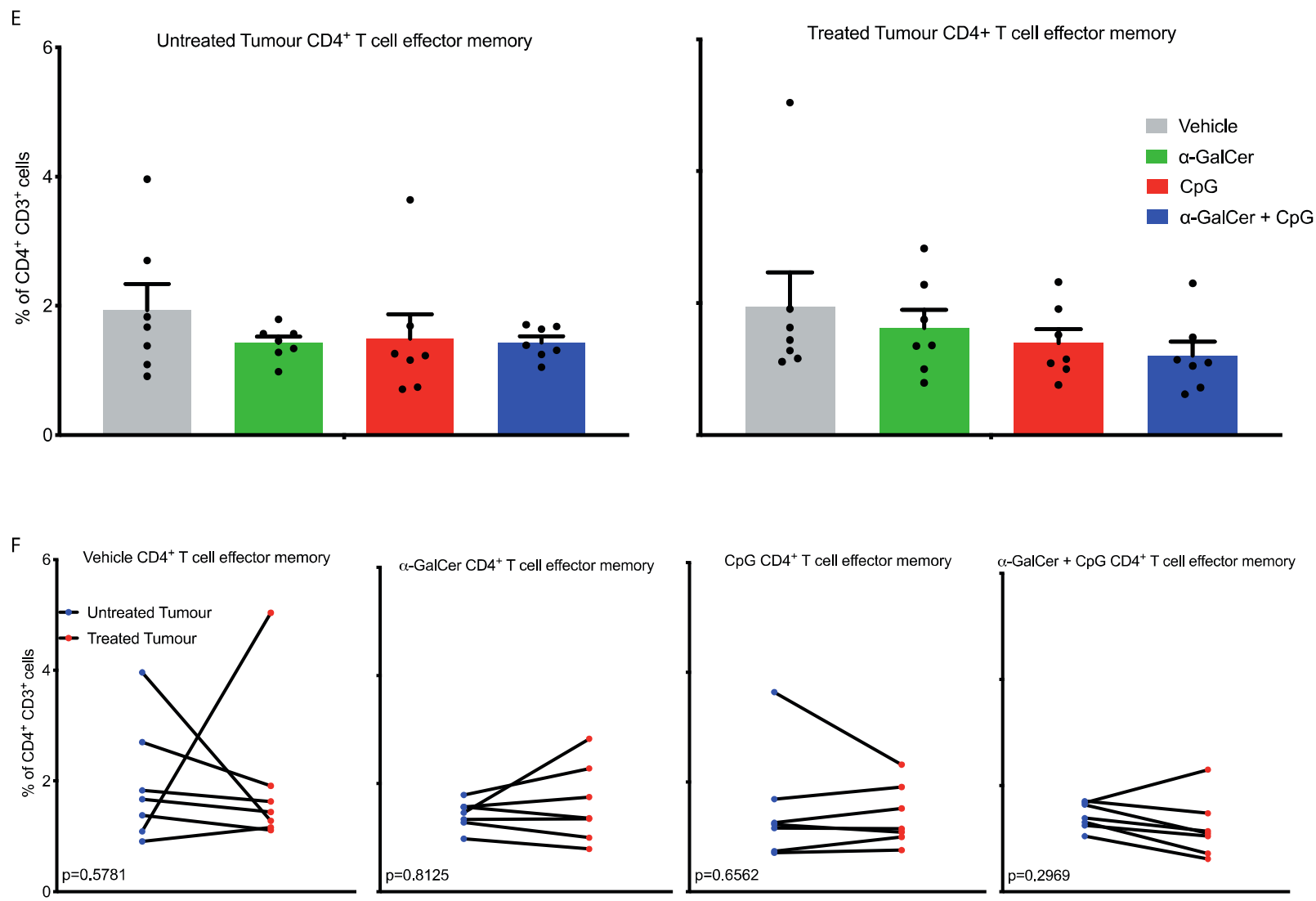

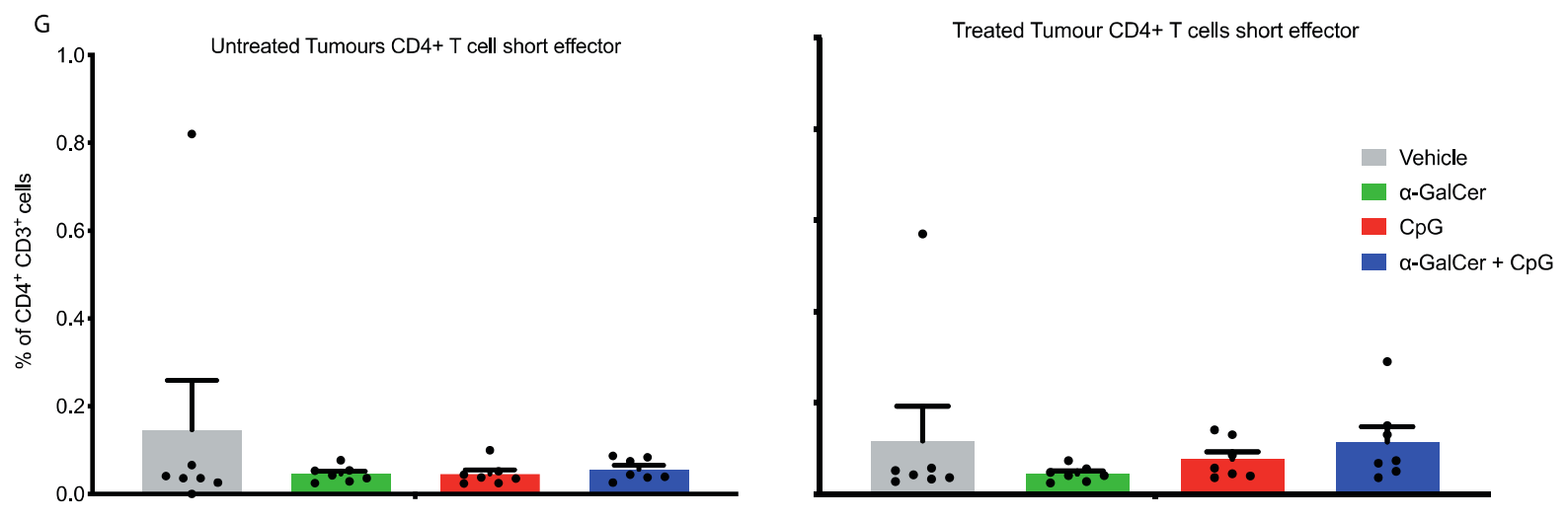

$\mathrm{H}$
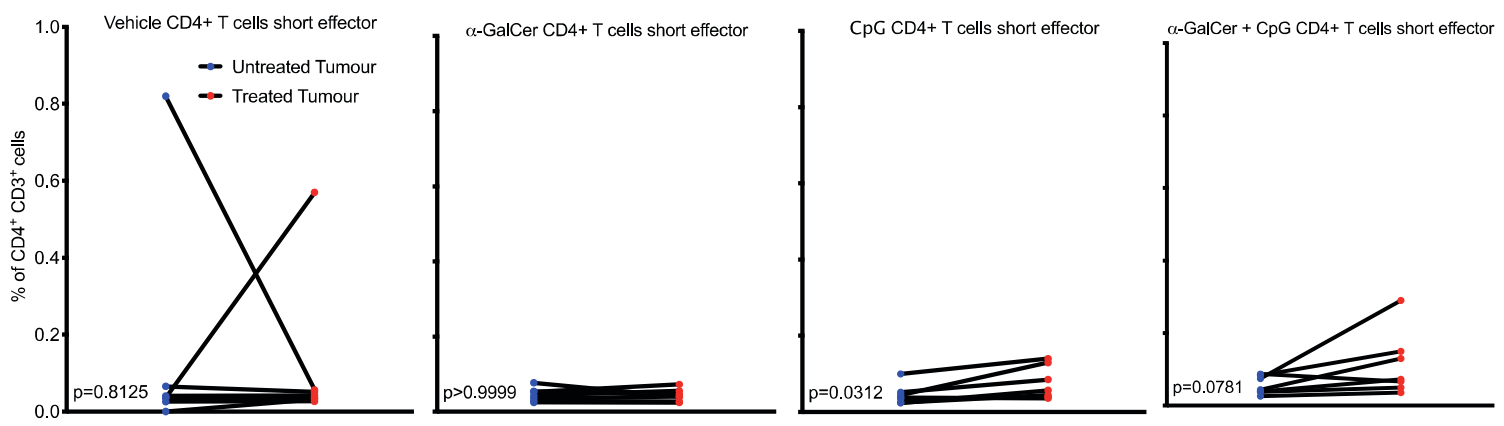

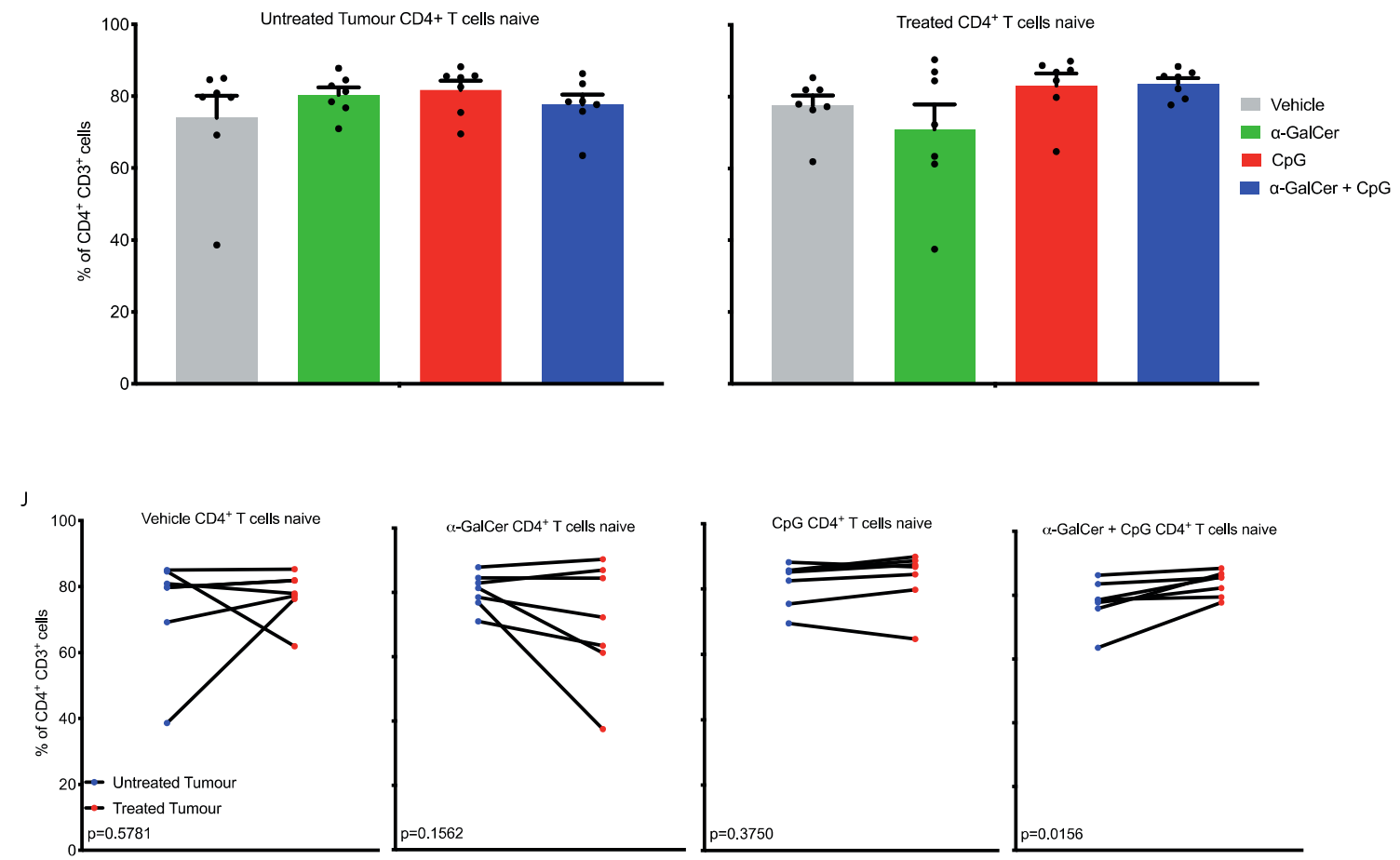

Figure 28 Intratumoural combination therapy of $\alpha$-GalCer and $C p G$ does not induce significant changes in the CD4 ${ }^{+} T$ cell populations in $d L N$. A) $d L N$ near treated and untreated tumours saw no changes in the overall $C D 4^{+} T$ cell population. B) $d L N$ proximal to treated tumours showed no significant differences to dLN near untreated (contralateral tumours) in terms of the overall $C D 4^{+} T$ cell population. C) Treated and untreated tumours saw no changes in the $C D 4^{+} T$ cell central memory population. D) dLN near treated tumours showed no significant differences to dLN proximal to untreated (contralateral tumours) in the $\mathrm{CD}^{+}$T cell central memory population. E) Combination therapy did not induce changes in the effector memory population. F) dLN near treated tumours did not show significant differences to dLN near untreated tumours in the effector memory population. $G)$ Treatment did not induce significant changes in the short-lived effector population. $H) C p G$ treatment induced increases in CD4+ T cell short-lived effectors in the dLN of treated tumours compared to the dLN of untreated tumours. I) Combination therapy did not induce changes in the naïve $C D 4^{+} T$ cell population in the $d L N$ of both treated and untreated tumours. J) Combination therapy increased the frequency of naïve CD4 ${ }^{+} T$ cells in the $\mathrm{dLN}$ of treated tumours compared to the $d L N$ of untreated tumours. Statistically significant differences are indicated $* P<0.05$, $* * P<0.01, * * * P<0.001, * * * * P<0.0001$ (One-Way ANOVA with Tukey's Post-Hoc Test). Statistically significant differences are indicated (Wilcoxon Signed-Rank Test). 

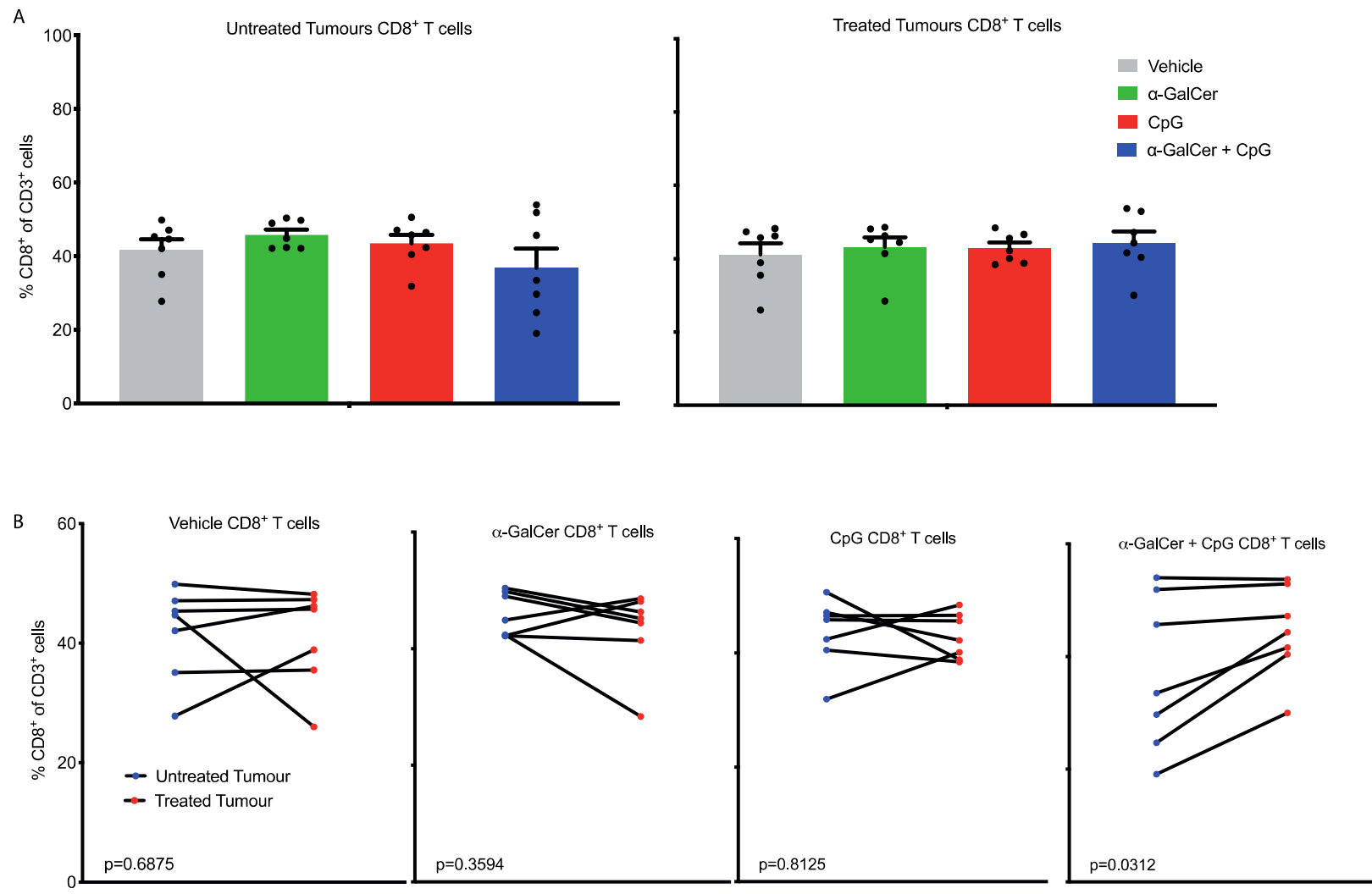

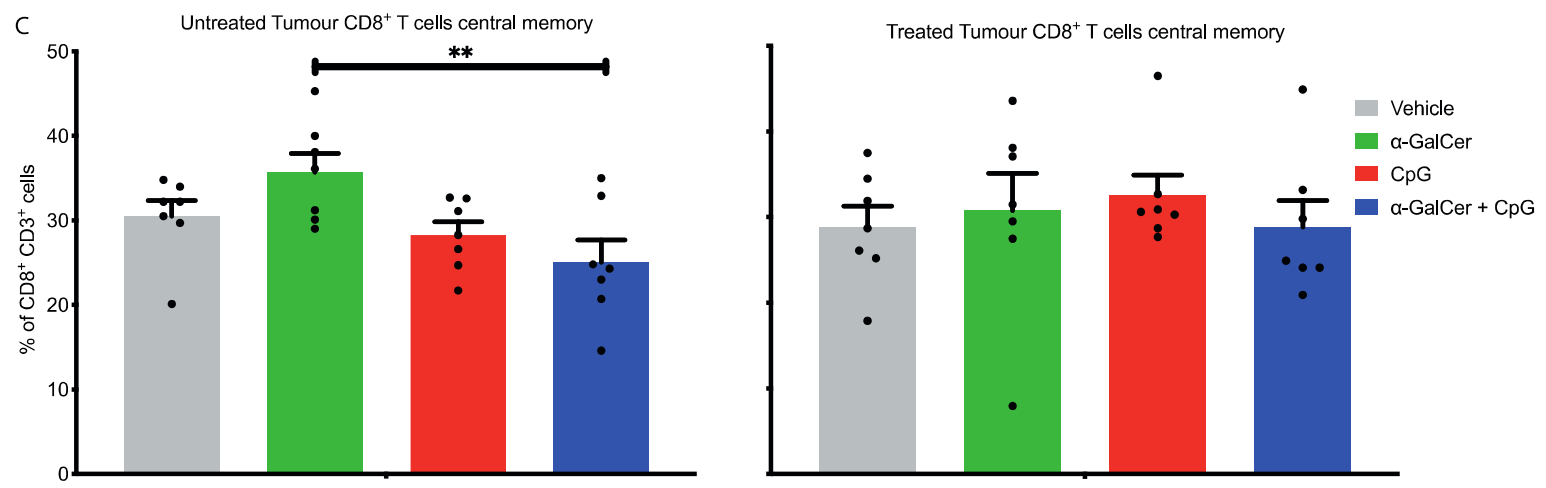

D
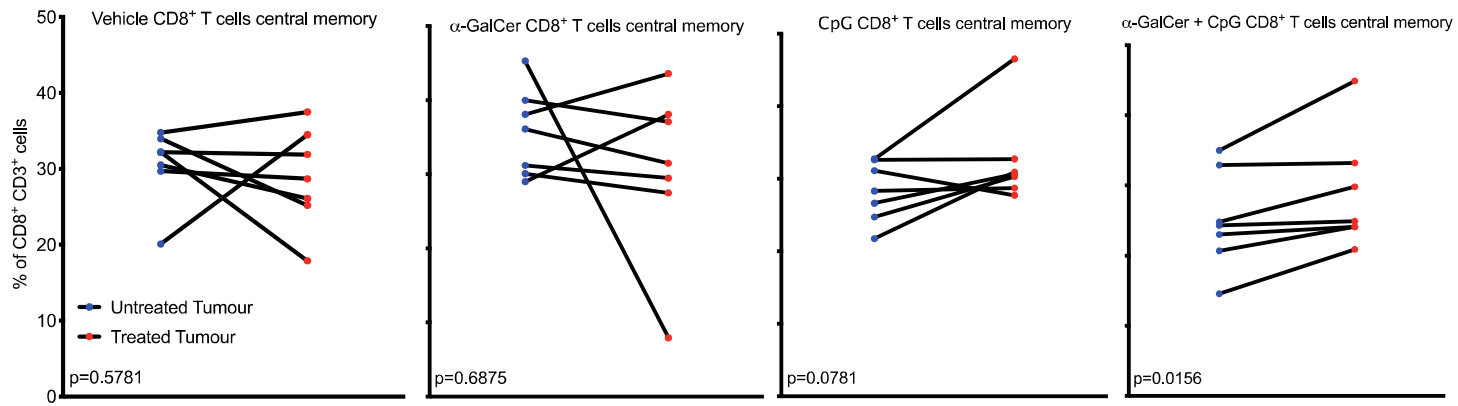

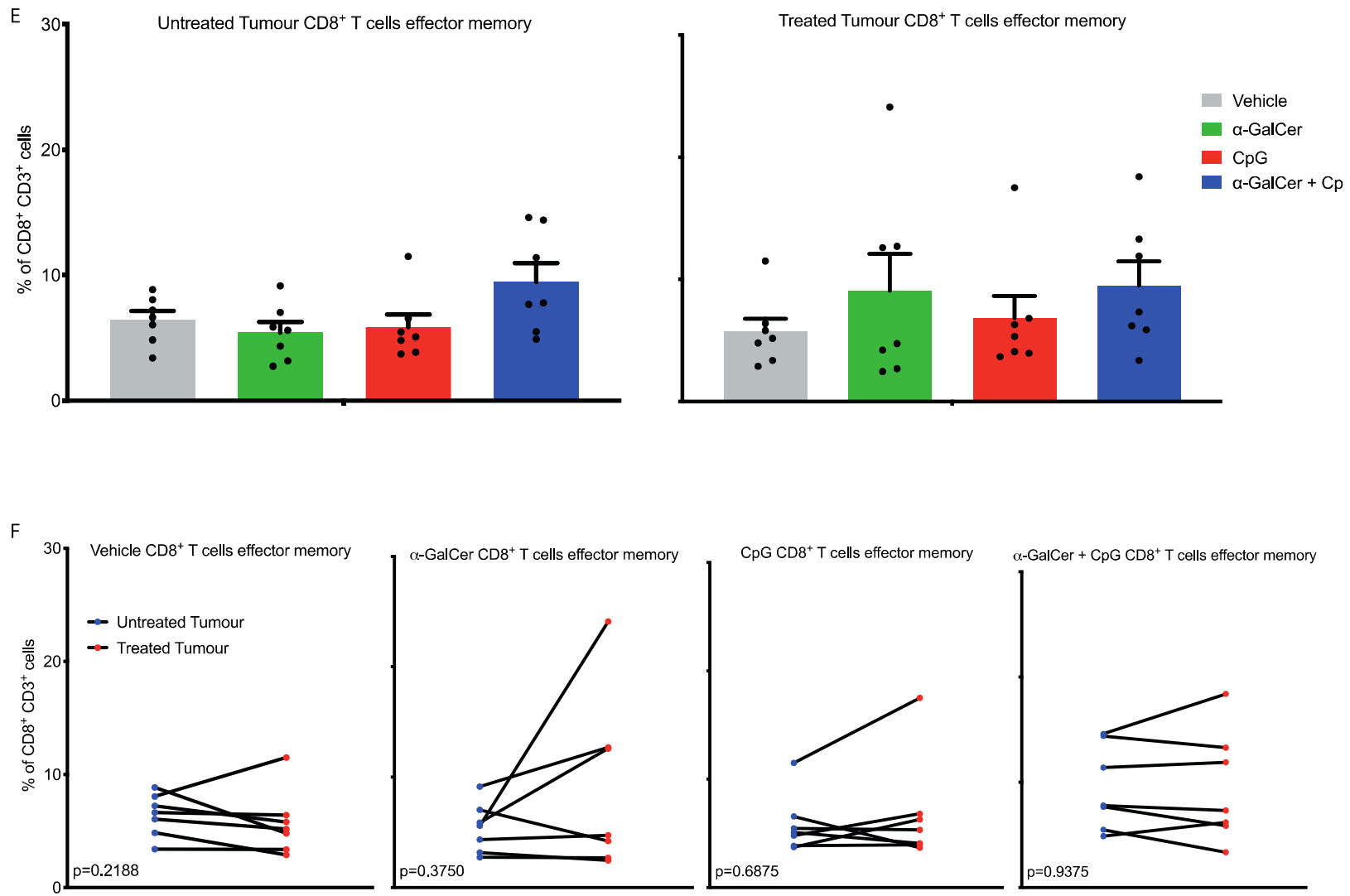
G

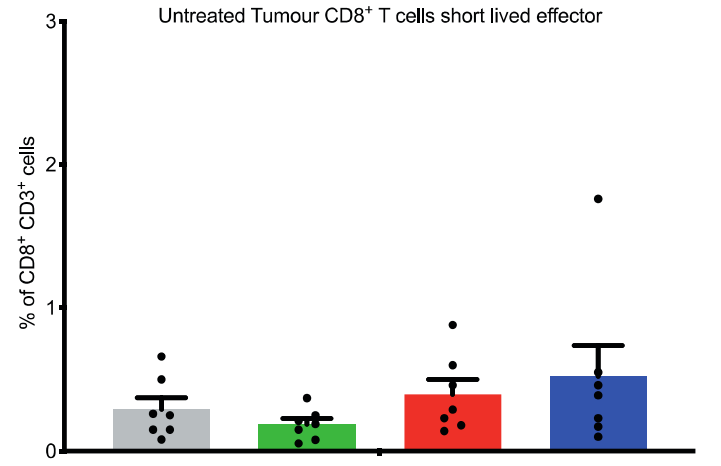

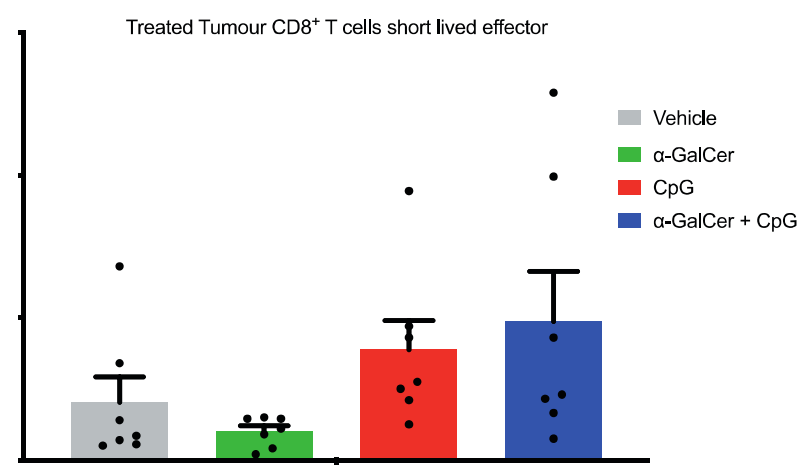

H

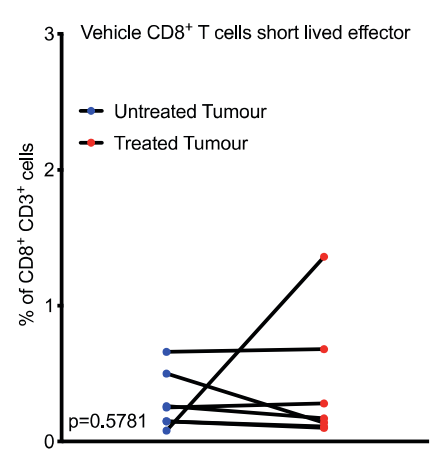

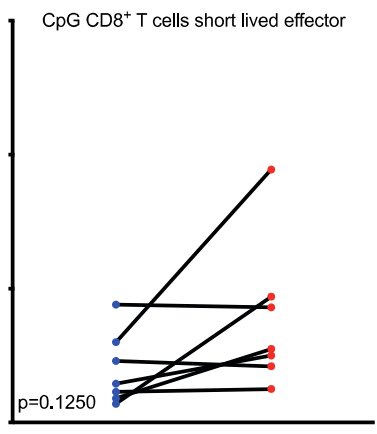

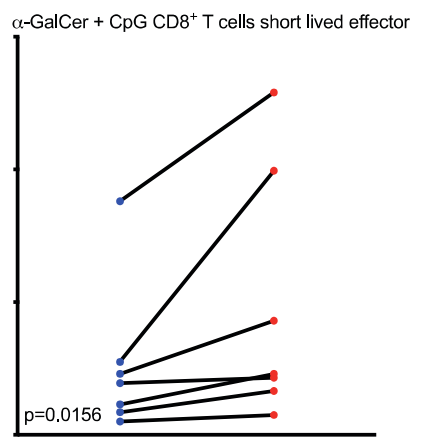



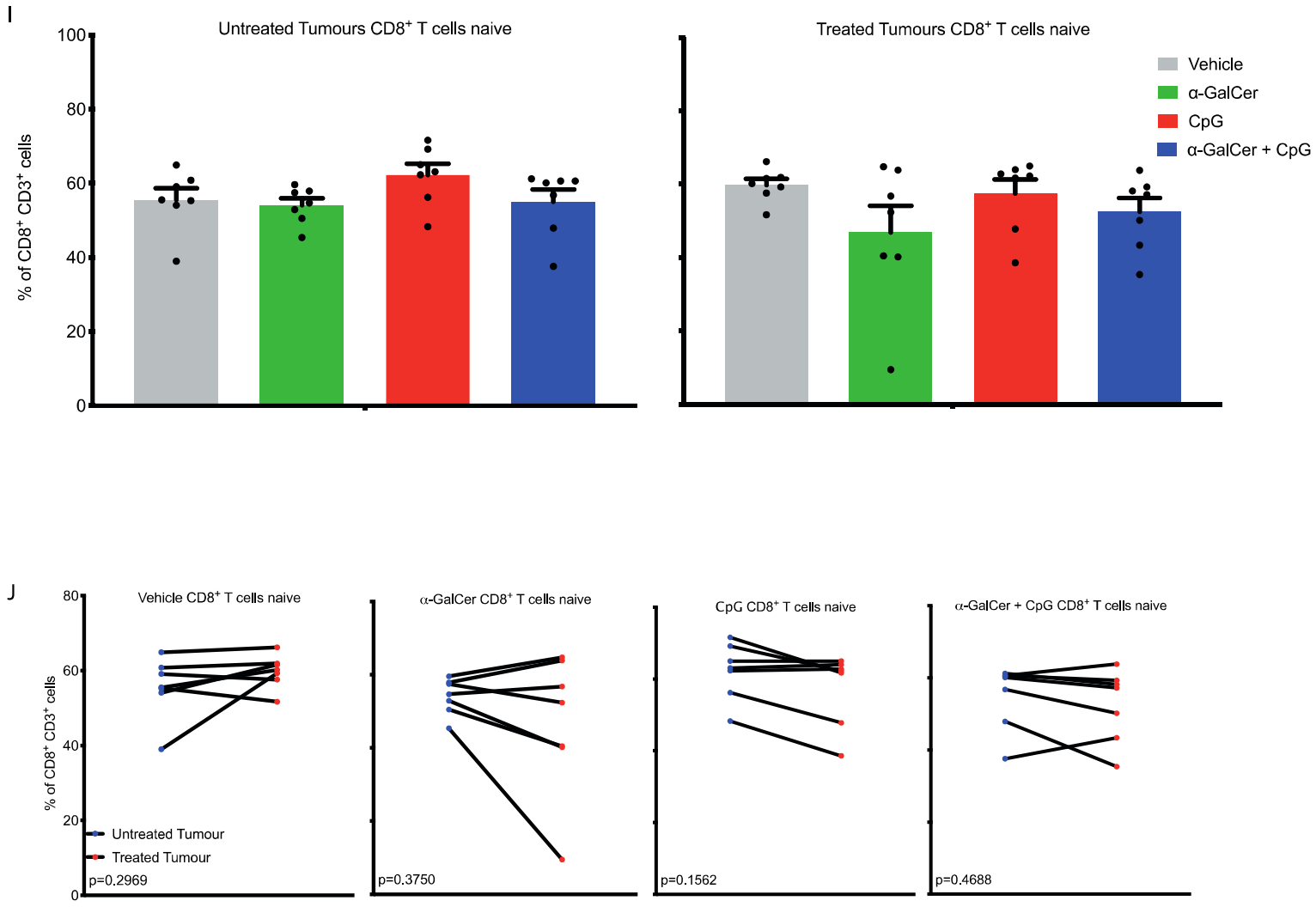

Figure 29 Intratumoural combination therapy of $\alpha$-GalCer and $C p G$ does not induce significant changes in the CD8 $8^{+} T$ cell populations in $d L N$. A) $d L N$ near treated and untreated tumours saw no changes in the overall $C D 8^{+} T$ cell population. B) $d L N$ near treated tumours showed significant increases in $C D 8^{+} T$ cells relative to dLN near untreated (contralateral tumours). C) Combination therapy induced a reduction in central memory $C D 8^{+} T$ cells in $d L N$ near untreated tumours compared to $\alpha$ GalCer treated animals. D) dLN near combination therapy treated tumours showed a significant increase in central memory $C D 8^{+}$T cells compared to untreated (contralateral tumours). E) Combination therapy did not induce changes in the effector memory population. $F$ ) dLN near treated tumours did not show significant differences to untreated tumours in the effector memory population. $G$ ) Combination therapy did not induce changes in the short-lived effector population. H) Combination therapy induced a significant increase in the frequency of short-lived effector $C D 8^{+} T$ cells in dLN near treated tumours compared to dLN near untreated (contralateral tumours). I) Combination therapy did not induce changes in the naïve $C D 8^{+} T$ cell population. J) $d L N$ near treated tumours showed no differences in the nä̈ve $C D 8^{+} T$ cell population compared to $d L N$ near untreated tumours. Statistically significant differences are indicated $* P<0.05, * * P<0.01, * * * P<0.001$, $* * * * P<0.0001$ (OneWay ANOVA with Tukey's Post-Hoc Test). Statistically significant differences are indicated (Wilcoxon Signed-Rank Test).

The combination induced increases in the iNKT cell population in the $\mathrm{dLN}$ of treated tumours compared to the dLN of either vehicle or CpG treated tumours (Fig 30A). Furthermore, combination therapy treated mice saw an increase in iNKT cells in the dLN of the treated tumour compared to their untreated tumours (Fig 30B). Of note, there were also associated increases in $\mathrm{CD}^{+}{ }^{+}$iNKT cells (Fig 30E) with increases also being seen when comparing untreated dLN to 
treated dLN of combination therapy, and $\mathrm{CpG}$ treated mice, perhaps indicating this increase is due to $\mathrm{CpG}$ (Fig 30F). There was also a reduction in $\mathrm{NK} 1.1^{+\mathrm{i}} \mathrm{NKT}$ cells compared to $\mathrm{CpG}$ alone (Fig 30G), which seems to be $\alpha$-GalCer treatment mediated as there is a decrease in NK1.1 $1^{+}$ iNKT cells in the dLN of tumours treated with either $\alpha$-GalCer or combination therapy (Fig $30 \mathrm{H})$. This may be due to the downregulation of NK1.1 due to iNKT cells being activated. As for the $\mathrm{CD} 4^{+} \mathrm{NK} 1.1^{+} \mathrm{iNKT}$ cells, there was a significant decrease when the population of $\mathrm{CD} 4^{+} \mathrm{NK} 1.1^{+}$iNKT cells in the dLN of treated tumours compared to the dLN of untreated tumours for $\alpha$-GalCer, and combination therapy treated mice (Fig 30J).
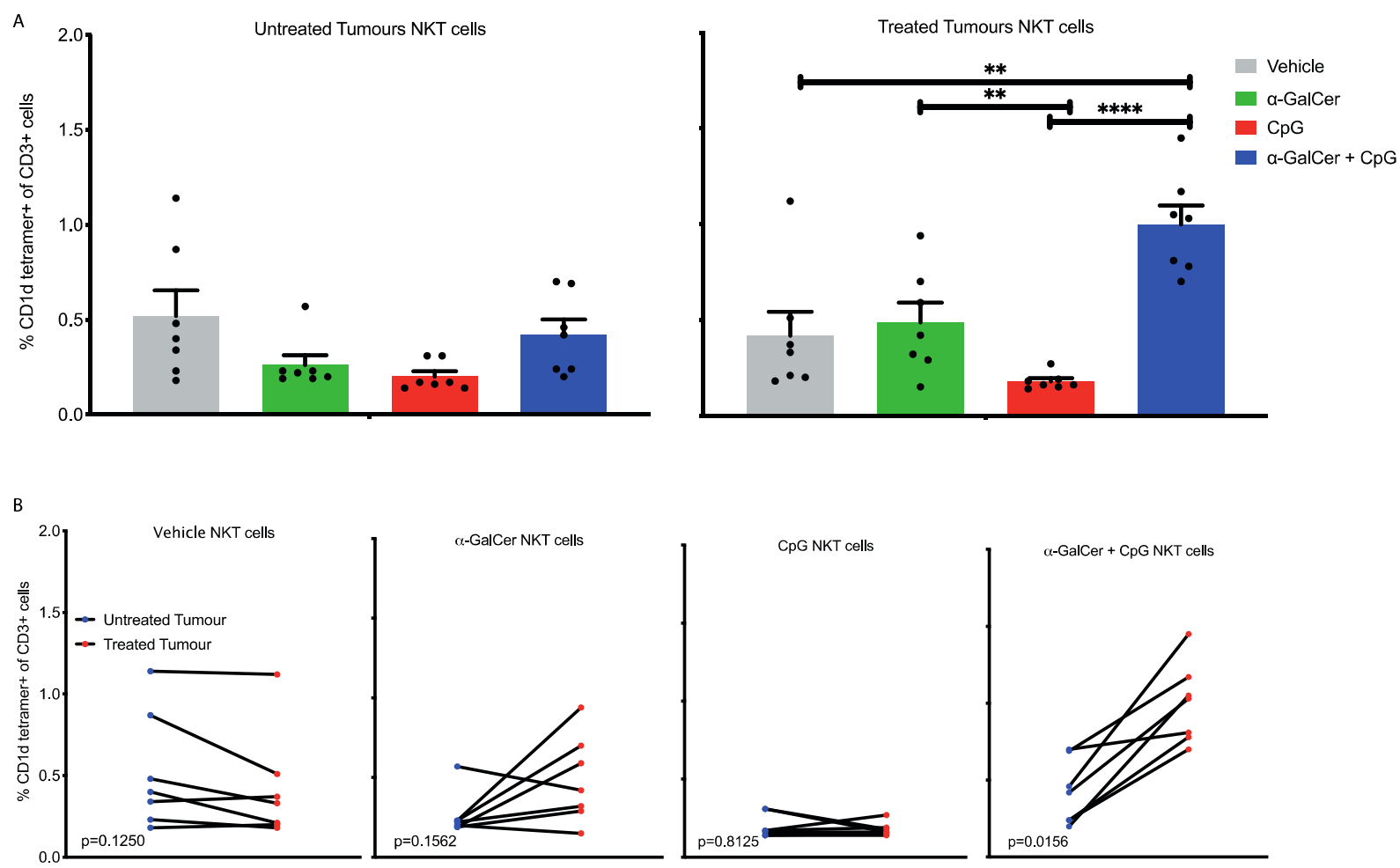
C

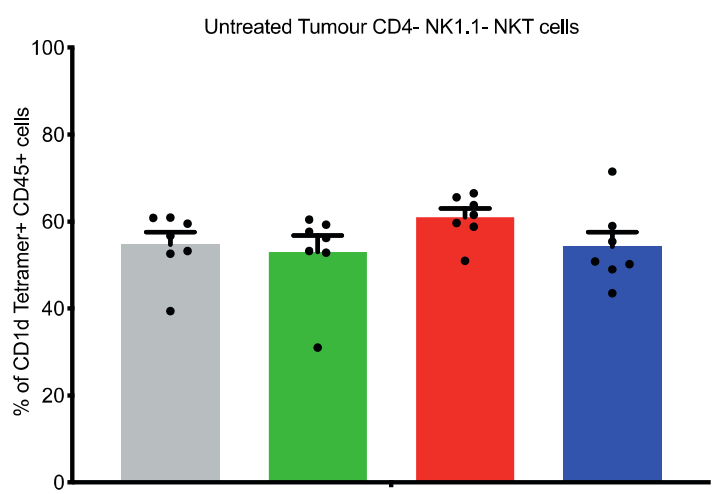

Treated Tumour CD4- NK1.1- NKT cells

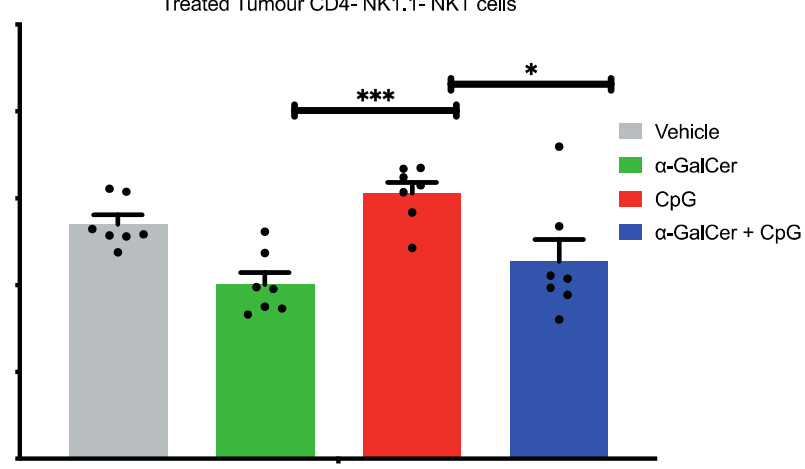

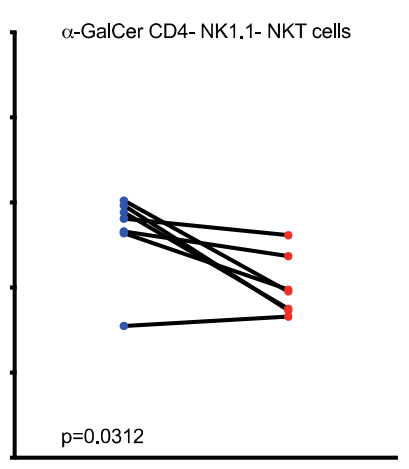

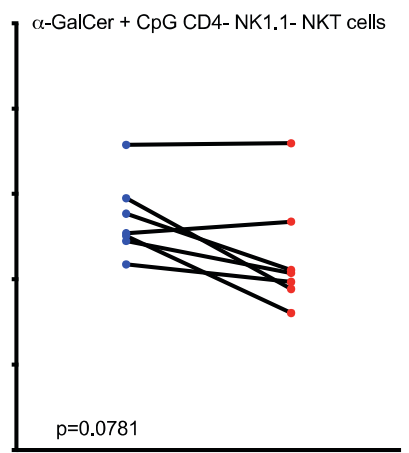

CpG CD4- NK1.1- NKT cells

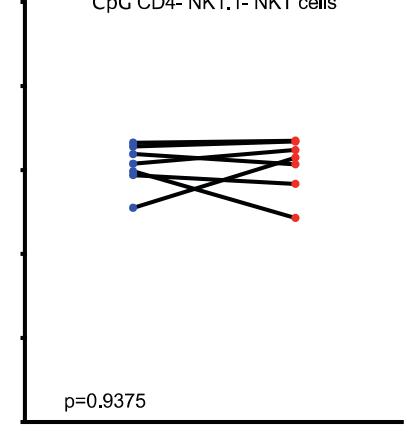



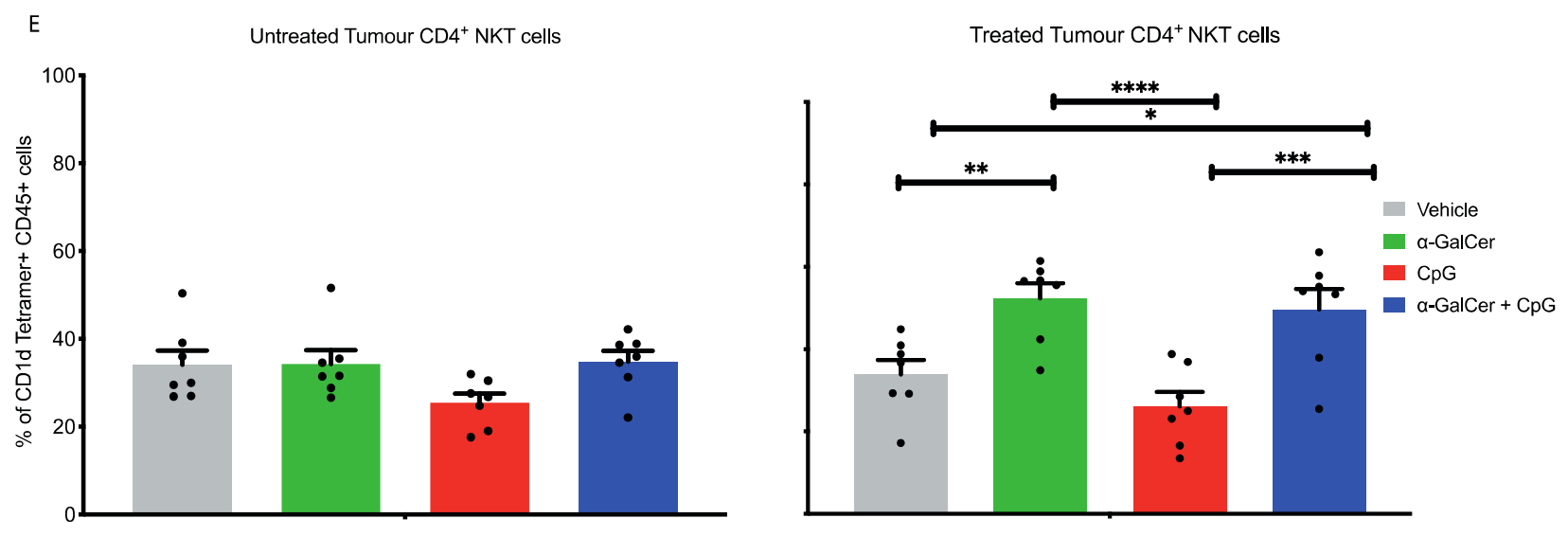

F

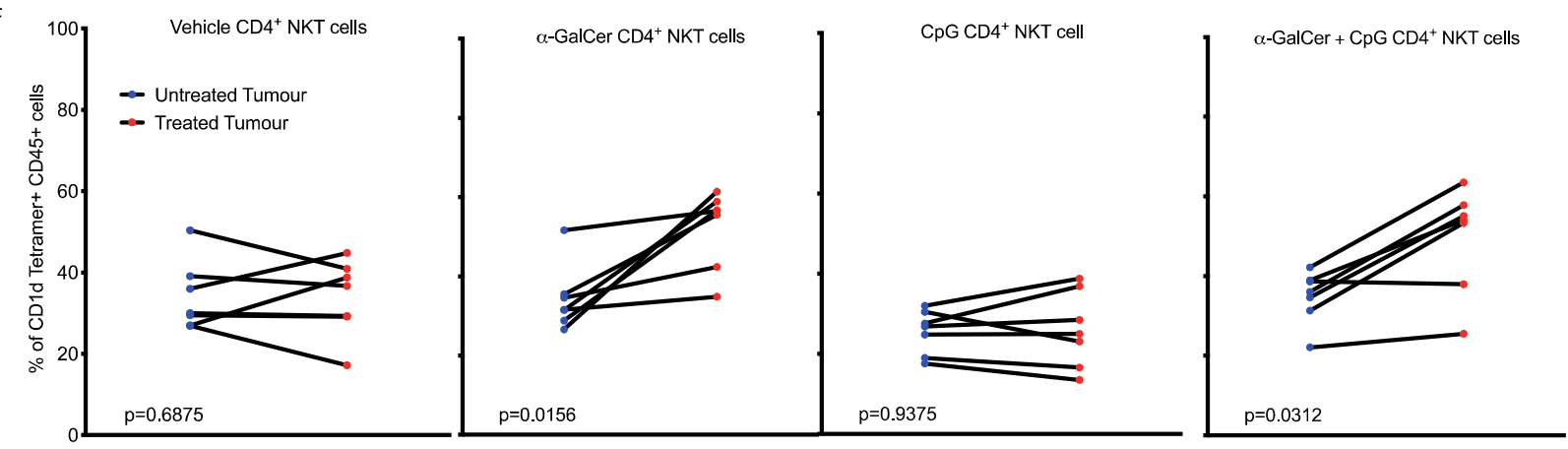

G
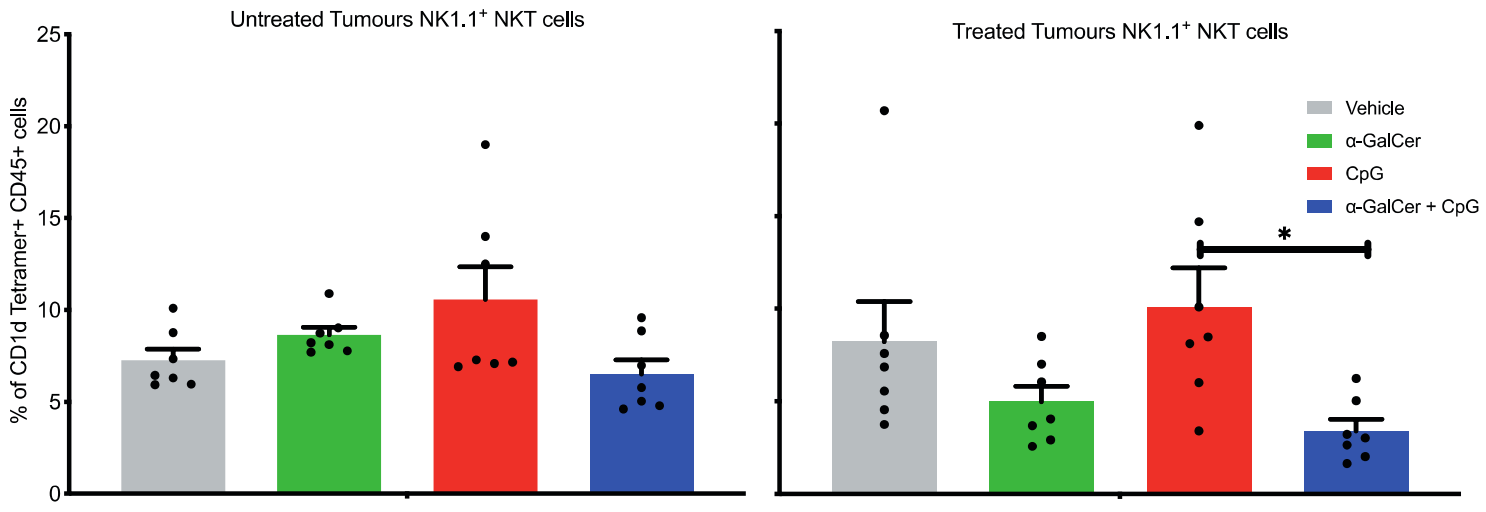

H
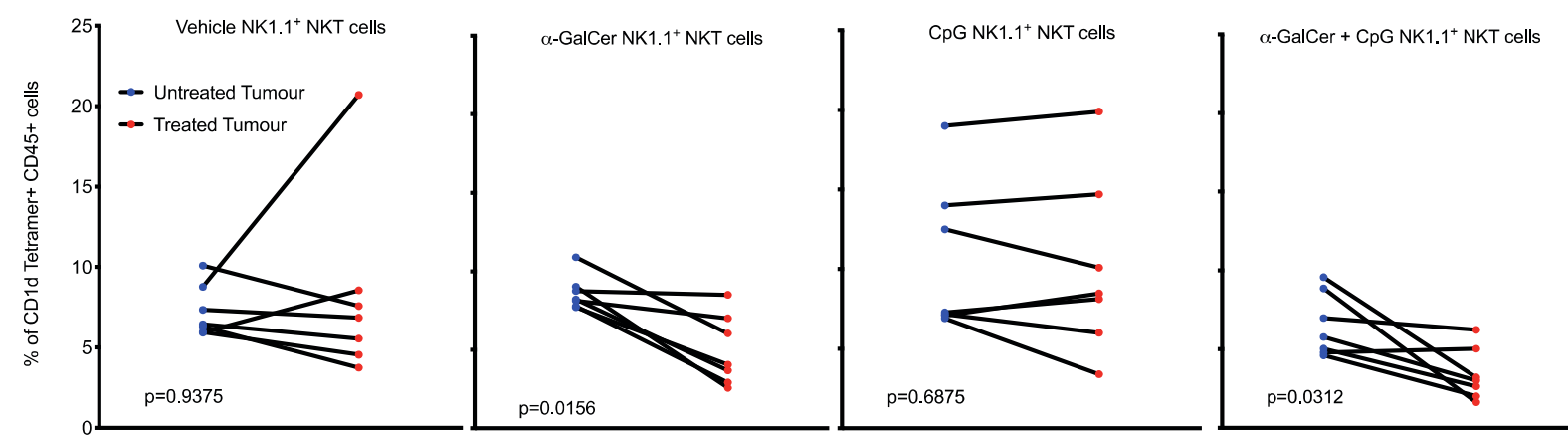
I
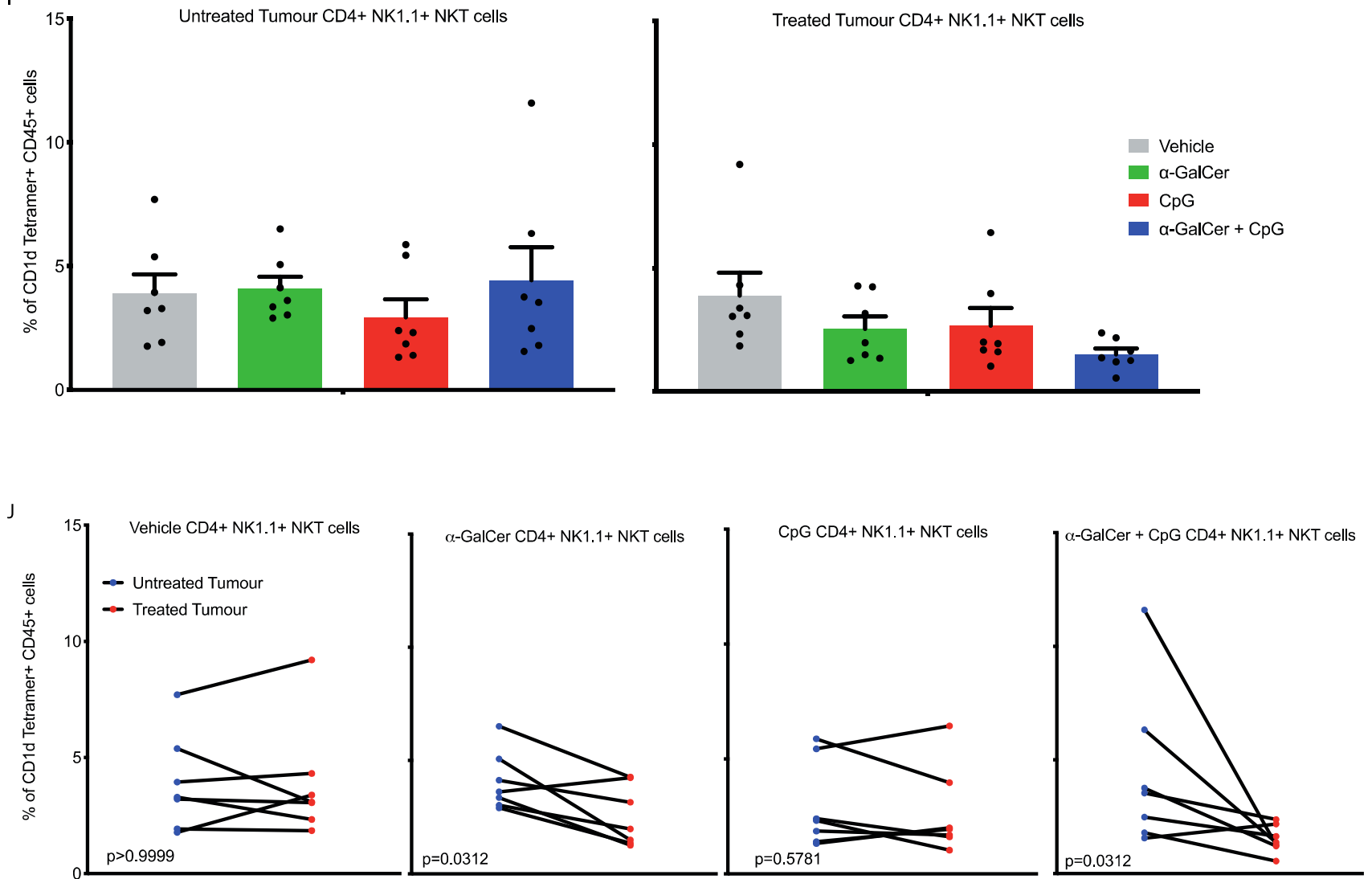

Figure 30 Intratumoural combination therapy of $\alpha$-GalCer and $C p G$ induces significant changes to the iNKT cell populations in the draining lymph node. A) The dLN of combination therapy treated tumours saw a significant increase in the frequency of iNKT cells compared to vehicle and CpG treated tumours. B) The dLN of combination therapy treated tumours showed a significant increase in iNKT cells compared to the dLN of untreated tumours. C) The dLN of CpG treated tumours saw a significant increase in the CD4- NK1.1- iNKT cell population compared to the $d L N$ of $\alpha$-GalCer or combination therapy treated tumours. D) The dLN of CPG treated tumours saw a significant decrease in CD4- NK1.1- ${ }^{-}$iNKT cells compared to the dLN of untreated (contralateral) tumours. E) Combination therapy and $\alpha$-GalCer mono-therapy induced an increase in $C D 4^{+} i N K T$ cell frequency in the $d L N$ of tumours compared to the dLN of treated tumours in CpG and vehicle-treated animals. F) The dLN of $\alpha$-GalCer and combination therapy treated tumours had a significant increase in $\mathrm{CD}^{+}{ }^{+} \mathrm{iNKT}$ cells compared to the $d L N$ untreated (contralateral) tumours in the $C D 4^{+} i N K T$ cell population. G) The dLN of combination therapy treated tumours induced a significant reduction in NK1.1 ${ }^{+}$ iNKT cells compared to the dLN of CpG treated tumours. H) ) The dLN of $\alpha$-GalCer and combination therapy treated tumours had a significant decrease in NK1.1 $1^{+}$iNKT cells compared to untreated (contralateral tumours). I) Combination therapy did not induce significant changes in $C D 4^{+} N K 1.1^{+}$iNKT cell population in the dlN of both treated and untreated tumours. J) The dLN of

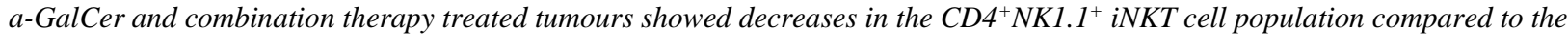
$d L N$ of untreated (contralateral tumours). Statistically significant differences are indicated $* P<0.05, * * P<0.01, * * * P<0.001$, $* * * * P<0.0001$ (One-Way ANOVA with Tukey's Post-Hoc Test). Statistically significant differences are indicated (Wilcoxon Signed-Rank Test). 
Interestingly, the $\mathrm{CpG}$ treated groups had an increase in the frequency of B cells in the dLN of treated tumours (Fig 31A). There were increases in the frequency of B cells in the dLN of treated tumours in mice treated with $\alpha$-GalCer, $\mathrm{CpG}$, or combination therapy treated mice, compared to the dLN of untreated tumours (Fig 31B).

A
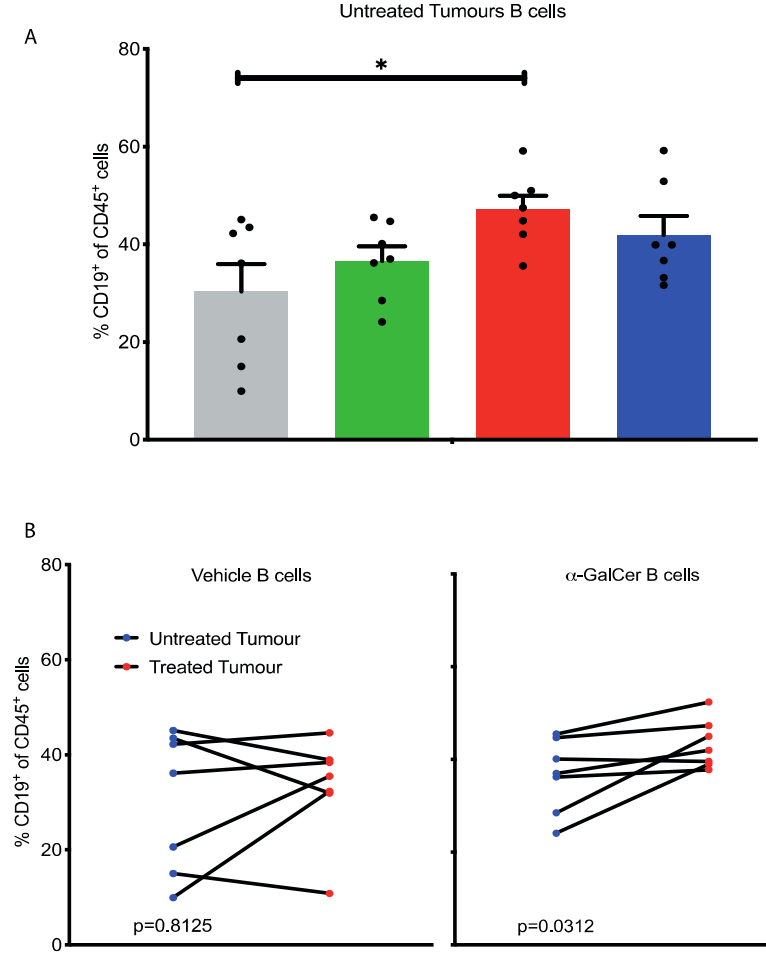

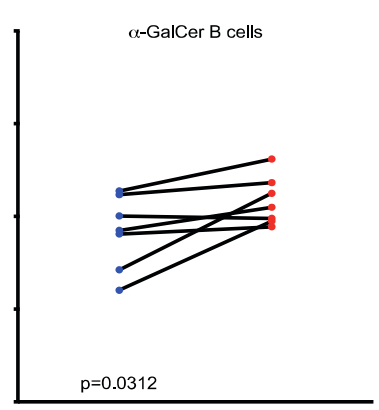

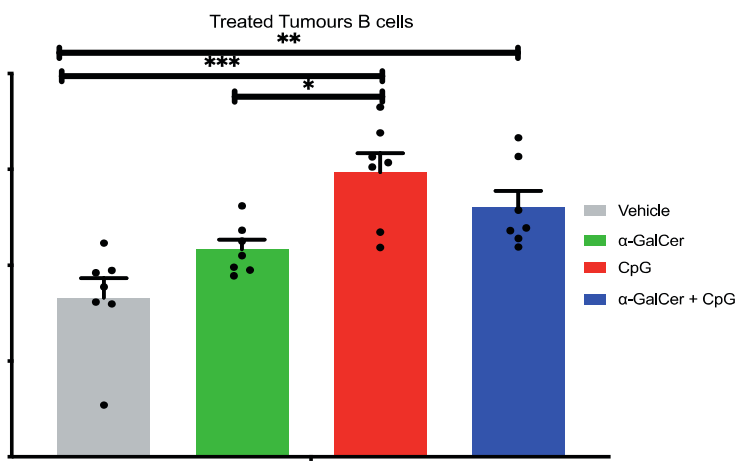

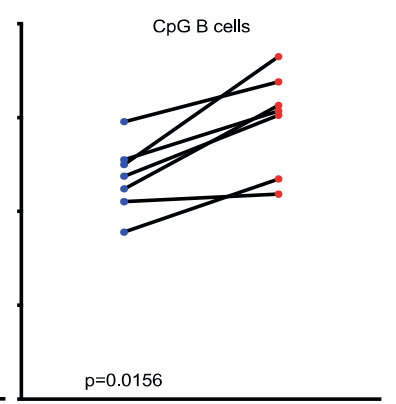

$\alpha-$ GalCer + CpG B cells

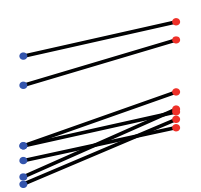

$\mathrm{p}=0.0156$

Figure 31 Intratumoural combination therapy of $\alpha$-GalCer and $C p G$ increases the frequency of $B$ cells in the dLN of treated tumours. A) In the dLN of treated tumours, combination therapy induced a significant increase in $B$ cells compared to vehicle in a similar manner as $C p G$; while $C p G$ induced and increase in B cells over $\alpha$-GalCer and vehicle. In untreated tumours, $C p G$ induced an increase in B cells compared to vehicle. Statistically significant differences are indicated $* P<0.05$, $* * P<0.01$, $* * * P<0.001$, ****P<0.0001 (One-Way ANOVA with Tukey's Post-Hoc Test). B) $\alpha$-GalCer and CpG combination therapy, as well as $C p G$ or $\alpha$-GalCer monotherapy, induce significant increases in B cells in the dLN of treated tumours and compared to the dLN of untreated tumours. Statistically differences are indicated (Wilcoxon Signed-Rank Test). 


\subsubsection{Immune profile of the spleen}

In the spleen, there were also no significant changes in the frequency of OVA-specific T cells (Fig 32). Similarly, there was also no change in the Treg population (Fig 33). The $\mathrm{CD}^{+} \mathrm{T}$ cell population saw a reduction when treated with either the combination of $\alpha$-GalCer and $\mathrm{CpG}$ or when treated with $\mathrm{CpG}$ alone (Fig 34A); however, there was no significant difference within the $\mathrm{CD}^{+} \mathrm{T}$ cell subpopulations (Fig 34B-E). As for the $\mathrm{CD} 8^{+} \mathrm{T}$ cell population, the combination of $\alpha$-GalCer and $\mathrm{CpG}$ induced an increase in the frequency of central memory $\mathrm{CD} 8^{+} \mathrm{T}$ cells (Fig 35B) compared to $\alpha$-GalCer alone, an increase in effector memory $\mathrm{CD} 8^{+} \mathrm{T}$ cells compared to vehicle (Fig 35C), and a reduction in naïve $\mathrm{CD} 8^{+} \mathrm{T}$ cells compared to vehicle (Fig 35E). In general, these data indicate activation of $\mathrm{CD} 8^{+} \mathrm{T}$ cells is a consequence of therapy, which tends to be enhanced with the combined treatment.

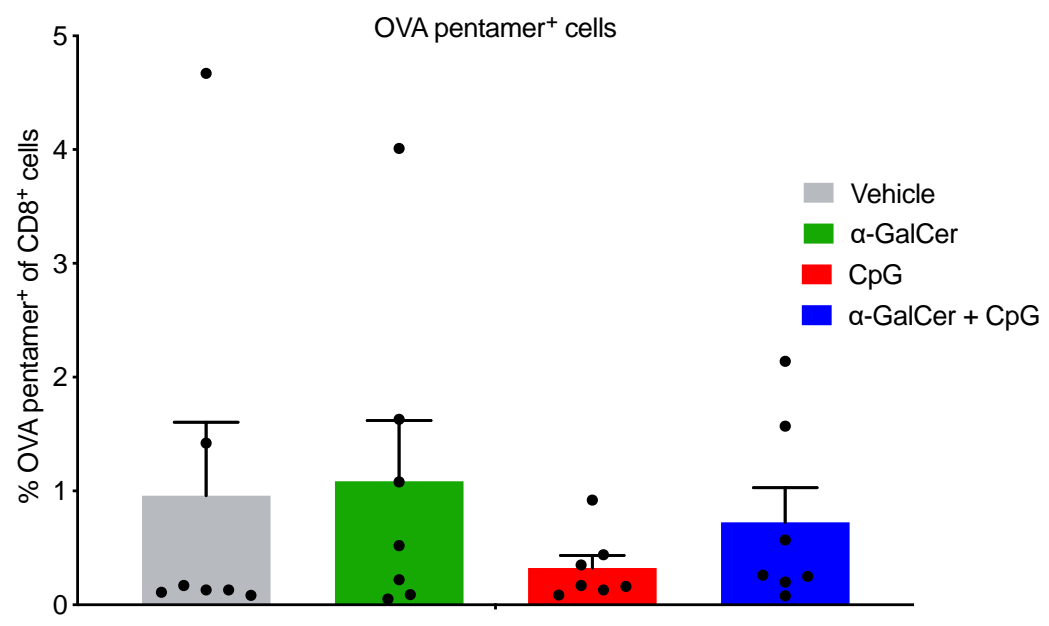

Figure 32 Intratumoural combination therapy of $\alpha$-GalCer and $C p G$ does not increase the frequency of $O V A$-specific $C D 8^{+} T$ cells in the spleen. A) Treatment did not induce changes in OVA-specific T cells in the spleen. Statistically significant differences are indicated $* P<0.05$, $* * P<0.01, * * * P<0.001$, ****P<0.0001 (One-Way ANOVA with Tukey's Post-Hoc Test). 


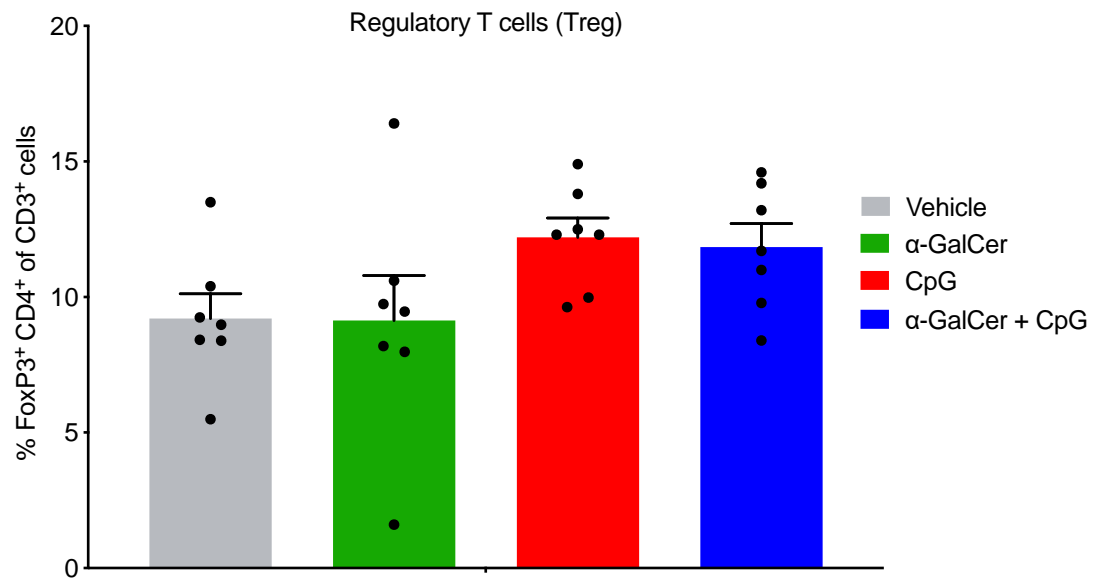

Figure 33 Intratumoural combination therapy of $\alpha$-GalCer and $C p G$ does not induce changes in the proportion of regulatory $T$ cells (Tregs) in the spleen. A) a-GalCer and CpG combination therapy did not induce significant changes in the Treg population in the spleen. Statistically significant differences are indicated $* P<0.05, * * P<0.01, * * * P<0.001, * * * * P<0.0001$ (One-Way ANOVA with Tukey's Post-Hoc Test). 

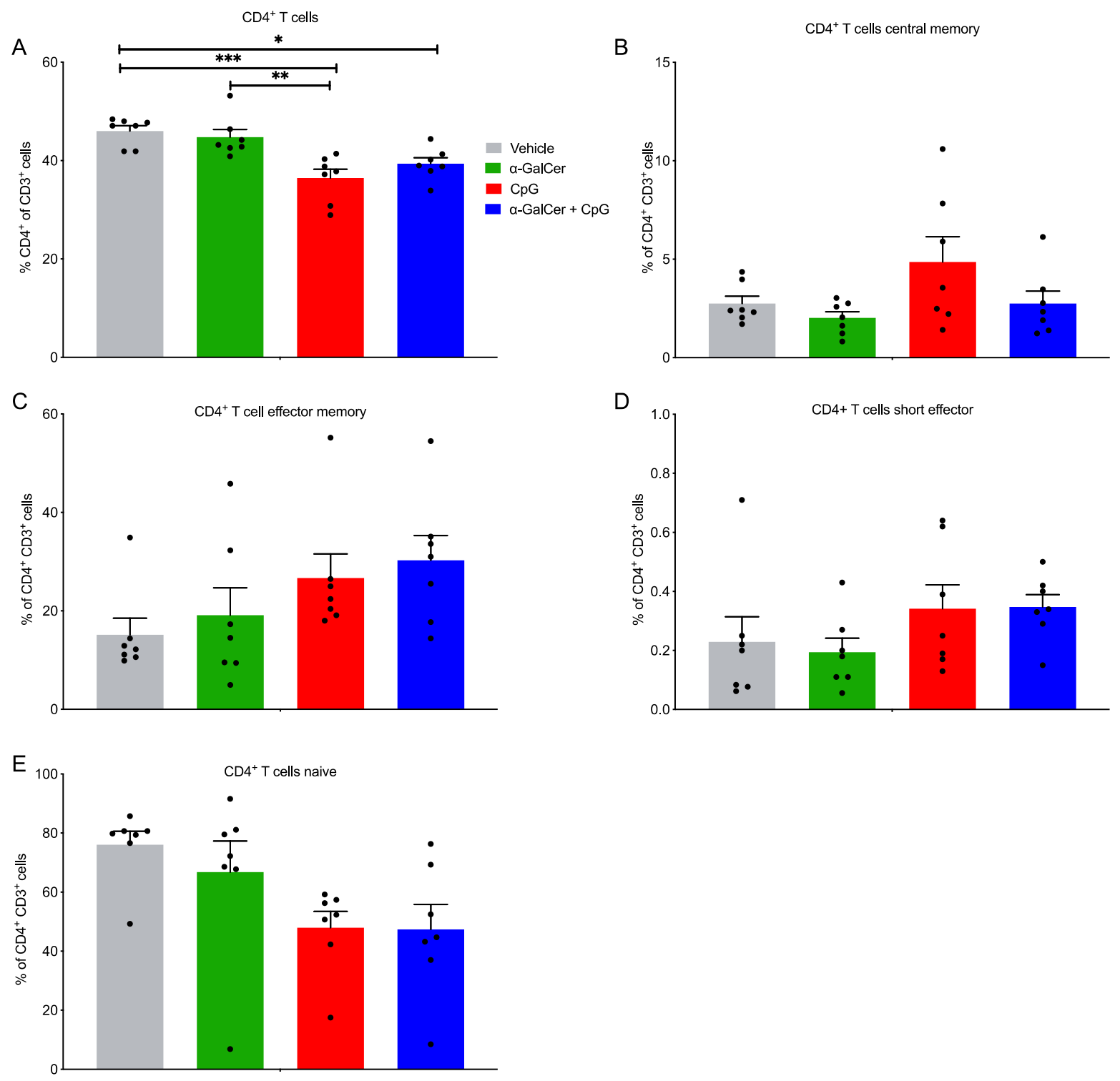

Figure 34 Intratumoural combination therapy of $\alpha$-GalCer and $C p G$ induces a reduction in the $C D 4^{+} T$ cells in the spleen. A) Treatment with $\mathrm{CpG}$ or combination therapy induced a significant reduction in the frequency of $C D 4^{+} T$ cells in the spleen. B) Treatment induced no significant differences in the $\mathrm{CD}^{+} \mathrm{T}$ cell central memory population. C) Treatment induced no significant differences in the $C D 4^{+} T$ cell effector memory population. D) Treatment induced no significant differences in the CD4 ${ }^{+} T$ cell short-lived effector population. E) Treatment induced no significant differences in the naïve $C D 4^{+} T$ cell Statistically significant differences are indicated $* P<0.05$, $* * P<0.01$, $* * * P<0.001$, ****P<0.0001 (One-Way ANOVA with Tukey's Post-Hoc Test). 

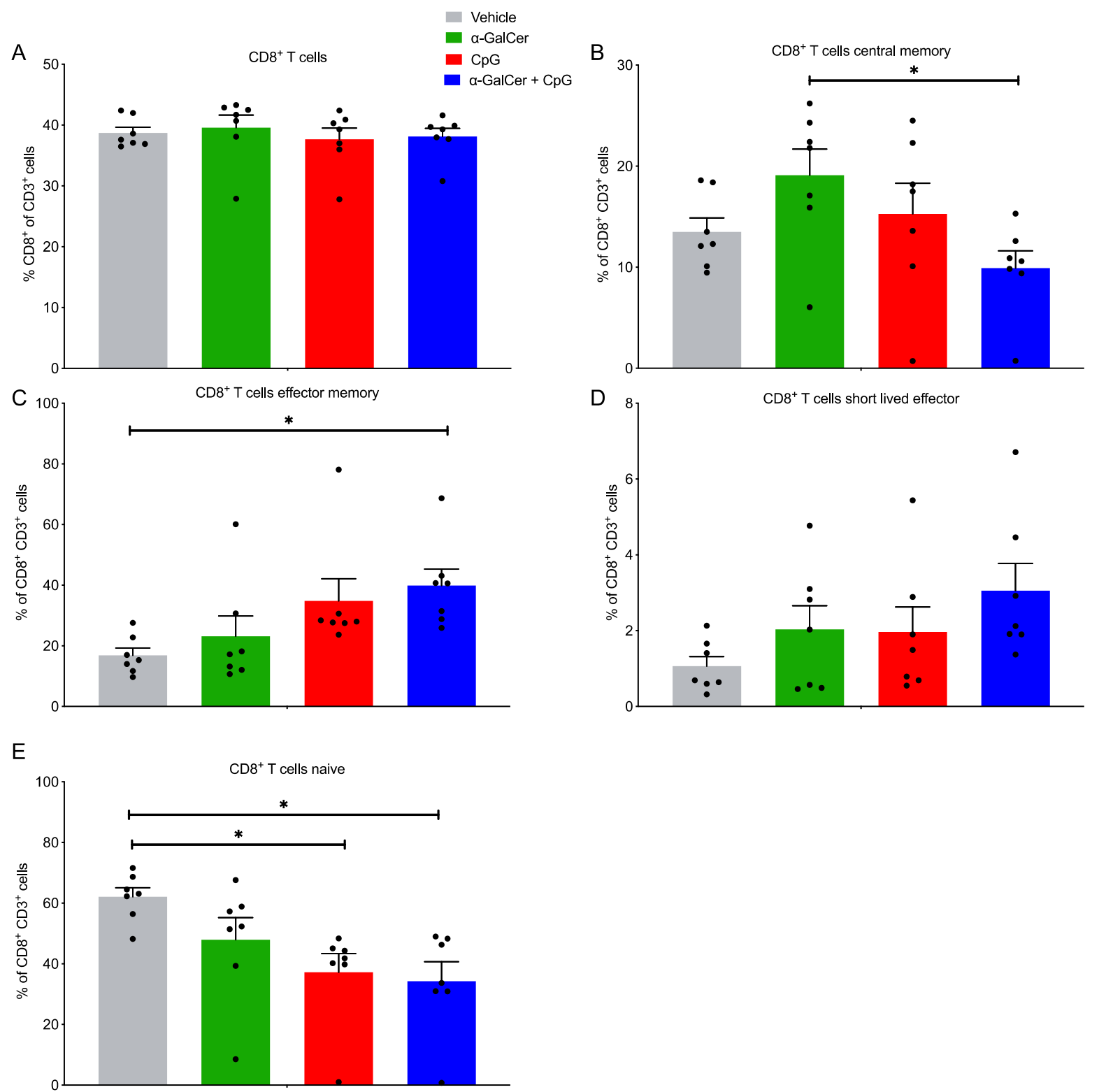

Figure 35 Intratumoural combination therapy of $\alpha$-GalCer and $C p G$ does not induce significant changes in the CD $8^{+} T$ cell subpopulations in the spleen. A) Treatment induced no changes in the overall $C D 8^{+} T$ cell population. B) Combination therapy induced significant increases in central memory $C D 8^{+} T$ cells compared to $\alpha$-GalCer treated animals. C) Combination therapy induced a significant increase in $C D 8^{+}$effector memory $T$ cells in the spleen relative to vehicle. D) Treatment induced no significant changes in the $C D 8^{+} T$ cell short-lived effector population. E) Combination therapy induced a decrease in the naïve $C D 8+T$ cell population in the spleen relative to vehicle, with $C p G$ showing a similar decrease. Statistically significant differences are indicated $* P<0.05$, $* * P<0.01$, ***P<0.001, ****P<0.0001 (One-Way ANOVA with Tukey's Post-Hoc Test). 
Similarly to in the tumour, the combination led to a significant increase in the frequency of splenic iNKT cells (Fig 36A). Within the iNKT cell subpopulations, there was an increased frequency of CD4+ ${ }^{+}$iNKT cells (Fig 36B) and CD4-NK1.1- iNKT cells (Fig 34C). However, there was a reduction in $\mathrm{CD}^{+} \mathrm{NK} 1.1^{+}$iNKT cells (Fig 36D) and NK1.1 ${ }^{+}$iNKT cells (Fig 36E), which fits with the concept that iNKT cell activation is associated with downregulated expression of NK1.1. 

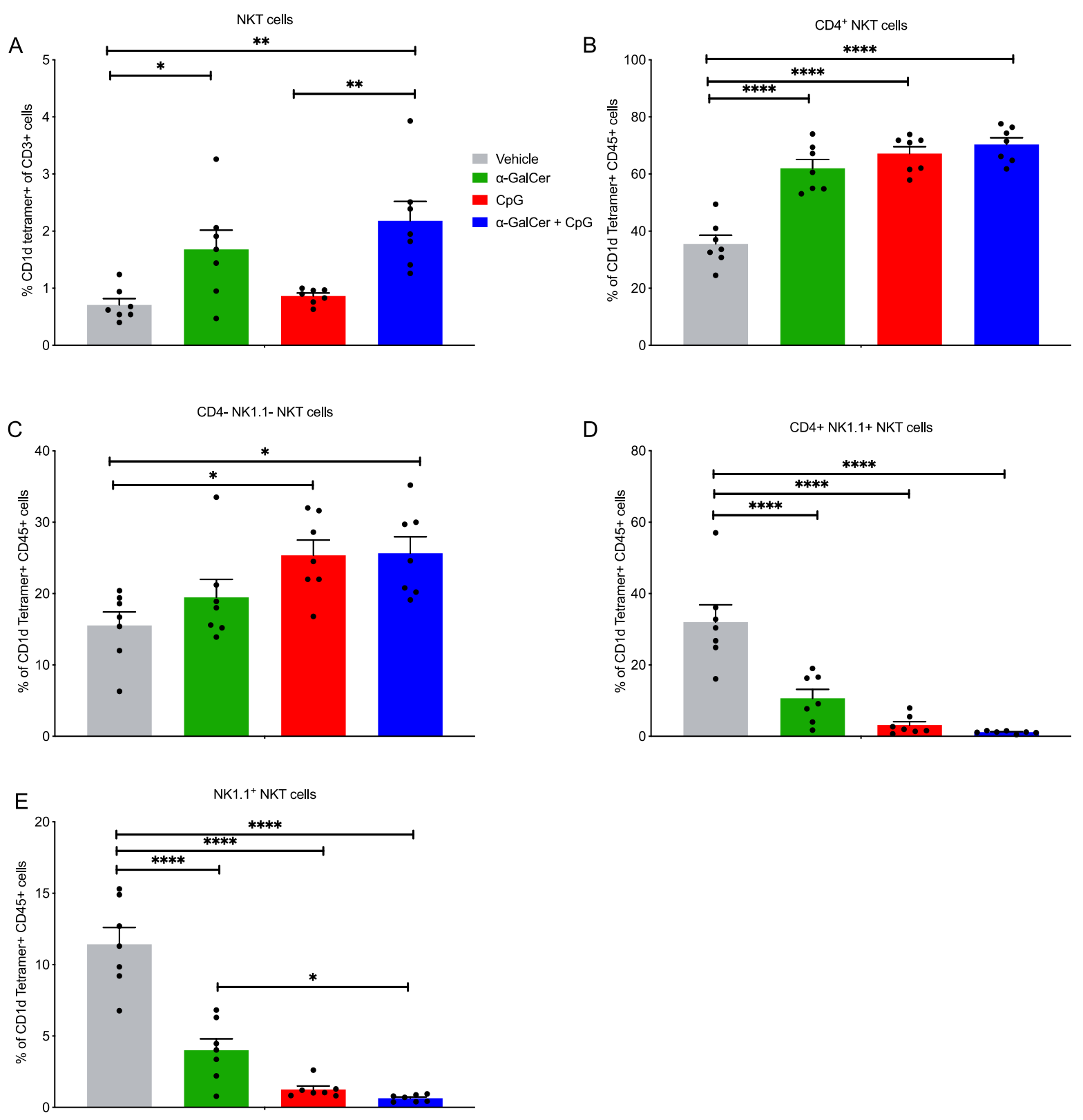

Figure 36 Intratumoural combination therapy of $\alpha$-GalCer and $C p G$ induces significant changes to the iNKT cell populations in the spleen. A) Combination therapy significantly increased the frequency of iNKT cells in the spleen compared to CpG and vehicle. $\alpha$-GalCer induced a statistically significant increase in iNKT cells over vehicle. B) Treatment with $\alpha$-GalCer, CpG or combination therapy resulted in an increase in $C D 4^{+} i N K T$ cells in the spleen. C) Treatment with $C p G$ and combination therapy induced a significant increase in CD4-NK1.1- iNKT cells in the spleen. D) Treatment with a-GalCer, CpG or combination therapy significantly reduced the frequency of $C D 4^{+} N K 1.1^{+} i N K T$ cells compared to vehicle. E) Treatment with $\alpha$-GalCer, CpG or combination therapy significantly reduced the frequency of $N K 1.1^{+}$iNKT cells compared to vehicle, in addition, combination therapy induced a significant reduction relative to $\alpha$-GalCer. Statistically significant differences are indicated $* P<0.05$, $* * P<0.01, * * * P<0.001, * * * * P<0.0001$ (One-Way ANOVA with Tukey’s Post-Hoc Test). 
Unlike in the tumours and associated dLNs, there were no significant differences in the frequency of B cells in the spleen between the treatment groups (Fig 37). This may suggest that B cell priming by the treatments may be a localised phenomenon.

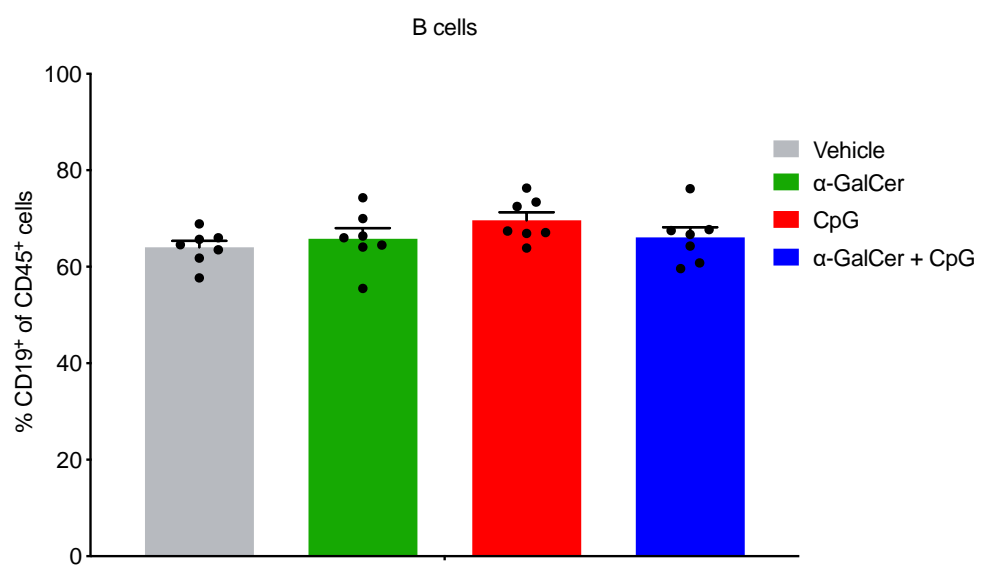

Figure 37 Intratumoural combination therapy of $\alpha$-GalCer and $C p G$ does not increase the frequency of $B$ cells in the spleen. The spleen exhibited no change in $B$ cell frequency as a result of treatment. Statistically significant differences are indicated $* P<0.05, * * P<0.01, * * * P<0.001$, ****P<0.0001 (One-Way ANOVA with Tukey's Post-Hoc Test). 


\subsubsection{Influx of immune cells into the tumour microenvironment}

To follow up on the flow cytometry findings, in a preliminary experiment, the immune cells that were infiltrating the tumour were examined by immunohistochemistry on EG7.OVA tumours that had been treated with either PBS $(\mathrm{N}=1)$ or the combination of $\alpha-\mathrm{GalCer}$ and $\mathrm{CpG}$ intratumourally 24 hours after the end of the last treatment cycle $(\mathrm{N}=1)$ (day 16) so as to give time from initial treatment for a $\mathrm{T}$ cell response to be initiated and home to the tumour. The tumours were snap-frozen using liquid nitrogen and were sectioned on a cryostat and placed on slides before immunohistochemistry was performed with antibodies to CD4 (BV421, Blue) and CD8 (BB515, Green) to visualise T cells (although some myeloid cells can express these markers), and to CD64 as a marker of the macrophage/monocyte lineage (AF647, Red).

The images generated are in Fig 38, with $100 \mu \mathrm{m}$ scale shown. These are randomly selected sections. While this experiment was performed only once with 2 animals, the observed difference between treated and untreated samples was dramatic. The control, a PBS treated EG7.OVA tumour was largely devoid of immune cells (left panel). Compared to PBS vehicle, the tumour treated with the combination of $\alpha-G a l C e r$ and $\mathrm{CpG}$ had a major influx of immune cells. This suggested that treatment with the combination induced immune infiltration of the tumour, prompting a larger study.

To follow up, in a larger experiment, tumours were prepared as above, and immunohistochemistry was performed by Professor Rod Dunbar's lab (University of Auckland), as they have expertise in this area. The tumours were harvested on day 11 after the start of treatment so as to coincide with the flow cytometry data described previously (Fig 19-37). The 4 quadrants are the 4 experimental conditions marked, with each row being one of 3 sites randomly selected in each tumour (to show any variability) and each column a different stain as marked 
(Fig 39). In the vehicle treated primary tumour (upper left quadrant), the untreated tumour cells are cyan (blue + green) in columns $1 \& 2$, staining for CD45.2 and CD3, and blue/white in column 3, staining for only DAPI and CD45.2. The bottom 2 rows show the tumour mass (cyan). There appear to be no $\mathrm{T}$ cells $\left(\mathrm{CD}^{+} \mathrm{CD}^{2} 5.2^{-}\right)$in these tumour masses. In addition, there are no NK cells, iNKTs or B cells (NK1.1 and CD45R respectively) infiltrating the main tumour body, although one segment of the edge of the tumour, shown in the bottom row, does have some NK1.1 cells and the occasional T cell. There are plenty of $\mathrm{F} 4 / 80^{+}$macrophages in some parts of the tumour mass which are CD206: The upper row shows the non-tumour area. This has some macrophages and NK1.1 cells, and the occasional T cell, but notably the macrophages are often $\mathrm{CD} 206^{+} \mathrm{F} 4 / 80^{+}$, so different from those in the tumour. Thus it appears that the vehicle-treated tumour has an abundance of tumour cells, but lacks T cell infiltrates, NK cells or B cells (except for $\mathrm{NK} 1.1^{+}$cells along one segment of the edge); however some zones are infiltrated by $\mathrm{F} 4 / 80^{+}$ macrophages.

Looking at the lower left quadrant, the untreated contralateral tumour of vehicle-treated mice looks mostly similar to what is described above. However, there is a large "slash" across the tumour that has a large amount of $\mathrm{NK} 1.1^{+} \mathrm{CD} 3^{-}$cells and $\mathrm{F} 4 / 80^{+} \mathrm{CD} 206^{-}$macrophages, as well as some $\mathrm{CD}_{206^{+}}$macrophages mingled among the $\mathrm{F} 4 / 80^{+}$macrophages, but few were double-positive within the tumours. As these were sequential sections, we are uncertain as to whether they are the same cells or intermingled.

Next, looking at the $\alpha-\mathrm{GalCer} / \mathrm{CpG}$ treated primary tumour in the upper right quadrant there are massive changes relative to the vehicle-treated tumours. In the top 2 rows the tumour cells are largely obliterated and replaced by $\mathrm{T}$ cells and NK1.1 cells (but not CD3 ${ }^{+} \mathrm{NK} 1.1^{+}$ double-positives). These cells surround voids suggesting massive necrosis, and there is a mix of 
macrophages that are largely F4/80+ CD206 ${ }^{+}$cells, but there are also some F4/80 ${ }^{+}$CD206- cells. The bottom row shows a zone where the $\mathrm{CD} 3^{+} \mathrm{CD} 45.2^{+}$tumour cells are surviving better, and although there is not much in the way of inflammatory infiltrate, there are possibly voids opening up, suggesting early necrosis.

Finally, looking at the treated contralateral tumour of the $\alpha$-GalCer/CpG treated mice (lower right quadrant), there were similar changes as seen in the treated tumour but weaker and less abundant. The bottom rows show areas where the tumour cells are largely untroubled, even in the presence of $\mathrm{F} 4 / 80^{+}$macrophages. However, the top row shows areas that look more like the treated tumour with T cell and NK1.1 infiltrates, CD206 ${ }^{+} \mathrm{F} 4 / 80^{+}$macrophages, and voids opening up again, suggesting necrosis. 


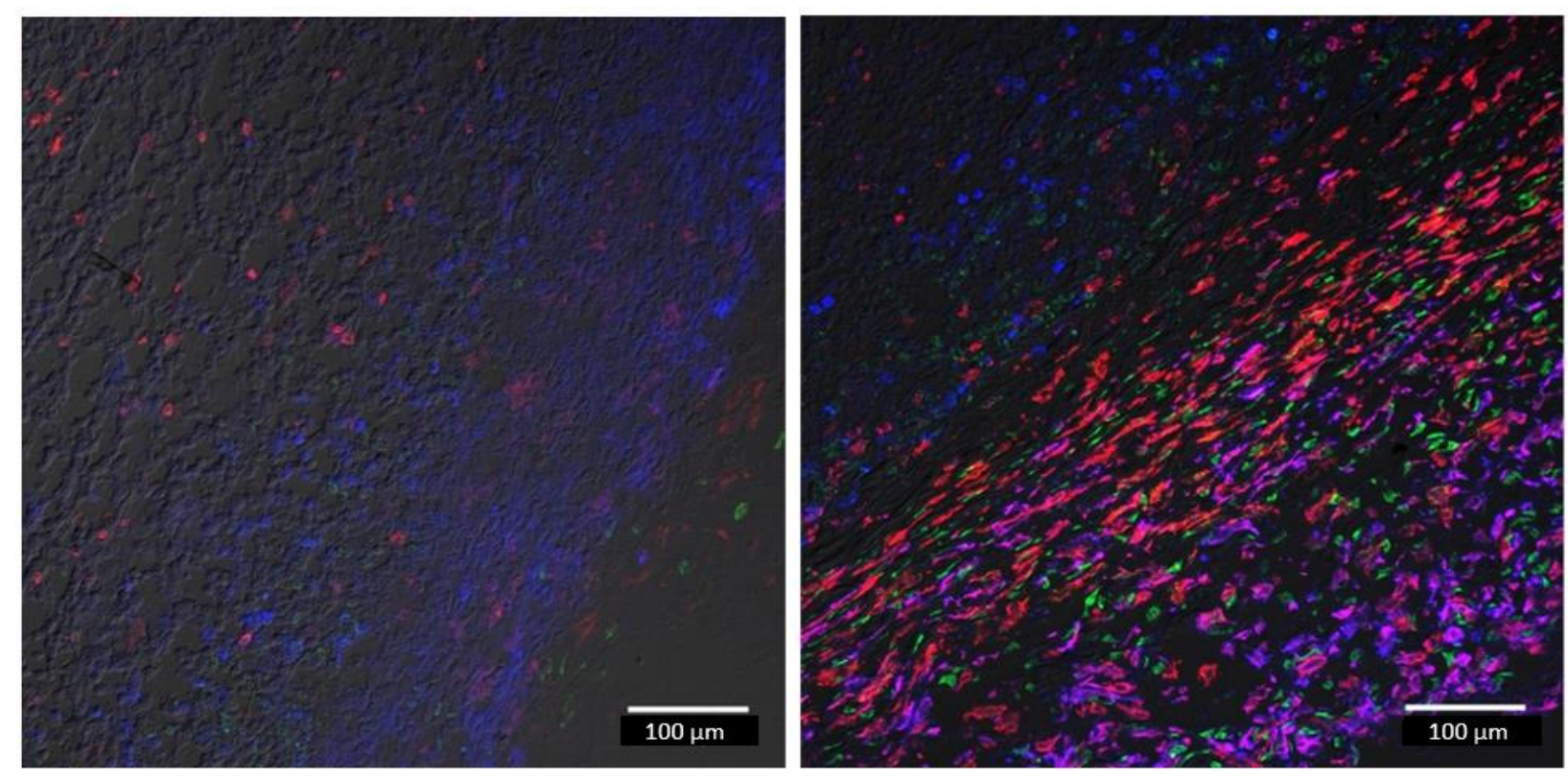

Figure 38 Intratumoural combination of a-GalCer and $\mathrm{CpG}$ induces an influx of $\mathrm{CD} 4^{+}, \mathrm{CD} 8^{+}, \mathrm{CD} 64^{+}$and $\mathrm{CD} 4^{+} \mathrm{CD} 64^{+}$cells. Tumours from treated and vehicle-treated mice were harvested on day 16 and snap-frozen in liquid nitrogen. Sections were then made and mounted on slides and stained with CD8-BB515, CD64-AF647, and CD4-BV421 antibodies. A) The vehicle-injected tumour shows minimal immune cell infiltration. B) Intratumoural combination therapy induces immune cell infiltration. CD4 (BV421, Blue), CD8 (BB515, Green) and CD64 (AF647, Red). Representative of 2 randomly selected sections from 2 animals (In collaboration with Alfonso Schmidt). The scale is $100 \mu \mathrm{m}$. 
Control primary tumour D11
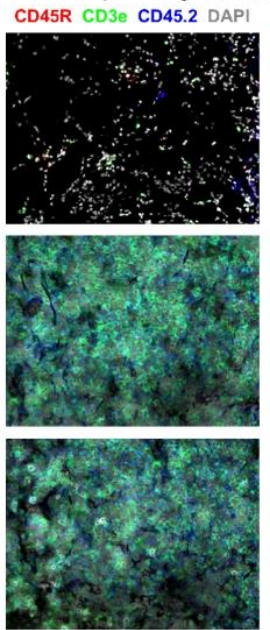

Control contralateral tumour D11
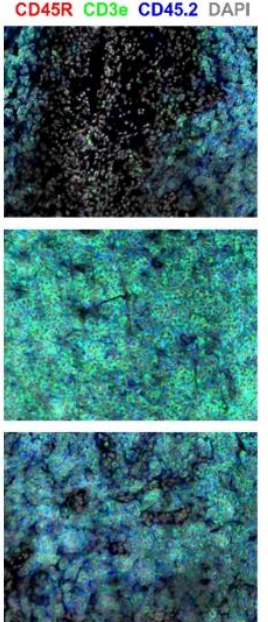
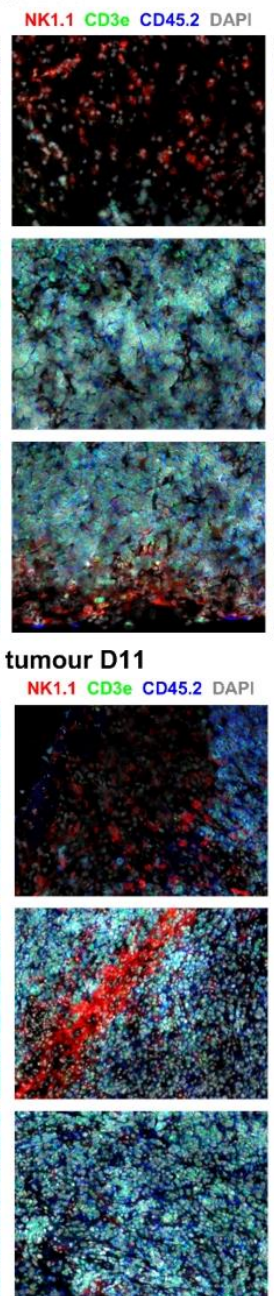

CD206 F4/80 CD45.2 DAPI
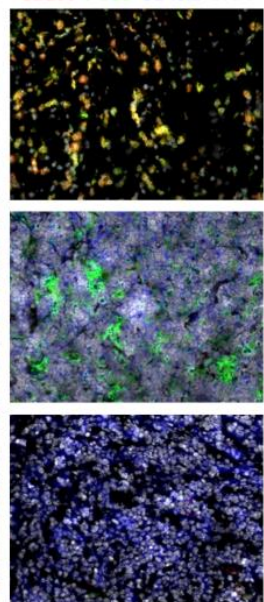

CD206 F $4 / 80$ CD45.2 DAP|
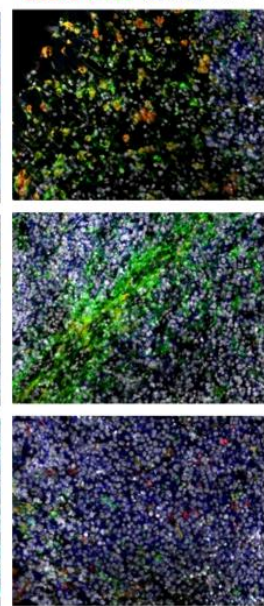

Treated primary tumour D11
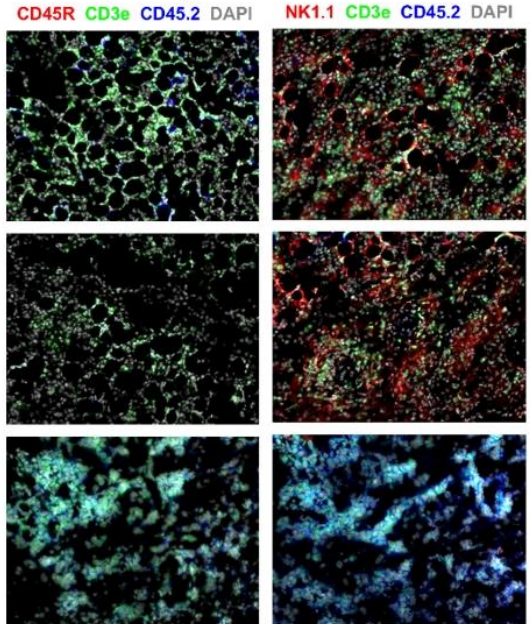

Treated contralateral tumour D11
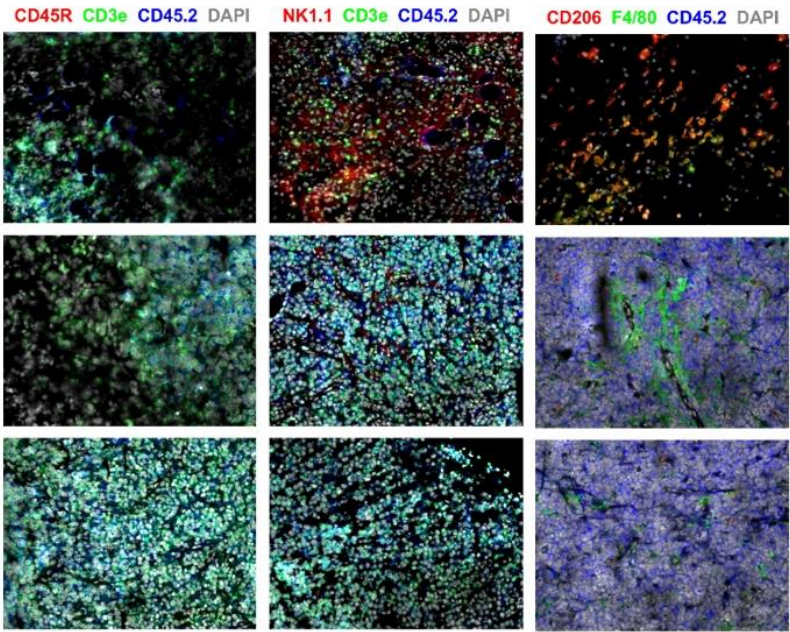

Figure 39 Treatment with intratumourally administered a-GalCer and $C p G$ induces infiltration by NK, T cells and macrophages in treated tumours and to a lesser extent the untreated contralateral tumour. The 4 quadrants are the 4 experimental conditions marked, with each row being one of 3 sites selected by Jennifer Chen in each tumour (to showing any variability) and each column a different stain as marked. Upper left quadrant: In the vehicle-treated primary tumour (upper left quadrant), the There appears to be no T cells $\left(C D 3^{+} C D 45.2^{-}\right)$in these tumour masses. In addition, there are no NK cells, iNKTs or B cells (NK1.1 and CD45R respectively) infiltrating the main tumour body, although one segment of the edge of the tumour, shown in the bottom row, does have some NK1.1 cells and the occasional T cell. There are plenty of F4/80+ macrophages in some parts of the tumour mass, and they're CD206. The upper row shows the non-tumour area. This has some macrophages and NK1.1 cells, and the occasional $T$ cell - but notably the macrophages are often $\mathrm{CD}^{206^{+}} \mathrm{F}_{4} / 80^{+}$, so different from those in the tumour. Thus it appears that the vehicle-treated tumour has an abundance of tumour cells, but lacks $T$ cell infiltrates, NK cells or B cells (except for $\mathrm{NK} 1.1^{+}$cells along one segment of the edge); however some zones are infiltrated by $\mathrm{F}^{2} / 8 \mathrm{O}^{+}$macrophages. Lower left quadrant: the untreated contralateral tumour of vehicle-treated mice. Similar to the vehicle-treated tumour, however, there is a large "slash" across the tumour that has a large amount of NK1.1 ${ }^{+} \mathrm{CD} 3^{-}$cells and F4/80 ${ }^{+} \mathrm{CD} 206^{-}$macrophages, as well as some $\mathrm{CD}_{206^{+}}$macrophages mingled among the $\mathrm{F} 4 / 80^{+}$macrophages, but few were double-positive within the tumours. Upper right quadrant: $\alpha$-GalCer and $C p G$ treated primary tumour. There are massive changes relative to the vehicle-treated tumours. In the top 2 rows the tumour cells are largely obliterated and replaced by T cells and NK1.1 cells (but not CD $3^{+} N K 1.1^{+}$doublepositives). These cells surround voids suggesting massive necrosis, and there's a mix of macrophages that are largely F4/80 ${ }^{+}$ $\mathrm{CD}_{206^{+}}$cells, but there are also some $\mathrm{F} 4 / 80^{+}$cells. The bottom row shows a zone where the $\mathrm{CD} 3^{+} \mathrm{CD} 45.2^{+}$tumour cells are surviving better, and although there is not much in the way of inflammatory infiltrate, there are possibly voids opening up, suggesting early necrosis. Lower right quadrant: untreated contralateral tumour of the $\alpha$-GalCer and CpG treated mice. There were similar changes as seen in the treated tumour but much weaker and less abundant. The bottom rows show areas where the tumour cells are largely untroubled, even in the presence of $F 4 / 80^{+}$macrophages. However, the top row shows areas that look 
more like the treated tumour with T cell and NK1.1 infiltrates, CD206 ${ }^{+} \mathrm{F}^{\prime} / 80^{+}$macrophages, and voids opening up again, suggesting necrosis.

\subsubsection{Exploring the role of $\mathrm{CD8}^{+} \mathrm{T}$ cells}

The flow cytometry data, perhaps surprisingly, did not reveal substantial changes in the conventional $\mathrm{T}$ cell population with the combined treatment, with only slight trends towards increased $\mathrm{CD}^{+} \mathrm{T}$ memory cells in tumour and spleen apparent. The preliminary histological analysis did show an influx of $\mathrm{T}$ cells with the combined treatment, but this work needs further validation. Therefore, to explore the role of T cells in combined treatment in more detail, specifically, the activity of $\mathrm{CD} 8^{+} \mathrm{T}$ cells, the functional consequence of depleting these CD8 expressing cells was explored.

First, given that memory formation is a hallmark of adaptive immunity, and a memory response was previously shown to be initiated in mice that had successfully rejected tumours, the role of $\mathrm{CD} 8^{+} \mathrm{T}$ cells in this memory response was evaluated. Thus, mice that had rejected EL4.LA tumours were assigned to either receive vehicle (PBS) or CD8 depleting antibody intraperitoneally (i.p.) days -2 and -1 prior to rechallenge with $1 \times 10^{6}$ EL4 into the opposite flank. Successful depletion of $\mathrm{CD}^{+}$cells was confirmed using flow cytometry (CD8-BV421, CD3FITC) on day 0 (Fig 40a). Naïve mice challenged with EL4.LA were used as controls for tumour growth. Mice challenged with tumours that had fully regressed from multiple previous experiments and were not subject to depletion of $\mathrm{CD} 8^{+}$cells, were able to reject the rechallenge, while those that were depleted of their $\mathrm{CD}^{+}$cells lost their anti-tumour memory (Fig 40b/c). This suggests that long term anti-tumour memory induced by the combination of $\alpha$-GalCer and $\mathrm{CpG}$ is $\mathrm{CD} 8^{+}$cell-mediated ${ }^{249}$.

Having established that $\mathrm{CD} 8^{+} \mathrm{T}$ cells were involved in the memory response to successful treatment, it was next investigated whether CD8-expressing cells were also the main 
effectors in the immediate anti-tumour response to treatment. To this end, mice were challenged with $1 \times 10^{6}$ EG7.OVA cells on both flanks and treatment was started once tumours reached $6 \times 6$ mm. The anti-CD8 antibody was given i.p. on days 8, 9, and 12 post tumour inoculation and isotype antibody was used as control. The mice were given either vehicle, isotype mAb with $\alpha$ GalCer and 6 hours delayed $\mathrm{CpG}$ or CD8 mAb with $\alpha$-GalCer 6 hours delayed CpG. The depletion of $\mathrm{CD}^{+} \mathrm{T}$ cell was determined via flow cytometry analysis on day 11 . The majority of mice that receive $\alpha-\mathrm{GalCer}$ and $\mathrm{CpG}$ with isotype control mAb saw regression in both their treated and untreated tumours, while all the mice that were depleted of their $\mathrm{CD}^{+} \mathrm{T}$ cells succumbed to both tumours and did not see any form of abscopal effect (Fig 41). This suggests that the anti-tumour response to both the treated and distal untreated tumour is $\mathrm{CD}^{+} \mathrm{T}$ cellmediated. 
A)

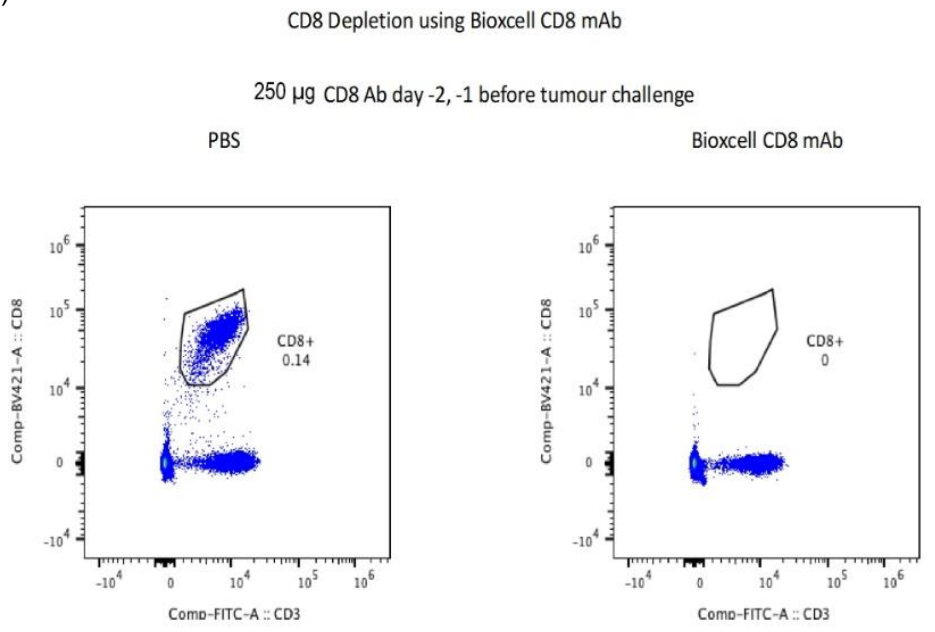

EL4 Rechallenge with CD8+ Cell Depletion

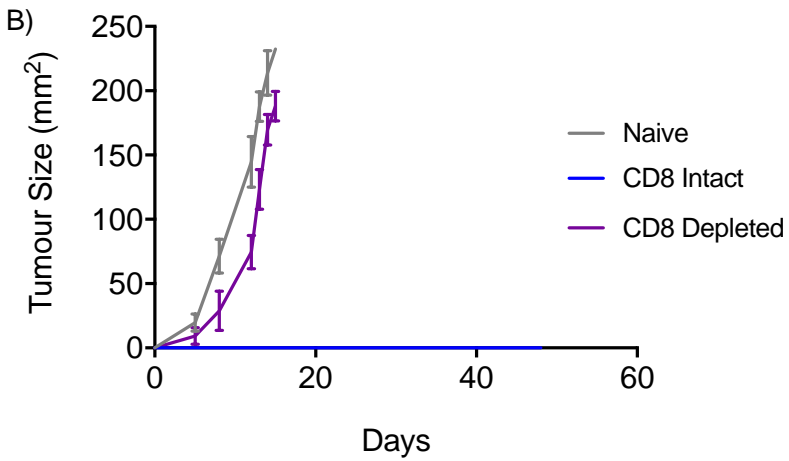

EL4 Rechallenge with CD8 ${ }^{+}$Cell Depletion Survival

C)

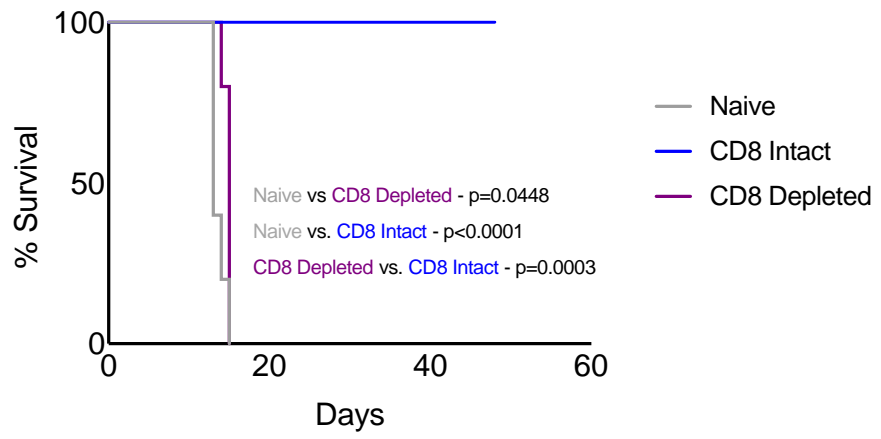

Figure 40:Memory against ELA.LA is CD8 mediated as mice depleted of CD8+ cells lose memory against rechallenge. A) Flow plots for CD8 depletion Left panel-cured mice with CD8 ${ }^{+}$cell; Right panel-Cured mice whose CD8 ${ }^{+}$cells were depleted with CD8 mAb from Bioxcell. Antibodies (CD3-FITC, CD8-BV421). B) Tumour growth curves plotted for each mouse. C) Survival curves for the different treatment groups, with statistics indicated (log-rank Mantel-Cox test). $N=8-9$ animals 
A
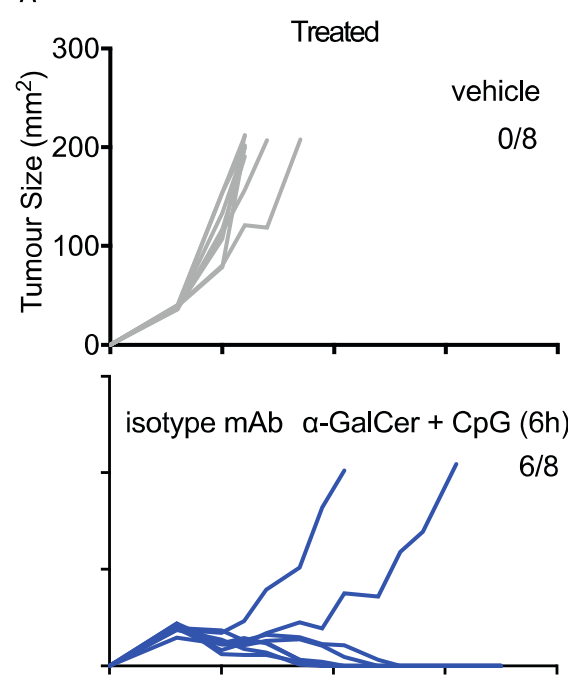

anti-CD8 $\alpha-$ GalCer + CpG (6h)

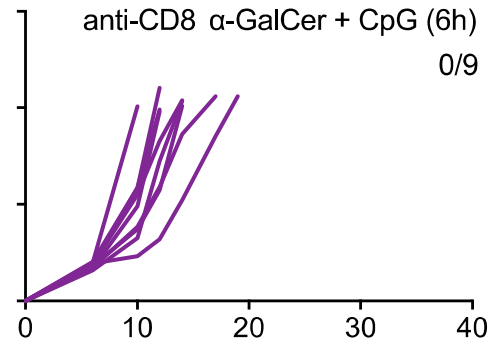

B
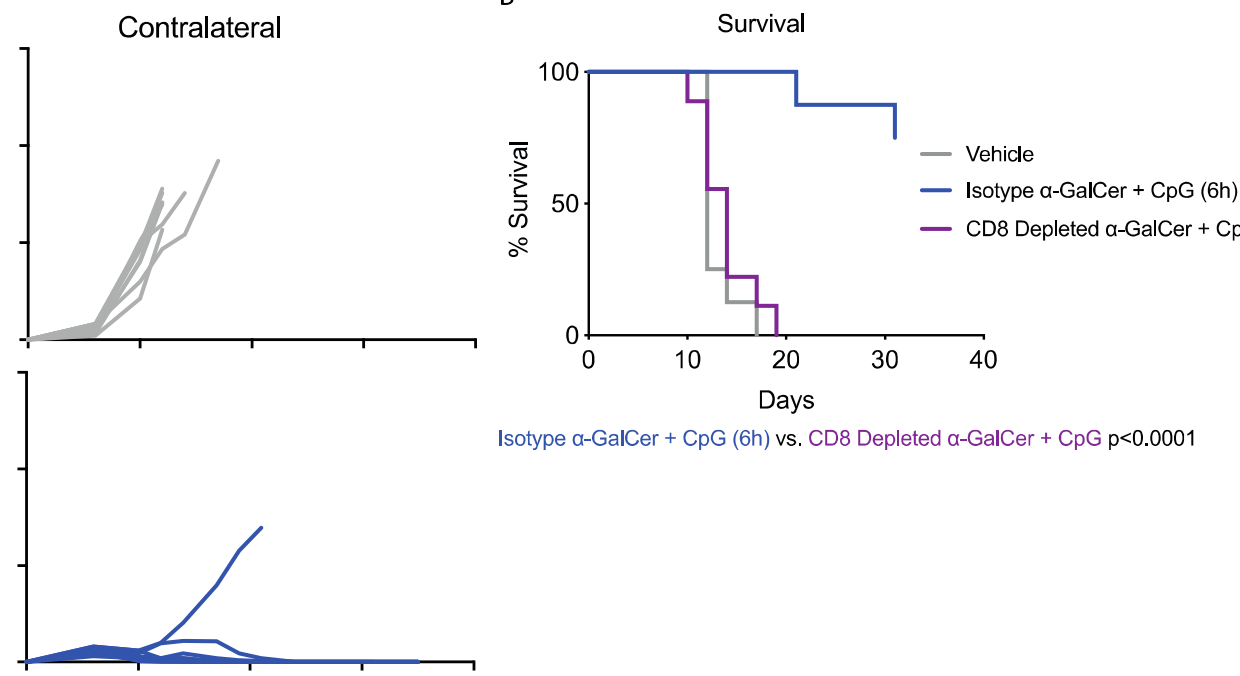

- CD8 Depleted $\alpha-G a l C e r+C p G(6 h)$

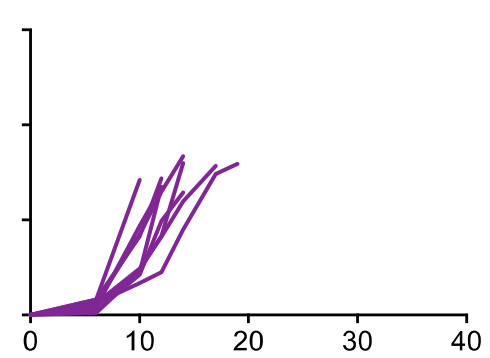

Days

Figure $41 \alpha$-GalCer/CpG combination therapy requires $C D 8^{+}$cells. A) Tumour growth curves plotted for each mouse. B) Survival curves for the different treatment groups, with statistics indicated (log-rank Mantel-Cox test). 


\subsubsection{Exploring the role of iNKT cells}

It was expected that iNKT cells should have some importance to the combination of $\alpha$ GalCer and CpG, since $\alpha$-GalCer activates iNKT cells which leads to improved DC activation and antigen-cross presentation. The flow cytometry data also showed an increase in the frequency of iNKT cells in the tumour after treatment, which was significant with the combined treatment, suggesting iNKT cells may have additional roles in the anti-tumour effect, perhaps as effectors themselves, or to support on-going immunity. To assess whether iNKT cells were indeed critical to the response, CD1d blocking antibody was administered i.p. to mice undergoing treatment. Mice were challenged with $1 \times 10^{6}$ EG7.OVA cells on both flanks and treatment began when the tumours reached approximately $6 \times 6 \mathrm{~mm}$ in size (day 6). The mice were given either vehicle, isotype mAb with $\alpha-G a l C e r$ and $\mathrm{CpG}(6 \mathrm{~h})$ or CD1d mAb with $\alpha$ GalCer and $\mathrm{CpG}$ (6h). Importantly, because iNKT cells were thought to be involved in licensing DCs for T cell priming, anti-CD1d (or isotype) was given on days 8, 9 and 11 post tumour challenge, which should block CD1d mediated activity after these initial activation events had taken place; it was therefore aimed to establish whether iNKT cells were also required at the effector phase of the response. Doses of antibody used were selected based on literature precedent ${ }^{250}$.

The majority of mice that were administered antibody to block interactions between iNKT cells and CD1d were able to induce some regression in their $\alpha$-GalCer and CpG treated tumours, as indicated by the slower individual growth curves in Fig 39. However, little regression was observed in the untreated distal tumours, and the animals ultimately succumbed to these tumours, so that overall survival was not improved with the combined treatment. This is 
in contrast to the isotype where both treated and untreated tumours experienced a regression in the majority of treated mice, and survival was significantly improved (Fig 42).

Similar results were obtained when using host mice that were devoid of iNKT cells throughout treatment. Two strains of mice were used; CD1d-deficient animals $\left(\right.$ CD1d $\left.{ }^{-/}\right)$, which cannot support the positive selection of iNKT cells in the thymus, and mice without the ability to form the TCR- $\alpha$ chain expressed by type I NKT cells (Traj18 $8^{-/-}$). In either case, the treated tumours in these mice saw some regression, but the mice eventually succumbed to their untreated, contralateral tumours (Fig 42). Overall these data suggest that iNKT cells are implicated in both the priming of local anti-tumour responses (which were weaker in the absence of these cells throughout treatment) and for the abscopal effect, with interactions via CD1d required in the effector phase for this effect. 
A
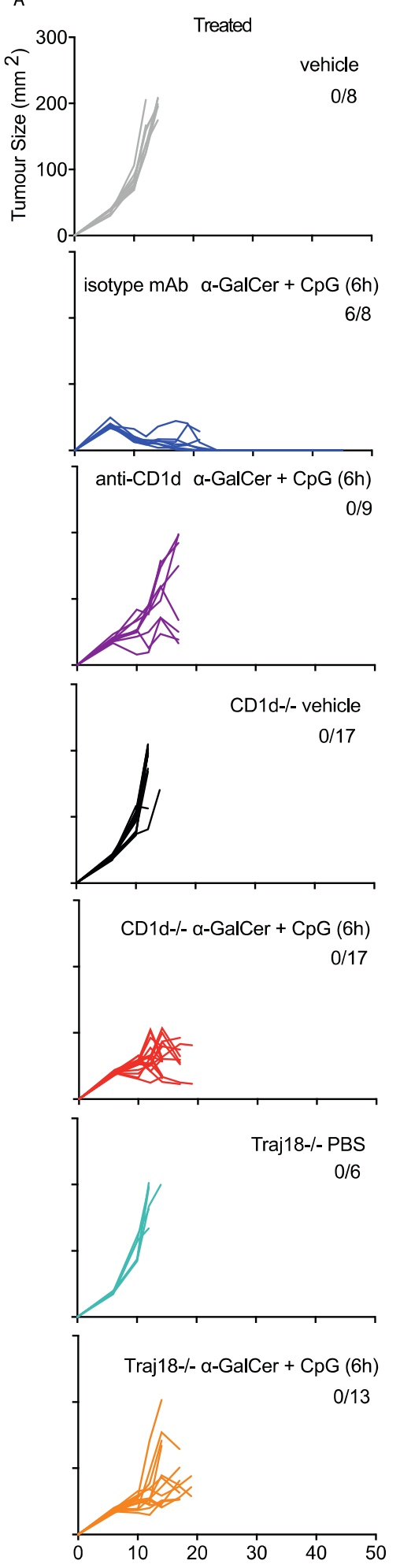
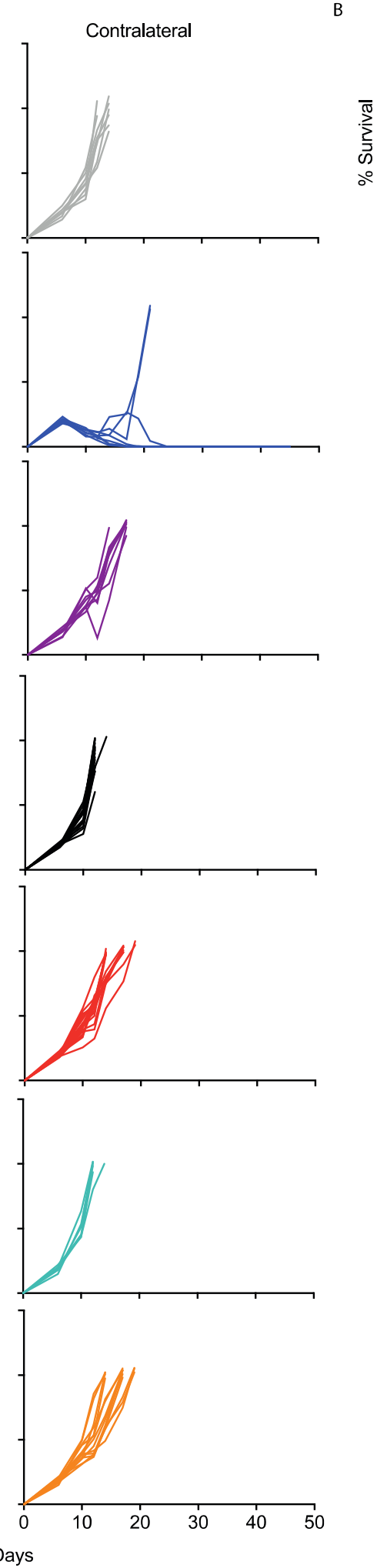

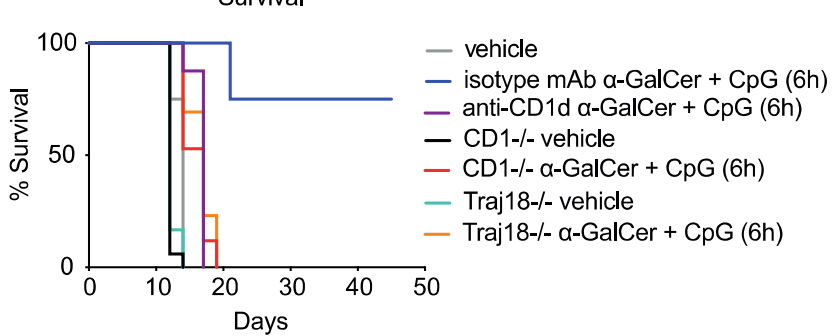

isotype $\alpha-G a l C e r+C p G(6 h)$ vs. anti-CD1d $\alpha-G a l C e r+C p G(6 h) \quad p=0.0001$ isotype $\alpha-\mathrm{GalCer}+\mathrm{CpG}(6 \mathrm{~h})$ vs. CD1-/- $\alpha-\mathrm{GalCer}+\mathrm{CpG}(6 \mathrm{~h}) \quad \mathrm{p}<0.0001$ isotype $a-G a l C e r+C p G(6 h)$ vs. Traj18-/- $\alpha-G a l C e r+C p G(6 h) \quad p<0.0001$

Figure $42 \alpha$-GalCer/CpG combination therapy requires iNKT cells. A) Tumour growth curves plotted for each mouse. B) Survival curves for the different treatment groups, with statistics indicated (log-rank Mantel-Cox test). 


\subsubsection{Exploring the role of DC subsets}

The previous data point to a role of $\mathrm{CD} 8^{+} \mathrm{T}$ cells and iNKT cells in the anti-tumour response induced with $\mathrm{CpG}$ and $\alpha-\mathrm{GalCer}$. Both of these cellular responses have previously been reported to be dependent to some degree on $\mathrm{cDC} 1$ cells, which have a heightened capacity for cross-priming $\mathrm{CD}^{+} \mathrm{T}$ cells, are involved in activating iNKT cells, and are the primary producers of IL-12p70 when $\alpha$-GalCer is administered ${ }^{251}$. Also, given that CpG oligonucleotides are known to induce the release of type 1 IFN, which can enhance adaptive immune responses (including $\mathrm{CD}^{+} \mathrm{T}$ cells responses), and pDCs are the primary producers of type $1 \mathrm{IFN}$, it was possible that pDCs were also involved in the anti-tumour response.

In order to delineate the role of these different DC subsets, we utilised Clec9a and Siglec$\mathrm{H}$ diphtheria toxin receptor (DTR) mice to deplete $\mathrm{cDC} 1$ or pDCs respectively. Clec9a is a group V C-type-lectin-like receptor that is expressed on myeloid lineage cells and functions as an activation receptor ${ }^{252}$. Siglec-H, on the other hand, is a murine CD33-related siglec-like molecule with 2 Ig-domains, which is explicitly expressed on BST2 ${ }^{+}$pDCs in bone marrow, spleen, blood and lymph node, though it is not present on other leukocytes including cDC subsets ${ }^{253}$. Mice are known to be largely resistant to diphtheria toxin (DT), and thus these transgenic mice have been modified to express the human DTR on specific cell subsets (on either the Clec9a or Siglec- $\mathrm{H}^{+}$promoters) ${ }^{246}$. In this way, administering diphtheria toxin will result in selected cell populations being knocked down while the rest of the mouse's immune system remains untouched. 


\subsubsection{Effect of depletion of cDC1 cells}

To test the involvement of $\mathrm{cDC} 1$ cells, Clec9a DTR mice were inoculated with $1 \times 10^{6}$ EG7.OVA subcutaneously into the right flank. On days 4 and 5 (days -2 and -1 prior to the start of treatment), the mice were given 15ng of DT per gram of body weight or vehicle. A couple of mice were set aside to determine the level of depletion via flow cytometry on the spleen, with a reduction of $87 \%$ of $\mathrm{CD} 11 \mathrm{c}^{+} \mathrm{CD}^{+}$cells observed relative to untreated controls (Supplementary Fig 4). Once the tumours reached $\sim 6 \times 6 \mathrm{~mm}$, mice were treated with vehicle (PBS), $\mathrm{CpG}$, or $\alpha$ GalCer with 6 hours delayed CpG, with or without DT.

When the mice were depleted of their Clec9 $\mathrm{a}^{+}$DCs there was a blunted anti-tumour response to the combination therapy; although there was a statistically significant growth delay compared to vehicle, no animals survived treatment. In contrast, most of the mice that retained their $\mathrm{Clec} 9 \mathrm{a}^{+}$DCs had their tumours regress to the baseline as a result of intratumoural $\alpha$-GalCer and CpG therapy (Fig 43). This suggests that $\mathrm{Clec} 9 \mathrm{a}^{+} \mathrm{DCs}$ are essential for an effective antitumour response, and by extension, that antigen presentation via this specialised DC subset is likely to be highly important. 
A
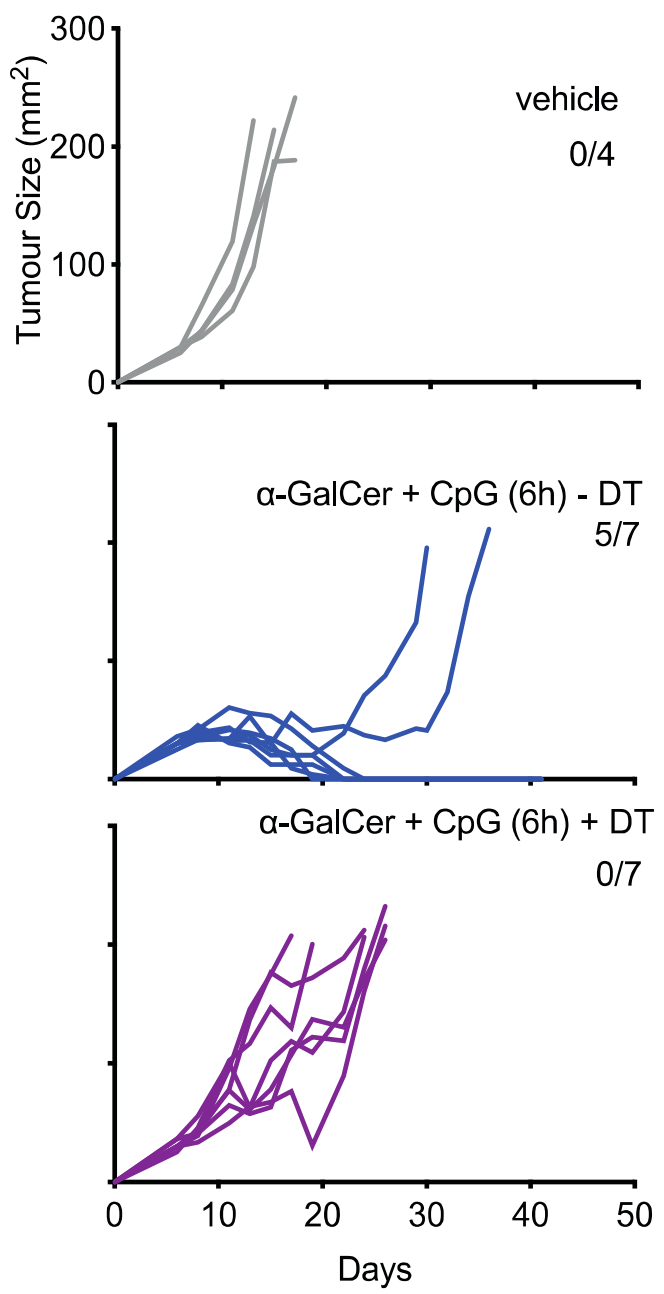

B

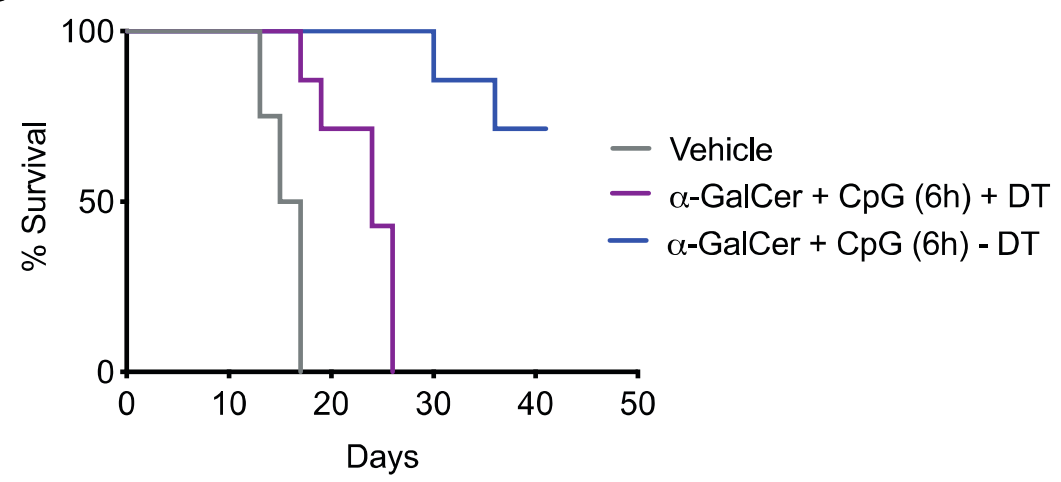

$\alpha-$ GalCer + CpG (6h) + DT vs $\alpha-$ GalCer + CpG (6h) $-D T p=0.0003$ Vehicle vs $\alpha$-GalCer + CpG (6h) + DT $p=0.0038$

Figure $43 \alpha$-GalCer and CpG mediated anti-tumour response requires Clec $9 a^{+}$DCs. A) Tumour growth curves plotted for each mouse. B) Survival curves for the different treatment groups, with statistical differences indicated (log-rank Mantel-Cox test). Representative of 2 separate experiments. 


\subsubsection{Effect of depletion of pDCs}

To investigate the role of pDCs in the combination treatment, Siglec-H DTR mice were inoculated with $1 \times 10^{6}$ EG7.OVA subcutaneously into the right flank. On days 4 and 5 (days -2 and -1 before the start of treatment), the mice were given $15 \mathrm{ng}$ of DT per gram of body weight or vehicle. A couple of mice were set aside to determine the level of depletion via flow cytometry on the spleen, with a reduction of $79 \%$ of Siglec- $\mathrm{H}^{+}$cells observed relative to untreated controls (Supplementary Fig 6). Once the tumours reached $\sim 6 \times 6 \mathrm{~mm}$, mice were treated with vehicle (PBS), CpG, or $\alpha$-GalCer with 6 hours delayed $\mathrm{CpG}$ with or without DT.

None of the mice that had their Siglec- $\mathrm{H}^{+}$cells depleted underwent regression to baseline after receiving an intratumoural combination of $\alpha$-GalCer and 6 hours delayed $\mathrm{CpG}$, though there was a statistically significant growth delay relative to vehicle; while treatment on the Siglec-H DTR mice that retained their Siglec- $\mathrm{H}^{+}$resulted in complete regression in the majority of the mice (Fig 44). This demonstrates that Siglec-H $\mathrm{H}^{+}$cells (pDCs) are important for the combination of $\alpha$-GalCer and $\mathrm{CpG}$ to function effectively, with their presence required to induce responses capable of complete regression. 


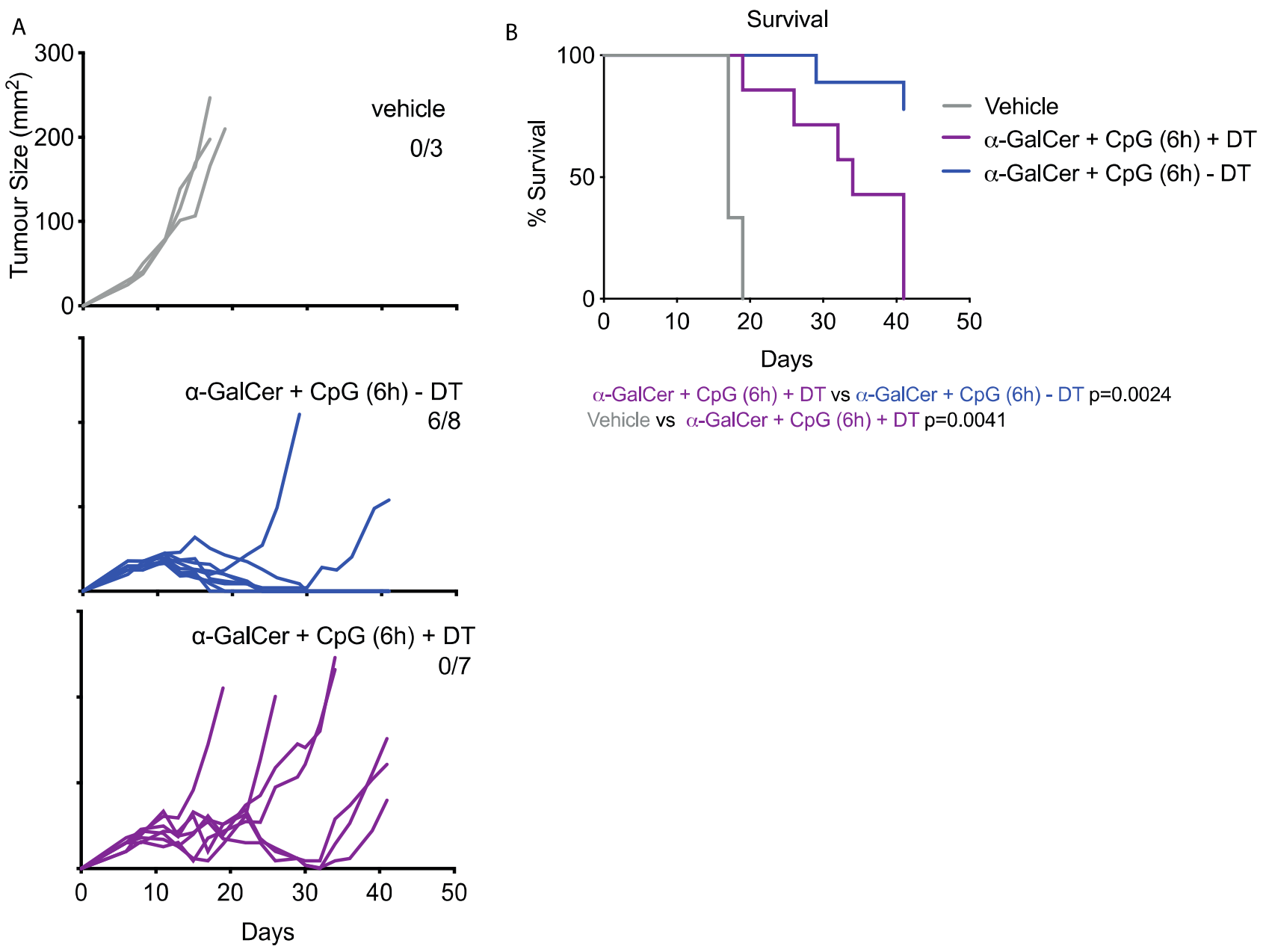

Figure $44 \alpha$-GalCer and CpG mediated anti-tumour response requires Siglec- $H^{+}$DCs. A) Tumour growth curves plotted for each mouse. B) Survival curves for the different treatment groups, with statistics indicated (log-rank Mantel-Cox test).

Representative of 2 separate experiments. 


\subsubsection{Role of B-cells}

Unlike Class A CpG ODNs which induce the production of large amounts of IFN- $\alpha$ from pDCs but weakly stimulate TLR9-dependent NF-KB signalling and pro-inflammatory cytokine (e.g. IL-6) production, CpG-B (1826) (which was used in these studies) strongly activates B cells and TLR9-dependent NF- $\kappa B$ signalling but weakly stimulate IFN- $\alpha$ secretion ${ }^{254}$. It was therefore investigated how B cells were affected by the intratumoural combination of $\alpha-\mathrm{GalCer}$ and $\mathrm{CpG}$. The function of activated B cells is to secrete antibodies which are the final products of the complex response to antigens. They specifically recognize their target antigen and have the function to clear the tumour or pathogen and to prevent its recurrence ${ }^{255}$. Therefore, we wanted to test whether the antibodies in the serum of mice cured via intratumoural $\alpha-G a l C e r$ and $\mathrm{CpG}$ therapy would protect against tumour challenge. In order to do this, serum was collected from the blood of intratumourally treated survivors from a previous experiment, where the blood was collected via terminal cardiac puncture 2 weeks after the resolution of disease. This is a procedure whereby the mice are culled using carbon dioxide, and then a 27 gauge needle is inserted into the left ventricle, and the blood is aspirated through the heart to maximise the blood collected. The blood was allowed to clot, and then serum was harvested, and $200 \mu \mathrm{L}$ was then transferred intravenously (i.v.) into naïve mice that were then challenged with $1 \times 10^{6}$ EG7.OVA cells in the flank 24 hours later. Serum from naïve mice was also transferred as controls.

Mice that had been given serum from the previously successfully treated mice experienced a tumour growth delay compared to vehicle, with a small proportion being completely protected from tumour challenge and never developing a tumour (Fig 45). These data suggest that circulating antibodies can contribute to the antitumour response. 
A)

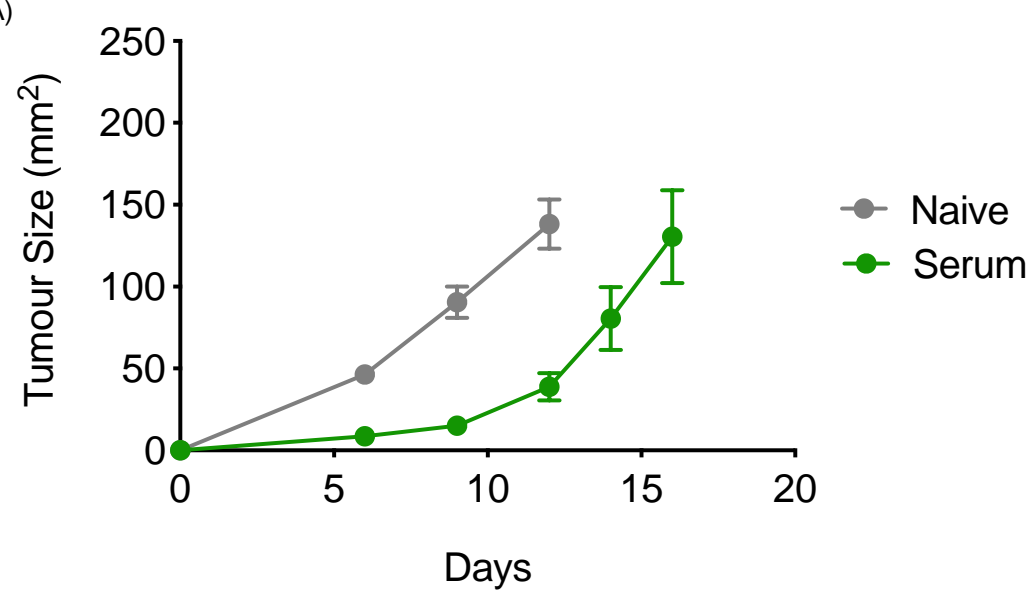

Survival

B)

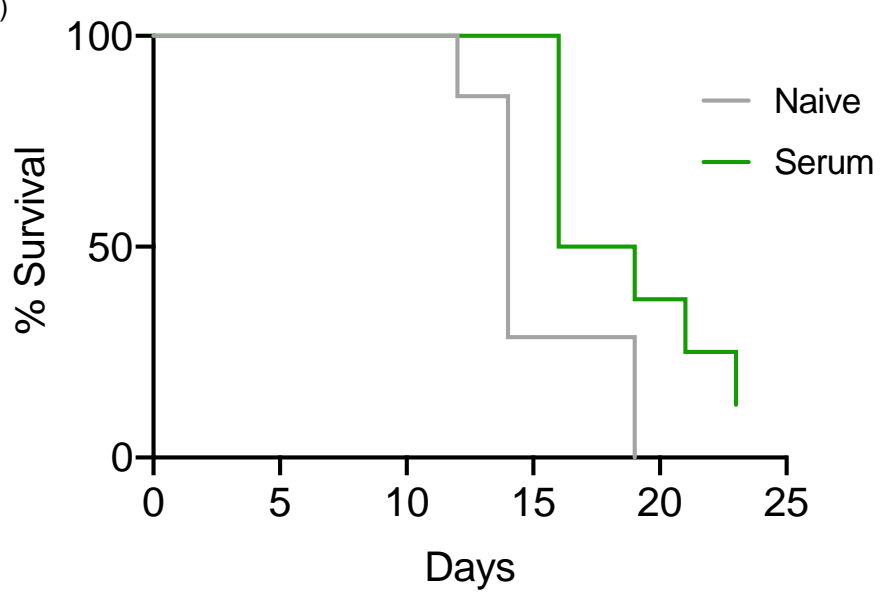

Naive vs. Serum $p=0.0256$

Figure 45 Transfer of serum from mice cured of EG7.OVA into naïve mice protects against EG7.OVA challenge. 200mL of serum was transferred from naïve or previously cured mice into naïve mice i.v. These mice were challenged with EG7.OVA tumours 24 hours after serum transfer. A) Average tumour growth curves plotted. B) Survival curves for the different treatment groups, with statistics indicated (log-rank Mantel-Cox test). Representative of 2 separate experiments $(n=7-8$ per group per experiment). 


\subsection{Discussion}

All the cell types studied to date, including $\mathrm{CD}^{+} \mathrm{T}$ cells, $\mathrm{cDC} 1 \mathrm{~s}, \mathrm{pDCs}$, iNKT cells and B cells are important for the treatment of cancer and the prevention of relapse. Each cell type serves a different function in the sequence of the immune response, and as such, removal of any of these can significantly hamper the efficacy of the anti-tumour response. Some cells appear to be less critical than others in tumour control, and their removal only partially affect the antitumour activity while others are crucial.

In this chapter, the role of different immune cell subsets in the effectiveness of the intratumourally administered combination of $\alpha$-GalCer and $\mathrm{CpG}$ was assessed. Through a combination of flow cytometry, immunohistochemistry, and the utilisation of depleting antibodies, efficacy was shown to be driven by $\mathrm{CD}^{+} \mathrm{T}$ cells, which in turn were likely to have been stimulated primarily through the activity of cDC1 cells. Optimal efficacy, resulting in full regressions with the abscopal effect, required additional contributions from iNKT cells, which were found to be increased in the tumour after combined treatment. The response was also most effective when pDCs were present, with these cells perhaps providing additional signals to shape the adaptive response. The activity of T cells was not the only adaptive response induced, as some anti-tumour activity could be transferred in the serum from treated animals, suggesting that anti-tumour antibody responses were also contributing to the overall anti-tumour activity.

The combination of the efficacy data and the flow cytometry data demonstrated that OVA-specific T cell responses were surprisingly not involved; however, this may be confounded due to there being a small number of cells evaluated further making it difficult to establish phenotype. There were small increases in the immune infiltrate, however most changes were 
only within $\mathrm{CD}^{+} \mathrm{T}$ cell subsets, which varied between the different organs that were being analysed. A potential reason for not detecting too many immune changes is that we potentially missed the window for detecting large changes based on the day we chose to harvest the organs, as tumours were still progressing. The regression phase may more accurately represent immune cell activity as the cells would potentially be present in order to induce regression via tumour cell killing.

With flow cytometry, we observed a slight increase in $\mathrm{CD}^{+}$cells, but these were not OVA-specific; this indicates that $\mathrm{CD}^{+}$cells are involved but that OVA is not the dominant antigen. While the lack of an increase in OVA-specific T cell was initially unexpected, it is consistent with a recent report which demonstrated that there were no $\mathrm{CD}^{+} \mathrm{T}$ cell responses to SIINFEKL detected in C57BL/6J mice with growing EG7.OVA or mice immunised with the tumour. They went on to show that the OVA-specific $\mathrm{CD}^{+} \mathrm{T}$ cells remained functional as they expressed IFN- $\gamma$ and upregulated CD44 when stimulated non-specifically with PMA and ionomycin ${ }^{256}$. In this case, the depletion of all $\mathrm{CD}^{+}$cells rendered the treatment ineffective, which is similar to the situation here. In fact, the non-OVA-specific $\mathrm{CD}^{+} \mathrm{T}$ cell populations in tumour and spleen were phenotypically different from those in vehicle, with small increases in memory cells at the expense of naïve cells. The involvement of $\mathrm{CD} 8^{+} \mathrm{T}$ cells was further confirmed in the antibody-mediated depletion experiments, where depleting CD8 ${ }^{+}$cells in animals that have been cured, but before rechallenge with the same tumour, ablated the mice of their anti-tumour memory. Similarly, knocking out $\mathrm{CD}^{+}$cells during therapy abrogated the antitumour efficacy of intratumoural $\alpha-$ GalCer and $\mathrm{CpG}$, suggesting that $\mathrm{CD}^{+}$cells are an essential cell type in mediating anti-tumour memory responses. 
Interestingly, we only observed a change in the $\mathrm{CD}^{+} \mathrm{T}$ cell population in the spleen using flow cytometry, but not in any other organ. There was a decrease in CD4 ${ }^{+} \mathrm{T}$ cells in the spleen of mice that had undergone either combination therapy or when $\mathrm{CpG}$ was given as a single agent. This would suggest $\mathrm{CD}^{+} \mathrm{T}$ cell egress from the spleen, but there was no associated increase in the other tested organs. This either suggests the $\mathrm{CD} 4^{+}$cells were lost in tissue preparation (it was notable that immunohistochemistry did reveal them in the tumour), they recirculated to other sites or potentially points towards activation-induced cell death. Similar to the $\mathrm{CD}^{+} \mathrm{T}$ cell population, the regulatory $\mathrm{T}$ cell population also saw a decrease in the spleen as a result of combination or $\mathrm{CpG}$ therapy as a single agent. This suggests that $\mathrm{CpG}$ may be inducing a reduction in Tregs either through activation-induced cell death or egress.

One consistent change in the immune infiltrate was that the combination therapy resulted in an increase in the frequency of iNKT cells with the CD4 ${ }^{+}$iNKT cell subpopulation also increasing, while NK1.1 ${ }^{+}$iNKT cells decreased. The downregulation of NK1.1 is welldocumented to occur when iNKT cells are activated ${ }^{257}$. Another finding was that either blocking CD1d-iNKT cell interactions or knocking out iNKT cells resulted in the loss of control of the untreated contralateral tumour, and to a lesser extent the treated tumour. This suggests that iNKT cells play a significant role in the abscopal effect and, by definition, the adaptive immune response. The fact that this was seen in Traj18-deficient animals suggests that this phenomenon was mediated by iNKT cells, and not other CD1d-restricted cells. The response seen in the treated tumours, as opposed to distal tumours, did not appear to be wholly dependent on the presence of iNKT cells, which suggests that innate immunity plays a dominant role in the control of the injected treated tumour, perhaps through the production of cytokines and stimulated lymphocytes. The loss of the abscopal effect in the absence or blockade of iNKT cell interactions 
suggests the possibility that iNKT cells may help create a local environment that favours infiltration of tumours and facilitates destruction as they are remarkable producers of both cytokines and chemokines. In addition, they may be able to assist with $\mathrm{T}$ cell boosting at the distal site, so long as the CD1d molecules are not blocked. This, in turn, may lead to the release of more antigen to the tumour dLN which can be processed and presented by $\mathrm{cDC} 1 \mathrm{~s}$ to mount $\mathrm{CD}^{+} \mathrm{T}$ cell responses that eliminate the abscopal tumour.

Knocking out cDC1 cells by ablating Clec $9 \mathrm{a}^{+}$cells before treatment abrogated the antitumour response during treatment; however, some residual activity was seen in the form of a tumour growth delay. This indicates that antigen presentation by the specialised DC subset is highly critical to the mechanism of action, with $\mathrm{Clec} 9 \mathrm{a}^{+}$cells known to be major antigen crosspresenters. Furthermore, it follows that a $\mathrm{CD} 8^{+} \mathrm{T}$ cell response would be hampered in the absence of antigen cross-presentation. The residual activity potentially points to other DCs compensating for the lack of $\mathrm{Clec} 9 \mathrm{a}^{+}$cells and participating in antigen-cross presentation. This data is directionally consistent with the recently described importance of $\mathrm{Clec} 9 \mathrm{a}^{+} \mathrm{cDC} 1 \mathrm{~s}$ which have been proposed to be the main human cross-presenting cDCs and may represent the human homologue of murine $\mathrm{CD}^{+} \mathrm{DCs}(\mathrm{cDC} 1)^{252}$. In addition to antigen cross-presentation, $\mathrm{cDC} 1$ cells also have higher basal levels of co-stimulatory molecules and these molecules are the main producers of IL-12p70 which aids in the activation and differentiation of T cells ${ }^{258}$. Furthermore, this data suggests that it may be possible to increase the efficacy of the therapy in less immunogenic tumours by the introduction of exogenous antigen or neoantigen into the system, provided that the $\mathrm{Clec} 9 \mathrm{a}^{+} \mathrm{DCs}$ are intact.

Interestingly, partially knocking out pDCs via Siglec-H initially had minimal impact on the anti-tumour response, but the mice eventually lost their anti-tumour response, and the 
tumours escaped. There was still some anti-tumour activity which resulted in a growth delay relative to vehicle, which could be potentially explained by the incomplete knockdown of pDCs when diphtheria toxin was administered. The reduced anti-tumour activity seen in the absence of pDCs may be simply a reflection of reduced initial $\mathrm{T}$ cell burst size that is not maintained for a substantial period of time. Another possible explanation is that pDCs, via type 1 IFN and downstream cytokines, are essential for improving the longevity and activity of an induced effector response ${ }^{259}$. This suggests that type 1 IFN is an important component of the mechanism for the effectiveness of the combination treatment and other cells that produce type 1 IFN may also play a strong role.

The combined treatment increased B cells in the $\mathrm{dLN}$, which requires further investigation. Nonetheless, a role for B cells was indicated when serum from cured EG7.OVA mice was shown to protect naive mice from EG7.OVA challenge, suggesting that anti-tumour memory is in part an antibody-mediated response. By extension, B cells may be activated by the combination of $\alpha$-GalCer and CpG. This would be consistent with the other works that found that $\mathrm{CpG}$ mediated activation of $\mathrm{B}$ cells results in antibody production ${ }^{255}$. The antibodies may be specific to recognising tumour-specific antigens and induce antibody-dependent cell-mediated cytotoxicity (ADCC) with the help of natural killer (NK) cells ${ }^{260}$ or phagocytosis by macrophages ${ }^{261}$. It would be interesting to study the system further using CD19 knockout mice as CD19 is expressed in all B lineage cells.

As mentioned above, all the cell types studied so far, including $\mathrm{CD} 8^{+} \mathrm{T}$ cells, cDC1s, pDCs, iNKT cells and B cells are important in tumour control. Each cell type serves a different role in the sequence of the immune response, and they co-ordinate to elicit the best result. However, $\mathrm{CD}^{+} \mathrm{T}$ cells appear to be the pillar of the anti-tumour activity as their depletion show 
conclusively that they are a crucial effector and contributor to memory that will be necessary for effective immunity in the clinic.

The preliminary immunohistochemistry data suggested that treatment with intratumourally administered $\alpha$-GalCer and $\mathrm{CpG}$ induces immune infiltration of tumours. This was further supported by the data generated from Professor Rod Dunbar's lab which revealed that treatment-induced infiltration is largely due to NK cells, T cells and macrophages in treated tumours when compared to the tumours of vehicle-treated mice. These immune infiltrate appeared to be associated with void formation, which suggests that treatment is inducing tumour rejection via immune cell recruitment to the tumour sites to induce tumour cell killing. 


\section{Chapter 5}

\section{The role of secondary lymphoid organs in the efficacy of intratumoural CpG and $\alpha$-GalCer}

\subsection{Introduction}

The combination of $\alpha$-GalCer and $\mathrm{CpG}$ delivered intratumourally can eradicate both the treated tumour as well as the untreated, distal tumour, suggesting that systemic responses are initiated, with the response involving $\mathrm{CD} 8^{+} \mathrm{T}$ cells with immunological memory. Given that $\mathrm{T}$ cell priming is generally regarded as taking place in lymphoid tissues, we wanted to determine what lymphatic organs were required for this anti-tumour response to be effective. Looking both locally near the tumour microenvironment and systemically, we hypothesised that the tumour draining lymph node and spleen (which are secondary lymphoid organs found in the vertebrate immune system) might be implicated in this therapy.

The lymph node (LN) provides a highly organised microarchitecture that supports optimal immune surveillance of the contents of lymphatic fluid. Lymphatic fluid is derived from the fluid that drains from body tissues into lymph vessels; this collected fluid, called lymph, contains foreign material and cells, including APCs, and is transported to the draining lymph node for analysis. It is known that the tumour draining $\mathrm{LN}$ is an important staging ground for mounting an immune response against the proximal tumour when treated with $\mathrm{CpG}^{262,263}$. If a significant proportion of the antigen presentation and T-cell priming in response to $\alpha$-GalCer and CpG is occurring in the tumour draining lymph node (dLN), this may be compromised if the 
most apparent LN to be involved in drainage from the tumour was removed before treatment. Of course, distal LNs could be involved, but the efficiency will likely be lower.

On the other hand, the spleen is the primary filter for blood-borne pathogens and antigens and is involved in the regulation of innate and adaptive immune responses against pathogens locally and in the whole body. A diverse network of splenic APCs is capable of trapping blood-borne antigens, either for their removal, or to initiate immunity. As opposed to in LNs, DCs in the spleen are only resident DCs specialising in the antigen processing and presentation required to initiate $\mathrm{T}$ cell immunity ${ }^{264}$. On the other hand, the tolerogenic potential of DCs has been proposed when there is an absence of the activation of DCs with proinflammatory signals, with several studies reporting the role of DCs in the induction of peripheral tolerance $251,265,266$. This is supported by in vitro experiments that have demonstrated that DCs with an immature phenotype induce the differentiation of anergic/suppressive $\mathrm{T}$ cells ${ }^{267}$. Upon the recognition of cognate antigens on activated $\mathrm{DCs}, \mathrm{CD} 4^{+}$and $\mathrm{CD} 8^{+} \mathrm{T}$ cells in the spleen are activated and relocate via the blood to tissues where they are needed to fight pathogens or prevent the growth of malignant tissue. Of particular relevance to the treatment being considered here, the spleen is home to a vast reservoir of iNKT cells and previous data from our lab has demonstrated that $\alpha$-GalCer loses adjuvancy of i.v. injected soluble antigens in the absence of the spleen ${ }^{11}$. This points towards the idea that free $\alpha$-GalCer and antigen is accessing the blood to traffic to the spleen. It is known that dying and apoptotic bodies end up in the marginal zone (MZ) of the spleen, potentially leading to $\mathrm{T}$ cell tolerance ${ }^{268}$. However, it has been demonstrated that $\alpha$-GalCer can accompany these dying cells and the strong signalling to iNKT cells can reverse this tolerance process ${ }^{269,270}$. In addition, the spleen has been shown to be required for robust $\mathrm{T}$ cell responses to viral infection, as it is a significant source of effectors that 
contribute to the response ${ }^{271}$. Therefore, it was possible that the efficacy of the combination therapy will be compromised by removing the spleen.

The experiments presented in this chapter were, therefore conducted to evaluate the role of these lymphoid organs in $\alpha$-GalCer and $\mathrm{CpG}$ immunotherapy. Thus it was determined whether their absence negatively impacts outcome. 


\subsection{Aims}

- Determine the importance of different secondary lymphoid organs to $\alpha$-GalCer and CpG therapy via surgical excision and post-surgery tumour challenge.

Specifically, we wanted to:

- Determine the role of the tumour draining lymph node and spleen. 


\subsection{Results}

\subsubsection{Importance of tumour draining lymph node}

\subsubsection{Intratumoural dye injection-identification of draining lymph node}

In order to determine which lymph nodes drain from the tumour, 20 - $40 \mu \mathrm{L}$ of black calligraphy dye was injected into the tumours of mice inoculated with $1 \times 10^{6}$ EG7.OVA cells so that the movement of the suspension of black particles could then be traced visually. This kind of analysis has a long history of clinical use as an anatomic marker for surgery. When injecting 40 $\mu \mathrm{L}$ of calligraphy dye, the inguinal lymph node (groin) was the only node coloured 1-hour postinjection, suggesting that the inguinal lymph node is the primary draining lymph node for the tumour that was implanted on the flank. It should be noted that the dye did not diffuse evenly when less dye $(20 \mu \mathrm{L})$ was used. 


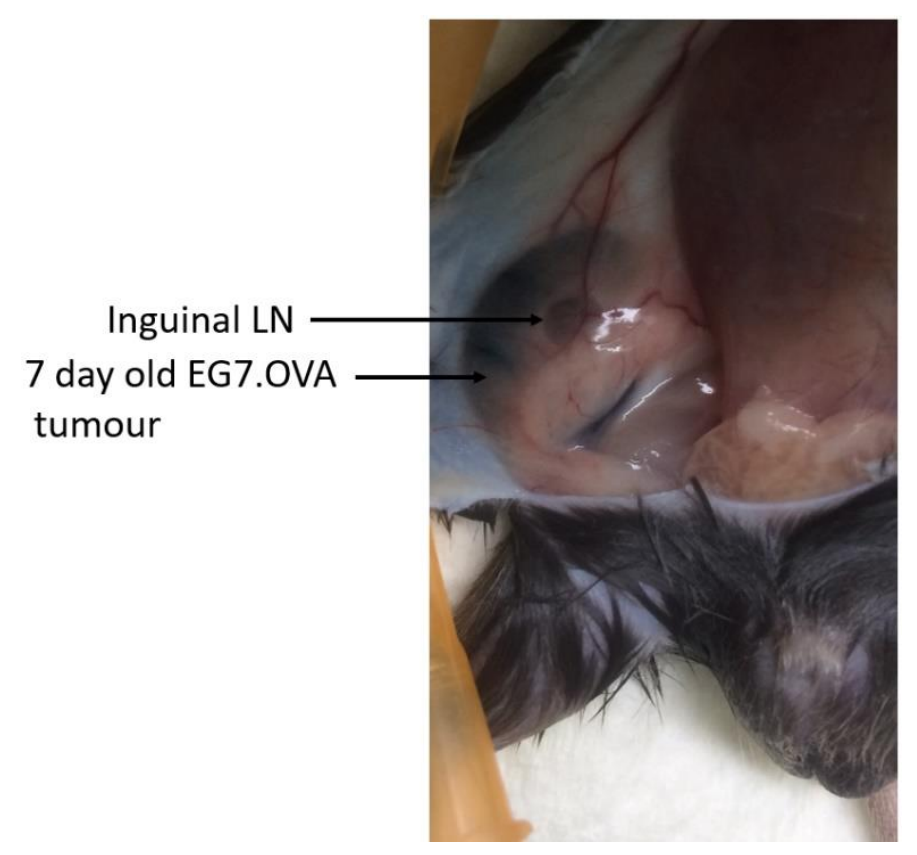

Figure 46 Intratumoural injection of 40uL calligraphy dye drains that the inguinal lymph nodes within 1 hour of injection, indicating the inguinal lymph node is the primary draining lymph node. C57BL/6J mice bearing EG7.OVA tumours were intratumourally injected with 40uL of calligraphy dye. After 1 hour, and the mouse was culled and opened to determine lymphatic drainage by visually tracking the dye. (With help from Johannes Mayer) 


\subsubsection{Lymph Node Removal Surgery}

In order to determine the importance of the tumour draining lymph node on the efficacy of intratumourally delivered $\alpha-\mathrm{GalCer}$ and $\mathrm{CpG}$, the inguinal lymph node was removed using a keyhole surgery technique. In this surgery procedure, C57BL/6J mice were anaesthetised with isoflurane, and then a keyhole incision made above the inguinal lymph node for the lymph node to be removed gently using forceps. The incision was then sutured closed. In order to control the potential side effects of the surgery on anti-tumour responses, control animals were subjected to sham surgeries, where the incision was made and then sutured closed. Mice were monitored daily for a week for pain, wound condition and general health and behaviour. After a week, the mice were examined by an animal welfare technician, and upon approval were inoculated with $1 \times 10^{6}$ EG7.OVA subcutaneously into the same flank as the surgery where the lymph node was. Once the tumours reached approximately $6 \times 6 \mathrm{~mm}$ mice were intratumourally treated with either PBS or $\alpha$-GalCer with 6 hours delayed CpG as previously.

Mice that had their draining lymph node (dLN) removed and received treatment experienced a tumour growth delay and regression similar to sham surgeries in the first 35 days or so. However, their tumours eventually escaped resulting in no cures. In contrast, some of the mice that underwent sham surgeries and received treatment showed complete regression without tumour rebound (Fig 47). While there was a trend, the difference in survival between the groups was not statistically significant. Intriguingly, the efficacy of the treatment in the sham animals was low compared to previous experiments in naïve animals. Typically, the rate of complete regression of EG7.OVA tumours with the combination treatment is $60-70 \%$; the $40 \%$ complete regression rate observed here could be due to surgery-induced immune suppression. To make some allowances for the surgery-related immunosuppression, a similar experiment was 
conducted with the more immunogenic EL4.LA tumour cell line instead of EG7.OVA. In this model, all of the mice that had their dLN removed lost their anti-tumour response, while the majority of the mice that underwent sham surgery successfully rejected their tumours (Fig 48). The regression rate was again low for this cell line, as previous experiments had shown close to $100 \%$ of treated animals completely rejected EL4.LA. In any event, this suggests that the tumour $\mathrm{dLN}$ is important for a successful anti-tumour response. 
A

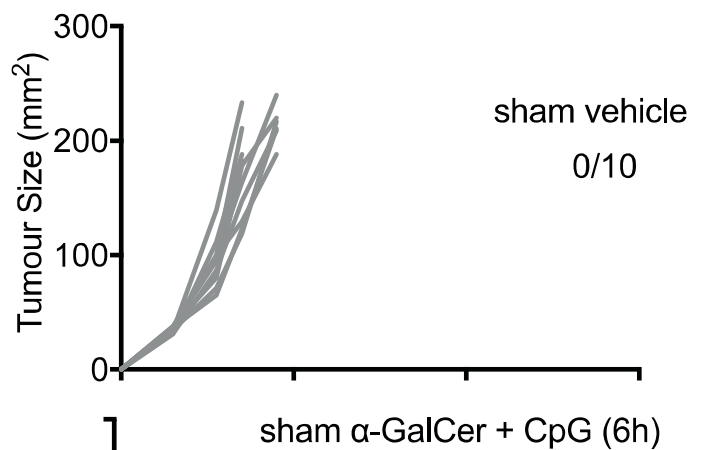

$4 / 10$
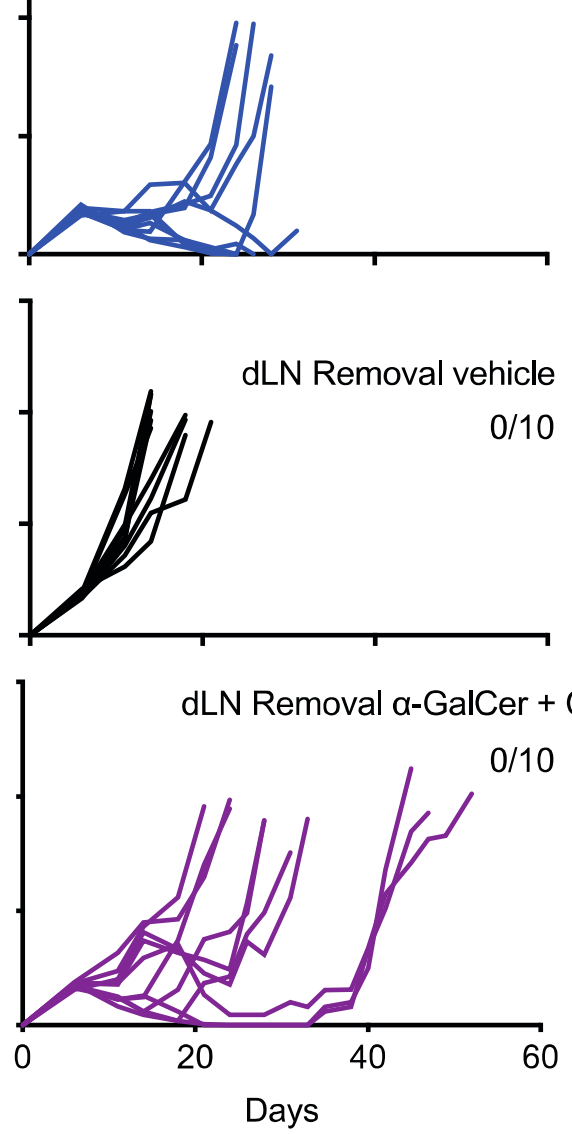

B

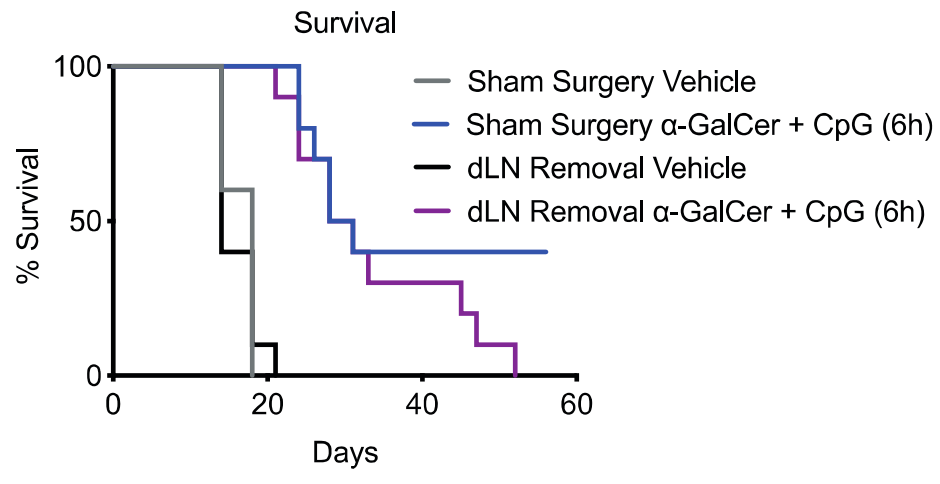

Sham Surgery $\alpha$-GalCer + CpG (6h) vs. dLN Removal $\alpha$-GalCer + CpG p $=0.1478$

Figure 47 Removal of draining lymph node partially abolishes anti-tumour immunity in an EG7.OVA tumour model. A) Tumour growth curves plotted for each mouse (1 sham $\alpha$-GalCer $+C p G$ mouse sacrificed early due to tumour ulceration). B) Survival plot with statistics indicated (log-rank Mantel-Cox test). 
A

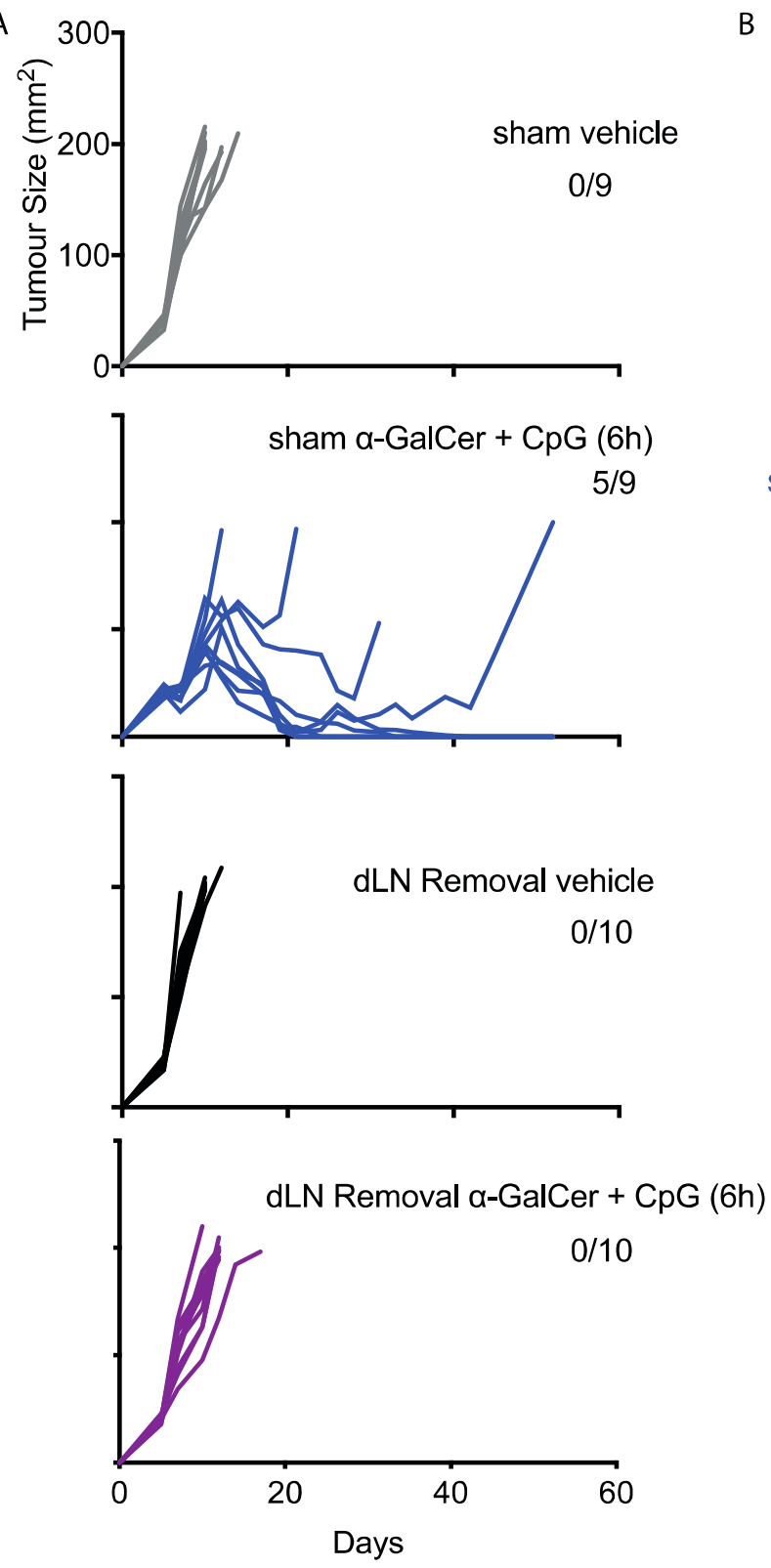

Survival

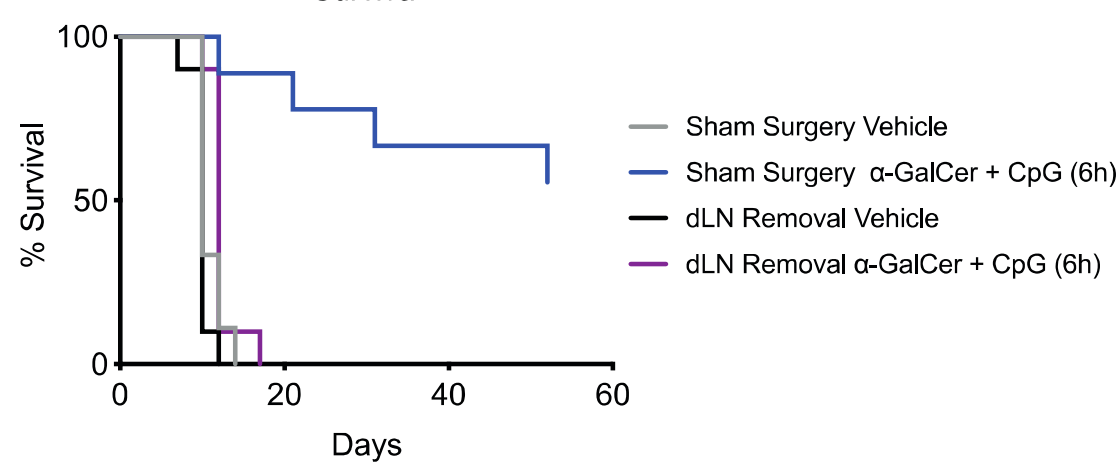

Sham Surgery a-GalCer + CpG (6h) vs. dLN Removal a-GalCer + CpG p $<0.0001$

Figure 48 Removal of the tumour draining lymph node diminishes the anti-tumour effect of intratumoural $\alpha$-GalCer and CpG in the EL4.LA tumour model. A) Tumour growth curves plotted for each mouse. B) Survival plot with statistics indicated (log-rank Mantel-Cox test). 


\subsubsection{Requirement of the spleen for treatment efficacy}

In order to elucidate the role of the spleen on the anti-tumour response elicited with $\mathrm{CpG}$ and $\alpha$-GalCer, spleens were removed from C57BL/6J mice by surgery before tumour challenge and treatment. In the splenectomy procedure, the spleens were removed via an incision into the peritoneum followed by suturing to close up the wound. Sham surgery was again used as a control, whereby a wound was created and sutured up without removing the spleen.

The mice were given one week to recover from surgery and were inspected by an animal welfare specialist before further manipulations were performed. Once approved, the mice were challenged with $1 \times 10^{6}$ EG7.OVA tumours into the right flank (the opposite flank from the spleen). Once the tumours were $\sim 6 \times 6 \mathrm{~mm}$ (day 6 ), the mice were treated intratumourally with either PBS or $\alpha$-GalCer with 6 hours delayed $\mathrm{CpG}$ as conducted in earlier experiments. Most of the mice that underwent sham surgery survived tumour challenge as a result of treatment in a statistically significant manner (Fig 49). However, mice that underwent the splenectomy procedure did not fare as well, though there was a slight statistically significant delay in tumour growth. This suggests that the spleen is strongly implicated in mounting an effective immune response against tumours. 
A

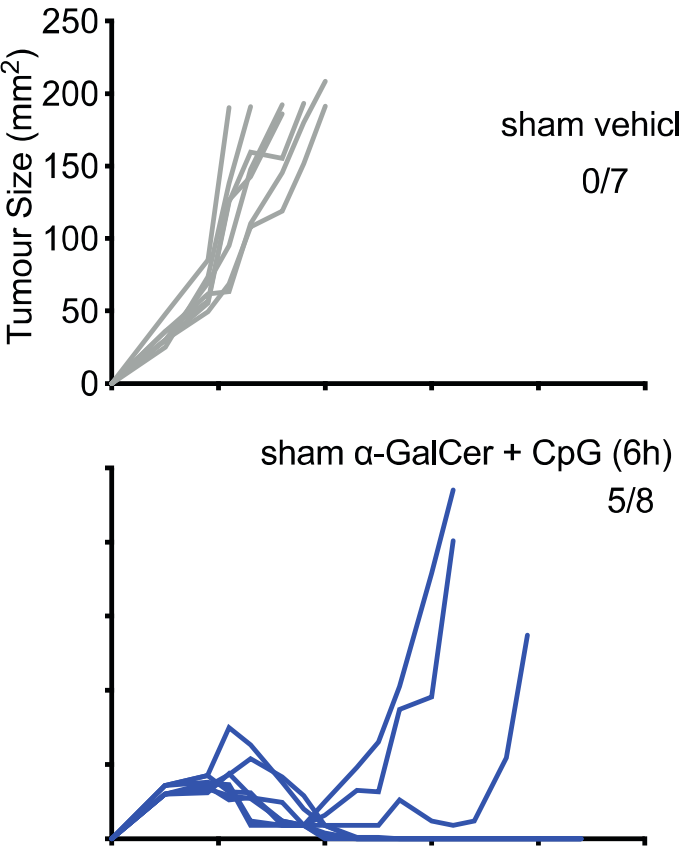

B

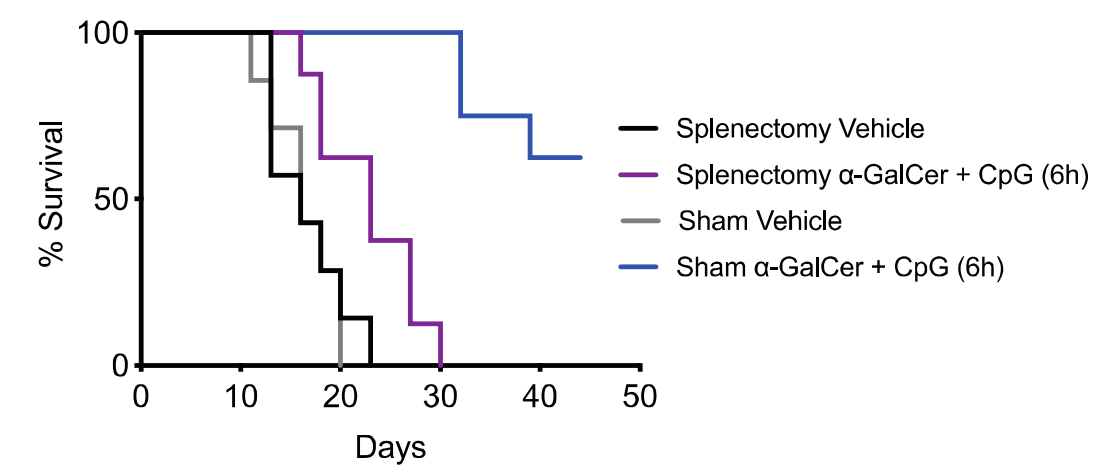

Splenectomy $\alpha-G a l C e r+C p G(6 h)$ vs. Sham Surgery $\alpha-G a l C e r+C p G(6 h) p<0.0001$ Splenectomy Vehicle vs Splenectomy $a-G a l C e r+C p G(6 h) p=0.0244$
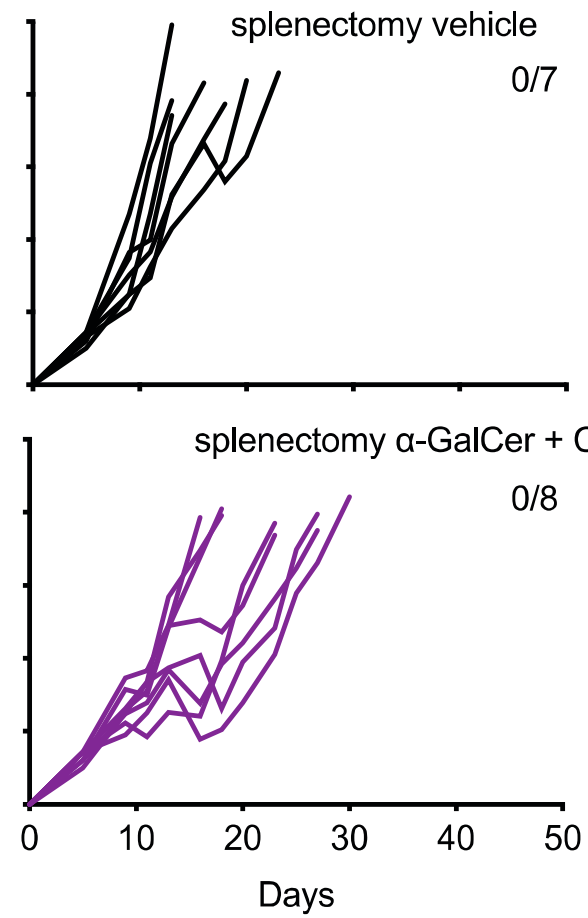

$(6 h)$

Figure 49 Intratumoural $\alpha$-GalCer and $C p G$ is ineffective after the removal of the spleen compared to sham surgery. A) Tumour growth curves plotted for each mouse. B) Survival curves for the different treatment groups, with statistical differences indicated (log-rank Mantel-Cox test). 


\subsection{Discussion}

In this chapter, it was examined how the anti-tumour response that is induced with $\mathrm{CpG}$ and $\alpha$-GalCer treatment was affected by the removal of two critical lymphoid organs, spleen and tumour draining lymph node, which were hypothesised to be essential sites for the immune priming of the anti-tumour response.

In order to determine which lymph nodes the tumour drained to, we used intratumourally injected calligraphy dye. Within 1 hour, only the inguinal lymph node adjacent to the tumour was stained; no other lymph nodes were stained by waiting prolonged periods. This indicates that the inguinal lymph node adjacent to the implanted tumour is the primary draining lymph node. The next step was to excise the $\mathrm{dLN}$ and determine how the anti-tumour response would be affected.

In this murine model, it was hypothesised that APCs enter the tumours, capture TAA, and subsequently migrate to the dLN to present to T cells. After either excision of the tumour dLN or sham surgery, with the EG7.OVA tumour line, it initially appeared that the rate of tumour regression was similar for mice with and without dLN. However, with time, the tumours eventually escaped control in mice whose dLN had been excised. The tumour escape is consistent with the hypothesis that a weaker immune response is initiated in the absence of this dLN. This is presumably because other remaining lymphoid tissues are unable to fully compensate for the loss of this critical draining site, as the antigens will have to traverse further to more distal, lymphoid tissues in order for them to be available for presentation.

With the faster growing EL4 tumour line, the importance of dLN is more dramatic, as all mice lost the ability to eradicate tumours when dLNs were removed compared to the sham group. A simple explanation for the observed weakened anti-tumour response is probably due to 
the speed at which tumours grew and overwhelmed any remaining immune response. This is in addition to the fact that fewer antigens would be able to travel to other secondary lymphoid sites in the shortened timeframe.

The spleen can be a site of innate and adaptive immune processes. The white pulp of the spleen is structurally similar to a lymph node, containing T-cell and B-cell zones, and allows for the generation of antigen-specific immune responses. Here it was shown that mice that had their spleen removed before treatment significantly lost their anti-tumour response, indicating that the anti-tumour response is dependent on systemic immune responses involving the spleen. The spleen may be a significant source of immune effector cells for the anti-tumour response, and by ablating this population, there are insufficient immune cells to clear the tumour. It is known that splenectomy predisposes human patients to increased susceptibility to infection due to a loss of effectors, as the spleen serves as a source of many immune cells including myeloid cells, T cells and iNKT cells which can be recruited as effectors for anti-tumour immunity ${ }^{272,273}$. The mechanism involved in the recruitment of these cells, especially $\alpha$-GalCer-mediated recruitment of iNKT cells, can be explained in two different ways. The first hypothesis posits that the antigen and $\alpha$-GalCer arrived at the spleen as free agents through leakage into the circulation. The second hypothesis is that these agents could be carried on certain cells, namely APCs. While DCs do not migrate from the site of inflammation to the spleen, it has been reported that monocytes can be recruited to the spleen and handoff antigen to resident professional APCs such as $\mathrm{cDC} 1 \mathrm{~s}$ in the context of infection and inflammation ${ }^{274}$. In light of this, it is possible that in addition to $\alpha$-GalCer being transported on monocytes, phagocytosed antigens can also be couriered by monocytes to the spleen and transferred to resident $\mathrm{cDC} 1 \mathrm{~s}$ for cross-priming to occur. It is also possible that the repeated injection of $\mathrm{CpG}$ and its associated local inflammation 
increased the release of dead and dying cells into the bloodstream whereby they travel to MZ of the spleen. Without the spleen, the dLN may compensate, but its overall efficiency in activating the immune response to clear pathogens will be significantly lower, hence the inability to eradicate established tumours.

An interesting observation in these lymphoid organ excision experiments was that the regression rates for the treated sham control arms in both experiments are lower than that observed in earlier experiments using the same cell lines where surgeries were not involved. A point of discussion is whether the surgery caused immunosuppression in the vicinity of the wound. There have been numerous reports in the literature that the wound healing process is immune-suppressive ${ }^{275}$. This immune-suppression could be driven by the immunosuppressive cytokine such as TGF $\beta$ which is essential for the wound healing process ${ }^{276}$. Of course, this could also be the normal variation from experiment to experiment; however, it is not inconceivable that implanting the tumour near the site of the lymph node removal surgery may have subjected the tumour to surgery-induced immune suppression, so that the negative impact of dLN removal per se may be exaggerated. Interestingly, treatment did not suffer similarly when the spleen was removed. However, this may have been ameliorated by the use of two extra doses of carprofen for pain control during that experiment (as a splenectomy is a more invasive procedure). Carprofen is a non-steroidal anti-inflammatory drug (NSAID), and it inhibits the production of the immunosuppressive prostaglandin E2 (PGE2). It has been reported that the use of NSAIDs, and thus the inhibition of immunosuppressive PGE2, can abrogate the immune suppression associated with surgery such that surgery-induced metastasis does not occur ${ }^{277}$. This suggests that surgery-induced immune suppression is localised to the site of surgery and may not affect tumours growing far from the surgery site. This is consistent with the fact that PGE2 is 
relatively unstable and has a short half-life and therefore does not travel over long distance ${ }^{278}$. Therefore, consideration should be taken when utilising immunotherapy post-surgery near the site of a tumour as localised immunotherapy may be hampered by the immunosuppressive microenvironment generated as part of the wound healing process. 


\section{Chapter 6}

\section{Role of type I IFN in $\alpha$-GalCer + CpG combination therapy}

\subsection{Introduction}

Type I IFNs are hallmark cytokines produced in immune responses to pathogens. In the literature, it is known that CpG can induce the release of type I IFN from pDCs ${ }^{279,280}$. Type I IFNs comprise a large family of cytokines that have been studied extensively in the context of virus infections, but they are now also known as key drivers of inflammation within the tumour microenvironment ${ }^{281}$. Type I IFNs, including multiple IFN- $\alpha$ species, IFN- $\beta$, and certain other IFNs, are potent regulators of both innate (direct tumour cell inhibition) and adaptive immunity (indirect anti-tumour immune responses) ${ }^{282}$. Type 1 IFN confers resistance to viral infections and promotes NK cell, DC, T cell, and B cell functions ${ }^{283}$. Moreover, IFN- $\alpha$ promotes the production of antibodies ${ }^{284}$. While IFN- $\beta$ is produced by most cells, IFN- $\alpha$ is primarily released by pDCs in response to DNA or RNA viruses, but can also be induced by synthetic TLR agonists such as CpG. ${ }^{285}$ It is known that when pDCs are depleted (as seen in chapter 4, Siglec-H ${ }^{+}$cells), it negatively impacts treatment outcome. In addition to secreting IFN- $\alpha$, pDCs produce IL-12, IL-6, and chemokines that contribute to innate and adaptive immune responses ${ }^{286}$. This broad activation of the immune system is beneficial during infections but can be deleterious if the IFN$\alpha$ production is not adequately downregulated after clearance of pathogens. Prolonged exposure of the immune system to IFN- $\alpha$ increases the risk for loss of self-tolerance. Development of autoimmune diseases, including systemic lupus erythematosus (SLE), has been frequently described in patients during long-term treatment with type I IFN ${ }^{287}$. Type 1 IFN can also exacerbate an existing autoimmune tendency ${ }^{288}$. 
It has been observed that intratumoural cytokine concentration correlates with tumour immune responses ${ }^{289}$. Thus, the intratumoural delivery of $\mathrm{CpG}$ is equivalent to selectively delivering type I IFNs to the tumour bed, increasing the intratumoural concentration of type I IFNs, in a way that results in superior immunostimulatory effects but that avoids possibly detrimental outcomes.

As we have previously demonstrated that pDCs were strongly implicated in the maintenance of the anti-tumour response generated by the intratumoural combination of $\alpha$ GalCer and CpG, we hypothesised that type I IFN would play a central role in our combination therapy's effectiveness. Therefore, we anticipated that the abrogation of IFN $\alpha \mathrm{R}$ receptor through IFN $\alpha \mathrm{R}$ antibody, as well as IFN $\alpha \mathrm{R}$ conditional receptor knockout (KO), will negatively impact the effectiveness of our combination treatment. Furthermore, we wanted to illuminate further what cells would be affected by type 1 IFN. 


\subsection{Aims}

The goal for this section was to determine whether type 1 IFN played a role in the effective intratumoral therapy. The aims were to:

- Examine type 1 IFN release in the tumour and blood in response to therapy

- Investigate the impact of blocking signalling via the type I IFN receptor

- Determine whether type IFN signalling by DCs is essential for treatment efficacy 


\subsection{Results}

\subsubsection{Determining levels of IFN- $\alpha$ in response to therapy}

To determine whether the production of IFN- $\alpha$ is a feature of the anti-tumour response to intratumoural treatment, the levels of IFN- $\alpha$ were determined in tumour and serum after treatment of established EG7.OVA tumours was initiated. Once the tumours had reached $\sim 10$ $\mathrm{x} 10 \mathrm{~mm}$ (in order to ensure there is enough tissue available to process) the mice were treated once intratumourally with PBS, $\alpha$-GalCer, $\mathrm{CpG}$, or an admix $\alpha$-GalCer and $\mathrm{CpG}$; no further intratumoural treatment given. Six hours later, the tumours were removed and homogenised in the presence of a protease inhibitor to prevent degradation of protein. The rationale for collecting tumours at 6 hours was because it is the optimum time point at which IFN- $\alpha$ production was observed after $\alpha$-GalCer and a TLR7 agonist were injected systemically in mice ${ }^{11}$. The material was then centrifuged and the supernatant collected for analysis. Blood was collected at the same time as tumour removal, and serum prepared; both tumour supernatant and serum were tested for IFN- $\alpha$ levels by ELISA.

IFN- $\alpha$ was detected in all tumour samples, treated and untreated, and the value for all samples fell within the recommended range on the standard curve. Neither $\alpha$-GalCer nor CpG alone demonstrated an increase in IFN- $\alpha$ compared to untreated tumours. On the other hand, the anti-tumour combination showed a statistically significant increase in IFN- $\alpha$ versus both untreated tumours as well as $\alpha$-GalCer as a single agent. In addition, IFN- $\alpha$ was absent in the serum under all conditions, including the combination of $\alpha-\mathrm{GalCer} / \mathrm{CpG}$ (Fig 50), indicating that IFN- $\alpha$ did not significantly leak into circulation. 


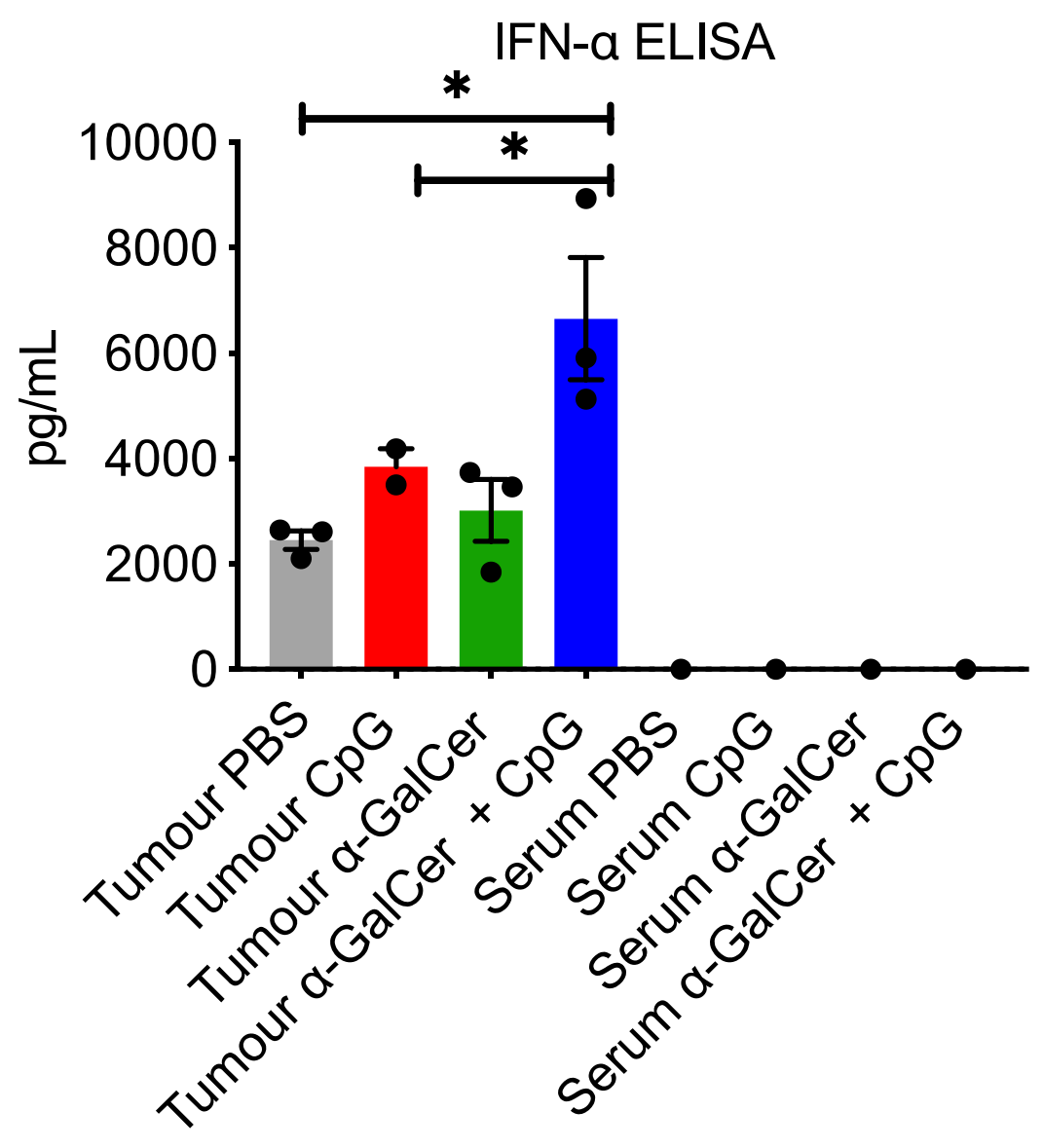

Figure 50 Type 1 IFN detected by ELISA is increased in the tumour but not the serum 6 hours after intratumoural treatment. A) IFN- $\alpha$ ELISA of tumour lysate and serum 6 hours after intratumoural treatment with either PBS, CpG, $\alpha$-GalCer, CpG, or admix combination of $\alpha$-GalCer and $C p G$, statistical significance indicated repeated twice $* P<0.05$ (One-Way ANOVA with Tukey's Post-Hoc Test; SEM for error). Experiment is representative of 2 separate experiments. 


\subsubsection{Investigating impact of the blockade of type I IFN signalling}

Next, it was determined how blocking the activity of IFN- $\alpha$ would affect intratumoural $\alpha$ GalCer and $\mathrm{CpG}$ mediated therapy. The IFN- $\alpha / \beta$ receptor (IFN $\alpha \mathrm{R})$ is a receptor which binds type I IFNs including IFN- $\alpha$ and $-\beta$. It is a heteromeric cell surface receptor composed of one chain with two subunits referred to as IFNAR1 and IFNAR2. Upon binding of type I IFNs, IFN $\alpha$ R activates the JAK-STAT signalling pathway. Therefore, to examine the role of type I IFNs during intratumoural treatment, an antibody for the IFN- $\alpha$ receptor (IFN $\alpha$ R) was administered to block signalling. The dose used $(250 \mu \mathrm{g})$ was based on previous work done by colleagues looking at the role of IFN- $\alpha$ in atopic dermatitis (C. Pellefigues, personal communication, 2018). For control, an IgG control antibody was administered to control for nonspecific effects of antibody binding.

Mice were inoculated with $1 \times 10^{6}$ EG7.OVA s.c into the right flank, on days 4 and 5 they received $250 \mu \mathrm{g}$ of either anti-IFN $\alpha \mathrm{R}$ or IgG control. On day 6 , when the tumours were approximately $6 \times 6 \mathrm{~mm}$, the mice were treated with either intratumourally delivered PBS or $\alpha$ GalCer with 6 hours delayed $\mathrm{CpG}$ as previously described. All the mice that were given the antiIFN $\alpha \mathrm{R}$ antibody had their tumours grow unabated; in contrast, the majority of the mice that received the IgG control had their tumours regress to baseline (Fig 51). Type I IFN signalling was therefore critically involved in the treatment-induced anti-tumour response. An interesting note is that the mice that were given anti-IFN $\alpha \mathrm{R}$ antibody had their tumours grow faster in a statistically significant manner than ones that received just vehicle. This suggests that IFN $\alpha \mathrm{R}$ is not only crucial for the efficacy of the combination of $\alpha$-GalCer and CpG but also plays an essential role in naïve tumour immunity. 
A

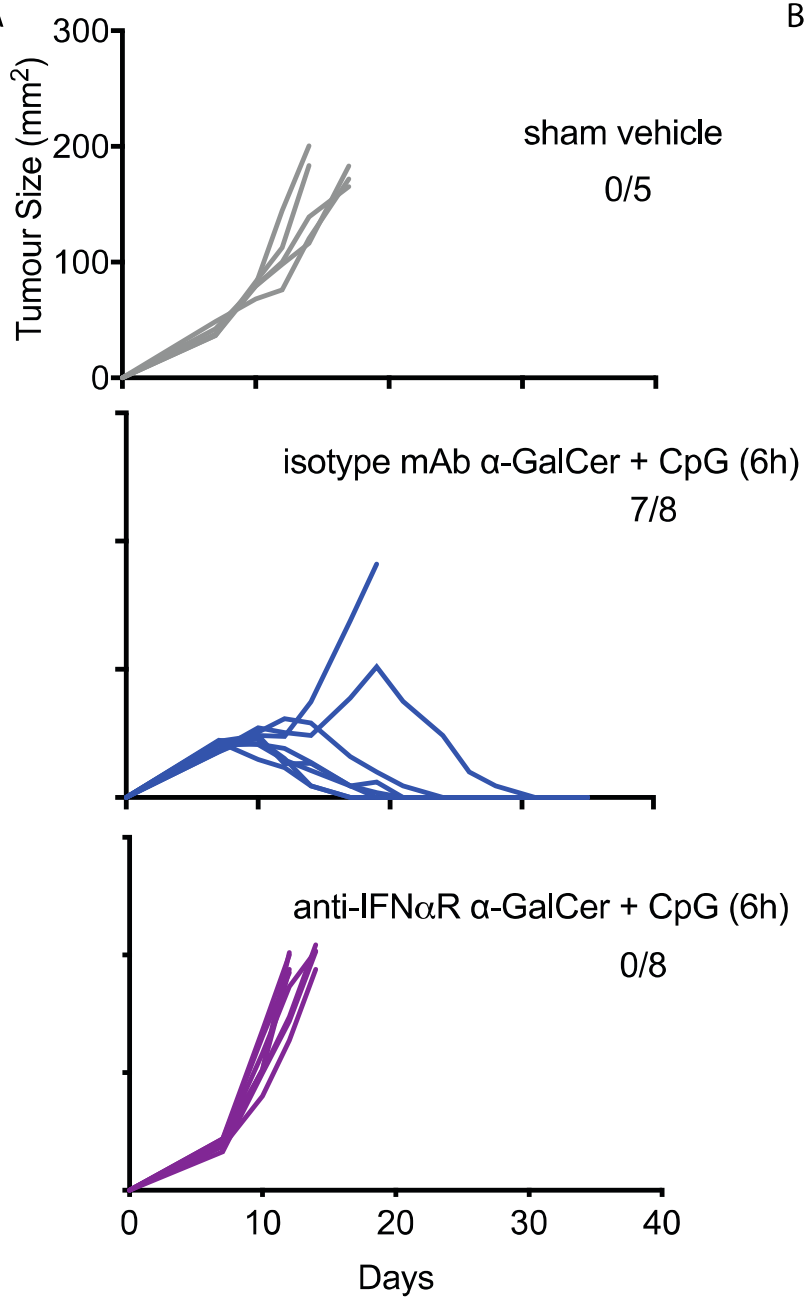

B

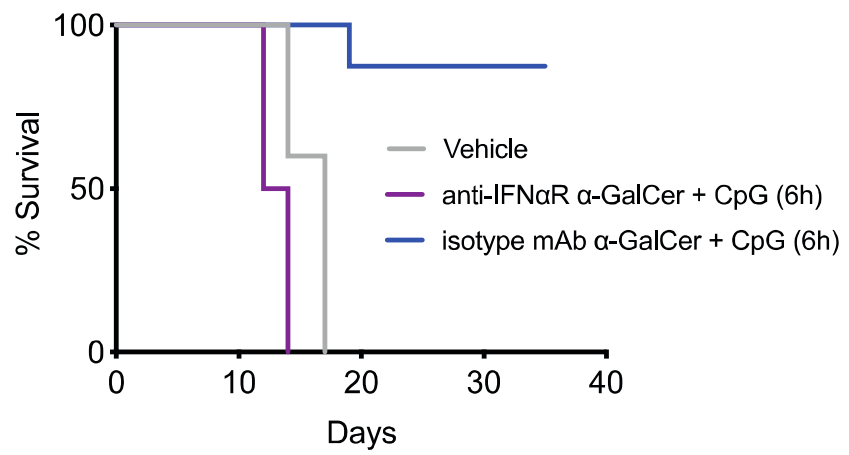

Vehicle vs anti-IFNaR $\alpha-$ GalCer + CpG (6h) p=0.0107

anti-IFNaR $\alpha-G a l C e r+C p G(6 h)$ vs + isotype mAb $\alpha-G a l C e r+C p G(6 h) p=0.0001$

Figure 51 IFN $\alpha$ R blockade leads to complete abrogation of $\alpha$-GalCer and CpG mediated anti-tumour responses. A) Tumour growth curves plotted for each mouse. B) Survival curves for the different treatment groups, with statistical differences indicated (log-rank Mantel-Cox test). 


\subsubsection{Requirement for type I IFN signalling by DCs}

Type 1 IFN is known to be capable of conditioning DCs to help prime T cell responses $203,254,280,290$. It was therefore hypothesised that a critical function of type I IFN was to improve DC function. To investigate this, mice that had IFN $\alpha$ R deleted specifically on DCs were used. To achieve this, a cross between IFN $\alpha \mathrm{R}^{\mathrm{fl} / \mathrm{fl}}$ and $\mathrm{CD} 11 \mathrm{c}-\mathrm{Cre}^{+/-}$mice were generated. In these mice, expression of Cre on the CD11c promoter ensures the deletion of exon 3 of the type I IFN$\alpha \beta$ receptor gene leading to a non-functional IFN $\alpha \mathrm{R} 1$ receptor on $\mathrm{CD} 11 \mathrm{c}^{+}$cells. These mice have the IFN $\alpha \mathrm{R}$ receptor knocked out $(\mathrm{KO})$ on $\mathrm{CD}_{11} \mathrm{c}^{+}$cells. Mice expressing the IFN $\alpha \mathrm{R} \mathrm{KO}$ were inoculated with $1 \times 10^{6}$ EG7.OVA s.c. into the right flank, while littermates not expressing the IFN $\alpha \mathrm{R} \mathrm{KO}$ were also given tumours and used as controls.

When the tumours were $\sim 6 x 6 \mathrm{~mm}$ in size, the mice were treated with either intratumoural PBS or $\alpha$-GalCer with 6 hours delayed $\mathrm{CpG}$, as previously described. Mice that had IFN $\alpha$ R deleted on their CD11 $\mathrm{c}^{+}$cells, and so IFN signalling was prevented in DCs, completely lost their anti-tumour response, whereas those that retained the IFN $\alpha \mathrm{R}$ receptor on all cells were able to induce full regression to baseline (Fig 52). This suggests that type 1 IFN is essential to $\alpha$-GalCer with 6 hours delayed CpG mediated therapy and that the ability for type 1 IFN to act on DCs is an essential mechanism by which the therapy functions. 

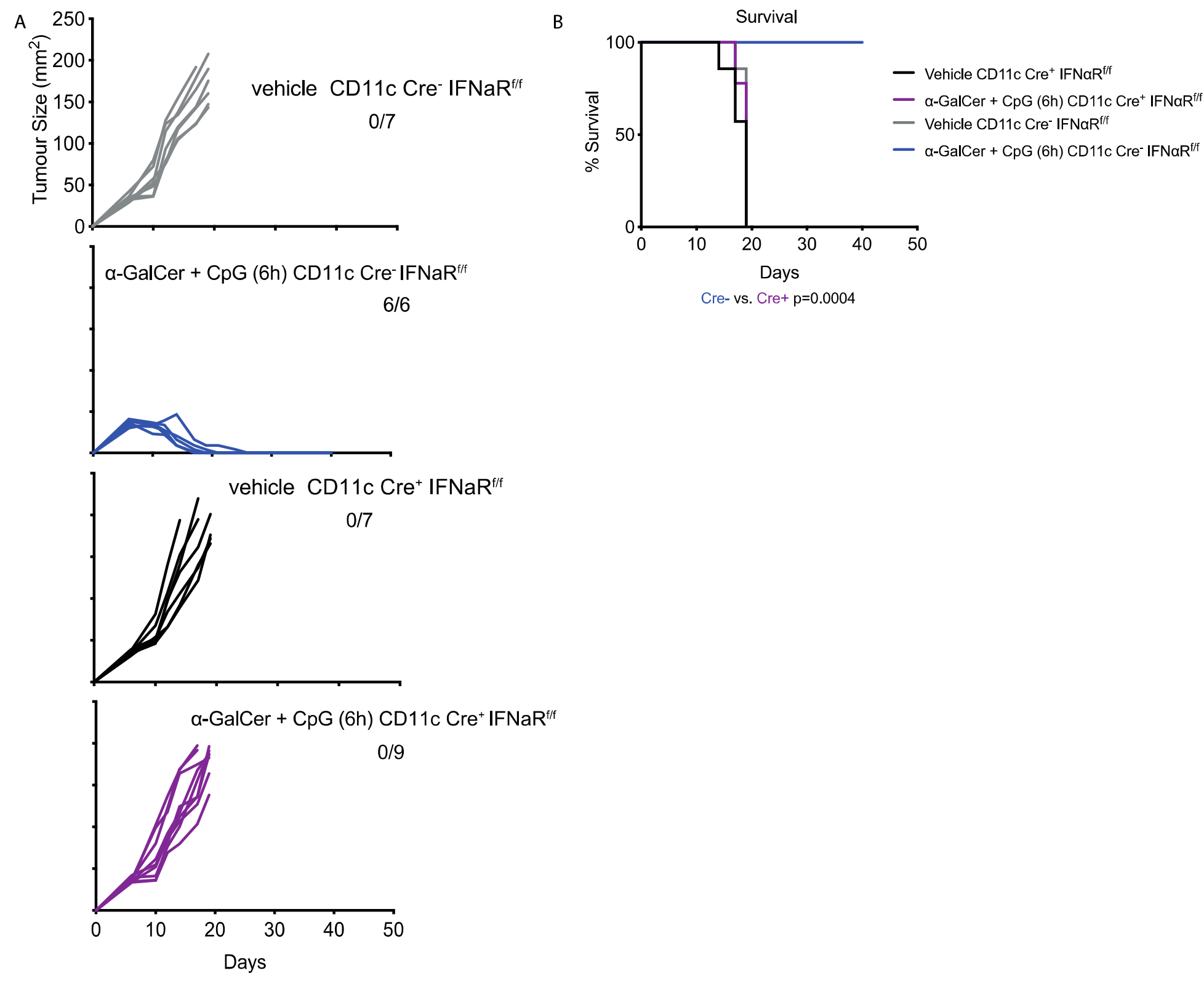

Figure 52 IFNaR knockout on CD11 ${ }^{+}$cells leads to loss of $\alpha$-GalCer and CpG mediated anti-tumour responses. A) Tumour growth curves plotted for each mouse. B) Survival curves for the different treatment groups, with statistical differences indicated (log-rank Mantel-Cox test). 


\subsection{Discussion}

The work presented in this chapter addressed the role of type I IFN in the anti-tumour efficacy of intratumoural $\alpha-\mathrm{GalCer}$ and $\mathrm{CpG}$ treatment. It is shown that effective treatment is associated with an increase of IFN- $\alpha$ and that when IFN signalling is blocked, specifically on DCs, the effective anti-tumour activity of the combined treatment is lost.

Initial experiments in this chapter showed that the combination of $\alpha-\mathrm{GalCer}$ and $\mathrm{CpG}$ increased the amount of IFN- $\alpha$ released in the tumour compared to that seen in untreated tumours. In contrast, neither $\alpha$-GalCer nor $\mathrm{CpG}$ alone were able to induce significant increases in production of IFN- $\alpha$. However, a most striking result was that IFN- $\alpha$ levels were already detectable in tumours without treatment, this basal level of IFN- $\alpha$ is likely being produced by immune cells within the tumour and perhaps is indicative of the high level of immunogenicity seen with EG7.OVA tumours in general. This may also indicate that tumour challenge itself can induce an immune response. In contrast to the measurable levels detected in tumours, IFN- $\alpha$ was below the detection limit in the serum of any of the conditions tested, including the combination

treatment. This suggests that the source of IFN- $\alpha$ is likely to be from cells within the tumour, and despite the increased production when the combined intratumoural treatment was used, there was minimal, if any, systemic exposure (below the level of detection). However, given that the intratumoural combination of $\alpha-\mathrm{GalCer} / \mathrm{CpG}$ induced lower serum ALT levels compared to intravenous $\alpha$-GalCer (Chapter 3.3.8), intratumoural delivery may provide high local cytokine concentrations within the tumour microenvironment that helps induce tumour regression without much systemic leakage. Furthermore, it is also hard to attribute the abscopal effect to systemic cytokines such as IFN- $\alpha$ as there were no detectable circulating cytokines in the bloodstream. This points to local activation of immune cells, such as APCs, that lay the foundation for a 
systemic cellular immune response, which the experiment in the DC-specific IFNaR knockout supports.

It is anticipated that type I IFNs produced by DCs and other immune cells within the tumour microenvironment will not only offer superior efficacy (higher concentration at the site of action) but also better tolerability as these IFNs are localised within the tumour microenvironment rather than present mainly in the circulatory system. In this context, type I IFN has been tested clinically for the potential treatment of breast cancer, melanoma and renal carcinoma and have shown moderate success in terms of clinical responses. However, the side effects associated with systemic administration of IFNs, such as nausea, fatigue, fever and dizziness, were dose-limiting ${ }^{291}$; therefore, there was an insufficient concentration of IFNs in the tumour bed. Intratumoral induction of local type I IFN may get around these problems A critical role for type I IFN in the anti-tumour response was established by blockade of IFN $\alpha$ R, which lead to a loss in anti-tumour response. Interestingly, administration of anti-IFN $\alpha$ R blocking antibody also slightly increased the tumour growth rate compared to that in mice treated with vehicle only. This indicates that IFN- $\alpha$ is not only crucial for our treatment to be effective but is required at baseline to retard tumour growth. This is supported by the fact that untreated tumours have a significant level of IFN- $\alpha$, suggesting that tumour challenge alone invokes an immune response, and may explain why the EL4 thymomas used in this thesis were reasonably immunogenic when compared to the other tumours tested.

Mice that had the IFN $\alpha$ R receptor knocked out on their DCs completely lost their antitumour response to the $\alpha-\mathrm{GalCer} / \mathrm{CpG}$ combination. Previous experiments in chapter 4 had shown that ablation of pDCs, a significant producer of type I IFN, led to a weakened anti-tumour response. Of note, preliminary interpretation of a CyTOF analysis conducted with 43 different 
antibodies for cell-surface markers on immune cells in this treatment model, conducted by a colleague in the laboratory in association with the Sydney Cytometry Facility, demonstrated increases in CD69 and SCA-1 on cDC1s, CD4 ${ }^{+}$, and $\mathrm{CD}^{+}{ }^{+} \mathrm{T}$ cells (L. Ferrer-Font, personal communication, 2018). Upregulation of CD69 and SCA-1 has been associated with exposure to type I IFN ${ }^{285,292}$. It was demonstrated in Chapter 4 that ablation of cDC1s had a significant negative impact on the anti-tumour response. Other laboratories have found that cDC1s exposed to type I IFN become activated and matured ${ }^{293}$. It is, therefore, possible that type IFN released by pDCs acts on $\mathrm{CDC} 1$ cells, thereby promoting the specialist cross-priming activity of these cells. It has been previously demonstrated that IFN- $\alpha$ stimulation of $\mathrm{cDC} 1 \mathrm{~s}$ can lead to improved IL-12 production for T cell priming ${ }^{294}$. Supporting this, another study showed that cDC1s were unable to enhance the production of IL-12p40 when pDCs were depleted, in this case in response to $\alpha$-GalCer and delayed administration the TLR7 agonist resiquimod (which normally produces copious amounts of IFN- $\alpha$ ). A follow-up on this experiment found that depleting mice of langerin $^{+} \mathrm{cDC} 1 \mathrm{~s}$ prevented the induction of a $\mathrm{CD}^{+} \mathrm{T}$ cell response ${ }^{11}$. As $\mathrm{CpG}$ also activates pDCs in a similar manner as resiquimod (TLR9 and TLR7/8 agonists respectively), it suggests a similar pathway whereby pDCs producing IFN- $\alpha$ was associated with the enhanced capability for $\mathrm{cDC} 1 \mathrm{~s}$ to cross-prime a $\mathrm{T}$ cell response. Thus, a potential mechanism may be presented: $\mathrm{CpG}$ directly ligates pDCs, leading to the release of type I IFN. This type I IFN directly stimulates cDC1s thereby increasing antigen cross-presentation and cross-priming of a $\mathrm{CD}^{+} \mathrm{T}$ cell response. The type I IFN also acts upon $\mathrm{CD}^{+}$and $\mathrm{CD}^{+} \mathrm{T}$ cells directly leading to increased cytotoxic capacity. There are multiple ways in which to test this hypothesis in the future. We could deplete pDCs or block IFN- $\alpha$ binding and see how it affects the induction of a CD8 ${ }^{+} \mathrm{T}$ cell response. Another method, similar to what was described above, could be to measure IL-12p40 
responses in mice depleted of pDCs to see how the lack of pDCs affects IL-12p40 production by cDC1s. The final method, would involve depleting $\mathrm{cDC} 1 \mathrm{~s}$ prior to treatment and measuring the induction of $\mathrm{CD}^{+} \mathrm{T}$ cells after treatment to determine if $\mathrm{cDC} 1 \mathrm{~s}$ are responsible for crosspriming in this model. 


\section{Chapter 7}

\section{Overcoming non-responsive tumours - developing rational combination strategies}

\subsection{Introduction}

As noted earlier in Chapter 3, unlike EL4.LA tumours, tumours such as B16 melanoma and TC1 lung carcinoma (a mouse epithelial cancer cell line that expresses HPV-16 E6 and E7 proteins) proved to be more resistant to the therapeutic effects $\alpha$-GalCer and $\mathrm{CpG}$ combination therapy. With B16 melanoma and TC1 lung carcinoma, while tumour growth delay was observed, no animal achieved tumour eradication. Studies have shown that immune-related gene expression is low in B16 melanoma, suggesting it is poorly immunogenic. Low levels of immune-related genes were also seen in CT26 colon carcinoma in BALB/c mice, with B16 tumours being the most poorly immunogenic of the two models by this criterion ${ }^{295}$. Other work has demonstrated that the B16 melanoma cell line is depleted of costimulatory molecules such as OX40L, CD40, CD137L (4-1BB) and others ${ }^{295}$. In addition, TC1 and B16 lines have been shown to have lower expression of MHC class I and II pathway genes through transcriptomic analysis compared to naïve animals. It also has been shown that these two tumour models are poorly infiltrated by immune cells ${ }^{296}$ compared to other tumour types such as EL4 and MC38. TC1 tumours are also known to contain large numbers of immunosuppressive Tregs, whose systemic depletion can lead to immunotherapy being successful in the TC1 model ${ }^{297}$. Nonetheless, the tumours can respond to immunotherapy to some extent $139,202,203,217,254,298$ suggesting that both B16 and TC1 tumours are antigenic (that is, they express TAAs) but are not immunogenic due to the variety of immunosuppressive mechanisms outlined above. 
It has been shown that tumour sections harvested two weeks after the subcutaneous implantation of TC1 cells into C57BL/6 mice were not a complete immune desert, but there were a small amount of $\mathrm{CD}^{+} \mathrm{T}$ cells, and these $\mathrm{T}$ cells are within the tumours and not outside in the margin ${ }^{299}$. Therefore, it was possible that immune responses were either too weak to have an effect or were effectively suppressed. If too weak, this may be due to a lack of TAAs presented to the immune system in a stimulatory context for the initiation and maintenance of a robust adaptive anti-tumour immune response in the TC1 model. Although the intratumoural combination therapy stimulates activation and maturation of professional APCs, this alone is insufficient to induce a powerful curative response. However, by increasing the visibility of TAAs within the tumour, it could increase $\mathrm{T}$ cell activation, and perhaps also attract additional antigen-specific T cells to the tumour tissue. Such a strategy to exogenously add antigen to the treatment can only be used where TAA have been defined, such as the case for the HPV antigens in TC1 ${ }^{300}$. This could be achieved by incorporating TAA into a suitably adjuvanted vaccine such that higher antigen doses are loaded onto APCs in a stimulatory context, which is the basis for several vaccine approaches ${ }^{151,301} 302-306$. Of note, anti-tumour responses have been seen in animal models by intratumourally injecting a peptide antigen in combination with a $\mathrm{CpG}$ adjuvant, suggesting this strategy could work, and it was possible that this could be amplified if iNKT cell activation was also incorporated into the regimen. ${ }^{188,290}$

In this context, previous work in our laboratory had shown that covalently attaching antigens to $\alpha$-GalCer improves immunogenicity by co-delivering antigen and adjuvant to the same APCs ${ }^{136,307}$. It is, therefore, possible that such peptide antigen/ $\alpha$-GalCer conjugates injected intratumourally will be delivered to APCs within the tumour (and also possibly drain to lymphoid tissues). Such attached peptides can be cross-presented to stimulate antigen-specific 
$\mathrm{CD}^{+} \mathrm{T}$ cell responses by the same DCs that present $\alpha-$ GalCer via CD1d to stimulate iNKT cell help ${ }^{307}$. Conjugation is achieved using a linker that is designed to self-immolate after enzymatic cleavage by intracellular cathepsins, allowing the release of native peptide and $\alpha$-GalCer structures that can bind the relevant antigen-presenting molecules expressed by APCs ${ }^{307}$. By combining this form of vaccine treatment with $\mathrm{CpG}$, it is, therefore, possible that responses of sufficient magnitude and quality could be achieved that induce regression in models of low immunogenicity where TAA are known.

Alternatively, low dose chemotherapy such as doxorubicin or cyclophosphamide can be used to elicit immunogenic cell death (ICD) with the dead and dying cells containing TAAs that are released to APCs in an immunostimulatory context ${ }^{308}$. This is in contrast to high dose chemotherapy, which is not only cytotoxic to tumour cells that divide quickly but can kill off fast proliferating immune cells such as those in the bone marrow, wiping out any immunogenicity boost ${ }^{309}$. It is important to note that not all chemotherapeutic agents are ICD inducers, as those that do are characterised by the exposure of "eat-me" signals on the cell surface through the release of DAMPs. The crucial DAMPs, which can induce ICD, include calreticulin (CRT), high mobility group box 1 (HMGB1), adenosine-5' -triphosphate (ATP) and heat shock protein (HSP) 70 and $90^{308}$. Although not explored in this thesis, it has also been reported that radiation or infection with oncolytic viruses can stimulate the release of tumour antigens and initiate an immune response ${ }^{302-306,310}$. In addition to doxorubicin, other chemotherapeutic ICD inducers are mitoxantrone, bortezomib and cyclophosphamide ${ }^{311}$.

Another main culprit for failed anti-tumour immune responses is that tumour cells learn to evade host immune surveillance through the expression of ligands for inhibitory immune checkpoints. In this scenario, it is anticipated that antagonists to these ligands such as anti-PD-1 
and anti-CTLA4 should prove useful. The PD-1/PD-L1 signalling pathway is crucial in dampening tumour immunosurveillance via over-expression of PD-L1, which is known to negatively regulate immune responses by interacting with PD-1 on T cells, essentially "turning off" the T cells and allowing the tumours to escape host immune surveillance ${ }^{312}$. Indeed, data from clinical samples indicate that the high expression of PD-1 ligands on tumours correlated with a poor prognosis ${ }^{313}$. Under this scenario, responses to TAA have to have already been initiated but have been constrained. Indeed the use of a PD-1 antagonist in combination with standard of care has proven to be the new cornerstone of cancer treatment ${ }^{314}$. It was possible, therefore, that responses to TAA in TC1 and B16 were under this immunosuppressive constraint, and $\mathrm{CpG}$ and $\alpha$-GalCer were unable to reverse this. Therefore, it is possible that $\alpha-\mathrm{GalCer} / \mathrm{CpG}$ treatment would perform better in combination with a PD-1/PD-L1 antagonist as the immune response may have been hampered by increased PD-L1 binding to PD-1 leading to exhaustion of effectors. Other strategies to overcome low immunogenicity, but not tested in this chapter, include depletion of immune suppressor cell populations such as Tregs, MDSCs, and TAMs to reduce the hostility of the TME to immune attack. 


\subsection{Aims}

Can the addition of exogenous antigen enhance the efficacy of the treatment for tumours that have known antigens such as EG7.OVA, TC1, and B16? Alternatively, can antagonism of upregulated immunosuppressive proteins improve the efficacy of the treatment, such as in the CT26 tumour model? The aims were therefore to:

- Determine the efficacy of combining intratumoural $\mathrm{CpG}$ with intratumoural administration of $\alpha$-GalCer with a peptide epitope from a TAA

- Determine if combining intratumoural $\alpha-$ GalCer and $\mathrm{CpG}$ therapy with anti-PD-1 can improve anti-tumour immunity

- Determine if combining intratumoural $\alpha$-GalCer and $\mathrm{CpG}$ therapy with low dose doxorubicin therapy can improve anti-tumour immunity 


\subsection{Results}

\subsubsection{Impact of the addition of tumour associated antigens to $\alpha$-GalCer/CpG: Addition of OVA peptide in EG7.OVA tumour model}

To test whether intratumoural co-delivery of an antigenic peptide could improve the efficacy of treatment involving iNKT cell activation and TLR9 agonism, a conjugate of $\alpha$ GalCer and OVA peptide containing both $\mathrm{CD}^{+}$and $\mathrm{CD}^{+} \mathrm{T}$ cell epitopes on an $\mathrm{H}-2^{\mathrm{b}}$ background ( $\alpha$-GalCer-OVA) was prepared so that analyses could be conducted initially in the relatively immunogenic EG7.OVA model. This $\alpha$-GalCer-OVA conjugate was synthesized as published ${ }^{315}$, and those that follow, were made and supplied by our synthetic chemistry collaborators in the laboratory of Prof Gavin Painter, Ferrier Research Institute. To accomplish conjugation, a rearranged $\alpha$-GalCer derivative with a free amino group was used to provide a synthetically selective and stable point of attachment. A cathepsin-sensitive valine-citrulline-pamino-benzyl carbamate within the linker ensured intracellular enzymatic cleavage between the valine and citrulline groups, followed by immolation of the surrounding linker to release the glycolipid and peptide. The released migrated $\alpha$-GalCer derivative then spontaneously rearranges to form $\alpha$-GalCer, to be presented via CD1d, while the peptide is processed to be presented via MHC class I and class II ${ }^{138}$. The initial aim was to determine whether conjugation of TAA epitopes expressed by the tumour to $\alpha$-GalCer would be effective when administered alongside intratumoural CpG. To do this, C57BL/6J mice were inoculated with $1 \times 10^{6}$ EG7.OVA s.c. into the right flank, and then when the tumours reached about $6 \times 6 \mathrm{~mm}$ the mice were treated intratumourally with either PBS, $\alpha$-GalCer-OVA conjugate, $\mathrm{CpG}$, admix $\alpha$-GalCer-OVA conjugate and $\mathrm{CpG}$, or $\alpha$-GalCer-OVA conjugate with 6 hours delayed $\mathrm{CpG}$. 
Intratumoural administration of the $\alpha$-GalCer-OVA conjugate as a single agent induced some growth delay and regression, while the administration of $\mathrm{CpG}$ alone, as usual, only induced growth delay. For the combination therapy, the admix $\alpha$-GalCer-OVA conjugate and $\mathrm{CpG}$ resulted in the majority of tumours regressing to baseline, while $\alpha$-GalCer-OVA conjugate with 6 hours delayed $\mathrm{CpG}$ resulted in all tumours regressing to baseline (Fig 53). The conjugation of $\alpha$-GalCer to the OVA epitope appeared to enhance the anti-tumour response in EG7.OVA tumour model when delivered 6 hours before $\mathrm{CpG}$, as had been observed in experiments presented in Chapter 3, although this was not significant; nonetheless, this regimen was included in later studies. While this experiment was not performed side by side with the use of $\alpha$-GalCer and $\mathrm{CpG}$, the conjugation certainly did not impair efficacy, justifying further experiments to explore the use of conjugates in a less immunogenic model. 
A

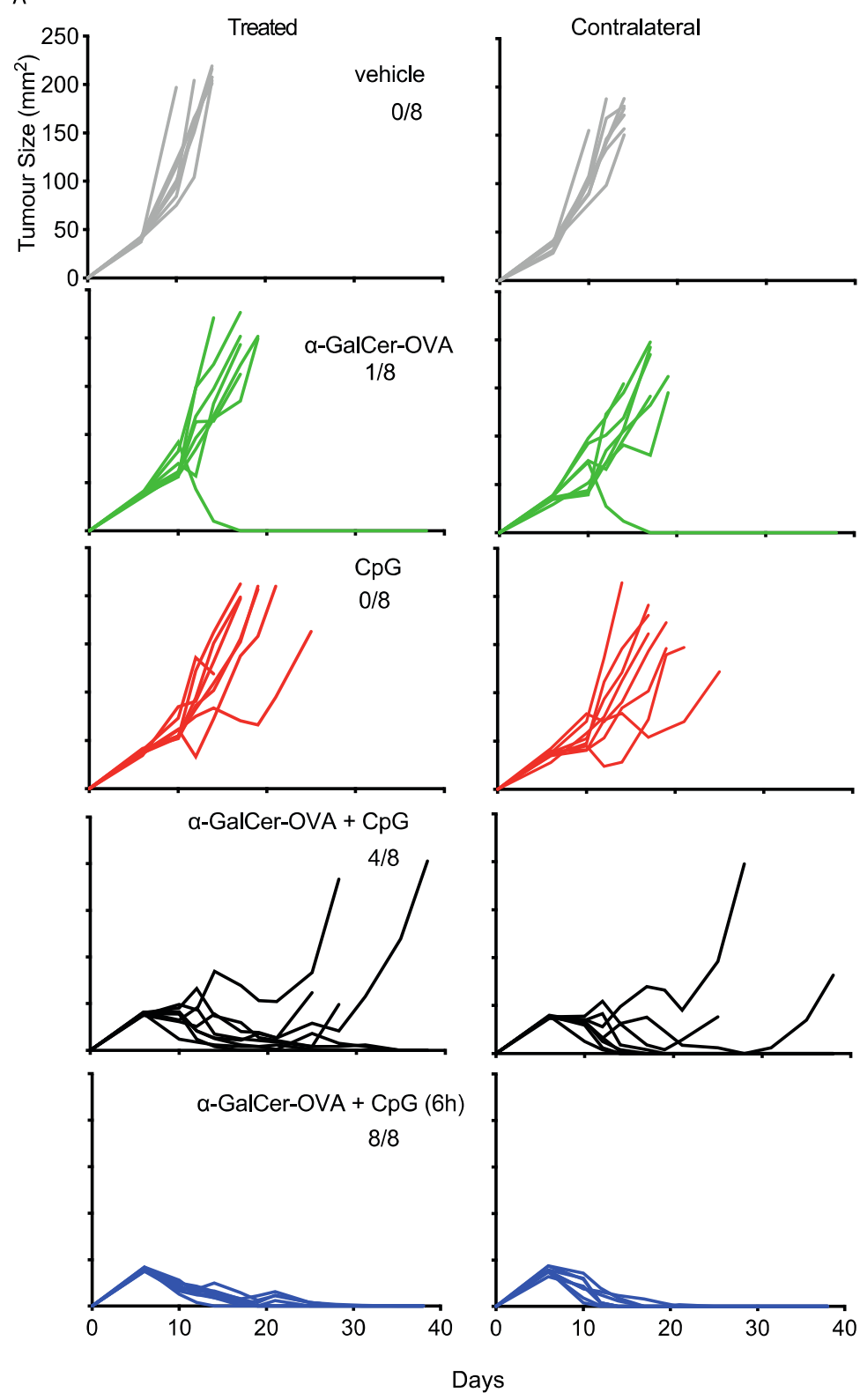

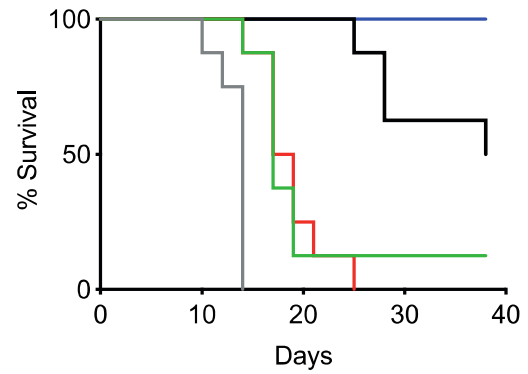

- Vehicle

- $a$-GalCer-OVA

- $\mathrm{CpG}$

- $\alpha$-GalCer-OVA + CpG

- $\alpha$-GalCer-OVA + CpG (6h)

$\alpha$-GalCer-OVA vs. $\alpha$-GalCer-OVA + CpG $p=0.0082$ $\alpha$-GalCer-OVA vs. $\alpha$-GalCer-OVA + CpG (6h) $p=0.0005$ CpG vs. $\alpha$-GalCer-OVA + CpG $p<0.0001$

CpG vs. $\alpha$-GalCer-OVA + CpG (6h) p<0.0001

$\alpha$-GalCer-OVA + CpG vs. $\alpha$-GalCer-OVA + CpG (6h) p=0.0513

Figure 53 Intratumoural administration of $\alpha$-GalCer-OVA conjugate and CpG combine to induce regression in EG7.OVA model. A) Tumour growth curves plotted for each mouse. B) Survival curves for the different treatment groups, with statistics indicated (log-rank Mantel-Cox test). 


\subsubsection{Impact of the addition of tumour associated antigens to $\alpha$-GalCer/CpG: Addition of HPV E7 epitope in the TC1 tumour model}

As mentioned in chapter 3, the TC1 tumour cell line is a transformed murine lung epithelial cancer cell line co-transfected with HPV-16 E6/E7 genes and the activated human Haras $(\mathrm{G} 12 \mathrm{~V})$ oncogene DNA ${ }^{216,217}$. In this earlier chapter, it was demonstrated that the intratumoural combination of $\alpha-\mathrm{GalCer}$ and $\mathrm{CpG}$, both as an admix and with delayed $\mathrm{CpG}$, only resulted in a tumour growth delay relative to PBS, with no baseline regressions achieved.

The constitutively expressed HPV oncoproteins E6 and E7 are used as target antigens for HPV therapeutic vaccines. These early (E) proteins are regarded as being crucial for HPV immune escape and malignant progression; and are constitutively expressed in both premalignant and advanced lesions, making them ideal targets for immunotherapeutic approaches for HPVinduced malignancies ${ }^{316}$. Recently our group has shown that the $\alpha$-GalCer-HPV E7 conjugate, $\alpha$-GalCer-E749-57 given intravenously exhibited a significant delay in tumour growth relative to saline or unconjugated component-treated control animals in mice implanted with the TC1 tumour cell line. The anti-tumour activity only occurred when the tumour associated antigen was covalently linked to $\alpha$-GalCer, as injection of unconjugated components alone or as an admix did not provide anti-tumour activity ${ }^{136}$. This study showed that, as expected, the glycolipid-peptide conjugate vaccine required cathepsin cleavage in vivo and induced CD1d-dependent iNKT cell proliferation. The anti-tumour activity of $\alpha$-GalCer-E749-57 was due to induction of peptidespecific $\mathrm{CD}^{+} \mathrm{T}$ cell activation with cytotoxic potential. However, while the anti-tumour response was robust, it did not eradicate tumours at the doses tested ${ }^{136}$.

In this study, the conjugate employed used a long peptide that incorporated the E7 $49-57$ (bolded) epitope (i.e. GQAEPDRAHYNIVTFCCKCDS) ${ }^{317,318}$. To test the anti-tumour activity 
of intratumoural $\alpha$-GalCer-HPV E7 conjugate with $\mathrm{CpG}, 1 \times 10^{5} \mathrm{TC} 1$ tumour cells were inoculated into the right flank of C57BL/6J mice. Once the tumours reached approximately $6 \times 6$ $\mathrm{mm}$ the mice were treated intratumourally with either PBS, CpG, $\alpha$-GalCer-HPV E7 conjugate, an admix $\alpha$-GalCer-HPV E7 conjugate and CpG, or with $\alpha$-GalCer-HPV E7 conjugate with 6 hours delayed $\mathrm{CpG}$.

The intratumoural administration of $\alpha$-GalCer-HPV E7 conjugate alone induced a growth delay relative to PBS with some mice regressing to baseline, while $\mathrm{CpG}$ did not induce any baseline regressions. Admixed $\alpha$-GalCer-HPV E7 conjugate and CpG resulted in the majority of mice undergoing regression, with similar results being achieved with $\alpha$-GalCer-HPV E7 conjugate combined with 6 hours delayed CpG (Fig 54). As regression was rare in earlier studies using the $\alpha-\mathrm{GalCer} / \mathrm{CpG}$ combination, this initial data was highly suggestive that the addition of a tumour associated antigen as part of a conjugate enhances anti-tumour activity over iNKT cell activation and TLR9 agonism alone in the TC1 model.

To confirm a role for an adaptive response with memory in this treatment, mice that survived tumour challenge were rechallenged with a double inoculum $(200,000)$ TC1 cells into the opposite flank. Similar to our previous findings with $\alpha-\mathrm{GalCer}$ and $\mathrm{CpG}$, all of the cured mice rejected their rechallenge tumours, whereas all of the naïve mice succumbed to their tumours (Fig 55). This includes the lone mouse that was cured by the $\alpha$-GalCer-HPV E7 conjugate without CpG. Overall this supports our previous finding that the cures generated from $\alpha-\mathrm{GalCer} / \mathrm{CpG}$ therapy are durable, and suggests an adaptive response with memory was initiated. 
A
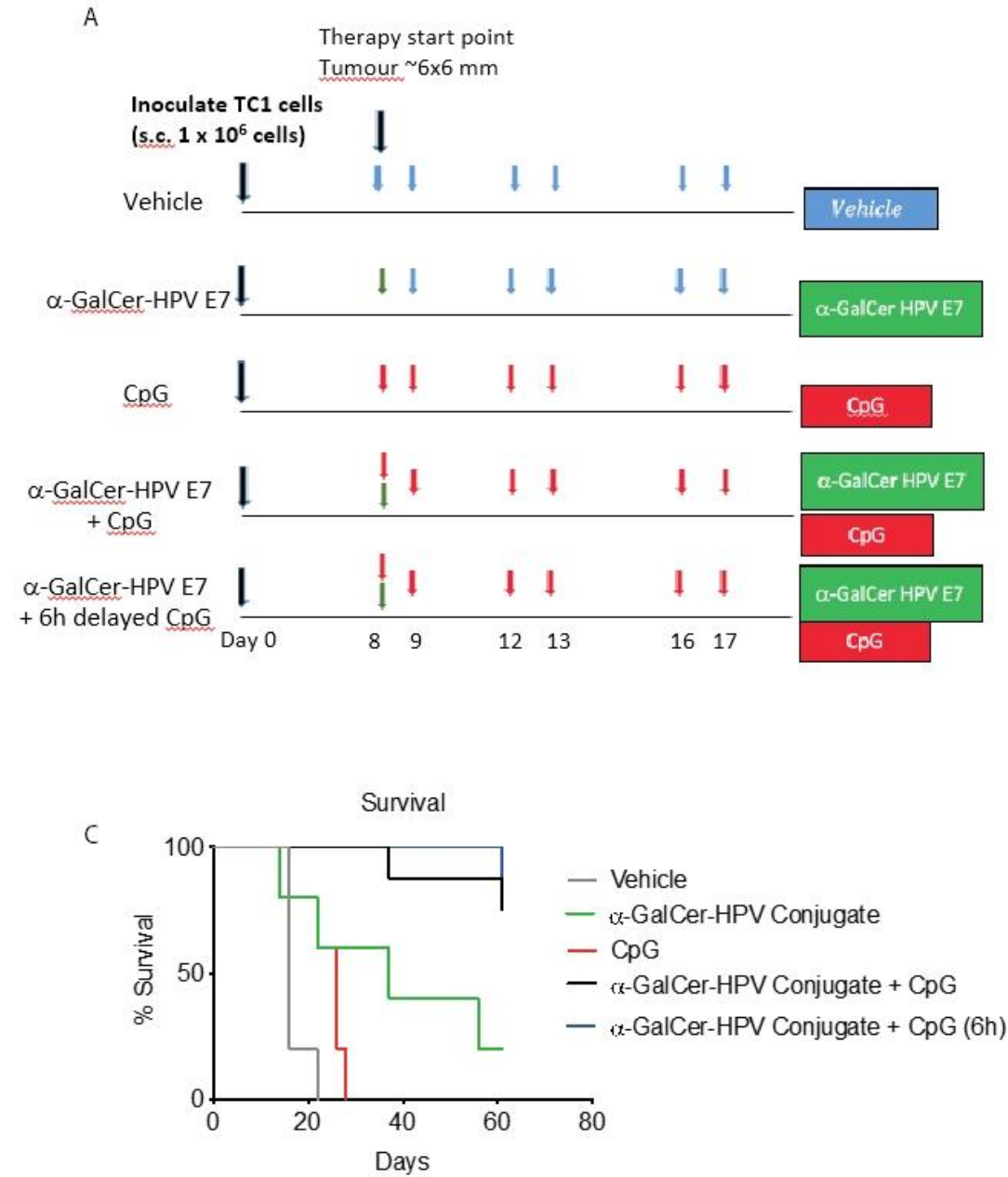

a-GalCer-HPV Conjugate vs $\alpha$-GalCer-HPV Conjugate $+\mathrm{CpG} p=0.0226$ $a t-$ GalCer-HPV Conjugate vs $x$-GalCer-HPV Conjugate $+\mathrm{CpG}(6 \mathrm{~h}) \mathrm{p}=0.0048$ CpG vs $a-$ GalCer-HPV Conjugate $+\mathrm{CpG} p=0.0002$ CpG vs rz-GalCer-HPV Conjugate + CpG (6h) p $=0.0002$
B

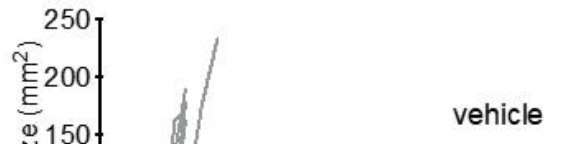

$0 / 5$ 売 100 至 50
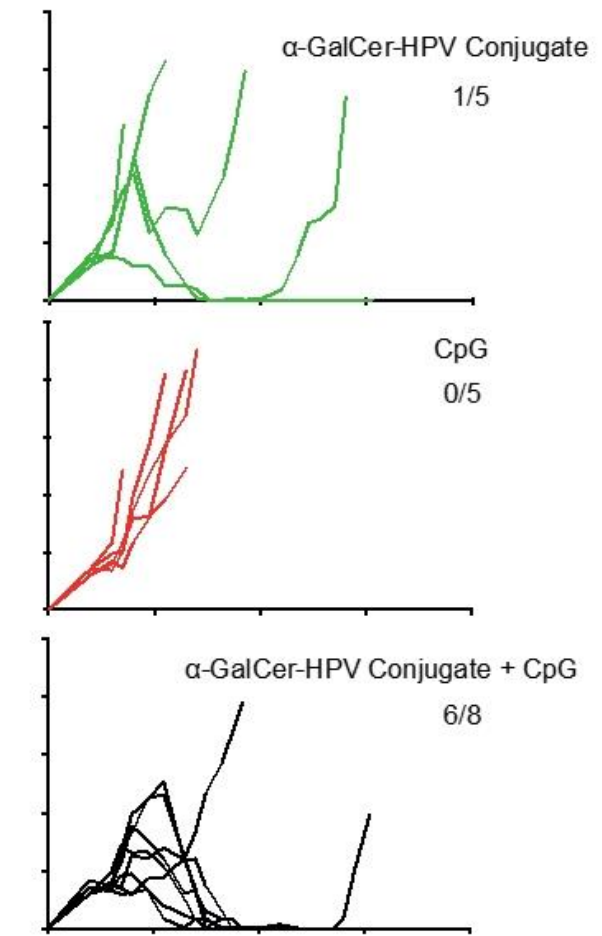

$\alpha-$ GalCer-HPV Conjugate + CpG (6h) $7 / 8$

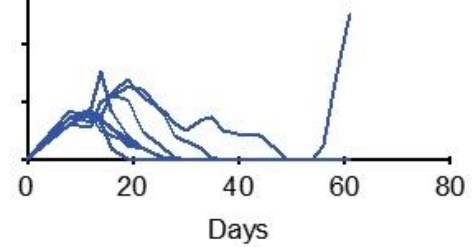

Figure 54 Intatumoural combination of $\alpha$-GalCer-E6 conjugate and CpG induces regression and cure in TC1 tumours. A) Treatment protocol. B) Tumour growth curves plotted for each mouse. C) Survival curves for the different treatment groups, with statistics indicated (log-rank Mantel-Cox test). 
A

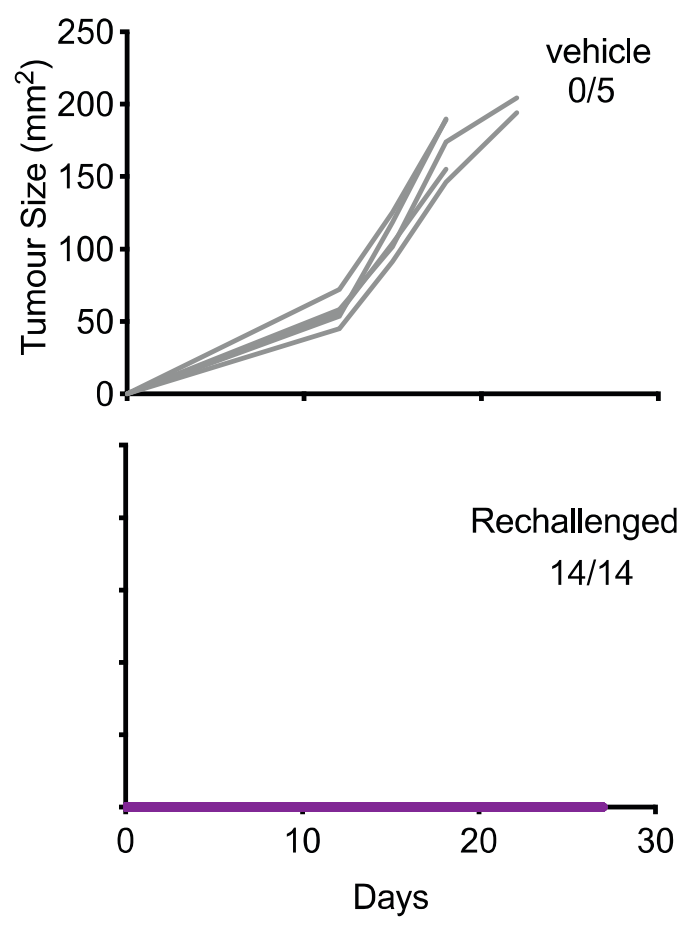

B

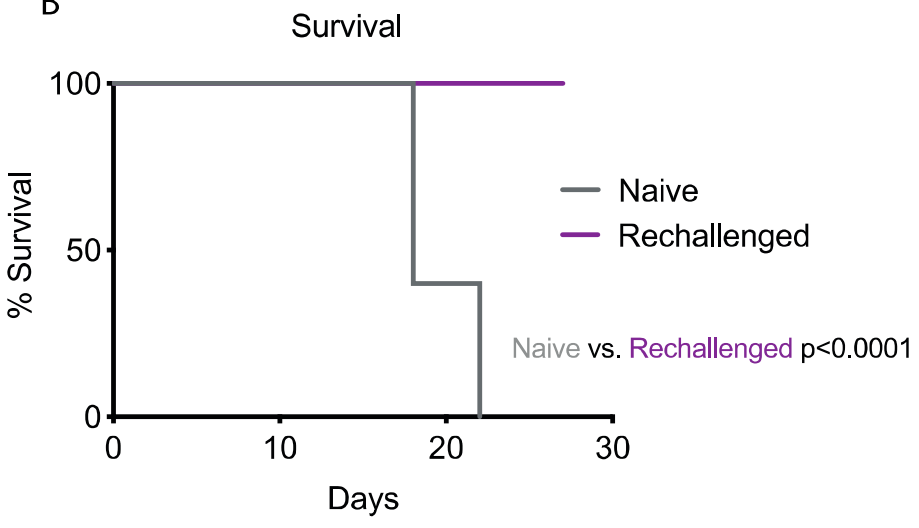

Figure 55 Mice cured of TC1 tumours are immune to rechallenge. A) Tumour growth curves plotted for each mouse. B) Survival curves for the different treatment groups, with statistical differences indicated (log-rank Mantel-Cox test). 


\subsubsection{Inducing abscopal effect of $\alpha$-GalCer-HPV conjugate + CpG in TC1 and comparison of the conjugate with admix of individual components}

Having shown regression in the TC1 model with $\alpha$-GalCer-HPV conjugate plus $\mathrm{CpG}$, it needed to be established whether the peptide had to be conjugated, or indeed if peptide alone could explain the enhanced activity. In addition, it remained to be established whether the treatment resulted in an abscopal effect. To test this, C57BL/6J mice were inoculated with $1 \times 10^{5}$ $\mathrm{TC} 1$ cells into both the left and right flanks. Once the tumours reached approximately $6 \times 6 \mathrm{~mm}$ in size in both flanks, the mice were treated with PBS, HPV E7 epitope alone, an admix of $\alpha$ GalCer with HPV E7 epitope, $\alpha$-GalCer with 6 hours delayed CpG, an admix of $\alpha$-GalCer and HPV E7 epitope with 6 hours delayed CpG, or $\alpha$-GalCer-HPV E7 conjugate with 6 hours delayed $\mathrm{CpG}$.

Intratumoural administration of antigen alone in the form of HPV E7 epitope did not produce a significant growth delay, and adding $\alpha-$ GalCer to serve as an adjuvant to HPV E7 epitope did not improve activity significantly. As expected, $\alpha$-GalCer with 6 hours delayed CpG induced a significant growth delay, but with no survivors. However, some of the mice underwent regression when the HPV E7 epitope was added in the mix, leading to a significantly improved therapeutic outcome (Fig 56). In cured mice, both primary and contralateral tumours fully regressed, showing that the abscopal effect was induced. The use of the $\alpha$-GalCer-HPV E7 conjugate with 6 hours delayed $\mathrm{CpG}$ was superior to all of the other treatments, including the addition of exogenous antigen, with almost all of the mice undergoing regression (Fig 56). However, this improved activity with conjugation (as opposed to free antigen) did not reach statistical significance when looking at overall survival. 
To confirm that complete responses were durable, and to show that activity was dependent on $\mathrm{CD}^{+} \mathrm{T}$ cells, mice that had survived tumour challenge were either given PBS or CD8 depleting antibody and rechallenged onto the back with $2 \times 10^{5} \mathrm{TC} 1$ tumour cells. Depletion of $\mathrm{CD}^{+}$cells was confirmed via flow cytometry, and naïve mice were used as growth controls. As seen with our previous depletion experiments, the mice that had their $\mathrm{CD}^{+}$cells depleted completely lost their anti-tumour response, whereas the mice that retained their $\mathrm{CD}^{+}$cells rejected the tumour rechallenge (Fig 57).

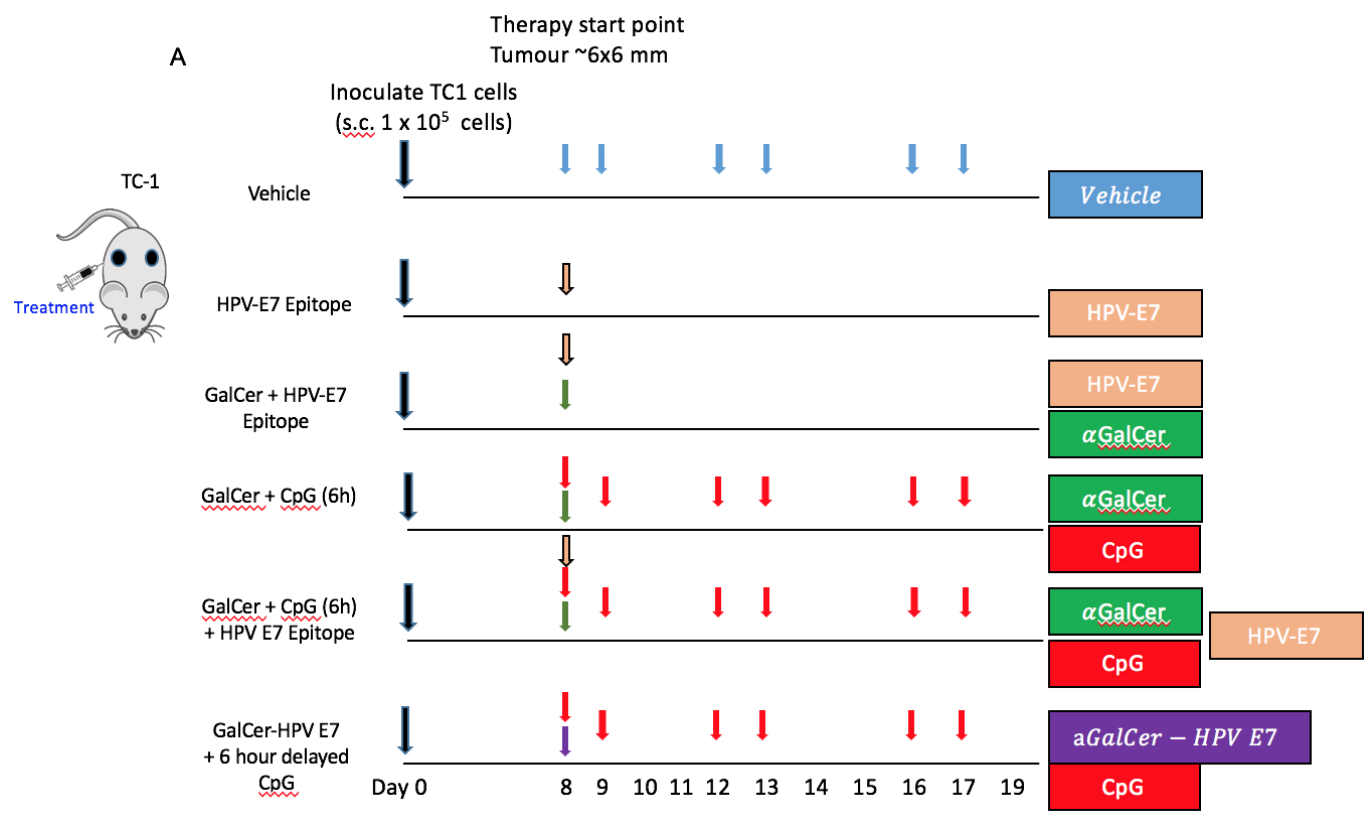


B
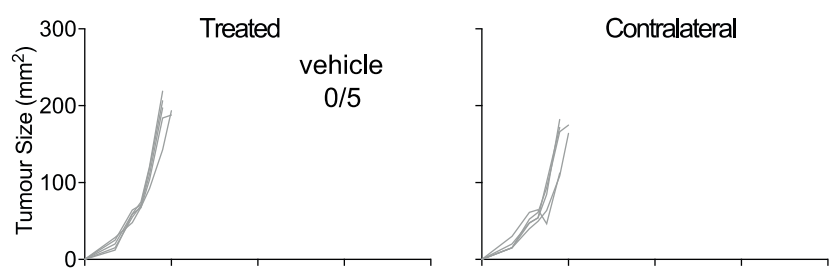

HPV-E7 Epitope
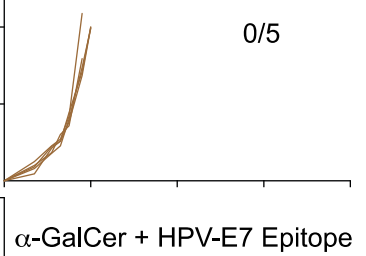

O $0 / 5$
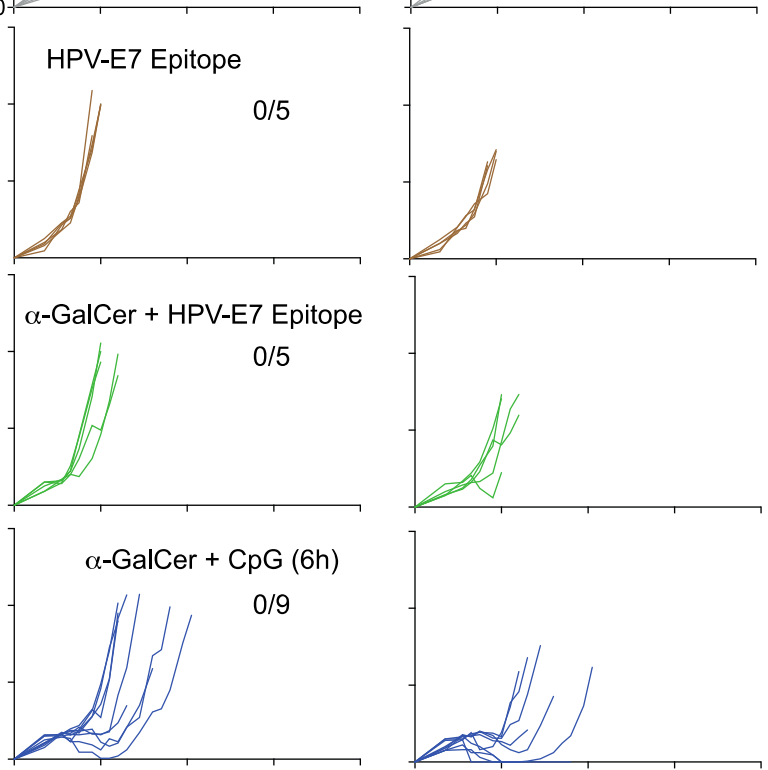

$\alpha$-GalCer + CpG (6h) + HPV-E7 Epitope
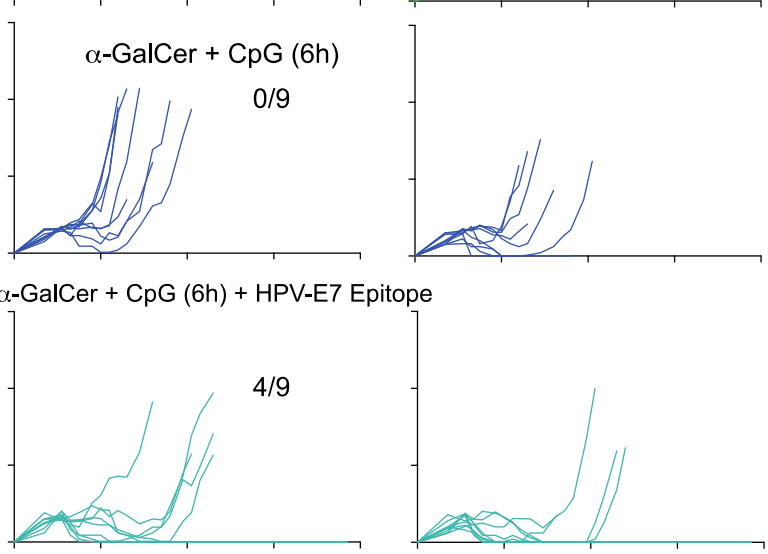

$\alpha-$ GalCer HPV-E7 Conjugate + CpG (6h)
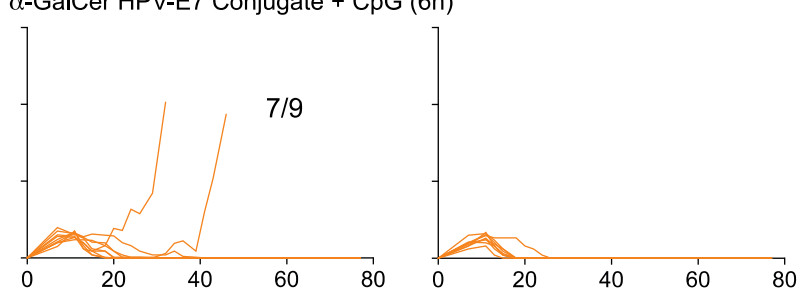

C

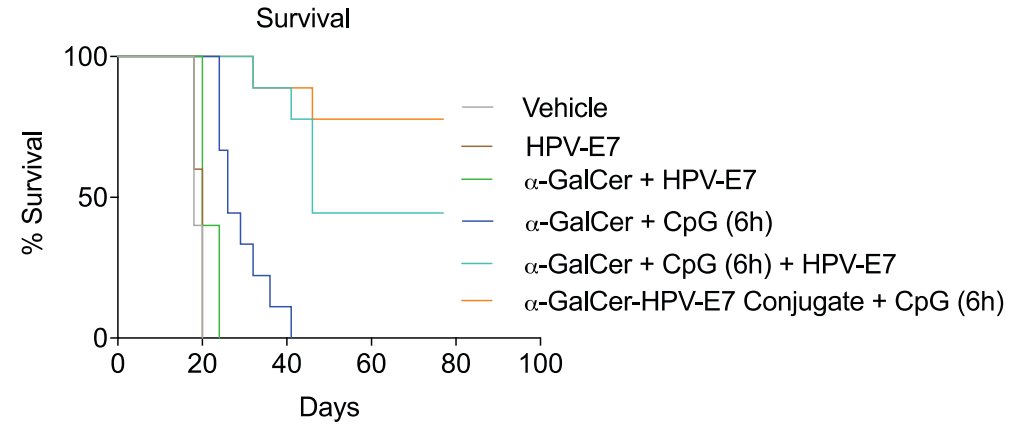

HPV-E7 vs. $\alpha-G a l C e r+C p G(6 h) p=0.0002$

HPV-E7 vs. $\alpha-G a l C e r+C p G(6 h)+H P V-E 7 p=0.0002$

HPV-E7 vs. $\alpha$-GalCer-HPV-E7 Conjugate $+C p G(6 h) p=0.0002$

$\alpha-$ GalCer + HPV-E7 vs. $\alpha-G a l C e r+C p G(6 h) p=0.0029$

$\alpha$-GalCer+ HPV-E7 vs. $\alpha-$ GalCer + CpG (6h) + HPV-E7 $p=0.0001$

$\alpha$-GalCer + HPV-E7 vs. $\alpha$-GalCer-HPV-E7 Conjugate + CpG (6h) p $=0.0001$

$\alpha-$ GalCer + CpG (6h) vs. $\alpha-G a l C e r+C p G(6 h)+H P V-E 7 p=0.0001$

$\alpha-$ GalCer + CpG (6h) vs. $\alpha$-GalCer-HPV-E7 Conjugate + CpG (6h) $p<0.0001$

$\alpha-G a l C e r+C p G(6 h)+H P V-E 7$ vs. $\alpha-G a l C e r-H P V-E 7$ Conjugate $+C p G(6 h) p=0.1827$

Days

Figure 56 Intratumoural combination of $\alpha$-GalCer-E7 conjugate and $C p G$ induces regression and cure in bilaterally implanted TC1 tumours. A) Treatment protocol. B) Tumour growth curves plotted for each mouse. C) Survival curves for the different treatment groups, with statistical differences indicated (log-rank Mantel-Cox test). 
A

TC1 Tumour Rechallenge with CD8 Depletion

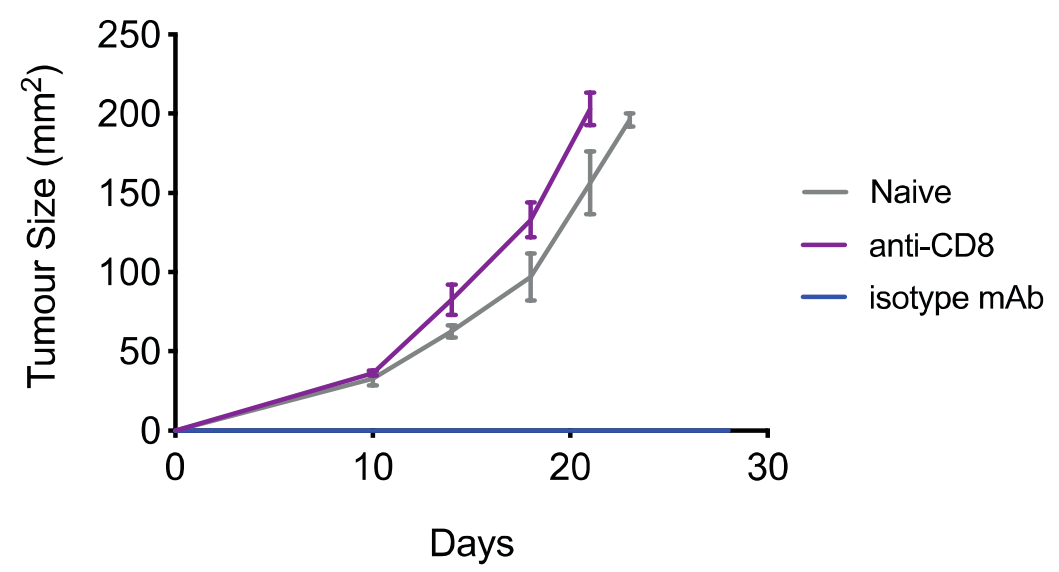

Survival

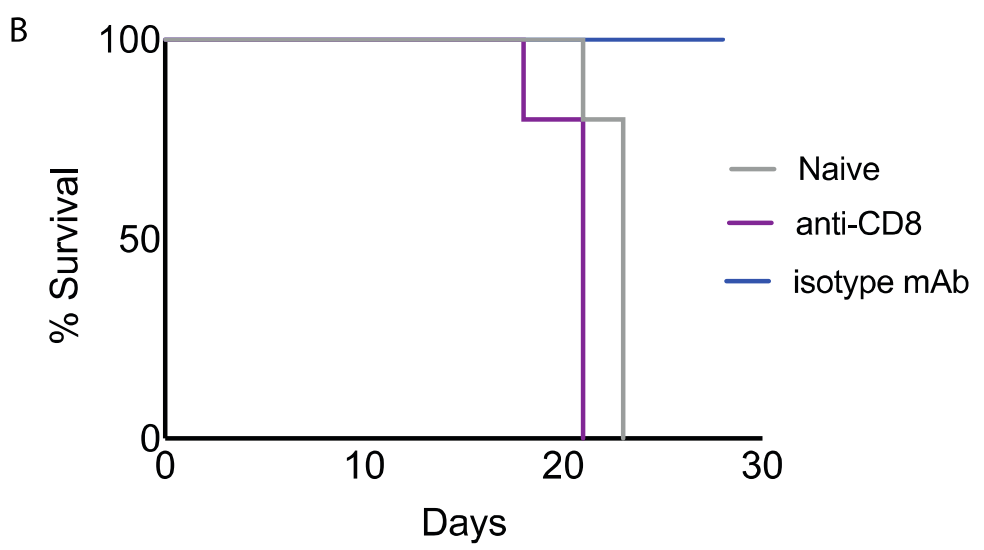

Naive vs. anti-CD8 $p=0.0145$

Naive vs. isotype $p=0.0018$

anti-CD8 vs isotype $p=0.0018$

Figure 57 TC1 anti-tumour memory is CD8 mediated. A) Tumour growth curve. C) Survival plot with statistics indicated (logrank Mantel-Cox test). 


\subsubsection{Impact of $\alpha$-GalCer-Neoepitope conjugate + CpG in B16.OVA tumours}

In order to design a vaccine against a cancer, a TAA must be first identified to use as a target for the immune response to home in on. While there have been a number of TAAs defined that are often expressed in some malignancies, recent studies on people responding to checkpoint blockade have highlighted the importance of patient-specific antigenic profiles, including neoepitopes resulting from mutation. To define these TAA in the clinic, patient biopsies would have to be sequenced, and MHC binding algorithms used to predict whether they are presented ${ }^{7}$. Alternatively, mass spectrometry can be used to establish the sequence of novel peptides eluted from a patient's tumour material ${ }^{319}$. With this information, vaccines could be prepared for treatment to initiate responses to ignored patient-specific antigens, or to boost weak responses. Along this line, after the publication of Kreiter et al. (2015), ${ }^{8}$ where they identified mutations in B16 tumours that resulted in TAAs, it was hypothesised that if B16 melanoma TAA were synthesised and conjugated to $\alpha$-GalCer, it might improve the anti-tumour efficacy against B16 tumours. A total of 21 different $\alpha$-GalCer-neoepitope conjugates were generated ${ }^{317,318}$, and in order to maximise the therapeutic potential by broadening the response, all were admixed for treatment. A dose of 2.33 nmol per conjugate per mouse was used to keep the overall amount of $\alpha$-GalCer consistent with previous experiments.

C57BL/6J mice were inoculated with $3.5 \times 10^{5} \mathrm{~B} 16-\mathrm{OVA}$ cells into the right flank and when the tumours were approximately $6 \times 6 \mathrm{~mm}$ on day 7 the mice were treated intratumourally with PBS, $\alpha$-GalCer with 6 hours delayed $\mathrm{CpG}$, and $\alpha$-GalCer-neoepitope conjugate with 6 hours delayed CpG. Intratumoural treatment with $\alpha$-GalCer with 6 hours delayed CpG was able to induce a significant growth delay, with some full regressions, whereas the $\alpha$-GalCerneoepitope conjugate with 6 hours delayed $\mathrm{CpG}$ cured most of the mice (Fig 58). Although 
suggesting an advantage of supplementing exogenous TAAs to the treatment strategy, this did not reach statistical significance.
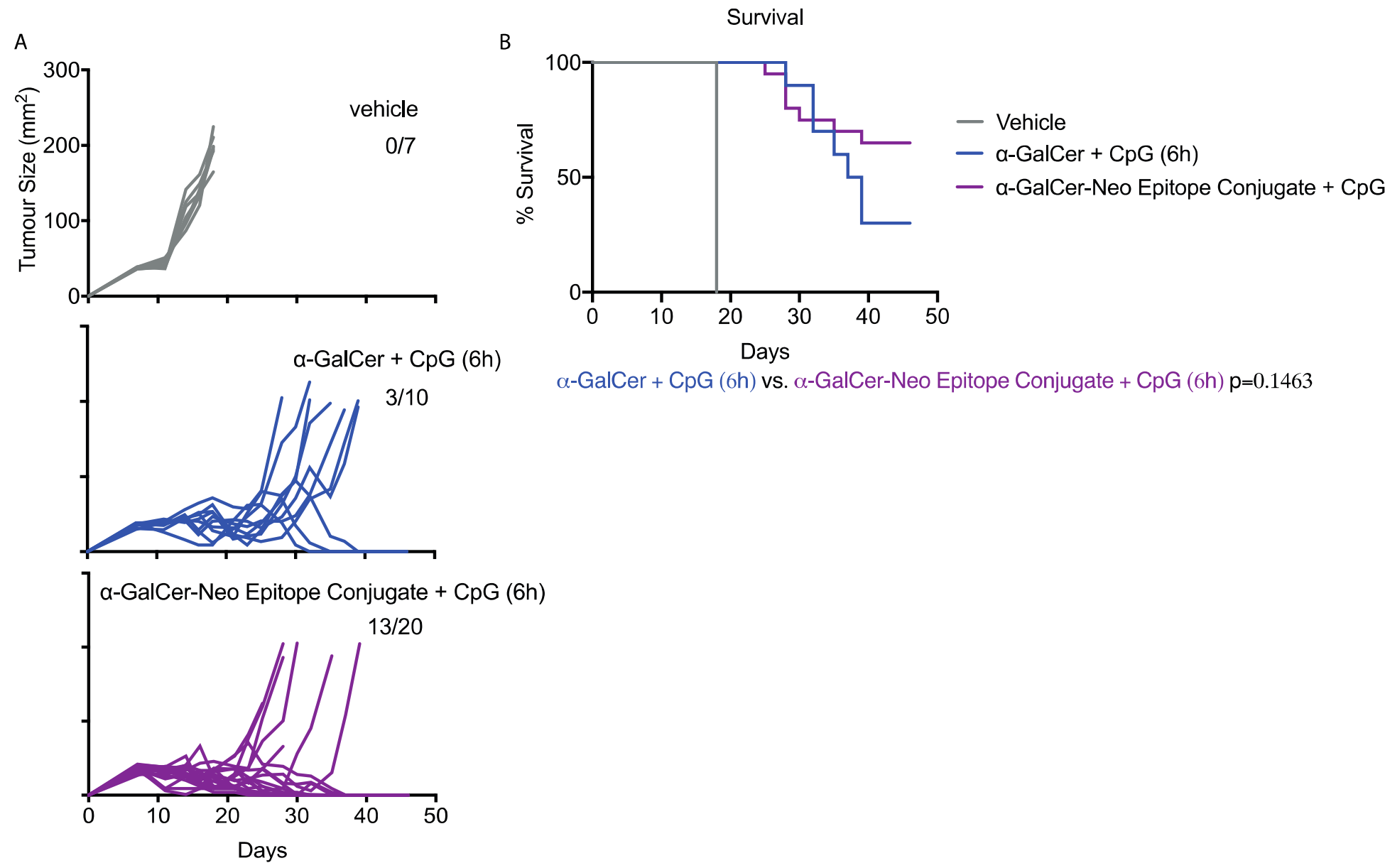

(6h)

$\alpha$-GalCer + CpG (6h) vs. $\alpha$-GalCer-Neo Epitope Conjugate + CpG (6h) $p=0.1463$

Figure 58 Intatumoural combination of $\alpha$-GalCer-neoepitope conjugate and $C p G$ induces regression and cure in B16.OVA tumours. A) Tumour growth curves plotted for each mouse. B) Survival curves for the different treatment groups, with statistics indicated (log-rank Mantel-Cox test). 


\subsubsection{Taking the brake off: Impact of $\alpha$-GalCer + CpG + checkpoint blockade (PD-1) in}

\section{CT26 tumours}

Next, it was investigated whether the anti-tumour activity of intratumoural iNKT cell agonists and $\mathrm{CpG}$ combines with checkpoint blockade. To do this, the CT26 tumour model was used because anti-PD-1 has previously been shown to only very weakly inhibit the growth of this tumour, but this can be improved with combination therapies ${ }^{203,217,320-322}$. As such, this is similar to the clinic, where the vast majority of cancer patients do not respond to checkpoint blockade as a standalone therapy. Treatment of CT26 with anti-PD-1 mAb has been shown to reduce the number of Tregs and MDSCs in the dLNs and the spleen ${ }^{323}$. Since CT26 colon cancer tumours express high levels of PD-L1, and anti-PD-1 mAb was shown to reduce the number of immunosuppressive cells, we anticipated that anti-PD-1 antagonist would be additive to $\alpha$ GalCer and $\mathrm{CpG}$, as the combination should increase the influx of immune cells into the TME, as well as decreasing immunosuppressive cells such as Tregs and MDSCs, for the eradication of CT26 tumours. It was therefore assessed firstly whether the combination of $\alpha$-GalCer and CpG would be able to induce an anti-tumour response in the CT26 colorectal cancer model, as had been seen with the other tumours tested, and importantly, whether treatment with anti-PD-1 mAb could improve this.

For this experiment, $2 \times 10^{5} \mathrm{CT} 26$ tumour cells were injected into the right flank of BALB/CJ. When the tumours were approximately $6 \times 6 \mathrm{~mm}$ on day 7 the mice were treated with either intratumoural PBS, $200 \mu \mathrm{g}$ anti-PD-1 given i.p. in $200 \mu \mathrm{L}$ on the last day of every dosing cycle, $\alpha$-GalCer alone as per standard dosing regimen, $\mathrm{CpG}, \alpha$-GalCer with 6 hours delayed $\mathrm{CpG}$, or anti-PD-1 and $\alpha$-GalCer with 6 hours delayed $\mathrm{CpG}$. 
Not surprisingly based on published reports, anti-PD-1 as a single agent was ineffective. So too were $\alpha$-GalCer and $\mathrm{CpG}$ as single agents. However, the combination of $\alpha$-GalCer and 6 hours delayed $\mathrm{CpG}$ induced complete regression in some mice, while the addition of anti-PD-1 doubled the rate of full regressions. However, this improvement did not reach statistical significance (Fig 59). As had been seen in earlier models, all mice that rejected the tumour were able to reject a rechallenge of the double inoculum of CT26 cells, while those that had been depleted of their $\mathrm{CD} 8^{+}$cells lost their anti-tumour memory (Fig 60). The anti-tumour memory elicited by $\alpha-\mathrm{GalCer} / \mathrm{CpG}$ and anti-PD- 1 is, therefore, $\mathrm{CD} 8^{+} \mathrm{T}$ cell-mediated.

A similar experiment performed using half the amount of anti-PD-1 mAb, not shown here, also showed a similar trend in that the triple combination had a slightly better rate of cure. Overall, this preliminary experiment suggests that intratumoural treatment using the combination of $\alpha$-GalCer and $\mathrm{CpG}$ can be combined with anti-PD- 1 treatment. The combined treatment is safe, with no additional adverse events seen, and showed a trend towards additivity. However, additional experiments, to increase the $\mathrm{n}$ value, are required to show that the additivity is statistically significant. 


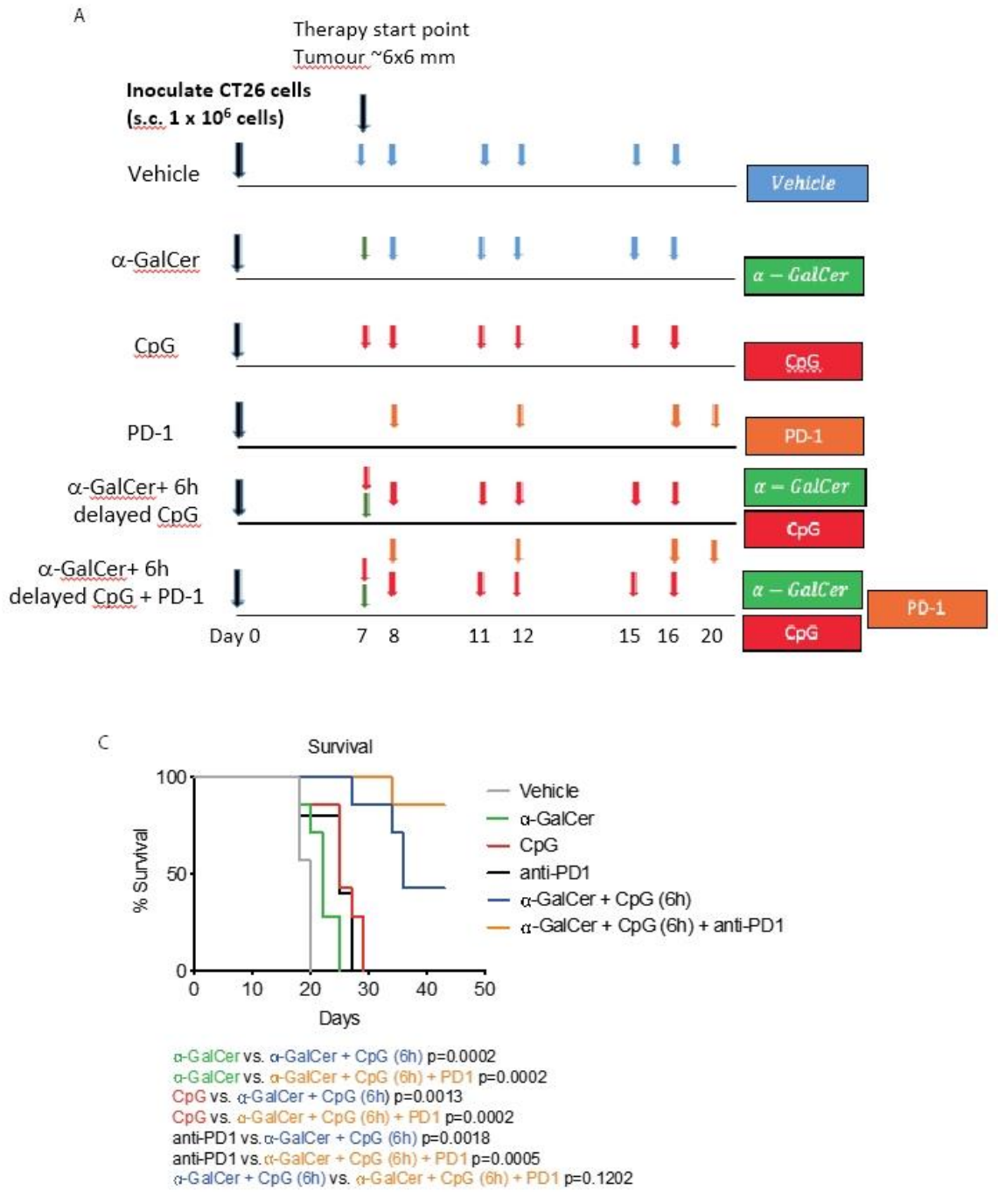

B

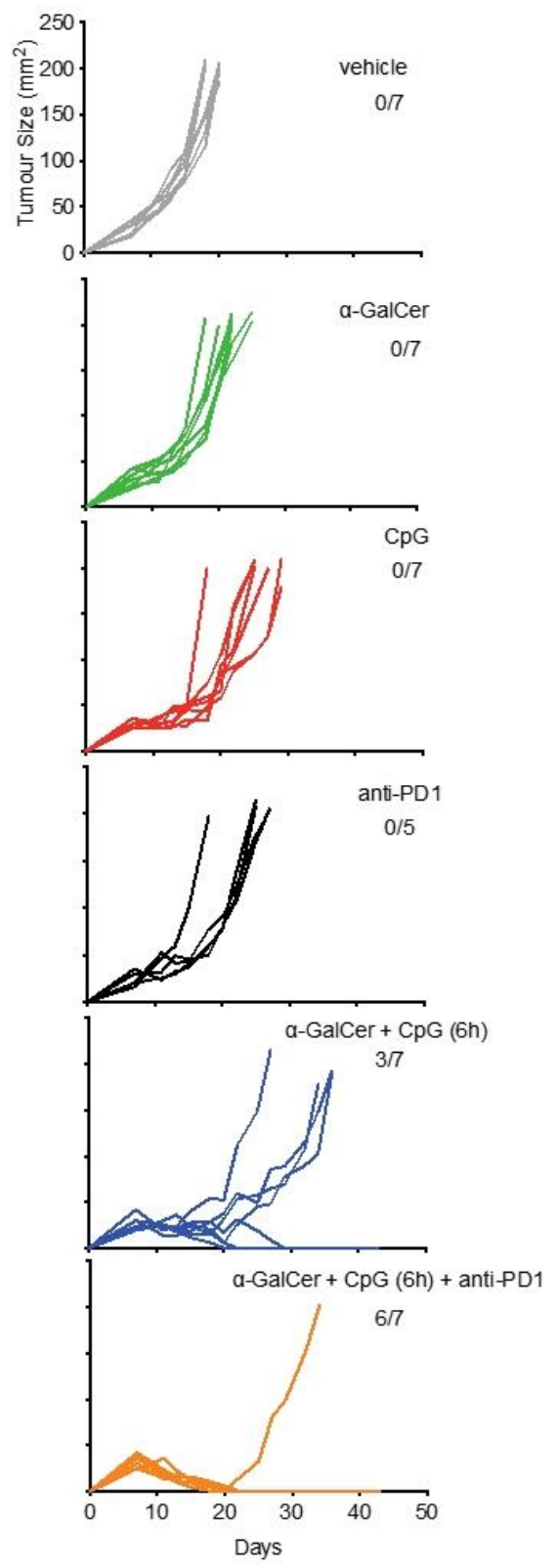

Figure 59 In situ vaccination with $\alpha$-GalCer and CpG in a CT26 tumour model. A) Treatment protocol. B) Tumour growth curves plotted for each mouse. C) Survival curves for the different treatment groups, with statistics indicated (log-rank Mantel-Cox test). 
A

Rechallenge CT26 Tumours with CD8 Depletion

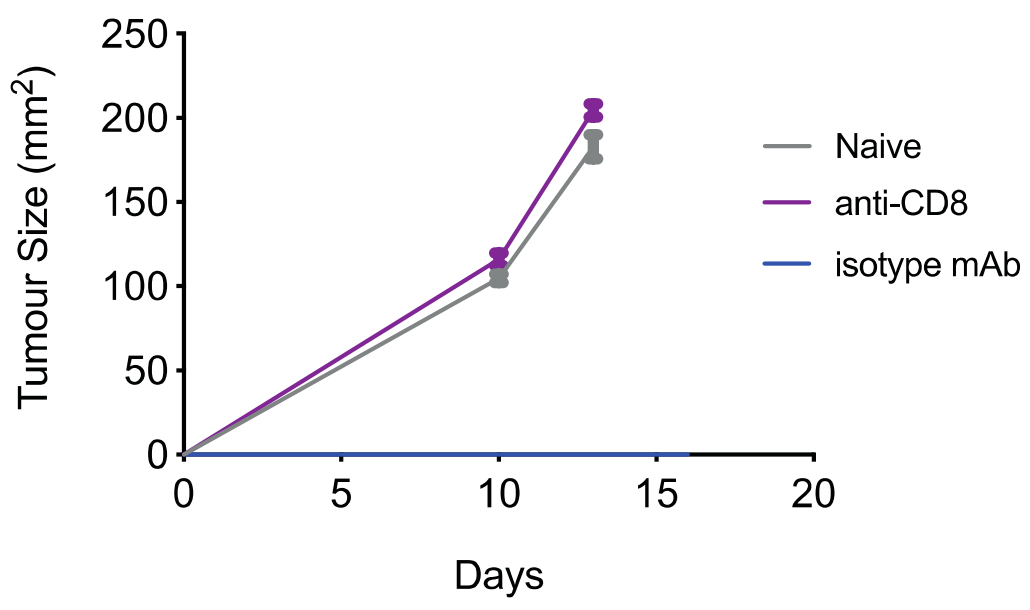

B

Survival

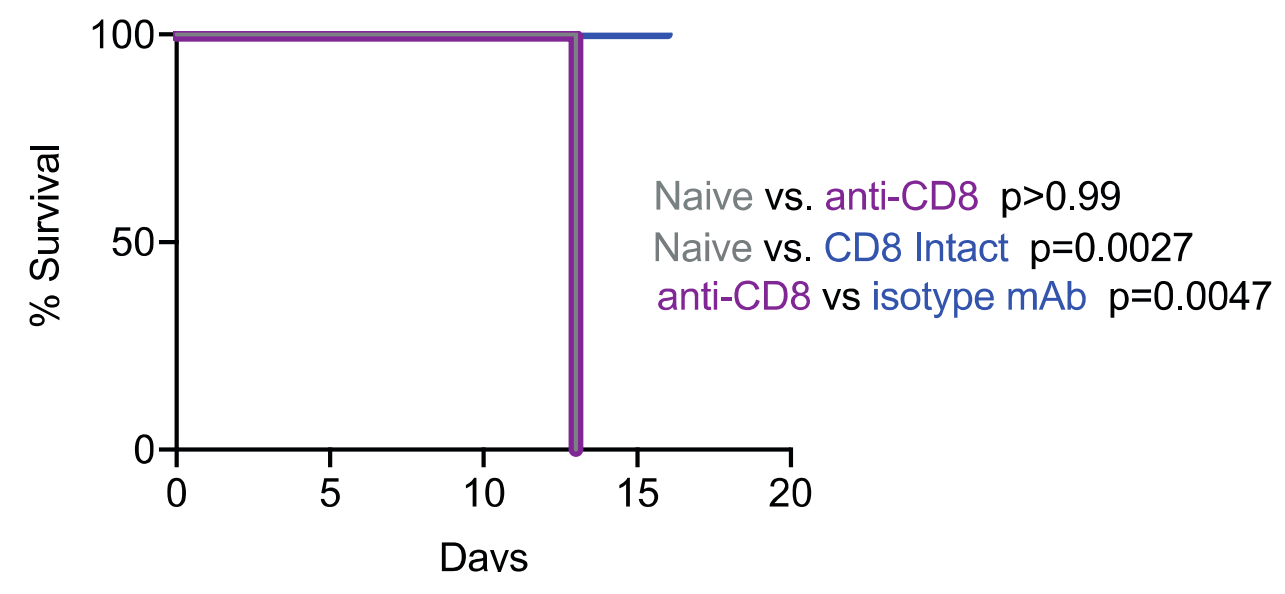

Figure 60 in situ vaccination induced anti-tumour memory is CD8 mediated. A) Tumour Plots. B) Survival plots with statistics indicated (log-rank Mantel-Cox test). 


\subsubsection{Increasing immunogenicity by inducing immunogenic cell death}

The pronounced increase in the effectiveness of the $\alpha$-GalCer/CpG treatment in less immunogenic tumours with the addition of TAA is very encouraging. For example, the complete response rate observed with TC1 tumours was significantly increased in the presence of the antigen conjugated with $\alpha$-GalCer. However, a potential barrier to this strategy becoming a universal practice is that currently few universal tumour antigens are known. Technological advances such as next-generation sequencing and the development of algorithms for predicting patient-specific TAA has the potential to allow for the development of personalised cancer therapies and vaccines. However, these technologies are in their infancy, and for now, computational prediction of neoantigens capable of eliciting effective anti-tumour responses in patients remains a hit-or-miss affair. In addition, a critical challenge for the clinical application of personalised vaccines is being able to manufacture and deliver individually tailored vaccines in a quick and timely manner, without being cost-prohibitive ${ }^{324}$. Despite these issues, the successful use of TAA peptide conjugates did point to an advantage in increasing TAA dose to improve efficacy. A simpler strategy, using chemotherapy to achieve increased release of TAA in an immunogenic context in situ, was therefore explored.

The proof of concept strategy was, therefore, to use a low dose of the ICD-inducing agent doxorubicin in order to liberate TAA along with DAMPs, which will help prime or boost an adaptive immune response. Doxorubicin was used as the ICD agent because of its reported efficiency as an ICD inducer in the poorly immunogenic B16.F10 model ${ }^{325}$. The doxorubicin dose chosen was adapted from a new modality of drug administration called "metronomic 
chemotherapy". Such regimens involve the frequent administration of conventional chemotherapeutic agents at very low doses $\left(\sim 1 / 10^{\text {th }} \text { of standard maximum tolerated dose }\right)^{311}$. The advantages of this modality include minimal adverse effects to the host, notably reduced rate of loss of immune cells ${ }^{326}$ and a reduction in acquired drug resistance during the prolonged drugfree breaks required to recover from host toxicity ${ }^{327}$. Previously it was thought that they act by targeting angiogenesis, but recently additional immune-related mechanisms have been discovered ${ }^{328}$. However, rather than subject the host to chemotherapy continuously throughout the treatment, we chose to give only two doses of doxorubicin to generate the release of enough TAA to support adaptive immune responses without risking a significant loss of immune cells; however, in principle, the low dose chemotherapy could potentially be continued throughout the treatment. In the same experiment, the $\alpha$-GalCer-TAA conjugate strategy described earlier was also used to see how the different approaches to increasing immunogenicity of TAAs compared.

Mice were inoculated with $1 \times 10^{5}$ B16.F10 tumours into the left flank. Relevant mice were treated with doxorubicin on days 6 and 7 , and when the tumours were approximate $6 \times 6 \mathrm{~mm}$ on day 8 , they were treated intratumourally with either PBS, the admix of $\alpha$-GalCer-TAA conjugates, $\mathrm{CpG}, \alpha$-GalCer with 6 hours delayed CpG, or $\alpha$-GalCer-TAA conjugates with 6 hours delayed CpG. An additional group involved combining initial i.p. treatment with 0.3 $\mathrm{mg} / \mathrm{kg}$ doxorubicin $\left(1 / 10^{\text {th }}\right.$ of standard dose for preclinical models) on days 6 and 7 with intratumoural $\alpha$-GalCer and 6 hours delayed CpG. A doxorubicin alone group was also used as a control.

As single agents, the $\alpha$-GalCer-TAA conjugate, $\mathrm{CpG}$, and doxorubicin did not produce significant growth delays. As for combinations, intratumoural $\alpha$-GalCer with 6 hours delayed CpG produced a growth delay, but ultimately no mice rejected their tumours. However, when $\alpha-$ 
GalCer was substituted with the $\alpha$-GalCer-neoepitope conjugates, some mice did reject their tumours, with statistically significant results showing that the addition of antigen in the form of a conjugate can improve the anti-tumour response, as had been suggested by a trend in the previous experiment in B16.OVA (Fig 58). Intriguingly, a statistically significant improvement over $\alpha$-GalCer and 6 hours delayed $\mathrm{CpG}$ was also seen when it was combined with doxorubicin treatment (Fig 61). This suggests that priming the tumours with doxorubicin to produce an improved anti-tumour response is an effective combination strategy. While there was a trend, it did not perform statistically better than utilising the $\alpha$-GalCer-TAA conjugate with CpG.

However, the ease of using an ICD agent makes this off-the-shelf treatment strategy simple and highly attractive in the absence of well characterised TAA. 
A

Inoculate B16.F10 cells

(s.c. $1 \times 10^{5}$ cells)

Vehicle

I $1 \quad 11 \quad$ ।

Vehicle
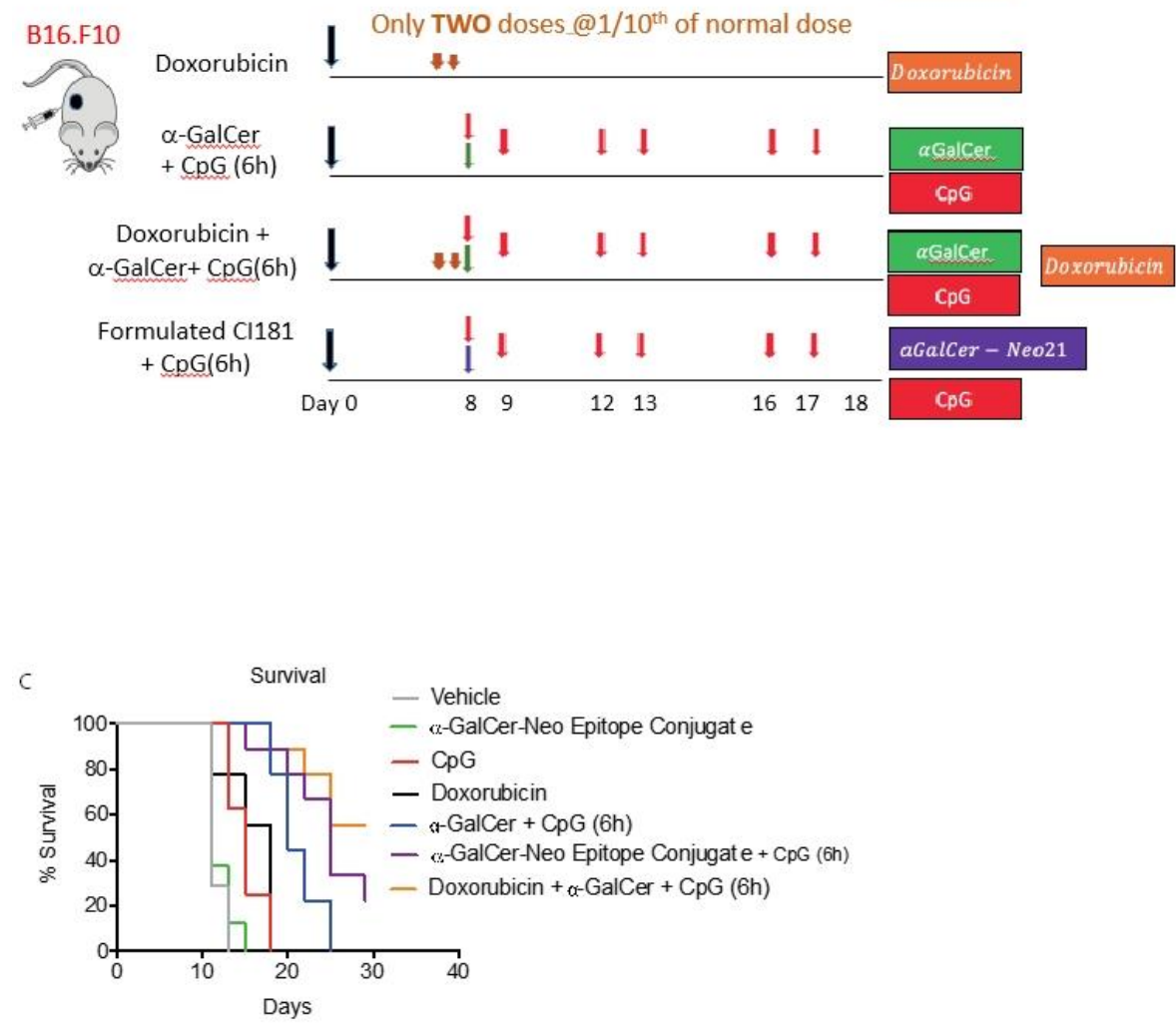

4a-GalCer-Neo Epitope Conjugate vs. $a-G a l C e r+C p G(6 h) p<0.0001$ a-GalCer-Neo Epitope Conjugate vs. Cl181 + CpG (6h) p<0.0001 w-GalCer-Neo Epitope Conjugate vs. Doxorubicin $+(x-G a l C e r+C p G(6 h) p<0.0001$ CpG vs. $a-G a l C e r+C p G(6 h) p=0.0002$

CpG vs. $\mathrm{Cl} 181+\mathrm{CpG}(6 \mathrm{~h}) \mathrm{p}=0.0002$

CpG vs. Doxorubicin + a-GalCer + CpG (6h) $p<0.0001$

Doxorubicin vs. $(\mathrm{a}-\mathrm{GalCer}+\mathrm{CpG}(6 \mathrm{~h}) \mathrm{p}=0.0006$

Doxorubicin vs. $\mathrm{Cl} 181+\mathrm{CpG}(6 \mathrm{~h}) \mathrm{p}=0.0005$

Doxorubicin vs. Doxorubicin $+e_{-G a l C e r}+\mathrm{CpG}(6 \mathrm{~h}) \mathrm{p}=0.0002$

at-GalCer + CpG (6h) vs. Cl $181+\mathrm{CpG}(6 \mathrm{~h}) \mathrm{p}=0.0391$

a-GalCer + CpG (6h) vs. Doxorubicin $+a-G a l C e r+C p G(6 h) p=0.0053$

$\mathrm{Cl} 181+\mathrm{CpG}(6 \mathrm{~h})$ vs. Doxorubicin $+a-G a l C e r+\mathrm{CpG}(6 \mathrm{~h}) \mathrm{p}=0.2045$
B

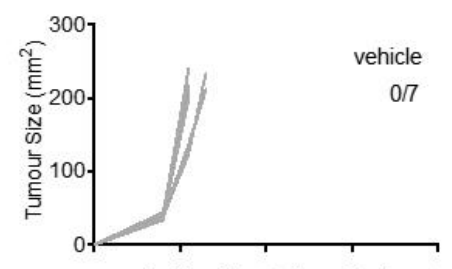
a-GalCer-Neo Epitope Conjugate

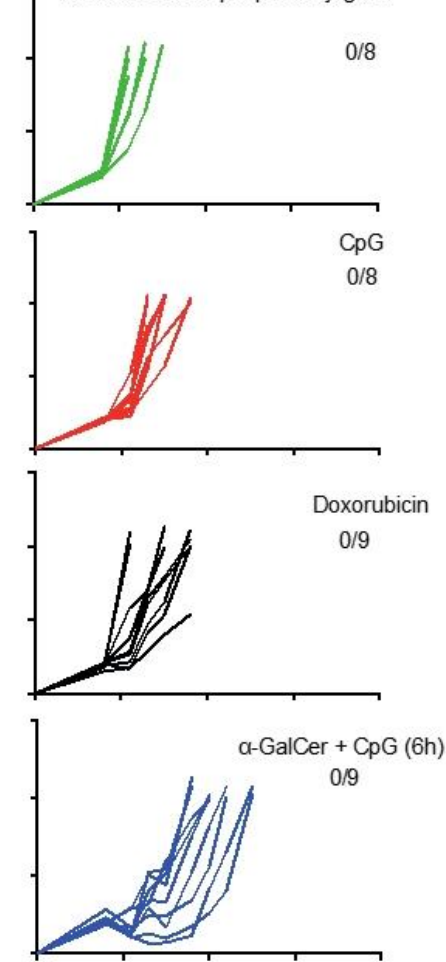

a-GalCer-Neo Epitope Conjugate $+\mathrm{CpG}(6 \mathrm{~h}$ )

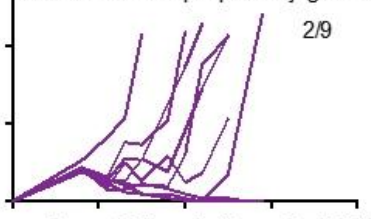

Doxorubicin $\alpha-$ GalCer + CpG (6h)

$5 / 9$

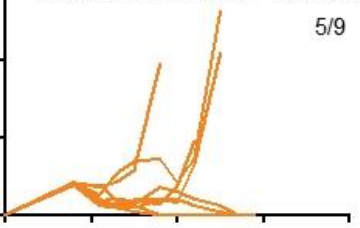

Figure 61 Intatumoural combination of $\alpha$-GalCer and $C p G$ with low dose doxorubicin induces regression and cure in B16.F10 tumours. A) Treatment protocol. B) Tumour growth curves plotted for each mouse. C) Survival curves for the different treatment groups, with statistical differences indicated (log-rank Mantel-Cox test). 


\subsection{Discussion}

Despite the unprecedented durable response rates observed with cancer immunotherapies, the majority of patients $(80-85 \%)$ do not benefit from the treatment and some responders relapse after a period of response. Several common cancer types such as breast, prostate, and colon have shown very low frequencies of responses and heterogeneous responses have even been seen between distinct tumours within the same patient ${ }^{329}$. The lack of sufficient or suitable TAA, impaired TAA processing, and impaired presentation of TAA can all lead to impaired formation of tumour-reactive T cells. Whether a tumour is non-immunogenic or immunogenic likely depends largely on the degree of immune cell infiltration ${ }^{330}$. Non-immunogenic tumours have been characterised by the sparse presence of immune cells in the TME, most notably cytotoxic $\mathrm{T}$ cells, possibly due to an impaired ability to present tumour antigens to T cells ${ }^{331,332}$. They also may lack expression of key chemokines that recruit immune cells to the tumours and are thus less able to promote tumour-specific T-cell infiltration ${ }^{331}$. Together, these factors limit cytotoxic T-cell activation and migration to the tumour, ultimately preventing tumour cell elimination. Immunogenic tumours, on the other hand, are marked by the presence of immune cells and can be an indicator of a pre-existing immune response, which is known to be positively associated with survival in the clinic ${ }^{333,334}$. These cancers have a high tumour mutational burden (TMB), producing a plethora of tumour antigens, which can facilitate the recruitment of cytotoxic T cells. Although the accumulation of mutations in tumour cells can provide a selective advantage by increasing genetic diversity and adaptability, it also poses a tremendous risk for the tumour cells, as they can become more easily recognised by the immune system ${ }^{335}$. Unlike non-immunogenic tumours, antigen presentation and T-cell activation, are active processes in immunogenic tumours and are more likely to respond to checkpoint inhibition ${ }^{336}$. As would be expected, some 
tumours exhibit features that are in-between these two extremes, displaying low immune cell infiltration or immune cells failing to enter tumours due to being blocked at the invasive margin. Different strategies are needed to tackle different types of tumours, for example, when treating tumours that are devoid of immune cells, the initial strategy would require attracting immune cells to the TME as checkpoint inhibition would be futile in this instance.

In the experiments presented in this chapter, it was shown that the addition of exogenous intratumoural tumour antigen to help support the adaptive immune response increases the effectiveness of the $\alpha-\mathrm{GalCer} / \mathrm{CpG}$ combination in certain tumours, particularly when the antigen is covalently linked to $\alpha$-GalCer. This perhaps suggests that the tumour associated antigens expressed in situ are potentially weakly immunogenic in the TC1 tumour model and that the induction of strong immunity required the addition of exogenous of antigen in close proximity to adjuvant to initiate an effective adaptive immune response. For antigens like the HPV oncoproteins, which are expressed in a broad range of tumours, it is possible to envisage an off-the-shelf conjugate vaccine for this purpose in the clinic, perhaps one incorporating multiple epitopes to give population-level coverage based on the diverse HLA background in the human population. Other antigens that could be used in this context are the cancer-testis antigens that are often expressed in a wide range of tumours. Conjugation is a useful strategy for increasing tumour immunogenicity by allowing for adjuvant and antigen to be simultaneously processed by APCs. A potential issue associated with injecting unconjugated minimal epitopes is that, without processing steps required (which are heightened in professional APCs), they may end up being presented by non-professional APCs to promote tolerance. On the other hand, conjugation should enforce uptake of $\alpha$-GalCer and TAA into the same cell, and because uptake and processing is 
enhanced in APCs, presentation of the cleaved components should be favoured by the professional cells most likely to promote $\mathrm{T}$ cell responses.

However, in the absence of useful "universal" antigens, the patient's tumour would have to be sequenced for TAA, which would then be synthesised and conjugated to $\alpha$-GalCer for use in the patients' treatment. Multiple TAAs will likely need to be used, increasing the complexity of the process. The challenge with this approach is the difficulty in identifying the relevant immunogenic TAAs and the synthesis of the complex conjugates. Here it is shown in B16 melanoma that $\alpha$-GalCer-neoepitope conjugates for several reported neoepitopes (C.W. Tang, personal communication, 2019) can improve the anti-tumour response over adjuvanted, free antigen with similar trends being seen in repeated experiments. However, significance was only achieved in one experiment. It is important to note that twenty-one conjugates were used even though at the time this work was done not all twenty-one antigens were shown to be immunogenic in the model used in this laboratory. A colleague subsequently found that significant anti-tumour was limited to only 3 of the epitopes used. This may reflect the fact that the $\alpha$-GalCer-conjugates favour $\mathrm{CD}^{+} \mathrm{T}$ cell responses (I.F. Hermans, personal communication, 2019) and that the published TAA sequences are reported to induce CD4+ $4^{+}$cell responses primarily ${ }^{8}$. An assessment of the antitumour activity of combining the three active conjugates with $\mathrm{CpG}$ intratumourally is therefore warranted.

The strategy of promoting ICD to liberate relevant immunogenic TAA in tumours with a non-inflamed, immune cell poor TME in situ is very appealing and appears to be very effective in our hands. In addition, the use of low dose chemotherapeutics will reduce the toxic side effects and reduce depletion of immune cells through cytotoxicity, which is usually associated with these molecules used at high doses. Based on the efficacy observed with $\alpha-\mathrm{GalCer} / \mathrm{CpG}$ 
combination with low dose doxorubicin, it is possible that the ICD agent delivered the relevant TAA into the TME where $\alpha$-GalCer/CpG was physically delivered, and subsequently supported T cell immunity locally, or through drainage to lymphoid tissues. Since this was a proof of concept study where doxorubicin was utilised only at the start of the experiment, it would be interesting to see how effective the treatment would be if low dose doxorubicin were used throughout the experiment to continually churn out TAAs as tumours age (true metronomic treatment). However, even in a metronomic setting, the negative impact of chemotherapy on immune cells number cannot always be avoided ${ }^{337}$.

The proposed principal mechanism of doxorubicin cardiotoxicity is increased oxidative stress, as evident from increased levels of reactive oxygen species and lipid peroxidation ${ }^{338}$. In the case of high dose doxorubicin, the most dangerous side effect clinically is dilated cardiomyopathy, leading to congestive heart failure. The rate of cardiomyopathy is dependent on its cumulative dose, which can be as high as $36 \%$ when the dose exceeds $600 \mathrm{mg} / \mathrm{m}^{2}$. When congestive heart failure develops, mortality is approximately $50 \%{ }^{339}$. A clinical strategy based on low dose use, as used here, would, therefore, be favourable.

It would be interesting to see how effective other low dose ICD inducing agents such as mitoxantrone, cyclophosphamide or radiation are since some of these have less severe side effects compared to doxorubicin. In principle, these ICD agents can also be administered intratumourally with $\alpha$-GalCer/CpG to avoid systemic toxicity, as well as the ease of administering all reagents with a single needle. Moreover, the total amount of ICD agents can further be reduced for intratumoural delivery.

Immune checkpoint inhibitors have revolutionised cancer treatment, but not all patients benefit from monotherapy. To overcome this, combinations of immunotherapy are increasingly 
being explored as a strategy to improve outcomes. According to the Cancer Research Institute, there are now over 1,500 clinical trials testing checkpoint inhibitors in combination with one or more agents. However, with so many potential combinatorial strategies, identifying optimal approaches and obtaining randomised data is paramount to maximise benefit and minimise toxicity. Ideal combinations should have complementary, not overlapping mechanisms of immune activation to maximise effectiveness.

Here, anti-PD-1 was used along with the $\alpha$-GalCer/CpG combination in the CT26 colon cancer model. The CT26 tumour cell line is derived from an undifferentiated colorectal carcinoma induced in a BALB/c mouse by repeated intrarectal instillations of N-nitroso-Nmethylurethan and shown to be modestly immunogenic ${ }^{340,341}$. It was recently shown that three weeks post-engraftment with the colon cancer line CT26, the macrophages in the tumour expressed high levels of surface PD-1 ( 50\%), which correlates with decreased phagocytosis by tumour-associated macrophages (TAM) ${ }^{342}$. Thus it was logical to add anti-PD-1 mAb to the $\alpha$ GalCer/CpG combination in the CT26. While combining anti-PD-1 mAb with $\alpha-G a l C e r / C p G$ did cause tumours in the majority of the mice to regress, this did not reach statistical significance over $\alpha-G a l C e r / C p G$ without checkpoint blockade, albeit there was a trend in the right direction. Similarly, others have demonstrated that a combination of a TLR9 agonist (SD101) in combination with anti-PD-1 mAb provided additivity in the tumour regression and survival of CT26 and MC38 (another colon cell line) bearing mice in a statistically significant manner ${ }^{203}$. We did not observe a dramatic enhancement of the cure rate in a similar experiment. However, the starting tumour sizes in their experiments were much smaller than those in our experiment. In any event, the totality of the data suggests that the inclusion of $\alpha-G a l C e r / C p G$ rendered a checkpoint blockade-resistant tumour susceptible to immune attack. Thus there is a rationale for 
pursuing this combination clinically as checkpoint blockade is rapidly becoming the default treatment for an increasing number of cancer conditions.

These promising preclinical results build a rationale for the clinical development of $\alpha$ GalCer/CpG treatment in combination with a judicious choice of a third agent which has a complementary, but not overlapping mechanism of immune activation to maximise therapeutic impact while minimising toxicity. In this chapter, we have provided a preclinical rationale to investigate novel approaches to the treatment of cancer in patients who are not responding to treatment with checkpoint inhibitors, the emerging standard of care for cancer. 


\section{Chapter 8}

\section{General Discussion}

\subsection{Summary:}

In this thesis, an intratumoural treatment model was developed and optimised by combining the stimulatory activity of the TLR9 agonist, $\mathrm{CpG}$ (a clinically promising intratumoural agent), with the activation of iNKT cells through intratumoural administration of $\alpha$-GalCer. The treatment regimen showed anti-tumour efficacy in several different in vivo models representing different types of cancer. The combination of $\alpha$-GalCer and $\mathrm{CpG}$ was highly effective in inducing the rejection of both treated and untreated tumours through the induction of a systemic adaptive immune response with the associated abscopal effect. This effect was shown to be critically dependent on the activation of $\mathrm{CD} 8^{+} \mathrm{T}$ cells, which were capable of mounting a memory response against rechallenge with the same, or similar, tumours. The evidence for a systemic adaptive immune response includes 1) The ablation of secondary lymphoid organs results in significantly reduced treatment effect, probably since lymphoid organs are home to a diverse population of lymphocytes and are where $\mathrm{T}$ and $\mathrm{B}$ cells encounter antigens to generate effector cells. 2) The response was mediated by $\mathrm{CD}^{+} \mathrm{T}$ cells which are known to recirculate around the body 3) Some anti-tumour activity could be transferred to another host via serum, suggesting a systemic antibody response had been induced. 4) Evidence for an abscopal effect, which is most likely caused by recirculation of cellular effectors and antibody to the distal tumours. However, despite this evidence of the response being systemic, an additional element is explicitly provided by the intratumoural administration, as subcutaneous and peritumoural routes of administration minimally affected tumours even though lymphoid 
organs were perhaps even more likely to come into contact with the systemically injected reagents than when the reagents were administered locally inside the tumours. It is likely, therefore, that the repeated intratumoural administration of $\mathrm{CpG}$ creates an inflammatory context that permits the induced anti-tumour response to flourish, both locally and distally. Intratumoural administration of the agents was associated with increases in IFN- $\alpha$ in the tumour (rather than the serum) (Fig 50), and this may be one of the key signals.

The critical involvement of the dLN and spleen in anti-tumour activity (as shown by excision of these organs), and residual ALT responses, suggests that some of the agonists and antigens may have dispersed into the lymphoid organs and liver, perhaps as free agents or loaded onto migratory APCs. This dispersal could potentially be through lymphatics (to reach the dLN) and access to the blood. This must occur in sufficient quantities to activate iNKT cells and promote activation of antigen-bearing APCs, thereby driving stimulation of antigen-specific T cells. Through the use of various techniques such as knockout mice, neutralising mAb, confocal, flow cytometry (and cyTOF data conducted by a colleague not presented here), we were able to decipher that iNKT cells, $\mathrm{CD} 8^{+}$cells, $\mathrm{pDCs}$ and $\mathrm{cDC} 1 \mathrm{~s}$ were recruited by the combination of $\alpha-$ GalCer and $\mathrm{CpG}$ to mount the anti-tumour response within the tumour and in the systemic circulation (spleen and blood). With less immunogenic tumours, we showed that the addition of TAA improves the anti-tumour response. The added antigen can be added in the form of an admix, but better responses were obtained when TAA was conjugated to $\alpha$-GalCer. Increasing the release of TAA in situ in an immunostimulatory context by exploiting the concept of ICD was shown to be another way to improve the response. Combining the treatment with anti-PD-1 $\mathrm{mAb}$, particularly those tumours that have demonstrated an increased expression of PD-1 or PDL1, also requires further investigation as a simple way to improve efficacy. Provided below is a 
discussion on each of the above points, a proposed overall mechanism, and comparisons of the approach with other approaches that are at the leading edge in the field and a summary of potential logical future directions. 


\subsection{The abscopal effect:}

The abscopal effect is essential for the intratumoural treatment to be clinically useful as it is not practical to inject into every tumour. When a tumour cell dies, it leads to the liberation of neoantigens or tumour-associated antigens (TAAs). The diverse TAA can be taken up by antigen-presenting cells (APCs), which travel to the draining lymph node where they are presented to $\mathrm{CD} 8^{+}$and $\mathrm{CD} 4^{+} \mathrm{T}$ cells ${ }^{343}$. It is also possible that dead or dying tumour cells provide a source of TAAs that access APCs in the spleen via the blood. In these lymphoid organs, TAA-specific $\mathrm{CD} 8^{+} \mathrm{T}$ cells, perhaps with help from TAA-specific $\mathrm{CD} 4^{+} \mathrm{T}$ cell, become activated. However, this outcome is also dependent on the activation of the APCs. It is possible that this is triggered directly by the $\mathrm{CpG}$ in the tumour before the APCs migrate, or that some CpG drains with antigen to the tumour. Dying tumour cells may also release danger-associated molecular patterns (DAMPs) and cytokines that enhance the trafficking of immune cells. For iNKT cells to contribute to T cell activation as "cellular adjuvants", some $\alpha$-GalCer and antigen must reach the APCs in lymphoid tissues concomitantly ${ }^{344,345}$. Low serum ALT levels were observed with intratumoural delivery compared to $\alpha$-GalCer given intravenously as a single agent; nonetheless, some ALT elevation was still observed, with the simplest interpretation being that some $\alpha$-GalCer did reach the liver to activate iNKT cells, causing the characteristic transient liver inflammation. Thus, some of the injected material is likely to have entered systemic circulation. This may be crucial, as removal of distal lymphoid organs, the dLN and spleen, significantly reduced the anti-tumour effect (with the spleen being particularly rich in the iNKT cells required for an adjuvant effect on $\mathrm{T}$ cell activation). It is possible that this occurs by $\alpha$ GalCer inserting into the membranes of dead or dying tumour cells that enter the blood, and are then trafficked to the spleen. Given that both dLN and spleen are required for optimal activity, it 
is likely that a combination of the events above ultimately promotes tumour cell elimination by primed $\mathrm{CD} 8^{+} \mathrm{T}$ cells throughout the host.

Evidence of an abscopal effect was seen in all of the models tested, whereby mice challenged with tumours on both flanks were able to successfully reject both tumours after receiving intratumoural treatment into only one of their tumours. The most obvious explanation for this abscopal effect was that systemic adaptive effectors recirculated to, and eliminated, the distal tumour. However, another hypothesis was that the abscopal effect was due to systemic leakage of the reagents to the distal site, and a similar process of anti-tumour activity was initiated at both sites. This may be a possibility in a small animal with low blood volume, and perhaps both hypotheses may be contributing to the observed anti-tumour response, rather than just a single pathway. However, to test whether the combination was solely dependent on "leakage" of the reagents, we compared the effectiveness of the $\alpha$-GalCer and CpG combination administered via different routes. If the leakage out of the tumour to the lymphoid organs (presumably with accompanying tumour antigens) was the only mechanism to induce the systemic response observed, then peritumoural and subcutaneous administration should perform equally as well as intratumoural treatment. However, since intratumoural delivery was demonstrated to be far superior, this suggests that local effects shaped the induced systemic responses and that this advantage translates to effective anti-tumour responses that could also function at distal tumour sites. As noted above, the repeated nature of the intratumoural treatment, notably the continued $\mathrm{CpG}$ administration, may serve to condition the immune response, perhaps providing better and longer-lived effectors through local exposure to type 1 IFNs. Based on this notion, there may be some benefit to optimising the physical properties such as the size and hydrophobicity of the active ingredients to allow for longer retention time in the 
tumour after intratumoural injection by making the molecule less soluble in water ${ }^{346}$. Finding the optimal balance between sufficient tumour retention, and allowing the agents to perform their adjuvant function is likely to be a key to therapeutic success with minimal toxicity.

As already noted, mice that had their spleen removed completely lost their anti-tumour response, and this organ is therefore likely to be involved in the abscopal effect, perhaps mainly through facilitating the activation of iNKT cells, as iNKT cells were not present in large numbers in the tumour before treatment. Interestingly, while depletion of iNKT cells understandably reduced the anti-tumour effect of the combined treatment down to that of $\mathrm{CpG}$ alone, the abscopal effect typically seen with $\mathrm{CpG}$ alone, although typically limited, was completely abrogated in the absence of these cells. Therefore, even in the absence of $\alpha$-GalCer, iNKT cells may contribute to the abscopal effect, perhaps interacting with an endogenous agonist in the TME. It has been demonstrated that mammalian cells contain endogenous lysosomal glycosphingolipids, namely $\alpha$-linked monoglycosylceramides, that can stimulate iNKT cells ${ }^{347}$. The expression of these endogenous iNKT cell ligands by DCs can be induced via stimulation by cytokines such as tumour necrosis factor (TNF) ${ }^{347}$ or by TLR mediated stimulation associated with bacterial infection ${ }^{348-350}$. This suggests stimulating iNKT cells may be required to get the response above the threshold for abscopal activity, and factors made by activated iNKT cells may facilitate $\mathrm{T}$ cell entry and effector function, including the development of anti-tumour memory. Notably, one of the few clear observations from the flow cytometry data was that the $\alpha-\mathrm{GalCer} / \mathrm{CpG}$ combination served to facilitate iNKT cell accumulation in the tumour, the site of injection, whereas $\alpha$-GalCer alone did not. This may be due to the combination of $\alpha$-GalCer and CpG activating iNKT cells both via direct ligation and indirectly via TLR mediated stress, leading to increased stimulation by endogenous iNKT cell ligands and cytokines. 


\subsection{Tumour antigen-specific $T$ cell responses with memory:}

Intratumourally injecting $\alpha-\mathrm{GalCer}$ and $\mathrm{CpG}$ resulted in a robust, systemic anti-tumour response that can eradicate large established tumours and provided a memory response against rechallenge mediated by $\mathrm{CD}^{+} \mathrm{T}$ cells. In the EG7.OVA lymphoma model, it was expected that the introduced OVA antigen would have been a major rejection antigen because it would not have been subjected to immune tolerance, and it is readily expressed in a soluble form, so it is very likely to be exposed to the immune system. However, there was no evidence that significant responses to OVA were induced, and the rejection response was effective against rechallenge with EL4.LA without OVA. Therefore there must have been at least one, or potentially multiple, TAAs that were sufficiently immunogenic to elicit a targeted immune response and long-term immune-memory. Perhaps, the effective rejection antigens may be those to which the host is already showing some endogenous immunogenicity and an immunostimulatory boost was required to get over the threshold for stronger anti-tumour immunity. It is possible that OVA was immunologically ignored, as demonstrated by a lack of OVA-specific T cells, which has been reported for a variety of TAAs in tumour models ${ }^{11,142}$. Indeed, later in the thesis, it was shown that OVA could be turned into a rejection antigen through manipulation via intratumoural vaccination with an $\alpha$-GalCer-OVA conjugate.

As type I IFN has been reported to assist in the formation of memory, the ablation of pDCs in Siglec-H DTR mice may have resulted in decreased type I IFN which may account for the reduction in the late anti-tumour effect. Reports have shown that pDCs produced higher amounts of IFN- $\alpha$ and IL-12p40 upon treatment with $\mathrm{CpG}^{253,351}$. As previously noted, these cytokines are essential for improving antigen-cross priming ability of $\mathrm{cDC} 1$ to $\mathrm{CD} 8^{+} \mathrm{T}$ cells. This supports the observation that mice with depleted pDCs, while initially able to regress 
tumours to the baseline, eventually allowed tumours to escape, potentially due to a reduced ability to prime $\mathrm{CD} 8^{+} \mathrm{T}$ cells efficiently. Thus, the reduction in type I IFN may, therefore, reduce initial "burst size" of the response, or have a negative impact on differentiation into memory cells; currently, it is difficult to differentiate the two. Additionally, pDCs have been reported to enhance iNKT cell function via IFN- $\alpha$ and cell to cell CD40-CD40L interactions ${ }^{352}$. This may be a potential explanation for the additivity of $\alpha$-GalCer and $\mathrm{CpG}$, as well as the strong reliance on iNKT cells for the therapy to have an anti-tumour response. 


\subsection{Quality of $\mathrm{T}$ cells and other effector immune cells:}

The most convincing evidence that $\mathrm{CD}^{+} \mathrm{T}$ cells were key effectors were the antibody depletion studies. These demonstrated that they were strongly involved in both the immediate anti-tumour activity in treated tumours and for memory. As $\mathrm{CD}^{+} \mathrm{T}$ cells likely require crosspriming, this data supports the observed loss of activity when cDC1s were depleted. Further, as ablation of pDCs lowered the efficacy of the anti-tumour response, as stated above, it supports the hypothesis that the lowered type 1 IFN levels resulted in a smaller T cell burst size and potentially limited the differentiation of $\mathrm{CD}^{+} \mathrm{T}$ cells into a memory phenotype. Studies in the literature have shown that intratumourally administered $\mathrm{CpG}$ is strongly reliant on a $\mathrm{CD}^{+} \mathrm{T}$ cell

response, with help from B cells and macrophages ${ }^{353}$. Surprisingly, our flow cytometry data saw only mild effects on the $\mathrm{CD}^{+} \mathrm{T}$ cell population; there were some phenotypic changes, with increases in short-lived effector and effector memory $\mathrm{CD} 8^{+} \mathrm{T}$ cells being seen, with an appropriate reduction in naïve $\mathrm{CD}^{+} \mathrm{T}$ cells. However, there were not any dramatic changes in any of the tested organs. A potential reason for this was that the timing of tissue harvest missed the window where we would have observed a large burst in T cell proliferation, processing time may have also been a factor as the cells may die while waiting for each sample to be manually processed, or cells may have also been lost during the CD45.2 selection process and may have been aggregated with $\mathrm{CD} 45.2^{-}$cells. In contrast to the flow data, the preliminary confocal data at a slightly later timepoint showed a promising level of $\mathrm{T}$ cell infiltration, though further investigations are warranted. It would be interesting to see the proportion of T cells to tumour cells and see where they are geographically compared to myeloid populations. Furthermore, looking at the level of B cell infiltration would also be informative. It would also be interesting 
to see if there are tertiary lymphoid structures present in the tumour that can provide an important staging ground for the immune response.

The anti-tumour data also convincingly demonstrates that iNKT cells are not only implicated in the anti-tumour response but are vital for the production of an abscopal effect. In the flow cytometry data, iNKT cells were the only cell type to see consistent changes in the tumour, further implicating them in the anti-tumour response. NK cells are also thought to be involved as iNKT cells are known to transactivate these cells. NK cell activity could be explored by using PK-136 or GM1 blocking antibody to deplete NK cells, or using E4BP4 knockout mice (which are deficient in NK cells) ${ }^{354}$ in the context of a tumour experiment. Mice receiving serum from mice that had been in remission for over 2 weeks (ensuring that cytokine levels had tapered off) were resistant to tumour challenge, implying that antibodies have transferred protection. This data also implies that B cells may be key effectors. $\mathrm{CpG}$ is known to activate B cells to produce antibodies and differentiate into a memory phenotype ${ }^{355,356}$. On the other hand, $\alpha$-GalCer can induce iNKT cells to license B cells to prime cytotoxic T lymphocytes with peptide to generate anti-tumour immunity ${ }^{357}$. iNKT cells can also boost the production of antibodies by interacting with B cells presenting glycolipid antigens on CD1d, though this can also occur in a non-cognate fashion whereby follicular helper $\mathrm{T}$ cells are activated by cDC1s and provide help to B cells ${ }^{355-357}$.

In addition, there was a massive influx of M1-type pro-inflammatory macrophages, as seen in the preliminary confocal data. It is unclear if the macrophages were already present as immunosuppressive TAMs and were repolarised by the treatment or if they were recruited to the tumours as a result of the treatment. In any event, these macrophages can undoubtedly act as additional APCs and, together with effector T cells, potentially cooperate to generate a large 
scale, effective immune responses. Macrophages and other APCs are known to be directly stimulated by $\mathrm{CpG}^{358}$ and can boost $\mathrm{T}$ cell responses via interaction with the TCR and production of cytokines ${ }^{359,360}$. However, a broader panel of antibodies is required to look extensively at changes in the myeloid population with a different timeframe being necessary to see optimal expansion. A potential way of investigating the myeloid population further would be to deplete macrophages using clodronate liposomes and determine how the anti-tumour response is affected. 


\subsection{Treating non-immunogenic tumours:}

The use of immunotherapy to treat cancer over the past decade has revolutionised the way many cancers are treated, especially lung cancer and melanoma, as well as some blood cancers, including leukaemia and lymphoma, drastically improving outcomes for many patients with these diseases, even in the metastatic setting. However, for most common cancers, such as colorectal and breast cancers, immunotherapy has been disappointing. These tumours are considered to be non-immunogenic tumours; for various reasons, these cancers are not recognised and do not provoke a strong response from the immune system, making them difficult to treat with current immunotherapies. The challenge becomes determining how to enhance immunotherapy and activate the immune system to destroy these cancer cells.

This conundrum may also depend on the circumstances surrounding the tumour in question. As the intratumoural combination of $\alpha-\mathrm{GalCer}$ and $\mathrm{CpG}$ requires a $\mathrm{T}$ cell target, low antigenicity or tumours with antigen-presenting problems will not benefit due to a lack of targets. Tumours that have initiated responses that have been constrained by different forms of immunosuppression may benefit from a "boost" via the combination. From this point of view, it makes sense for the therapy to be additive to tumours that express high levels of to PD-1 or PDL1. However, combining the intratumoural combination of $\alpha-\mathrm{GalCer}$ and $\mathrm{CpG}$ with i.p. anti-PD$1 \mathrm{mAb}$ did not show significant additivity in the CT26 colon cancer model, which is known to express a high level of PD-1 on the tumour surface, although there was a positive trend. This suggests that other constraints may be involved such as Tregs, MDSCs, or CTLA-4 mediated T cell suppression. Another scenario where the combination may work is when tumour antigen has been recognised, but the response is insufficient for clearing the tumour due to too few effectors. The use of the combination therapy may help to boost the current response by inducing the 
proliferation of more effectors or recruiting more effectors to help clear the tumour. In addition, the combination therapy may prime new responses in tumours that have antigens that have been entirely ignored by the endogenous immune response.

As noted before, tumours may contain useful but ignored antigens, but they still do not get stimulated. ICD may "expose" these antigens to the immune system in an immunogenic manner, thereby reversing ignorance. The intratumoural vaccine approach using an antigenconjugate vaccine can also be used to induce robust responses to ignored antigens if they are known, as boosting antigen presentation by APCs can increase the visibility of the TAA. In addition, significant vaccine-induced killing may also facilitate "epitope spreading" further enhancing the anti-tumour response. Combining intratumoural vaccination and ICD approaches may have the advantage of stimulating all of these different ways at once.

Inducing ICD to release relevant and immunogenic antigens in situ with tumours that have a non-inflamed, immune cell poor TME is a potential workaround for this issue in the absence of known TAA. Using low dose chemotherapeutics (metronomic dosing) will potentially reduce the traditional toxic side effects of these compounds, notably on the immune system. Furthermore, these ICD agents can potentially be administered intratumourally with $\alpha$ GalCer/CpG to further avoid systemic toxicity and simplify the treatment strategy ${ }^{361}$. In a proof of concept experiment, in chapter 7, doxorubicin was used as an ICD inducing agent at $1 / 10^{\text {th }}$ of the standard efficacious dose used in preclinical tumour models. This supports the hypothesis that making the relevant antigens available at the site of action, whether in the form of exogenously supplied antigens or generation in situ allows the immune system to recognise the tumours. 
The ease of using an ICD agent makes this off-the-shelf treatment strategy simple and readily translatable in the clinic. It would be interesting to see the effectiveness of the treatment if low dose doxorubicin was used throughout the experiment rather than just the beginning so that TAAs are continually generated as tumours develop.

These promising early preclinical results provide a strong rationale for further exploration of $\alpha-\mathrm{GalCer} / \mathrm{CpG}$ combination alongside a third agent that has a complementary, but not overlapping mechanism such that anti-tumour efficacy is maximised while toxicity is limited. 


\subsection{Overall mechanism:}

We can tailor and refine the mechanistic model of the anti-tumour activity of the $\alpha$-GalCer/CpG combination as follows:

1. Tumours must be antigenic, and must have exhibited some limited immunogenicity for activity to work; this may involve some limited influx of immune cells to the tumour, but with these cells having limited effect on tumour growth.

2. Intratumoural $\mathrm{CpG}$ can activate local APCs, notably pDCs which produce type I IFN. Local activation of B cells, macrophages and other myeloid cells may also occur, either via $\mathrm{CpG}$ directly, or through type I IFN. Activated migratory APCs that have acquired tumour antigens drain to the lymph nodes to initiate, or boost, an adaptive response. Some limited CpG may simply leech to the lymphoid tissues with tumour antigens in soluble form, or as dead or dying tumour cells. The injection process itself may account for some extra cell death to induce this process. Antigen arriving in the lymphoid tissues with $\mathrm{CpG}$ by these different routes can also lead to stimulation of an adaptive response. Key APCs involved appear to be cDC1, which have a high propensity for cross-priming of $\mathrm{CD}^{+} \mathrm{T}$ cells, which is reinforced by type I IFN.

3. Local stimulation by $\mathrm{CpG}$ may be sufficient to locally "reinvigorate" activated immune cells that had already been in the tumour, perhaps by relieving immunosuppression through activating local APCs.

4. Because there are few, if any, iNKT cells in the tumour before treatment, the local effect of $\alpha$-GalCer is likely to be weak, although only a few cells may be necessary to interact with local APCs together with CpG to drive an increase in type I IFN. Throughout the treatment, iNKT cells increase in the tumour, which appears to be supported by repeated 
CpG. These cells may contribute to a local inflammatory environment to support the ongoing anti-tumour response.

5. To access the main sources of iNKT cells, some $\alpha$-GalCer must reach the lymphoid tissues, possibly incorporated into dead or dying tumours that enter the blood and make it to the spleen, though some limited $\alpha$-GalCer can make it to the liver too. The arrival of $\alpha$ GalCer and antigen in lymphoid tissues can then lead to activation of lymphoid-resident APCs and drive T cell responses. This process involves $\alpha$-GalCer/CD1d complexes being presented to iNKT cells which produce IFN $\gamma$ and induce maturation of DCs via CD40CD40L binding. Again, key APCs involved appear to be cDC1 (which have a known role in activating iNKT cells to support cross-priming). Importantly, at the initiation of treatment, this whole process could be happening concurrently with the impact of $\mathrm{CpG}$ on APC function as described above.

6. B cells can be stimulated both by $\mathrm{CpG}$ and iNKT cells; where and how this helps tumour-specific antibody production has not been explored in this thesis

7. The enhanced activation of $\mathrm{T}$ cells in lymphoid tissues leads to egress of tumour-specific effector $\mathrm{T}$ cells into peripheral blood and trafficking to tumour tissues

8. Constant restimulation with $\mathrm{CpG}$ sustains $\mathrm{T}$ cell and iNKT cell homing to tumour sites, with tumour recognition and tumour lysis; this benefits not only the impact on the injected tumour but improves responses at distal tumours. The impact on distal tumours is likely a reflection of the overall strength of response (i.e. T cell burst size), but also appears to be facilitated by iNKT cells through an unknown mechanism. Although not explored here, the ongoing tumour killing could potentially lead to the release of more tumour antigen and epitope spreading to broaden the response. 
9. The whole process results in the generation of tumour-specific memory $\mathrm{T}$ cells that can reject rechallenge with tumours.

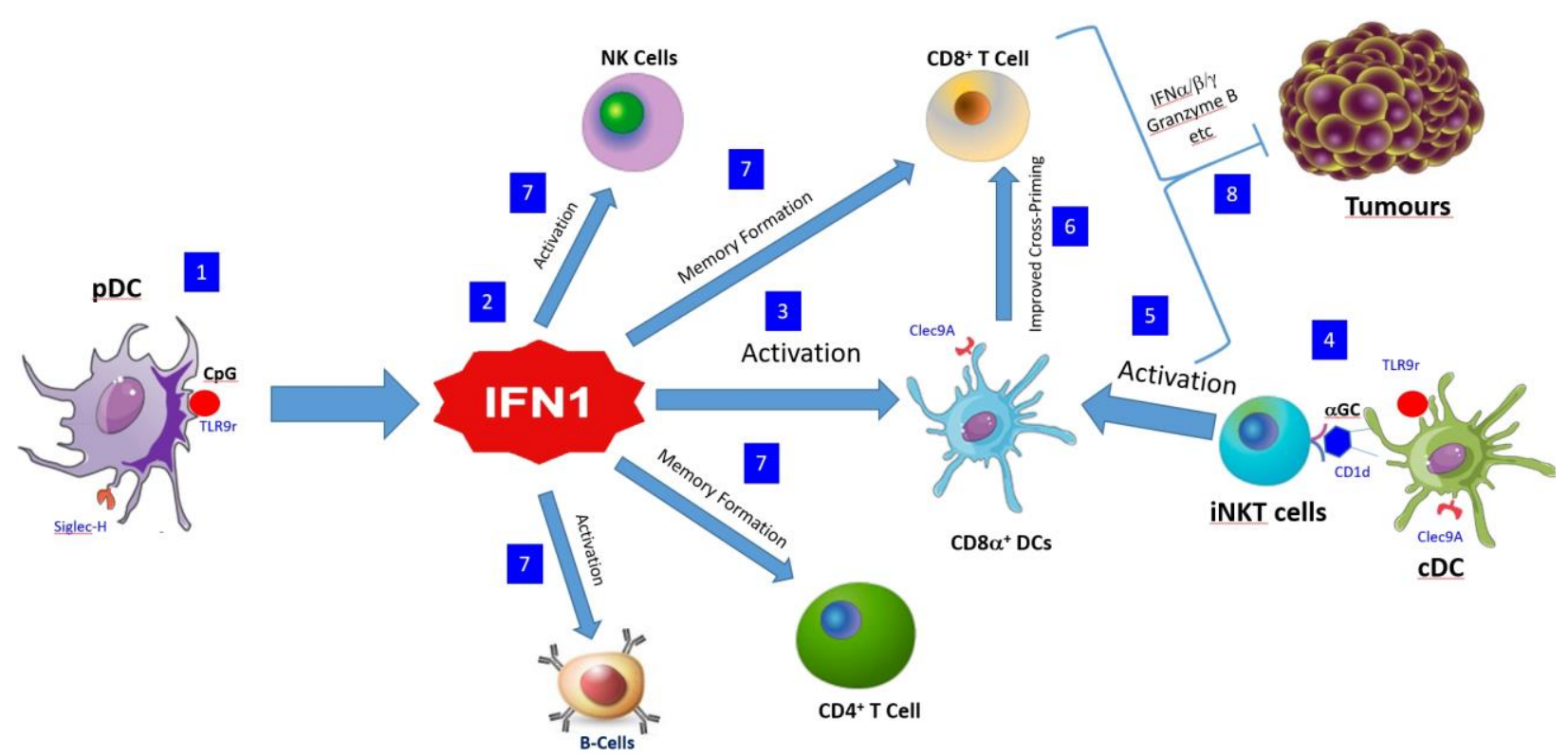

Figure 62 Simplified Mechanistic Model. 1) CpG binds to the TLR9 receptor on pDCs. 2) Type 1 IFN is released by pDCs. 3) Type 1 IFN activates CD8 $\alpha^{+}$DCs. 4) $\alpha$-GalCer binds to CDId receptor and the galactose moiety is presented to iNKT cells leading to their activation and production of IFN- $\gamma$. 5) iNKT cells induce the maturation of CD8 $\alpha^{+}$DCs via CD40-CD40L binding. 6) Improved cross-priming of a $C D 8^{+} T$ cell response occurs as a result of maturation of CD8 $\alpha^{+} D C s$ in concert with type 1 IFN based activation. 7) B cells and other myeloid cells can be stimulated by both CpG, iNKT cells, and type 1 IFN. Type 1 IFN also induces the generation of $C D 8^{+}$and $C D 4^{+} T$ cell memory populations. 8)Improved $C D 8^{+} T$ cell responses along with the release of soluble factors by activated cells leads to tumour cell killing. Tumour cell killing coupled with type 1 IFN mediated memory formation in the $\mathrm{CD}^{+}$and $\mathrm{CD}^{+}$T cell populations results in tumour-specific memory that can reject the rechallenge of the same tumour type.

\subsection{Comparison with closely related approaches:}

There are a couple of recently published reports that are similar to what is described in this thesis. The first used a combination of CpG and anti-OX40 agonist antibody (CD134) to treat lymphoma and other tumour types with the anti-OX40 antibody enhancing the activation of T cells ${ }^{189}$. A salient point of comparison is the syngeneic lymphoma models used, where here EG7.OVA was used in C57BL/6J, and in the published study, A20 was used in BALB/c. The efficacy of both treatments and the starting tumour sizes ( $~ 6 \mathrm{~mm}$ in diameter) are comparable. Much like what has been described here, the combination of $\mathrm{CpG}$ and anti-OX40 was very 
effective in eradicating primary tumours and displayed an abscopal effect with the distal tumours. However, it turned out that in this model injection of $\mathrm{CpG}$ stimulated release of cytokines by local myeloid cells that in turn induced the expression of OX40 on pre-existing intratumoral $\mathrm{CD}^{+} \mathrm{T}$ cells; hence the effective combination of the reagents relies on stimulating these $\mathrm{OX} 40^{+} \mathrm{CD} 4^{+} \mathrm{T}$ cells. It was concluded that the antigen-specific immune response elicited by the intratumoural combination of $\mathrm{CpG}$ and $\mathrm{OX} 40 \mathrm{~L}$ was initiated at the site of the injected tumour. This response was shown to be antigen-specific, and becomes systemic, as shown in an experiment that involved implanting two A20 lymphoma tumours and one CT26 tumour in the same mouse but injecting the agents into one of the A20 lymphoma tumours. They found that regression occurred in both A20 tumours, but not the CT26 tumour. It is possible, in this case, that the whole response was initiated (or boosted) within the tumour, with the $\mathrm{CD} 4^{+} \mathrm{T}$ cells facilitating a response that recirculates to distal tumours. However, no investigation of the involvement of lymphoid tissues was conducted by removing these tissues, as was done here. Through the use of depleting antibodies, they showed that the final effector response involved both $\mathrm{CD}^{+}$and $\mathrm{CD} 8^{+} \mathrm{T}$ cells. TIL analysis of tumours in the early phases of treatment (within 24 h) found that the combination was associated with an increase in myeloid cell infiltration (macrophages), increased activation of CD8 (CD69) and NK (CD137) cells. These early changes in the microenvironment only occurred in the treated tumour and were not reflected in distal tumour sites. They also found that Treg inhibition by OX40L was required and that neutralisation of IL-12, IFN- $\gamma$, and TNF- $\alpha$ prevented CpG induced upregulation of OX40 on T cells. This early response was not investigated in the experiments performed in this thesis, and this should be investigated further. Given that the OX40 stimulation is acting on cells within the tumour, whereas $\alpha$-GalCer likely has to function through the activity of iNKT cells in the 
lymphoid tissues, it is possible that changes in immune profiles in the tumour will not be as immediate. Finally, the $\mathrm{CpG}$ and anti-OX40 anti-tumour response was, as shown here, also dependent on $\mathrm{cDC} 1 \mathrm{~s}$, which may contribute to the $\mathrm{CD}^{+} \mathrm{T}$ cell involvement ${ }^{189}$.

Another similar study was the use of a combination of $\alpha$-GalCer with 4-1BB agonist antibody and E6/E7 peptide in the TC1 model, although this was not intratumoural ${ }^{202}$. The group used a triple combination of $\alpha$-GalCer, 4-1BB agonist antibody and HPV E6/E7 peptides administered intranasally - similar to the triple combination of $\alpha-\mathrm{GalCer} / \mathrm{CpG}$ with HPV E6/E7 intratumourally in chapter 7. It is known that 4-1BB agonists (CD137, TNF receptor superfamily 9) provide potent $\mathrm{T}$ cell co-stimulation, which can restore the effector functions of ligated $\mathrm{T}$ cells 362. While $\mathrm{CpG}$ directly activates APCs, it is known that $\mathrm{CpG}$ also mediates co-stimulation of $\mathrm{T}$ cells through TLR9 ${ }^{363}$, which explains part of the potent adjuvant effects of CpG-ODN in vivo 363. Their combination of 4-1BB, $\alpha$-GalCer and HPV E6/E7 peptides was similarly efficacious as our intratumoural combination of $\alpha-\mathrm{GalCer}$ and $\mathrm{CpG}$ with both combinations producing the desired abscopal effect.

Another study found that intratumoural treatment of CT26 tumours with SD-101, a TLR9 agonist, and anti-PD-1 mAb correlated with increased T- and B-cell infiltration and IFN expression. Furthermore, they found that combining intratumoural injection of SD-101 with antiPD-1 mAb in anti-PD-1 non-responders resulted in the rejection of most injected tumours and a majority of uninjected, distal tumours ${ }^{203}$. In contrast, when we injected $\alpha-G a l C e r$ and $\mathrm{CpG}$ intratumourally, the addition of i.p. anti-PD-1 mAb did not significantly improve anti-tumour responses, though there was a trend. To an extent, this result can be explained by the fact that $\alpha$ GalCer and $\mathrm{CpG}$ already induced improved anti-tumour responses compared to anti-PD-1 as a single agent in CT26 tumours. Thus intratumoural $\alpha$-GalCer and $\mathrm{CpG}$ combination therapy 
appears to induce a similar quality of responses compared to intratumoural SD-101 and i.p. antiPD-1.

Therapeutic efficacy of the combination of SD-101 and anti-PD-1 was also observed with the TSA mammary adenocarcinoma and MCA38 colon carcinoma tumour models that show little response to PD-1 blockade alone. Intratumoural SD-101 substantially increased leukocyte infiltration and IFN-regulated gene expression, and its activity was dependent on $\mathrm{CD}^{+} \mathrm{T}$ cells and type I IFN signalling. Anti-PD-1 mAb with intratumoural SD-101 promoted infiltration of activated, proliferating $\mathrm{CD}^{+} \mathrm{T}$ cells and led to an increase in the total and tumour antigenspecific $\mathrm{CD}^{+} \mathrm{T}$ cells expressing both IFN- $\gamma$ and TNF- $\alpha$. Additionally, PD-1 blockade could alter the SD-101 mediated differentiation of tumour-specific $\mathrm{CD}^{+} \mathrm{T}$ cells into CD127 ${ }^{\text {low }} \mathrm{KLRG} 1^{\text {high }}$ short-lived effector cells, preferentially expanding the CD $127^{\text {high }}$ KLRG $1^{\text {low }}$ long-lived memory precursors. Tumour control and intratumoural T-cell proliferation in the combined treatment was independent of T-cell trafficking from secondary lymphoid organs. These findings in preclinical models suggest that a $\mathrm{CpG}$ oligonucleotide given intratumourally may increase the response of cancer patients to PD-1 blockade in the clinic, increasing the quantity and the quality of tumourspecific $\mathrm{CD}^{+} \mathrm{T}$ cells ${ }^{203}$. Similar to our experiments, they found that $\mathrm{CpG}$ treatment is dependent on $\mathrm{CD}^{+} \mathrm{T}$ cells and type 1 IFN.

The density of endogenously presented antigen-derived peptides on tumour cells is generally sparse, resulting in the inability of antigen-specific CTLs to work effectively ${ }^{364-366}$. Nobuoka et al. found that intratumoural injection of a peptide leads to the loading of additional peptide onto MHC I molecules of tumour cells, which enhanced the recognition of tumour cells by antigen-specific CTLs ${ }^{365}$. Specifically, intratumoural injection of OVA257-264 peptide (SIINFEKL) was sufficient for inducing tumour growth inhibition and survival against OVA- 
negative tumours without adverse reactions. Of note, this antigen-spreading effect was demonstrated to have occurred after the injection of the peptide. Intratumoural peptide injection appears to enhance tumour cell antigenicity and may potentially work by increasing antigen density ${ }^{365}$. This is a potential explanation for how the addition of exogenous antigen increases the anti-tumour response to $\alpha$-GalCer/CpG therapy in that it increases tumour antigen density, leading to improved CTL responses. It is possible that lipidating antigen (in a similar manner as our conjugate vaccines) can protect the peptide and prolong the kinetics of this presentation.

Since ligation of $\mathrm{CpG}$ to APCs produced a large amount of type 1 IFN, it may be logical to bypass the use of $\mathrm{CpG}$ and directly use IFN- $\alpha$. The caveat is that the systemic use of IFN- $\alpha$ is dose-limiting (i.e. cytokine storm). Indeed, a combination of intratumoural injections of mouse IFN- $\alpha$ and intraperitoneal injections of anti-CD137 mAb induced tumour regression in MC38 colon carcinoma tumours, which are resistant to either treatment as a single agent. The therapeutic activity was achieved against both directly injected tumours and distal concomitant tumours. Similar effects of this combination were also seen in subcutaneous TC1 tumours that had been established for 24 days before the start of treatment ${ }^{298}$. However, using IFN- $\alpha$ directly instead of by ligating pDCs, through TLR9, will likely bypass and prevent the recruitment of other cell types. Thus, using CpG instead of direct IFN- $\alpha$ may lead to improved anti-tumour effects because it modulates and recruits a broader range of immune cells through TLR9 agonism, which is upstream from IFN- $\alpha$.

Using these latest published examples as benchmarks, the treatment strategy developed in this thesis compares very favourably with the latest from across the world. It would be interesting to see how these approaches compare side by side in the same laboratory. The limitation of all of these approaches is that some method of introducing or releasing more TAA 
will be required for the treatment of less immunogenic tumours, and if the tumours are not antigenic to start with, these intratumoural therapies are unlikely to work. Intratumoural loading of exogenous peptides onto tumour cells that do not express TAA, but have retained MHC expression, may go some way to dealing with this issue, but further investigation is needed. 


\subsection{Future Directions}

The next logical step is to elucidate further the mechanism by which the combination of $\alpha-\mathrm{GalCer}$ and $\mathrm{CpG}$ are additive. While $\mathrm{CD} 8^{+} \mathrm{T}$ cells are critical for the anti-tumour response, this was hard to demonstrate by flow cytometry. This could have been due to a variety of reasons including the timing of tissue harvesting, quality of the antibodies, and other reasons. Of paramount importance is to get the timing of the flow analysis correct so that we can start looking at the evolving immune response, starting with the early initiating events. Further work is required on examining the influx of immune cells, including $\mathrm{CD} 8^{+} \mathrm{T}$ cells as well as monocytes and macrophages, and their physical relationship to one another, by confocal microscopy.

To further elucidate the mechanisms involved, we could test to see if treatment is affected by IFN- $\gamma$ blockade, as we have previously demonstrated that iNKT and $\mathrm{CD}^{+} \mathrm{T}$ cells are essential to the treatment and one of the primary cytokines used for tumour cell killing is IFN- $\gamma$. If this has a small impact, it may point to other cytokines such as TNF- $\alpha$ having a more significant role. We could also test for the potential role of the STING pathway using STING knockout mice, which has been reported to be activated as DAMPs are released due to cell death. As for other cell types, B cells could be depleted using an anti-CD20 antibody.

It would be interesting to see if the combination treatment produces a response in a metastasis model. In this experiment, B16.OVA tumour would be subcutaneously implanted as the primary tumour. Meanwhile, B16.OVA tumours cells would be injected intravenously into the tail vein to generate tumours in the lung in the same animals, mimicking the extravasion component of lung metastasis. Treatment would occur as usual by only injecting the primary tumour, but the mice would be culled and the lungs examined for metastatic nodules at day 21 
post-tumour inoculation. As tumours typically have a heterogeneous antigen repertoire, another approach would be to determine if the treatment was effective in tumours that spontaneously developed in mice genetically predisposed to cancer such as mammary breast cancer ${ }^{367}$, pancreatic ductal adenocarcinoma and other models ${ }^{368}$.

To help further elucidate where the immune response is initiated, we could look at the different lymphoid organs and tumour much earlier using flow cytometry. It would be interesting to see in which organs the iNKT cells and APCs get initially activated. Furthermore, we could inject fluorescent $\alpha$-GalCer or $\mathrm{CpG}$ and determine where the compounds end up. Another method may be to transfer CFSE-labelled OT-I cells at different times of treating an OVAexpressing tumour. While this was not observed to be a strong rejection antigen, nonetheless it may be possible to use this technique to determine where tumour antigens distribute. The OT-I cells would proliferate when they come into contact with OVA, irrespective of the level of APC activation, although the proliferative response would be sustained if activated APCs are encountered ${ }^{11}$. Thus, the various lymphoid organs and tumour could be harvested at different times to determine which location is the first site of OT-I cell proliferation, and hence the presence of antigen.

Other experiments could be performed to see if the intratumoural combination of $\alpha$ GalCer and CpG could be combined with other therapies that have complementary, but not overlapping mechanisms. A promising combination would be with $\mathrm{OX} 40 \mathrm{~L}$ as $\mathrm{CpG}$ increases the expression of OX40 on T cells. Similarly, as 4-1BB has previously been demonstrated to be complementary to $\alpha$-GalCer, it would also make sense to combine with an agonistic 4-1BB antibody. In a different vein, as tumour cell death can lead to the release of various DAMPs, it would be interesting to see how combining $\alpha$-GalCer with other PRR agonists performs. Given 
that tumours induce angiogenesis as a way of propagating and the subsequent rampant growth of blood vessels becomes convoluted leading to the development of immunosuppressive hypoxic zones it would be logical to see how intratumoural $\alpha$-GalCer/CpG would combine with antiangiogenic agents or agents that were able to reduce hypoxia. In terms of combining with the standard of care, a logical combination would be to see how intratumoural $\alpha$-GalCer and CpG therapy would perform in combination with low-dose radiation (which is known to cause ICD) and with surgical tumour resection (treating residual disease). Along the same line, $\alpha$-GalCer and CpG could be combined with low dose chemotherapy, such as doxorubicin as we have demonstrated above, but in a strategy where low dose doxorubicin is given throughout the treatment.

The one area that could help minimise the risk of going into the clinic will be to explore if this combination has a chance to work in humans by performing tumour studies in humanised CD1d mice which have a similar number of iNKT cells as humans ${ }^{369}$. This is a concern because humans have less iNKT cells than mice, and, therefore, it remains an open question as to whether iNKT cell-mediated maturation of APCs and cross-priming will be as efficient in humans. Encouragingly, recent studies in our laboratory with $\alpha$-GalCer-peptide conjugate vaccines have shown that induced responses are not reduced in these animals (A. Mooney, personal communication, 2020).

As for TLR agonists, both mice and humans express TLRs 1-9 ${ }^{370}$; therefore, the use of TLR9 in humans should not present a problem so long as a human-specific $\mathrm{CpG}$ is used, though it will be interesting to explore the efficacy of other TLRs such as TLR7. Our preliminary data (not shown) suggested that it should work as well and is supported by the literature ${ }^{181}$. 
While intratumoural injections should be feasible for any tumours that can be accessed by the operator, a strategy to make this approach more convenient would be to explore how the combination can be injected less frequently through the use of various formulation and delivery techniques for slow release such as encapsulating nanoparticles to create a longer-lasting depot of the reagents at the tumour ${ }^{371,372}$. Creating a depot effect or having a slow release of the reagents would make the intratumoural combination of $\alpha-\mathrm{GalCer}$ and $\mathrm{CpG}$ a more efficient and clinically translatable therapy. 


\section{References}

1. Riedel S. Edward Jenner and the history of smallpox and vaccination. Baylor Univ Med Cent Proc. 2005;18:21-25.

2. Smith KA. Louis Pasteur, the father of immunology? Front Immunol. 2012;3(APR):1-10. doi:10.3389/fimmu.2012.00068

3. Berinstein, N.L., Spaner D. Therapeutic cancer vaccines. In: Vaccines. 5th ed. Philadelphia: Saunders; 2008:72-88.

4. Sela M, Arnon R, Schechter B. Therapeutic vaccines: Realities of today and hopes for the future. Drug Discov Today. 2002;7(12):664-673. doi:10.1016/S1359-6446(02)02296-1

5. Hollingsworth RE, Jansen K. Turning the corner on therapeutic cancer vaccines. $n p j$ Vaccines. 2019;4(1):1-10. doi:10.1038/s41541-019-0103-y

6. Small EJ, Schellhammer PF, Higano CS, et al. Placebo-controlled phase III trial of immunologic therapy with Sipuleucel-T (APC8015) in patients with metastatic, asymptomatic hormone refractory prostate cancer. J Clin Oncol. 2006;24(19):3089-3094. doi:10.1200/JCO.2005.04.5252

7. Richters MM, Xia H, Campbell KM, Gillanders WE, Griffith OL, Griffith M. Best practices for bioinformatic characterization of neoantigens for clinical utility. Genome Med. 2019;11(1):1-21. doi:10.1186/s13073-019-0666-2

8. Kreiter S, Vormehr M, Van de Roemer N, et al. Mutant MHC class II epitopes drive 
therapeutic immune responses to cancer. Nature. 2015;46(5):1247-1262.

doi:10.1038/nature14426

9. Montico B, Nigro A, Casolaro V, Dal Col J. Immunogenic apoptosis as a novel tool for anticancer vaccine development. Int J Mol Sci. 2018;19(594):1-16.

doi:10.3390/ijms19020594

10. Martin K, Schreiner J, Zippelius A. Modulation of APC function and anti-tumor immunity by anti-cancer drugs. Front Immunol. 2015;6(501):1-7. doi:10.3389/fimmu.2015.00501

11. Osmond TL, Farrand KJ, Painter GF, Ruedl C, Petersen TR, Hermans IF. Activated NKT Cells Can Condition Different Splenic Dendritic Cell Subsets To Respond More Effectively to TLR Engagement and Enhance Cross-Priming. J Immunol. 2015;195(3):821-831. doi:10.4049/jimmunol.1401751

12. Milling L, Zhang Y, Irvine DJ. Delivering safer immunotherapies for cancer. Adv Drug Deliv Rev. 2017;114:79-101. doi:10.1016/j.addr.2017.05.011

13. Hori S, Nomura T, Sakaguchi S. Control of regulatory T cell development by the transcription factor Foxp3. Science (80- ). 2003;299(1057):1057-1061. doi:10.1126/science.1079490

14. Bogen B, Fauskanger M, Haabeth OA, Tveita A. CD4+ T cells indirectly kill tumor cells via induction of cytotoxic macrophages in mouse models. Cancer Immunol Immunother. 2019;68(11):1865-1873. doi:10.1007/s00262-019-02374-0

15. Doorduijn EM, Sluijter M, Salvatori DC, et al. CD4 + T Cell and NK Cell Interplay Key to Regression of MHC Class I low Tumors upon TLR7/8 Agonist Therapy . Cancer 
Immunol Res. 2017;5(8):642-653. doi:10.1158/2326-6066.cir-16-0334

16. Ostroumov D, Fekete-Drimusz N, Saborowski M, Kühnel F, Woller N. CD4 and CD8 T lymphocyte interplay in controlling tumor growth. Cell Mol Life Sci. 2018;75(4):689-713. doi:10.1007/s00018-017-2686-7

17. Sant AJ, McMichael A. Revealing the role of CD4+ T cells in viral immunity. J Exp Med. 2012;209(8):1391-1395. doi:10.1084/jem.20121517

18. Kronenberg M. When Less Is More: T Lymphocyte Populations with Restricted Antigen Receptor Diversity. J Immunol. 2014;193(3):975-976. doi:10.4049/jimmunol.1401491

19. Chinn IK, Blackburn CC, Manley NR, Sempowski GD. Changes in primary lymphoid organs with aging. Semin Immunol. 2012;24(5):309-320. doi:10.1016/j.smim.2012.04.005

20. Witmer MD, Steinman RM. The anatomy of peripheral lymphoid organs with emphasis on accessory cells: Light-microscopic immunocytochemical studies of mouse spleen, lymph node, and peyer's patch. Am J Anat. 1984;170(3):465-481. doi:10.1002/aja.1001700318

21. Fujita T, Matsushita M, Endo Y. The lectin-complement pathway - Its role in innate immunity and evolution. Immunol Rev. 2004;198:185-202. doi:10.1111/j.01052896.2004.0123.x

22. Netea MG, van de Veerdonk FL, van der Meer JWM. Primary immunodeficiencies of pattern recognition receptors. J Intern Med. 2012;272(6):517-527. doi:10.1111/j.13652796.2012.02583.x

23. Bonilla FA, Oettgen HC. Adaptive Immunity. J Allergy Clin Immunol. 2010;1-2(2):20-29. 
doi:10.1016/j.jaci.2009.09.017

24. Kasturi SP, Skountzou I, Albrecht RA, et al. Programming the magnitude and persistence of antibody responses with innate immunity. Nature. 2011;470(7335):543-550. doi:10.1038/nature09737

25. Karakawa WW, Sutton A, Schneerson R, Karpas A, Vann WF. Capsular antibodies induce type-specific phagocytosis of capsulated Staphylococcus aureus by human polymorphonuclear leukocytes. Infect Immun. 1988;56(5):1090-1095.

26. Zhu J, Paul WE. CD4 T cells : fates, functions , and faults. Blood. 2008;112(5):15571569. doi:10.1182/blood-2008-05-078154

27. Mosmann TR, Coffman RL. TH1 and TH2 Cells: Different Patterns of Lymphokine Secretion Lead to Different Functional Properties. Аnпи Rev Immunol. 2003;7(1):145-173. doi:10.1146/annurev.iy.07.040189.001045

28. Kurts C. Cross-presentation: Inducing CD8 T cell immunity and tolerance. J Mol Med. 2000;78(6):326-332. doi:10.1007/s001090000108

29. Lambolez F, Jooss K, Vasseur F, Sarukhan A. Tolerance induction to self antigens by peripheral dendritic cells. Eur J Immunol. 2002;32(9):2588-2597. doi:10.1002/15214141(200209)32:9<2588::AID-IMMU2588>3.0.CO;2-1

30. Curtsinger JM, Schmidt CS, Mondino A, et al. Inflammatory cytokines provide a third signal for activation of naive CD4+ and CD8+ T cells. J Immunol. 1999;162(6):32563262. http://www.ncbi.nlm.nih.gov/pubmed/10092777.

31. Curtsinger JM, Valenzuela JO, Agarwal P, Lins D, Mescher MF. Type I IFNs provide a 
third signal to CD8 $\mathrm{T}$ cells to stimulate clonal expansion and differentiation. J Immunol. 2005;174(8):4465-4469. doi:10.4049/jimmunol.174.8.4465

32. Chouaib S, Chehimi J, Bani L, et al. Interleukin 12 induces the differentiation of major histocompatibility complex class I-primed cytotoxic T-lymphocyte precursors into allospecific cytotoxic effectors. Proc Natl Acad Sci. 2006;91(26):12659-12663. doi:10.1073/pnas.91.26.12659

33. BLUM A, MILLER H. The Major Histocompatibility Complex and Inflammation. South Med J. 2000;93(2):169-172. doi:10.1097/00007611-200002000-00002

34. Hewitt EW. The MHC class I antigen presentation pathway: strategies for viral immune evasion. Immunology. 2003;(110):163-169. doi:10.1046/j.1365-2567.2003.01738.x

35. Aderem A, Underhill DM. Mechanisms of Phagocytosis in Macrophages. Annu Rev Immunol. 2002;17(1):593-623. doi:10.1146/annurev.immunol.17.1.593

36. Cruz FM, Colbert JD, Merino E, Kriegsman BA, Rock KL. The Biology and Underlying Mechanisms of Cross-Presentation of Exogenous Antigens on MHC-I Molecules. Annu Rev Immunol. 2017;35(1):149-176. doi:10.1146/annurev-immunol-041015-055254

37. Trombetta ES, Mellman I. Cell Biology of Antigen Processing in Vitro and in Vivo. Annu Rev Immunol. 2005;23(1):975-1028. doi:10.1146/annurev.immunol.22.012703.104538

38. Banchereau J, Steinman RM. Dendritic cells and the control of immunology. Nature. 1998;392(March):245-252. doi:10.1038/32588

39. Mackey MF, Barth RJ, Noelle RJ. The role of CD40/CD154 interactions in the priming, differentiation, and effector function of helper and cytotoxic T cells. J Leukoc Biol. 
1998;63(4):418-428. doi:10.1002/jlb.63.4.418

40. Ma DY, Clark EA. The role of CD40 and CD154/CD40L in dendritic cells. Semin Immunol. 2009;21(5):265-272. doi:10.1016/j.smim.2009.05.010

41. Hamza T, Barnett JB, Li B. Interleukin 12 a key immunoregulatory cytokine in infection applications. Int J Mol Sci. 2010;11(3):789-806. doi:10.3390/ijms11030789

42. Nair S, Dhodapkar M V. Natural killer T cells in cancer immunotherapy. Front Immunol. 2017;8(1178):1-18. doi:10.3389/fimmu.2017.01178

43. Chaplin DD. Overview of the immune response. J Allergy Clin Immunol. 2010;125(2 SUPPL. 2):S3-S23. doi:10.1016/j.jaci.2009.12.980

44. Albert ML, Sauter B, Bhardwaj N. Dendritic cells acquire antigen from apoptotic cells and induce class I-restricted CTLs. Nature. 1998;392(March):86-89. doi:10.1038/32183

45. Huang F-P, Platt N, Wykes M, et al. A Discrete Subpopulation of Dendritic Cells Transports Apoptotic Intestinal Epithelial Cells to T Cell Areas of Mesenteric Lymph Nodes. J Exp Med. 2002;191(3):435-444. doi:10.1084/jem.191.3.435

46. Kono H, Rock KL. How dying cells alert the immune system to danger. Nat Rev Immunol. 2008;8(4):279-289. doi:10.1038/nri2215

47. Cannon GJ, Swanson JA. The macrophage capacity for phagocytosis. J Cell Sci. 1992;101 ( Pt 4:907-913. http://www.ncbi.nlm.nih.gov/pubmed/1527185.

48. Inaba K, Witmer-Pack M, Inaba M, et al. The Tissue Distribution of the B7-2 Costimulator in Mice: Abundant Expression on Dendritic Cells In Situ and During Maturation In Vitro. J Exp Med. 1994;180(November):1128-1131. 
doi:10.1084/jem.180.5.1849

49. Uanue ER. Antigen-presenting function of the macrophage. Annu Rev Immunol. 1984;2:395-428. doi:10.1146/annurev.iy.02.040184.002143

50. Watts C. Capture and Processing of Exogenous Antigens for Presentation on Mhc Molecules. Аnпu Rev Immunol. 2002;15(1):821-850.

doi:10.1146/annurev.immunol.15.1.821

51. Denzin LK, Cresswell P. HLA-DM induces clip dissociation from MHC class II $\alpha \beta$ dimers and facilitates peptide loading. Cell. 1995;82(1):155-165. doi:10.1016/00928674(95)90061-6

52. Kropshofer H, Vogt AB, Moldenhauer G, Hammer J, Blum JS, Hämmerling GJ. Editing of the HLA-DR-peptide repertoire by HLA-DM. EMBO J. 1996;15(22):6144-6154. http://www.ncbi.nlm.nih.gov/pubmed/8947036\%0Ahttp://www.pubmedcentral.nih.gov/art iclerender.fcgi?artid=PMC452435.

53. Chow A, Toomre D, Garrett W, Mellman I. Dendritic cell maturation triggers retrograde MHC class II transport from lysosomes to the plasma membrane. Nature. 2002;418(6901):988-994. doi:10.1038/nature01006

54. Wubbolts R, Fernandez-Borja M, Oomen L, et al. Direct vesicular transport of MHC class II molecules from lysosomal structures to the cell surface. J Cell Biol. 1996;135(3):611622. doi:10.1083/jcb.135.3.611

55. Heath WR, Carbone FR, Walter T, Hall E, Royal PO. Cross-presentation in viral immunity and self-tolerance. Nat Rev Immunol. 2001;1(November):126-135. 
doi:10.1038/35100512

56. Neefjes J, Jongsma MLM, Paul P, Bakke O. Towards a systems understanding of MHC class i and MHC class II antigen presentation. Nat Rev Immunol. 2011;11(12):823-836. doi:10.1038/nri3084

57. Serwold T, Gonzalez F, Kim J, Jacob R, Shastri N. ERAAP customizes peptides for MHC class I molecules in the endoplasmic reticulum. Nature. 2002;419(6906):480-483. doi:10.1038/nature01074

58. Shastri N, Nagarajan N, Lind KC, Kanaseki T. Monitoring peptide processing for MHC class I molecules in the endoplasmic reticulum. Curr Opin Immunol. 2014;0:123-127. doi:10.1016/j.coi.2013.11.006

59. Kagi D, Ledermanno B, Burkin K, Hengartner H, Zinkernagel RM. CD8+ T cell-mediated protection against an intracellular bacterium by perforin-dependent cytotoxicity. Eur $J$ Immunol. 1994;24:3068-3072.

60. Gulzar N, Copeland KFT. CD8+ T-cells: function and response to HIV infection. Curr HIV Res. 2004;2(1):23-37. http://www.ncbi.nlm.nih.gov/pubmed/15053338.

61. Thomas C, Tampé R. Proofreading of peptide-MHC complexes through dynamic multivalent interactions. Front Immunol. 2017;8(65):1-10. doi:10.3389/fimmu.2017.00065

62. Thomas C, Tampé R. Structure of the TAPBPR-MHC I complex defines the mechanism of peptide loading and editing. Science (80- ). 2017;358(6366):1060-1064. doi:10.1126/science.aao6001 
63. Rock KL, Shen L. Cross-presentation: underlying mechanisms and role in immune surveillance. Immunol Rev. 2005;207:166-183. doi:10.1111/j.0105-2896.2005.00301.x

64. Hughes EA, Hammond C, Cresswell P. Misfolded major histocompatibility complex class I heavy chains are translocated into the cytoplasm and degraded by the proteasome. Proc Natl Acad Sci U S A. 1997;94(5):1896-1901.

http://www.ncbi.nlm.nih.gov/pubmed/9050876\%0Ahttp://www.pubmedcentral.nih.gov/art iclerender.fcgi?artid=PMC20014.

65. Koopmann JO, Hu E, Bulbuc N, et al. Export of antigenic peptides from the endoplasmic reticulum intersects with retrograde protein translocation through the Sec61p channel. Immunity. 2000;13(1):117-127. http://www.ncbi.nlm.nih.gov/pubmed/10933400.

66. Bevan MJ. Cross-priming for a secondary cytotoxic response to minor $\mathrm{H}$ antigens with $\mathrm{H}-$ 2 congenic cells which do not cross-react in the cytotoxic assay. J Exp Med. 1976;143:1283-1288. http://hydra.icgeb.trieste.it/library/Group-Publications/PAPERS PDF ONLY/22.pdf.

67. Bevan MJ. Minor $\mathrm{H}$ antigens introduced on $\mathrm{H}-2$ different stimulating cells cross-react at the cytotoxic T cell level during in vivo priming. J Immunol. 1976;117(6):2233-2238. http://www.ncbi.nlm.nih.gov/pubmed/825578. Accessed July 4, 2019.

68. Mantegazza AR, Magalhaes JG, Amigorena S, Marks MS. Presentation of Phagocytosed Antigens by MHC Class I and II. Traffic. 2013;14(2):135-152. doi:10.1111/tra.12026

69. Shen L, Sigal LJ, Boes M, Rock KL. Important role of cathepsin S in generating peptides for TAP-independent MHC class I crosspresentation in vivo. Immunity. 2004;21:155-165. doi:10.1016/j.immuni.2004.07.004 
70. Hashimoto D, Miller J, Merad M. Dendritic Cell and Macrophage Heterogeneity In Vivo. Immunity. 2011;35(3):323-335. doi:10.1016/j.immuni.2011.09.007

71. Liu K, Nussenzweig MC. Origin and development of dendritic cells. Immunol Rev. 2010;234:45-54. doi:10.1111/j.0105-2896.2009.00879.x

72. Sallusto F, Lanzavecchia A. Efficient Presentation of Soluble Antigen by Cultured Human Dendritic Cells Is Maintained by Granulocyte/Macrophage Colony-stimulating Factor Plus Iuterleukin 4 and Downregulated by Tumor Necrosis Factor alpha. $J$ Exp Med. 1994;179(April):1109-1118.

73. Lim SN, Kuhn S, Hyde E, Ronchese F. Combined TLR Stimulation With Pam3Cys and Poly I. J Immunother. 2012;35(9):670-679. doi:10.1097/cji.0b013e318270e135

74. Schimmelpfennig $\mathrm{CH}$, Schulz S, Arber C, et al. Ex vivo expanded dendritic cells home to T-cell zones of lymphoid organs and survive in vivo after allogeneic bone marrow transplantation. Am J Pathol. 2005;167(5):1321-1331. doi:10.1016/S00029440(10)61219-9

75. Yamana T, Kitajima K. Standardized generation of fully mature p70 IL-12 secreting monocyte-derived dendritic cells for clinical use. Cancer Immunol Immunother. 2001;50(8):417-427. doi:10.1007/s002620100215

76. Ohradanova-Repic A, Machacek C, Fischer MB, Stockinger H. Differentiation of human monocytes and derived subsets of macrophages and dendritic cells by the HLDA10 monoclonal antibody panel. Clin Transl Immunol. 2016;5(1):e55-9.

doi:10.1038/cti.2015.39 
77. Tel J, Van Der Leun AM, Figdor CG, Torensma R, De Vries IJM. Harnessing human plasmacytoid dendritic cells as professional APCs. Cancer Immunol Immunother. 2012;61(8):1279-1288. doi:10.1007/s00262-012-1210-z

78. Zhang J, Raper A, Sugita N, et al. Characterization of Siglec-H as a novel endocytic receptor expressed on murine plasmacytoid dendritic cell precursors. Blood. 2006;107(9):3600-3608. doi:10.1182/blood-2005-09-3842

79. Asselin-Paturel C, Brizard G, Chemin K, et al. Type I interferon dependence of plasmacytoid dendritic cell activation and migration. J Exp Med. 2005;201(7):1157-1167. doi:10.1084/jem.20041930

80. Kwissa M, Nakaya HI, Oluoch H, Pulendran B. Distinct TLR adjuvants differentially stimulate systemic and local innate immune responses in nonhuman primates. Blood. 2012;119(9):2044-2055. doi:10.1182/blood-2011-10-388579

81. Guilliams M, Ginhoux F, Jakubzick C, et al. Dendritic cells, monocytes and macrophages: A unified nomenclature based on ontogeny. Nat Rev Immunol. 2014;14(8):571-578. doi:10.1038/nri3712

82. Steinman RM, Kaplan G, Witmer MD, Cohn ZA. Identification of a novel cell type in peripheral lymphoid organs of mice V. Purification of spleen dendritic cells, new surface markers, and maintenance in vitro. $J$ Exp Med. 1979;149:1-16. doi:10.1084/jem.149.1.1

83. Metlay JP, Witmer-pack MD, Agger R, Crowley MT, Lawless D, Steinman RM. The distinct leukocyte integrins of mouse spleen dendritic cells as identified with new hamster monoclonal antibodies. J Exp Med. 1990;171(May):1753-1771.

doi:10.1084/jem.171.5.1753 
84. Guilliams M, Dutertre CA, Scott CL, et al. Unsupervised High-Dimensional Analysis Aligns Dendritic Cells across Tissues and Species. Immunity. 2016;45(3):669-684. doi:10.1016/j.immuni.2016.08.015

85. Blasius AL, Giurisato E, Cella M, Schreiber RD, Shaw AS, Colonna M. Bone Marrow Stromal Cell Antigen 2 Is a Specific Marker of Type I IFN-Producing Cells in the Naive Mouse, but a Promiscuous Cell Surface Antigen following IFN Stimulation. J Immunol. 2006;177(5):3260-3265. doi:10.4049/jimmunol.177.5.3260

86. Tussiwand R, Lee WL, Murphy TL, et al. Compensatory dendritic cell development mediated by BATF-IRF interactions. Nature. 2012;490(7421):502-507. doi:10.1038/nature11531

87. Lewis KL, Caton ML, Bogunovic M, et al. Notch2 receptor signaling controls functional differentiation of dendritic cells in the spleen and intestine. Immunity. 2011;35(5):780791. doi:10.1016/j.immuni.2011.08.013

88. Satpathy AT, Briseño CG, Lee JS, et al. Notch2-dependent classical dendritic cells orchestrate intestinal immunity against attaching and effacing bacterial pathogens Ansuman. Nat Immunol. 2013;14(9):937-948. doi:10.1038/ni.2679.Notch2-dependent

89. Linsley PS, Brady W, Grosmaire L, Aruffo A, Damle NK, Ledbetter JA. Binding of the B cell activation antigen $\mathrm{B} 7$ to CD28 costimulates T cell proliferation and interleukin 2 mRNA accumulation. $J$ Exp Med. 1991;173(3):721-730.

http://www.ncbi.nlm.nih.gov/pubmed/1847722\%0Ahttp://www.pubmedcentral.nih.gov/art iclerender.fcgi?artid=PMC2118836.

90. Gross JA, Callas E, Allison JP. Identification and distribution of the costimulatory 
receptor CD28 in the mouse. J Immunol. 1992;149(2):380-388.

http://www.ncbi.nlm.nih.gov/pubmed/1320641. Accessed July 4, 2019.

91. Caux BC, Massacrier C, Vanbervliet B, et al. Activation of Human Dendritic Cells through CD40 Cross-linking. J Exp Med. 1994;180:1263-1272.

doi:10.1084/jem.180.4.1263

92. Manetti R. Interleukin 12 induces stable priming for interferon gamma (IFN-gamma) production during differentiation of human T helper (Th) cells and transient IFN-gamma production in established Th2 cell clones. J Exp Med. 2004;179(4):1273-1283. doi:10.1084/jem.179.4.1273

93. Flynn JL, Chan J, Triebold KJ, Dalton DK, Stewart TA, Bloom BR. An essential role for interferon gamma in resistance to Mycobacterium tuberculosis infection. J Exp Med. 2004;178(6):2249-2254. doi:10.1084/jem.178.6.2249

94. Zhang D, Beresford PJ, Greenberg AH, Lieberman J. Granzymes A and B directly cleave lamins and disrupt the nuclear lamina during granule-mediated cytolysis. Proc Natl Acad Sci. 2001;98(10):5746-5751. doi:10.1073/pnas.101329598

95. Darmon AJ, Ley TJ, Nicholson DW, Bleackley RC. Cleavage of CPP32 by granzyme B represents a critical role for granzyme B in the induction of target cell DNA fragmentation. J Biol Chem. 1996;271(36):21709-21712. doi:10.1074/jbc.271.36.21709

96. Medema JP, Toes REM, Scaffidi C, et al. Cleavage of FLICE (caspase-8) by granzyme B during cytotoxic T lymphocyte-induced apoptosis. Eur J Immunol. 1997;27(12):34923498. doi:10.1002/eji.1830271250 
97. Regner M, Müllbacher A. Granzymes in cytolytic lymphocytes - To kill a killer? Immunol Cell Biol. 2004;82(2):161-169. doi:10.1046/j.0818-9641.2004.01225.x

98. Nakajima H, Henkart PA. Cytotoxic lymphocyte granzymes trigger a target cell internal disintegration pathway leading to cytolysis and DNA breakdown. J Immunol. 1994;152(3):1057-1063. http://www.ncbi.nlm.nih.gov/pubmed/7507956. Accessed July 4, 2019.

99. Cao W, Tykodi SS, Esser MT, Braciale VL, Braciale TJ. Partial activation of CD8+ T cells by a self-derived peptide. Nature. 1995;378(6554):295-298. doi:10.1038/378295a0

100. Dianzani U, Chiocchetti A, Ramenghi U. Role of inherited defects decreasing Fas function in autoimmunity. Life Sci. 2003;72(25):2803-2824. doi:10.1016/S00243205(03)00196-6

101. Xu X, Fu XY, Plate J, Chong ASF. IFN- $\gamma$ induces cell growth inhibition by fas-mediated apoptosis: Requirement of STAT1 protein for up-regulation of Fas and FasL expression. Cancer Res. 1998;58(13):2832-2837.

102. Liao T, Kaufmann AM, Qian X, et al. Susceptibility to cytotoxic T cell lysis of cancer stem cells derived from cervical and head and neck tumor cell lines. J Cancer Res Clin Oncol. 2013;139(1):159-170. doi:10.1007/s00432-012-1311-2

103. Schlaepfer E, Speck RF. Anti-HIV activity mediated by natural killer and CD8+ cells after toll-like receptor 7/8 triggering. PLoS One. 2008;3(4):1-13. doi:10.1371/journal.pone.0001999

104. Andersen BM, Ohlfest JR. Increasing the efficacy of tumor cell vaccines by enhancing 
cross priming. Cancer Lett. 2012;325(2):155-164. doi:10.1016/j.canlet.2012.07.012.

105. Jiménez-Dalmaroni MJ, Gerswhin ME, Adamopoulos IE. The critical role of toll-like receptors - From microbial recognition to autoimmunity: A comprehensive review. Autoimmun Rev. 2016;15(1):1-8. doi:10.1016/j.autrev.2015.08.009

106. O’Neill LAJ, Golenbock D, Bowie AG. The history of Toll-like receptors-redefining innate immunity. Nat Rev Immunol. 2013;13(6):453-460. doi:10.1038/nri3446

107. 'Mac' Cheever MA. Twelve immunotherapy drugs that could cure cancers. Immunol Rev. 2008;222(1):357-368. doi:10.1111/j.1600-065X.2008.00604.x

108. Vacchelli E, Galluzzi L, Eggermont A, et al. Trial watch: FDA-approved toll-like receptor agonists for cancer therapy. Oncoimmunology. 2012;1(6):894-907. doi:10.4161/onci.20931

109. Bagchi A, Herrup EA, Warren HS, et al. MyD88-Dependent and MyD88-Independent Pathways in Synergy, Priming, and Tolerance between TLR Agonists. J Immunol. 2007;178(2):1164-1171. doi:10.4049/jimmunol.178.2.1164

110. Sun L, Jiang Q, Zhang Y, Liang H, Ren H, Zhang D. Toll-like receptors and breast cancer. Integr Cancer Sci Ther. 2016;3(2):432-436. doi:10.15761/icst.1000183

111. Kawai T, Akira S. Signaling to NF-אB by Toll-like receptors. Trends Mol Med. 2007;13(11):460-469. doi:10.1016/j.molmed.2007.09.002

112. Behar SM, Porcelli SA. CD1-restricted T cells in host defense to infectious diseases. Curr Top Microbiol Immunol. 2007;314:215-250. doi:10.1007/978-3-540-69511-0-9

113. Crowe NY, Coquet JM, Berzins SP, et al. Differential antitumor immunity mediated by 
NKT cell subsets in vivo. $J$ Exp Med. 2005;202(9):1279-1288. doi:10.1084/jem.20050953

114. Zajonc DM, Girardi E. Recognition of microbial glycolipids by Natural Killer T cells. Front Immunol. 2015;6(JUL):1-11. doi:10.3389/fimmu.2015.00400

115. Nandakumar S, Woolard SN, Yuan D, Rouse BT, Kumaraguru U. Natural Killer Cells as Novel Helpers in Anti-Herpes Simplex Virus Immune Response. J Virol. 2008;82(21):10820-10831. doi:10.1128/jvi.00365-08

116. Wang T, Gao Y, Scully E, et al. Gamma Delta T Cells Facilitate Adaptive Immunity against West Nile Virus Infection in Mice. J Immunol. 2014;177(3):1825-1832. doi:10.4049/jimmunol.177.3.1825

117. Fujii SI, Shimizu K, Hemmi H, Steinman RM. Innate Va14+ natural killer T cells mature dendritic cells, leading to strong adaptive immunity. Immunol Rev. 2007;220(1):183-198. doi:10.1111/j.1600-065X.2007.00561.x

118. Bricard G, Porcelli SA. Antigen presentation by CD1 molecules and the generation of lipid-specific T cell immunity. Cell Mol Life Sci. 2007;64(14):1824-1840. doi:10.1007/s00018-007-7007-0

119. Kawano T, Cui J, Koezuka Y, et al. CDl d-Restricted and TCR-Mediated Activation of Val 4 NKT Cells by Glycosylceramides. Science (80- ). 1997;278(14):12-15.

120. Taniguchi M, Harada M, Kojo S, Nakayama T, Wakao H. The regulatory role of Valpha14 NKT cells in innate and acquired immune response. Annи Rev Immunol. 2003;21(1):483-513. doi:10.1146/annurev.immunol.21.120601.141057

121. Bendelac A, Savage PB, Teyton L. The Biology of NKT Cells. Annu Rev Immunol. 
2007;25(1):297-336. doi:10.1146/annurev.immunol.25.022106.141711

122. Kitamura H, Iwakabe K, Yahata T, et al. The natural killer T (NKT) cell ligand alphagalactosylceramide demonstrates its immunopotentiating effect by inducing interleukin (IL)-12 production by dendritic cells and IL-12 receptor expression on NKT cells. $J$ Exp Med. 1999;189(7):1121-1128.

123. Yoshimoto T, Bendelac A, Watson C, Hu-li J, Paul WE. Role of NKI .I T Cells in a T,2 Response and in immunoglobulin E Production. Sci Rep. 1995;270(December):18451847.

124. Kawano T, Cui J, Koezuka Y, et al. Natural killer-like nonspecific tumor cell lysis mediated by specific ligand-activated Va14 NKT cells. Proc Natl Acad Sci. 1998;95(10):5690-5693. doi:10.1073/pnas.95.10.5690

125. Slauenwhite D, Johnston B. Regulation of NKT cell localization in homeostasis and infection. Front Immunol. 2015;6(255):1-19. doi:10.3389/fimmu.2015.00255

126. Bendelac A, Rivera MN, Park S-H, Roark JH. Mouse CD1-specific NK1 T cells: development, specificity, and function. Anпи Rev Immunol. 1997;15(1):535-562. doi:10.1146/annurev.immunol.15.1.535

127. Im JS, Kang TJ, Lee SB, et al. Alteration of the relative levels of iNKT cell subsets is associated with chronic mycobacterial infections. Clin Immunol. 2008;127(2):214-224. doi:10.1016/j.clim.2007.12.005

128. Artiaga BL, Whitener RL, Staples CR, Driver JP. Adjuvant effects of therapeutic glycolipids administered to a cohort of NKT cell-diverse pigs. Vet Immunol 
Immunopathol. 2014;162(1-2):1-13. doi:10.1016/j.vetimm.2014.09.006

129. Im JS, Yu KOA, Illarionov PA, et al. Direct Measurement of Antigen Binding Properties of CD1 Proteins Using Fluorescent Lipid Probes. J Biol Chem. 2004;279(1):299-310. doi:10.1074/jbc.M308803200

130. Sidobre S, Naidenko O V., Sim B-C, Gascoigne NRJ, Garcia KC, Kronenberg M. The V 14 NKT Cell TCR Exhibits High-Affinity Binding to a Glycolipid/CD1d Complex. $J$ Immunol. 2002;169(3):1340-1348. doi:10.4049/jimmunol.169.3.1340

131. Yu KKQ, Wilburn DB, Hackney JA, et al. Conservation of molecular and cellular phenotypes of invariant NKT cells between humans and non-human primates. Immunogenetics. 2019;71(7):465-478. doi:10.1007/s00251-019-01118-9

132. Godfrey DI, Le Nours J, Andrews DM, Uldrich AP, Rossjohn J. Unconventional T Cell Targets for Cancer Immunotherapy. Immunity. 2018;48(3):453-473. doi:10.1016/j.immuni.2018.03.009

133. Ishikawa A, Motohashi S, Ishikawa E, et al. A phase I study of alpha-galactosylceramide (KRN7000)-pulsed dendritic cells in patients with advanced and recurrent non-small cell lung cancer. Clin Cancer Res. 2005;11(5):1910-1917. http://ovidsp.ovid.com/ovidweb.cgi?T=JS\&PAGE=reference\&D=emed7\&NEWS=N\&AN $=2005157427$.

134. Koch M, Stronge VS, Shepherd D, et al. The crystal structure of human CD1d with and without $\alpha$-galactosylceramide. Nat Immunol. 2005;6(8):819-826. doi:10.1038/ni1225

135. Mattarollo SR, West AC, Steegh K, et al. NKT cell adjuvant-based tumor vaccine for 
treatment of myc oncogene-driven mouse B-cell lymphoma. Blood. 2012;120(15):30193029. doi:10.1182/blood-2012-04-426643

136. Speir M, Authier-Hall A, Brooks CR, et al. Glycolipid-peptide conjugate vaccines enhance CD8+ T cell responses against human viral proteins. Sci Rep. 2017;7(1):1-12. doi:10.1038/s41598-017-14690-5

137. Gasser O, Sharples KJ, Barrow C, et al. A phase I vaccination study with dendritic cells loaded with NY-ESO-1 and $\alpha$-galactosylceramide: induction of polyfunctional T cells in high-risk melanoma patients. Cancer Immunol Immunother. 2018;67(2):285-298. doi:10.1007/s00262-017-2085-9

138. Anderson RJ, Compton BJ, Tang CW, et al. NKT cell-dependent glycolipid-peptide vaccines with potent anti-tumour activity. Chem Sci Harnessing NKT cells to Improv situ vaccination_KPedit-14.docx. 2015;6(9):5120-5127.doi:10.1039/c4sc03599b

139. Nakagawa R, Serizawa I, Motoki K, et al. Antitumor activity of alpha-galactosylceramide, KRN7000, in mice with the melanoma B16 hepatic metastasis and immunohistological study of tumor infiltrating cells. Oncol Res. 2000;12(2):51-58. http://www.ncbi.nlm.nih.gov/pubmed/11132924. Accessed September 3, 2019.

140. Nakagawa R, Motoki K, Ueno H, et al. Treatment of hepatic metastasis of the Colon26 adenocarcinoma with an $\alpha$-Galactosylceramide, KRN7000. Cancer Res. 1998;58(6):12021207.

141. Fujii S, Shimizu K, Smith C, Bonifaz L, Steinman RM. Activation of Natural Killer T Cells by $\alpha$-Galactosylceramide Rapidly Induces the Full Maturation of Dendritic Cells In Vivo and Thereby Acts as an Adjuvant for Combined CD4 and CD8 T Cell Immunity to a 
Coadministered Protein. J Exp Med. 2003;198(2):267-279. doi:10.1084/jem.20030324

142. Hermans IF, Silk JD, Gileadi U, et al. NKT Cells Enhance CD4 + and CD8 + T Cell Responses to Soluble Antigen In Vivo through Direct Interaction with Dendritic Cells . $J$ Immunol. 2003;171(10):5140-5147. doi:10.4049/jimmunol.171.10.5140

143. Stober D, Jomantaite I, Schirmbeck R, Reimann J. NKT Cells Provide Help for Dendritic Cell-Dependent Priming of MHC Class I-Restricted CD8+ T Cells In Vivo. J Immunol. 2003;170(5):2540-2548. doi:10.4049/jimmunol.170.5.2540

144. Fujii SI, Liu K, Smith C, Bonito AJ, Steinman RM. The linkage of innate to adaptive immunity via maturing dendritic cells in vivo requires CD40 ligation in addition to antigen presentation and CD80/86 costimulation. J Exp Med. 2004;199(12):1607-1618. doi:10.1084/jem.20040317

145. Hayakawa Y, Takeda K, Yagita H, et al. IFN- $\gamma$-mediated inhibition of tumor angiogenesis by natural killer T-cell ligand, $\alpha$-galactosylceramide. Blood. 2002;100(5):1728-1733. doi:10.1182/BLOOD.V100.5.1728.H81702001728_1728_1733

146. Smyth MJ, Wallace ME, Nutt SL, Yagita H, Godfrey DI, Hayakawa Y. Sequential activation of NKT cells and NK cells provides effective innate immunotherapy of cancer. J Exp Med. 2005;201(12):1973-1985. doi:10.1084/jem.20042280

147. Kobayashi E, Motoki K, Uchida T, Fukushima H, Koezuka Y. KRN7000, a novel immunomodulator, and its antitumor activities. Oncol Res. 1995;7(10-11):529-534.

148. Motoki K, Maeda K, Ueno H, et al. Antitumor activities of combined treatment with a novel immunomodulator, (2S,3S,4R)-1-O-(alpha-D-Galactopyranosyl)-2-(N- 
Hexacosanoylamino)-1,3,4 - octadecanetriol (KRN7000), and radiotherapy in tumorbearing mice. Oncol Res. 1996;8(4):155-162.

http://www.ncbi.nlm.nih.gov/pubmed/8869965. Accessed March 27, 2020.

149. Galli G, Nuti S, Tavarini S, et al. CD1d-restricted Help To B Cells By Human Invariant Natural Killer T Lymphocytes. J Exp Med. 2003;197(8):1051-1057. doi:10.1084/jem.20021616

150. Lang GA, Devera TS, Lang ML. Requirement for CD1d expression by B cells to stimulate NKT cell - enhanced antibody production. Blood. 2008;111(4):2158-2163. doi:10.1182/blood-2007-10- 117309.

151. Carreño LJ, Kharkwal SS, Porcelli SA. Optimizing NKT cell ligands as vaccine adjuvants. Immunotherapy. 2014;6(3):309-320. doi:10.2217/imt.13.175

152. Carreño LJ, Saavedra-Ávila NA, Porcelli SA. Synthetic glycolipid activators of natural killer T cells as immunotherapeutic agents. Clin Transl Immunol. 2016;5(4):1-9. doi:10.1038/cti.2016.14

153. Waldowska M, WaldoWska M, Bojarska-junak A, Roliński J. A brief review of clinical trials involving manipulation of invariant NKT cells as a promising approach in future cancer therapies. Cent Eur J Immunol. 2017;42(2):181-195. doi:10.5114/ceji.2017.69361

154. Chang DH, Osman K, Kukreja A, et al. Sustained expansion of NKT cells and antigenspecific $\mathrm{T}$ cells after injection of $\alpha$-galactosyl-ceramide loaded mature dendritic cells in cancer patients. J Exp Med. 2005;201(9):1503-1517. doi:10.1084/jem.20042592

155. Giaccone G, Punt CJA, Ando Y, et al. A phase I study of the natural killer T-cell ligand $\alpha$ - 
galactosylceramide (KRN7000) in patients with solid tumors. Clin Cancer Res. 2002;8(12):3702-3709.

156. Woltman AM, Ter Borg MJ, Binda RS, et al. $\alpha$-Galactosylceramide in chronic hepatitis B infection: Results from a randomized placebo-controlled Phase I/ II trial. Antivir Ther. 2009;14(6):809-818. doi:10.3851/IMP1295

157. Tefit JN, Crabe S, Orlandini B, et al. Efficacy of ABX196, a new NKT agonist, in prophylactic human vaccination. 2014;32(46):6138-6145. doi:10.1016/j.vaccine.2014.08.070

158. Palucka K, Banchereau J. Cancer immunotherapy via dendritic cells. Nat Rev Cancer. 2012;12(April):265-277. doi:10.1007/978-3-7091-1300-4_4

159. Bui JD, Uppaluri R, Hsieh CS, Schreiber RD. Comparative analysis of regulatory and effector $\mathrm{T}$ cells in progressively growing versus rejecting tumors of similar origins. Cancer Res. 2006;66(14):7301-7309. doi:10.1158/0008-5472.CAN-06-0556

160. Duthie MS, Windish HP, Fox CB, Reed SG. Use of defined TLR ligands as adjuvants within human vaccines. Immunol Rev. 2011;239(1):178-196. doi:10.1111/j.1600065X.2010.00978.X

161. Kulkarni RR, Villanueva AI, Elawadli I, et al. Costimulatory activation of murine invariant natural killer T cells by toll-like receptor agonists. Cell Immunol. 2012;277(12):33-43. doi:10.1016/j.cellimm.2012.06.002

162. Hermans IF, Silk JD, Gileadi U, et al. Dendritic Cell Function Can Be Modulated through Cooperative Actions of TLR Ligands and Invariant NKT Cells. J Immunol. 
2007;178(5):2721-2729. doi:10.4049/jimmunol.178.5.2721

163. Spranger S, Gajewski TF. Mechanisms of Tumor Cell-Intrinsic Immune Evasion. Annu Rev Cancer Biol. 2018;2(1):213-228. doi:10.1146/annurev-cancerbio-030617-050606

164. Marincola FM, Jaffee EM, Hickljn DJ, Ferrone S. Escape of human solid tumors from tcell recognition: molecular mechanisms and functional significance. Adv Immunol. 2000;74(74):181-273.

165. Garrido F, Cabrera T, Aptsiauri N. "Hard" and "soft" lesions underlying the HLA class I alterations in cancer cells: Implications for immunotherapy. Int J Cancer. 2010;127(2):249-256. doi:10.1002/ijc.25270

166. Setiadi AF, Omilusik K, David MD, et al. Epigenetic enhancement of antigen processing and presentation promotes immune recognition of tumors. Cancer Res. 2008;68(23):96019607. doi:10.1158/0008-5472.CAN-07-5270

167. Landsberg J, Kohlmeyer J, Renn M, et al. Melanomas resist T-cell therapy through inflammation-induced reversible dedifferentiation. Nature. 2012;490(7420):412-416. doi:10.1038/nature11538

168. Medema JP, De Jong J, Van Hall T, Melief CJM, Offringa R. Immune escape of tumors in vivo by expression of cellular FLICE- inhibitory protein. J Exp Med. 1999;190(7):10331038. doi:10.1084/jem.190.7.1033

169. Kythreotou A, Siddique A, Mauri FA, Bower M, Pinato DJ. PD-L1. J Clin Pathol. 2018;71(3):189-194. doi:10.1136/jclinpath-2017-204853

170. Tormoen GW, Crittenden MR, Gough MJ. Role of the immunosuppressive 
microenvironment in immunotherapy. Adv Radiat Oncol. 2018;3(4):520-526.

doi:10.1016/j.adro.2018.08.018

171. Munn DH, Mellor AL. Indoleamine 2,3 dioxygenase and metabolic control of immune responses The IDO pathway and immune regulation. 2014;34(3):137-143. doi:10.1016/j.it.2012.10.001

172. Van Der Burg SH, Arens R, Ossendorp F, Van Hall T, Melief CJM. Vaccines for established cancer: Overcoming the challenges posed by immune evasion. Nat Rev Cancer. 2016;16(4):219-233. doi:10.1038/nrc.2016.16

173. Corgnac S, Perret R, Zhang L, Mach JP, Romero P, Donda A. iNKT/CD1d-antitumor immunotherapy significantly increases the efficacy of therapeutic $\mathrm{CpG} /$ peptide-based cancer vaccine. J Immunother Cancer. 2014;2(1):1-12.

doi:https://doi.org/10.1186/s40425-014-0039-8

174. Hoffman ES, Smith RET, Renaud RC. From the analyst's couch: TLR-targeted therapeutics. Nat Rev Drug Discov. 2005;4(11):879-880. doi:10.1038/nrd1880

175. Redelman-Sidi G, Glickman MS, Bochner BH. The mechanism of action of BCG therapy for bladder cancer-A current perspective. Nat Rev Urol. 2014;11(3):153-162. doi:10.1038/nrurol.2014.15

176. Crozat K, Beutler B. TLR7: A new sensor of viral infection. Proc Natl Acad Sci U S A. 2004;101(18):6835-6836. doi:10.1073/pnas.0401347101

177. Paavonen J, Naud P, Salmerón J, et al. Efficacy of human papillomavirus (HPV)-16/18 AS04-adjuvanted vaccine against cervical infection and precancer caused by oncogenic 
HPV types (PATRICIA): final analysis of a double-blind, randomised study in young women. Lancet. 2009;374(9686):301-314. doi:10.1016/S0140-6736(09)61248-4

178. Giannini SL, Hanon E, Moris P, et al. Enhanced humoral and memory B cellular immunity using HPV16/18 L1 VLP vaccine formulated with the MPL/aluminium salt combination (AS04) compared to aluminium salt only. Vaccine. 2006;24(33-34):59375949. doi:10.1016/j.vaccine.2006.06.005

179. Smith M, García-Martínez E, Pitter MR, et al. Trial Watch: Toll-like receptor agonists in cancer immunotherapy. Oncoimmunology. 2018;7(12):1-15. doi:10.1080/2162402X.2018.1526250

180. Hotz C, Treinies M, Mottas I, et al. Reprogramming of TLR7 signaling enhances antitumor NK and cytotoxic T cell responses. Oncoimmunology. 2016;5(11):1-34. doi:10.1080/2162402X.2016.1232219

181. Cho JH, Lee H-J, Ko H-J, et al. The TLR7 agonist imiquimod induces anti-cancer effects via autophagic cell death and enhances anti-tumoral and systemic immunity during radiotherapy for melanoma. Oncotarget. 2017;8(15):24932-24948. doi:10.18632/oncotarget.15326

182. Dhodapkar M V., Sznol M, Zhao B, et al. Induction of antigen-specific immunity with a vaccine targeting NY-ESO-1 to the dendritic cell receptor DEC-205. Sci Transl Med. 2014;6(232):1-10. doi:10.1126/scitranslmed.3008068

183. Rahman AH, Taylor DK, Turka LA. The contribution of direct TLR signaling to T cell responses. Immunol Res. 2009;45(1):25-36. doi:10.1007/s12026-009-8113-X 
184. Krieg AM. Therapeutic potential of Toll-like receptor 9 activation. Nat Rev Drug Discov. 2006;5(6):471-484. doi:10.1038/nrd2059

185. Krieg AM, Krieg AM. Development of TLR9 agonists for cancer therapy Find the latest version : Review series Development of TLR9 agonists for cancer therapy. J Clin Invest. 2007;117(5):1184-1194. doi:10.1172/JCI31414.1184

186. Dreno B, Thompson JF, Smithers BM, et al. MAGE-A3 immunotherapeutic as adjuvant therapy for patients with resected, MAGE-A3-positive, stage III melanoma (DERMA): a double-blind, randomised, placebo-controlled, phase 3 trial. Lancet Oncol. 2018;19(7):916-929. doi:10.1016/S1470-2045(18)30254-7

187. Manegold C, Gravenor D, Woytowitz D, et al. Randomized phase II trial of a toll-like receptor 9 agonist oligodeoxynucleotide, PF-3512676, in combination with first-line taxane plus platinum chemotherapy for advanced-stage non-small-cell lung cancer. $J$ Clin Oncol. 2008;26(24):3979-3986. doi:10.1200/JCO.2007.12.5807

188. Brody JD, Ai WZ, Czerwinski DK, et al. In situ vaccination with a TLR9 agonist induces systemic lymphoma regression: A phase I/II study. J Clin Oncol. 2010;28(28):4324-4332. doi:10.1200/JCO.2010.28.9793

189. Sagiv-Barfi I, Czerwinski DK, Levy S, et al. Eradication of spontaneous malignancy by local immunotherapy. Sci Transl Med. 2018;10(426):eaan4488. doi:10.1126/scitranslmed.aan4488

190. Temizoz B, Kuroda E, Ishii KJ. Vaccine adjuvants as potential cancer immunotherapeutics. Int Immunol. 2016;28(7):329-338. doi:10.1093/intimm/dxw015 
191. Krieg AM. Therapeutic potential of toll-like receptor 9 activation. Nat Rev Drug Discov. 2006;5(6):471-484. doi:10.1038/nrd2059

192. Kuan RK, Janssen R, Heyward W, Bennett S, Nordyke R. Cost-effectiveness of hepatitis B vaccination using HEPLISAV ${ }^{\mathrm{TM}}$ in selected adult populations compared to Engerix-B (® vaccine. Vaccine. 2013;31(37):4024-4032. doi:10.1016/j.vaccine.2013.05.014

193. Kruit WHJ, Suciu S, Dreno B, et al. Selection of immunostimulant AS15 for active immunization with MAGE-A3 protein: Results of a randomized phase II study of the European organisation for research and treatment of cancer melanoma group in metastatic melanoma. J Clin Oncol. 2013;31(19):2413-2420. doi:10.1200/JCO.2012.43.7111

194. Ursu R, Carpentier AF. Immunotherapeutic approach with oligodeoxynucleotides containing CpG motifs (CpG-ODN) in malignant glioma. Adv Exp Med Biol. 2012;746:95-108. doi:10.1007/978-1-4614-3146-6_8

195. Yin P, Liu X, Mansfield AS, et al. CpG-induced antitumor immunity requires IL-12 in expansion of effector cells and down-regulation of PD-1. Oncotarget. 2016;7(43):7022370231. doi:10.18632/oncotarget.11833

196. Marabelle A, Kohrt H, Caux C, Levy R. Intratumoral immunization: A new paradigm for cancer therapy. Clin Cancer Res. 2014;20(7):1747-1756. doi:10.1158/1078-0432.CCR$13-2116$

197. Morton DL, Eilber FR, Holmes EC, et al. BCG immunotherapy of malignant melanoma: Summary of a seven year experience. Ann Surg. 1974;180(4):635-643. doi:10.1097/00000658-197410000-00029 
198. Kidner TB, Morton DL, Lee DJ, et al. Combined intralesional bacille calmette-guérin (BCG) and topical imiquimod for in-transit melanoma. J Immunother. 2012;35(9):716720. doi:10.1097/CJI.0b013e31827457bd

199. Humbert M, Guery L, Brighouse D, Lemeille S, Hugues S. Intratumoral CpG-B promotes antitumoral neutrophil, $\mathrm{CDC}$, and T-cell cooperation without reprograming tolerogenic pDC. Cancer Res. 2018;78(12):3280-3292. doi:10.1158/0008-5472.CAN-17-2549

200. Heckelsmiller K, Rall K, Beck S, Schlamp A, Seiderer J. Peritumoral CpG DNA Elicits a Coordinated Response of CD8 T Cells and Innate Effectors to Cure Established Tumors in a Murine Colon Carcinoma Model. J Immunol. 2002;169(7):3892-3899. doi:10.4049/jimmunol.169.7.3892

201. Kramer K, Shields NJ, Poppe V, Young SL, Walker GF. Intracellular Cleavable CpG Oligodeoxynucleotide-Antigen Conjugate Enhances Anti-tumor Immunity. Mol Ther. 2017;25(1):62-70. doi:10.1016/j.ymthe.2016.10.001

202. Bartkowiak T, Singh S, Yang G, et al. Unique potential of 4-1BB agonist antibody to promote durable regression of HPV + tumors when combined with an E6/E7 peptide vaccine . Proc Natl Acad Sci. 2015;112(38):E5290-E5299. doi:10.1073/pnas.1514418112

203. Wang S, Campos J, Gallotta M, et al. Intratumoral injection of a $\mathrm{CpG}$ oligonucleotide reverts resistance to PD-1 blockade by expanding multifunctional CD8 ${ }^{+}$T cells. Proc Natl Acad Sci. 2016;113(46):E7240-E7249. doi:10.1073/pnas.1608555113

204. Ribas A, Medina T, Kummar S, et al. Sd-101 in combination with pembrolizumab in advanced melanoma: Results of a phase ib, multicenter study. Cancer Discov. 2018;8(10):1250-1257. doi:10.1158/2159-8290.CD-18-0280 
205. Levy R. TLR9 Agonist SD-101, Anti-OX40 Antibody BMS 986178, and Radiation Therapy in Treating Patients With Low-Grade B-Cell Non-Hodgkin Lymphomas - Full Text View - ClinicalTrials.gov. doi:NCT03410901

206. Kim E. SD-101, Nivolumab, and Radiation Therapy in Treating Patients With Chemotherapy-Refractory Metastatic Pancreatic Cancer - Full Text View ClinicalTrials.gov. doi:NCT04050085

207. Antonialli R, Sulczewski FB, Amorim KN da S, et al. CpG oligodeoxinucleotides and flagellin modulate the immune response to antigens targeted to CD8 $\alpha+$ and CD8 $\alpha-$ conventional dendritic cell subsets. Front Immunol. 2017;8(1727):1-16. doi:10.3389/fimmu.2017.01727

208. Marschner A, Rothenfusser S, Hornung V, et al. CpG ODN enhance antigen-specific NKT cell activation via plasmacytoid dendritic cells. Eur J Immunol. 2005;35(8):2347-2357. doi:10.1002/eji.200425721

209. Hermans IF, Silk JD, Gileadi U, et al. Dendritic Cell Function Can Be Modulated through Cooperative Actions of TLR Ligands and Invariant NKT Cells. J Immunol. 2014;178(5):2721-2729. doi:10.4049/jimmunol.178.5.2721

210. Humbert M, Guery L, Brighouse D, Lemeille S, Hugues S. Intratumoral CpG-B promotes antitumoral neutrophil, CDC, and T-cell cooperation without reprograming tolerogenic pDC. Cancer Res. 2018;78(12):3280-3292. doi:10.1158/0008-5472.CAN-17-2549

211. Houot R, Levy R. T-cell modulation combined with intratumoral CpG cures lymphoma in a mouse model without the need for chemotherapy. Blood. 2009;113(15):3546-3552. doi:10.1182/blood-2008-07-170274 
212. Kleffel S, Posch C, Barthel SR, et al. Melanoma Cell-Intrinsic PD-1 Receptor Functions Promote Tumor Growth. Cell. 2015;162(6):1242-1256. doi:10.1016/j.cell.2015.08.052

213. Wang JL, Saffold S, Cao XT, Krauss J, Chen W. Eliciting T cell immunity against poorly immunogenic tumors by immunization with dendritic cell-tumor fusion vaccines. $J$ Immunol. 1998;161(10):5516-5524.

214. Caisová V, Vieru A, Kumžáková Z, et al. Innate immunity based cancer immunotherapy: B16-F10 murine melanoma model. BMC Cancer. 2016;16(1):940. doi:10.1186/s12885016-2982-x

215. Diniz MO, Sales NS, Silva JR, Ferreira LCS. Protection against HPV-16-Associated tumors requires the activation of CD8+ effector memory $\mathrm{T}$ cells and the control of myeloid-derived suppressor cells. Mol Cancer Ther. 2016;15(8):1920-1930. doi:10.1158/1535-7163.MCT-15-0742

216. Wilcox RA, Flies DB, Zhu G, et al. Provision of antigen and CD137 signaling breaks immunological ignorance, promoting regression of poorly immunogenic tumors. J Clin Invest. 2002;109(5):651-659. doi:10.1172/JCI0214184

217. Mkrtichyan M, Chong N, Eid RA, et al. Anti-PD-1 antibody significantly increases therapeutic efficacy of Listeria monocytogenes (Lm)-LLO immunotherapy. J Immunother Cancer. 2013;1(1):1. doi:10.1186/2051-1426-1-15

218. Biburger M, Tiegs G. -Galactosylceramide-Induced Liver Injury in Mice Is Mediated by TNF- but Independent of Kupffer Cells. J Immunol. 2014;175(3):1540-1550. doi:10.4049/jimmunol.175.3.1540 
219. Hermans IF. ALT levels in murine models. Pers Commun.

220. Otto GP, Rathkolb B, Oestereicher MA, et al. Clinical chemistry reference intervals for C57BL/6J, C57BL/6N, and C3HeB/FeJ mice (Mus musculus). J Am Assoc Lab Anim Sci. 2016;55(4):375-386.

221. Schulz O, Edwards AD, Schito M, et al. CD40 triggering of heterodimeric IL-12 p70 production by dendritic cells in vivo requires a microbial priming signal. Immunity. 2000;13(4):453-462. doi:10.1016/S1074-7613(00)00045-5

222. Zhang $\mathrm{Y}$, Wang $\mathrm{F}$, Bhan $\mathrm{U}$, et al. TLR9 signaling is required for generation of the adaptive immune protection in Cryptococcus neoformans-infected lungs. Am J Pathol. 2010;177(2):754-765. doi:10.2353/ajpath.2010.091104

223. Nigar S, Shimosato T. Cooperation of Oligodeoxynucleotides and Synthetic Molecules as Enhanced Immune Modulators. Front Nutr. 2019;6(August):1-11. doi:10.3389/fnut.2019.00140

224. Lemoine R, Velge-Roussel F, Herr F, et al. Interferon gamma licensing of human dendritic cells in T-helper-independent CD8 + alloimmunity. Blood. 2010;116(16):30893098. doi:10.1182/blood-2010-02-268623

225. Seaman MS, Peyerl FW, Jackson SS, et al. Subsets of Memory Cytotoxic T Lymphocytes Elicited by Vaccination Influence the Efficiency of Secondary Expansion In Vivo. $J$ Virol. 2004;78(1):206-215. doi:10.1128/jvi.78.1.206-215.2004

226. Huster KM, Busch V, Schiemann M, et al. Selective expression of IL-7 receptor on memory $\mathrm{T}$ cells identifies early CD40L-dependent generation of distinct CD8+ memory $\mathrm{T}$ 
cell subsets. Proc Natl Acad Sci U S A. 2004;101(15):5610-5615.

doi:10.1073/pnas.0308054101

227. Chang J, Cho J-H, Lee S-W, Choi S-Y, Ha S-J, Sung Y-C. IL-12 Priming during In Vitro Antigenic Stimulation Changes Properties of CD8 T Cells and Increases Generation of Effector and Memory Cells. J Immunol. 2004;172(5):2818-2826.

doi:10.4049/jimmunol.172.5.2818

228. Lérias JR, de Sousa E, Paraschoudi G, et al. Trained Immunity for Personalized Cancer Immunotherapy: Current Knowledge and Future Opportunities. Front Microbiol. 2020;10(2924):1-12. doi:10.3389/fmicb.2019.02924

229. Shimizu K, Sato Y, Shinga J, et al. KLRG+ invariant natural killer T cells are long-lived effectors. Proc Natl Acad Sci U S A. 2014;111(34):12474-12479. doi:10.1073/pnas.1406240111

230. Breart B, Lemaître F, Celli S, Bousso P. Two-photon imaging of intratumoral CD8+ T cell cytotoxic activity during adoptive $\mathrm{T}$ cell therapy in mice. J Clin Invest. 2008;118(4):1390-1397. doi:10.1172/JCI34388

231. Kägi D, Ledermann B, Bürki K, et al. Cytotoxicity mediated by $\mathrm{T}$ cells and natural killer cells is greatly impaired in perforin-deficient mice. Nature. 1994;369(6475):31-37. doi:10.1038/369031a0

232. Barth RJ, Mule JJ, Spiess PJ, Rosenberg SA. Interferon $\gamma$ and tumor necrosis factor have a role in tumor regressions mediated by murine CD8+ tumor-infiltrating lymphocytes. $J$ Exp Med. 1991;173(3):647-658. doi:10.1084/jem.173.3.647 
233. Hollenbaugh JA, Dutton RW. IFN- $\gamma$ Regulates Donor CD8 T Cell Expansion, Migration, and Leads to Apoptosis of Cells of a Solid Tumor. J Immunol. 2006;177(5):3004-3011. doi:10.4049/jimmunol.177.5.3004

234. Collin M, Bigley V. Human dendritic cell subsets: an update. Immunology. 2018;154(1):320. doi:10.1111/imm.12888

235. Cance JC, Crozat K, Dalod M, Mattiuz R. Are conventional type 1 dendritic cells critical for protective antitomor immunity and how? Front Immunol. 2019;10(FEB):9. doi:10.3389/fimmu.2019.00009

236. Noubade R, Majri-Morrison S, Tarbell K V. Beyond cDC1: Emerging roles of DC crosstalk in cancer immunity. Front Immunol. 2019;10(1014):1-13. doi:10.3389/fimmu.2019.01014

237. Binnewies M, Mujal AM, Pollack JL, et al. Unleashing Type-2 Dendritic Cells to Drive Protective Antitumor CD4 + T Cell Immunity. Cell. 2019;177(3):556-571.e16. doi:10.1016/j.cell.2019.02.005

238. Fujii SI, Shimizu K, Smith C, Bonifaz L, Steinman RM. Activation of natural killer T cells by $\alpha$-galactosylceramide rapidly induces the full maturation of dendritic cells in vivo and thereby acts as an adjuvant for combined CD4 and CD8 T cell immunity to a coadministered protein. J Exp Med. 2003;198(2):267-279. doi:10.1084/jem.20030324

239. Asselin-Paturel C, Brizard G, Chemin K, et al. Type I interferon dependence of plasmacytoid dendritic cell activation and migration. J Exp Med. 2005;201(7):1157-1167. doi:10.1084/jem.20041930 
240. Sester DP, Brion K, Trieu A, et al. CpG DNA Activates Survival in Murine Macrophages through TLR9 and the Phosphatidylinositol 3-Kinase-Akt Pathway. J Immunol. 2006;177(7):4473-4480. doi:10.4049/jimmunol.177.7.4473

241. Jiang W, Lederman MM, Harding CV, Rodriguez B, Mohner RJ, Sieg SF. TLR9 stimulation drives naïve B cells to proliferate and to attain enhanced antigen presenting function. Eur J Immunol. 2007;37(8):2205-2213. doi:10.1002/eji.200636984

242. Roda JM, Parihar R, Carson WE. CpG-Containing Oligodeoxynucleotides Act through TLR9 to Enhance the NK Cell Cytokine Response to Antibody-Coated Tumor Cells. $J$ Immunol. 2005;175(3):1619-1627. doi:10.4049/jimmunol.175.3.1619

243. Fujii SI, Shimizu K. Immune Networks and Therapeutic Targeting of iNKT Cells in Cancer. Trends Immunol. 2019;40(11):984-997. doi:10.1016/j.it.2019.09.008

244. Gorbachev A V., Fairchild RL. Activated NKT cells increase dendritic cell migration and enhance CD8+ T cell responses in the skin. Eur J Immunol. 2006;36(9):2494-2503. doi:10.1002/eji.200636075

245. Deligne C, Milcent B, Josseaume N, Teillaud JL, Sibéril S. Impact of depleting therapeutic monoclonal antibodies on the host adaptive immunity: A bonus or a malus? Front Immunol. 2017;8(AUG):950. doi:10.3389/fimmu.2017.00950

246. Saito M, Iwawaki T, Taya C, et al. Diphtheria toxin receptor-mediated conditional and targeted cell ablation in transgenic mice. Nat Biotechnol. 2001;19(8):746-750. doi:10.1038/90795

247. Lee JH, Park JH, Nam TW, et al. Differences between immunodeficient mice generated 
by classical gene targeting and CRISPR/Cas9-mediated gene knockout. Transgenic Res. 2018;27(3):241-251. doi:10.1007/s11248-018-0069-y

248. Hall B, Limaye A, Kulkarni AB. Overview: Generation of Gene Knockout Mice. Curr Protoc Cell Biol. 2009;19(1217):1-23. doi:10.1002/0471143030.cb1912s44

249. Obar JJ, Lefranois L. Memory CD8+ T cell differentiation. Ann N Y Acad Sci. 2010;1183:251-266. doi:10.1111/j.1749-6632.2009.05126.x

250. Christaki E, Diza E, Giamarellos-Bourboulis EJ, et al. NK and NKT cell depletion alters the outcome of experimental pneumococcal pneumonia: Relationship with regulation of interferon- $\gamma$ production. J Immunol Res. 2015;2015. doi:10.1155/2015/532717

251. Fujii SI, Shimizu K, Hemmi H, et al. Glycolipid $\alpha$-C-galactosylceramide is a distinct inducer of dendritic cell function during innate and adaptive immune responses of mice. Proc Natl Acad Sci U S A. 2006;103(30):11252-11257. doi:10.1073/pnas.0604812103

252. Schreibelt G, Klinkenberg LJJ, Cruz LJ, et al. The C-type lectin receptor CLEC9A mediates antigen uptake and (cross-)presentation by human blood BDCA3+ myeloid dendritic cells. Blood. 2012;119(10):2284-2292. doi:10.1182/blood-2011-08-373944

253. Takagi H, Fukaya T, Eizumi K, et al. Plasmacytoid Dendritic Cells Are Crucial for the Initiation of Inflammation and T Cell Immunity In Vivo. Immunity. 2011;35(6):958-971. doi:10.1016/j.immuni.2011.10.014

254. Jordan M, Waxman DJ. CpG-1826 immunotherapy potentiates chemotherapeutic and antitumor immune responses to metronomic cyclophosphamide in a preclinical glioma model Marie. Cancer Lett. 2016;373(1):88-96. doi:10.1016/j.canlet.2015.11.029. CpG-1826 
255. Capolunghi F, Cascioli S, Giorda E, et al. CpG Drives Human Transitional B Cells to Terminal Differentiation and Production of Natural Antibodies. J Immunol. 2008;180(2):800-808. doi:10.4049/jimmunol.180.2.800

256. Karandikar SH, Sidney J, Sette A, Selby MJ, Korman AJ, Srivastava PK. Identification of epitopes in ovalbumin that provide insights for cancer neoepitopes. JCI insight. 2019;4(8):1-12. doi:https://doi.org/10.1172/jci.insight.127882

257. Das R, Sant'Angelo DB, Nichols KE. Transcriptional control of invariant NKT cell development. Immunol Rev. 2010;238(1):195-215. doi:10.1111/j.1600065X.2010.00962.X

258. Gee K, Guzzo C, Che Mat N, Ma W, Kumar A. The IL-12 Family of Cytokines in Infection, Inflammation and Autoimmune Disorders. Inflamm Allergy-Drug Targets. 2009;8(1):40-52. doi:10.2174/187152809787582507

259. Nayar S, Dasgupta P, Galustian C. Extending the lifespan and efficacies of immune cells used in adoptive transfer for cancer immunotherapies-A review. Oncoimmunology. 2015;4(4):1-12. doi:10.1080/2162402X.2014.1002720

260. Lo Nigro C, Macagno M, Sangiolo D, Bertolaccini L, Aglietta M, Merlano MC. NKmediated antibody-dependent cell-mediated cytotoxicity in solid tumors: biological evidence and clinical perspectives. Ann Transl Med. 2019;7(5):105-105. doi:10.21037/atm.2019.01.42

261. Yuen GJ, Demissie E, Pillai S, Burnet M. B lymphocytes and cancer: a love-hate relationship Grace. Trends Cancer. 2017;2(12):747-757. doi:10.1016/j.trecan.2016.10.010.B 
262. Thomas SN, Vokali E, Lund AW, Hubbell JA, Swartz MA. Targeting the tumor-draining lymph node with adjuvanted nanoparticles reshapes the anti-tumor immune response. Biomaterials. 2014;35:814-824. doi:10.1016/j.biomaterials.2013.10.003

263. Jeanbart L, Ballester M, De Titta A, et al. Enhancing efficacy of anticancer vaccines by targeted delivery to tumor-draining lymph nodes. Cancer Immunol Res. 2014;2(5):436447. doi:10.1158/2326-6066.CIR-14-0019-T

264. Bialecki E, Macho Fernandez E, Ivanov S, et al. Spleen-Resident CD4+ and CD4CD8 $\alpha$ - Dendritic Cell Subsets Differ in Their Ability to Prime Invariant Natural Killer T Lymphocytes. Combs C, ed. PLoS One. 2011;6(10):e26919. doi:10.1371/journal.pone.0026919

265. Wakkach A, Fournier N, Brun V, Breittmayer JP, Cottrez F, Groux H. Characterization of dendritic cells that induce tolerance and $\mathrm{T}$ regulatory 1 cell differentiation in vivo. Immunity. 2003;18(5):605-617. doi:10.1016/S1074-7613(03)00113-4

266. Steinman RM, Turley S, Mellman I, Inaba K. The induction of tolerance by dendritic cells that have captured apoptotic cells. J Exp Med. 2000;191(3):411-416. doi:10.1084/jem.191.3.411

267. Jonuleit H, Schmitt E, Schuler G, Knop J, Enk AH. Induction of interleukin 10-producing, nonproliferating CD4+ T cells with regulatory properties by repetitive stimulation with allogeneic immature human dendritic cells. J Exp Med. 2000;192(9):1213-1222. doi:10.1084/jem.192.9.1213

268. Mcgaha TL, Karlsson MCI. Apoptotic cell responses in the splenic marginal zone: A paradigm for immunologic reactions to apoptotic antigens with implications for 
autoimmunity. Immunol Rev. 2016;269(1):26-43. doi:10.1111/imr.12382

269. Backer R, Schwandt T, Greuter M, et al. Effective collaboration between marginal metallophilic macrophages and CD8+ dendritic cells in the generation of cytotoxic $\mathrm{T}$ cells. Proc Natl Acad Sci U S A. 2010;107(1):216-221. doi:10.1073/pnas.0909541107

270. Shimizu K, Kurosawa Y, Taniguchi M, Steinman RM, Fujii SI. Cross-presentation of glycolipid from tumor cells loaded with $\alpha$-galactosylceramide leads to potent and longlived T cell-mediated immunity via dendritic cells. J Exp Med. 2007;204(11):2641-2653. doi:10.1084/jem.20070458

271. Wu H, Kumar A, Miao H, et al. Modeling of Influenza-Specific CD8 + T Cells during the Primary Response Indicates that the Spleen Is a Major Source of Effectors . J Immunol. 2011;187(9):4474-4482. doi:10.4049/jimmunol.1101443

272. Ram S, Lewis LA, Rice PA. Infections of people with complement deficiencies and patients who have undergone splenectomy. Clin Microbiol Rev. 2010;23(4):740-780. doi:10.1128/CMR.00048-09

273. Newland A, Provan D, Myint S. Preventing severe infection after splenectomy. Br Med J. 2005;331(7514):417-418. doi:10.1136/bmj.331.7514.417

274. Shi C, Pamer EG. Monocyte recruitment during infection and inflammation. Nat Rev Immunol. 2011;11(11):762-774. doi:10.1038/nri3070

275. Coffey JC, Wang JH, Smith MJF, Bouchier-Hayes D, Cotter TG, Redmond HP. Excisional surgery for cancer cure: Therapy at a cost. Lancet Oncol. 2003;4(12):760-768. doi:10.1016/S1470-2045(03)01282-8 
276. Goldstein MR, Mascitelli L. Surgery and cancer promotion: Are we trading beauty for cancer? Qjm. 2011;104(9):811-815. doi:10.1093/qjmed/hcr039

277. Panigrahy D, Gartung A, Yang J, et al. Preoperative stimulation of resolution and inflammation blockade eradicates micrometastases. J Clin Invest. 2019;129(7):2964-2979. doi:10.1172/JCI127282

278. Markovič T, Jakopin Ž, Dolenc MS, Mlinarič-Raščan I. Structural features of subtypeselective EP receptor modulators. Drug Discov Today. 2017;22(1):57-71. doi:10.1016/j.drudis.2016.08.003

279. Krug A, Rothenfusser S, Hornung V, et al. Identification of CpG oligonucleotide sequences with high induction of IFN- $\alpha / \beta$ in plasmacytoid dendritic cells. Eur J Immunol. 2001;31(7):2154-2163. doi:10.1002/1521-4141(200107)31:7<2154::AIDIMMU2154>3.0.CO;2-U

280. Jeske S, Pries R, Wollenberg B. CpG-Induced IFN- $\alpha$ Production of Plasmacytoid Dendritic Cells: Time and Dosage Dependence and the Effect of Structural Modifications to the CpG Backbone. Nucleic Acid Ther. 2013;23(2):118-124. doi:10.1089/nat.2012.0384

281. Dunn GP, Koebel CM, Schreiber RD. Interferons, immunity and cancer immunoediting. Nat Rev Immunol. 2006;6(11):836-848. doi:10.1038/nri1961

282. Theofilopoulos AN, Baccala R, Beutler B, Kono DH. Type I Interferons (A/B) in Immunity and Autoimmunity. Annu Rev Immunol. 2005;23(1):307-335. doi:10.1146/annurev.immunol.23.021704.115843

283. García-Sastre A, Biron CA. Type 1 Interferons and the Virus-Host Relationship: A Lesson 
in Détente. Am Nat. 2002;23(274):935-939. doi:10.1086/275028

284. Jego G, Palucka AK, Blanck JP, Chalouni C, Pascual V, Banchereau J. Plasmacytoid dendritic cells induce plasma cell differentiation through type I interferon and interleukin 6. Immunity. 2003;19(2):225-234. doi:10.1016/S1074-7613(03)00208-5

285. DeLong JH, Hall AO, Konradt C, et al. Cytokine- and TCR-Mediated Regulation of T Cell Expression of Ly6C and Sca-1. J Immunol. 2018:ji1701154. doi:10.4049/jimmunol.1701154

286. Trinchieri G. Type I interferon: friend or foe? J Exp Med. 2010;207(10):2053-2063. doi:10.1084/jem.20101664

287. Borg FAY, Isenberg DA. Syndromes and complications of interferon therapy. Curr Opin Rheumatol. 2007;19(1):61-66. doi:10.1097/BOR.0b013e328010c547

288. Tison A, Quéré G, Misery L, et al. Safety and Efficacy of Immune Checkpoint Inhibitors in Patients With Cancer and Preexisting Autoimmune Disease: A Nationwide, Multicenter Cohort Study. Arthritis Rheumatol. 2019;71(12):2100-2111. doi:10.1002/art.41068

289. Van der Jeught K, Bialkowski L, Daszkiewicz L, et al. Targeting the tumor microenvironment to enhance antitumor immune responses. Oncotarget. 2015;6(3):13591381. doi:10.18632/oncotarget. 3204

290. Humbert M, Guery L, Brighouse D, Lemeille S, Hugues S. Intratumoral CpG-B promotes antitumoral neutrophil, CDC, and T-cell cooperation without reprograming tolerogenic pDC. Cancer Res. 2018;78(12):3280-3292. doi:10.1158/0008-5472.CAN-17-2549

291. Ma C, Armstrong AW. Severe adverse events from the treatment of advanced melanoma: 
A systematic review of severe side effects associated with ipilimumab, vemurafenib, interferon alfa-2b, dacarbazine and interleukin-2. J Dermatolog Treat. 2014;25(5):401408. doi:10.3109/09546634.2013.813897

292. Niederquell M, Kurig S, Fischer JAA, et al. Sca-1 expression defines developmental stages of mouse pDCs that show functional heterogeneity in the endosomal but not lysosomal TLR9 response. Eur J Immunol. 2013;43(11):2993-3005. doi:10.1002/eji.201343498

293. Swiecki M, Colonna M. Type I interferons: diversity of sources, production pathways and effects on immune responses Melissa. Curr Opin Virol. 2011;1(6):463-475. doi:10.10.1016/j.coviro.2011.10.026

294. Longman RS, Braun D, Pellegrini S, Rice CM, Darnell RB, Albert ML. Dendritic-cell maturation alters intracellular signaling networks, enabling differential effects of IFN- $\alpha / \beta$ on antigen cross-presentation. Blood. 2007;109(3):1113-1122. doi:10.1182/blood-200605-023465

295. Lechner MG, Karimi SS, Barry-Holson K, et al. Immunogenicity of murine solid tumor models as a defining feature of in vivo behavior and response to immunotherapy. $J$ Immunother. 2013;36(9):477-489. doi:10.1097/01.cji.0000436722.46675.4a

296. Mosely SIS, Prime JE, Sainson RCA, et al. Rational selection of syngeneic preclinical tumor models for immunotherapeutic drug discovery. Cancer Immunol Res. 2017;5(1):2941. doi:10.1158/2326-6066.CIR-16-0114

297. Tuve S, Chen BM, Liu Y, et al. Combination of tumor site-located CTL-associated antigen-4 blockade and systemic regulatory T-cell depletion induces tumor-destructive 
immune responses. Cancer Res. 2007;67(12):5929-5939. doi:10.1158/0008-5472.CAN$06-4296$

298. Dubrot J, Palazón A, Alfaro C, et al. Intratumoral injection of interferon- $\alpha$ and systemic delivery of agonist anti-CD137 monoclonal antibodies synergize for immunotherapy. Int $J$ Cancer. 2011;128(1):105-118. doi:10.1002/ijc.25333

299. Ying L, Tuve S, Persson J, et al. Adenovirus-mediated intratumoral expression of immunostimulatory proteins in combination with systemic Treg inactivation induces tumor-destructive immune responses in mouse models. Cancer Gene Ther. 2011;18(6):407-418. doi:10.1038/cgt.2011.8.Adenovirus-mediated

300. Lin K, Guarnieri FG, Carroll KFS, et al. Treatment of Established Tumors with a Novel Vaccine That Enhances Major Histocompatibility Class II Presentation of Tumor Antigen Advances in Brief Treatment of Established Tumors with a Novel Vaccine That Enhances Major Histocompatibility Class II Prese. Cancer Res. 1996;(410):21-26.

301. Anderson RJ, Compton BJ, Tang CW, et al. NKT cell-dependent glycolipid-peptide vaccines with potent anti-tumour activity. Chem Sci. 2015;6(9):5120-5127. doi: $10.1039 / \mathrm{c} 4 \mathrm{sc} 03599 \mathrm{~b}$

302. Nowak AK, Lake RA, Marzo AL, et al. Induction of Tumor Cell Apoptosis In Vivo Increases Tumor Antigen Cross-Presentation, Cross-Priming Rather than Cross-Tolerizing Host Tumor-Specific CD8 T Cells. J Immunol. 2014;170(10):4905-4913. doi:10.4049/jimmunol.170.10.4905

303. Anyaegbu CC, Lake RA, Heel K, Robinson BW, Fisher SA. Chemotherapy enhances cross-presentation of nuclear tumor antigens. PLoS One. 2014;9(9):1-8. 
doi:10.1371/journal.pone.0107894

304. Kaur P, Asea A. Radiation-induced effects and the immune system in cancer. Front Oncol. 2012;2(December):1-10. doi:10.3389/fonc.2012.00191

305. Adkins I, Fucikova J, Garg AD, Agostinis P, Špíšek R. Physical modalities inducing immunogenic tumor cell death for cancer immunotherapy. Oncoimmunology. 2014;3(12):1-12. doi:10.4161/21624011.2014.968434

306. Aurelian L. Oncolytic viruses as immunotherapy: progress and remaining challenges. Onco Targets Ther. 2016;9:2627-2637. doi:10.2147/OTT.S63049

307. Anderson RJ, Tang CW, Daniels NJ, et al. A self-adjuvanting vaccine induces cytotoxic T lymphocytes that suppress allergy. Nat Chem Biol. 2014;10(11):943-949. doi:10.1038/nchembio.1640

308. Kepp O, Galluzzi L, Martins I, et al. Molecular determinants of immunogenic cell death elicited by anticancer chemotherapy. Cancer Metastasis Rev. 2011;30(1):61-69. doi:10.1007/s10555-011-9273-4

309. Verma R, Foster RE, Horgan K, et al. Lymphocyte depletion and repopulation after chemotherapy for primary breast cancer. Breast Cancer Res. 2016;18(1):10. doi:10.1186/s13058-015-0669-x

310. Liao Y-P, Wang C-C, Butterfield LH, et al. Ionizing Radiation Affects Human MART-1 Melanoma Antigen Processing and Presentation by Dendritic Cells. J Immunol. 2014;173(4):2462-2469. doi:10.4049/jimmunol.173.4.2462

311. Wu J, Waxman DJ. Immunogenic chemotherapy: Dose and schedule dependence and 
combination with immunotherapy. Cancer Lett. 2018;419:210-221.

doi:10.1016/j.canlet.2018.01.050

312. Iwai Y, Ishida M, Tanaka Y, Okazaki T, Honjo T, Minato N. Involvement of PD-L1 on tumor cells in the escape from host immune system and tumor immunotherapy by PD-L1 blockade. Proc Natl Acad Sci. 2002;99(19):12293-12297. doi:10.1073/pnas.192461099

313. Thompson RH, Gillett MD, Cheville JC, et al. Costimulatory B7-H1 in renal cell carcinoma patients: Indicator of tumor aggressiveness and potential therapeutic target. Proc Natl Acad Sci. 2004;101(49):17174-17179. doi:10.1073/pnas.0406351101

314. Lazzari C, Karachaliou N, Bulotta A, et al. Combination of immunotherapy with chemotherapy and radiotherapy in lung cancer: is this the beginning of the end for cancer? Ther Adv Med Oncol. 2018;10:1-12. doi:10.1177/1758835918762094

315. Anderson RJ, Li J, Kedzierski L, et al. Augmenting Influenza-Specific T Cell Memory Generation with a Natural Killer T Cell-Dependent Glycolipid-Peptide Vaccine. ACS Chem Biol. 2017;12(11):2898-2905. doi:10.1021/acschembio.7b00845

316. Tan S, G.E. de Vries E, G.J. van der Zee A, de Jong S. Anticancer Drugs Aimed at E6 and E7 Activity in HPV-Positive Cervical Cancer. Curr Cancer Drug Targets. 2012;12(2):170-184. doi:10.2174/156800912799095135

317. US Patent Application for CONJUGATE COMPOUNDS Patent Application (Application \#20150352219 issued December 10, 2015) - Justia Patents Search. https://patents.justia.com/patent/20150352219. Accessed May 15, 2020.

318. US Patent for Conjugate compounds Patent (Patent \# 9,764,037 issued September 19, 
2017) - Justia Patents Search. https://patents.justia.com/patent/9764037. Accessed May $15,2020$.

319. Zhang X, Qi Y, Zhang Q, Liu W. Application of mass spectrometry-based MHC immunopeptidome profiling in neoantigen identification for tumor immunotherapy. Biomed Pharmacother. 2019;120:109542. doi:10.1016/j.biopha.2019.109542

320. O’Donnell JS, Long G V., Scolyer RA, Teng MWL, Smyth MJ. Resistance to PD1/PDL1 checkpoint inhibition. Cancer Treat Rev. 2017;52(November):71-81. doi:10.1016/j.ctrv.2016.11.007

321. Kyi C, Postow M. Immune checkpoint inhibitor combinations in solid tumors: opportunities and challenges. Futur Med. 2016;8:821-837.

322. Lee J, Ahn E, Kissick HT, Ahmed R. Reinvigorating exhausted T cells by blockade of the PD-1 pathway. For Immunopathol Dis Therap. 2015;6(1-2):7-18. doi:10.1615/ForumImmunDisTher.2015014188

323. Shindo Y, Yoshimura K, Kuramasu A, et al. Combination immunotherapy with 4-1BB activation and PD-1 blockade enhances antitumor efficacy in a mouse model of subcutaneous tumor. Anticancer Res. 2015;35(1):129-136.

324. Brennick C, George M, Corwin W, Srivastava P, Ebrahimi-Nik H. Neoepitopes as cancer immunotherapy targets: key challenges and opportunities. Immunotherapy. 2014;9:361371. doi:10.1016/j.jscs.2014.12.002

325. Romano S, Nappo G, Calì G, et al. Synergy between enzastaurin doxorubicin in inducing melanoma apoptosis. Pigment Cell Melanoma Res. 2013;26(6):900-911. 
doi:10.1111/pcmr.12144

326. Litterman AJ, Zellmer DM, Grinnen KL, et al. Profound Impairment of Adaptive Immune Responses by Alkylating Chemotherapy. J Immunol. 2013;190(12):6259-6268. doi:10.4049/jimmunol.1203539

327. Holohan C, Van Schaeybroeck S, Longley DB, Johnston PG. Cancer drug resistance: An evolving paradigm. Nat Rev Cancer. 2013;13(10):714-726. doi:10.1038/nrc3599

328. Klement G, Baruchel S, Rak J, et al. Continuous low-dose therapy with vinblastine and VEGF receptor-2 antibody induces sustained tumor regression without overt toxicity. $J$ Clin Invest. 2006;116(10):2827. doi:10.1172/JCI08829C1

329. Rosenberg SA, Yang JC, Restifo NP. Cancer immunotherapy: moving beyond current vaccines. Nat Med. 2004;10(9):909-915. doi:10.1038/nm1100

330. Hegde PS, Karanikas V, Evers S. The where, the when, and the how of immune monitoring for cancer immunotherapies in the era of checkpoint inhibition. Clin Cancer Res. 2016;22(8):1865-1874. doi:10.1158/1078-0432.CCR-15-1507

331. Harlin H, Meng Y, Peterson AC, et al. Chemokine expression in melnma metastasis with CD8+ T-cell recruitment. Cancer Res. 2009;69(7):1-18. doi:10.1158/0008-5472.CAN-082281.Chemokine

332. Spranger S, Bao R, Gajewski TF. Melanoma-intrinsic $\beta$-catenin signalling prevents antitumour immunity. Nature. 2015;523(7559):231-235. doi:10.1038/nature14404

333. Gajewski TF, Woo SR, Zha Y, et al. Cancer immunotherapy strategies based on overcoming barriers within the tumor microenvironment. Curr Opin Immunol. 
2013;25(2):268-276. doi:10.1016/j.coi.2013.02.009

334. Lam M, Tie J, Lee B, Desai J, Gibbs P, Tran B. Systemic Inflammation -“ Impact on Tumor Biology and Outcomes in Colorectal Cancer. J Clin Cell Immunol. 2016;06(06). doi:10.4172/2155-9899.1000377

335. Samstein RM, Lee $\mathrm{CH}$, Shoushtari AN, et al. Tumor mutational load predicts survival after immunotherapy across multiple cancer types. Nat Genet. 2019;51(2):202-206. doi:10.1038/s41588-018-0312-8

336. Giannakis M, Mu XJ, Shukla SA, et al. Genomic Correlates of Immune-Cell Infiltrates in Colorectal Carcinoma. Cell Rep. 2016;15(4):857-865. doi:10.1016/j.celrep.2016.03.075

337. Hermans IF, Chong TW, Palmowski MJ, Harris AL, Cerundolo V. Synergistic Effect of Metronomic Dosing of Cyclophosphamide Combined with Specific Antitumor Immunotherapy in a Murine Melanoma Model. Cancer Res. 2003;63(23):8408-8413.

338. Singal PK, Deally CMR, Weinberg LE. Subcellular effects of adriamycin in the heart: A concise review. J Mol Cell Cardiol. 1987;19(8):817-828. doi:10.1016/S00222828(87)80392-9

339. Chatterjee K, Zhang J, Honbo N, Karliner JS. Doxorubicin cardiomyopathy. Cardiology. 2010;115(2):155-162. doi:10.1159/000265166

340. Corbett TH, Griswold DP, Roberts BJ, Peckham JC, Schabel FM. Tumor induction relationships in development of transplantable cancers of the colon in mice for chemotherapy assays, with a note on carcinogen structure. Cancer Res. 1975;35(9):24342439. http://www.ncbi.nlm.nih.gov/pubmed/1149045. 
341. Cleveland PH, Colmerauer MEM, Barone RM. Immunogenicity of Chemically Induced Murine Colon Cancers. Cancer Res. 1979;39(4):1174-1179.

342. Gordon S, Maute R, Dulken B, et al. PD-1 expression by tumor-associated macrophages inhibits phagocytosis and tumor immunity. Nature. 2017;37(4):784-790. doi:10.1183/09031936.00063810.The

343. Ngwa W, Irabor OC, Schoenfeld JD, Hesser J, Demaria S, Formenti SC. Using immunotherapy to boost the abscopal effect. Nat Rev Cancer. 2018;18(5):313-322. doi:10.1038/nrc.2018.6

344. Petersen TR, Sika-Paotonu D, Knight DA, Simkins HMA, Hermans IF. Exploiting the role of endogenous lymphoid-resident dendritic cells in the priming of NKT cells and CD8+ T cells to dendritic cell-based vaccines. PLoS One. 2011;6(3). doi:10.1371/journal.pone.0017657

345. Fujii S ichiro, Shimizu K, Okamoto Y, et al. NKT cells as an ideal anti-tumor immunotherapeutic. Front Immunol. 2013;4(DEC):1-7. doi:10.3389/fimmu.2013.00409

346. McDaniel JR, Callahan DJ, Chilkoti A. Drug delivery to solid tumors by elastin-like polypeptides. Adv Drug Deliv Rev. 2010;62(15):1456-1467. doi:10.1016/j.addr.2010.05.004

347. Kain L, Webb B, Anderson BL, et al. The Identification of the Endogenous Ligands of Natural Killer T Cells Reveals the Presence of Mammalian $\alpha$-Linked Glycosylceramides. Immunity. 2014;41(4):543-554. doi:10.1016/j.immuni.2014.08.017

348. Brigl M, Bry L, Kent SC, Gumperz JE, Brenner MB. Mechanism of CD1d-restricted 
natural killer T cell activation during microbial infection. Nat Immunol. 2003;4(12):12301237. doi:10.1038/ni1002

349. Mattner J, DeBord KL, Ismail N, et al. Exogenous and endogenous glycolipid antigens activate NKT cells during microbial infections. Nature. 2005;434(7032):525-529. doi:https://doi.org/10.1038/nature03408

350. Brigl M, Brenner MB. How invariant natural killer $\mathrm{T}$ cells respond to infection by recognizing microbial or endogenous lipid antigens. Semin Immunol. 2010;22(2):79-86. doi:10.1016/j.smim.2009.10.006

351. Gilliet M, Cao W, Liu YJ. Plasmacytoid dendritic cells: Sensing nucleic acids in viral infection and autoimmune diseases. Nat Rev Immunol. 2008;8(8):594-606. doi:10.1038/nri2358

352. Kolumam GA, Thomas S, Thompson LJ, Sprent J, Murali-Krishna K. Type I interferons act directly on CD8 $\mathrm{T}$ cells to allow clonal expansion and memory formation in response to viral infection. $J$ Exp Med. 2005;202(5):637-650. doi:10.1084/jem.20050821

353. VanOosten RL, Griffith TS. Activation of tumor-specific CD8+ T cells after intratumoral Ad5-TRAIL/CpG oligodeoxynucleotide combination therapy. Cancer Res. 2007;67(24):11980-11990. doi:10.1158/0008-5472.CAN-07-1526

354. Lee BC, Kim MS, Pae M, et al. Adipose Natural Killer Cells Regulate Adipose Tissue Macrophages to Promote Insulin Resistance in Obesity. Cell Metab. 2016;23(4):685-698. doi:10.1016/j.cmet.2016.03.002

355. Kitamura H, Ohta A, Sekimoto M, et al. $\alpha$-galactosylceramide induces early B-cell 
activation through IL-4 production by NKT cells. Cell Immunol. 2000;199(1):37-42. doi:10.1006/cimm.1999.1602

356. Doherty DG, Melo AM, Moreno-Olivera A, Solomos AC. Activation and regulation of B cell responses by invariant natural killer T cells. Front Immunol. 2018;9(JUN):1360. doi:10.3389/fimmu.2018.01360

357. Kim YJ, Ko HJ, Kim YS, et al. $\alpha$-Galactosylceramide-loaded, antigen-expressing B cells prime a wide spectrum of antitumor immunity. Int J Cancer. 2008;122(12):2774-2783. doi:10.1002/ijc.23444

358. Roberts TL, Dunn JA, Terry TD, et al. Differences in Macrophage Activation by Bacterial DNA and CpG-Containing Oligonucleotides. J Immunol. 2005;175(6):3569-3576. doi:10.4049/jimmunol.175.6.3569

359. Kranzer K, Bauer M, Lipford GB, Heeg K, Wagner H, Lang R. CpGoligodeoxynucleotides enhance T-cell receptor-triggered interferon- $\gamma$ production and upregulation of CD69 via induction of antigen-presenting cell-derived interferon type $\mathrm{T}$ and interleukin-12. Immunology. 2000;99(2):170-178. doi:10.1046/j.1365-2567.2000.00964.x

360. Dibra D, Li S. The cell-to-cell coordination between activated T cells and CpG-stimulated macrophages synergistically induce elevated levels of IL-10 via NF-kB1, STAT3, and CD40/CD154. Cell Commun Signal. 2013;11(1):95. doi:10.1186/1478-811X-11-95

361. Pitt JM, Vétizou M, Daillère R, et al. Resistance Mechanisms to Immune-Checkpoint Blockade in Cancer: Tumor-Intrinsic and -Extrinsic Factors. Immunity. 2016;44(6):12551269. doi:10.1016/j.immuni.2016.06.001 
362. Chester C, Sanmamed MF, Wang J, Melero I. Immunotherapy targeting 4-1BB:

Mechanistic rationale, clinical results, and future strategies. Blood. 2018;131(1):49-57. doi:10.1182/blood-2017-06-741041

363. Bendigs S, Salzer U, Lipford GB, Wagner H, Heeg K. CpG-oligodeoxynucleotides costimulate primary $\mathrm{T}$ cells in the absence of antigen-presenting cells. Eur J Immunol. 1999;29(4):1209-1218. doi:10.1002/(SICI)1521-4141(199904)29:04<1209::AIDIMMU1209>3.0.CO;2-J

364. Velders MP, Van Rhijn CM, Oskam E, Fleuren GJ, Warnaar SO, Litvinov S V. The impact of antigen density and antibody affinity on antibody dependent cellular cytotoxicity: Relevance for immunotherapy of carcinomas. Br J Cancer. 1998;78(4):478483. doi:10.1038/bjc. 1998.518

365. Nobuoka D, Yoshikawa T, Takahashi M, et al. Intratumoral peptide injection enhances tumor cell antigenicity recognized by cytotoxic $\mathrm{T}$ lymphocytes: A potential option for improvement in antigen-specific cancer immunotherapy. Cancer Immunol Immunother. 2013;62(4):639-652. doi:10.1007/s00262-012-1366-6

366. Chang CC, Campoli M, Ferrone S. HLA class I antigen expression in malignant cells: Why does it not always correlate with CTL-mediated lysis? Curr Opin Immunol. 2004;16(5):644-650. doi:10.1016/j.coi.2004.07.015

367. Guy CT, Webster MA, Schaller M, Parsons TJ, Cardiff RD, Muller WJ. Expression of the neu protooncogene in the mammary epithelium of transgenic mice induces metastatic disease. Proc Natl Acad Sci U S A. 1992;89(22):10578-10582.

doi:10.1073/pnas.89.22.10578 
368. Ardito CM, Grüner BM, Takeuchi KK, et al. EGF Receptor Is Required for KRASInduced Pancreatic Tumorigenesis. Cancer Cell. 2012;22(3):304-317. doi:10.1016/j.ccr.2012.07.024

369. Wen X, Kim S, Xiong R, et al. A Subset of CD $8 \alpha \beta+$ Invariant NKT Cells in a Humanized Mouse Model. J Immunol. 2015;195(4):1459-1469. doi:10.4049/jimmunol.1500574

370. Huhta H, Helminen O, Kauppila JH, et al. The Expression of Toll-like Receptors in Normal Human and Murine Gastrointestinal Organs and the Effect of Microbiome and Cancer. J Histochem Cytochem. 2016;64(8):470-482. doi:10.1369/0022155416656154

371. Krause AE, Adler BL, Makdisi J, et al. Nanoparticle-Encapsulated Doxorubicin Demonstrates Superior Tumor Cell Kill in Triple Negative Breast Cancer Subtypes Intrinsically Resistant to Doxorubicin. Precis Nanomedicine. 2018;1(3):173-182. doi:10.33218/prnano1(3).181029.1

372. Hassan AH, Hosny KM, Murshid ZA, Alhadlaq A, Alyamani A, Naguib G. Depot injectable biodegradable nanoparticles loaded with recombinant human bone morphogenetic protein-2: Preparation, characterization, and in vivo evaluation. Drug Des Devel Ther. 2015;9:3599-3606. doi:10.2147/DDDT.S79812 


\section{Supplementary Data}

\section{CpG Dose Curve}

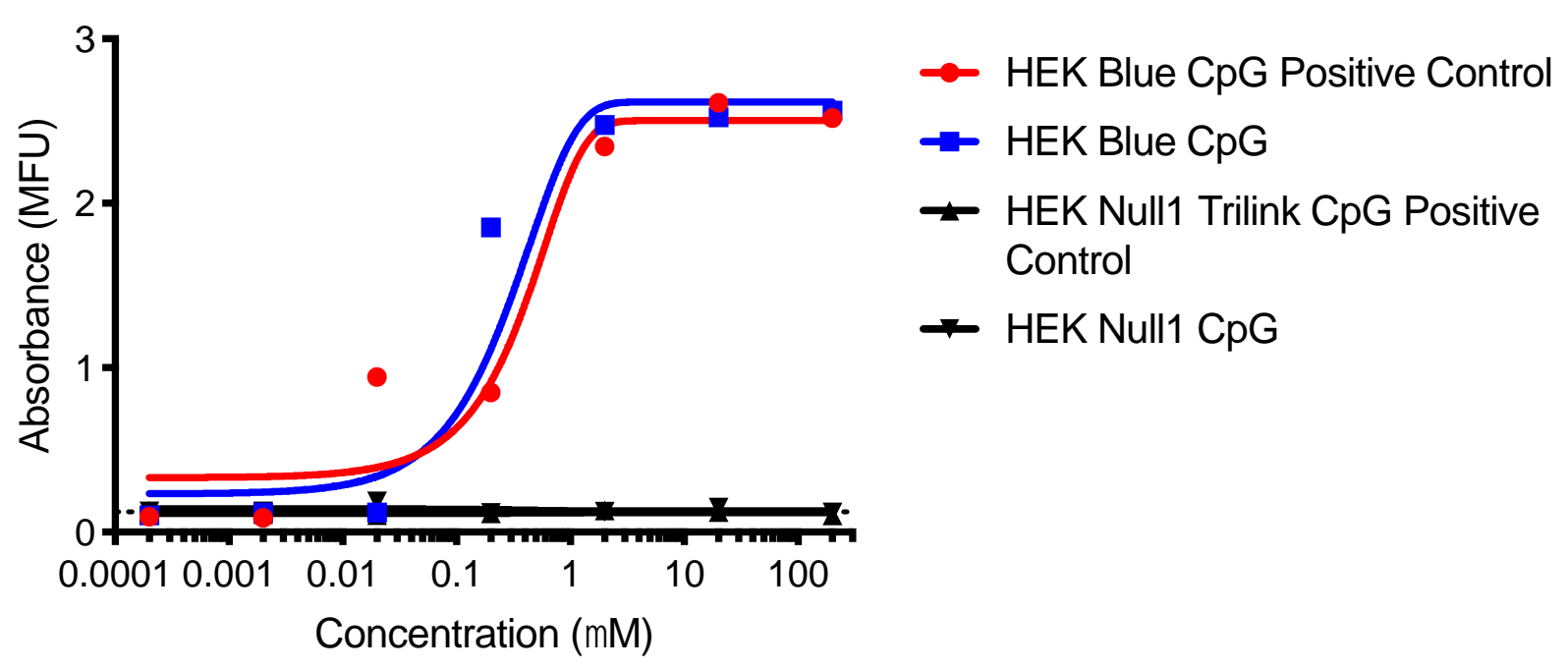

Supplementary Figure 1 CpG dose titration shows an EC50 of approximately 0.4uM. HEK-Blue TLR9 cells were treated with 7 different doses (10 fold dilutions with $200 \mu \mathrm{M}$ and $0.0002 \mu \mathrm{M}$ being the highest and lowest doses). The HEK Null1 (not expressing the TLR9 reporter) was used to control for false positives. 

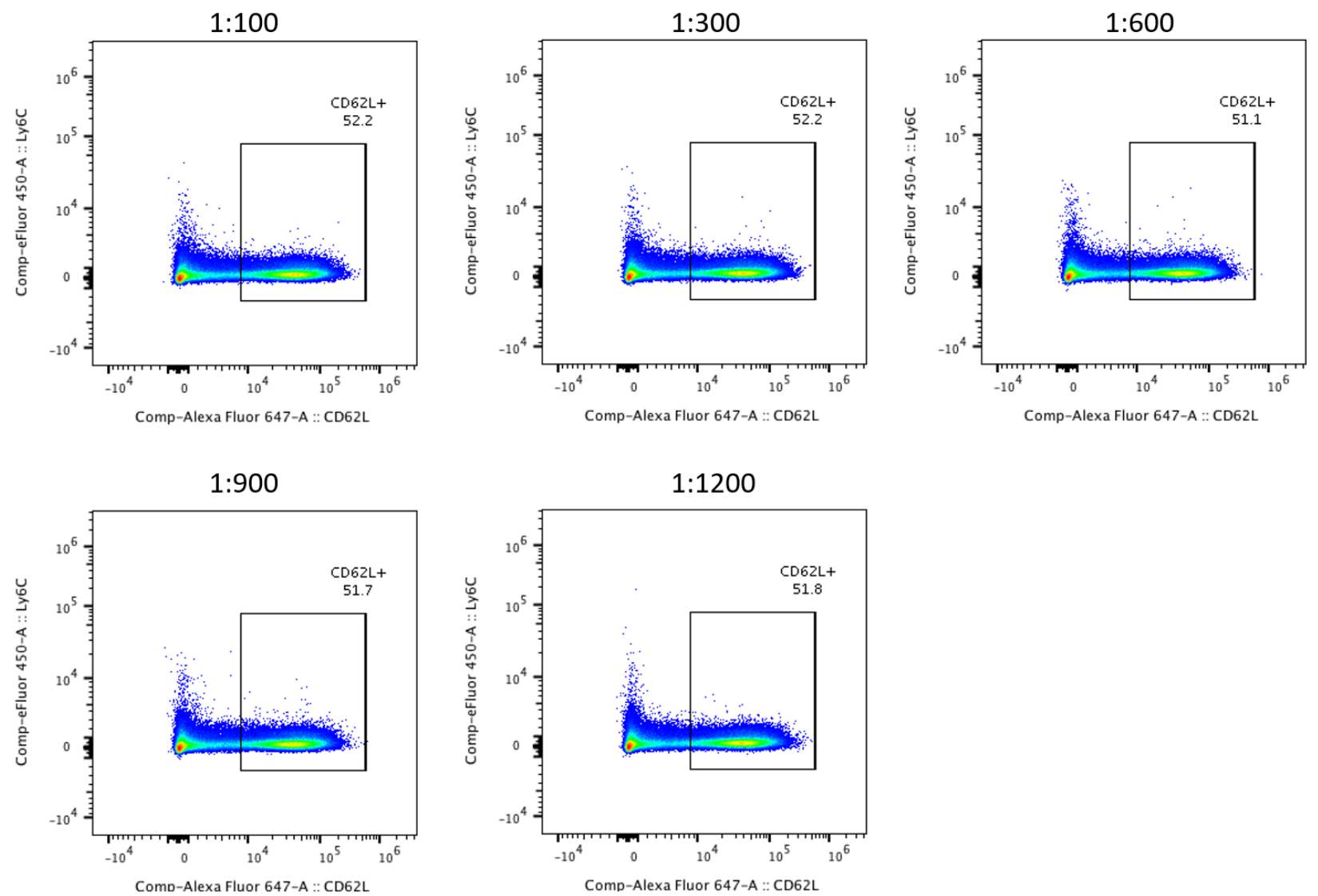

Supplmentary Figure 2 Example titration with dilutions noted above each graph. Antibody (CD62-Alexa Fluor 647). Dilution chosen would be based on equivalent staining with the use of less antibody (i.e. 1:1200). 

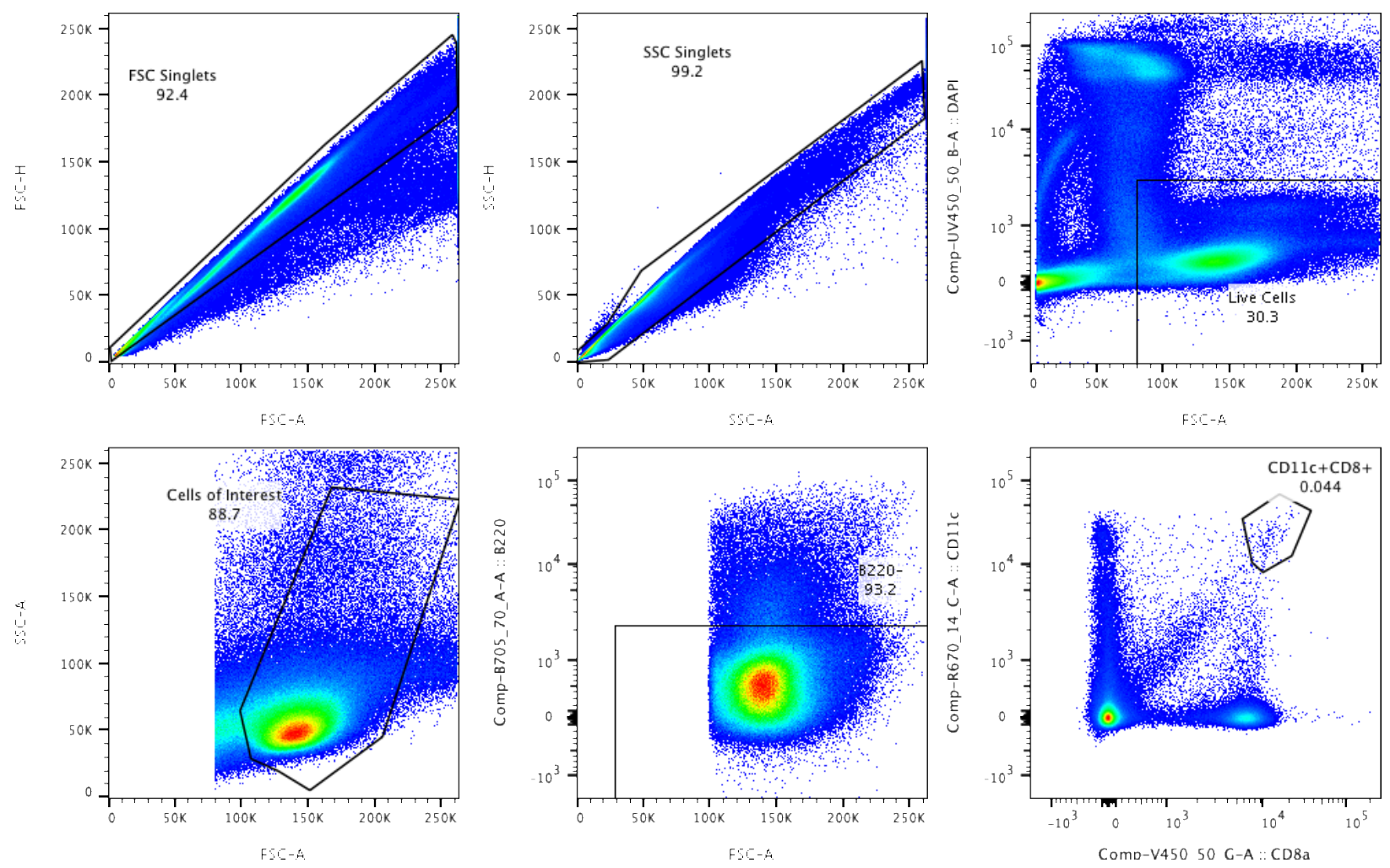

Supplementary Figure 3 Gating strategy for cDCl cells $\left(C l e c 9 a^{+}\right)$. Antibodies are noted in the methods section. Antibodies (CD8-BV421, CD11C-APC, MHCII-A488, CD103-PE, B220-APC, CD11b-APC-Cy7). 

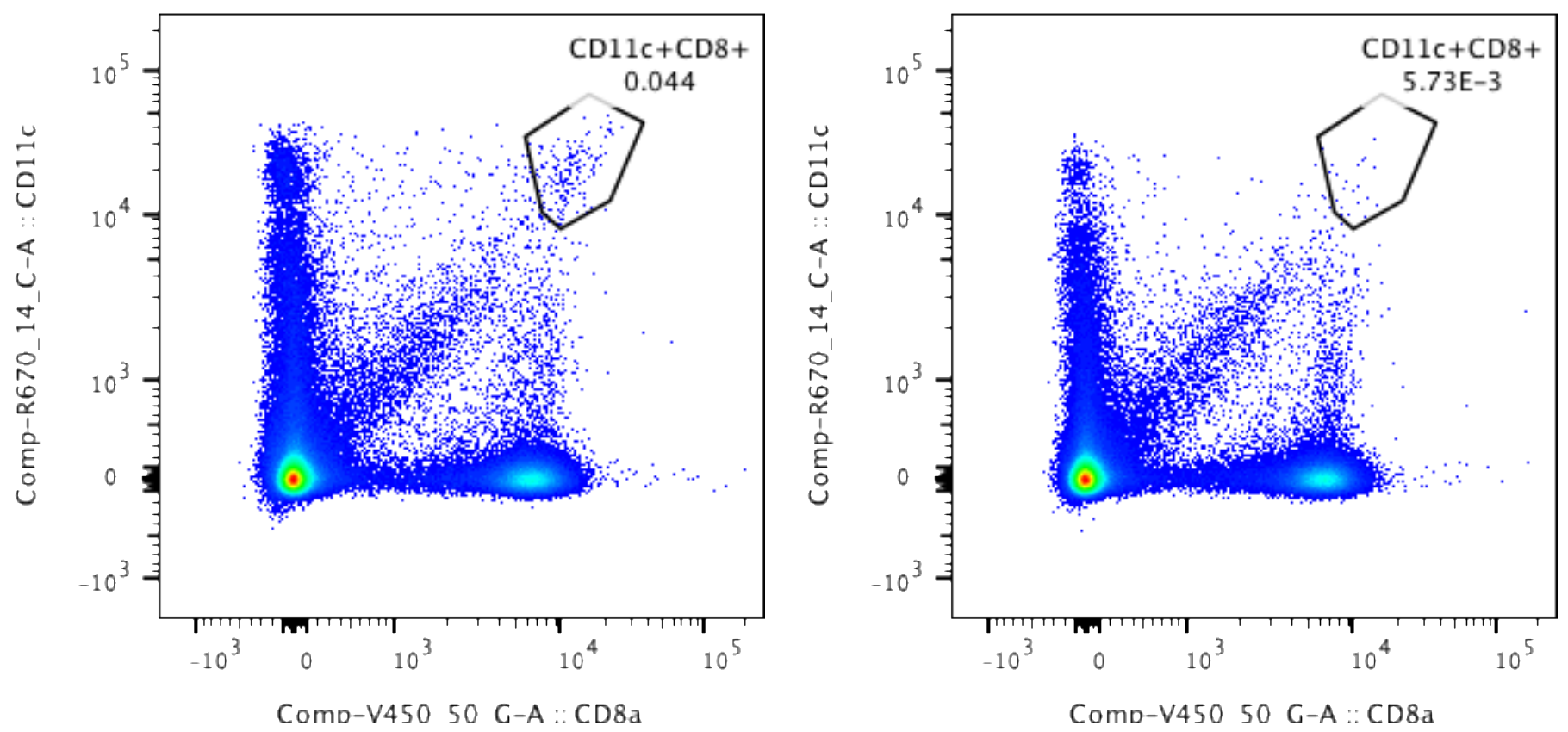

Supplementary Figure 4 Depletion of Clec $9 a^{+}$cell using diptheria toxin. Left Panel: PBS control. Right panel: Diptheria toxin administered i.p. days -2 and -1 prior to treatment. Antibodies (CD8-BV421, CD11C-APC, MHCII-A488, CD103-PE, B220-APC, CD11b$A P C-C y 7)$ 

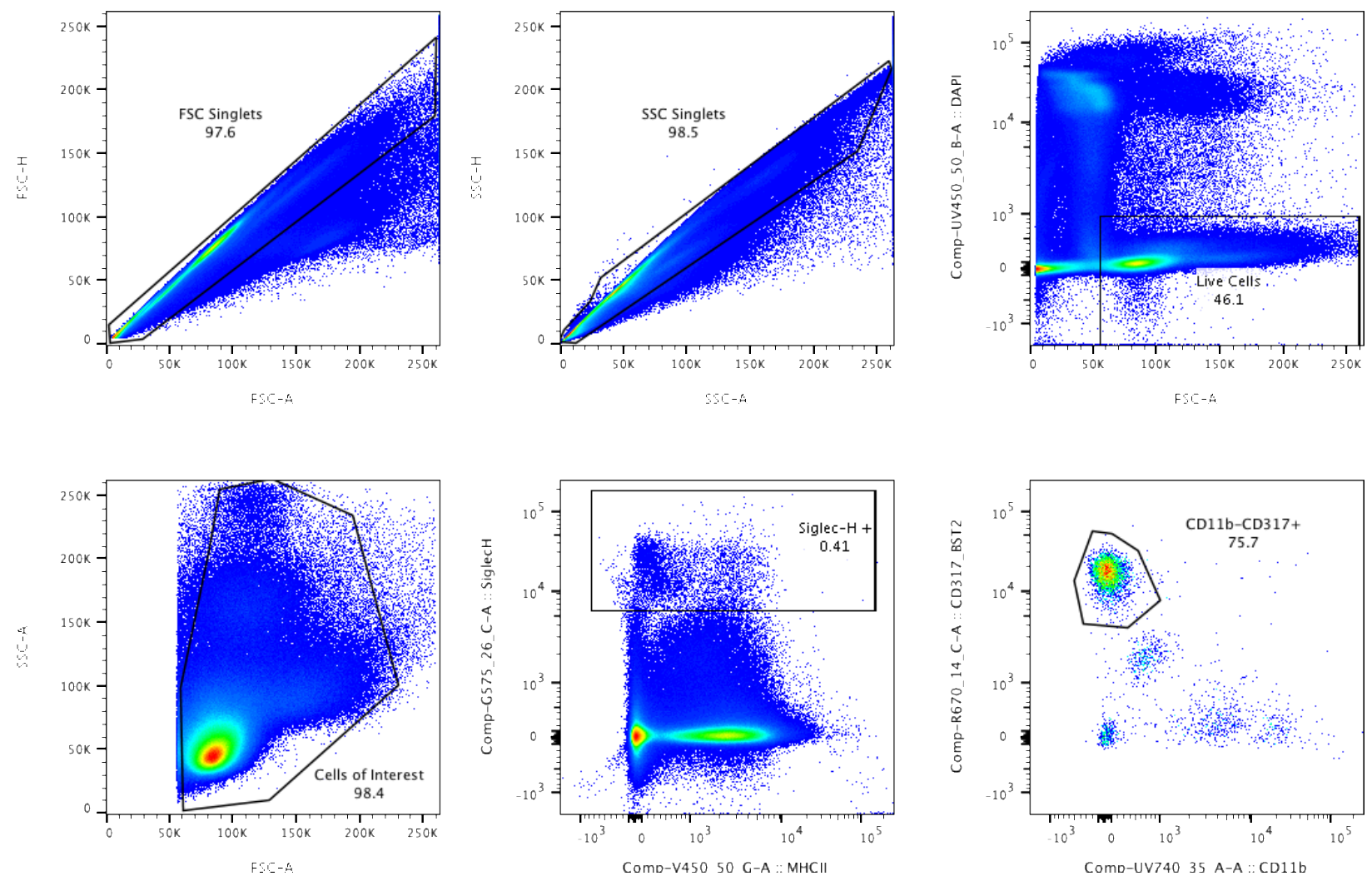

Supplementary Figure 5 Gating strategy for pDCs (siglec- $H^{+}$). Antibodies (CD11c-BV786, MHCII-BV421, CD11b-BUV737, Ly6C-PE-Cy7, CD317-APC, Siglec-H-PE, CD103-FITC). 

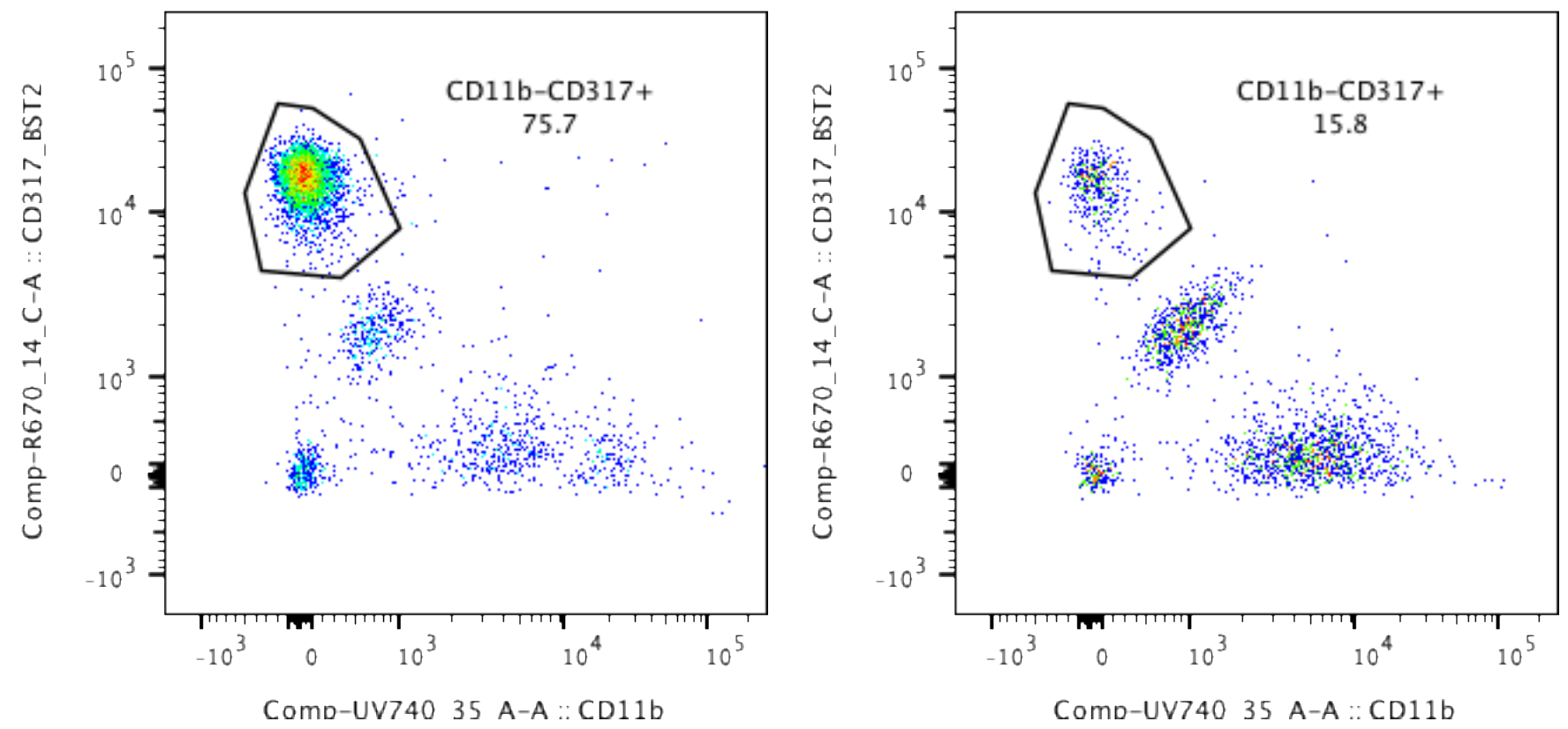

Supplementary Figure 6 Depletion of pDCs using diphtheria toxin. Left Panel: PBS control. Right panel: Diptheria toxin administered i.p. days -2 and -1 prior to treatment. Antibodies (CD11c-BV786, MHCII-BV421, CD11b-BUV737, Ly6C-PE-Cy7, CD317-APC, Siglec-H-PE, CD103-FITC). 